\title{
Synthesis of Isomerically Pure (Z)-Alkenes from Terminal Alkynes and Terminal Alkenes: Silver-Catalyzed Hydroalkylation of Alkynes
}

\author{
Mitchell T. Lee, ${ }^{\dagger}$ Madison B. Goodstein,${ }^{\dagger}$ and Gojko Lalic ${ }^{*}$ \\ Department of Chemistry, University of Washington, Seattle, Washington 98195, United States \\ email: lalic@chem.washington.edu
}




\section{Table of Contents}

General Information $\quad 3$

Reaction Development $\quad 3$

Verification of Product Stereochemistry and Determination of the E/Z Selectivity 4

Synthesis of TriAgCl and IPrAgCl 17

Hydroalkylation of Terminal Alkynes: General Procedure 17

Characterization of Z-Selective Hydroalkylation Products: Alkynes 18

Characterization of Z-Selective Hydroalkylation Products: Alkylboranes 27

Gram Scale Reaction 35

Alkyne Starting Materials $\quad 35$

Alkene Starting Materials for Alkylboranes $\quad 40$

Mechanistic Studies $\quad 43$

References $\quad 46$

$\begin{array}{ll}\text { Spectroscopic Data } & 49\end{array}$ 


\section{General Information}

All reactions were performed under a nitrogen atmosphere with flame-dried or oven-dried (120 ${ }^{\circ} \mathrm{C}$ ) glassware, using standard Schlenk techniques, or in a glovebox (Nexus II from Vacuum Atmospheres). Column chromatography was performed using a Biotage Iso-1SV flash purification

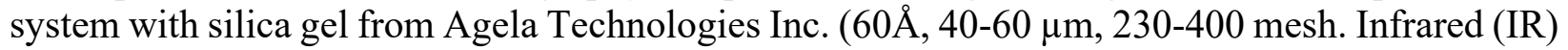
spectra were recorded on a Perkin Elmer Spectrum RX I spectrometer. IR peak absorbencies are represented as follows: $\mathrm{s}=$ strong, $\mathrm{m}=$ medium, $\mathrm{w}=$ weak, $\mathrm{br}=$ broad. ${ }^{1} \mathrm{H}$ - and ${ }^{13} \mathrm{C}-\mathrm{NMR}$ spectra were recorded on a Bruker AV-300 or AV-500 spectrometer. ${ }^{1} \mathrm{H}$ NMR chemical shifts $(\delta)$ are reported in parts per million (ppm) downfield of TMS and are referenced relative to residual solvent peak $\left(\mathrm{CDCl}_{3}(7.26 \mathrm{ppm})\right) .{ }^{13} \mathrm{C} \mathrm{NMR}$ chemical shifts are reported in parts per million downfield of TMS and are referenced to the carbon resonance of the solvent $\left(\mathrm{CDCl}_{3}(77.2\right.$ ppm)). ${ }^{19} \mathrm{~F}$ NMR chemical shifts $(\delta)$ are reported in parts per million (ppm) and are referenced relative to the internal standard, hexafluorobenzene. Data are represented as follows: chemical shift, multiplicity $(\mathrm{s}=$ singlet, $\mathrm{d}=$ doublet, $\mathrm{t}=$ triplet, $\mathrm{q}=$ quartet, $\mathrm{p}=$ pentet, hept $=$ heptet, $\mathrm{m}=$ multiplet), coupling constants in Hertz $(\mathrm{Hz})$, intergration. Mass spectra were collected on a JEOL HX-110 mass spectrometer. Gas Chromatography (GC) analysis was performed on a Shimadzu GC-2010 instrument with a flame ionization detector and a SHRXI-5MS column (15 m, $0.25 \mathrm{~mm}$ inner diameter, $0.25 \mu \mathrm{m}$ film thickness). The following temperature program was used: 2 min @ $60{ }^{\circ} \mathrm{C}, 13{ }^{\circ} \mathrm{C} / \min$ to $160{ }^{\circ} \mathrm{C}, 30{ }^{\circ} \mathrm{C} / \min$ to $250{ }^{\circ} \mathrm{C}, 5.5 \min @ 250{ }^{\circ} \mathrm{C}$.

Materials: THF, $\mathrm{CH}_{2} \mathrm{Cl}_{2}$, ether, benzene, and toluene were degassed and dried by passing through columns of neutral alumina. Anhydrous methanol was purchased from Millipore Sigmaand was degassed and stored over $4 \AA$ molecular sieves. Isooctane was purchased from Fisher Scientific and was degassed and stored over $4 \AA \AA$ molecular sieves. Deuterated solvents were purchased from Cambridge Isotope Laboratories, Inc. and were stored over $4 \AA$ molecular sieves prior to use. Commercial reagents were purchased from Millipore Sigma, TCI America, GFS-Chemicals, ArkPharm, Combi-Blocks, Oakwood Chemicals, Strem Chemicals and Alfa Aesar. 9-BBN Dimer was purchased from Millipore Sigma and recrystallized from THF.

\section{Reaction Development}

All reactions were performed on a $0.05 \mathrm{mmol}$ scale with the stoichiometry shown in Table S1. In a nitrogen-filled glovebox a dram vial was charged with a stir bar, $\mathrm{LiO} t$-Bu (1.5 equiv), catalyst, methyl 4-(pent-4-yn-1-yloxy)benzoate, 1,3,5-trimethoxy benzene (TMB, internal standard), 9-(3phenylpropyl)-9-borabicyclo[3.3.1]nonane (1.3 equiv), methanol (1.1 equiv) and solvent. The reaction mixture was stirred at $45{ }^{\circ} \mathrm{C}$ and monitored by Gas Chromatography for reaction completion. An aliquot was taken every 24 hours. 


\section{Table S1.}

Reaction Development and Control Experiments

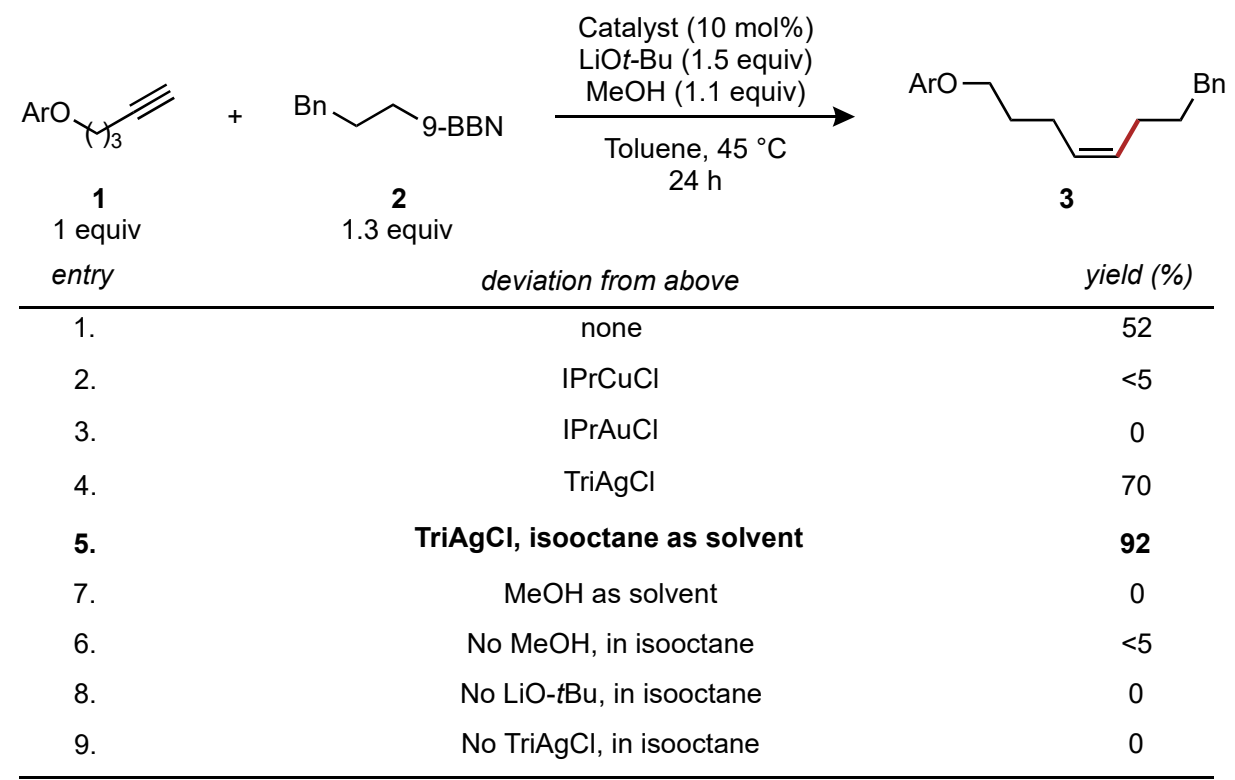

Ar = methyl 4-benzoate

\section{Verification of Product Stereochemistry and Determination of the E/Z Selectivity}

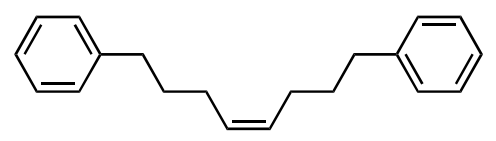

(Z)-1,8-diphenyloct-4-ene

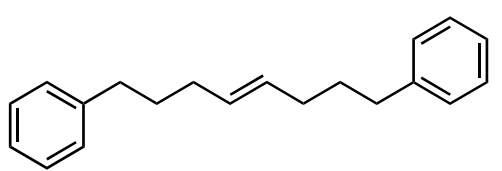

(E)-1,8-diphenyloct-4-ene

(Z)-1,8-diphenyloct-4-ene (product 4) and (E)-1,8-diphenyloct-4-ene (E isomer of 4) have been previously synthesized and characterized ${ }^{1}$. Provided are GC traces of pure (Z)-1,8-diphenyloct-4ene, pure $(E)$-1,8-diphenyloct-4-ene, a mixture of both pure isomers, and a GC trace of the crude reaction mixture of the synthesis of $(Z)$-1,8-diphenyloct-4-ene using our method. 
GC trace of isolated (Z)-1,8-diphenyloct-4-ene (product 4).

Intensity

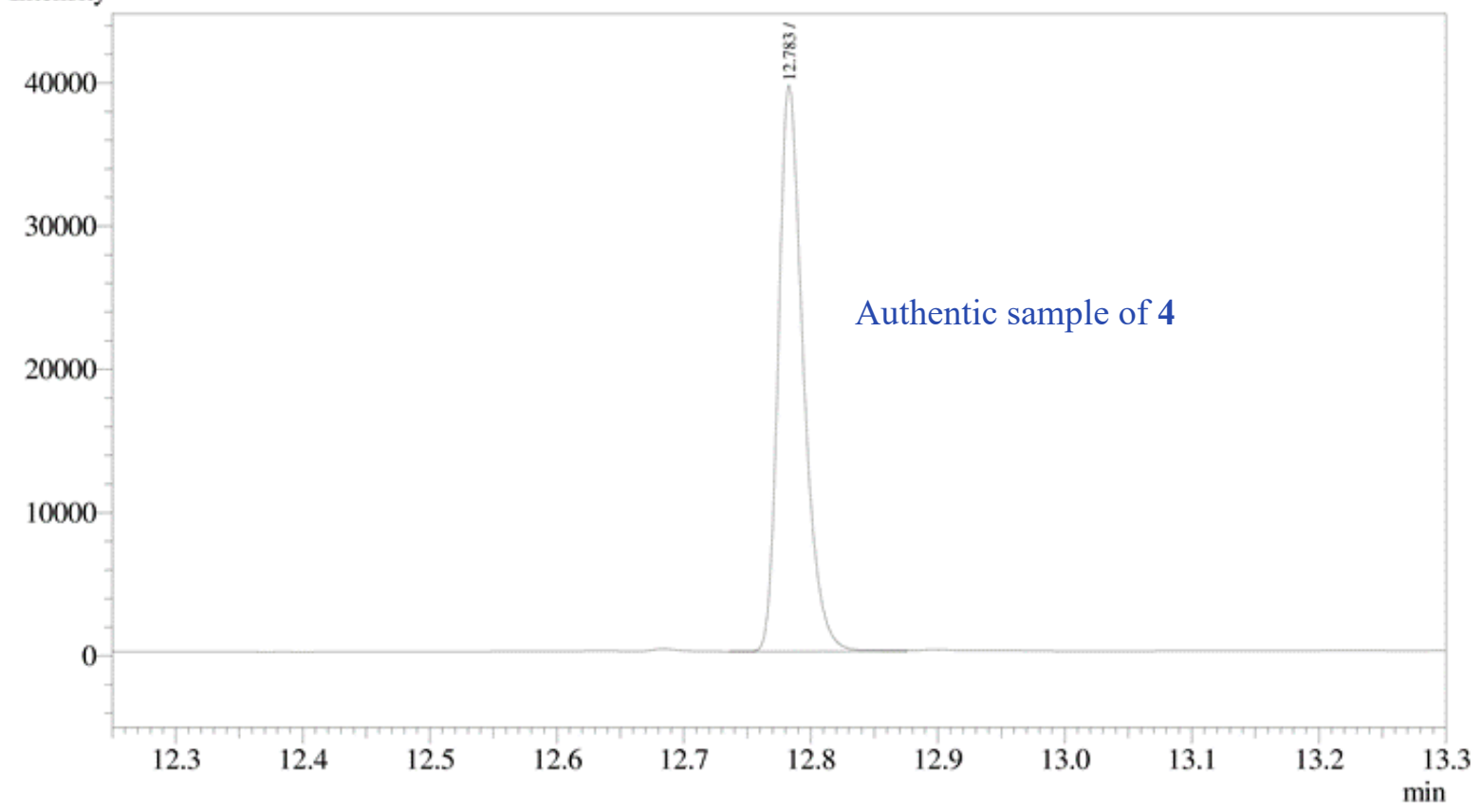

\begin{tabular}{lrrrrl} 
Peak\# & Ret.Time & \multicolumn{1}{c}{ Area } & Height & Conc. \\
& 1 & 12.783 & 53920 & 37688 & 100.000 \\
\hline Total & & 53920 & 37688 & \\
\hline
\end{tabular}

GC trace of isolated (E)-1,8-diphenyloct-4-ene (E isomer of 4). Intensity

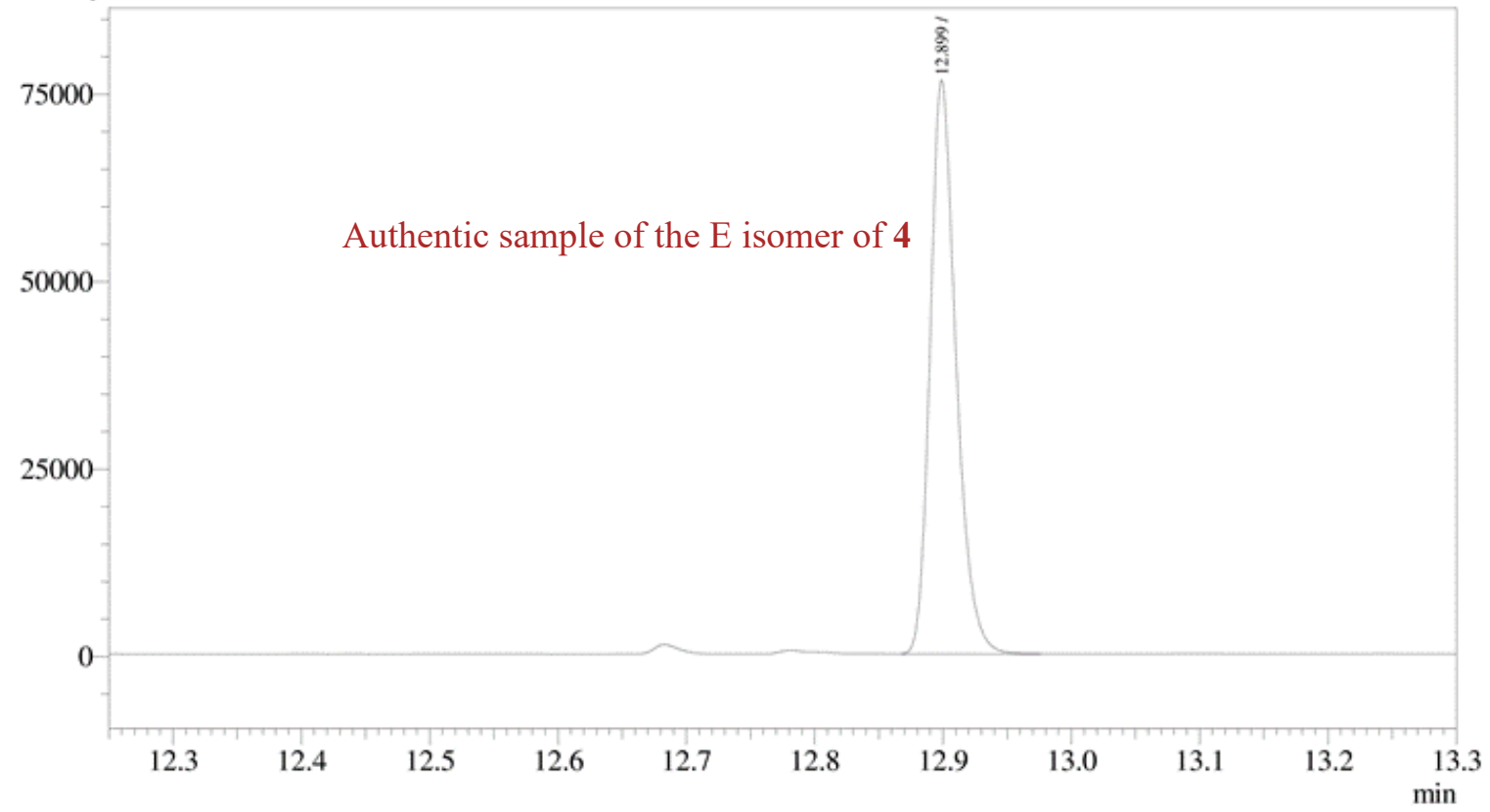

\begin{tabular}{|c|c|c|c|c|}
\hline${ }_{1}$ Peak\# & $\begin{array}{r}\text { Ret.Time } \\
12.899\end{array}$ & $\begin{array}{l}\text { Area } \\
\quad 107803\end{array}$ & $\begin{array}{l}\text { Height } \\
74075\end{array}$ & $\begin{array}{l}\text { Conc. } \\
100.000\end{array}$ \\
\hline Total & & 107803 & 74075 & \\
\hline
\end{tabular}


GC trace of a mixture of the authentic samples of (Z)-1,8-diphenyloct-4-ene (product 4) and $(E)$ 1,8-diphenyloct-4-ene ( $\mathrm{E}$ isomer of 4).

Intensity

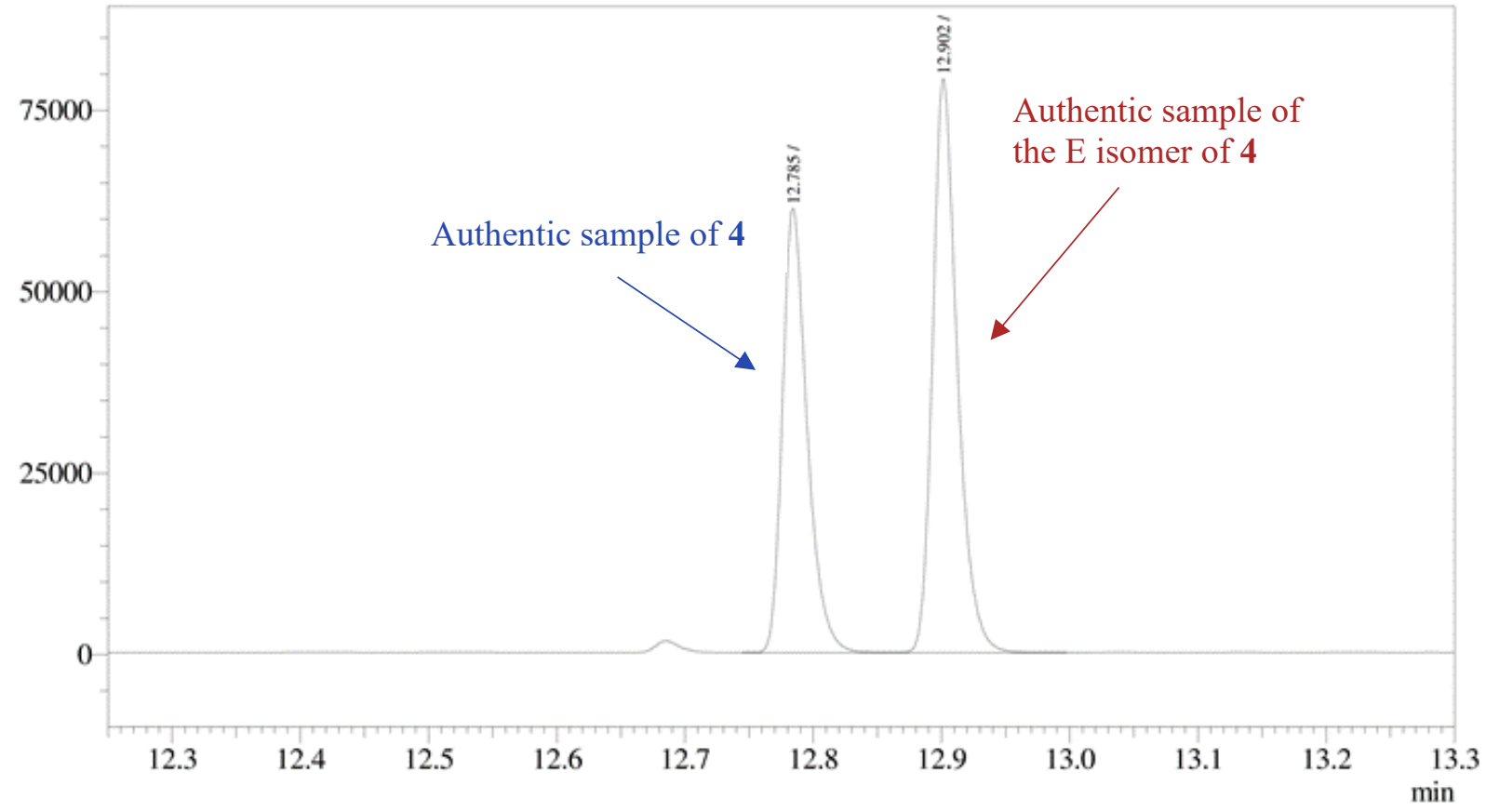

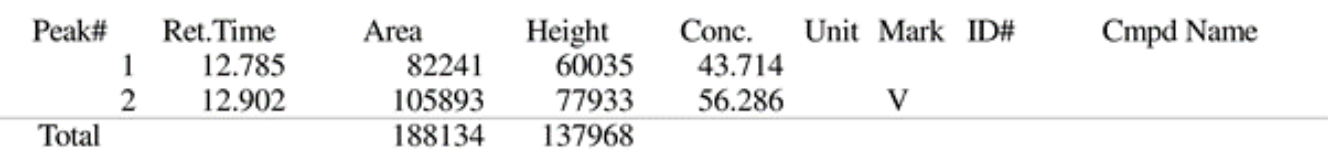

$\mathrm{E} / \mathrm{Z}$ selectivity of the following reaction was determined by GC/FID analysis of an aliquot taken from a crude reaction mixture at the end of the reaction:
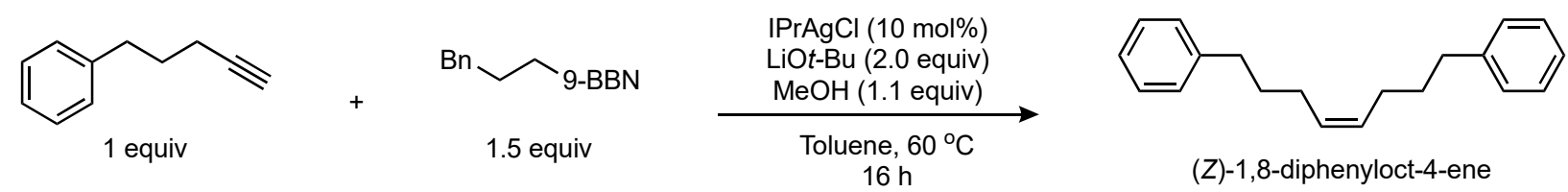

GC/FID trace obtained by analysis of a crude reaction mixture

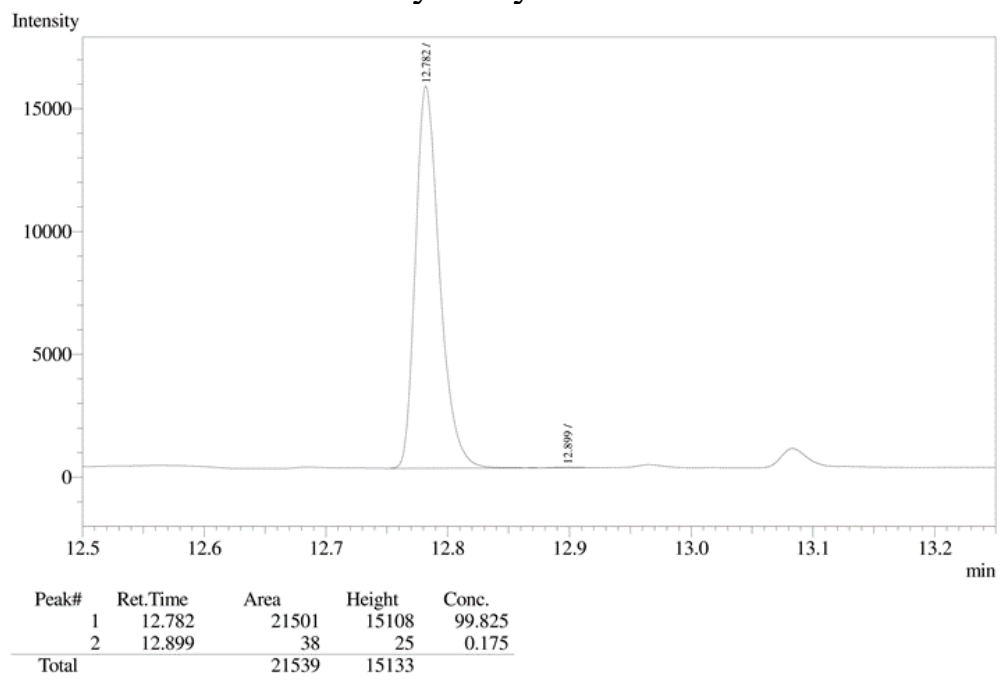


Expansion of the product peak:

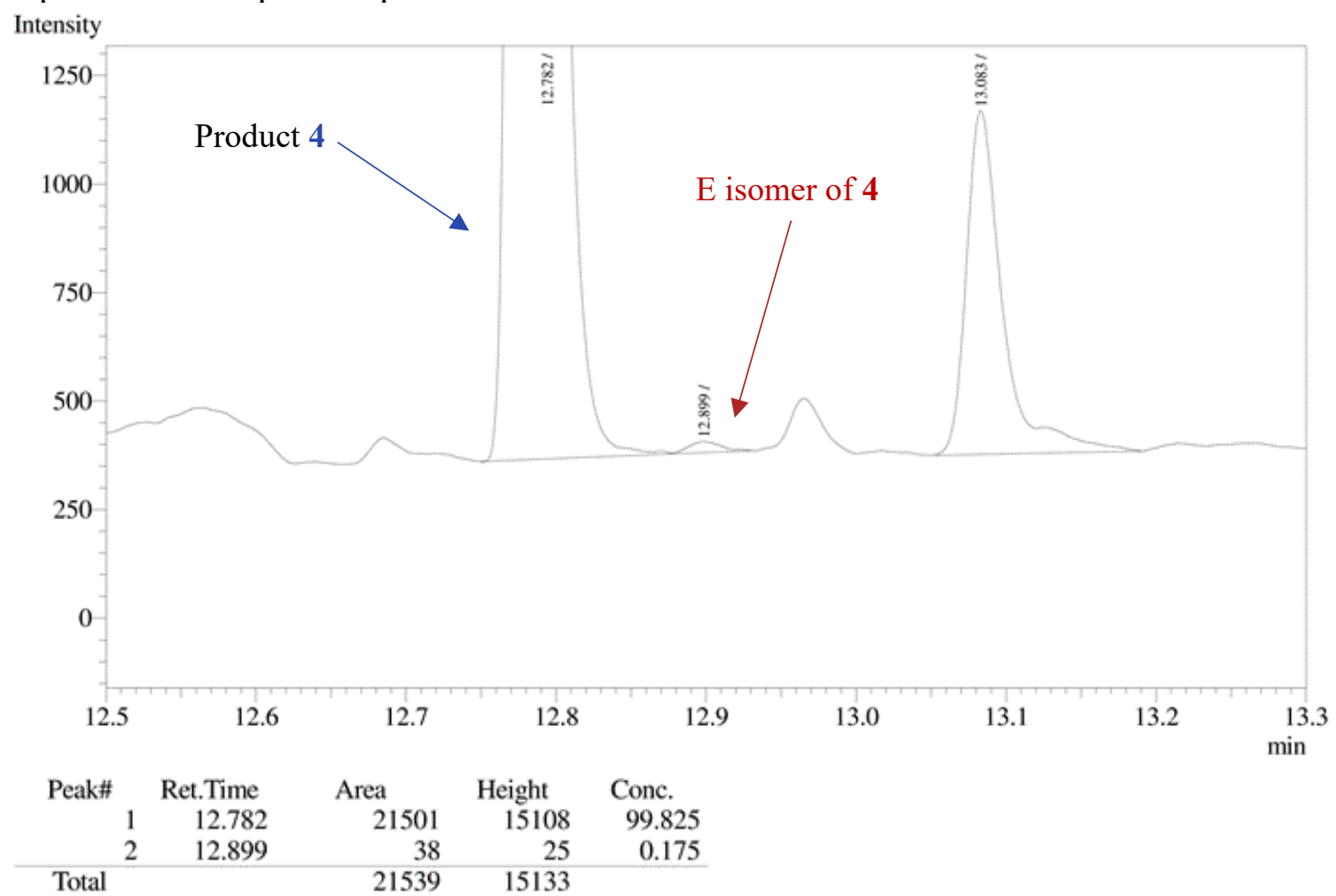

Relative concentrations of the two isomers are $570: 1 \mathrm{Z}$ to $\mathrm{E}$.

\section{Determination of $\mathrm{Z} / \mathbf{E}$ selectivity:}

The selectivity of the hydroalkylation reaction was determined for several substrates. The $\mathrm{E}$ isomer of product 17 was synthesized according to a known procedure. ${ }^{2}$ For other substrates, purified Zalkene products isolated from the hydroalkylation reaction were subjected to a known isomerization procedure ${ }^{3}$ to obtain mixtures of $\mathrm{Z}$ and $\mathrm{E}$ isomers. These mixtures were then used to determine $\mathrm{GC}$ retention times of the $\mathrm{E}$ isomer in order to analyze the selectivity in the crude reaction mixtures. Analysis was done using GC/FID instrument.

Preparation of samples for the analysis of the reaction selectivity: After completion of the catalytic reaction, an aliquot of the crude reaction mixture was taken, passed through a plug of silica with ethyl acetate and analyzed by GC.

\section{$\mathrm{Z} / \mathrm{E}$ selectivity in the formation of 3:}<smiles>COc1ccc(OCCC/C=C\CCCc2ccccc2)cc1</smiles> 
GC trace from the analysis of purified $\mathbf{3}$ :

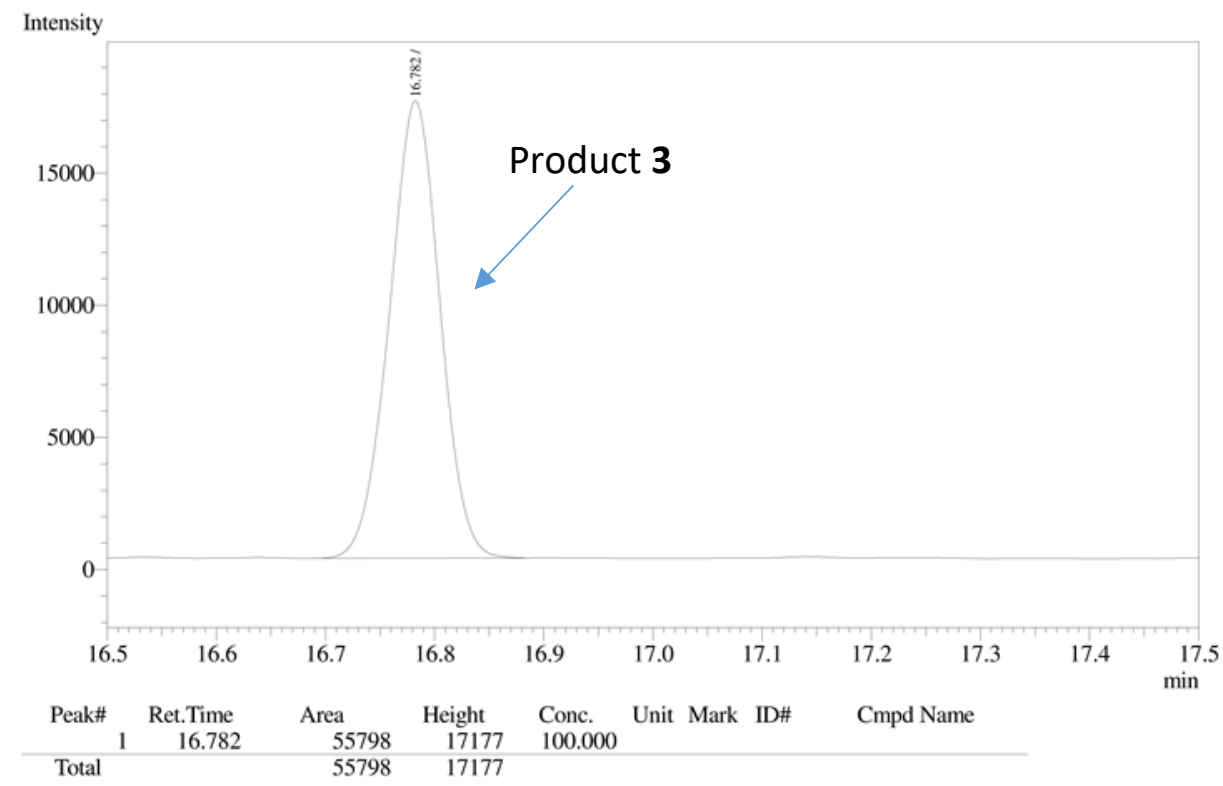

GC trace from the analysis of $\mathbf{3}$ after isomerization (see above):

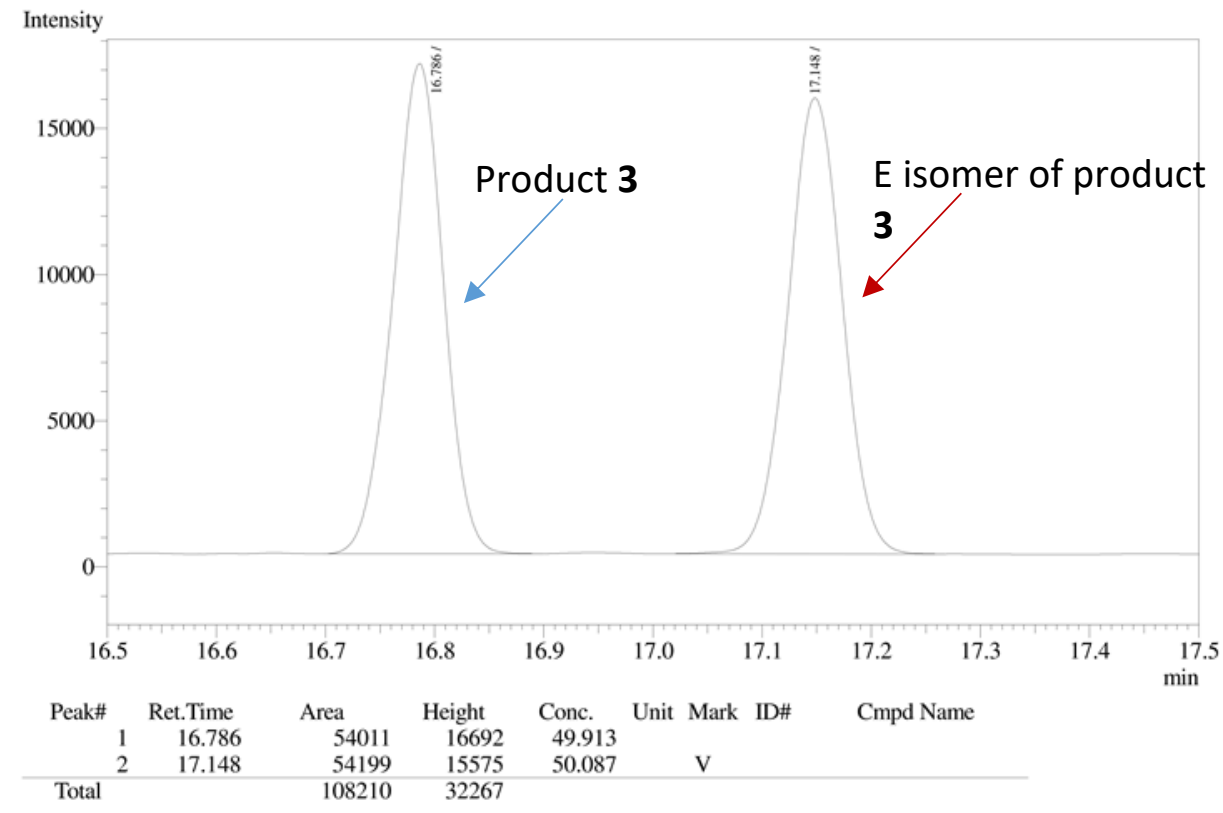


GC trace from the analysis of the crude reaction mixture:

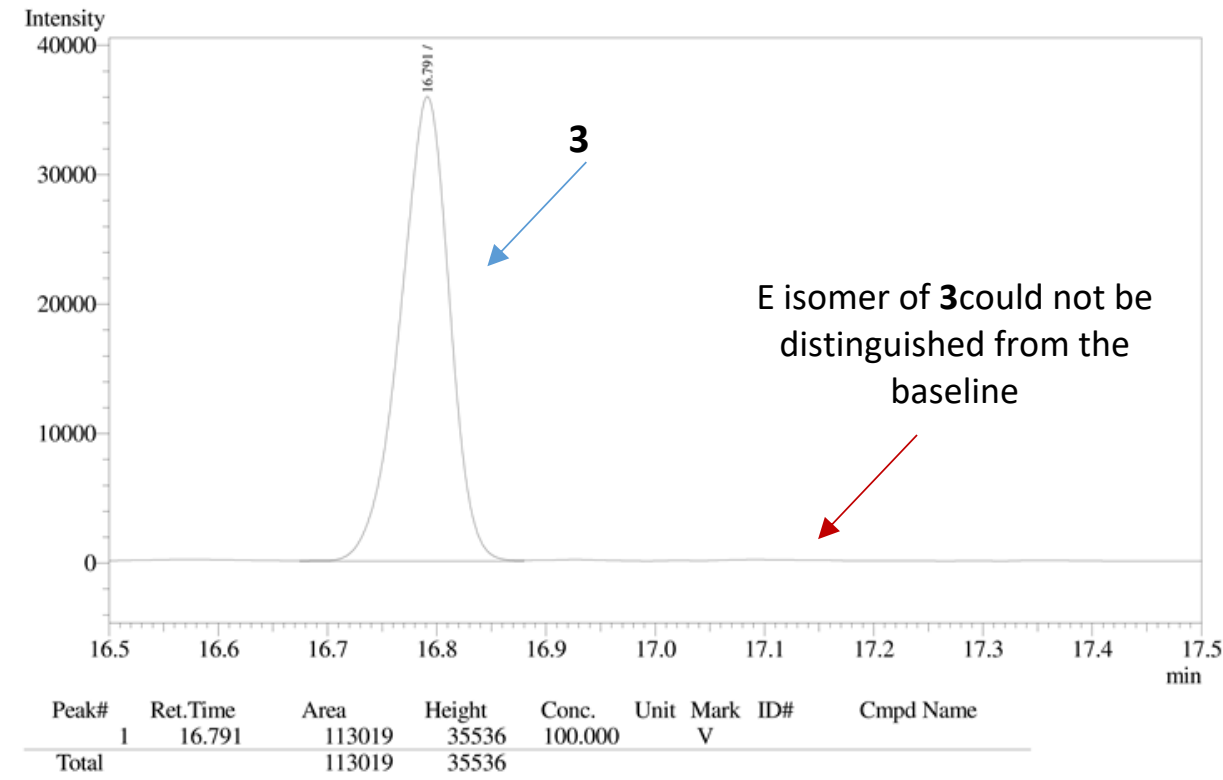

The Z:E ration $>500: 1$

\section{$\mathrm{Z} / \mathrm{E}$ selectivity in the formation of 17 :}

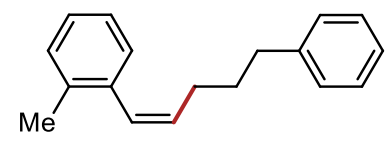

17

GC trace from the analysis of purified $\mathbf{1 7}$ :

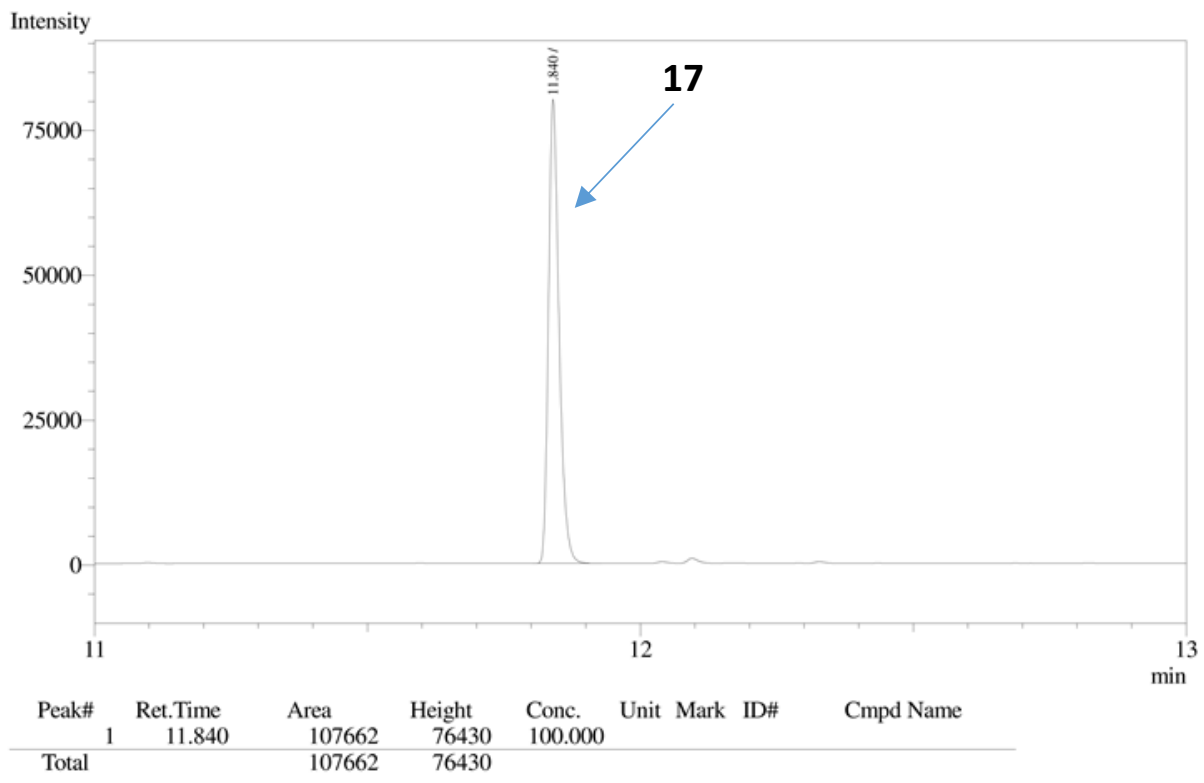


$\mathrm{GC}$ trace from the analysis of the $\mathrm{E}$ isomer of $\mathbf{1 7}$ :

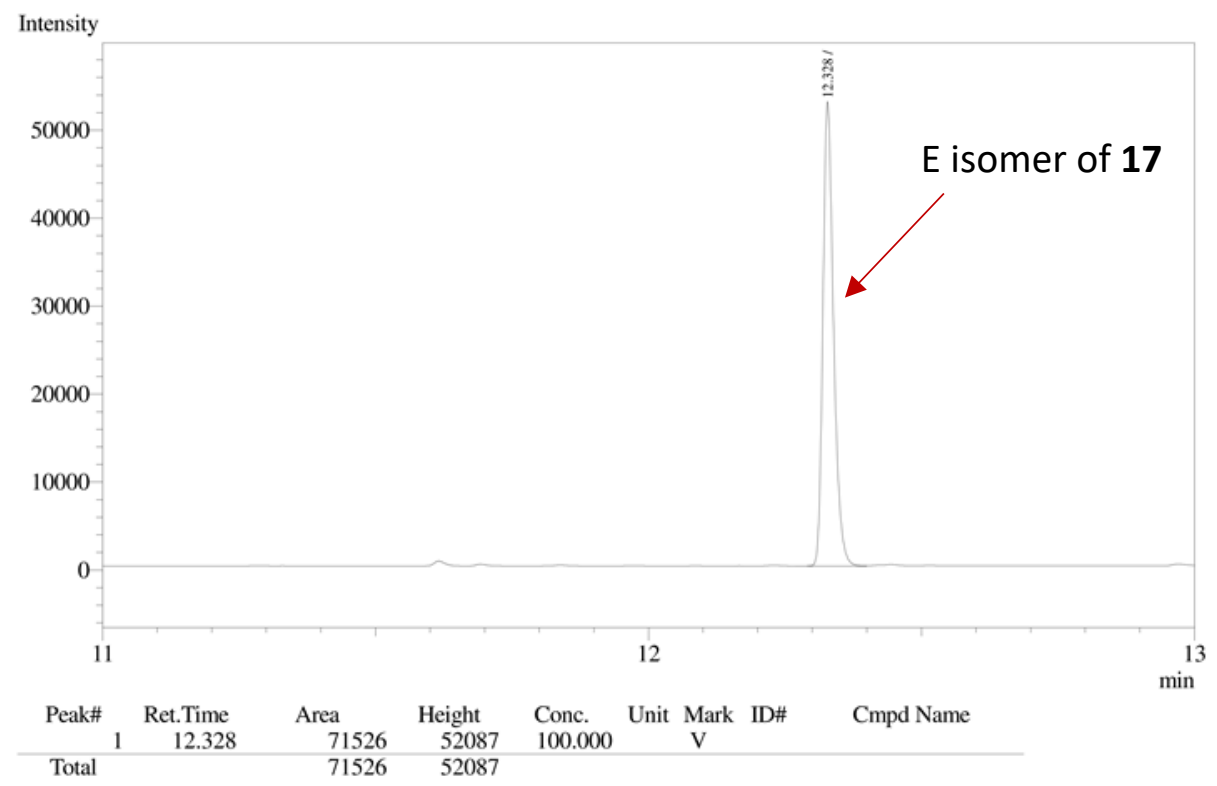

GC trace from the analysis of the crude reaction mixture:

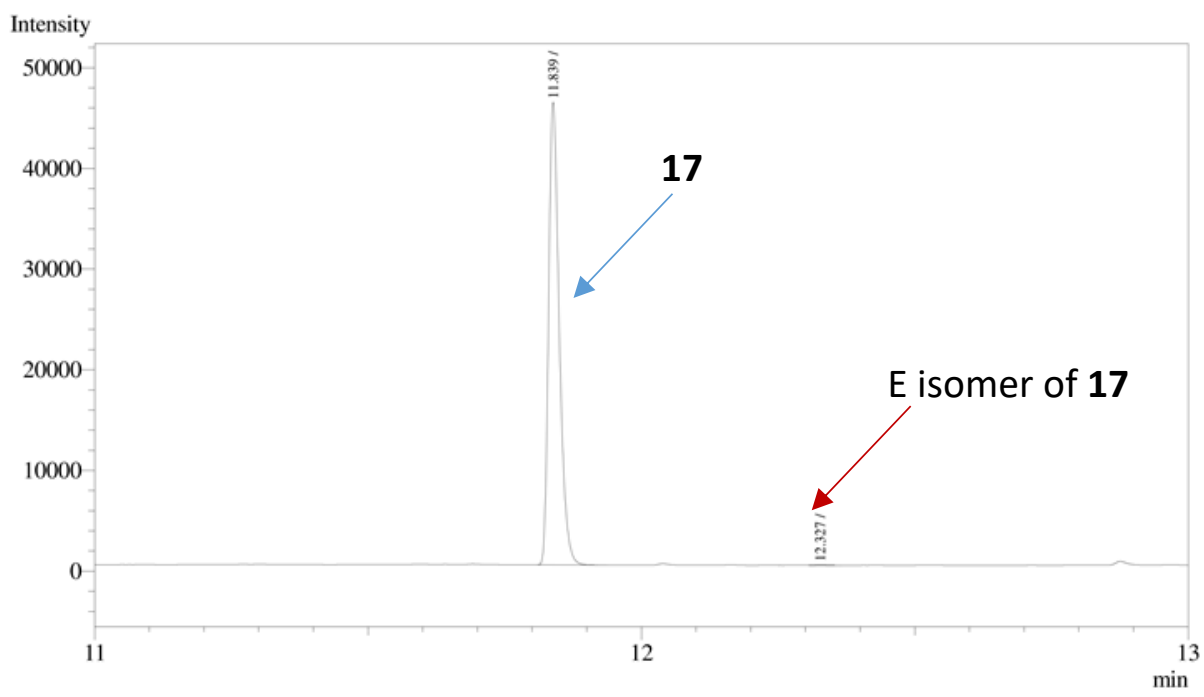


Close up of the GC trace from the analysis of a sample from the crude reaction mixture:

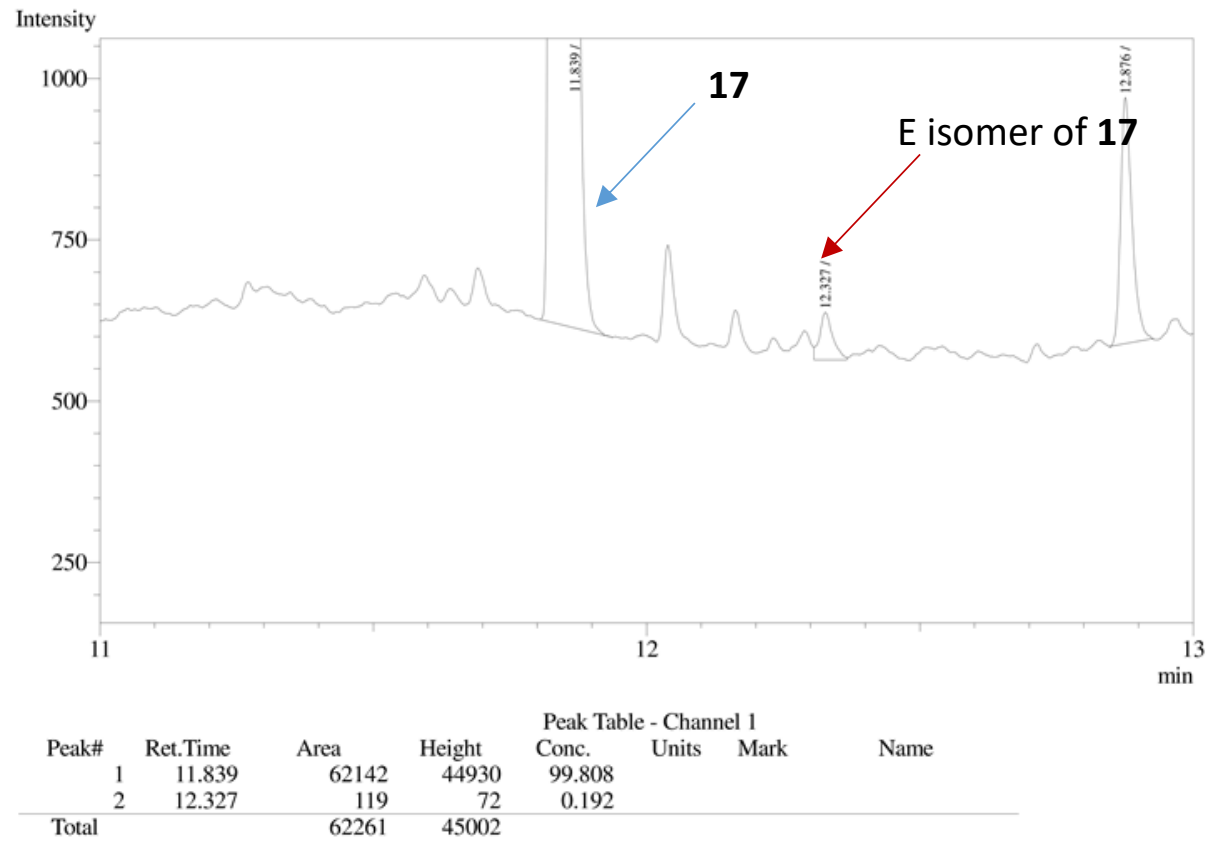

The ratio of concentrations of the two isomers is 519:1 (Z:E).

\section{$\mathrm{Z} / \mathrm{E}$ selectivity in the formation of 35 :}

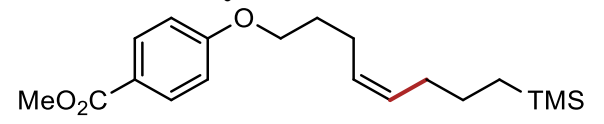

35

$\mathrm{GC}$ trace from the analysis of purified $\mathbf{3 5}$ :

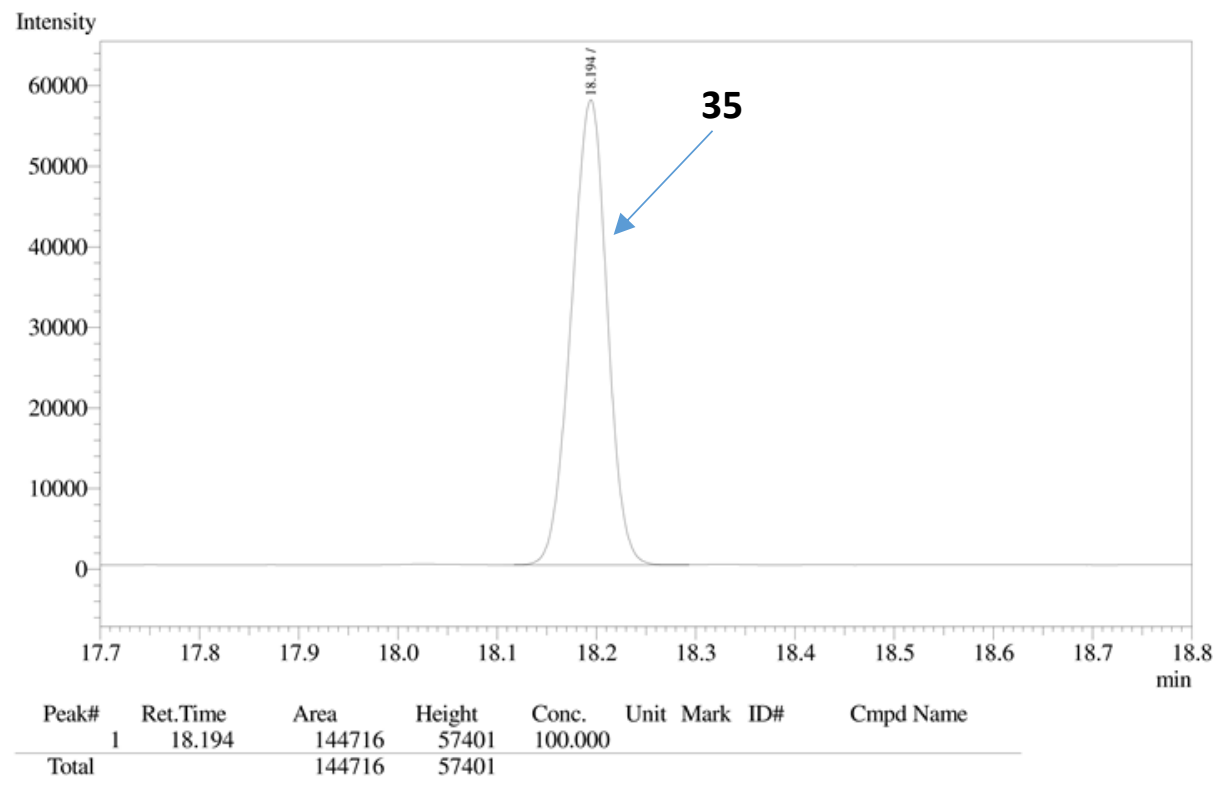


GC trace from the analysis of a mixture of $\mathbf{3 5}$ and the $\mathrm{E}$ isomer of $\mathbf{3 5}$ :

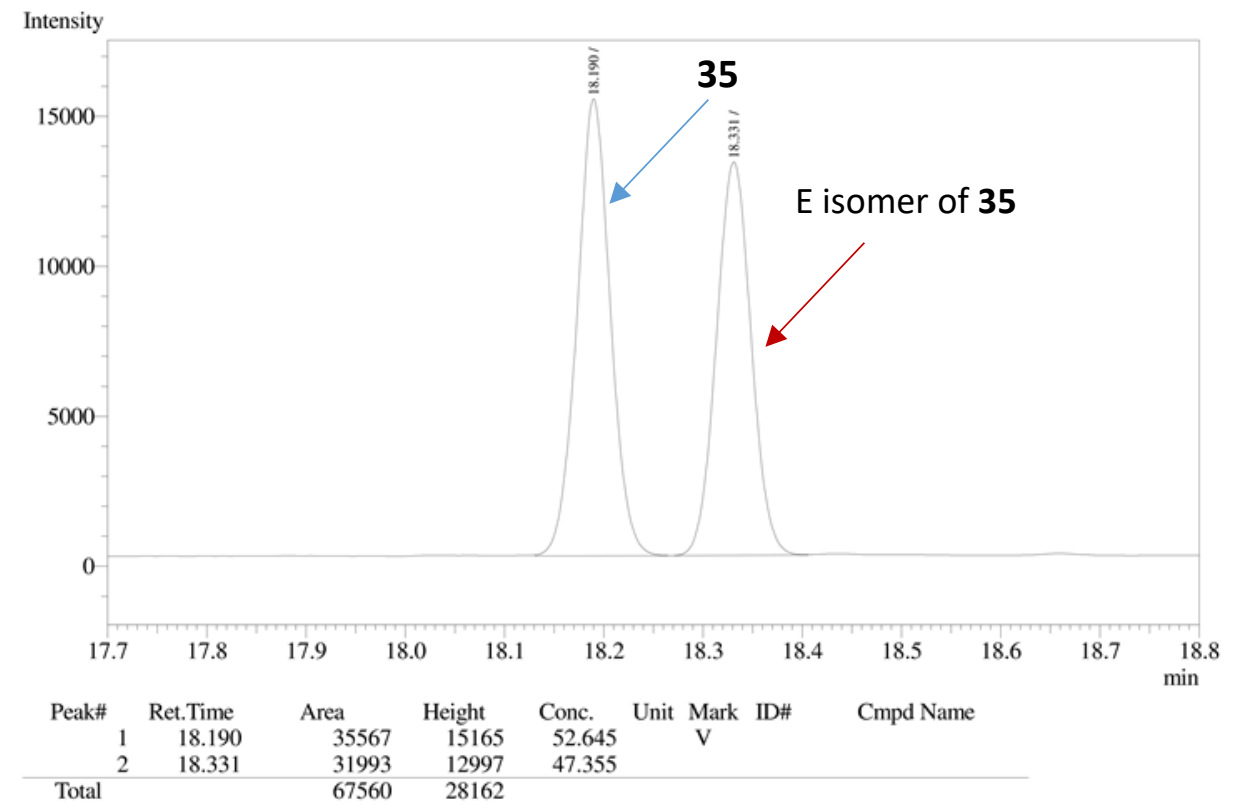

GC trace from the analysis of the crude reaction mixture:

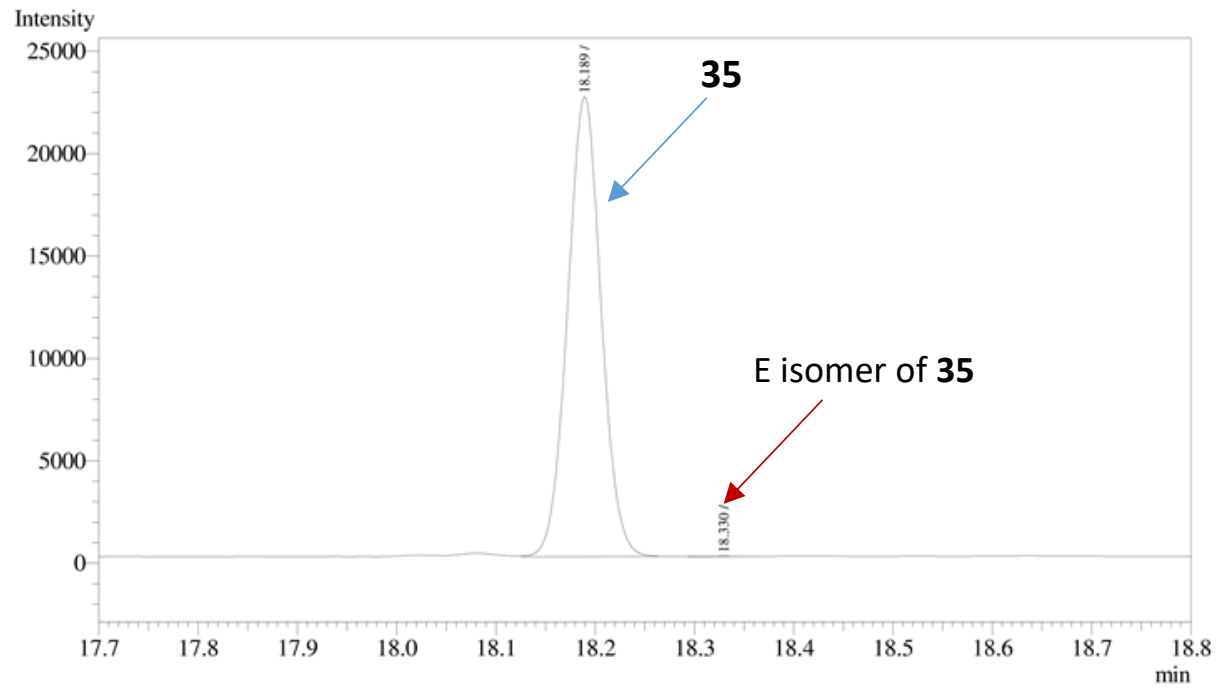


Close up of the GC trace from the analysis of the crude reaction mixture:

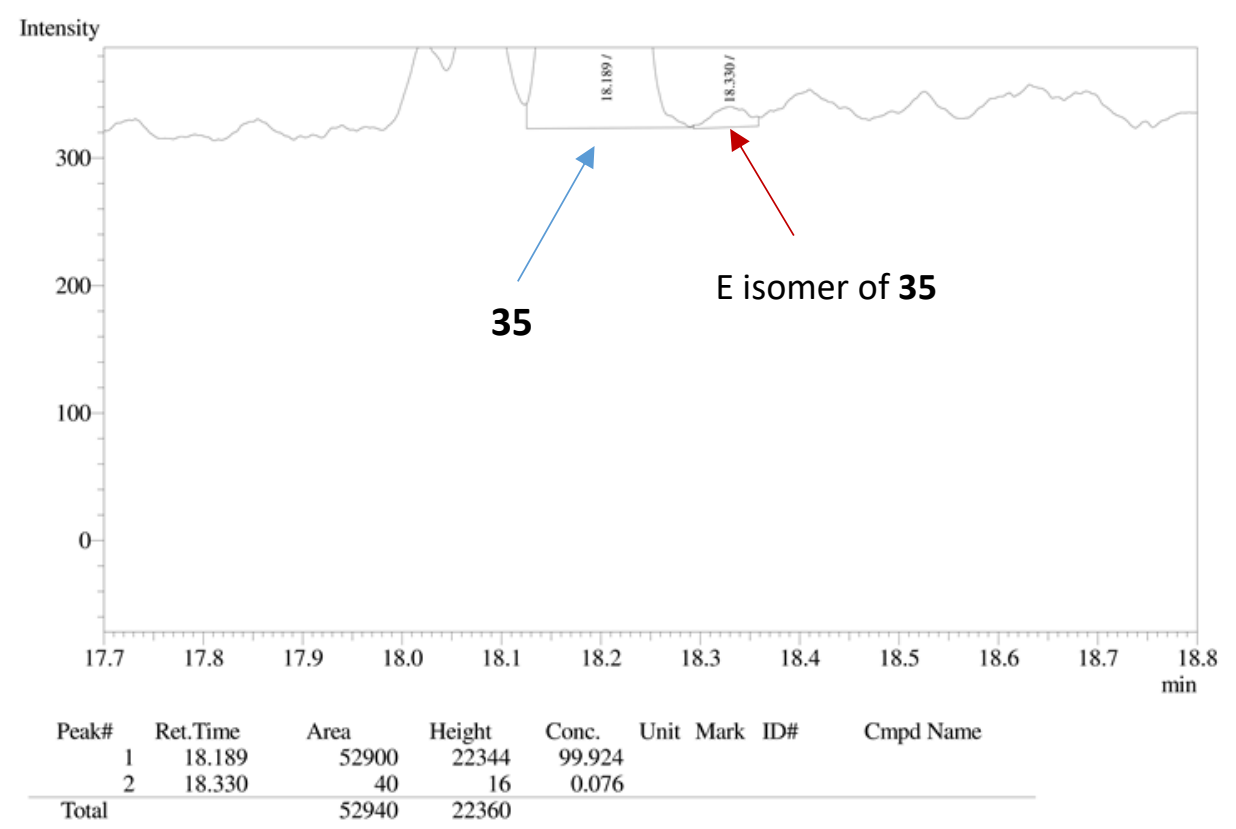

The ratio of concentrations of the two isomers is $>500: 1$ (Z:E).

Z/E selectivity in the formation of 39:

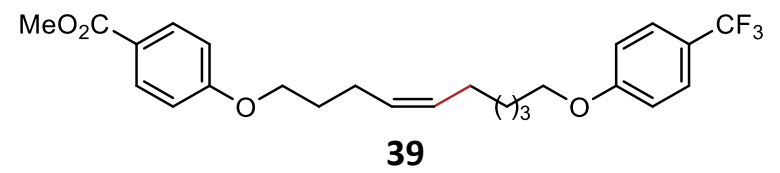

GC trace from the analysis of purified 39:

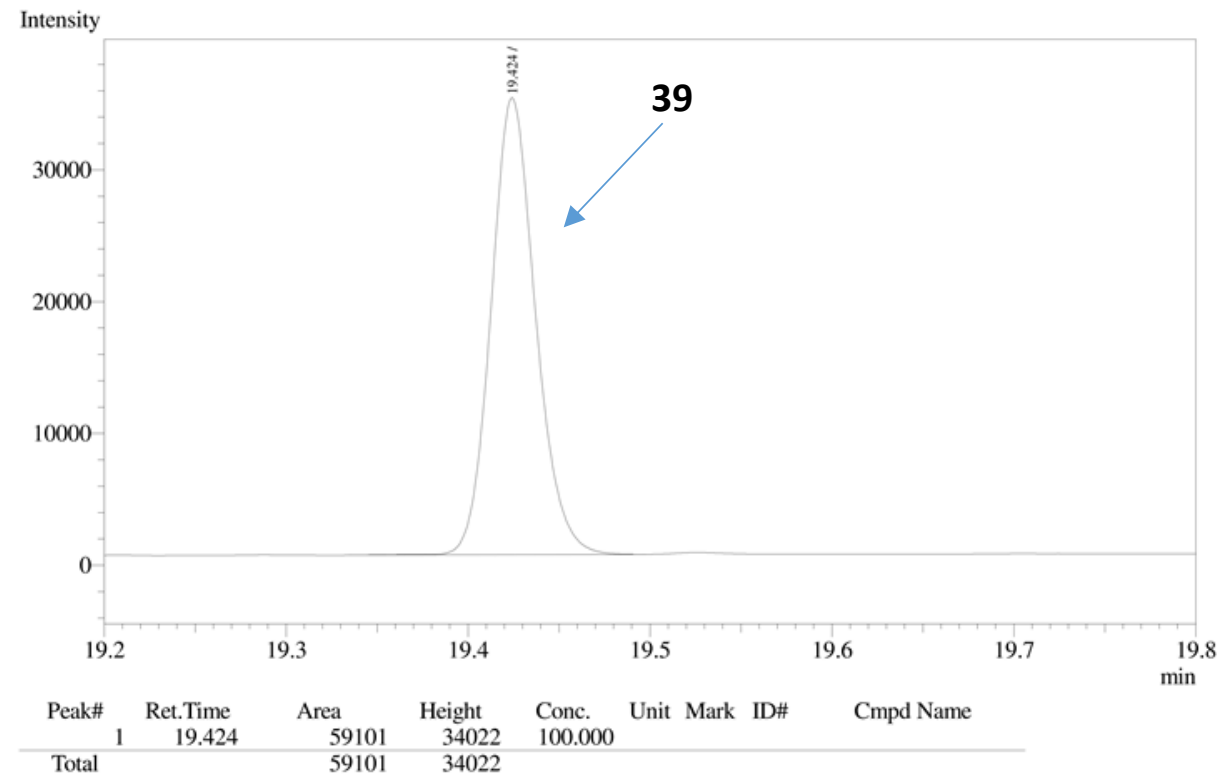

$\mathrm{GC}$ trace from the analysis of a mixture of $\mathbf{3 9}$ and the $\mathrm{E}$ isomer of $\mathbf{3 9}$ : 


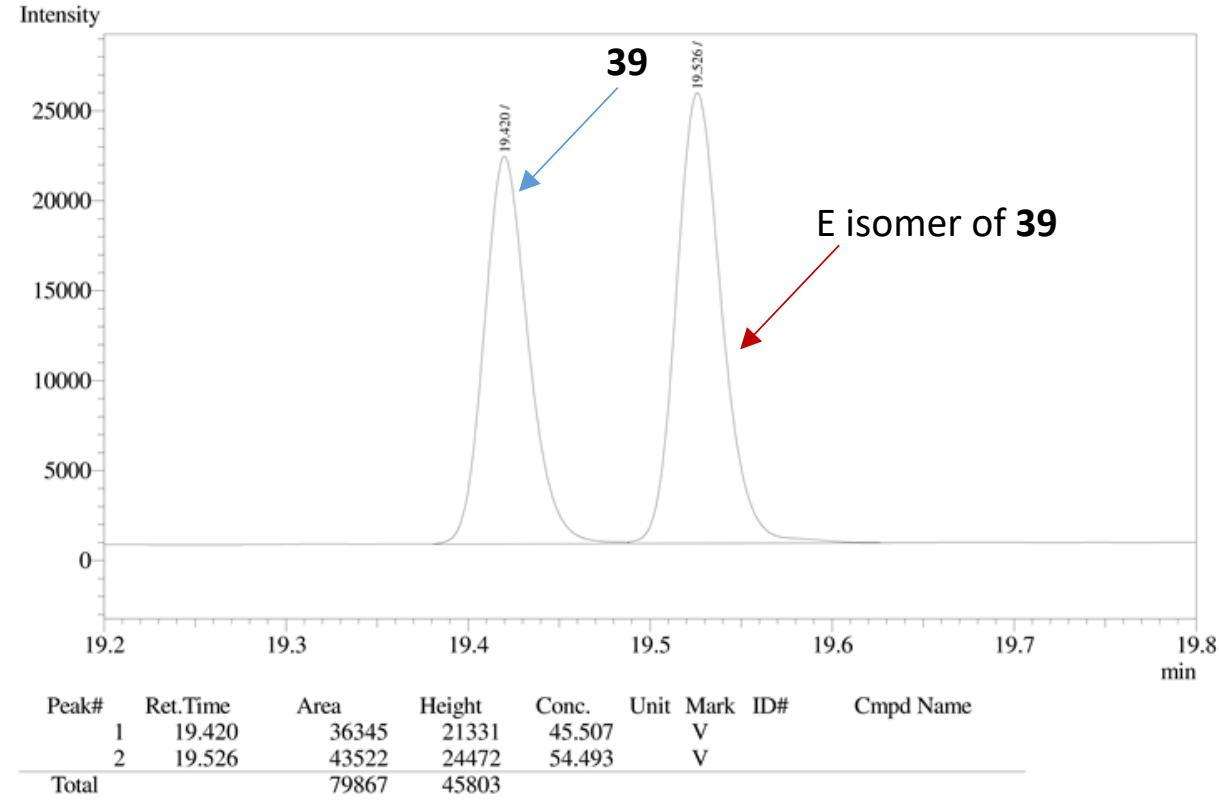

$\mathrm{GC}$ trace from the analysis of the crude reaction mixture:

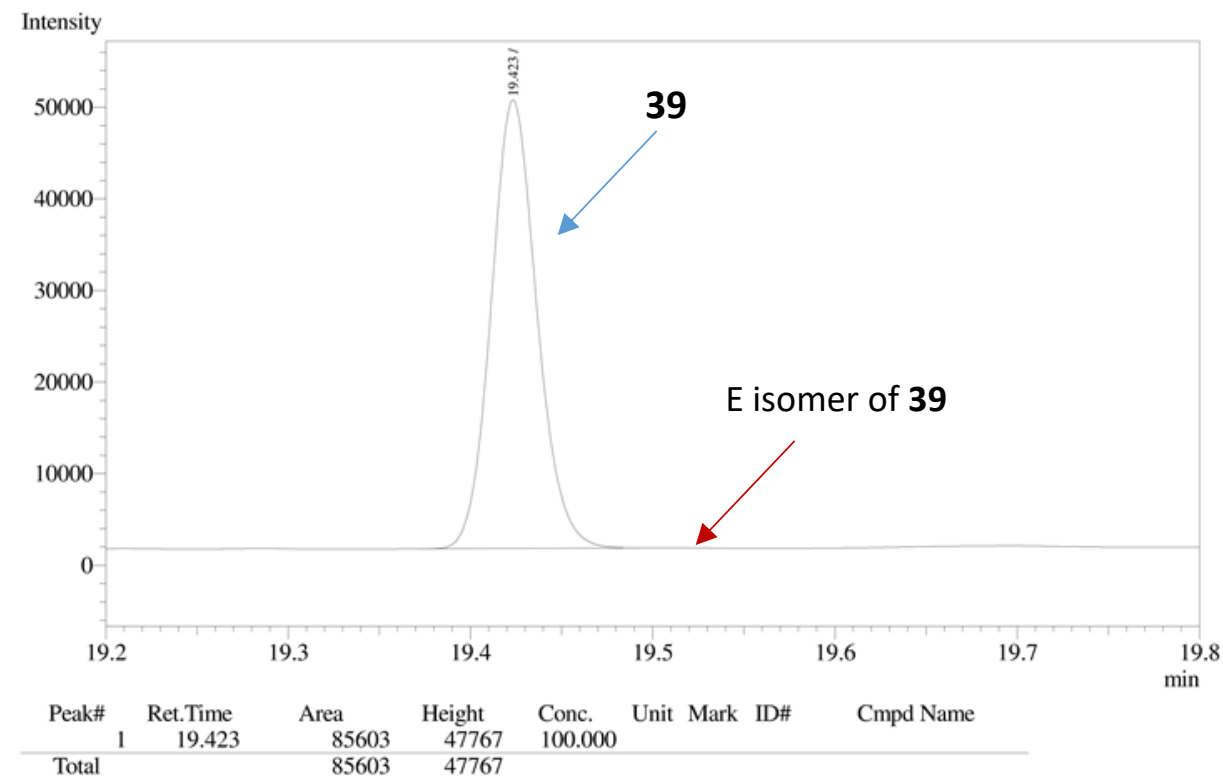


Close up of the GC trace from th analysis of the crude reaction mixture:

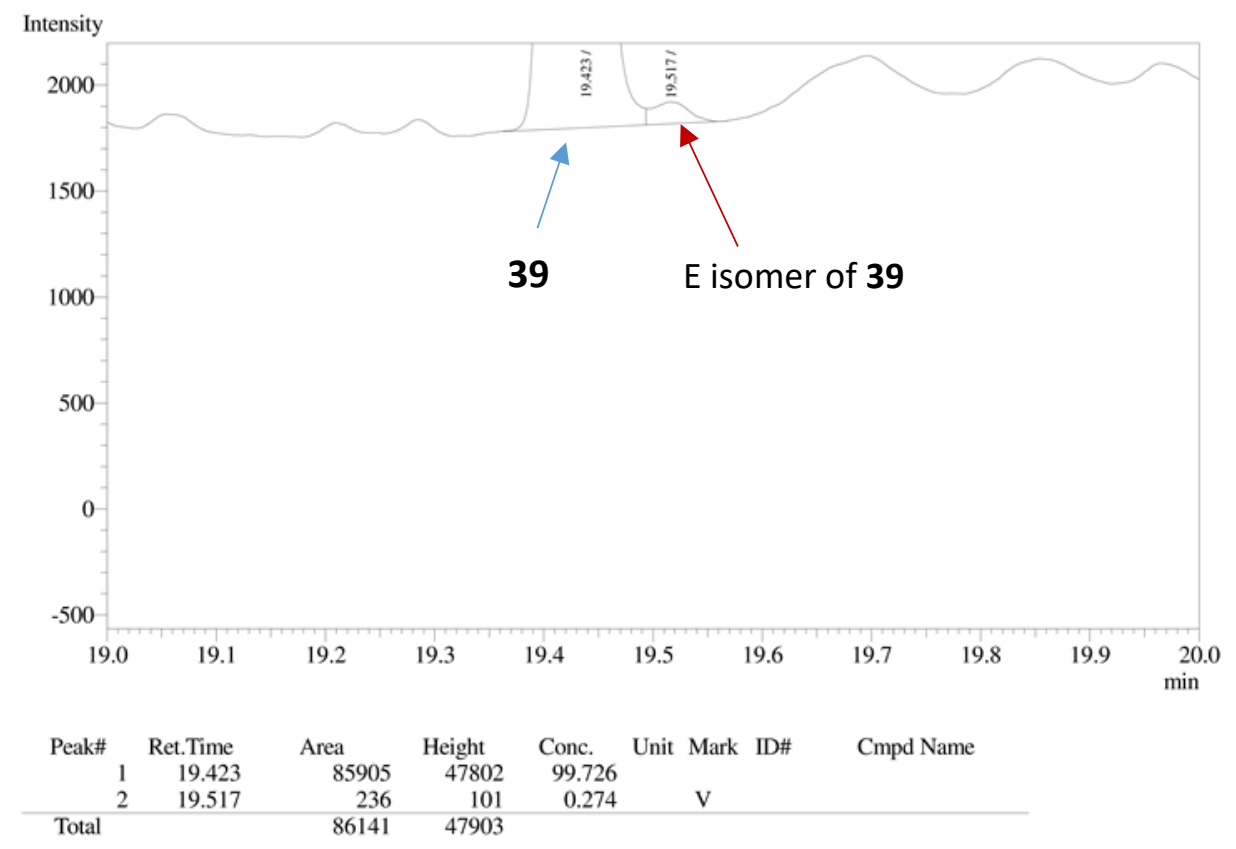

The ratio of concentrations of the two isomers is $363: 1(\mathrm{Z}: \mathrm{E})$.

$\mathrm{Z} / \mathrm{E}$ selectivity in the formation of 41 :

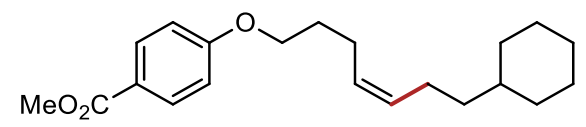

41

GC trace from the analysis of purified $\mathbf{4 1}$ :

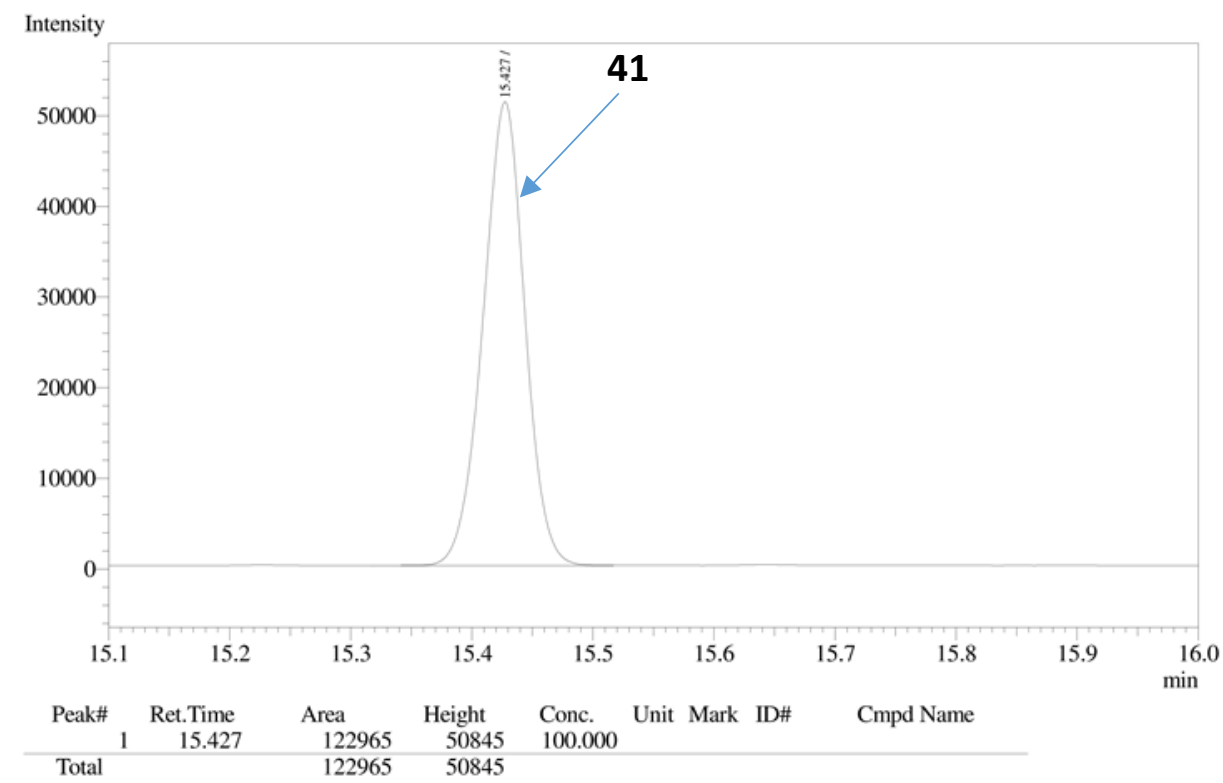


$\mathrm{GC}$ trace from the analysis of a mixture of $\mathbf{4 1}$ and the $\mathrm{E}$ isomer of $\mathbf{4 1}$ :

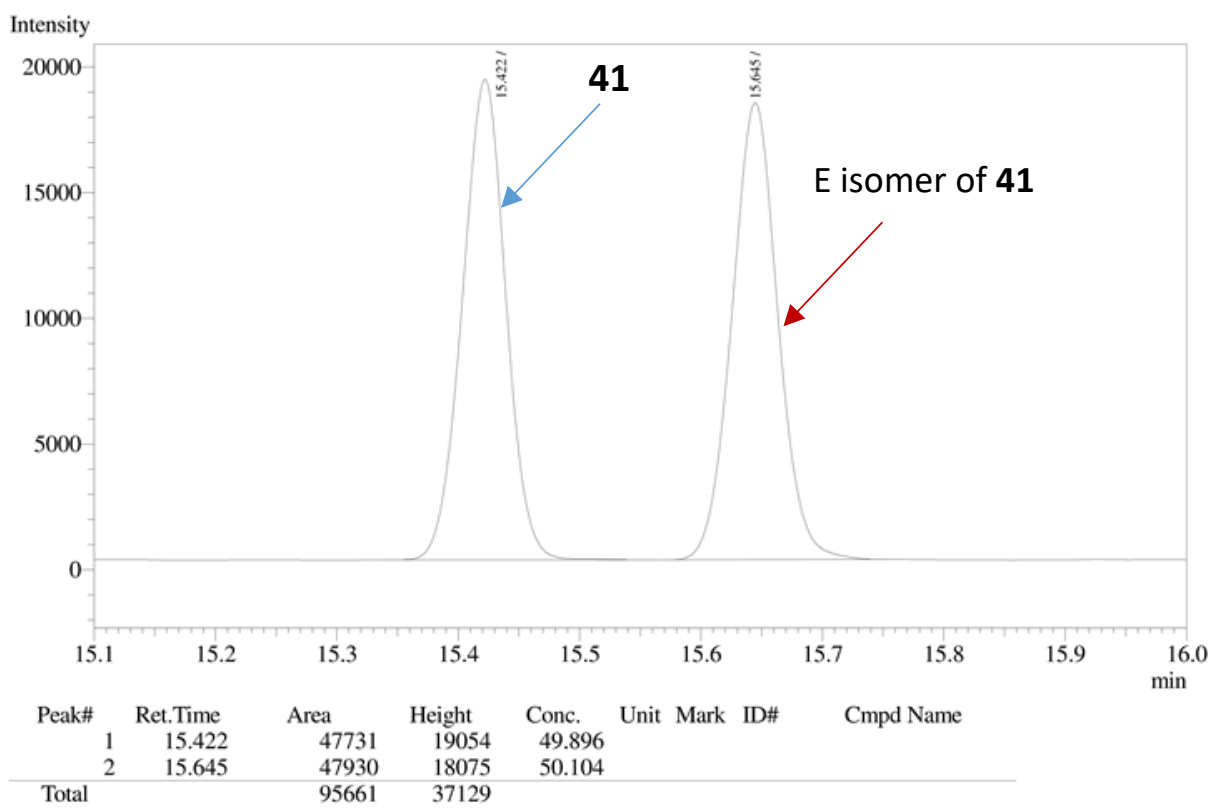

$\mathrm{GC}$ trace from the analysis of the crude reaction mixture:

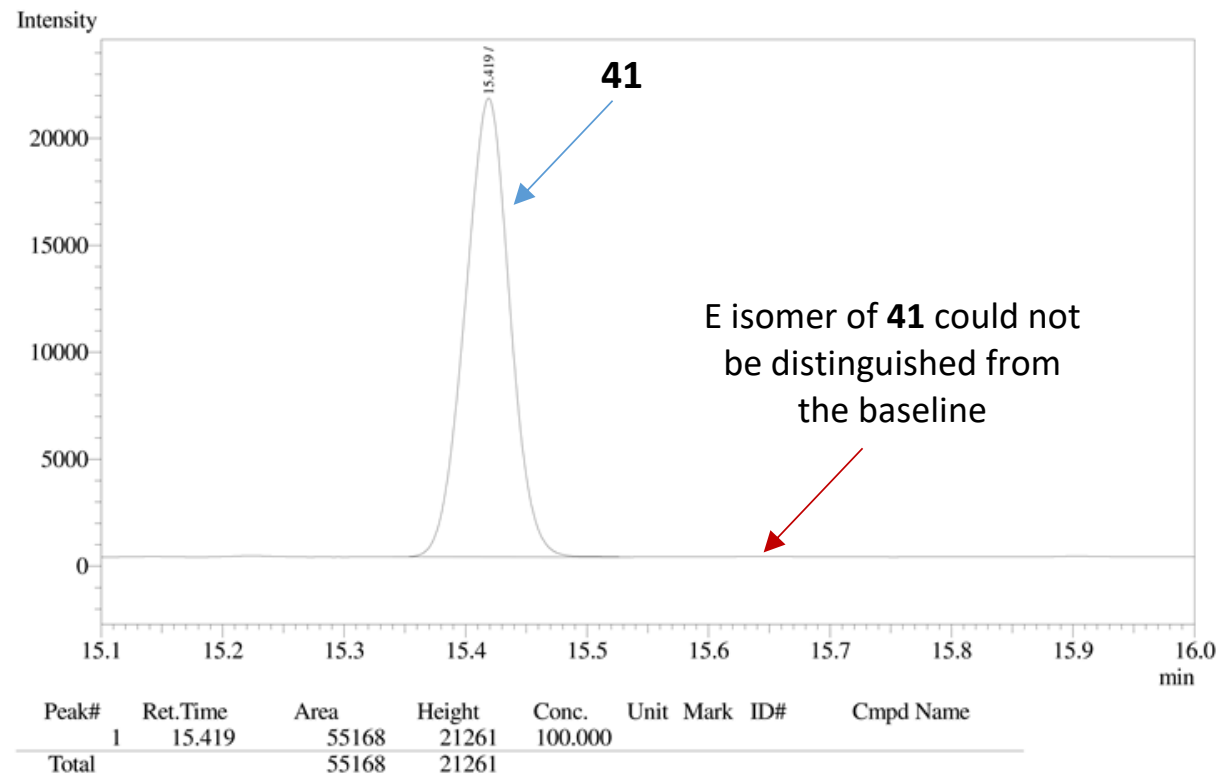

The $\mathrm{Z}: \mathrm{E}$ ration $>500: 1$ 


\section{Synthesis of TriAgCl and IPrAgCI}

The silver catalysts were synthesized using an adapted procedure from Sadighi et. al. that was used for the synthesis of SIPrAgCl. ${ }^{4}$<smiles>CC(C)c1cccc(C(C)C)c1N1C=CN(c2c(C(C)C)cccc2C(C)C)C1C(=O)Cl</smiles>

IPrAgCl, complex has been previously characterized. ${ }^{5}$<smiles>CC(C)c1ccccc1C1=NN(c2c(C(C)C)cccc2C(C)C)C(C(=O)c2ccccc2)N1c1c(C(C)C)cccc1C(C)C</smiles>

TriAgCl, complex was synthesized from TriHCl, ${ }^{6}$ and isolated as a white solid $(563.0 \mathrm{mg} 77 \%$ yield). ${ }^{1} \mathrm{H} \mathrm{NMR}\left(300 \mathrm{MHz}, \mathrm{CDCl}_{3}\right) \delta 7.64-7.50(\mathrm{~m}, 2 \mathrm{H}), 7.48-7.39(\mathrm{~m}, 3 \mathrm{H}), 7.39-7.28(\mathrm{~m}$, $6 \mathrm{H}), 2.71-2.55(\mathrm{~m}, 2 \mathrm{H}), 2.55-2.40(\mathrm{~m}, 2 \mathrm{H}), 1.41-1.17(\mathrm{~m}, 18 \mathrm{H}), 1.00(\mathrm{~d}, J=6.8 \mathrm{~Hz}, 6 \mathrm{H})$. ${ }^{13} \mathrm{C} \mathrm{NMR}\left(126 \mathrm{MHz}, \mathrm{CDCl}_{3}\right) \delta 188.0\left(\mathrm{dd}, J\left({ }^{109} \mathrm{Ag}^{13} \mathrm{C}\right)=265.2 \mathrm{~Hz}, J\left({ }^{107} \mathrm{Ag}^{13} \mathrm{C}\right)=229.2 \mathrm{~Hz}\right)$, 153.6, 153.6, 145.7, 145.3, 134.9, 131.8, 131.7, 131.5, 131.5, 129.1, 128.0, 125.3, 124.4, 29.1, $25.1,24.5,23.9,22.9$.

\section{Hydroalkylation of Terminal Alkynes}

\section{General Procedure:}

In a nitrogen filled glovebox, a scintillation vial was charged with a stir bar and $\mathrm{LiO} t-\mathrm{Bu}(1.5$ or 2.0 equiv). To this was added silver catalyst ( $0.05 \mathrm{mmol}, 0.10$ equiv), alkyne $(0.50 \mathrm{mmol}, 1.0$ equiv), alkylborane (1.3 or 1.5 equiv), methanol (17.6 mg, $0.55 \mathrm{mmol}, 1.10$ equiv), and solvent $(5 \mathrm{~mL})$. The reaction mixture was heated and stirred for 16 hours. After 16 hours, an aliquot of the crude reaction mixture was analyzed by $\mathrm{GC}$, and the reaction was quenched with the addition of sodium perborate ( $150 \mathrm{mg}, 0.75 \mathrm{mmol}, 1.5$ equiv) in $5 \mathrm{~mL}$ THF and $5 \mathrm{~mL}$ deionized water. The mixture was stirred at room temperature for 1 hour, and then extracted with ether $(3 \times 10$ $\mathrm{mL}$ ) and dried over $\mathrm{MgSO}_{4}$. The crude mixture was concentrated under reduced pressure and purified by silica gel chromatography.

A: The general procedure was followed using TriAgCl (30.4 mg, $0.05 \mathrm{mmol}, 0.10$ equiv) as catalyst, 1.5 equivalents of $\mathrm{LiO}$ - $\mathrm{Bu}(60 \mathrm{mg}, 0.75 \mathrm{mmol})$ and 1.3 equivalents of alkylborane $(0.65 \mathrm{mmol})$ and the reaction was stirred in isooctane at $45^{\circ} \mathrm{C}$ overnight. 
B: The general procedure was followed using $\operatorname{IPrAgCl}(26.7 \mathrm{mg}, 0.05 \mathrm{mmol}, 0.10$ equiv) as catalyst, 1.5 equivalents of $\mathrm{LiOt}$ - $\mathrm{Bu}(80 \mathrm{mg}, 1.00 \mathrm{mmol})$ and 1.5 equivalents of alkylborane $(0.75 \mathrm{mmol})$ and the reaction was stirred in toluene at $60^{\circ} \mathrm{C}$ overnight.

Most reactions were performed using general procedure A, however products 4, 18, 19, 22, 23, 27 , 42, 52, and 54, were synthesized using general procedure B.

\section{Characterization of $Z$-selective Hydroalkylation Products: Alkynes}

9-(3-phenylpropyl)-9-borabicyclo[3.3.1]nonane was used neat and was synthesized according to literature procedure. ${ }^{7}$

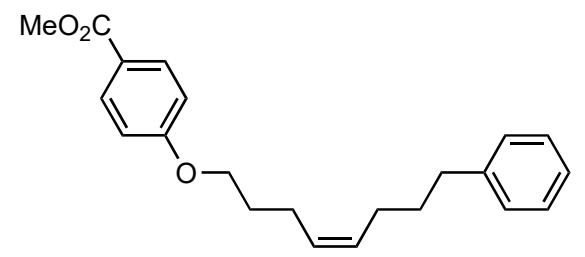

methyl 4-\{[(4Z)-8-phenyloct-4-en-1-yl]oxy\}benzoate (3), compound was prepared according to general procedure $\mathrm{A}$. The compound was purified by silica gel chromatography with EtOAc/Hex $(0 \rightarrow 30 \%)$ and isolated as a colorless oil $(147.6 \mathrm{mg}, 87 \%$ yield $) .{ }^{1} \mathrm{H} \mathrm{NMR}\left(300 \mathrm{MHz}, \mathrm{CDCl}_{3}\right) \delta$ $7.98(\mathrm{~d}, J=8.9 \mathrm{~Hz}, 2 \mathrm{H}), 7.36-7.27(\mathrm{~m}, 1 \mathrm{H}), 7.25-7.21(\mathrm{~m}, 1 \mathrm{H}), 7.20-7.09(\mathrm{~m}, 3 \mathrm{H}), 6.89(\mathrm{~d}$, $J=8.9 \mathrm{~Hz}, 2 \mathrm{H}), 5.64-5.31(\mathrm{~m}, 2 \mathrm{H}), 3.99(\mathrm{t}, J=6.3 \mathrm{~Hz}, 2 \mathrm{H}), 3.88(\mathrm{~s}, 3 \mathrm{H}), 2.64-2.50(\mathrm{~m}, 2 \mathrm{H})$, $2.28-2.15(\mathrm{~m}, 2 \mathrm{H}), 2.12-2.01(\mathrm{~m}, 2 \mathrm{H}), 1.92-1.78(\mathrm{~m}, 2 \mathrm{H}), 1.70-1.59(\mathrm{~m}, 2 \mathrm{H}) .{ }^{13} \mathrm{C} \mathrm{NMR}$ $\left(126 \mathrm{MHz} \mathrm{CDCl}_{3}\right) \delta 167.0,163.0,142.5,131.7,130.9,128.8,128.5,128.4,125.8,122.5,114.2$, 67.3, 51.9, 35.6, 31.5, 29.1, 26.9, 23.6.GCMS (EI) calculated for [ $\mathrm{M}^{]^{+}} 338.19$, found 338.2. FTIR (neat, $\left.\mathrm{cm}^{-1}\right)$ : 2929(m), 2849(m), 2360(m), 1719(s), 1604(s), 1510(s), 1437(s), 1280(s), 1253(s), 1167(s), 1104(s).

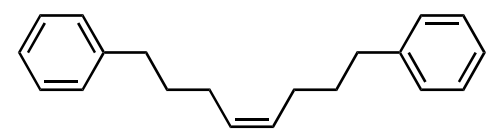

[(4Z)-8-phenyloct-4-en-1-yl]benzene (4), compound was prepared according to general procedure B. The compound was purified by silica gel chromatography with EtOAc/Hex $(0 \rightarrow$ $10 \%)$ and isolated as a colorless oil $(116.3 \mathrm{mg}, 88 \%$ yield $) .{ }^{1} \mathrm{H}$ NMR $\left(300 \mathrm{MHz}, \mathrm{CDCl}_{3}\right) \delta 7.33-$ $7.26(\mathrm{~m}, 4 \mathrm{H}), 7.21-7.14(\mathrm{~m}, 6 \mathrm{H}), 5.54-5.30(\mathrm{~m}, 2 \mathrm{H}), 2.68-2.54(\mathrm{~m}, 4 \mathrm{H}), 2.12-2.00(\mathrm{~m}$, 4H), $1.76-1.60(\mathrm{~m}, 4 \mathrm{H}) .{ }^{13} \mathrm{C}$ NMR $\left(126 \mathrm{MHz}, \mathrm{CDCl}_{3}\right) \delta 142.6,130.0,128.6,128.4,125.8,35.6$, 31.6, 27.0. GCMS (EI) calculated for $[\mathrm{M}]^{+}$264.19, found 264.2. FTIR (neat, $\left.\mathrm{cm}^{-1}\right)$ : $3061(\mathrm{~m})$, 3005(m), 2931(m), 2856(m), 1602(m) 1495(s), 1452(s), 1217(s), 1030(s), 906(w).

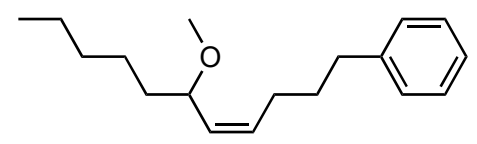

[(4Z)-6-methoxyundec-4-en-1-yl]benzene (5), compound was prepared according to general procedure A. The compound was purified by silica gel chromatography with EtOAc/Hex $(0 \rightarrow$ $20 \%)$ and isolated as a colorless oil $(116.6 \mathrm{mg}, 90 \%$ yield $) .{ }^{1} \mathrm{H}$ NMR $\left(300 \mathrm{MHz}, \mathrm{CDCl}_{3}\right) \delta 7.36-$ $7.26(\mathrm{~m}, 2 \mathrm{H}), 7.23-7.13(\mathrm{~m}, 3 \mathrm{H}), 5.63(\mathrm{dt}, J=10.9,7.4 \mathrm{~Hz}, 1 \mathrm{H}), 5.23(\mathrm{dd}, J=10.9,9.2 \mathrm{~Hz}$, $1 \mathrm{H}), 3.88(\mathrm{dt}, J=9.2,6.3 \mathrm{~Hz}, 1 \mathrm{H}), 3.23(\mathrm{~s}, 3 \mathrm{H}), 2.73-2.54(\mathrm{~m}, 2 \mathrm{H}), 2.24-2.01(\mathrm{~m}, 2 \mathrm{H}), 1.80-$ 
$1.64(\mathrm{~m}, 2 \mathrm{H}), 1.41-1.19(\mathrm{~m}, 8 \mathrm{H}), 0.88(\mathrm{t}, J=6.6 \mathrm{~Hz}, 3 \mathrm{H}) .{ }^{13} \mathrm{C} \mathrm{NMR}\left(126 \mathrm{MHz}, \mathrm{CDCl}_{3}\right) \delta 142.3$, 133.0, 131.4, 128.5, 128.5, 125.9, 76.6, 56.0, 35.7, 35.6, 32.0, 31.6, 27.5, 25.1, 22.8, 14.2.

GCMS (EI) calculated for [M] $]^{+} 260.21$, found 260.3. FTIR (neat, $\left.\mathrm{cm}^{-1}\right): 3025(\mathrm{~m}), 2929(\mathrm{~m})$, 2857(m), 1603(s), 1496(s), 1452(s), 1332(m), 1124(s), 1097(s).

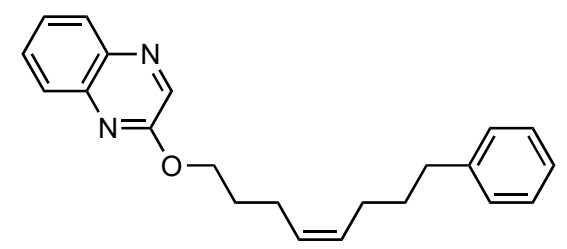

2-\{[(4Z)-8-phenyloct-4-en-1-yl]oxy\} quinoxaline (6), compound was prepared according to general procedure $\mathrm{A}$. The compound was purified by silica gel chromatography with EtOAc/Hex $(0 \rightarrow 40 \%)$ and isolated as a colorless oil $(133.5 \mathrm{mg}, 80 \%$ yield $) .{ }^{1} \mathrm{H}$ NMR $\left(300 \mathrm{MHz}, \mathrm{CDCl}_{3}\right) \delta$ $8.46(\mathrm{~s}, 1 \mathrm{H}), 8.01(\mathrm{dd}, J=8.3,1.5 \mathrm{~Hz}, 1 \mathrm{H}), 7.81(\mathrm{dd}, J=8.4,1.4 \mathrm{~Hz}, 1 \mathrm{H}), 7.66(\mathrm{ddd}, J=8.4,7.0$, $1.5 \mathrm{~Hz}, 1 \mathrm{H}), 7.55(\mathrm{ddd}, J=8.3,7.0,1.4 \mathrm{~Hz}, 1 \mathrm{H}), 7.26-7.19(\mathrm{~m}, 2 \mathrm{H}), 7.19-7.08(\mathrm{~m}, 3 \mathrm{H}), 5.57$ $-5.34(\mathrm{~m}, 2 \mathrm{H}), 4.47(\mathrm{t}, J=6.5 \mathrm{~Hz}, 2 \mathrm{H}), 2.67-2.50(\mathrm{~m}, 2 \mathrm{H}), 2.33-2.16(\mathrm{~m}, 2 \mathrm{H}), 2.16-2.01$ $(\mathrm{m}, 2 \mathrm{H}), 1.98-1.83(\mathrm{~m}, 2 \mathrm{H}), 1.76-1.60(\mathrm{~m}, 2 \mathrm{H}) .{ }^{13} \mathrm{C} \mathrm{NMR}\left(75 \mathrm{MHz}, \mathrm{CDCl}_{3}\right) \delta 157.6,142.5$, 140.6, 139.9, 139.0, 130.7, 130.2, 129.1, 129.0, 128.5, 128.4, 127.3, 126.5, 125.8, 66.0, 35.6, 31.5, 28.8, 27.0, 23.8. GCMS (EI) calculated for [M]+332.19, found 332.2. FTIR (neat, $\mathrm{cm}^{-1}$ ): 3004(m), 2928(m), 2855(m), 1944(w), 1571(s), 1497(s), 1414(s), 1305(s), 1221(s), 1136(s), 1019(m), 911(s).

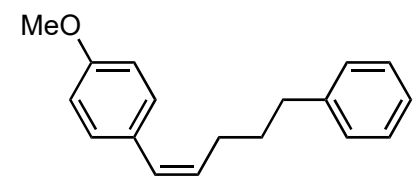

1-methoxy-4-[(1Z)-5-phenylpent-1-en-1-yl]benzene (7), compound was prepared according to general procedure A. The compound was purified by silica gel chromatography with EtOAc/Hex $(0 \rightarrow 20 \%)$ and isolated as a colorless oil $(117.9 \mathrm{mg}, 93 \%$ yield $) .{ }^{1} \mathrm{H} \mathrm{NMR}\left(300 \mathrm{MHz}, \mathrm{CDCl}_{3}\right) \delta$ $7.34-7.27(\mathrm{~m}, 1 \mathrm{H}), 7.24-7.12(\mathrm{~m}, 6 \mathrm{H}), 6.85(\mathrm{~d}, J=8.7 \mathrm{~Hz}, 2 \mathrm{H}), 6.37(\mathrm{~d}, J=11.6 \mathrm{~Hz}, 1 \mathrm{H})$, $5.60(\mathrm{dt}, J=11.6,7.2 \mathrm{~Hz}, 1 \mathrm{H}), 3.82(\mathrm{~s}, 3 \mathrm{H}), 2.70-2.60(\mathrm{~m}, 2 \mathrm{H}), 2.45-2.30(\mathrm{~m}, 2 \mathrm{H}), 1.85-$ $1.70(\mathrm{~m}, 2 \mathrm{H}) .{ }^{13} \mathrm{C}$ NMR $\left(126 \mathrm{MHz}, \mathrm{CDCl}_{3}\right) \delta 158.3,142.5,131.1,130.5,130.1,128.7,128.6$, 128.4, 125.8, 113.7, 55.4, 35.6, 31.9, 28.3. GCMS (EI) calculated for [M] ${ }^{+} 252.15$, found 252.2. FTIR (neat, $\left.\mathrm{cm}^{-1}\right): 3292(\mathrm{~m}), 3061(\mathrm{~m}), 3003(\mathrm{~m}), 2929(\mathrm{~m}), 2855(\mathrm{~m}), 1605(\mathrm{~s}), 1503(\mathrm{~s}), 1452(\mathrm{~s})$, 1300(s), 1250(s), 1174(s), 1108(s), 1033(s), 837(s).

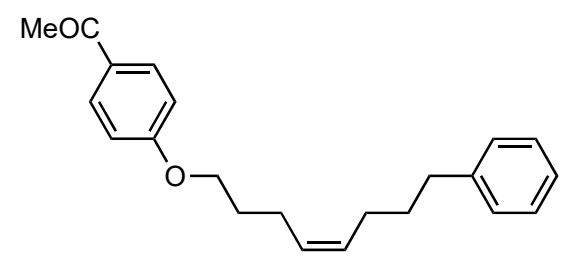

1-(4-\{[(4Z)-8-phenyloct-4-en-1-yl]oxy\}phenyl)ethan-1-one (8), compound was prepared according to general procedure $\mathrm{A}$. The compound was purified by silica gel chromatography with EtOAc/Hex $(0 \rightarrow 30 \%)$ and isolated as a colorless oil $(97.8 \mathrm{mg}, 61 \%$ yield $) .{ }^{1} \mathrm{H}$ NMR $(300$ $\left.\mathrm{MHz} \mathrm{CDCl}_{3}\right) \delta 7.92(\mathrm{~d}, J=8.8 \mathrm{~Hz}, 2 \mathrm{H}), 7.28(\mathrm{~s}, 1 \mathrm{H}), 7.23(\mathrm{~s}, 1 \mathrm{H}), 7.20-7.08(\mathrm{~m}, 3 \mathrm{H}), 6.91(\mathrm{~d}$, $J=8.8 \mathrm{~Hz}, 2 \mathrm{H}), 5.56-5.30(\mathrm{~m}, 2 \mathrm{H}), 4.01(\mathrm{t}, J=6.3 \mathrm{~Hz}, 2 \mathrm{H}), 2.69-2.47(\mathrm{~m}, 5 \mathrm{H}), 2.29-2.15$ (m, 2H), $2.11-1.98(\mathrm{~m}, 2 \mathrm{H}), 1.91-1.82(\mathrm{~m}, 2 \mathrm{H}), 1.72-1.59(\mathrm{~m}, 2 \mathrm{H}) .{ }^{13} \mathrm{C} \mathrm{NMR}(126 \mathrm{MHz}$, 
$\left.\mathrm{CDCl}_{3}\right) \delta$ 196.9, 163.2, 142.5, 130.9, 130.7, 130.3, 128.7, 128.5, 128.4, 125.8, 114.2, 67.4, 35.6,

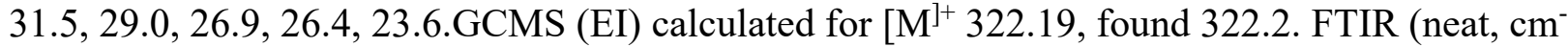
$\left.{ }^{1}\right): 3060(\mathrm{~m}), 3002(\mathrm{~m}), 2932(\mathrm{~m}), 2855(\mathrm{~m}), 1676(\mathrm{~s}), 1600(\mathrm{~s}), 1508(\mathrm{~s}), 1357(\mathrm{~s}), 1305(\mathrm{~s}), 1255(\mathrm{~s})$, $1170(\mathrm{~s}), 1029(\mathrm{~m}), 955(\mathrm{~s}), 833(\mathrm{~s})$.

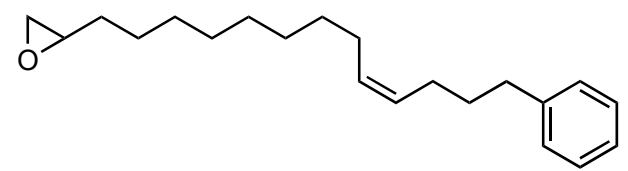

2-[(10Z)-14-phenyltetradec-10-en-1-yl]oxirane (9), compound was prepared according to general procedure $\mathrm{A}$. The compound was purified by silica gel chromatography with $\mathrm{EtOAc} / \mathrm{Hex}$ $(0 \rightarrow 20 \%)$ and isolated as a colorless oil $\left(122.0 \mathrm{mg}, 81 \%\right.$ yield) ${ }^{1} \mathrm{H} \mathrm{NMR}\left(300 \mathrm{MHz}, \mathrm{CDCl}_{3}\right) \delta$ $7.31-7.26(\mathrm{~m}, 2 \mathrm{H}), 7.21-7.14(\mathrm{~m}, 3 \mathrm{H}), 5.51-5.26(\mathrm{~m}, 2 \mathrm{H}), 2.95-2.85(\mathrm{~m}, 1 \mathrm{H}), 2.78-2.72$ $(\mathrm{m}, 1 \mathrm{H}), 2.66-2.57(\mathrm{~m}, 2 \mathrm{H}), 2.46(\mathrm{dd}, J=5.0,2.7 \mathrm{~Hz}, 1 \mathrm{H}), 2.12-1.95(\mathrm{~m}, 4 \mathrm{H}), 1.74-1.61(\mathrm{~m}$, $2 \mathrm{H}), 1.52-1.41(\mathrm{~m}, 4 \mathrm{H}), 1.29(\mathrm{~s}, 10 \mathrm{H}){ }^{13} \mathrm{C}$ NMR $\left(126 \mathrm{MHz}, \mathrm{CDCl}_{3}\right) \delta 142.7,130.6,129.4$, 128.5, 128.4, 125.7, 52.5, 47.2, 35.6, 32.6, 31.6, 29.8, 29.7, 29.6, 29.4, 27.4, 27.0, 26.1. GCMS (EI) calculated for $[\mathrm{M}]^{+} 300.25$, found 300.3. FTIR (neat, $\left.\mathrm{cm}^{-1}\right): 3001(\mathrm{~m}), 2926(\mathrm{~m}), 2853(\mathrm{~m})$, 1725(m), 1603(s), 1495(s), 1453(s), 1258(w), 1029(s).

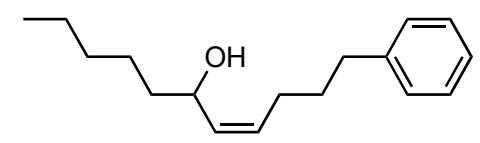

(4Z)-1-phenylundec-4-en-6-ol (10), compound was prepared according to general procedure A. The compound was purified by silica gel chromatography with EtOAc/Hex $(0 \rightarrow 60 \%)$ and isolated as a colorless oil (78.2 $\mathrm{mg}, 63 \%$ yield). ${ }^{1} \mathrm{H}$ NMR $\left(300 \mathrm{MHz}, \mathrm{CDCl}_{3}\right) \delta 7.35-7.27(\mathrm{~m}$, $2 \mathrm{H}), 7.24-7.12(\mathrm{~m}, 3 \mathrm{H}), 5.64-5.26(\mathrm{~m}, 2 \mathrm{H}), 4.44-4.29(\mathrm{~m}, 1 \mathrm{H}), 2.75-2.55(\mathrm{~m}, 2 \mathrm{H}), 2.24-$ $2.00(\mathrm{~m}, 2 \mathrm{H}), 1.78-1.64(\mathrm{~m}, 2 \mathrm{H}), 1.59-1.38(\mathrm{~m}, 2 \mathrm{H}), 1.35-1.22(\mathrm{~m}, 6 \mathrm{H}), 0.88(\mathrm{t}, J=5.5 \mathrm{~Hz}$, $3 \mathrm{H}) .{ }^{13} \mathrm{C}$ NMR $\left(126 \mathrm{MHz}, \mathrm{CDCl}_{3}\right) \delta 142.2,133.3,131.6,128.5,128.4,125.9,67.8,37.6,35.5$, 31.9, 31.4, 27.3, 25.1, 22.7, 14.1. GCMS (EI) calculated for $[\mathrm{M}]^{+} 246.20$, found 246.2. FTIR (neat, $\left.\mathrm{cm}^{-1}\right)$ : 3349(b), 3025(m), 3004(m), 2929(m), 2857(m), 1603(s), 1496(s), 1453(s), 1378(s), 1303(m), 1123(s), 1028(s), 910(s).

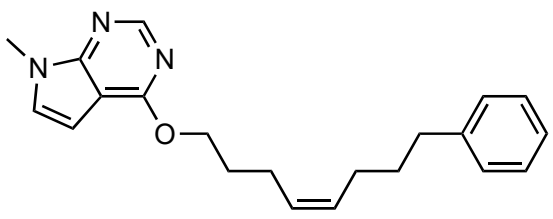

7-methyl-4-\{[(4Z)-8-phenyloct-4-en-1-yl]oxy\}-7H-pyrrolo[2,3-d]pyrimidine (11), compound was prepared according to general procedure A. The compound was purified by silica gel chromatography with EtOAc/Hex $(0 \rightarrow 40 \%)$ and isolated as a colorless oil $(146.7 \mathrm{mg}, 87 \%$ yield). ${ }^{1} \mathrm{H}$ NMR $\left(300 \mathrm{MHz}, \mathrm{CDCl}_{3}\right) \delta 8.46(\mathrm{~s}, 1 \mathrm{H}), 7.25-7.19(\mathrm{~m}, 2 \mathrm{H}), 7.20-7.06(\mathrm{~m}, 3 \mathrm{H}), 6.99$ $(\mathrm{d}, J=3.4 \mathrm{~Hz}, 1 \mathrm{H}), 6.52(\mathrm{~d}, J=3.4 \mathrm{~Hz}, 1 \mathrm{H}), 5.58-5.33(\mathrm{~m}, 2 \mathrm{H}), 4.51(\mathrm{t}, J=6.6 \mathrm{~Hz}, 2 \mathrm{H}), 3.84$ $(\mathrm{s}, 3 \mathrm{H}), 2.63-2.46(\mathrm{~m}, 2 \mathrm{H}), 2.31-2.16(\mathrm{~m}, 2 \mathrm{H}), 2.14-2.00(\mathrm{~m}, 2 \mathrm{H}), 1.96-1.86(\mathrm{~m}, 2 \mathrm{H}), 1.69$ $-1.60(\mathrm{~m}, 2 \mathrm{H}) .{ }^{13} \mathrm{C} \mathrm{NMR}\left(126 \mathrm{MHz}, \mathrm{CDCl}_{3}\right) \delta 162.9,151.9,150.9,142.4,130.5,129.0,128.4$, 128.2, 126.6, 125.6, 105.5, 98.2, 65.7, 35.5, 31.4, 31.4, 28.9, 26.8, 23.7. GCMS (EI) calculated for $[\mathrm{M}]^{+}$335.20, found 335.2. FTIR (neat, $\left.\mathrm{cm}^{-1}\right)$ : 3003(m), 2926(m), 2856(m), 1873(w), 1595(s), 1561(s), 1441(s), 1367(s), 1317(s), 1247(s), 1055(s), 1006(m), 888(m). 


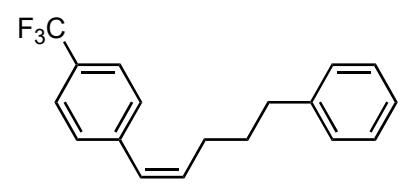

1-[(1Z)-5-phenylpent-1-en-1-yl]-4-(trifluoromethyl)benzene (12), compound was prepared according to general procedure $\mathrm{A}$. The compound was purified by silica gel chromatography with EtOAc/Hex $(0 \rightarrow 20 \%)$ and isolated as a colorless oil $\left(124.3 \mathrm{mg}, 86 \%\right.$ yield). ${ }^{1} \mathrm{H}$ NMR (300 $\left.\mathrm{MHz}, \mathrm{CDCl}_{3}\right) \delta 7.55(\mathrm{~d}, J=8.2 \mathrm{~Hz}, 2 \mathrm{H}), 7.38-7.27(\mathrm{~m}, 3 \mathrm{H}), 7.24-7.09(\mathrm{~m}, 4 \mathrm{H}), 6.46(\mathrm{~d}, J=$ $11.6 \mathrm{~Hz}, 1 \mathrm{H}), 5.80(\mathrm{dt}, J=11.6,7.4 \mathrm{~Hz}, 1 \mathrm{H}), 2.72-2.57(\mathrm{~m}, 2 \mathrm{H}), 2.43-2.26(\mathrm{~m}, 2 \mathrm{H}), 1.87-$ $1.71(\mathrm{~m}, 2 \mathrm{H}){ }^{13} \mathrm{C}$ NMR $\left(126 \mathrm{MHz} \mathrm{CDCl}_{3}\right) \delta 142.1,141.3,134.8,129.0,128.6(\mathrm{q}, J=27.6 \mathrm{~Hz})$, 128.6, 128.5, 128.2, 126.0, $125.2(\mathrm{q}, J=3.8 \mathrm{~Hz}), 124.5(\mathrm{q}, J=271.9 \mathrm{~Hz}), 35.5,31.6,28.1 .{ }^{19} \mathrm{~F}$ NMR $\left(470 \mathrm{MHz}, \mathrm{CDCl}_{3}\right) \delta$-65.4. GCMS (EI) calculated for $[\mathrm{M}]^{+}$290.13, found 290.1. FTIR (neat, $\left.\mathrm{cm}^{-1}\right):$ 3062(m), 3025(m), 2931(m), 2858(m), 1614(s), 1496(s), 1453(s), $1420(\mathrm{~m}), 1326(\mathrm{~s})$, 1164(s), 1124(s), 1067(s), 1016(s), 837(s).

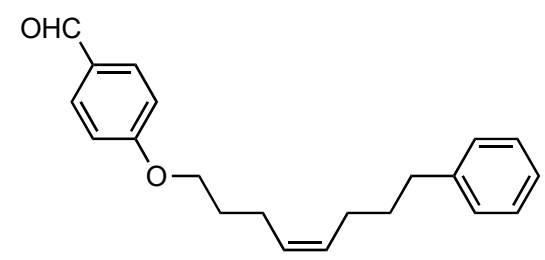

4-\{[(4Z)-8-phenyloct-4-en-1-yl]oxy $\}$ benzaldehyde (13), compound was prepared according to general procedure $\mathrm{A}$. The compound was purified by silica gel chromatography with EtOAc/Hex $(0 \rightarrow 20 \%)$ and isolated as a colorless oil $(108.2 \mathrm{mg}, 70 \%$ yield $) .{ }^{1} \mathrm{H} \mathrm{NMR}\left(300 \mathrm{MHz}, \mathrm{CDCl}_{3}\right) \delta$ $9.87(\mathrm{~s}, 1 \mathrm{H}), 7.82(\mathrm{~d}, J=8.7 \mathrm{~Hz}, 2 \mathrm{H}), 7.28(\mathrm{~s}, 1 \mathrm{H}), 7.23(\mathrm{~s}, 1 \mathrm{H}), 7.20-7.07(\mathrm{~m}, 3 \mathrm{H}), 6.98(\mathrm{~d}, J=$ $8.7 \mathrm{~Hz}, 2 \mathrm{H}), 5.57-5.30(\mathrm{~m}, 2 \mathrm{H}), 4.03(\mathrm{t}, J=6.3 \mathrm{~Hz}, 2 \mathrm{H}), 2.68-2.50(\mathrm{~m}, 2 \mathrm{H}), 2.30-2.16(\mathrm{~m}$, 2H), $2.13-1.99(\mathrm{~m}, 2 \mathrm{H}), 1.97-1.81(\mathrm{~m}, 2 \mathrm{H}), 1.74-1.59(\mathrm{~m}, 2 \mathrm{H}) .{ }^{13} \mathrm{C} \mathrm{NMR}\left(126 \mathrm{MHz}, \mathrm{CDCl}_{3}\right)$ $\delta 190.9,164.3,142.5,132.1,131.0,129.9,128.7,128.5,128.4,125.8,114.9,67.6,35.6,31.5$, 29.0, 27.0, 23.6. GCMS (EI) calculated for $[\mathrm{M}]^{+}$308.18, found 308.2. FTIR (neat, $\mathrm{cm}^{-1}$ ): 3016(m), 3003(m), 2924(m), 2854(m), 2735(m), 1694(s), 1590(s), 1574(s), 1497(s), 1393(s), 1310(m), 1257(m), 1158(s), 1029(s), 832(s), 750(s), 698(s).

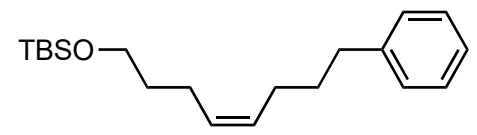

tert-butyldimethyl $\{[(4 Z)-8$-phenyloct-4-en-1-yl]oxy $\}$ silane (14), compound was prepared according to general procedure A. The compound was purified by silica gel chromatography with EtOAc/Hex $(0 \rightarrow 20 \%)$ and isolated as a colorless oil $\left(150.4 \mathrm{mg}, 95 \%\right.$ yield). ${ }^{1} \mathrm{H}$ NMR (300 $\left.\mathrm{MHz}, \mathrm{CDCl}_{3}\right) \delta 7.31-7.26(\mathrm{~m}, 1 \mathrm{H}), 7.26-7.23(\mathrm{~m}, 1 \mathrm{H}), 7.22-7.13(\mathrm{~m}, 3 \mathrm{H}), 5.50-5.31(\mathrm{~m}$, $2 \mathrm{H}), 3.60(\mathrm{t}, J=6.5 \mathrm{~Hz}, 2 \mathrm{H}), 2.68-2.51(\mathrm{~m}, 2 \mathrm{H}), 2.15-2.00(\mathrm{~m}, 4 \mathrm{H}), 1.75-1.62(\mathrm{~m}, 2 \mathrm{H}), 1.61$ $-1.50(\mathrm{~m}, 2 \mathrm{H}), 0.90(\mathrm{~s}, 9 \mathrm{H}), 0.05(\mathrm{~s}, 6 \mathrm{H}) .{ }^{13} \mathrm{C} \mathrm{NMR}\left(126 \mathrm{MHz}, \mathrm{CDCl}_{3}\right) \delta 142.7,129.9,128.6$, $128.4,125.8,62.8,35.7,33.0,31.6,27.0,26.1,23.7,18.5,-5.1$. GCMS (EI) calculated for $[\mathrm{M}]^{+}$ 318.24, found 318.3. FTIR (neat, $\left.\mathrm{cm}^{-1}\right): 3062(\mathrm{~m}), 3026(\mathrm{~m}), 3004(\mathrm{~m}), 2927(\mathrm{~m}), 2856(\mathrm{~m})$, 1604(s), 1496(s), 1471(s), 1388(s), 1360(s), 1255(s), 1102(s), 836(s), 774(s), 698(s). 


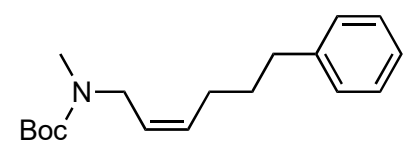

tert-butyl N-methyl-N-[(2Z)-6-phenylhex-2-en-1-yl]carbamate (15), compound was prepared according to general procedure $\mathrm{A}$. The compound was purified by silica gel chromatography with EtOAc/Hex $(0 \rightarrow 20 \%)$ and isolated as a colorless oil $(127.7 \mathrm{mg}, 88 \%$ yield $) .{ }^{1} \mathrm{H}$ NMR (300 $\left.\mathrm{MHz} \mathrm{CDCl}_{3}\right) \delta 7.34-7.26(\mathrm{~m}, 2 \mathrm{H}), 7.23-7.11(\mathrm{~m}, 3 \mathrm{H}), 5.66-5.48(\mathrm{~m}, 1 \mathrm{H}), 5.48-5.31(\mathrm{~m}$, $1 \mathrm{H}), 3.83(\mathrm{~d}, J=5.0 \mathrm{~Hz}, 2 \mathrm{H}), 2.78(\mathrm{~s}, 3 \mathrm{H}), 2.69-2.54(\mathrm{~m}, 2 \mathrm{H}), 2.20-2.05(\mathrm{~m}, 2 \mathrm{H}), 1.80-1.61$ $(\mathrm{m}, 2 \mathrm{H}), 1.46(\mathrm{~s}, 9 \mathrm{H}) .{ }^{13} \mathrm{C} \mathrm{NMR}\left(126 \mathrm{MHz}, \mathrm{CDCl}_{3}\right) \delta 155.8,142.2,132.4,128.4,128.4,125.8$, 79.4, 45.2, 35.5, 33.5, 31.4, 28.5, 26.9. GCMS (EI) calculated for [M] ${ }^{+} 289.20$, found 289.4. FTIR (neat, $\left.\mathrm{cm}^{-1}\right):$ 3061(w), 3024(m), 2974(m), 2930(m), 2857(m), 1699(s), 1480(s), 1390(s) 1365(s), 1238(s), 1141(s), 1030(w), 879(s).

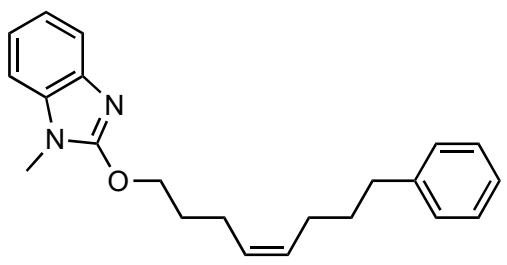

1-methyl-2-\{[(4Z)-8-phenyloct-4-en-1-yl]oxy\}-1H-1,3-benzodiazole (16), compound was prepared according to general procedure A. The compound was purified by silica gel chromatography with EtOAc/Hex $(0 \rightarrow 40 \%)$ and isolated as a colorless oil $(127.6 \mathrm{mg}, 76 \%$ yield). ${ }^{1} \mathrm{H}$ NMR $\left(300 \mathrm{MHz}, \mathrm{CDCl}_{3}\right) \delta 7.60-7.50(\mathrm{~m}, 1 \mathrm{H}), 7.30-7.27(\mathrm{~m}, 2 \mathrm{H}), 7.25-7.22(\mathrm{~m}$, $1 \mathrm{H}), 7.22-7.09(\mathrm{~m}, 6 \mathrm{H}), 5.55-5.37(\mathrm{~m}, 2 \mathrm{H}), 4.55(\mathrm{t}, J=6.5 \mathrm{~Hz}, 2 \mathrm{H}), 3.54(\mathrm{~s}, 3 \mathrm{H}), 2.67-2.55$ $(\mathrm{m}, 2 \mathrm{H}), 2.29-2.17(\mathrm{~m}, 2 \mathrm{H}), 2.16-2.04(\mathrm{~m}, 2 \mathrm{H}), 1.99-1.86(\mathrm{~m}, 2 \mathrm{H}), 1.76-1.63(\mathrm{~m}, 2 \mathrm{H}) .{ }^{13} \mathrm{C}$ NMR $\left(126 \mathrm{MHz}, \mathrm{CDCl}_{3}\right) \delta 157.7,142.5,140.2,134.4,130.8,128.8,128.5,128.4,125.8,121.6$, 120.9, 117.7, 108.0, 69.8, 35.6, 31.4, 29.1, 28.1, 26.9, 23.7 GCMS (EI) calculated for [M] ${ }^{+}$ 334.20, found 334.2. FTIR (neat, $\left.\mathrm{cm}^{-1}\right): 3003(\mathrm{~m}), 2923(\mathrm{~m}), 2855(\mathrm{~m}), 1603(\mathrm{w}), 1514(\mathrm{~s})$, 1452(m), 1021(m), 808(s).

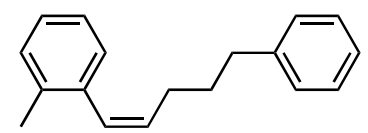

1-methyl-2-[(1Z)-5-phenylpent-1-en-1-yl]benzene (17), compound was prepared according to general procedure A. The compound was purified by silica gel chromatography with EtOAc/Hex $(0 \rightarrow 10 \%)$ and isolated as a colorless oil $(99.5 \mathrm{mg}, 84 \%$ yield $) .{ }^{1} \mathrm{H}$ NMR $\left(300 \mathrm{MHz}, \mathrm{CDCl}_{3}\right) \delta$ $7.35-7.27(\mathrm{~m}, 2 \mathrm{H}), 7.25-7.11(\mathrm{~m}, 7 \mathrm{H}), 6.51(\mathrm{~d}, J=11.5 \mathrm{~Hz}, 1 \mathrm{H}), 5.78(\mathrm{dt}, J=11.5,7.4 \mathrm{~Hz}$, 1H), $2.72-2.56(\mathrm{~m}, 2 \mathrm{H}), 2.30(\mathrm{~s}, 3 \mathrm{H}), 2.29-2.18(\mathrm{~m}, 2 \mathrm{H}), 1.85-1.68(\mathrm{~m}, 2 \mathrm{H}) .{ }^{13} \mathrm{C}$ NMR $(126$ $\left.\mathrm{MHz}_{2} \mathrm{CDCl}_{3}\right) \delta 142.5,136.9,136.3,132.4,129.9,129.2,128.5,128.5,128.4,126.9,125.8$, 125.4, 35.6, 31.8, 28.1, 20.1. GCMS (EI) calculated for $[\mathrm{M}]^{+} 236.16$, found 236.2. FTIR (neat, $\left.\mathrm{cm}^{-1}\right)$ : 3024(m), 2920(m), 2855(m), 1602(s), 1485(s), 1453(s), 1435(m), 1104(s0, 1083(s), $1030(\mathrm{~s})$. 


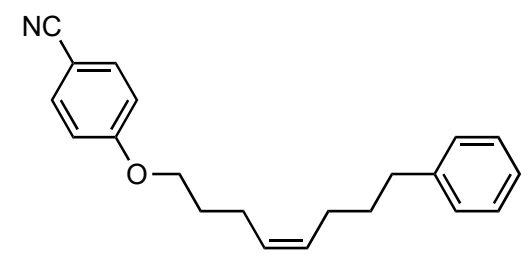

4-\{[(4Z)-8-phenyloct-4-en-1-yl]oxy\}benzonitrile (18), compound was prepared according to general procedure B. The compound was purified by silica gel chromatography with EtOAc/Hex $(0 \rightarrow 20 \%)$ and isolated as a colorless oil (146.2 mg, 96\% yield). ${ }^{1} \mathrm{H}$ NMR $\left(300 \mathrm{MHz}, \mathrm{CDCl}_{3}\right) \delta$ $7.56(\mathrm{~d}, J=8.8 \mathrm{~Hz}, 2 \mathrm{H}), 7.34-7.26(\mathrm{~m}, 1 \mathrm{H}), 7.26-7.23(\mathrm{~m}, 1 \mathrm{H}), 7.21-7.10(\mathrm{~m}, 3 \mathrm{H}), 6.91(\mathrm{~d}$, $J=8.8 \mathrm{~Hz}, 2 \mathrm{H}), 5.58-5.31(\mathrm{~m}, 2 \mathrm{H}), 3.98(\mathrm{t}, J=6.3 \mathrm{~Hz}, 2 \mathrm{H}), 2.64-2.52(\mathrm{~m}, 2 \mathrm{H}), 2.26-2.16$ $(\mathrm{m}, 2 \mathrm{H}), 2.12-2.01(\mathrm{~m}, 2 \mathrm{H}), 1.91-1.80(\mathrm{~m}, 2 \mathrm{H}), 1.71-1.59(\mathrm{~m}, 2 \mathrm{H}) .{ }^{13} \mathrm{C} \mathrm{NMR}(126 \mathrm{MHz}$, $\left.\mathrm{CDCl}_{3}\right) \delta 162.4,142.3,134.0,130.9,128.5,128.4,128.3,125.7,119.3,115.2,103.7,67.4,35.5$, 31.3, 28.8, 26.8, 23.4. GCMS (EI) calculated for [M] $]^{+} 305.18$, found 305.1. FTIR (neat, $\mathrm{cm}^{-1}$ ): 3024(m), 3004(m), 2934(m), 2856(m), 2224(s), 1605(s), 1508(s), 1452(s), 1302(s), 1258(s), 1171(s), 1112(s), 1029(s).

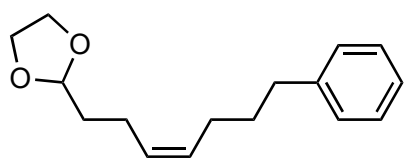

2-[(3Z)-7-phenylhept-3-en-1-yl]-1,3-dioxolane (19), compound was prepared according to general procedure B. The compound was purified by silica gel chromatography with EtOAc/Hex $(0 \rightarrow 20 \%)$ and isolated as a colorless oil $(104.4 \mathrm{mg}, 85 \%$ yield $) .{ }^{1} \mathrm{H} \mathrm{NMR}\left(300 \mathrm{MHz}, \mathrm{CDCl}_{3}\right) \delta$ $7.33-7.26(\mathrm{~m}, 2 \mathrm{H}), 7.21-7.13(\mathrm{~m}, 3 \mathrm{H}), 5.50-5.32(\mathrm{~m}, 2 \mathrm{H}), 4.86(\mathrm{t}, J=4.8 \mathrm{~Hz}, 1 \mathrm{H}), 4.02-$ $3.91(\mathrm{~m}, 2 \mathrm{H}), 3.91-3.79(\mathrm{~m}, 2 \mathrm{H}), 2.67-2.56(\mathrm{~m}, 2 \mathrm{H}), 2.22-2.04(\mathrm{~m}, 4 \mathrm{H}), 1.78-1.62(\mathrm{~m}$, $4 \mathrm{H}) .{ }^{13} \mathrm{C} \mathrm{NMR}\left(126 \mathrm{MHz}, \mathrm{CDCl}_{3}\right) \delta 142.7,130.2,129.2,128.5,128.4,125.8,104.3,65.0,35.6$, 34.0, 31.5, 26.9, 22.1. GCMS (EI) calculated for [M] $]^{+}$246.16, found 246.2. FTIR (neat, $\mathrm{cm}^{-1}$ ): 3061(m), 3024(m), 3003(m), 2928(m), 2857(m), 1602(s), 1495(s), 1452(s), 1359(s), 1138(s), 1055(s), 1030(s), 738(s), 699(s).

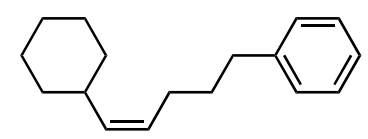

[(4Z)-5-cyclohexylpent-4-en-1-yl]benzene (20), compound was prepared according to general procedure A. The compound was purified by silica gel chromatography with EtOAc/Hex $(0 \rightarrow$ $10 \%)$ and isolated as a colorless oil $\left(83.7 \mathrm{mg}, 73 \%\right.$ yield). ${ }^{1} \mathrm{H}$ NMR $\left(300 \mathrm{MHz}, \mathrm{CDCl}_{3}\right) \delta 7.35-$ $7.26(\mathrm{~m}, 2 \mathrm{H}), 7.24-7.10(\mathrm{~m}, 3 \mathrm{H}), 5.37-5.12(\mathrm{~m}, 2 \mathrm{H}), 2.72-2.52(\mathrm{~m}, 2 \mathrm{H}), 2.30-2.00(\mathrm{~m}$, $3 \mathrm{H}), 1.75-1.51(\mathrm{~m}, 6 \mathrm{H}), 1.35-0.96(\mathrm{~m}, 6 \mathrm{H}) .{ }^{13} \mathrm{C} \mathrm{NMR}\left(126 \mathrm{MHz}, \mathrm{CDCl}_{3}\right) \delta 142.7,136.7$, 128.6, 128.4, 127.6, 125.8, 110.1, 36.5, 35.6, 33.5, 31.8, 27.2, 26.2, 26.1. GCMS (EI) calculated for $[\mathrm{M}]^{+}$228.19, found 228.2. FTIR (neat, $\left.\mathrm{cm}^{-1}\right)$ : 3062(m), 3024(m), 2999(m), 2921(m), 2847(m), 1603(s), 1495(s), 1446(s), 1435(m), 1349(s), 1258(m), 1074(s), 1030(s). 


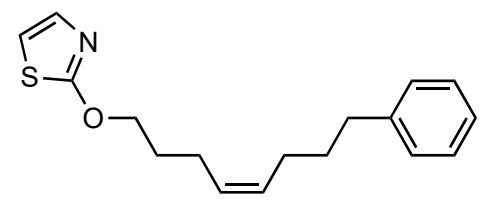

2-\{[(4Z)-8-phenyloct-4-en-1-yl]oxy\}-1,3-thiazole (21), compound was prepared according to general procedure $\mathrm{A}$. The compound was purified by silica gel chromatography with EtOAc/Hex $(0 \rightarrow 40 \%)$ and isolated as a colorless oil $(118.2 \mathrm{mg}, 82 \%$ yield $) .{ }^{1} \mathrm{H}$ NMR $\left(300 \mathrm{MHz}, \mathrm{CDCl}_{3}\right) \delta$ $7.33-7.26(\mathrm{~m}, 2 \mathrm{H}), 7.22-7.14(\mathrm{~m}, 3 \mathrm{H}), 7.13(\mathrm{~d}, J=3.8 \mathrm{~Hz}, 1 \mathrm{H}), 6.67(\mathrm{~d}, J=3.8 \mathrm{~Hz}, 1 \mathrm{H}), 5.60$ $-5.34(\mathrm{~m}, 2 \mathrm{H}), 4.40(\mathrm{t}, J=6.4 \mathrm{~Hz}, 2 \mathrm{H}), 2.68-2.55(\mathrm{~m}, 2 \mathrm{H}), 2.26-2.14(\mathrm{~m}, 2 \mathrm{H}), 2.14-2.02$ $(\mathrm{m}, 2 \mathrm{H}), 1.94-1.81(\mathrm{~m}, 2 \mathrm{H}), 1.75-1.62(\mathrm{~m}, 2 \mathrm{H}) .{ }^{13} \mathrm{C} \mathrm{NMR}\left(126 \mathrm{MHz}, \mathrm{CDCl}_{3}\right) \delta 175.2,142.5$, 137.0, 130.8, 128.6, 128.5, 128.3, 125.7, 110.8, 71.1, 35.5, 31.4, 28.8, 26.9, 23.5. GCMS (EI) calculated for $[\mathrm{M}]^{+}$287.13, found 287.1. FTIR (neat, $\left.\mathrm{cm}^{-1}\right): 3003(\mathrm{~m}), 2931(\mathrm{~m}), 2855(\mathrm{~m})$, 1523(s), 1461(m), 1380(s), 1308(s), 1236(s), 1162(s), 978(m), 907(m).

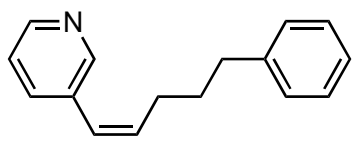

3-[(1Z)-5-phenylpent-1-en-1-yl]pyridine (22), compound was prepared according to general procedure B. The compound was purified by silica gel chromatography with EtOAc/Hex $(0 \rightarrow$ $40 \%)$ and isolated as a colorless oil $\left(94.6 \mathrm{mg}, 85 \%\right.$ yield). ${ }^{1} \mathrm{H}$ NMR $\left(300 \mathrm{MHz}, \mathrm{CDCl}_{3}\right) \delta 8.52(\mathrm{~s}$, $1 \mathrm{H}), 8.45(\mathrm{~d}, J=3.7 \mathrm{~Hz}, 1 \mathrm{H}), 7.52(\mathrm{~d}, J=7.9 \mathrm{~Hz}, 1 \mathrm{H}), 7.25-7.09(\mathrm{~m}, 5 \mathrm{H}), 6.39(\mathrm{~d}, J=11.7 \mathrm{~Hz}$, $1 \mathrm{H}), 5.83(\mathrm{dt}, J=11.7,7.4 \mathrm{~Hz}, 1 \mathrm{H}), 2.70-2.56(\mathrm{~m}, 2 \mathrm{H}), 2.41-2.28(\mathrm{~m}, 2 \mathrm{H}), 1.87-1.71(\mathrm{~m}$, 2H). ${ }^{13} \mathrm{C} \mathrm{NMR}\left(126 \mathrm{MHz}, \mathrm{CDCl}_{3}\right) \delta 149.8,147.4,142.1,136.0,135.2,133.5,128.6,128.5$, 126.0, 125.7, 123.3, 35.5, 31.6, 28.2. GCMS (EI) calculated for $[\mathrm{M}]^{+} 223.14$, found 223.1. FTIR (neat, $\left.\mathrm{cm}^{-1}\right):$ 3048(m), 3026(m), 2931(m), 2857(m), 1602(s), 1566(s), 1474(s), 1420(s), 1399(s), 1265(s), 1176(s), 1024(s).

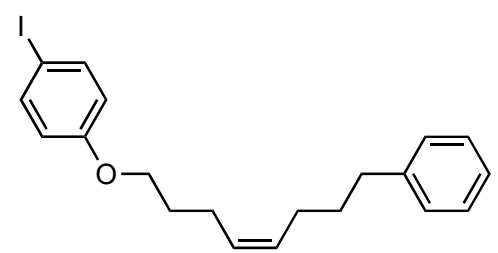

1-iodo-4-\{[(4Z)-8-phenyloct-4-en-1-yl]oxy\}benzene (23), compound was prepared according to general procedure $\mathrm{B}$. The compound was purified by silica gel chromatography with EtOAc/Hex $(0 \rightarrow 10 \%)$ and isolated as a colorless oil $187.0 \mathrm{mg}, 92 \%$ yield $).{ }^{1} \mathrm{H}$ NMR $\left(300 \mathrm{MHz}, \mathrm{CDCl}_{3}\right) \delta$ $7.55(\mathrm{~d}, J=8.9 \mathrm{~Hz}, 2 \mathrm{H}), 7.32-7.27(\mathrm{~m}, 2 \mathrm{H}), 7.23-7.11(\mathrm{~m}, 3 \mathrm{H}), 6.67(\mathrm{~d}, J=8.9 \mathrm{~Hz}, 2 \mathrm{H}), 5.56$ $-5.31(\mathrm{~m}, 2 \mathrm{H}), 3.91(\mathrm{t}, J=6.3 \mathrm{~Hz}, 2 \mathrm{H}), 2.66-2.52(\mathrm{~m}, 2 \mathrm{H}), 2.26-2.16(\mathrm{~m}, 2 \mathrm{H}), 2.13-2.03$ $(\mathrm{m}, 2 \mathrm{H}), 1.89-1.78(\mathrm{~m}, 2 \mathrm{H}), 1.73-1.59(\mathrm{~m}, 2 \mathrm{H}) .{ }^{13} \mathrm{C}$ NMR $\left(126 \mathrm{MHz}, \mathrm{CDCl}_{3}\right) \delta 159.1,142.5$, $138.3,130.8,128.9,128.5,128.4,125.8,117.0,82.6,67.2$, 35.6, 31.5, 29.1, 27.0, 23.7. GCMS (EI) calculated for $[\mathrm{M}]^{+}$406.08, found 406.1. FTIR (neat, $\left.\mathrm{cm}^{-1}\right): 3060(\mathrm{~m}), 3024(\mathrm{~m}), 3001(\mathrm{~m})$, 2933(m), 2855(m), 1586(s), 1485(s), 1467(s), 1282(s), 1243(s), 1174(s), 819(s), 698(s). 


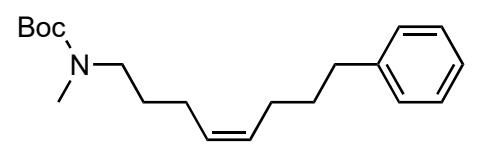

tert-butyl N-methyl-N-[(4Z)-8-phenyloct-4-en-1-yl]carbamate (24), compound was prepared according to general procedure $\mathrm{A}$. The compound was purified by silica gel chromatography with EtOAc/Hex $(0 \rightarrow 20 \%)$ and isolated as a colorless oil $(123.2 \mathrm{mg}, 78 \%$ yield $) .{ }^{1} \mathrm{H}$ NMR (300 $\left.\mathrm{MHz} \mathrm{CDCl}_{3}\right) \delta 7.33-7.26(\mathrm{~m}, 2 \mathrm{H}), 7.23-7.13(\mathrm{~m}, 3 \mathrm{H}), 5.53-5.31(\mathrm{~m}, 2 \mathrm{H}), 3.26-3.11(\mathrm{~m}$, $2 \mathrm{H}), 2.84(\mathrm{~s}, 3 \mathrm{H}), 2.71-2.50(\mathrm{~m}, 2 \mathrm{H}), 2.13-1.94(\mathrm{~m}, 4 \mathrm{H}), 1.75-1.63(\mathrm{~m}, 2 \mathrm{H}), 1.61-1.53(\mathrm{~m}$, 2H), $1.46(\mathrm{~s}, 9 \mathrm{H}) .{ }^{13} \mathrm{C}$ NMR $\left(126 \mathrm{MHz}, \mathrm{CDCl}_{3}\right) \delta 155.9,142.5,130.1,129.4,128.5,128.4,125.8$, 79.2, 48.8, 35.6, 34.2, 31.5, 28.6, 28.6, 26.9, 24.6. GCMS (EI) calculated for [M] 317.24 , found 317.2. FTIR (neat, $\left.\mathrm{cm}^{-1}\right): 3062(\mathrm{~m}), 3005(\mathrm{~m}), 2974(\mathrm{~m}), 2929(\mathrm{~m}), 2858(\mathrm{~m}), 1695(\mathrm{~s}), 1479(\mathrm{~s})$, 1391(s), 1365(s), 1303(s), 1166(s), 879(s), 754(s).

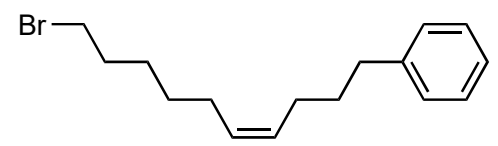

[(4Z)-10-bromodec-4-en-1-yl]benzene (25), compound was prepared according to general procedure A. The compound was purified by silica gel chromatography with EtOAc/Hex $(0 \rightarrow$ $10 \%)$ and isolated as a colorless oil $(96.9 \mathrm{mg}, 66 \%$ yield $) .{ }^{1} \mathrm{H} \mathrm{NMR}\left(300 \mathrm{MHz}, \mathrm{CDCl}_{3}\right) \delta 7.36-$ $7.26(\mathrm{~m}, 2 \mathrm{H}), 7.22-7.12(\mathrm{~m}, 3 \mathrm{H}), 5.49-5.29(\mathrm{~m}, 2 \mathrm{H}), 3.40(\mathrm{t}, J=6.8 \mathrm{~Hz}, 2 \mathrm{H}), 2.69-2.55(\mathrm{~m}$, $2 \mathrm{H}), 2.12-1.97(\mathrm{~m}, 4 \mathrm{H}), 1.92-1.79(\mathrm{~m}, 2 \mathrm{H}), 1.75-1.61(\mathrm{~m}, 2 \mathrm{H}), 1.47-1.33(\mathrm{~m}, 4 \mathrm{H}) .{ }^{13} \mathrm{C}$ NMR $\left(126 \mathrm{MHz}, \mathrm{CDCl}_{3}\right) \delta 142.6,130.0,129.9,128.6,128.4,125.8,35.6,34.0,32.9,31.6,29.0$, 28.0, 27.2, 27.0. GCMS (EI) calculated for [M] ${ }^{+}$294.10, found 294.1. FTIR (neat, $\mathrm{cm}^{-1}$ ): 3060(m), 3002(m), 2930(m), 2855(m), 1602(s), 1495(s), 1452(s), 970(s).

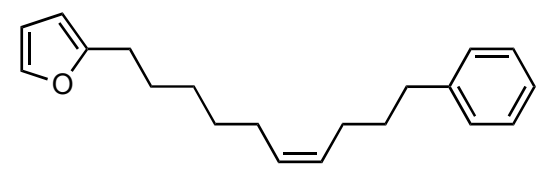

2-[(6Z)-10-phenyldec-6-en-1-yl]furan (26), compound was prepared according to general procedure A. The compound was purified by silica gel chromatography with EtOAc/Hex $(0 \rightarrow$ $20 \%)$ and isolated as a colorless oil $\left(127.3 \mathrm{mg}, 90 \%\right.$ yield). ${ }^{1} \mathrm{H} \mathrm{NMR}\left(300 \mathrm{MHz}, \mathrm{CDCl}_{3}\right) \delta 7.33-$ $7.26(\mathrm{~m}, 3 \mathrm{H}), 7.23-7.14(\mathrm{~m}, 3 \mathrm{H}), 6.28(\mathrm{dd}, J=2.9,2.0 \mathrm{~Hz}, 1 \mathrm{H}), 6.00-5.95(\mathrm{~m}, 1 \mathrm{H}), 5.47-$ $5.32(\mathrm{~m}, 2 \mathrm{H}), 2.62(\mathrm{t}, J=7.6 \mathrm{~Hz}, 4 \mathrm{H}), 2.13-1.97(\mathrm{~m}, 4 \mathrm{H}), 1.73-1.60(\mathrm{~m}, 4 \mathrm{H}), 1.41-1.32(\mathrm{~m}$, $4 \mathrm{H}) .{ }^{13} \mathrm{C}$ NMR $\left(126 \mathrm{MHz}, \mathrm{CDCl}_{3}\right) \delta 156.3,142.4,140.5,130.1,129.3,128.3,128.1,125.5$, 109.9, 104.4, 35.3, 31.3, 29.3, 28.7, 27.8, 27.0, 26.7. GCMS (EI) calculated for $[\mathrm{M}]^{+} 282.20$, found 282.1. FTIR (neat, $\left.\mathrm{cm}^{-1}\right): 3025(\mathrm{~m}), 3002(\mathrm{~m}), 2928(\mathrm{~m}), 2855(\mathrm{~m}), 1655(\mathrm{~m}), 1597(\mathrm{~s})$, 1507(s), 1460(s), 1350(w), 1147(s), 1079(s), 1007(s), 920(m), 796(s).

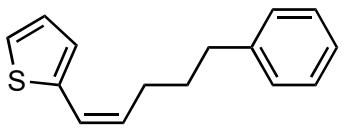

2-[(1Z)-5-phenylpent-1-en-1-yl]thiophene (27), compound was prepared according to general procedure B. The compound was purified by silica gel chromatography with EtOAc/Hex $(0 \rightarrow$ $20 \%$ ) and isolated as a colorless oil (107.4 mg, 94\% yield). ${ }^{1} \mathrm{H}$ NMR $\left(300 \mathrm{MHz}, \mathrm{CDCl}_{3}\right) \delta 7.34-$ 
$7.26(\mathrm{~m}, 3 \mathrm{H}), 7.24-7.14(\mathrm{~m}, 3 \mathrm{H}), 7.10(\mathrm{~d}, J=2.3 \mathrm{~Hz}, 1 \mathrm{H}), 7.07(\mathrm{~d}, J=5.0 \mathrm{~Hz}, 1 \mathrm{H}), 6.40(\mathrm{~d}, J=$ $11.6 \mathrm{~Hz}, 1 \mathrm{H}), 5.65(\mathrm{dt}, J=11.6,7.3 \mathrm{~Hz}, 1 \mathrm{H}), 2.79-2.57(\mathrm{~m}, 2 \mathrm{H}), 2.41(\mathrm{q}, J=7.3 \mathrm{~Hz}, 2 \mathrm{H}), 1.91$ - $1.73(\mathrm{~m}, 2 \mathrm{H}) .{ }^{13} \mathrm{C}$ NMR $\left(126 \mathrm{MHz}, \mathrm{CDCl}_{3}\right) \delta 142.4,138.9,131.7,128.7,128.6,128.4,125.9$, 125.0, 123.5, 122.8, 35.7, 31.6, 28.7. GCMS (EI) calculated for [M] ${ }^{+} 228.10$, found 228.1. FTIR (neat, $\left.\mathrm{cm}^{-1}\right)$ : 3061(m), 3024(m), 2923(m), 2855(m), 1602(s), 1494(s), 1452(s), 1350(s), 1251(m), 1149(s), 1080(s), 1029(s).

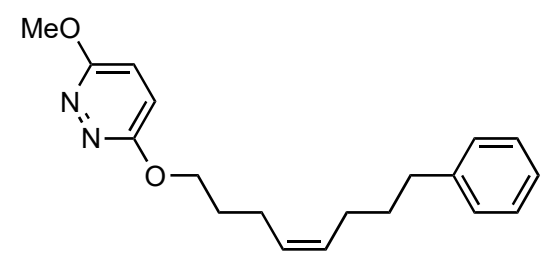

3-methoxy-6-\{[(4Z)-8-phenyloct-4-en-1-yl]oxy\}pyridazine (28), compound was prepared according to general procedure A. The compound was purified by silica gel chromatography with EtOAc/Hex $(0 \rightarrow 40 \%)$ and isolated as a colorless oil $\left(118.6 \mathrm{mg}, 76 \%\right.$ yield). ${ }^{1} \mathrm{H}$ NMR (300 $\left.\mathrm{MHz}, \mathrm{CDCl}_{3}\right) \delta 7.30-7.26(\mathrm{~m}, 1 \mathrm{H}), 7.26-7.22(\mathrm{~m}, 1 \mathrm{H}), 7.21-7.11(\mathrm{~m}, 3 \mathrm{H}), 6.98-6.79(\mathrm{~m}$, $2 \mathrm{H}), 5.57-5.26(\mathrm{~m}, 2 \mathrm{H}), 4.40(\mathrm{t}, J=6.5 \mathrm{~Hz}, 2 \mathrm{H}), 4.04(\mathrm{~s}, 3 \mathrm{H}), 2.66-2.52(\mathrm{~m}, 2 \mathrm{H}), 2.25-2.13$ $(\mathrm{m}, 2 \mathrm{H}), 2.13-2.00(\mathrm{~m}, 2 \mathrm{H}), 1.92-1.81(\mathrm{~m}, 2 \mathrm{H}), 1.75-1.57(\mathrm{~m}, 2 \mathrm{H}) .{ }^{13} \mathrm{C}$ NMR $(75 \mathrm{MHz}$, $\left.\mathrm{CDCl}_{3}\right) \delta 162.0,161.9,142.6,130.5,129.1,128.5,128.4,125.8,121.6,121.4,66.7,54.6,35.6$, 31.5, 28.9, 26.9, 23.8. GCMS (EI) calculated for $[\mathrm{M}]^{+} 312.18$, found 312.2. FTIR (neat, $\mathrm{cm}^{-1}$ ): 3005(m), 2936(m), 2856(m), 1696(s), 1602(s), 1463(m), 1421(s), 1383(s), 1267(s), 1015(s), 838(s), 750(s), 699(s).

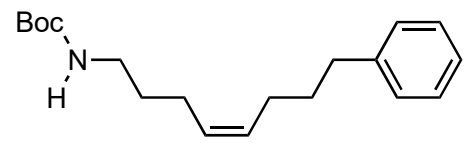

tert-butyl N-[(4Z)-8-phenyloct-4-en-1-yl]carbamate (29), compound was prepared according to general procedure $\mathrm{A}$. The compound was purified by silica gel chromatography with EtOAc/Hex $(0 \rightarrow 50 \%)$ and isolated as a colorless oil $(109.7 \mathrm{mg}, 72 \%$ yield $) .{ }^{1} \mathrm{H} \mathrm{NMR} \mathrm{(300}$ $\left.\mathrm{MHz}, \mathrm{CDCl}_{3}\right) \delta 7.34-7.26(\mathrm{~m}, 2 \mathrm{H}), 7.24-7.10(\mathrm{~m}, 3 \mathrm{H}), 5.53-5.24(\mathrm{~m}, 2 \mathrm{H}), 4.49(\mathrm{~s}, 1 \mathrm{H}), 3.22$ $-2.95(\mathrm{~m}, 2 \mathrm{H}), 2.69-2.53(\mathrm{~m}, 2 \mathrm{H}), 2.15-1.96(\mathrm{~m}, 4 \mathrm{H}), 1.74-1.61(\mathrm{~m}, 2 \mathrm{H}), 1.56-1.50(\mathrm{~m}$, 2H), 1.45 (s, 9H). ${ }^{13} \mathrm{C}$ NMR $\left(126 \mathrm{MHz}, \mathrm{CDCl}_{3}\right) \delta 170.4,142.6,130.4,129.2,128.5,128.4,125.8$, 79.2, 40.4, 35.6, 31.5, 30.1, 28.6, 26.9, 24.7. GCMS (EI) calculated for $[\mathrm{M}]^{+} 303.22$, found 303.1. FTIR (neat, $\left.\mathrm{cm}^{-1}\right): 3438(\mathrm{~b}), 3355(\mathrm{~b}), 3005(\mathrm{~m}), 2927(\mathrm{~m}), 2857(\mathrm{~m}), 2247(\mathrm{~s}), 1694(\mathrm{~s})$, 1524(s), 1455(s), 1363(s), 1250(m), 1168(m), 1030(m), 910(s), 852(m).

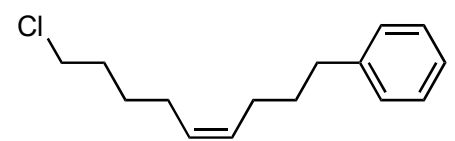

[(4Z)-9-chloronon-4-en-1-yl]benzene (30), compound was prepared according to general procedure $\mathrm{A}$. The compound was purified by silica gel chromatography with EtOAc/Hex $(0 \rightarrow$ $10 \%)$ and isolated as a colorless oil $\left(109.6 \mathrm{mg}, 93 \%\right.$ yield). ${ }^{1} \mathrm{H}$ NMR $\left(300 \mathrm{MHz}, \mathrm{CDCl}_{3}\right) \delta 7.35-$ $7.26(\mathrm{~m}, 2 \mathrm{H}), 7.24-7.13(\mathrm{~m}, 3 \mathrm{H}), 5.54-5.25(\mathrm{~m}, 2 \mathrm{H}), 3.53(\mathrm{t}, J=6.7 \mathrm{~Hz}, 2 \mathrm{H}), 2.72-2.51(\mathrm{~m}$, 2H), $2.17-1.95(\mathrm{~m}, 4 \mathrm{H}), 1.83-1.63(\mathrm{~m}, 4 \mathrm{H}), 1.53-1.41(\mathrm{~m}, 2 \mathrm{H}) .{ }^{13} \mathrm{C} \mathrm{NMR}\left(126 \mathrm{MHz}, \mathrm{CDCl}_{3}\right)$ 
$\delta 142.5,130.2,129.5,128.5,128.4,125.8,45.0,35.5,32.2,31.5,27.0,26.9,26.5$. GCMS (EI) calculated for $[\mathrm{M}]^{+}$236.13, found 236.1. FTIR (neat, $\left.\mathrm{cm}^{-1}\right): 3061(\mathrm{~m}), 3002(\mathrm{~m}), 2925(\mathrm{~m})$, 2855(m), 1602(s), 1494(s), 1452(s), 1433(s), 1309(s), $1275(\mathrm{~m}), 1029(\mathrm{~s})$.

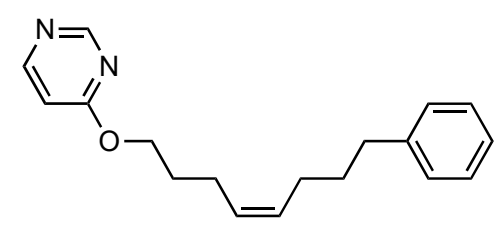

4-\{[(4Z)-8-phenyloct-4-en-1-yl]oxy\}pyrimidine (31), compound was prepared according to general procedure $\mathrm{A}$. The compound was purified by silica gel chromatography with $\mathrm{EtOAc} / \mathrm{Hex}$ $(0 \rightarrow 40 \%)$ and isolated as a colorless oil $\left(112.8 \mathrm{mg}, 80 \%\right.$ yield). ${ }^{1} \mathrm{H} \mathrm{NMR}\left(300 \mathrm{MHz}, \mathrm{CDCl}_{3}\right) \delta$ $8.75(\mathrm{~s}, 1 \mathrm{H}), 8.40(\mathrm{~d}, J=5.8 \mathrm{~Hz}, 1 \mathrm{H}), 7.32-7.27(\mathrm{~m}, 1 \mathrm{H}), 7.25-7.21(\mathrm{~m}, 1 \mathrm{H}), 7.20-7.12(\mathrm{~m}$, $3 \mathrm{H}), 6.70(\mathrm{dd}, J=5.8,1.1 \mathrm{~Hz}, 1 \mathrm{H}), 5.57-5.27(\mathrm{~m}, 2 \mathrm{H}), 4.35(\mathrm{t}, J=6.6 \mathrm{~Hz}, 2 \mathrm{H}), 2.65-2.54(\mathrm{~m}$, $2 \mathrm{H}), 2.24-2.12(\mathrm{~m}, 2 \mathrm{H}), 2.12-2.01(\mathrm{~m}, 2 \mathrm{H}), 1.88-1.77(\mathrm{~m}, 2 \mathrm{H}), 1.74-1.60(\mathrm{~m}, 2 \mathrm{H}) .{ }^{13} \mathrm{C}$ NMR (126 MHz, $\left.\mathrm{CDCl}_{3}\right) \delta 169.4,158.9,157.3,142.8,131.0,129.1,128.8,128.7,126.1,109.1$, $66.3,35.8,31.7,29.0,27.2,23.9$. GCMS (EI) calculated for [M] ${ }^{+} 282.17$, found 282.1. FTIR (neat, $\left.\mathrm{cm}^{-1}\right): 3005(\mathrm{~m}), 2933(\mathrm{~m}), 2856(\mathrm{~m}), 1582(\mathrm{~s}), 1560(\mathrm{~s}), 1470(\mathrm{~s}), 1462(\mathrm{~s}), 1396(\mathrm{~s}), 1374(\mathrm{~s})$, 1305(s), 1163(m), 985(s), 834(s), 733(s).

\section{Characterization of $Z$-selective Hydroalkylation Products: Alkylboranes}

Alkylboranes were used as a $2 \mathrm{M}$ solution in toluene and were synthesized according to literature procedure the day before use. ${ }^{7}$<smiles>COC(=O)CCCCCCCCCC/C=C\CCCOc1ccc(C(C)=O)cc1</smiles>

methyl 4-\{[(4Z)-16-methoxy-16-oxohexadec-4-en-1-yl]oxy\}benzoate (32), compound was prepared according to general procedure A. The compound was purified by silica gel chromatography with EtOAc/Hex $(0 \rightarrow 30 \%)$ and isolated as a colorless oil $(163.3 \mathrm{mg}, 78 \%$ yield). ${ }^{1} \mathrm{H}$ NMR $\left(300 \mathrm{MHz}, \mathrm{CDCl}_{3}\right) \delta 7.97(\mathrm{~d}, J=8.9 \mathrm{~Hz}, 2 \mathrm{H}), 6.90(\mathrm{~d}, J=8.9 \mathrm{~Hz}, 2 \mathrm{H}), 5.52-$ $5.27(\mathrm{~m}, 2 \mathrm{H}), 4.00(\mathrm{t}, J=6.4 \mathrm{~Hz}, 2 \mathrm{H}), 3.88(\mathrm{~s}, 3 \mathrm{H}), 3.66(\mathrm{~s}, 3 \mathrm{H}), 2.30(\mathrm{t}, J=7.6 \mathrm{~Hz}, 2 \mathrm{H}), 2.25-$ $2.15(\mathrm{~m}, 2 \mathrm{H}), 2.06-1.95(\mathrm{~m}, 2 \mathrm{H}), 1.89-1.81(\mathrm{~m}, 2 \mathrm{H}), 1.64-1.57(\mathrm{~m}, 2 \mathrm{H}), 1.36-1.14(\mathrm{~m}$, 16H). ${ }^{13} \mathrm{C}$ NMR $\left(126 \mathrm{MHz}, \mathrm{CDCl}_{3}\right) \delta$ 174.4, 166.9, 163.0, 131.6, 131.5, 128.1, 122.4, 114.1, $67.3,51.9,51.5,42.0,34.2,29.8,29.6,29.5,29.4,29.2,29.1,27.3,27.2,25.0,23.5$. GCMS (EI) calculated for $[\mathrm{M}]^{+}$418.27, found 418.3. FTIR (neat, $\left.\mathrm{cm}^{-1}\right)$ : 2998(m), 2925(m), 2853(m), 1726(s), 1720(s), 1605(s), 1511(s), 1434(s), 1279(s), 1253(s), 1167(s), 1104(s), 1024(m), 913(s).<smiles>COc1ccc(OCCC/C=C\CCCCCOc2ccc(C3OCCO3)cc2)cc1</smiles>

methyl 4-\{[(4Z)-10-[4-(1,3-dioxolan-2-yl)phenoxy]dec-4-en-1-yl]oxy\}benzoate (33), compound was prepared according to general procedure A. The compound was purified by silica gel chromatography with EtOAc/Hex $(0 \rightarrow 30 \%)$ and isolated as a colorless oil $(153.2 \mathrm{mg}, 67 \%$ yield). ${ }^{1} \mathrm{H} \mathrm{NMR}\left(300 \mathrm{MHz}, \mathrm{CDCl}_{3}\right) \delta 7.97(\mathrm{~d}, J=8.8 \mathrm{~Hz}, 2 \mathrm{H}), 7.38(\mathrm{~d}, J=8.6 \mathrm{~Hz}, 2 \mathrm{H}), 6.96-$ 
$6.80(\mathrm{~m}, 84), 5.75(\mathrm{~s}, 1 \mathrm{H}), 5.50-5.30(\mathrm{~m}, 2 \mathrm{H}), 4.17-4.05(\mathrm{~m}, 2 \mathrm{H}), 4.03-3.98(\mathrm{~m}, 2 \mathrm{H}), 3.92(\mathrm{t}$, $J=6.5 \mathrm{~Hz}, 2 \mathrm{H}), 3.87(\mathrm{~s}, 3 \mathrm{H}), 2.29-2.17(\mathrm{~m}, 2 \mathrm{H}), 2.10-1.99(\mathrm{~m}, 2 \mathrm{H}), 1.91-1.79(\mathrm{~m}, 2 \mathrm{H})$, $1.76-1.68(\mathrm{~m}, 2 \mathrm{H}), 1.47-1.32(\mathrm{~m}, 4 \mathrm{H}) .{ }^{13} \mathrm{C} \mathrm{NMR}\left(126 \mathrm{MHz}, \mathrm{CDCl}_{3}\right) \delta 166.9,163.0,160.0$, $131.6,131.1,128.5,127.9,122.5,114.4,114.1,103.8,68.0,67.3,65.3,51.9,41.9,29.5,29.2$, 29.0, 27.2, 25.7, 23.6. GCMS (ESI) calculated for $[\mathrm{M}+\mathrm{H}]^{+} 455.24$, found 455.2. FTIR (neat, $\mathrm{cm}^{-}$ $\left.{ }^{1}\right)$ : 3006(m), 2945(m), 2864(m), 1919(w), 1720(s), 1605(s), 1511(s), 1435(s), 1393(s), 1252(m), 1168(s), 912(m), 847(s).<smiles>COc1ccc(OCCCCC/C=C\CCCOc2ccc(C(C)=O)cc2)cc1</smiles>

methyl 4-\{[(4Z)-10-(4-methoxyphenoxy)dec-4-en-1-yl]oxy\}benzoate (34), compound was prepared according to general procedure A. The compound was purified by silica gel chromatography with EtOAc/Hex $(0 \rightarrow 30 \%)$ and isolated as a colorless oil $(178.6 \mathrm{mg}, 87 \%$ yield). ${ }^{1} \mathrm{H}$ NMR $\left(300 \mathrm{MHz}, \mathrm{CDCl}_{3}\right) \delta 7.97(\mathrm{~d}, J=8.9 \mathrm{~Hz}, 2 \mathrm{H}), 6.90(\mathrm{~d}, J=8.9 \mathrm{~Hz}, 2 \mathrm{H}), 6.82(\mathrm{~s}$, $4 \mathrm{H}), 5.51-5.32(\mathrm{~m}, 2 \mathrm{H}), 4.00(\mathrm{t}, J=6.3 \mathrm{~Hz}, 2 \mathrm{H}), 3.91-3.82(\mathrm{~m}, 5 \mathrm{H}), 3.76(\mathrm{~s}, 3 \mathrm{H}), 2.29-2.18$ $(\mathrm{m}, 2 \mathrm{H}), 2.05(\mathrm{q}, J=6.5 \mathrm{~Hz}, 2 \mathrm{H}), 1.92-1.80(\mathrm{~m}, 2 \mathrm{H}), 1.78-1.65(\mathrm{~m}, 2 \mathrm{H}), 1.48-1.32(\mathrm{~m}, 4 \mathrm{H})$. ${ }^{13} \mathrm{C} \mathrm{NMR}\left(126 \mathrm{MHz}, \mathrm{CDCl}_{3}\right) \delta 167.0,163.0,153.8,153.4,131.7,131.2,128.5,122.5,115.5$, 114.7, 114.2, 68.6, 67.3, 55.8, 51.9, 29.5, 29.4, 29.1, 27.2, 25.8, 23.6. GCMS (EI) calculated for $[\mathrm{M}]^{+}$412.22, found 412.2. FTIR (neat, $\left.\mathrm{cm}^{-1}\right): 3053(\mathrm{~m}), 2986(\mathrm{~m}), 1711(\mathrm{~s}), 1606(\mathrm{~s}), 1502(\mathrm{~s})$, 1421(s), 1262(s), 1169(s), 895(s), 738(s).<smiles>CCCC/C=C\CCCOc1ccc(C(C)=O)cc1</smiles>

methyl 4-\{[(4Z)-8-(trimethylsilyl)oct-4-en-1-yl]oxy\}benzoate (35), compound was prepared according to general procedure $\mathrm{A}$. The compound was purified by silica gel chromatography with EtOAc/Hex $(0 \rightarrow 30 \%)$ and isolated as a colorless oil $\left(139.0 \mathrm{mg}, 83 \%\right.$ yield). ${ }^{1} \mathrm{H}$ NMR (300 $\left.\mathrm{MHz} \mathrm{CDCl}_{3}\right) \delta 7.98(\mathrm{~d}, J=8.8 \mathrm{~Hz}, 1 \mathrm{H}), 6.90(\mathrm{~d}, J=8.8 \mathrm{~Hz}, 1 \mathrm{H}), 5.49-5.32(\mathrm{~m}, 1 \mathrm{H}), 4.01(\mathrm{t}, J$ $=6.4 \mathrm{~Hz}, 1 \mathrm{H}), 3.88(\mathrm{~s}, 1 \mathrm{H}), 2.32-2.16(\mathrm{~m}, 1 \mathrm{H}), 2.10-1.97(\mathrm{~m}, 1 \mathrm{H}), 1.86(\mathrm{p}, J=6.7 \mathrm{~Hz}, 1 \mathrm{H})$, $1.37-1.23(\mathrm{~m}, 1 \mathrm{H}), 0.56-0.39(\mathrm{~m}, 1 \mathrm{H}),-0.05(\mathrm{~s}, 4 \mathrm{H}) .{ }^{13} \mathrm{C} \mathrm{NMR}\left(126 \mathrm{MHz}, \mathrm{CDCl}_{3}\right) \delta 142.7$, $130.2,129.2$, 128.5, 128.4, 125.8, 104.3, 65.0, 35.6, 34.0, 31.5, 26.9, 22.1. GCMS (EI) calculated for $[\mathrm{M}]^{+}$334.20, found 334.2. FTIR (neat, $\left.\mathrm{cm}^{-1}\right)$ : 3003(m), 2950(m), 2873(m), 1914(w), 1721(s), 1606(s), 1511(s), 1434(s), 1279(m), 1254(m), 1167(s), 1104(s), 969(s), 844(s).<smiles>CCCCCCCCC(CC/C=C\CCCOc1ccc(C(C)=O)cc1)OC</smiles>

methyl 4-\{[(4Z)-8-methoxyhexadec-4-en-1-yl]oxy\}benzoate (36), compound was prepared according to general procedure A. The compound was purified by silica gel chromatography with EtOAc/Hex $(0 \rightarrow 30 \%)$ and isolated as a colorless oil $\left(182.8 \mathrm{mg}, 90 \%\right.$ yield). ${ }^{1} \mathrm{H}$ NMR (300 $\left.\mathrm{MHz}_{2} \mathrm{CDCl}_{3}\right) \delta 7.97(\mathrm{~d}, J=8.8 \mathrm{~Hz}, 2 \mathrm{H}), 6.90(\mathrm{~d}, J=8.8 \mathrm{~Hz}, 2 \mathrm{H}), 5.54-5.26(\mathrm{~m}, 2 \mathrm{H}), 4.01(\mathrm{t}, J$ $=6.4 \mathrm{~Hz}, 2 \mathrm{H}), 3.88(\mathrm{~s}, 3 \mathrm{H}), 3.29(\mathrm{~s}, 3 \mathrm{H}), 3.18-3.01(\mathrm{~m}, 1 \mathrm{H}), 2.32-2.16(\mathrm{~m}, 2 \mathrm{H}), 2.15-2.01$ $(\mathrm{m}, 2 \mathrm{H}), 1.86(\mathrm{p}, J=6.8 \mathrm{~Hz}, 2 \mathrm{H}), 1.54-1.35(\mathrm{~m}, 4 \mathrm{H}), 1.27(\mathrm{~s}, 12 \mathrm{H}), 0.88(\mathrm{t}, J=6.6 \mathrm{~Hz}, 3 \mathrm{H})$. ${ }^{13} \mathrm{C} \mathrm{NMR}\left(126 \mathrm{MHz}, \mathrm{CDCl}_{3}\right) \delta 167.0,163.0,131.7,131.0,128.5,122.5,114.1,80.4,67.4,56.4$, 
51.9, 33.5, 33.4, 32.0, 30.0, 29.7, 29.4, 29.1, 25.3, 23.6, 23.1, 22.8, 14.2. GCMS (EI) calculated for $[\mathrm{M}]^{+}$404.29, found 404.3. FTIR (neat, $\left.\mathrm{cm}^{-1}\right): 3004(\mathrm{~m}), 2927(\mathrm{~m}), 2854(\mathrm{~m}), 1913(\mathrm{w}), 1719(\mathrm{~s})$, 1606(s), 1511(s), 1434(s), 1280(s), 1253(s), 1167(s), 1103(s), 1037(m), 970(s), 846(s), 757(s).

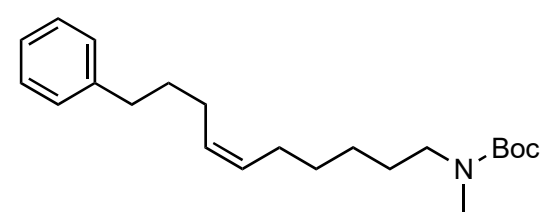

tert-butyl N-methyl-N-[(6Z)-10-phenyldec-6-en-1-yl]carbamate (37), compound was prepared according to general procedure $\mathrm{A}$. The compound was purified by silica gel chromatography with EtOAc/Hex $(0 \rightarrow 30 \%)$ and isolated as a colorless oil (As determined by NMR, 64\% yield). ${ }^{1} \mathrm{H}$ NMR $\left(300 \mathrm{MHz}, \mathrm{CDCl}_{3}\right) \delta 7.31-7.26(\mathrm{~m}, 1 \mathrm{H}), 7.26-7.22(\mathrm{~m}, 1 \mathrm{H}), 7.20-7.13(\mathrm{~m}, 3 \mathrm{H})$, $5.50-5.27(\mathrm{~m}, 2 \mathrm{H}), 3.18(\mathrm{t}, J=7.1 \mathrm{~Hz}, 2 \mathrm{H}), 2.82(\mathrm{~s}, 3 \mathrm{H}), 2.68-2.56(\mathrm{~m}, 2 \mathrm{H}), 2.15-1.91(\mathrm{~m}$, $4 \mathrm{H}), 1.74-1.61(\mathrm{~m}, 2 \mathrm{H}), 1.50-1.43(\mathrm{~m}, 11 \mathrm{H}), 1.36-1.24(\mathrm{~m}, 4 \mathrm{H}) .{ }^{13} \mathrm{C}$ NMR $(126 \mathrm{MHz}$, $\left.\mathrm{CDCl}_{3}\right) \delta 156.0,142.7,130.3,129.6,128.6,128.4,125.8,79.2,48.9,35.6,34.2,32.2,31.6,29.6$, 28.6, 27.4, 27.0, 26.5. GCMS (EI) calculated for [M] $]^{+} 345.27$, found 345.3. FTIR (neat, $\mathrm{cm}^{-1}$ ): 3084(w), 3062(w), 3025(m), 3003(m), 2929(s) 2858(s), 1696(s), 1480(m), 1453(m), 1394(s), 1365(s), 1308(m), 1247(m), 1215(m), 1161(s).<smiles>COC(=O)c1ccc(OCCCC/C=C\CCCCCOc2ccc(C(C)=O)cc2)cc1</smiles>

methyl 4-\{[(4Z)-10-[4-(methoxycarbonyl)phenoxy]dec-4-en-1-yl]oxy\}benzoate (38), compound was prepared according to general procedure A. The compound was purified by silica gel chromatography with EtOAc/Hex $(0 \rightarrow 30 \%)$ and isolated as a colorless oil $(198.4 \mathrm{mg}, 90 \%$ yield). ${ }^{1} \mathrm{H}$ NMR $\left(300 \mathrm{MHz}, \mathrm{CDCl}_{3}\right) \delta 7.97(\mathrm{~d}, J=8.7 \mathrm{~Hz}, 4 \mathrm{H}), 6.95-6.80(\mathrm{~m}, 4 \mathrm{H}), 5.51-5.31$ $(\mathrm{m}, 2 \mathrm{H}), 4.04-3.92(\mathrm{~m}, 4 \mathrm{H}), 3.88(\mathrm{~s}, 3 \mathrm{H}), 3.87(\mathrm{~s}, 3 \mathrm{H}), 2.31-2.16(\mathrm{~m}, 2 \mathrm{H}), 2.05(\mathrm{q}, J=6.4 \mathrm{~Hz}$, $2 \mathrm{H}), 1.93-1.80(\mathrm{~m}, 2 \mathrm{H}), 1.80-1.67(\mathrm{~m}, 2 \mathrm{H}), 1.45-1.29(\mathrm{~m}, 4 \mathrm{H}) .{ }^{13} \mathrm{C} \mathrm{NMR}(126 \mathrm{MHz}$, $\left.\mathrm{CDCl}_{3}\right) \delta 166.8,166.8,162.9,162.9,131.6,130.9,128.5,122.4,122.3,114.0,68.0,67.1,51.8$, 29.4, 29.0, 28.9, 27.1, 25.6, 23.5. GCMS (EI) calculated for $[\mathrm{M}]^{+}$440.22, found 440.3. FTIR (neat, $\left.\mathrm{cm}^{-1}\right):$ 3002(m), 2946(m), 2857(m), 1716(s), 1606(s), 1511(s), 1434(s), 1280(s), 1254(s), 1168(s), 1104(s), 1010(m), 970(m).<smiles>CC(=O)c1ccc(OCCC/C=C\CCCCCOc2ccc(C(F)(F)F)cc2)cc1</smiles>

methyl 4-\{[(4Z)-10-[4-(trifluoromethyl)phenoxy]dec-4-en-1-yl]oxy\}benzoate (39), compound was prepared according to general procedure A. The compound was purified by silica gel chromatography with EtOAc/Hex $(0 \rightarrow 30 \%)$ and isolated as a colorless oil $(181.2 \mathrm{mg}, 80 \%$ yield). ${ }^{1} \mathrm{H} \mathrm{NMR}\left(300 \mathrm{MHz}, \mathrm{CDCl}_{3}\right) \delta 7.97(\mathrm{~d}, J=8.6 \mathrm{~Hz}, 2 \mathrm{H}), 7.52(\mathrm{~d}, J=8.7 \mathrm{~Hz}, 2 \mathrm{H}), 7.05-$ $6.70(\mathrm{~m}, 4 \mathrm{H}), 5.50-5.31(\mathrm{~m}, 2 \mathrm{H}), 4.00(\mathrm{t}, J=6.3 \mathrm{~Hz}, 2 \mathrm{H}), 3.94(\mathrm{t}, J=6.5 \mathrm{~Hz}, 2 \mathrm{H}), 3.87(\mathrm{~s}, 3 \mathrm{H})$, $2.33-2.16(\mathrm{~m}, 2 \mathrm{H}), 2.12-1.97(\mathrm{~m}, 2 \mathrm{H}), 1.94-1.80(\mathrm{~m}, 2 \mathrm{H}), 1.80-1.65(\mathrm{~m}, 2 \mathrm{H}), 1.47-1.30$ $(\mathrm{m}, 4 \mathrm{H}) .{ }^{13} \mathrm{C} \mathrm{NMR}\left(126 \mathrm{MHz}, \mathrm{CDCl}_{3}\right) \delta 166.9,163.0,161.7,131.7,131.0,128.6,126.9(\mathrm{q}, J=$ $3.7 \mathrm{~Hz}), 124.6(\mathrm{q}, J=271.0 \mathrm{~Hz}), 122.7(\mathrm{q}, J=32.8 \mathrm{~Hz}), 122.5,114.5,114.1,68.2,67.3,51.9$, 29.5, 29.1, 29.0, 27.2, 25.7, 23.6. $\left.{ }^{19} \mathrm{~F} \mathrm{NMR} \mathrm{(470} \mathrm{MHz,} \mathrm{CDCl}_{3}\right) \delta$-64.4. GCMS (EI) calculated for $[\mathrm{M}]^{+}$450.20, found 450.3. FTIR (neat, $\left.\mathrm{cm}^{-1}\right)$ : 3005(m), 2942(m), 2858(m), 1716(s), 1606(s), 1511(s), 1435(s), 1329(s), 1255(s), 1168(s), 1112(m), 1067(s), 1009(s), 836(s). 
<smiles>CC(=O)c1ccc(OCCC/C=C\CCCCCCl)cc1</smiles>

methyl 4-\{[(4Z)-10-chlorodec-4-en-1-yl]oxy\}benzoate (40), compound was prepared according to general procedure A. The compound was purified by silica gel chromatography with EtOAc/Hex $(0 \rightarrow 30 \%)$ and isolated as a colorless oil $\left(123.1 \mathrm{mg}, 76 \%\right.$ yield). ${ }^{1} \mathrm{H}$ NMR (300 $\left.\mathrm{MHz} \mathrm{CDCl}_{3}\right) \delta 7.98(\mathrm{~d}, J=8.9 \mathrm{~Hz}, 2 \mathrm{H}), 6.90(\mathrm{~d}, J=8.9 \mathrm{~Hz}, 2 \mathrm{H}), 5.49-5.32(\mathrm{~m}, 2 \mathrm{H}), 4.00(\mathrm{t}, J$ $=6.3 \mathrm{~Hz}, 2 \mathrm{H}), 3.88(\mathrm{~s}, 3 \mathrm{H}), 3.49(\mathrm{t}, J=6.7 \mathrm{~Hz}, 2 \mathrm{H}), 2.30-2.15(\mathrm{~m}, 2 \mathrm{H}), 2.09-1.96(\mathrm{~m}, 2 \mathrm{H})$, $1.93-1.79(\mathrm{~m}, 2 \mathrm{H}), 1.78-1.63(\mathrm{~m}, 2 \mathrm{H}), 1.42-1.26(\mathrm{~m}, 4 \mathrm{H}) .{ }^{13} \mathrm{C} \mathrm{NMR}\left(126 \mathrm{MHz}, \mathrm{CDCl}_{3}\right) \delta$ 166.9, 162.9, 131.6, 130.9, 128.6, 122.5, 114.1, 67.2, 51.8, 45.0, 32.5, 29.0, 27.1, 26.6, 23.5. GCMS (EI) calculated for $[\mathrm{M}]^{+} 324.15$, found 324.2. FTIR (neat, $\left.\mathrm{cm}^{-1}\right): 3003(\mathrm{~m}), 2934(\mathrm{~m})$, 2856(m), 1919(w), 1719(s), 1605(s), 1511(s), 1433(s), 1253(s), 1167(s), 1104(m), 846(s), 770(s).<smiles>CC(=O)c1ccc(OCCC/C=C\CCC2CCCCC2)cc1</smiles>

methyl 4-\{[(4Z)-7-cyclohexylhept-4-en-1-yl]oxy\}benzoate (41), compound was prepared according to general procedure $\mathrm{A}$. The compound was purified by silica gel chromatographywith EtOAc/Hex $(0 \rightarrow 30 \%)$ and isolated as a colorless oil $\left(155.2 \mathrm{mg}, 94 \%\right.$ yield). ${ }^{1} \mathrm{H}$ NMR (300 $\left.\mathrm{MHz}_{2} \mathrm{CDCl}_{3}\right) \delta 7.98(\mathrm{~d}, J=8.8 \mathrm{~Hz}, 2 \mathrm{H}), 6.90(\mathrm{~d}, J=8.8 \mathrm{~Hz}, 2 \mathrm{H}), 5.50-5.26(\mathrm{~m}, 2 \mathrm{H}), 4.00(\mathrm{t}, J$ $=6.3 \mathrm{~Hz}, 2 \mathrm{H}), 3.88(\mathrm{~s}, 3 \mathrm{H}), 2.23(\mathrm{q}, J=7.1 \mathrm{~Hz}, 2 \mathrm{H}), 2.09-1.94(\mathrm{~m}, 2 \mathrm{H}), 1.85(\mathrm{p}, J=6.8 \mathrm{~Hz}$, $2 \mathrm{H}), 1.74-1.57(\mathrm{~m}, 5 \mathrm{H}), 1.29-1.07(\mathrm{~m}, 6 \mathrm{H}), 0.94-0.71(\mathrm{~m}, 2 \mathrm{H}) .{ }^{13} \mathrm{C} \mathrm{NMR}\left(75 \mathrm{MHz}, \mathrm{CDCl}_{3}\right)$ $\delta$ 167.0, 163.0, 131.8, 131.7, 128.0, 122.5, 114.2, 67.4, 51.9, 37.6, 37.4, 33.4, 29.1, 26.8, 26.5, 24.7, 23.6. GCMS (EI) calculated for $[\mathrm{M}]^{+} 330.22$, found 330.2. FTIR (neat, $\left.\mathrm{cm}^{-1}\right): 3003(\mathrm{~m})$, 2919(m), 2848(m), 1914(w), 1720(s), 1605(s), 1511(s), 1435(m), 1252(s), 1167(s), 971(m) 845(s), 770(s).

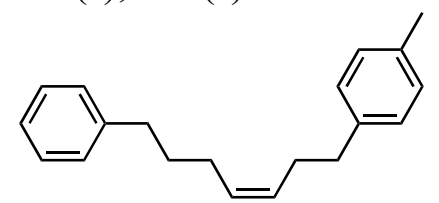

1-methyl-4-[(3Z)-7-phenylhept-3-en-1-yl]benzene (42), compound was prepared according to general procedure $\mathrm{B}$. The compound was purified by silica gel chromatography with EtOAc/Hex $(0 \rightarrow 10 \%)$ and isolated as a colorless oil (123.8 $\mathrm{mg}, 94 \%$ yield). ${ }^{1} \mathrm{H}$ NMR $\left(300 \mathrm{MHz}, \mathrm{CDCl}_{3}\right) \delta$ $7.32-7.23(\mathrm{~m}, 3 \mathrm{H}), 7.23-7.12(\mathrm{~m}, 3 \mathrm{H}), 7.12-6.34(\mathrm{~m}, 4 \mathrm{H}), 5.52-5.32(\mathrm{~m}, 2 \mathrm{H}), 2.67-2.51$ $(\mathrm{m}, 4 \mathrm{H}), 2.39-2.26(\mathrm{~m}, 5 \mathrm{H}), 2.10-1.95(\mathrm{~m}, 2 \mathrm{H}), 1.69-1.56(\mathrm{~m}, 2 \mathrm{H}) .{ }^{13} \mathrm{C} \mathrm{NMR}(126 \mathrm{MHz}$, $\left.\mathrm{CDCl}_{3}\right) \delta 142.6,139.1,135.3,130.1,129.4,129.1,128.5,128.5,128.4,125.8,35.7,35.6,31.5$, 29.5, 27.0, 21.1. GCMS (EI) calculated for [M] ${ }^{+}$264.19, found 264.2. FTIR (neat, $\mathrm{cm}^{-1}$ ): 3060(m), 3005(m), 2932(s), 2855(m), 1622(m), 1537(s), 1454(s), 1375(m) 1285(s), 1007(s), 909(m), 739(s).<smiles>CC(=O)c1ccc(OCCC/C=C\CCCCCOc2ccc(N(C)C)cc2)cc1</smiles>

methyl 4-\{[(4Z)-10-[4-(dimethylamino)phenoxy]dec-4-en-1-yl]oxy\}benzoate (43), compound was prepared according to general procedure A. The compound was purified by silica gel 
chromatography with EtOAc/Hex $(0 \rightarrow 40 \%)$ and isolated as a colorless oil $(189.7 \mathrm{mg}, 89 \%$ yield). ${ }^{1} \mathrm{H}$ NMR $\left(300 \mathrm{MHz}, \mathrm{CDCl}_{3}\right) \delta 7.97(\mathrm{~d}, J=8.9 \mathrm{~Hz}, 2 \mathrm{H}), 6.90(\mathrm{~d}, J=8.9 \mathrm{~Hz}, 2 \mathrm{H}), 6.82(\mathrm{~d}$, $J=9.1 \mathrm{~Hz}, 2 \mathrm{H}), 6.73(\mathrm{~d}, J=9.1 \mathrm{~Hz}, 2 \mathrm{H}), 5.49-5.33(\mathrm{~m}, 2 \mathrm{H}), 4.00(\mathrm{t}, J=6.3 \mathrm{~Hz}, 2 \mathrm{H}), 3.95-$ $3.77(\mathrm{~m}, 5 \mathrm{H}), 2.86(\mathrm{~s}, 6 \mathrm{H}), 2.31-2.16(\mathrm{~m}, 2 \mathrm{H}), 2.11-1.97(\mathrm{~m}, 2 \mathrm{H}), 1.92-1.76(\mathrm{~m}, 2 \mathrm{H}), 1.76-$ $1.63(\mathrm{~m}, 2 \mathrm{H}), 1.47-1.31(\mathrm{~m}, 4 \mathrm{H}) .{ }^{13} \mathrm{C} \mathrm{NMR}\left(126 \mathrm{MHz}, \mathrm{CDCl}_{3}\right) \delta$ 166.9, 163.0, 151.5, 145.8, 131.6, 131.1, 128.4, 122.4, 115.5, 114.9, 114.1, 68.6, 67.3, 51.8, 41.9, 29.5, 29.4, 29.1, 27.2, 25.8, 23.6. GCMS (EI) calculated for [M] ${ }^{+} 425.26$, found 425.3. FTIR (neat, $\left.\mathrm{cm}^{-1}\right)$ : $3000(\mathrm{~m})$, 2938(m), 2857(m), 2793(m), 1715(s), 1605(s), 1513(s), 1434(s), 1280(s), 1253(m), 1168(s), 1104(s), 1051(m), 856(s), 816(s).

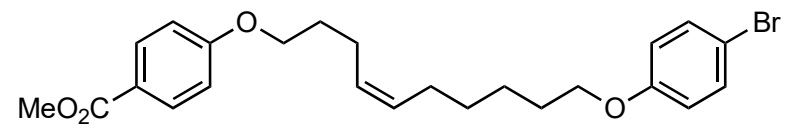

methyl 4-\{[(4Z)-10-(4-bromophenoxy)dec-4-en-1-yl]oxy\}benzoate (44), compound was prepared according to general procedure $\mathrm{A}$. The compound was purified by silica gel chromatography with EtOAc/Hex $(0 \rightarrow 30 \%)$ and isolated as a colorless oil $(177.3 \mathrm{mg}, 77 \%$ yield). ${ }^{1} \mathrm{H}$ NMR $\left(300 \mathrm{MHz}, \mathrm{CDCl}_{3}\right) \delta 7.97(\mathrm{~d}, J=8.7 \mathrm{~Hz}, 2 \mathrm{H}), 7.35(\mathrm{~d}, J=8.8 \mathrm{~Hz}, 2 \mathrm{H}), 6.89$ (d, $J=8.7 \mathrm{~Hz}, 2 \mathrm{H}), 6.75(\mathrm{~d}, J=8.8 \mathrm{~Hz}, 2 \mathrm{H}), 5.49-5.32(\mathrm{~m}, 2 \mathrm{H}), 4.00(\mathrm{t}, J=6.3 \mathrm{~Hz}, 2 \mathrm{H}), 3.94-$ $3.77(\mathrm{~m}, 5 \mathrm{H}), 2.31-2.17(\mathrm{~m}, 2 \mathrm{H}), 2.05(\mathrm{q}, J=6.2 \mathrm{~Hz}, 2 \mathrm{H}), 1.86(\mathrm{p}, J=6.6 \mathrm{~Hz}, 2 \mathrm{H}), 1.78-1.63$ $(\mathrm{m}, 2 \mathrm{H}), 1.47-1.30(\mathrm{~m}, 4 \mathrm{H}) .{ }^{13} \mathrm{C}$ NMR $\left(126 \mathrm{MHz}, \mathrm{CDCl}_{3}\right) \delta 167.0,163.0,158.3,132.3,131.7$, $131.1,128.6,122.5,116.4,114.2,112.7,68.2,67.3,52.0,29.5,29.2,29.1,27.2,25.8,23.6$. GCMS (EI) calculated for [M] ${ }^{+} 460.12$, found 460.1. FTIR (neat, $\left.\mathrm{cm}^{-1}\right): 3003(\mathrm{~m}), 2940(\mathrm{~m})$, 2857(m), 1716(s), 1605(s), 1510(s), 1488(s), 1282(m), 1253(m), 1168(s), 1104(s), 1002(s), 847(s), 738(s).<smiles>COC(=O)c1ccc(OCCC/C=C\CCCCCCc2cccs2)cc1</smiles>

methyl 4-\{[(4Z)-11-(thiophen-2-yl)undec-4-en-1-yl]oxy\}benzoate (45), compound was prepared according to general procedure $\mathrm{A}$. The compound was purified by silica gel chromatography with EtOAc/Hex $(0 \rightarrow 30 \%)$ and isolated as a colorless oil $(150.1 \mathrm{mg}, 78 \%$ yield). ${ }^{1} \mathrm{H}$ NMR $\left(300 \mathrm{MHz}, \mathrm{CDCl}_{3}\right) \delta 7.98(\mathrm{~d}, J=8.9 \mathrm{~Hz}, 2 \mathrm{H}), 7.10(\mathrm{dd}, J=5.1,1.0 \mathrm{~Hz}, 1 \mathrm{H})$, $6.99-6.83(\mathrm{~m}, 3 \mathrm{H}), 6.80-6.71(\mathrm{~m}, 1 \mathrm{H}), 5.53-5.28(\mathrm{~m}, 2 \mathrm{H}), 4.00(\mathrm{t}, J=6.3 \mathrm{~Hz}, 2 \mathrm{H}), 3.88(\mathrm{~s}$, $3 \mathrm{H}), 2.79(\mathrm{t}, J=7.6 \mathrm{~Hz}, 2 \mathrm{H}), 2.31-2.15(\mathrm{~m}, 2 \mathrm{H}), 2.08-1.93(\mathrm{~m}, 2 \mathrm{H}), 1.85(\mathrm{p}, J=6.7 \mathrm{~Hz}, 2 \mathrm{H})$, $1.72-1.58(\mathrm{~m}, 2 \mathrm{H}), 1.37-1.22(\mathrm{~m}, 6 \mathrm{H}) .{ }^{13} \mathrm{C} \mathrm{NMR}\left(126 \mathrm{MHz}, \mathrm{CDCl}_{3}\right) \delta 166.9,162.9,145.7$, 131.6, 131.3, 128.2, 126.7, 123.9, 122.7, 122.4, 114.1, 67.2, 51.8, 31.8, 29.9, 29.6, 29.0, 29.0, 27.2, 23.5. GCMS (EI) calculated for $[\mathrm{M}]^{+} 386.19$, found 386.2. FTIR (neat, $\left.\mathrm{cm}^{-1}\right): 3002(\mathrm{~m})$, 2928(m), 2853(m), 1720(s), 1605(s), 1510(s), 1434(s), 1253(s), 1167(s), 1104(s), 969(m), 846(s), 770(s), 695(s).

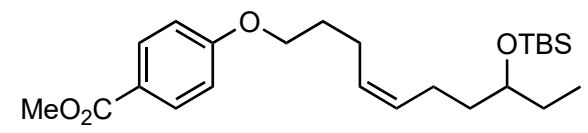

methyl 4-\{[(4Z)-8-[(tert-butyldimethylsilyl)oxy]dec-4-en-1-yl]oxy\}benzoate (46), compound was prepared according to general procedure A. The compound was purified by silica gel chromatography with EtOAc/Hex $(0 \rightarrow 30 \%)$ and isolated as a colorless oil $(190.4 \mathrm{mg}, 91 \%$ yield). ${ }^{1} \mathrm{H} \mathrm{NMR}\left(300 \mathrm{MHz}, \mathrm{CDCl}_{3}\right) \delta 7.97(\mathrm{~d}, J=8.7 \mathrm{~Hz}, 2 \mathrm{H}), 6.90(\mathrm{~d}, J=8.7 \mathrm{~Hz}, 2 \mathrm{H}), 5.51-$ 
$5.30(\mathrm{~m}, 2 \mathrm{H}), 4.00(\mathrm{t}, J=6.4 \mathrm{~Hz}, 2 \mathrm{H}), 3.88(\mathrm{~s}, 3 \mathrm{H}), 3.56(\mathrm{p}, J=5.6 \mathrm{~Hz}, 1 \mathrm{H}), 2.29-2.17(\mathrm{~m}$, $2 \mathrm{H}), 2.18-1.91(\mathrm{~m}, 2 \mathrm{H}), 1.86(\mathrm{p}, J=6.7 \mathrm{~Hz}, 2 \mathrm{H}), 1.50-1.37(\mathrm{~m}, 4 \mathrm{H}), 0.91-0.80(\mathrm{~m}, 12 \mathrm{H})$, $0.03(\mathrm{~s}, 6 \mathrm{H}) .{ }^{13} \mathrm{C} \mathrm{NMR}\left(126 \mathrm{MHz}, \mathrm{CDCl}_{3}\right) \delta 166.9,163.0,131.6,131.2,128.3,122.5,114.1$, 73.1, 67.3, 51.8, 36.6, 29.7, 29.1, 26.0, 23.6, 23.3, 18.2, 9.6, -4.3, -4.4. GCMS (EI) calculated for $[\mathrm{M}]^{+}$420.27, found 420.2. FTIR (neat, $\left.\mathrm{cm}^{-1}\right): 3005(\mathrm{~m}), 2953(\mathrm{~m}), 2855(\mathrm{~m}), 1914(\mathrm{w}), 1721(\mathrm{~s})$, 1606(s), 1511(s), 1435(m), 1253(m), 1167(s), 1103(m), 1050(m), 835(m), 770(s).

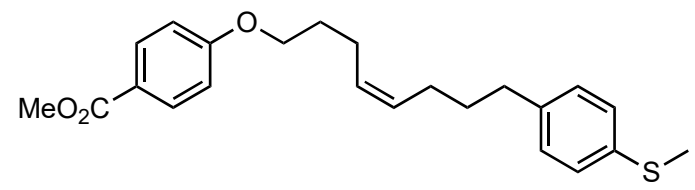

methyl 4-\{[(4Z)-8-[4-(methylsulfanyl)phenyl]oct-4-en-1-yl]oxy\}benzoate (47), compound was prepared according to general procedure A. The compound was purified by silica gel chromatography with EtOAc/Hex $(0 \rightarrow 30 \%)$ and isolated as a colorless oil $(161.0 \mathrm{mg}, 84 \%$ yield). ${ }^{1} \mathrm{H}$ NMR $\left(300 \mathrm{MHz}, \mathrm{CDCl}_{3}\right) \delta 7.98(\mathrm{~d}, J=8.7 \mathrm{~Hz}, 2 \mathrm{H}), 7.17(\mathrm{~d}, J=8.1 \mathrm{~Hz}, 2 \mathrm{H}), 7.04(\mathrm{~d}$, $J=8.1 \mathrm{~Hz}, 2 \mathrm{H}), 6.89(\mathrm{~d}, J=8.7 \mathrm{~Hz}, 2 \mathrm{H}), 5.51-5.33(\mathrm{~m}, 2 \mathrm{H}), 3.99(\mathrm{t}, J=6.3 \mathrm{~Hz}, 2 \mathrm{H}), 3.88(\mathrm{~s}$, $3 \mathrm{H}), 2.58-2.47(\mathrm{~m}, 2 \mathrm{H}), 2.46(\mathrm{~s}, 3 \mathrm{H}), 2.27-2.14(\mathrm{~m}, 2 \mathrm{H}), 2.12-1.99(\mathrm{~m}, 2 \mathrm{H}), 1.92-1.77(\mathrm{~m}$, 2H), $1.68-1.57(\mathrm{~m}, 2 \mathrm{H}) .{ }^{13} \mathrm{C}$ NMR $\left(126 \mathrm{MHz}, \mathrm{CDCl}_{3}\right) \delta 166.9,163.0,139.6,135.2,131.7$, $130.8,129.0,128.8,127.2,122.5,114.1,67.3,51.9,35.0,31.4,29.0,26.8,23.6,16.4$. GCMS (EI) calculated for $[\mathrm{M}]^{+} 384.18$, found 384.2. FTIR (neat, $\left.\mathrm{cm}^{-1}\right): 3003(\mathrm{~m}), 2921(\mathrm{~m}), 2855(\mathrm{~m})$, 1913(w), 1716(s), 1604(s), 1511(s), 1498(s), 1435(s), 1253(s), 1167(s), 1103(s), 1016(m), 835(s).

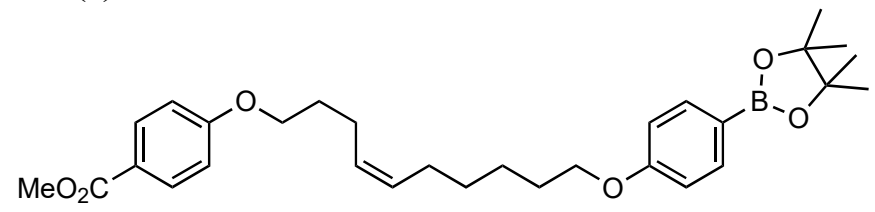

methyl 4-\{[(4Z)-10-[4-(4,4,5,5-tetramethyl-1,3,2-dioxaborolan-2-yl)phenoxy]dec-4-en-1yl]oxy\}benzoate (48), compound was prepared according to general procedure A. The compound was purified by silica gel chromatography with EtOAc/Hex $(0 \rightarrow 50 \%)$ and isolated as a colorless oil (198.7 mg, 78\% yield). ${ }^{1} \mathrm{H}$ NMR $\left(300 \mathrm{MHz}, \mathrm{CDCl}_{3}\right) \delta 7.97(\mathrm{~d}, J=8.8 \mathrm{~Hz}, 2 \mathrm{H})$, $7.73(\mathrm{~d}, J=8.5 \mathrm{~Hz}, 2 \mathrm{H}), 6.93-6.82(\mathrm{~m}, 4 \mathrm{H}), 5.50-5.31(\mathrm{~m}, 2 \mathrm{H}), 4.00(\mathrm{t}, J=6.3 \mathrm{~Hz}, 2 \mathrm{H}), 3.94$ $(\mathrm{t}, J=6.5 \mathrm{~Hz}, 2 \mathrm{H}), 3.87(\mathrm{~s}, 3 \mathrm{H}), 2.30-2.18(\mathrm{~m}, 2 \mathrm{H}), 2.09-2.00(\mathrm{~m}, 2 \mathrm{H}), 1.90-1.83(\mathrm{~m}, 2 \mathrm{H})$, $1.79-1.68(\mathrm{~m}, 2 \mathrm{H}), 1.48-1.29(\mathrm{~m}, 16 \mathrm{H}) .{ }^{13} \mathrm{C} \mathrm{NMR}\left(126 \mathrm{MHz}, \mathrm{CDCl}_{3}\right) \delta 166.9,163.0,161.8$, $136.5,131.6,131.1,128.5,122.5,114.1,113.9,83.6,67.7,67.3,51.9,42.0,29.5,29.12,29.0$, 27.2, 25.8, 24.9, 23.6. GCMS (ESI) calculated for $[\mathrm{M}+\mathrm{H}]^{+} 509.30$, found 509.2. FTIR (neat, $\mathrm{cm}^{-}$ 1): 3305(m), 2977(m), 2937(m), 2858(m), 1913(w), 1720(s), 1604(s), 1511(s), 1434(s), 1252(m), 1142(s), 962(s), 911(s), 846(s), 771(s), 734(s).<smiles>CC(=O)c1ccc(OCCC/C=C\CCCCCOc2ccc(I)cc2)cc1</smiles>

methyl 4-\{[(4Z)-10-(4-iodophenoxy)dec-4-en-1-yl]oxy\}benzoate (49), compound was prepared according to general procedure $\mathrm{A}$. The compound was purified by silica gel chromatography with EtOAc/Hex $(0 \rightarrow 30 \%)$ and isolated as a colorless oil $\left(202.4 \mathrm{mg}, 80 \%\right.$ yield). ${ }^{1} \mathrm{H}$ NMR (300 $\left.\mathrm{MHz}^{\mathrm{CDCl}} 3\right) \delta 7.97(\mathrm{~d}, J=8.8 \mathrm{~Hz}, 2 \mathrm{H}), 7.53(\mathrm{~d}, J=8.7 \mathrm{~Hz}, 2 \mathrm{H}), 6.90(\mathrm{~d}, J=8.8 \mathrm{~Hz}, 2 \mathrm{H}), 6.65$ $(\mathrm{d}, J=8.7 \mathrm{~Hz}, 2 \mathrm{H}), 5.50-5.32(\mathrm{~m}, 2 \mathrm{H}), 4.00(\mathrm{t}, J=6.3 \mathrm{~Hz}, 2 \mathrm{H}), 3.92-3.79(\mathrm{~m}, 5 \mathrm{H}), 2.29-$ 
$2.16(\mathrm{~m}, 2 \mathrm{H}), 2.05(\mathrm{q}, J=6.1 \mathrm{~Hz}, 2 \mathrm{H}), 1.86(\mathrm{p}, J=6.8 \mathrm{~Hz}, 2 \mathrm{H}), 1.78-1.62(\mathrm{~m}, 2 \mathrm{H}), 1.46-1.29$ (m, 4H). ${ }^{13} \mathrm{C}$ NMR $\left(126 \mathrm{MHz}, \mathrm{CDCl}_{3}\right) \delta 166.9,162.9,159.0,138.2,131.6,131.0,128.5,122.5$, 117.0, 114.1, 82.5, 68.0, 67.2, 51.9, 29.5, 29.1, 29.0, 27.2, 25.7, 23.6. GCMS (ESI) calculated for $[\mathrm{M}+\mathrm{H}]^{+}$509.11, found 509.0. FTIR (neat, $\left.\mathrm{cm}^{-1}\right): 3002(\mathrm{~m}), 2939(\mathrm{~m}), 2856(\mathrm{~m}), 1914(\mathrm{w}), 1716(\mathrm{~s})$, 1605(s), 1511(s), 1435(s), 1391(s), 1253(m), 1167(s), 1019(m), 909(s).<smiles>CC(=O)OCCCCC/C=C\CCCOc1ccc(C(C)=O)cc1</smiles>

methyl 4-\{[(4Z)-10-[(tert-butyldimethylsilyl)oxy]dec-4-en-1-yl] $0 x y\}$ benzoate (50), compound was prepared according to general procedure A. The compound was purified by silica gel chromatography with EtOAc/Hex $(0 \rightarrow 30 \%)$ and isolated as a colorless oil $(189.9 \mathrm{mg}, 90 \%$ yield). ${ }^{1} \mathrm{H}$ NMR $\left(300 \mathrm{MHz}, \mathrm{CDCl}_{3}\right) \delta 7.98(\mathrm{~d}, J=8.8 \mathrm{~Hz}, 2 \mathrm{H}), 6.90(\mathrm{~d}, J=8.8 \mathrm{~Hz}, 2 \mathrm{H}), 5.54-$ $5.24(\mathrm{~m}, 2 \mathrm{H}), 4.00(\mathrm{t}, J=6.4 \mathrm{~Hz}, 2 \mathrm{H}), 3.88(\mathrm{~s}, 3 \mathrm{H}), 3.57(\mathrm{t}, J=6.6 \mathrm{~Hz}, 2 \mathrm{H}), 2.29-2.15(\mathrm{~m}, 2 \mathrm{H})$, $2.02(\mathrm{q}, J=6.4 \mathrm{~Hz}, 2 \mathrm{H}), 1.85(\mathrm{p}, J=6.7 \mathrm{~Hz}, 2 \mathrm{H}), 1.52-1.42(\mathrm{~m}, 2 \mathrm{H}), 1.35-1.25(\mathrm{~m}, 4 \mathrm{H}), 0.89$ $(\mathrm{s}, 9 \mathrm{H}), 0.04(\mathrm{~s}, 6 \mathrm{H}) .{ }^{13} \mathrm{C} \mathrm{NMR}\left(126 \mathrm{MHz}, \mathrm{CDCl}_{3}\right) \delta 166.9,162.9,131.6,131.2,128.2,122.4$, 114.1, 67.3, 63.2, 51.8, 32.8, 29.5, 29.0, 27.2, 26.0, 25.5, 23.5, 18.4, -5.3. GCMS (EI) calculated for $[\mathrm{M}]^{+}$420.27, found 420.4. FTIR (neat, $\left.\mathrm{cm}^{-1}\right)$ : 3004(w), 2930(m), 2856(m), 1721(s), 1606(s), 1511(s), 1434(s), 1279(2), 1254(s), 1167(s), 1103(s) 836(s).

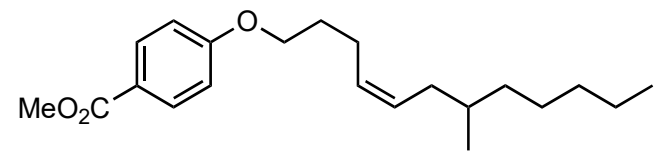

methyl 4-\{[(4Z)-7-methyldodec-4-en-1-yl]oxy\}benzoate (51), compound was prepared according to general procedure $\mathrm{A}$. The compound was purified by silica gel chromatography with EtOAc/Hex $(0 \rightarrow 30 \%)$ and isolated as a colorless oil (105.6 mg, 64\% yield). ${ }^{1} \mathrm{H}$ NMR (300 $\left.\mathrm{MHz}_{2} \mathrm{CDCl}_{3}\right) \delta 7.97(\mathrm{~d}, J=8.9 \mathrm{~Hz}, 2 \mathrm{H}), 6.90(\mathrm{~d}, J=8.9 \mathrm{~Hz}, 2 \mathrm{H}), 5.56-5.30(\mathrm{~m}, 2 \mathrm{H}), 4.00(\mathrm{t}, J$ $=6.4 \mathrm{~Hz}, 2 \mathrm{H}), 3.88(\mathrm{~s}, 3 \mathrm{H}), 2.32-2.15(\mathrm{~m}, 2 \mathrm{H}), 2.05-1.95(\mathrm{~m}, 1 \mathrm{H}), 1.91-1.81(\mathrm{~m}, 2 \mathrm{H}), 1.52$ $-1.35(\mathrm{~m}, 2 \mathrm{H}), 1.34-1.10(\mathrm{~m}, 8 \mathrm{H}), 0.93-0.76(\mathrm{~m}, 6 \mathrm{H}) .{ }^{13} \mathrm{C} \mathrm{NMR}\left(126 \mathrm{MHz}, \mathrm{CDCl}_{3}\right) \delta 167.1$, 163.1, 131.7, 130.1, 129.0, 122.5, 114.2, 67.5, 51.9, 36.8, 34.7, 33.5, 32.3, 29.1, 27.0, 23.7, 22.8, 19.7, 14.2. GCMS (EI) calculated for $[\mathrm{M}]^{+} 332.24$, found 332.2. FTIR (neat, $\left.\mathrm{cm}^{-1}\right): 2998(\mathrm{~m})$, 2925(m), 2857(m), 1913(w), 1720(s), 1606(s), 1511(s), 1433(m), 1253(s), 1167(s), 1103(s), 970(s), 845(s).

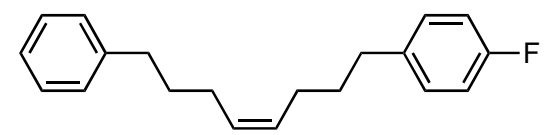

1-fluoro-4-[(4Z)-8-phenyloct-4-en-1-yl]benzene (52), compound was prepared according to general procedure B. The compound was purified by silica gel chromatography with EtOAc/Hex $(0 \rightarrow 10 \%)$ and isolated as a colorless oil $(118.6 \mathrm{mg}, 84 \%$ yield $) .{ }^{1} \mathrm{H} \mathrm{NMR}\left(300 \mathrm{MHz}, \mathrm{CDCl}_{3}\right) \delta$ $7.34-7.27(\mathrm{~m}, 2 \mathrm{H}), 7.24-7.17(\mathrm{~m}, 3 \mathrm{H}), 7.17-7.08(\mathrm{~m}, 2 \mathrm{H}), 7.03-6.91(\mathrm{~m}, 2 \mathrm{H}), 5.58-5.29$ $(\mathrm{m}, 2 \mathrm{H}), 2.68-2.54(\mathrm{~m}, 4 \mathrm{H}), 2.06(\mathrm{~s}, 4 \mathrm{H}), 1.78-1.58(\mathrm{~m}, 4 \mathrm{H}) .{ }^{13} \mathrm{C} \mathrm{NMR}\left(126 \mathrm{MHz}, \mathrm{CDCl}_{3}\right) \delta$ $161.34(\mathrm{~d}, J=243.0 \mathrm{~Hz}), 142.62,138.20(\mathrm{~d}, J=3.1 \mathrm{~Hz}), 130.11,129.83,129.83(\mathrm{~d}, J=7.7 \mathrm{~Hz})$, $128.56,128.41,125.82,115.09(\mathrm{~d}, J=21.0 \mathrm{~Hz}), 35.61,34.74,31.65,31.54,26.99,26.85 .{ }^{19} \mathrm{~F}$ NMR (470 MHz, $\left.\mathrm{CDCl}_{3}\right) \delta$-121.0. GCMS (EI) calculated for [M] ${ }^{+} 282.18$, found 282.2. FTIR (neat, $\left.\mathrm{cm}^{-1}\right):$ 3053(m), 2986(m), 2857(m), 2304(m), 1509(s), 1421(m), 1265(s), 895(s), 746(s). 
<smiles>CC(=O)c1ccc(OCCC/C=C\CCCC(C)CCC=C(C)C)cc1</smiles>

methyl 4-\{[(4Z)-9,13-dimethyltetradeca-4,12-dien-1-yl]oxy\}benzoate (53), compound was prepared according to general procedure $\mathrm{A}$. The compound was purified by silica gel chromatography with EtOAc/Hex $(0 \rightarrow 30 \%)$ and isolated as a colorless oil $(138.7 \mathrm{mg}, 75 \%$ yield). ${ }^{1} \mathrm{H}$ NMR $\left(300 \mathrm{MHz}, \mathrm{CDCl}_{3}\right) \delta 7.98(\mathrm{~d}, J=8.7 \mathrm{~Hz}, 2 \mathrm{H}), 6.90(\mathrm{~d}, J=8.7 \mathrm{~Hz}, 2 \mathrm{H}), 5.50-$ $5.30(\mathrm{~m}, 2 \mathrm{H}), 5.09(\mathrm{t}, J=7.1 \mathrm{~Hz}, 1 \mathrm{H}), 4.00(\mathrm{t}, J=6.4 \mathrm{~Hz}, 2 \mathrm{H}), 3.88(\mathrm{~s}, 3 \mathrm{H}), 2.30-2.14(\mathrm{~m}, 2 \mathrm{H})$, $2.06-1.79(\mathrm{~m}, 6 \mathrm{H}), 1.68(\mathrm{~s}, 3 \mathrm{H}), 1.60(\mathrm{~s}, 3 \mathrm{H}), 1.38-1.20(\mathrm{~m}, 5 \mathrm{H}), 1.17-1.01(\mathrm{~m}, 2 \mathrm{H}), 0.83(\mathrm{~d}$, $J=6.3 \mathrm{~Hz}, 3 \mathrm{H}) .{ }^{13} \mathrm{C} \mathrm{NMR}\left(126 \mathrm{MHz}, \mathrm{CDCl}_{3}\right) \delta 166.9,163.0,131.6,131.5,131.0,128.2,125.1$, 122.5, 114.1, 67.3, 51.8, 37.2, 36.7, 32.4, 29.1, 27.6, 27.2, 25.8, 25.6, 23.6, 19.6, 17.7. GCMS (EI) calculated for $[\mathrm{M}]^{+} 372.27$, found 372.3. FTIR (neat, $\left.\mathrm{cm}^{-1}\right): 3006(\mathrm{~m}), 2925(\mathrm{~m}), 2854(\mathrm{~m})$, 1720(s), 1606(s), 1510(s), 1434(s), 1279(s), 1253(s), 1167(s), 1104(s), 910(w), 846(s), 770(s).

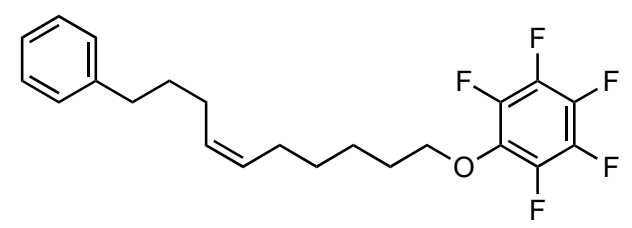

1,2,3,4,5-pentafluoro-6-\{[(6Z)-10-phenyldec-6-en-1-yl]oxy $\}$ benzene (54), compound was prepared according to general procedure B. The compound was purified by silica gel chromatography with EtOAc/Hex $(0 \rightarrow 20 \%)$ and isolated as a colorless oil $(158.8 \mathrm{mg}, 80 \%$ yield). ${ }^{1} \mathrm{H}$ NMR $\left(300 \mathrm{MHz}, \mathrm{CDCl}_{3}\right) \delta 7.34-7.26(\mathrm{~m}, 2 \mathrm{H}), 7.24-7.15(\mathrm{~m}, 3 \mathrm{H}), 5.57-5.27(\mathrm{~m}$, $2 \mathrm{H}), 4.16(\mathrm{t}, J=6.5 \mathrm{~Hz}, 2 \mathrm{H}), 2.71-2.56(\mathrm{~m}, 2 \mathrm{H}), 2.17-1.98(\mathrm{~m}, 4 \mathrm{H}), 1.87-1.61(\mathrm{~m}, 4 \mathrm{H}), 1.56$ $-1.36(\mathrm{~m}, 4 \mathrm{H}) .{ }^{13} \mathrm{C} \mathrm{NMR}\left(126 \mathrm{MHz}, \mathrm{CDCl}_{3}\right) \delta 142.7,130.0,129.9,128.5,128.4,125.8,75.9$, 35.6, 31.6, 29.9, 29.4, 27.2, 27.0, 25.3. $\left.{ }^{19} \mathrm{~F} \mathrm{NMR} \mathrm{(470} \mathrm{MHz,} \mathrm{CDCl}_{3}\right) \delta-159.9$ (d, $\left.J=20.5 \mathrm{~Hz}\right)$, $166.5(\mathrm{t}, J=21.3 \mathrm{~Hz}),-166.9(\mathrm{t}, J=21.7 \mathrm{~Hz})$. GCMS (EI) calculated for $[\mathrm{M}]^{+} 398.17$, found 398.2. FTIR (neat, $\left.\mathrm{cm}^{-1}\right): 3310(\mathrm{~m}), 3004(\mathrm{~m}), 2935(\mathrm{~m}), 2858(\mathrm{~m}), 2658(\mathrm{w}), 2467(\mathrm{w}), 1512(\mathrm{~s})$ 1453(m), 1387(s), 1312(s), 1159(s), 1028(m), 997(m), 746(s), 699(s).<smiles>COC(=O)CCCC/C=C\C(C)C</smiles>

methyl (6Z)-8-methylnon-6-enoate (57), compound was prepared according to general procedure A. The compound was purified by silica gel chromatography with EtOAc/Hex $(0 \rightarrow$ $30 \%$ ) and isolated as a colorless oil $(75.1 \mathrm{mg}, 82 \%$ yield). This compound has been previously synthesized and spectra match the literature values. ${ }^{8}$<smiles>C#CC#CC(/C=C\CCCCCCC)O[Mg]</smiles>

[(4Z)-3-[(tert-butyldimethylsilyl)oxy]dodec-4-en-1-yn-1-yl]tris(propan-2-yl)silane (60), compound was prepared according to general procedure A. The compound was purified by silica gel chromatography with EtOAc/Hex $(0 \rightarrow 30 \%)$ and isolated as a colorless oil $(209.0 \mathrm{mg}, 93 \%$ yield). ${ }^{1} \mathrm{H}$ NMR (300 MHz, Chloroform- $d$ ) $\delta 5.67-5.28(\mathrm{~m}, 2 \mathrm{H}), 5.14(\mathrm{dd}, J=7.8,1.0 \mathrm{~Hz}, 1 \mathrm{H})$, 2.09 (q, $J=7.2 \mathrm{~Hz}, 2 \mathrm{H}), 1.40-1.18(\mathrm{~m}, 10 \mathrm{H}), 1.06(\mathrm{~m}, 21 \mathrm{H}), 0.99-0.79(\mathrm{~m}, 12 \mathrm{H}), 0.14(\mathrm{~d}, J=$ 
$3.3 \mathrm{~Hz}, 6 \mathrm{H}) .{ }^{13} \mathrm{C} \mathrm{NMR}\left(126 \mathrm{MHz}, \mathrm{CDCl}_{3}\right) \delta 131.1,130.9,108.7,84.7,59.8,32.0,29.6,29.4$, 29.4, 27.9, 25.9, 22.8, 18.7, 18.4, 14.2, 11.4, -4.3, -4.5. GCMS (EI) calculated for $[\mathrm{M}]^{+} 345.27$, found 345.3. FTIR (neat, $\left.\mathrm{cm}^{-1}\right)$ : 3020(w), 2928(s), 2893(s), 2864(s), 2168(w), 1463(m), 1387(w), 1361(w), 1314(w), 1252(m), 1075(s).

\section{Gram Scale Reaction}

To a flame dried Schlenk flask filled with nitrogen and charged with a stir bar was added $\mathrm{LiO} t$ $\mathrm{Bu}$ (480.3 mg, $6 \mathrm{mmol}, 1.5$ equiv). To this was added TriAgCl (243.6 mg, $0.4 \mathrm{mmol}, 0.10$ equiv), methyl-4-(pent-4-yn-1-yloxy)benzoate ( $873.0 \mathrm{mg}, 4 \mathrm{mmol}, 1.0$ equiv), and isooctane (40 $\mathrm{mL}$ ). Then, 9-(3-phenylpropyl)-9-borabicyclo[3.3.1]nonane (2M in toluene, $2.6 \mathrm{~mL}, 1.3$ equiv) and methanol ( $2 \mathrm{M}$ in toluene, $2.2 \mathrm{~mL}, 1.1$ equiv) were added and the reaction mixture was heated to $45^{\circ} \mathrm{C}$ in an oil bath for 16 hours. After 16 hours, an aliquot of the crude reaction mixture was analyzed by $\mathrm{GC}$, and the reaction was quenched with the addition of sodium perborate (1.20 g, $12 \mathrm{mmol}, 3.0$ equiv) in $40 \mathrm{~mL}$ THF and $40 \mathrm{~mL}$ deionized water. The mixture was stirred at room temperature for 1 hour and then extracted with ether $(3 \mathrm{x} 100 \mathrm{~mL})$ and dried over $\mathrm{MgSO}_{4}$. The crude mixture was concentrated under reduced pressure and purified by silica gel chromatography.

\section{Alkyne Starting Materials}<smiles>C#CCCCOc1ccc(C(C)=O)cc1</smiles>

methyl 4-(pent-4-yn-1-yloxy)benzoate (1) was prepared according to a known procedure and has been previously characterized. ${ }^{9}$<smiles>C#CCCCc1ccccc1</smiles>

5-phenyl-1-pentyne (61) was purchased from GFS Chemical and distilled over calcium hydride under reduced pressure before use.<smiles>C#CC(CCCCC)OC</smiles>

3-methoxyoct-1-yne (S1) was prepared according to a known procedure and has been previously characterized. ${ }^{10}$<smiles>C#Cc1ccc(OC)cc1</smiles>

1-ethynyl-4-methoxybenzene (S2) was purchased from Millipore Sigma and distilled over calcium hydride under reduced pressure before use.<smiles>C#CCCCOc1ccc(C(C)=O)cc1</smiles>

1-[4-(pent-4-yn-1-yloxy)phenyl]ethan-1-one (S3) was prepared according to a known procedure and has been previously characterized. ${ }^{11}$ 
<smiles>C#CCC1CO1</smiles>

2-(dec-9-yn-1-yl)oxirane (S4) was prepared according to a known procedure and has been previously characterized. ${ }^{12}$<smiles>C#Cc1ccncn1</smiles>

3-ethynylpyridine (S5) was purchased from Ark Pharm and distilled over calcium hydride under reduced pressure before use.<smiles>C#Cc1ccc(C(F)(F)F)cc1</smiles>

1-ethynyl-4-(trifluoromethyl)benzene (S6) was purchased from Millipore Sigma and distilled over calcium hydride under reduced pressure before use.<smiles>C#CCCCOc1ccc(C=O)cc1</smiles>

4-(pent-4-yn-1-yloxy)benzaldehyde (S7) is commercially available from Aurora Building Blocks 2.<smiles>C#CCCCO[13CH3]</smiles>

tert-butyldimethyl(pent-4-yn-1-yloxy)silane (S8) was prepared according to a known procedure and has been previously characterized. ${ }^{13}$<smiles>C#Cc1cccs1</smiles>

2-ethynylthiophene (S9) was purchased from Combi-Blocks and distilled over calcium hydride under reduced pressure before use.<smiles>C#CCCCCCl</smiles>

6-chlorohex-1-yne (S10) was purchased from TCI America and distilled over calcium hydride under reduced pressure before use.<smiles>C#CCCCOc1ccc(N)cc1</smiles>

4-(pent-4-yn-1-yloxy)benzonitrile (S11) was prepared according to a known procedure and has been previously characterized. ${ }^{11}$<smiles>C=CCCC1OCCO1</smiles>

2-(but-3-yn-1-yl)-1,3-dioxolane (S12) was prepared according to a known procedure and has been previously characterized. ${ }^{14}$<smiles>C#CC1CCCCC1</smiles>

ethynylcyclohexane (S13) was purchased from Millipore Sigma and distilled over calcium hydride under reduced pressure before use.<smiles>C=CCCCCCBr</smiles> 
7-bromohept-1-yne (S14) was prepared according to a known procedure and has been previously characterized. ${ }^{11}$<smiles>C#CCCCOc1ccc(I)cc1</smiles>

1-iodo-4-(pent-4-yn-1-yloxy)benzene (S15) was prepared according to a known procedure and has been previously characterized. ${ }^{11}$<smiles>C#CCCCN(C)C(=O)c1ccccc1</smiles>

tert-butyl N-methyl-N-(pent-4-yn-1-yl)carbamate (S16) was prepared according to a known procedure and has been previously characterized. ${ }^{15}$<smiles>C#CC(O)CCCCC</smiles>

oct-1-yn-3-ol (S17) was purchased from TCI America and distilled over calcium hydride under reduced pressure before use.<smiles>C#CCCCCc1ccco1</smiles>

2-(hept-6-yn-1-yl)furan (S18) was prepared according to a known procedure and has been previously characterized. ${ }^{16}$<smiles>C#CCN(C)C(=O)c1ccccc1</smiles>

tert-butyl N-methyl-N-(prop-2-yn-1-yl)carbamate (S19) was prepared according to a known procedure and has been previously characterized. ${ }^{17}$<smiles>C#CCCCNC(=O)c1ccccc1</smiles>

tert-butyl N-(pent-4-yn-1-yl)carbamate (S20) was prepared according to a known procedure and has been previously characterized. ${ }^{18}$<smiles>C#Cc1ccccc1C</smiles>

1-ethynyl-2-methylbenzene (S21) was purchased from Millipore Sigma and distilled over calcium hydride under reduced pressure before use.<smiles>C#CCCCOc1ccncn1</smiles>

4-(pent-4-yn-1-yloxy)pyrimidine (S22). A reaction flask charged with a stir bar was flame-dried under vacuum and allowed to cool under nitrogen. The flask was then charged with triphenylphosphine (2.2 g, $24.0 \mathrm{mmol}, 1.2$ equiv), phenol $(7.7 \mathrm{mmol}, 1.1$ equiv), THF (14.0 mL, $0.5 \mathrm{M})$ and 4-pentyn-1-ol $(654.0 \mu \mathrm{L}, 7.0 \mathrm{mmol}, 1.0$ equiv). The reaction mixture was cooled to 0 ${ }^{\circ} \mathrm{C}$ with an ice bath. To the cooled reaction mixture was added DIAD (1.6 mL, $8.4 \mathrm{mmol}, 1.2$ equiv) dropwise. The reaction mixture was allowed to warm to $23{ }^{\circ} \mathrm{C}$ and stirred overnight. THF was removed under reduced pressure and the mixture was suspended in hexanes and stirred vigorously for $30 \mathrm{~min}$. The solid triphenylphosphine oxide was removed by passing the mixture through a plug of celite. The solvent was removed under reduced pressure and the crude product was purified by silica gel chromatography. Compound was isolated as a white solid. ${ }^{1} \mathrm{H}$ NMR (300 
$\left.\mathrm{MHz}, \mathrm{CDCl}_{3}\right) \delta 8.76(\mathrm{~s}, 1 \mathrm{H}), 8.41(\mathrm{~d}, J=5.9 \mathrm{~Hz}, 1 \mathrm{H}), 6.72(\mathrm{dd}, J=5.9,1.0 \mathrm{~Hz}, 1 \mathrm{H}), 4.46(\mathrm{t}, J=$ $6.2 \mathrm{~Hz}, 2 \mathrm{H}), 2.36(\mathrm{td}, J=7.0,2.6 \mathrm{~Hz}, 2 \mathrm{H}), 2.12-1.88(\mathrm{~m}, 3 \mathrm{H}) .{ }^{13} \mathrm{C}$ NMR $\left(75 \mathrm{MHz}, \mathrm{CDCl}_{3}\right) \delta$ $169.2,158.4,156.8,108.8,83.1,69.2,65.2,27.8,15.3$. GCMS (EI) calculated for $[\mathrm{M}]^{+} 162.08$, found 162.20 .<smiles>C#CC(C)C</smiles>

3-methylbut-1-yne (55) was purchased from Millipore Sigma and distilled over calcium hydride under reduced pressure before use.<smiles>C#CC(C#CP)O[Na]</smiles>

tert-butyldimethyl(\{1-[tris(propan-2-yl)silyl]penta-1,4-diyn-3-yl\}oxy)silane (58). The deprotected alcohol of $\mathbf{5 8}$ has been previously prepared ${ }^{19}$. TBS protection of the alcohol was performed according to a known literature procedure ${ }^{13}$. The compound was isolated as a yellow oil. ${ }^{1} \mathrm{H}$ NMR $\left(300 \mathrm{MHz}, \mathrm{CDCl}_{3}\right) \delta 5.22(\mathrm{~d}, J=2.3 \mathrm{~Hz}, 1 \mathrm{H}), 2.47(\mathrm{~d}, J=2.3 \mathrm{~Hz}, 1 \mathrm{H}), 1.08$ (s, $21 \mathrm{H}), 0.91(\mathrm{~s}, 9 \mathrm{H}), 0.19(\mathrm{~s}, 6 \mathrm{H}) .{ }^{13} \mathrm{C} \mathrm{NMR}\left(75 \mathrm{MHz}, \mathrm{CDCl}_{3}\right) \delta 104.7,85.5,82.0,71.7,53.4$, 25.8, 18.7, 18.4, 11.4, -4.4. GCMS (EI) calculated for $[\mathrm{M}]^{+} 350.25$, found 350.3. FTIR (neat, $\mathrm{cm}^{-}$ 1): 3313(s), 2944(m), 2865(m), 2716(w), 2176(w), 1623(w), 1464(s), 1287(s), 1252(s), 1087(m), 996(m), 882(s), 836(s), 780(s) 633(m).

\section{General Procedure for the Preparation of Heterocyclic Alkynes:}

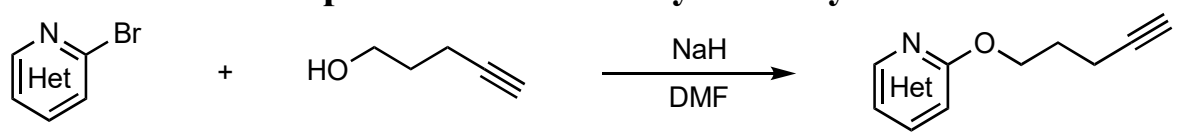

A reaction flask charged with stir bar was flame-dried under vacuum and allowed to cool under nitrogen. The flask was then charged with sodium hydride (1.5 equiv) and DMF $(0.1 \mathrm{M})$. The reaction mixture was cooled to $0{ }^{\circ} \mathrm{C}$ with an ice bath. To the cooled reaction mixture was added 4pentyn-1-ol (1.5 equiv) and the reaction mixture was allowed to stir for 30 minutes. After the indicated time, heterocyclic bromide (1.0 equiv) was added and the mixture was stirred for 2 hours. After 2 hours, the reaction mixture was quenched with water and extracted with diethyl ether. The extract was concentrated under reduced pressure and the crude product was purified by silica gel chromatography. The following alkynes were prepared using this procedure.<smiles>C#CCCCOc1cnc2ccccc2n1</smiles>

2-(pent-4-yn-1-yloxy)quinoxaline (S23), compound was isolated as a white solid. ${ }^{1} \mathrm{H}$ NMR $\left(300 \mathrm{MHz}, \mathrm{CDCl}_{3}\right) \delta 8.46(\mathrm{~s}, 1 \mathrm{H}), 8.01(\mathrm{~d}, J=8.1 \mathrm{~Hz}, 1 \mathrm{H}), 7.83(\mathrm{~d}, J=8.3 \mathrm{~Hz}, 1 \mathrm{H}), 7.73-7.62$ $(\mathrm{m}, 1 \mathrm{H}), 7.61-7.50(\mathrm{~m}, 1 \mathrm{H}), 4.59(\mathrm{t}, J=6.2 \mathrm{~Hz}, 2 \mathrm{H}), 2.44(\mathrm{td}, J=7.0,2.6 \mathrm{~Hz}, 2 \mathrm{H}), 2.09(\mathrm{p}, J=$ $6.6 \mathrm{~Hz}, 2 \mathrm{H}), 1.99(\mathrm{t}, J=2.6 \mathrm{~Hz}, 1 \mathrm{H}) .{ }^{13} \mathrm{C} \mathrm{NMR}\left(75 \mathrm{MHz}, \mathrm{CDCl}_{3}\right) \delta 157.5,140.6,139.8,139.2$, $130.2,129.2,127.4,126.7,83.4,69.1,65.1,27.9,15.5$. GCMS (EI) calculated for $[\mathrm{M}]^{+} 212.09$, found 212.1. FTIR (neat, $\left.\mathrm{cm}^{-1}\right): 3245(\mathrm{~s}), 3058(\mathrm{w}), 2960(\mathrm{~m}), 2852(\mathrm{~m}), 1849(\mathrm{w}), 1573(\mathrm{~s}), 1466(\mathrm{~s})$, 1416(s), 1314(s), 1223(s), 1141(s), 1029(s), 998(s), 959(s). 
<smiles>C#CCCCOc1ncnc2c1ccn2C</smiles>

7-methyl-4-(pent-4-yn-1-yloxy)-7H-pyrrolo[2,3-d]pyrimidine (S24), compound was isolated as a white solid. ${ }^{1} \mathrm{H}$ NMR $\left(300 \mathrm{MHz}, \mathrm{CDCl}_{3}\right) \delta 8.46(\mathrm{~s}, 1 \mathrm{H}), 7.00(\mathrm{~d}, J=3.4 \mathrm{~Hz}, 1 \mathrm{H}), 6.51(\mathrm{~d}, J$ $=3.4 \mathrm{~Hz}, 1 \mathrm{H}), 4.62(\mathrm{t}, J=6.2 \mathrm{~Hz}, 2 \mathrm{H}), 3.85(\mathrm{~s}, 3 \mathrm{H}), 2.43(\mathrm{td}, J=7.1,2.6 \mathrm{~Hz}, 2 \mathrm{H}), 2.15-2.04$ $(\mathrm{m}, 2 \mathrm{H}), 1.98(\mathrm{t}, J=2.6 \mathrm{~Hz}, 1 \mathrm{H}) .{ }^{13} \mathrm{C} \mathrm{NMR}\left(75 \mathrm{MHz}, \mathrm{CDCl}_{3}\right) \delta 162.8,152.3,151.0,126.8$, 105.6, 98.3, 83.5, 69.0, 64.8, 31.4, 28.2, 15.5. GCMS (EI) calculated for $[\mathrm{M}]^{+} 215.11$, found 215.1. FTIR (neat, $\left.\mathrm{cm}^{-1}\right): 3295(\mathrm{~m}), 3104(\mathrm{w}), 2951(\mathrm{~m}), 1597(\mathrm{~s}), 1560(\mathrm{~s}), 1445(\mathrm{~s}), 1370(\mathrm{~m})$, 1317(m), 1247(s), 1210(s), 1056(s), 884(m).<smiles>C#CCCCOc1nc2ccccc2n1C</smiles>

1-methyl-2-(pent-4-yn-1-yloxy)-1H-1,3-benzodiazole (S25), compound was isolated as a white solid. ${ }^{1} \mathrm{H}$ NMR $\left(300 \mathrm{MHz}, \mathrm{CDCl}_{3}\right) \delta 7.57-7.50(\mathrm{~m}, 1 \mathrm{H}), 7.20-7.11(\mathrm{~m}, 3 \mathrm{H}), 4.65(\mathrm{t}, J=6.2$ $\mathrm{Hz}, 2 \mathrm{H}), 3.56(\mathrm{~s}, 3 \mathrm{H}), 2.42(\mathrm{td}, J=7.0,2.6 \mathrm{~Hz}, 2 \mathrm{H}), 2.10(\mathrm{p}, J=6.6 \mathrm{~Hz}, 2 \mathrm{H}), 1.99$ (t, $J=2.6 \mathrm{~Hz}$, $1 \mathrm{H}) .{ }^{13} \mathrm{C} \mathrm{NMR}\left(75 \mathrm{MHz}, \mathrm{CDCl}_{3}\right) \delta 157.4,140.1,134.3,121.5,120.7,117.6,107.9,83.0,69.2$, 68.7, 28.2, 27.9, 15.2. GCMS (EI) calculated for [M] $]^{+}$214.11, found 214.1. FTIR (neat, $\mathrm{cm}^{-1}$ ): 3190(m), 2955(m), 2931(m), 1622(s), 1540(s), 1456(s), 1375(s), 1288(s), 1211(s), 1042(s), 975(s), 892(w), 741(s).<smiles>C#CCCCOc1nccs1</smiles>

2-(pent-4-yn-1-yloxy)-1,3-thiazole (S26), compound was isolated as colorless liquid. ${ }^{1} \mathrm{H}$ NMR $(300 \mathrm{MHz}$, Chloroform- $d) \delta 7.12(\mathrm{~d}, J=3.8 \mathrm{~Hz}, 1 \mathrm{H}), 6.67(\mathrm{~d}, J=3.8 \mathrm{~Hz}, 1 \mathrm{H}), 4.51(\mathrm{t}, J=6.1$ $\mathrm{Hz}, 2 \mathrm{H}), 2.44-2.30(\mathrm{~m}, 2 \mathrm{H}), 2.12-1.93(\mathrm{~m}, 3 \mathrm{H}) .{ }^{13} \mathrm{C} \mathrm{NMR}\left(75 \mathrm{MHz}, \mathrm{CDCl}_{3}\right) \delta 174.5,136.7$, 111.0, 82.7, 69.7, 69.2, 27.6, 14.9. GCMS (EI) calculated for [M] $]^{+}$167.04, found 167.10.<smiles>C#CCCCOc1ccc(OC)nn1</smiles>

3-methoxy-6-(pent-4-yn-1-yloxy)pyridazine (S27), compound was isolated as a white solid. ${ }^{1} \mathrm{H}$ NMR (300 MHz, Chloroform- $d) \delta 6.92-6.90(\mathrm{~m}, 2 \mathrm{H}), 4.52(\mathrm{t}, J=6.2 \mathrm{~Hz}, 2 \mathrm{H}), 4.04(\mathrm{~s}, 3 \mathrm{H}), 2.38$ $(\mathrm{td}, J=7.0,2.6 \mathrm{~Hz}, 2 \mathrm{H}), 2.16-1.91(\mathrm{~m}, 3 \mathrm{H}) .{ }^{13} \mathrm{C} \mathrm{NMR}\left(75 \mathrm{MHz}, \mathrm{CDCl}_{3}\right) \delta 161.9,161.7,121.4$, 121.3, 83.3, 68.9, 65.6, 54.5, 27.8, 15.2. GCMS (EI) calculated for [M] ${ }^{+} 192.09$, found 192.10. FTIR (neat, cm-1) 3328(s), 2852(s), 2284(s), 1495(s), 1444(s), 1423(s), 1384(s), 1337(m), 1268(s), 1098(m), 1038(s), 1013(s), 949(s), 913(s), 949(s), 913(s), 839(s), 796(m). 729(s).

\section{Alkene Starting Materials for Alkylboranes}<smiles>C=CCc1ccccc1</smiles> 
(prop-2-en-1-yl)benzene (S28) was purchased from Millipore Sigma and distilled over calcium hydride under reduced pressure before use.<smiles>C=C[14CH2]C(=O)OC</smiles>

methyl undec-10-enoate (S29) was prepared according to a known procedure and has been previously characterized. ${ }^{20}$<smiles>C=CCCCOc1ccc(OC)cc1</smiles>

1-methoxy-4-(pent-4-en-1-yloxy)benzene (S30) was prepared according to a known procedure and has been previously characterized. ${ }^{21}$

TMS

trimethyl(prop-2-en-1-yl)silane (S31) was purchased from Oakwood Chemicals and distilled over calcium hydride under reduced pressure before use.

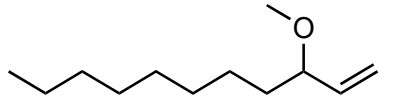

3-methoxyundec-1-ene (S32) was prepared according to a known procedure and has been previously characterized. ${ }^{22}$<smiles>C=CCCCN(C)C(=O)OC(C)(C)C</smiles>

tert-butyl N-methyl-N-(pent-4-en-1-yl)carbamate (S33) is commercially available from Enamine Building Blocks.<smiles>C=CCCCOc1ccc(C(C)=O)cc1</smiles>

methyl 4-(pent-4-en-1-yloxy)benzoate (S34) was prepared according to a known procedure and has been previously characterized. ${ }^{23}$<smiles>C=CCCCOc1ccc(C(F)(F)F)cc1</smiles>

1-(pent-4-en-1-yloxy)-4-(trifluoromethyl)benzene (S35) was prepared according to a known procedure and has been previously characterized. ${ }^{24}$

$\mathrm{Cl}$

5-chloropent-1-ene (S36) was purchased from Arctom Chemicals and distilled over calcium hydride under reduced pressure before use.<smiles>C=CC1CCCCC1</smiles>

ethenylcyclohexane (S37) was purchased from Alfa Aesar and distilled over calcium hydride under reduced pressure before use.<smiles>C=Cc1ccc(C)cc1</smiles>

1-ethenyl-4-methylbenzene (S38) was purchased from TCI America and distilled over calcium hydride under reduced pressure before use.<smiles>C=CCCCOc1ccc(Br)cc1</smiles> 
1-bromo-4-(pent-4-en-1-yloxy)benzene (S39) was prepared according to a known procedure and has been previously characterized. ${ }^{25}$<smiles>C=CCCCc1cccs1</smiles>

2-(hex-5-en-1-yl)thiophene (S40) was prepared according to a known procedure and has been previously characterized. ${ }^{26}$<smiles>C=CC(C)[Se-]</smiles>

tert-butyldimethyl(pent-1-en-3-yloxy)silane (S41) was prepared according to a known procedure and has been previously characterized. ${ }^{27}$<smiles>C=CCc1ccc(SC)cc1</smiles>

1-(methylsulfanyl)-4-(prop-2-en-1-yl)benzene (S42) was prepared according to a known procedure and has been previously characterized. ${ }^{28}$<smiles>C=CCCCOc1ccc(I)cc1</smiles>

1-iodo-4-(pent-4-en-1-yloxy)benzene (S43) was prepared according to a known procedure and has been previously characterized. ${ }^{21}$

TBSO

tert-butyldimethyl(pent-4-en-1-yloxy)silane (S44) was prepared according to a known procedure and has been previously characterized. ${ }^{29}$<smiles>C=C(C)CCCCC</smiles>

2-methylhept-1-ene (S45) was purchased from Millipore Sigma and distilled over calcium hydride under reduced pressure before use.<smiles>C=CCc1ccc(F)cc1</smiles>

1-fluoro-4-(prop-2-en-1-yl)benzene (S46) was prepared according to a known procedure and has been previously characterized. ${ }^{30}$<smiles>C=CCCC(C)CCC=C(C)C</smiles>

5,9-dimethyldeca-1,8-diene (S47) was prepared according to a known procedure and has been previously characterized. ${ }^{31}$

$\widehat{\sim}$

hept-1-ene (56) was purchased from Millipore Sigma and distilled over calcium hydride under reduced pressure before use.<smiles>C=CCCC(=O)OC</smiles>

methyl pent-4-enoate (59) was prepared according to a known procedure and has been previously characterized. ${ }^{32}$

General Procedure for the Preparation of Different Alkenes:<smiles>C=CCCCOc1ccc(CC(C)C(C)C)cc1</smiles> 
A reaction flask charged with stir bar was flame-dried under vacuum and allowed to cool under nitrogen. The flask was then charged with triphenylphosphine (2.2 g, $24.0 \mathrm{mmol}, 1.2 \mathrm{equiv})$, phenol (7.7 mmol, 1.1 equiv), THF (14.0 mL, 0.5 M) and 4-penten-1-ol (654.0 $\mu \mathrm{L}, 7.0 \mathrm{mmol}, 1.0$ equiv). The reaction mixture was cooled to $0^{\circ} \mathrm{C}$ with an ice bath. To the cooled reaction mixture was added DIAD (1.6 mL, $8.4 \mathrm{mmol}, 1.2$ equiv) dropwise. The reaction mixture was allowed to warm to $23{ }^{\circ} \mathrm{C}$ and stirred overnight. THF was removed under reduced pressure and the mixture was suspended in hexanes and stirred vigorously for $30 \mathrm{~min}$. The solid triphenylphosphine oxide was removed by passing the mixture through a plug of celite. The solvent was removed under reduced pressure and the crude product was purified by silica gel chromatography. The following alkenes were prepared using this procedure.<smiles>C=CCCCOc1ccc(C2OCCO2)cc1</smiles>

2-[4-(pent-4-en-1-yloxy)phenyl]-1,3-dioxolane (S48), compound was isolated as a colorless liquid. ${ }^{1} \mathrm{H}$ NMR $\left(300 \mathrm{MHz}, \mathrm{CDCl}_{3}\right) \delta 7.41(\mathrm{~d}, J=8.6 \mathrm{~Hz}, 2 \mathrm{H}), 6.91(\mathrm{~d}, J=8.6 \mathrm{~Hz}, 2 \mathrm{H}), 5.86$ (ddt, $J=16.9,10.1,6.6 \mathrm{~Hz}, 1 \mathrm{H}), 5.76(\mathrm{~s}, 1 \mathrm{H}), 5.13-4.96(\mathrm{~m}, 2 \mathrm{H}), 4.26-3.82(\mathrm{~m}, 6 \mathrm{H}), 2.35-$ $2.11(\mathrm{~m}, 2 \mathrm{H}), 2.00-1.76(\mathrm{~m}, 2 \mathrm{H}) .{ }^{13} \mathrm{C} \mathrm{NMR}\left(75 \mathrm{MHz}, \mathrm{CDCl}_{3}\right) \delta 159.9,137.8,130.1,127.9$, 115.2, 114.5, 103.8, 67.3, 65.3, 30.1, 28.5. GCMS (EI) calculated for [M] $]^{+} 234.13$, found 234.1. FTIR (neat, $\left.\mathrm{cm}^{-1}\right): 3075(\mathrm{~s}), 2944(\mathrm{~m}), 2881(\mathrm{~m}), 2759(\mathrm{~m}), 1640(\mathrm{~s}), 1615(\mathrm{~s}), 1515(\mathrm{~s}), 1435(\mathrm{~s})$, 1393(s), 1304(m), 1246(m), 1078(m), 1011(m), 942(m), 829(s).<smiles>C=CCCCOc1ccc(N(C)C)cc1</smiles>

N,N-dimethyl-4-(pent-4-en-1-yloxy)aniline (S49), compound was isolated as a light yellow liquid. ${ }^{1} \mathrm{H} \mathrm{NMR}\left(300 \mathrm{MHz}, \mathrm{CDCl}_{3}\right) \delta 6.87(\mathrm{~d}, J=9.1 \mathrm{~Hz}, 2 \mathrm{H}), 6.76(\mathrm{~d}, J=9.1 \mathrm{~Hz}, 2 \mathrm{H}), 5.88$ (ddt, $J=16.9,10.1,6.6 \mathrm{~Hz}, 1 \mathrm{H}), 5.16-4.94(\mathrm{~m}, 2 \mathrm{H}), 3.94(\mathrm{t}, J=6.5 \mathrm{~Hz}, 2 \mathrm{H}), 2.88(\mathrm{~s}, J=10.3$ $\mathrm{Hz}, 6 \mathrm{H}), 2.31-2.14(\mathrm{~m}, 2 \mathrm{H}), 1.95-1.75(\mathrm{~m}, 2 \mathrm{H}) .{ }^{13} \mathrm{C} \mathrm{NMR}\left(75 \mathrm{MHz}, \mathrm{CDCl}_{3}\right) \delta 151.6,145.9$, 138.1, 115.7, 115.1, 115.0, 68.1, 41.9, 30.3, 28.8. GCMS (EI) calculated for $[\mathrm{M}]^{+} 205.15$, found 205.2. FTIR (neat, $\left.\mathrm{cm}^{-1}\right): 3074(\mathrm{~m}), 2939(\mathrm{~m}), 2791(\mathrm{~m}), 1635(\mathrm{~m}), 1516(\mathrm{~s}), 1456(\mathrm{~s}), 1339(\mathrm{w})$, 1243(s), 1057(m), 947(s), 816(s), 757(s).<smiles>C=CCCCOc1ccc(B2OC(C)(C)C(C)(C)O2)cc1</smiles>

4,4,5,5-tetramethyl-2-[4-(pent-4-en-1-yloxy)phenyl]-1,3,2-dioxaborolane (S50), compound was isolated as a colorless liquid. ${ }^{1} \mathrm{H} \mathrm{NMR}\left(300 \mathrm{MHz}, \mathrm{CDCl}_{3}\right) \delta 7.77(\mathrm{~d}, J=8.6 \mathrm{~Hz}, 2 \mathrm{H}), 6.90$ $(\mathrm{d}, J=8.6 \mathrm{~Hz}, 2 \mathrm{H}), 5.86(\mathrm{ddt}, J=16.9,10.2,6.6 \mathrm{~Hz}, 1 \mathrm{H}), 5.16-4.92(\mathrm{~m}, 2 \mathrm{H}), 4.00(\mathrm{t}, J=6.4$ $\mathrm{Hz}, 2 \mathrm{H}), 2.34-2.16(\mathrm{~m}, 2 \mathrm{H}), 1.97-1.79(\mathrm{~m}, 2 \mathrm{H}), 1.35(\mathrm{~s}, 12 \mathrm{H}) .{ }^{13} \mathrm{C} \mathrm{NMR}\left(75 \mathrm{MHz}, \mathrm{CDCl}_{3}\right) \delta$ $161.8,137.9,136.6,115.3,114.0,83.6,77.4,67.1,30.2,28.5,25.0$. GCMS (EI) calculated for $\left[\mathrm{M}^{+}\right.$288.19, found 288.1. FTIR (neat, $\left.\mathrm{cm}^{-1}\right): 3076(\mathrm{~m}), 2977(\mathrm{~m}), 2944(\mathrm{~m}), 1605(\mathrm{~s}), 1558(\mathrm{~s})$, 1516(s), 1397(s), 1361(s), 1246(s), 1143(s), 1090(s), 962(m), 860(s), 757(s). 
<smiles>C=CCCCOc1c(F)c(F)c(F)c(F)c1F</smiles>

1,2,3,4,5-pentafluoro-6-(pent-4-en-1-yloxy)benzene (S51), compound was isolated as a colorless liquid. ${ }^{1} \mathrm{H}$ NMR $\left(300 \mathrm{MHz}, \mathrm{CDCl}_{3}\right) \delta 5.82(\mathrm{ddt}, J=16.9,10.2,6.7 \mathrm{~Hz}, 1 \mathrm{H}), 5.20-4.90$ $(\mathrm{m}, 2 \mathrm{H}), 4.17(\mathrm{t}, J=6.4 \mathrm{~Hz}, 2 \mathrm{H}), 2.37-2.15(\mathrm{~m}, 2 \mathrm{H}), 1.97-1.77(\mathrm{~m}, 2 \mathrm{H}) .{ }^{13} \mathrm{C} \mathrm{NMR}(75 \mathrm{MHz}$, $\left.\mathrm{CDCl}_{3}\right) \delta 137.4,115.7,75.1,29.7,29.2$. GCMS (EI) calculated for $[\mathrm{M}]^{+} 252.06$, found 252.1. FTIR (neat, $\left.\mathrm{cm}^{-1}\right):$ 3082(m), 2947(m), 2663(w), 1829(w), 1643(m), 1538(m), 1511(s), 1456(m), 1313(m), 1161(m), 1026(m), 995(m), 917(m).

\section{Mechanistic Studies}

\section{Synthesis of Silver Borate Complex 63}
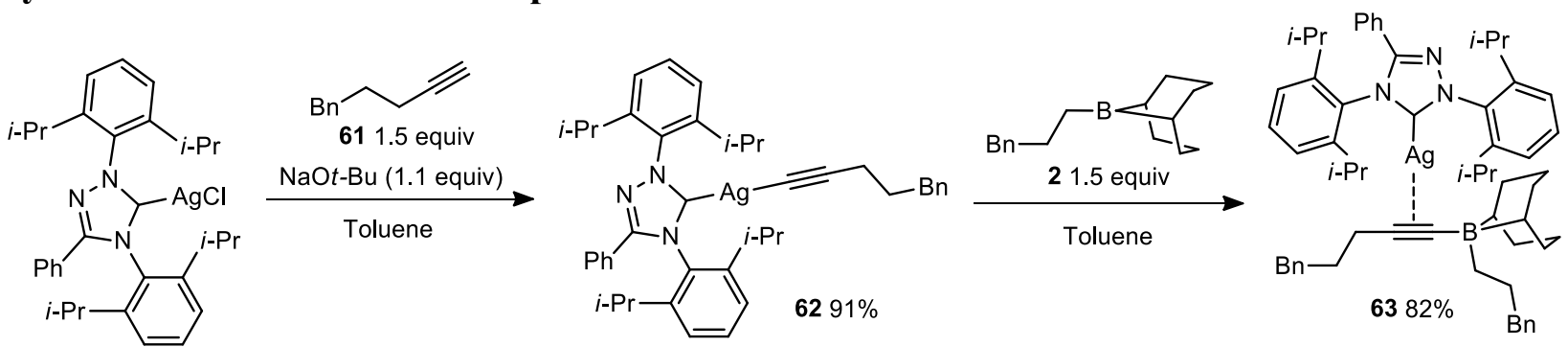

Synthesis of 62, In a nitrogen filled glovebox, a scintillation vial was charged with a stir bar and TriAgCl (91.3 mg, $0.150 \mathrm{mmol}, 1.0$ equiv). To this was added $\mathrm{NaO} t-\mathrm{Bu}$ (15.9 mg $0.165 \mathrm{mmol}$, 1.1 equiv), 5-phenyl-1-pentyne ( $32.4 \mathrm{mg} 0.225 \mathrm{mmol}, 1.5$ equiv) and toluene ( $3 \mathrm{~mL})$. The reaction mixture was stirred at $25{ }^{\circ} \mathrm{C}$ for 2 hours. After, 2 hours, the reaction mixture was filtered through a plug of celite and concentrated. The silver acetylide was then precipitated out with pentane, collected by vacuum filtration and washed several times with pentane. The product was isolated as a white powder $\left(98.2 \mathrm{mg}, 91 \%\right.$ yield). ${ }^{1} \mathrm{H} \mathrm{NMR}\left(300 \mathrm{MHz}, \mathrm{CDCl}_{3}\right) \delta 7.62-7.47(\mathrm{~m}$, 2H), $7.44-7.27(\mathrm{~m}, 9 \mathrm{H}), 7.24-7.17(\mathrm{~m}, 2 \mathrm{H}), 7.17-7.06(\mathrm{~m}, 3 \mathrm{H}), 2.70-2.56(\mathrm{~m}, 4 \mathrm{H}), 2.56-$ $2.41(\mathrm{~m}, 2 \mathrm{H}), 2.19(\mathrm{t}, J=7.6 \mathrm{~Hz}, 2 \mathrm{H}), 1.81-1.68(\mathrm{~m}, 2 \mathrm{H}), 1.43-1.27(\mathrm{~m}, 12 \mathrm{H}), 1.24(\mathrm{~d}, J=6.9$ $\mathrm{Hz}, 6 \mathrm{H}), 0.97(\mathrm{~d}, J=6.8 \mathrm{~Hz}, 6 \mathrm{H}) .{ }^{13} \mathrm{C}$ NMR $\left(126 \mathrm{MHz}, \mathrm{CDCl}_{3}\right) \delta 153.4,153.3,145.7,145.3$, $142.7,135.2,131.9,131.5,131.2,128.9,128.6,128.1,128.1,125.5,125.1,124.3,35.2,32.0$, $29.1,29.0,25.1,24.5,23.9,22.8,20.3$

Synthesis of 63, In a nitrogen filled glovebox, a scintillation vial was charged with a stir bar and Silver acetylide 62 (39.3 mg, $0.055 \mathrm{mmol}, 1.0$ equiv). To this was added alkylborane 2 (19.8 $\mathrm{mg}$ $0.083 \mathrm{mmol}, 1.1$ equiv) and toluene $(3 \mathrm{~mL})$. The reaction mixture was stirred at $25^{\circ} \mathrm{C}$ for 1 hours. After 1 hour, the solvent was removed under vacuum and the borate complex was then precipitated out with pentane. It was then collected by vacuum filtration and washed several times with pentane. The complex was isolated as an off-white powder (43.6 $\mathrm{mg}, 82 \%$ yield). Crystallographic information is below. ${ }^{1} \mathrm{H}$ NMR $\left(300 \mathrm{MHz}, \mathrm{C}_{6} \mathrm{D}_{6}\right) \delta 7.52-7.43(\mathrm{~m}, 2 \mathrm{H}), 7.42-$ $7.32(\mathrm{~m}, 2 \mathrm{H}), 7.31-7.17(\mathrm{~m}, 5 \mathrm{H}), 7.15-7.04(\mathrm{~m}, 7 \mathrm{H}), 7.03-6.94(\mathrm{~m}, 2 \mathrm{H}), 6.87-6.69(\mathrm{~m}$, $3 \mathrm{H}), 2.97-2.83(\mathrm{~m}, 2 \mathrm{H}), 2.83-2.69(\mathrm{~m}, 2 \mathrm{H}), 2.65-2.20(\mathrm{~m}, 10 \mathrm{H}), 2.04(\mathrm{~s}, 8 \mathrm{H}), 1.90-1.73$ $(\mathrm{m}, 2 \mathrm{H}), 1.42-1.24(\mathrm{~m}, 14 \mathrm{H}), 1.15(\mathrm{~d}, J=6.8 \mathrm{~Hz}, 6 \mathrm{H}), 0.87-0.63(\mathrm{~m}, 10 \mathrm{H})$. 


\section{Catalytic Competency of the Borate Complex}

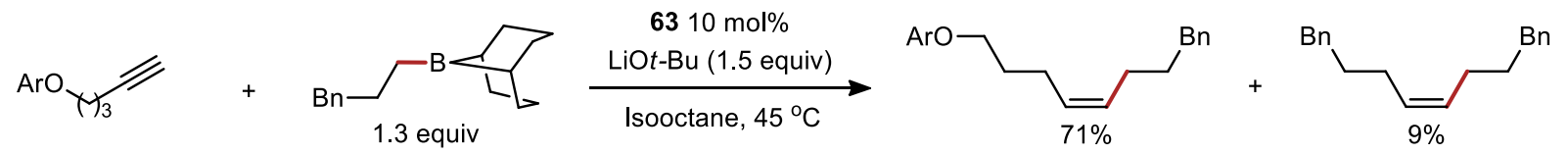

Ar = methyl-4-benzoate

In a nitrogen filled glovebox, a dram vial was charged with a stir bar and $\mathrm{LiO} t$ - $\mathrm{Bu}(6.0 \mathrm{mg}, 0.075$ mmol, 1.5 equiv). To this was added Borate complex 63 (4.9 mg 0.005 mmol, 0.005 equiv), alkyne 1 (10.9 mg $0.05 \mathrm{mmol}, 1.0$ equiv), alkylborane 2 (15.6 mg, $0.065 \mathrm{mmol}, 1.3$ equiv), methanol (1.8 mg, $0.055 \mathrm{mmol}, 1.10$ equiv), isooctane $(0.5 \mathrm{~mL})$ and internal standard, TMB (4.2 $\mathrm{mg}, 0.05 \mathrm{mmol}, 0.5$ equiv). The reaction mixture was heated at $45^{\circ} \mathrm{C}$ and an aliquot of the crude reaction mixture was analyzed by GC after $16 \mathrm{~h}$.

\section{Crystallization of 63 for X-ray Crystallography}

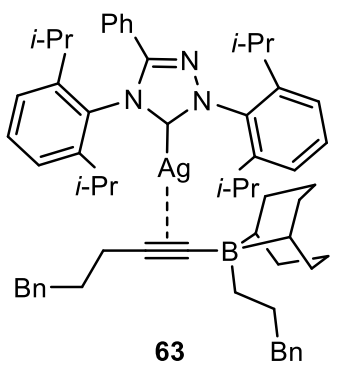

In a nitrogen filled glove box, a dram vial was charged with borate complex 63 (14.6 or $13.4 \mathrm{mg}$, $0.015 \mathrm{mmol}, 1$ equiv). The solid was then crystallized from benzene and pentane.

\section{Crystallography Data of 63}

A colorless prism, measuring $0.09 \times 0.07 \times 0.04 \mathrm{~mm}^{3}$ was mounted on a loop with oil. Data was collected at $-173^{\circ} \mathrm{C}$ on a Bruker APEX II single crystal X-ray diffractometer, Mo-radiation.

Crystal-to-detector distance was $40 \mathrm{~mm}$ and exposure time was 60 seconds per frame for all sets. The scan width was $0.5^{\circ}$. Data collection was $99.7 \%$ complete to $25^{\circ}$ in $\vartheta$. A total of 25311 merged reflections were collected covering the indices, $-23<=\mathrm{h}<=23,-17<=\mathrm{k}<=17,-29<=1<=29$. 12944 reflections were symmetry independent and the $\mathrm{R}_{\text {int }}=0.1029$ reflects the small sample size. Indexing and unit cell refinement indicated a primitive monoclinic lattice. The space group was found to be P $21 / \mathrm{n}$ (No. 14). 
The data was integrated and scaled using SAINT, ${ }^{33}$ SADABS $^{34}$ within the APEX2 ${ }^{35}$ software package by Bruker.

Solution by direct methods (SHELXT ${ }^{36}$ or SIR97 ${ }^{37,38}$ ) produced a complete heavy atom phasing model consistent with the proposed structure. The structure was completed by difference Fourier synthesis with SHELXL97. ${ }^{39-41}$ Scattering factors are from Waasmair and Kirfel. ${ }^{42}$ Hydrogen atoms were placed in geometrically idealised positions and constrained to ride on their parent atoms with $\mathrm{C}---\mathrm{H}$ distances in the range 0.95-1.00 Angstrom. Isotropic thermal parameters $\mathrm{U}_{\text {eq }}$ were fixed such that they were $1.2 \mathrm{U}_{\text {eq }}$ of their parent atom Ueq for $\mathrm{CH}$ 's and $1.5 \mathrm{U}_{\text {eq }}$ of their parent atom $U_{\text {eq }}$ in case of methyl groups. All non-hydrogen atoms were refined anisotropically by full-matrix least-squares.

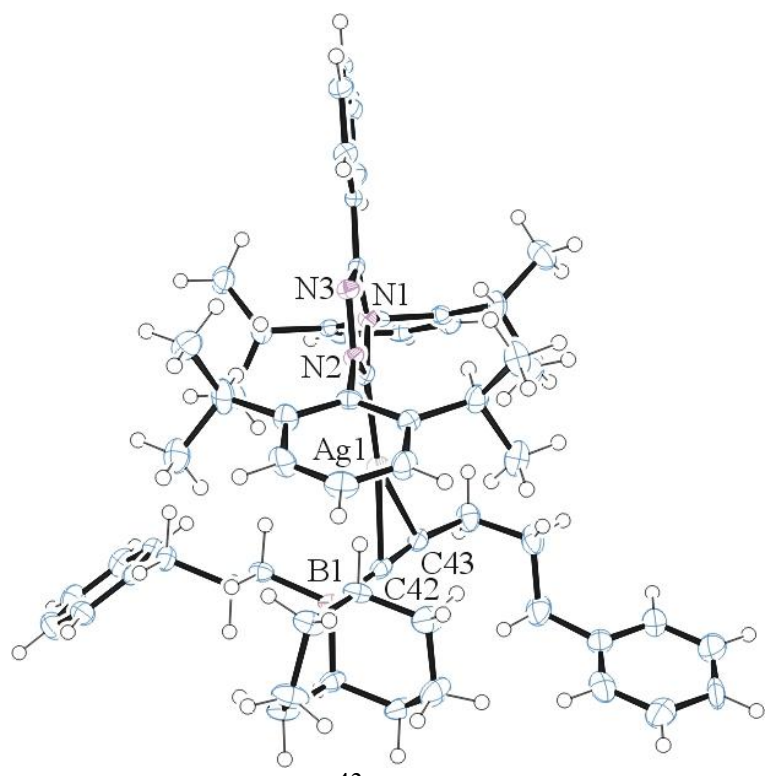

Figure S1. ORTEP ${ }^{43}$ of the structure with thermal ellipsoids at the $50 \%$ probability level. Disorder omitted for clarity. One phenyl and one $i$-Pr unit are disordered.

Crystallographic data for the structures provided.

Empirical formula

Formula weight

Temperature

Wavelength

Crystal system

Space group

Unit cell dimensions

Volume

Z

Density (calculated)
C60 H75 Ag B N3

956.91

$99(2) \mathrm{K}$

$0.71073 \AA$

Monoclinic

P $21 / \mathrm{n}$

$\mathrm{a}=17.5111(18) \AA \quad \alpha=90^{\circ}$.

$\mathrm{b}=13.3239(14) \AA \quad \beta=94.480(4)^{\circ}$.

$\mathrm{c}=22.382(2) \AA \quad \gamma=90^{\circ}$.

4

$1.221 \mathrm{Mg} / \mathrm{m}^{3}$ 
Absorption coefficient

$\mathrm{F}(000)$

Crystal size

Theta range for data collection

Index ranges

Reflections collected

Independent reflections

Completeness to theta $=25.000^{\circ}$

Refinement method

Data / restraints / parameters

Goodness-of-fit on $\mathrm{F}^{2}$

Final $R$ indices [I $>2 \operatorname{sigma}(\mathrm{I})]$

$\mathrm{R}$ indices (all data)

Largest diff. peak and hole
$0.427 \mathrm{~mm}^{-1}$

2032

$0.090 \times 0.070 \times 0.040 \mathrm{~mm}^{3}$

1.424 to $28.373^{\circ}$.

$-23<=\mathrm{h}<=23,-17<=\mathrm{k}<=17,-29<=\mathrm{l}<=29$

25311

$12944[\mathrm{R}(\mathrm{int})=0.1029]$

$99.7 \%$

Full-matrix least-squares on $\mathrm{F}^{2}$

12944 / 33 / 596

0.946

$\mathrm{R} 1=0.0480, \mathrm{wR} 2=0.0793$

$\mathrm{R} 1=0.1377, \mathrm{wR} 2=0.1037$

0.544 and -0.661 e. $\AA^{-3}$

\section{References}

(1) Molander, G. A.; Argintaru, O. A. Stereospecific Ni-Catalyzed Cross-Coupling of Potassium Alkenyltrifluoroborates with Alkyl Halides. Org. Lett. 2014, 16 (7), 1904 1907. https://doi.org/10.1021/ol500408a.

(2) Armstrong, M. K.; Goodstein, M. B.; Lalic, G. Diastereodivergent Reductive Cross Coupling of Alkynes through Tandem Catalysis: Z- and E-Selective Hydroarylation of Terminal Alkynes. J. Am. Chem. Soc. 2018, 140 (32), 10233-10241. https://doi.org/10.1021/jacs.8b05113.

(3) Biermann, U.; Butte, W.; Koch, R.; Fokou, P. A.; Türünç, O.; Meier, M. A. R.; Metzger, J. O. Initiation of Radical Chain Reactions of Thiol Compounds and Alkenes without Any Added Initiator: Thiol-Catalyzed Cis/Trans Isomerization of Methyl Oleate. Chem. - Eur. J. 2012, 18 (26), 8201-8207. https://doi.org/10.1002/chem.201103252.

(4) Laitar, D. S.; Müller, P.; Gray, T. G.; Sadighi, J. P. A Carbene-Stabilized Gold(I) Fluoride: Synthesis and Theory. Organometallics 2005, 24 (19), 4503-4505. https://doi.org/10.1021/om050619f.

(5) Yu, X.-Y.; Patrick, B. O.; James, B. R. New Rhodium(I) Carbene Complexes from Carbene Transfer Reactions. Organometallics 2006, 25 (9), 2359-2363. https://doi.org/10.1021/om060030w.

(6) Yatham, V. R.; Harnying, W.; Kootz, D.; Neudörfl, J.-M.; Schlörer, N. E.; Berkessel, A. 1,4-Bis-Dipp/Mes-1,2,4-Triazolylidenes: Carbene Catalysts That Efficiently Overcome Steric Hindrance in the Redox Esterification of $\alpha$ - and $\beta$-Substituted A, $\beta$-Enals. J. Am. Chem. Soc. 2016, 138 (8), 2670-2677. https://doi.org/10.1021/jacs.5b11796.

(7) Rucker, R. P.; Whittaker, A. M.; Dang, H.; Lalic, G. Synthesis of Tertiary Alkyl Amines from Terminal Alkenes: Copper-Catalyzed Amination of Alkyl Boranes. J. Am. Chem. Soc. 2012, 134 (15), 6571-6574. https://doi.org/10.1021/ja3023829.

(8) Kaga, H.; Miura, M.; Orito, K. A Facile Procedure for Synthesis of Capsaicin. J. Org. Chem. 1989, 54 (14), 3477-3478. https://doi.org/10.1021/jo00275a040. 
(9) Uehling, M. R.; Suess, A. M.; Lalic, G. Copper-Catalyzed Hydroalkylation of Terminal Alkynes. J. Am. Chem. Soc. 2015, 137 (4), 1424-1427. https://doi.org/10.1021/ja5124368.

(10) Hintermann, L.; Kribber, T.; Labonne, A.; Paciok, E. Aldol Synthesis by AntiMarkovnikov Hydration of Propargyloxy Substrates: Feasibility, Stereospecifity, and Reiterative Alkynylation-Hydration. Synlett 2009, 2009 (15), 2412-2416. https://doi.org/10.1055/s-0029-1217734.

(11) Mailig, M.; Hazra, A.; Armstrong, M. K.; Lalic, G. Catalytic Anti-Markovnikov Hydroallylation of Terminal and Functionalized Internal Alkynes: Synthesis of Skipped Dienes and Trisubstituted Alkenes. J. Am. Chem. Soc. 2017, 139 (20), 6969-6977. https://doi.org/10.1021/jacs.7b02104.

(12) Uehling, M. R.; Rucker, R. P.; Lalic, G. Catalytic Anti-Markovnikov Hydrobromination of Alkynes. J. Am. Chem. Soc. 2014, 136 (24), 8799-8803. https://doi.org/10.1021/ja503944n.

(13) Balas, L.; Bertrand-Michel, J.; Viars, F.; Faugere, J.; Lefort, C.; Caspar-Bauguil, S.; Langin, D.; Durand, T. Regiocontrolled Syntheses of FAHFAs and LC-MS/MS Differentiation of Regioisomers. Org. Biomol. Chem. 2016, 14 (38), 9012-9020. https://doi.org/10.1039/C6OB01597B.

(14) Abdel Ghani, S. B.; Chapman, J. M.; Figadère, B.; Herniman, J. M.; Langley, G. J.; Niemann, S.; Brown, R. C. D. Total Synthesis and Stereochemical Assignment of Cis Uvariamicin I and Cis -Reticulatacin. J. Org. Chem. 2009, 74 (18), 6924-6928. https://doi.org/10.1021/jo9012578.

(15) Lawson, E. C. Preparation of Indoylpropylaminobutanamide Derivatives and Analogs for Use as DPP-1 Inhibitors. U.S. Patent WO 2011/075634 A1, June 23, 2011.

(16) Harwood, L. M.; Leeming, S. A.; Isaacs, N. S.; Jones, G.; Pickard, J.; Thomas, R. M.; Watkin, D. The High Pressure Mediated Intramolecular Diels-Alder Reaction of Furans: Factors Controlling Cycloaddition with Monoactivated Dienophiles. Tetrahedron Lett. 1988, 29 (39), 5017-5020. https://doi.org/10.1016/S0040-4039(00)80668-1.

(17) Tayama, E.; Toma, Y. Stereoselective Preparation of (1Z)- and (1E)-N-Boc-1-Amino-1,3Dienes by Stereospecific Base-Promoted 1,4-Elimination. Tetrahedron 2015, 71 (4), 554559. https://doi.org/10.1016/j.tet.2014.12.039.

(18) Reed, N. L.; Herman, M. I.; Miltchev, V. P.; Yoon, T. P. Photocatalytic Oxyamination of Alkenes: Copper(II) Salts as Terminal Oxidants in Photoredox Catalysis. Org. Lett. 2018, 20 (22), 7345-7350. https://doi.org/10.1021/acs.orglett.8b03345.

(19) Cocq, K.; Saffon-Merceron, N.; Coppel, Y.; Poidevin, C.; Maraval, V.; Chauvin, R. Carbo -Naphthalene: A Polycyclic Carbo-Benzenoid Fragment of $\alpha$-Graphyne. Angew. Chem. Int. Ed. 2016, 55 (48), 15133-15136. https://doi.org/10.1002/anie.201608300.

(20) Narra, N.; Kaki, S. S.; Prasad, R. B. N.; Misra, S.; Dhevendar, K.; Kontham, V.; Korlipara, P. V. Synthesis and Evaluation of Anti-Oxidant and Cytotoxic Activities of Novel 10-Undecenoic Acid Methyl Ester Based Lipoconjugates of Phenolic Acids. Beilstein J. Org. Chem. 2017, 13, 26-32. https://doi.org/10.3762/bjoc.13.4.

(21) Chen, C.; Luo, Y.; Fu, L.; Chen, P.; Lan, Y.; Liu, G. Palladium-Catalyzed Intermolecular Ditrifluoromethoxylation of Unactivated Alkenes: $\mathrm{CF}_{3}$ O-Palladation Initiated by Pd(IV). J. Am. Chem. Soc. 2018, 140 (4), 1207-1210. https://doi.org/10.1021/jacs.7b11470.

(22) Park, S. R.; Kim, C.; Kim, D.; Thrimurtulu, N.; Yeom, H.-S.; Jun, J.; Shin, S.; Rhee, Y. H. Entry to $\beta$-Alkoxyacrylates via Gold-Catalyzed Intermolecular Coupling of Alkynoates and Allylic Ethers. Org. Lett. 2013, 15 (6), 1166-1169. https://doi.org/10.1021/ol4001087. 
(23) Wilkinson, M. C.; Higson, M. Improved Preparation of an Epoxy Substituted Reactive Mesogen. Org. Process Res. Dev. 2017, 21 (1), 75-78.

https://doi.org/10.1021/acs.oprd.6b00351.

(24) Wang, X.; Wu, Y. Direct Oxidative Isoperfluoropropylation of Terminal Alkenes via Hexafluoropropylene (HFP) and Silver Fluoride. Chem. Commun. 2018, 54 (15), $1877-$ 1880. https://doi.org/10.1039/C7CC07614B.

(25) Danon, J. J.; Krüger, A.; Leigh, D. A.; Lemonnier, J.-F.; Stephens, A. J.; VitoricaYrezabal, I. J.; Woltering, S. L. Braiding a Molecular Knot with Eight Crossings. Science 2017, 355 (6321), 159-162. https://doi.org/10.1126/science.aal1619.

(26) Ponomarenko, S. A.; Borshchev, O. V.; Meyer-Friedrichsen, T.; Pleshkova, A. P.; Setayesh, S.; Smits, E. C. P.; Mathijssen, S. G. J.; de Leeuw, D. M.; Kirchmeyer, S.; Muzafarov, A. M. Synthesis of Monochlorosilyl Derivatives of Dialkyloligothiophenes for Self-Assembling Monolayer Field-Effect Transistors. Organometallics 2010, 29 (19), 4213-4226. https://doi.org/10.1021/om100139y.

(27) Midland, M. M.; Koops, R. W. Asymmetric Hetero Diels-Alder Reaction of .alpha.Alkoxy Aldehydes with Activated Dienes. The Scope of Lewis Acid Chelation-Controlled Cycloadditions. J. Org. Chem. 1990, 55 (17), 5058-5065. https://doi.org/10.1021/jo00304a018.

(28) Lazzaroni, S.; Dondi, D.; Fagnoni, M.; Albini, A. Photochemical Arylation Reactions by 4-Chlorothioanisole. Eur. J. Org. Chem. 2007, 2007 (26), 4360-4365. https://doi.org/10.1002/ejoc.200700290.

(29) Solinski, A. E.; Koval, A. B.; Brzozowski, R. S.; Morrison, K. R.; Fraboni, A. J.; Carson, C. E.; Eshraghi, A. R.; Zhou, G.; Quivey, R. G.; Voelz, V. A.; et al. Diverted Total Synthesis of Carolacton-Inspired Analogs Yields Three Distinct Phenotypes in Streptococcus Mutans Biofilms. J. Am. Chem. Soc. 2017, 139 (21), 7188-7191. https://doi.org/10.1021/jacs.7b03879.

(30) Guo, Y.; Shen, Z. Palladium-Catalyzed Allylic C-H Oxidation under Simple Operation and Mild Conditions. Org. Biomol. Chem. 2019, 17 (12), 3103-3107. https://doi.org/10.1039/C9OB00209J.

(31) Ueno, Y.; Sano, H.; Okawara, M. Deoxygenation of Allylic Alcohols to Terminal Olefins via Stannylation/Destannylation. Tetrahedron Lett. 1980, 21 (18), 1767-1770. https://doi.org/10.1016/S0040-4039(00)77832-4.

(32) Bauer, J. M.; Frey, W.; Peters, R. Asymmetric Cascade Reaction to Allylic Sulfonamides from Allylic Alcohols by Palladium(II)/Base-Catalyzed Rearrangement of Allylic Carbamates. Angew. Chem. Int. Ed. 2014, 53 (29), 7634-7638.

https://doi.org/10.1002/anie.201403090.

(36) Sheldrick, G. M. SHELXT - Integrated Space-Group and Crystal-Structure Determination. Acta Crystallogr. Sect. Found. Adv. 2015, 71 (1), 3-8. https://doi.org/10.1107/S2053273314026370.

(37) Altomare, A.; Burla, M. C.; Camalli, M.; Cascarano, G. L.; Giacovazzo, C.; Guagliardi, A.; Moliterni, A. G. G.; Polidori, G.; Spagna, R. SIR97: A New Tool for Crystal Structure Determination and Refinement. J. Appl. Crystallogr. 1999, 32 (1), 115-119. https://doi.org/10.1107/S0021889898007717. 
(38) Altomare, A.; Cascarano, G.; Giacovazzo, C.; Guagliardi, A. Completion and Refinement of Crystal Structures with SIR92. J. Appl. Crystallogr. 1993, 26 (3), 343-350. https://doi.org/10.1107/S0021889892010331.

(39) G. M. Sheldrick. SHELXL-97, Program for the Refinement of Crystal Structures; University of Göttingen: Germany, 1997.

(40) Sheldrick, G. M. Crystal Structure Refinement with SHELXL. Acta Crystallogr. Sect. C Struct. Chem. 2015, 71 (1), 3-8. https://doi.org/10.1107/S2053229614024218.

(41) S. Mackay; C. Edwards; A. Henderson; C. Gilmore; K. Shankland. MaXus: A Computer Program for the Solution and Refinement of Crystal Structures from Diffraction Data; University of Glasgow: Scotland, 1997.

(42) Waasmaier, D.; Kirfel, A. New Analytical Scattering-Factor Functions for Free Atoms and Ions. Acta Crystallogr. A 1995, 51 (3), 416-431.

https://doi.org/10.1107/S0108767394013292.

(43) Farrugia, L. J. ORTEP-3 for Windows - a Version of ORTEP-III with a Graphical User Interface (GUI). J. Appl. Crystallogr. 1997, 30 (5), 565-565. https://doi.org/10.1107/S0021889897003117.

\section{3. $\underline{\text { Spectroscopic Data }}$}




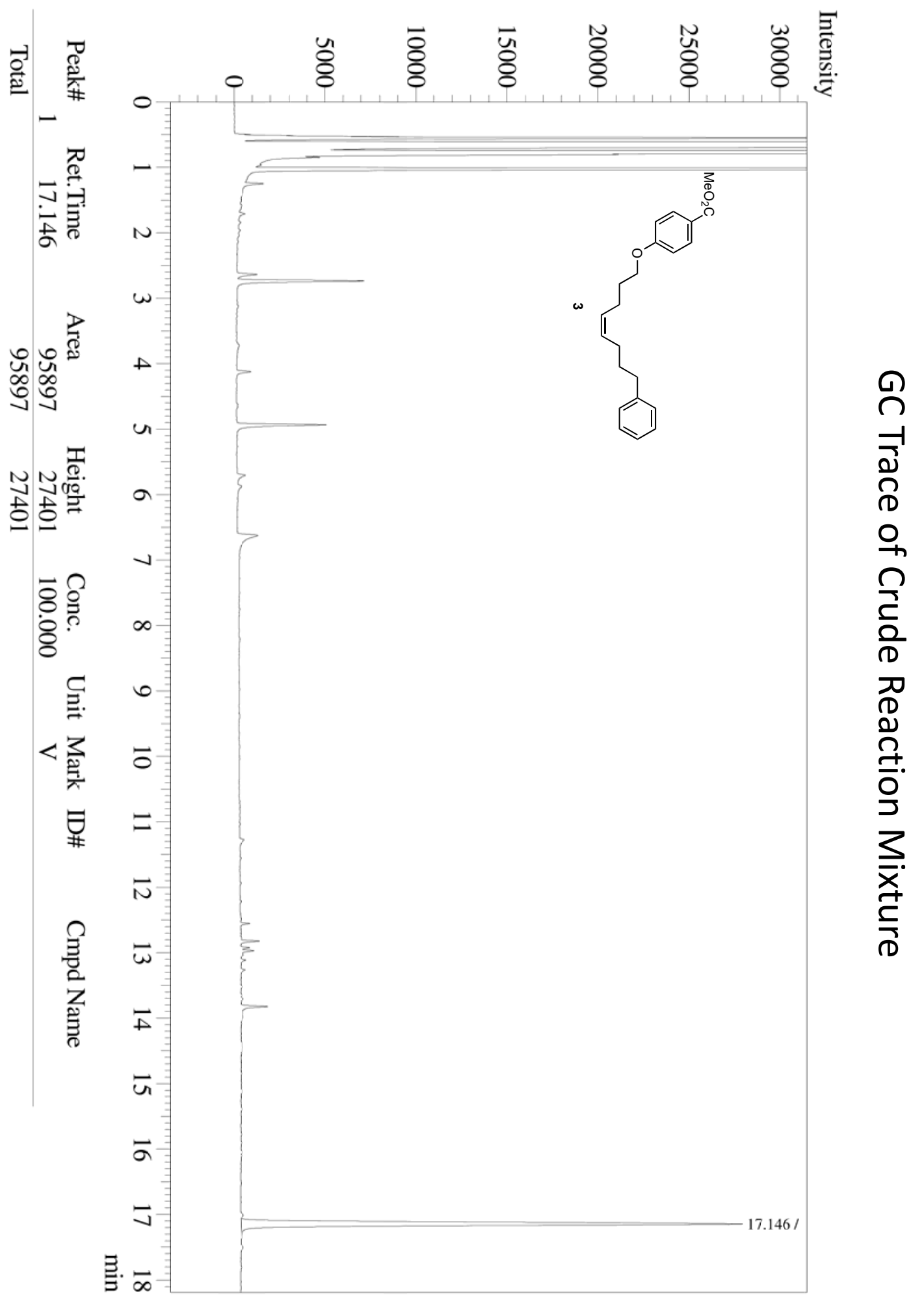



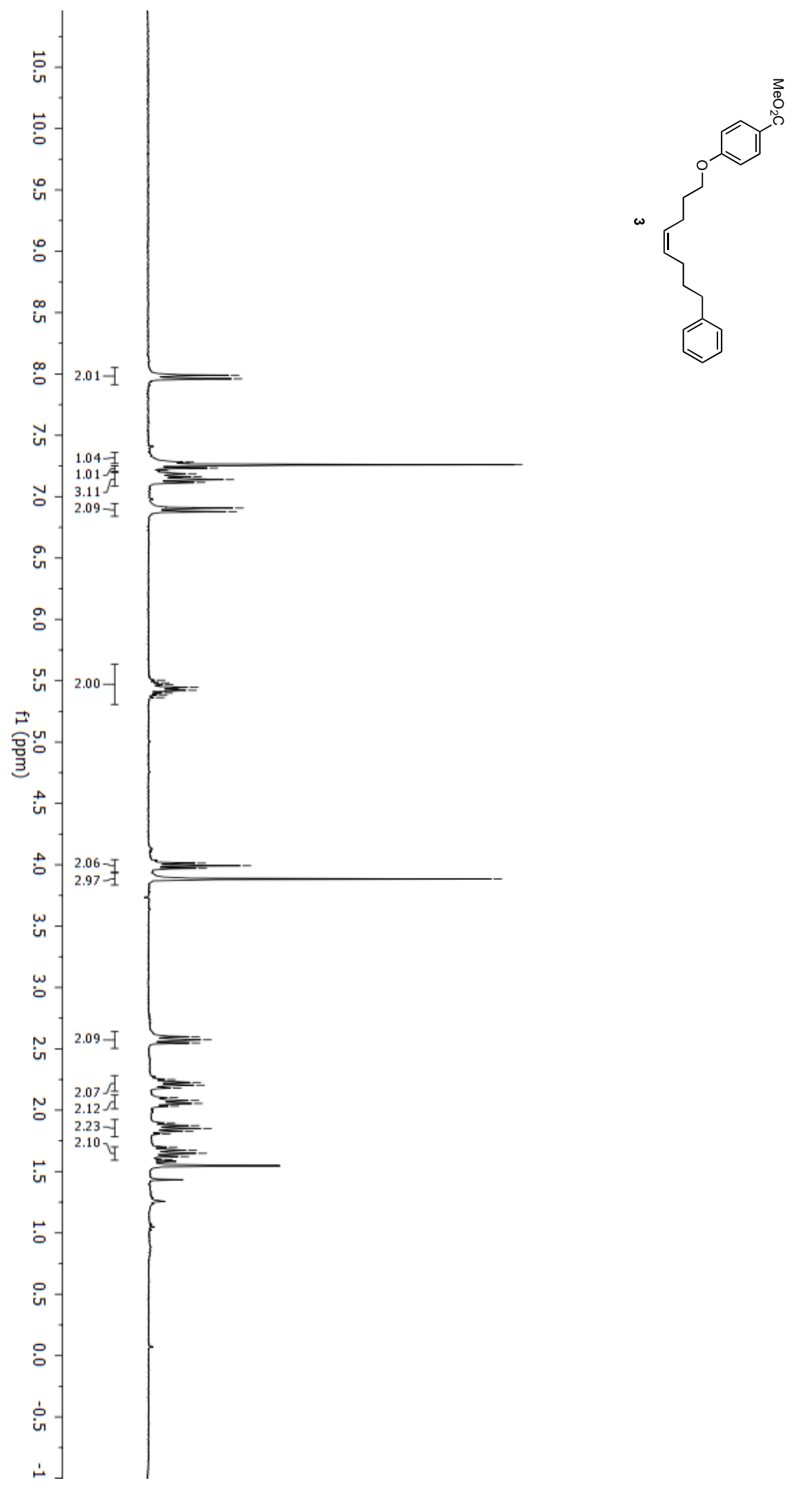

刍目

尊具

芯

家

苗学

岽是

봉

10

奂

苑

苟最

额

$\int_{7.28}^{7.28} \div$

$7.26 \mathrm{CDCl}$ के के

-7.22 证

7.21 缶合

- 7.16 夏

$-7.15 \quad$ i

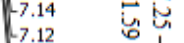

-7.12
6.91
6.88

5.50
5.48

5.47
-5.45

-5.45 जे

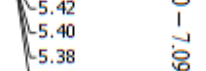

$-5.36$

$\int_{-3.99}^{4.01}-$

美

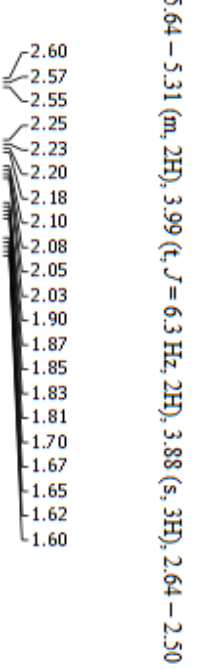



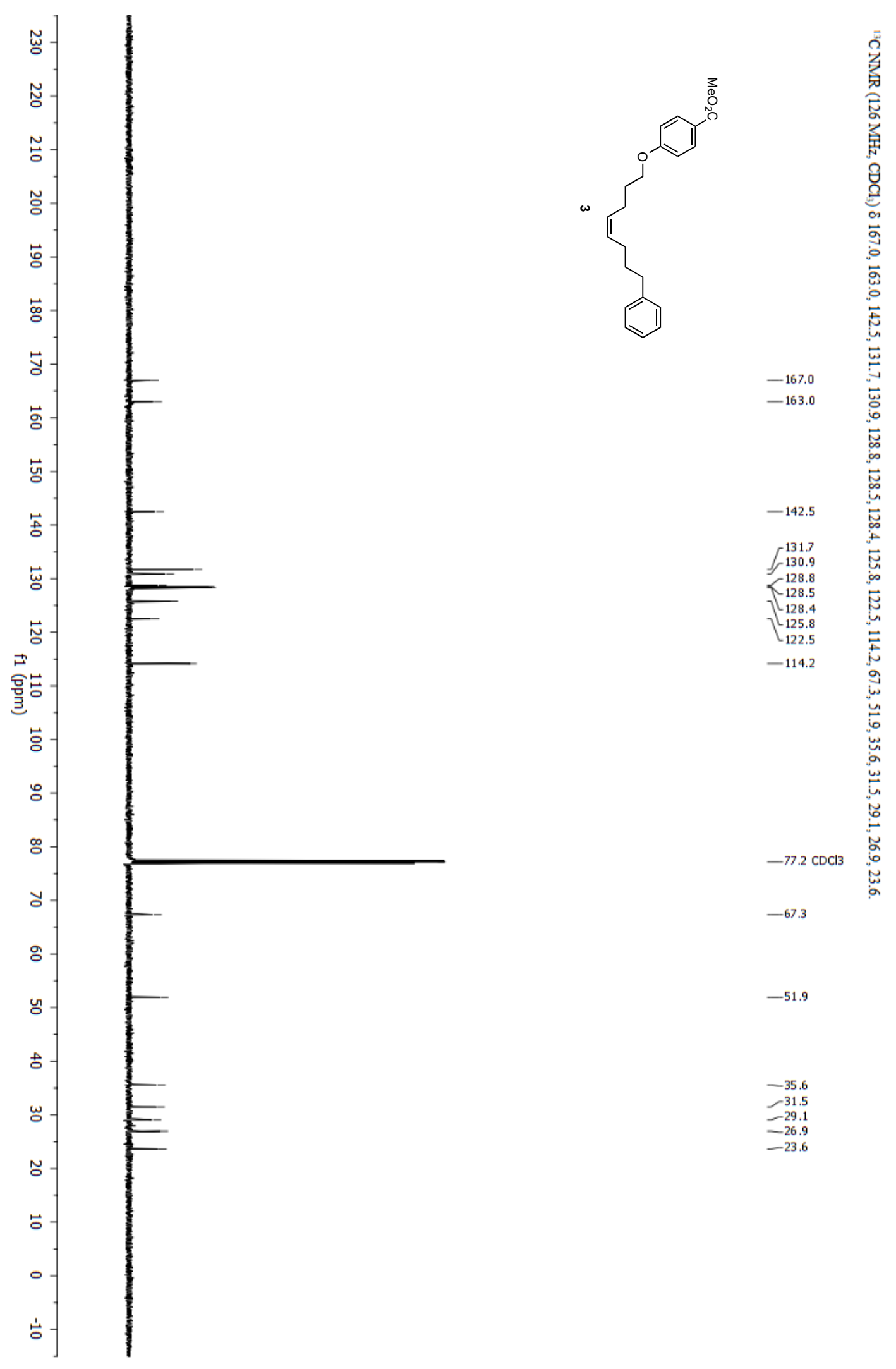

$-51.9$

-35.6
-31.5
-29.1
-26.9
-23.6 


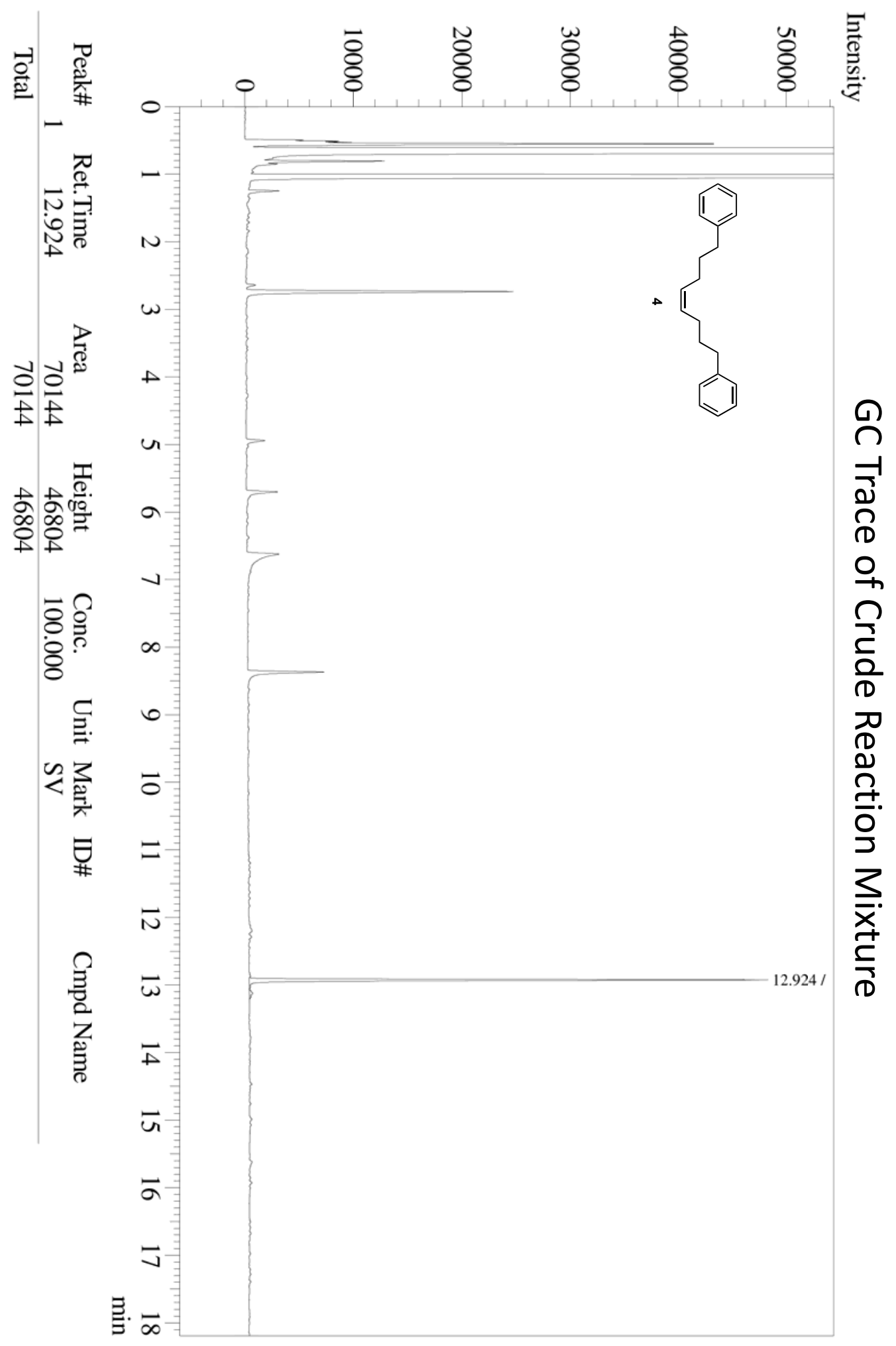



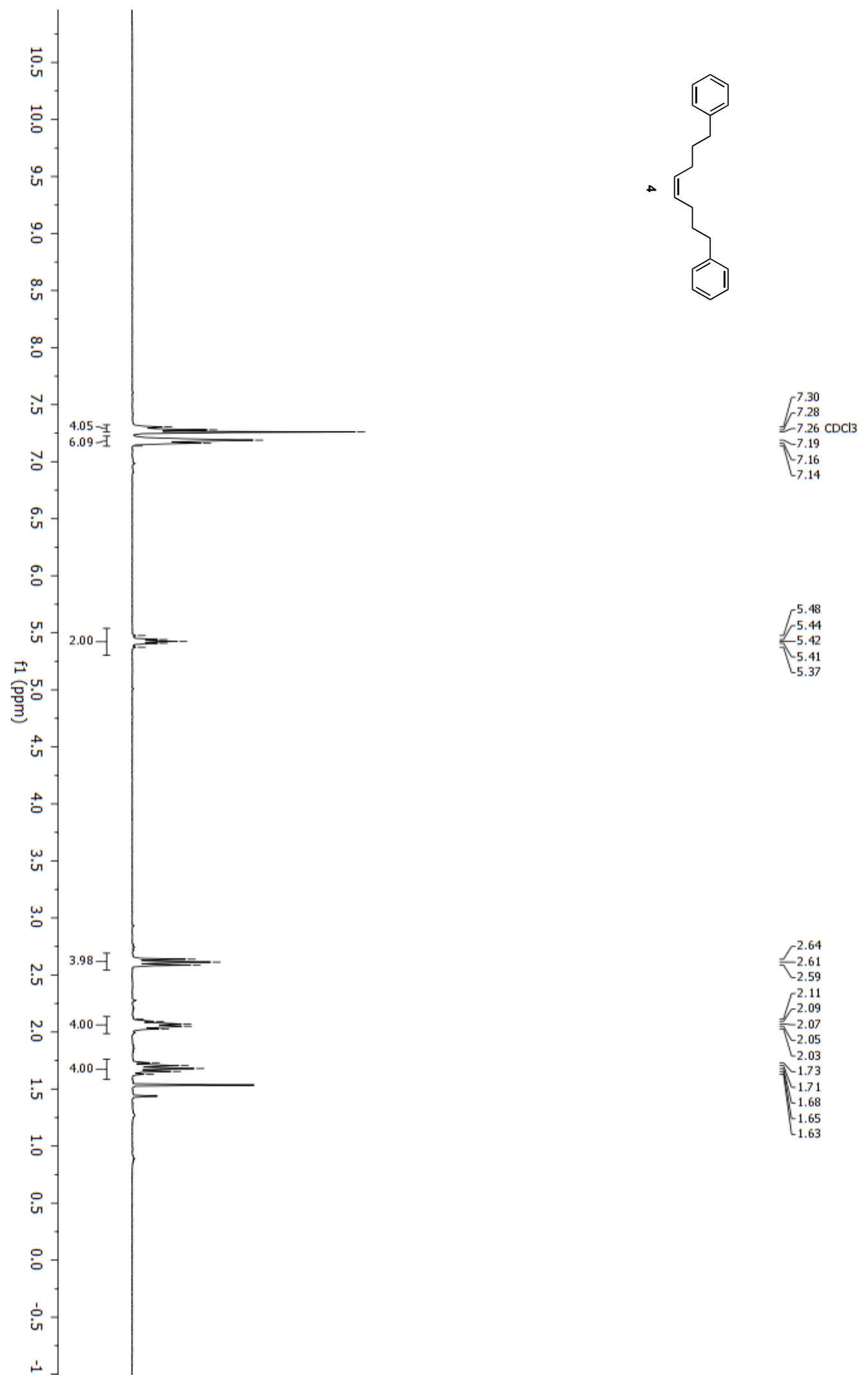

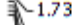

$\mathfrak{r}_{1.71}$

1.68
-1.65

-1.65
1.63 

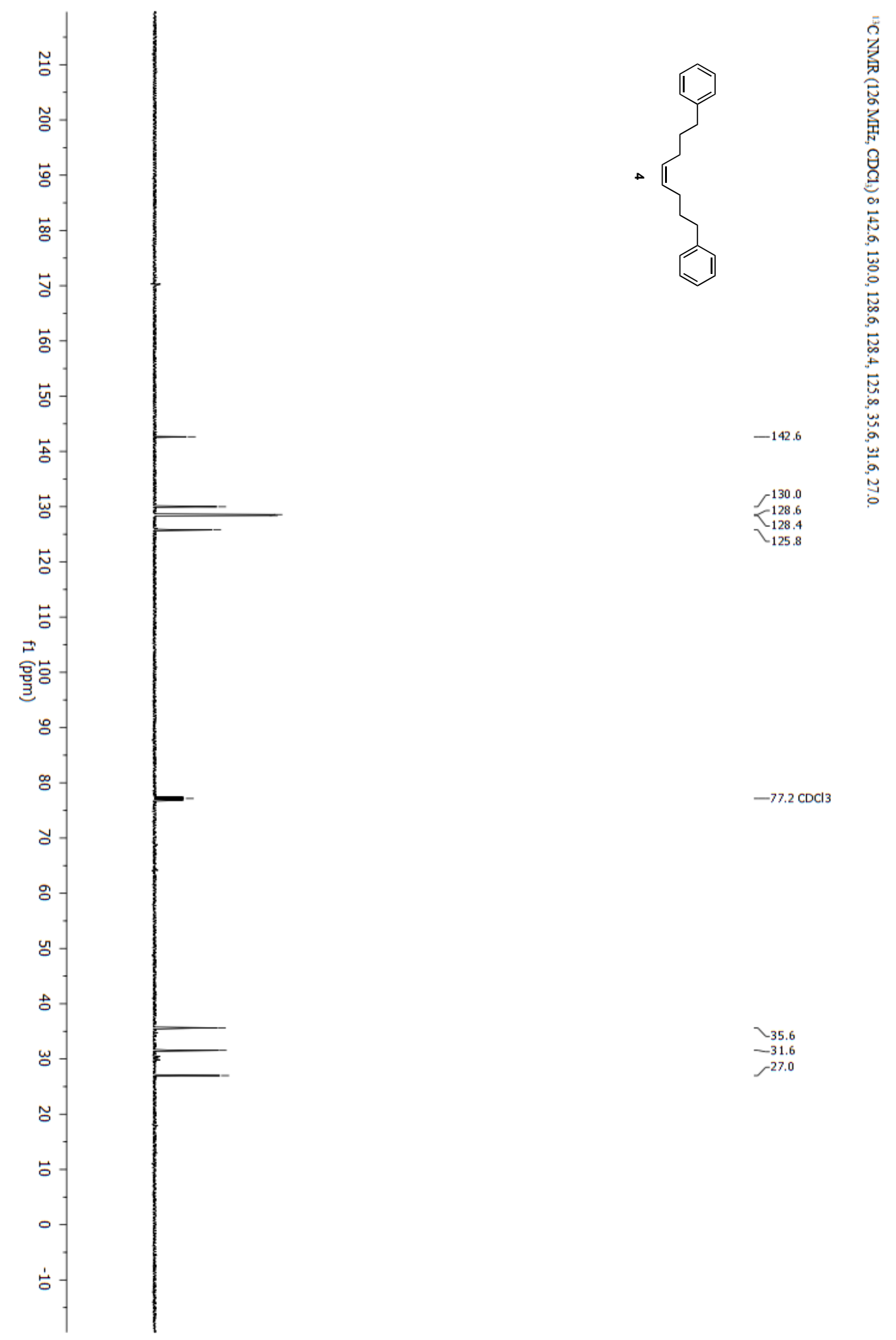


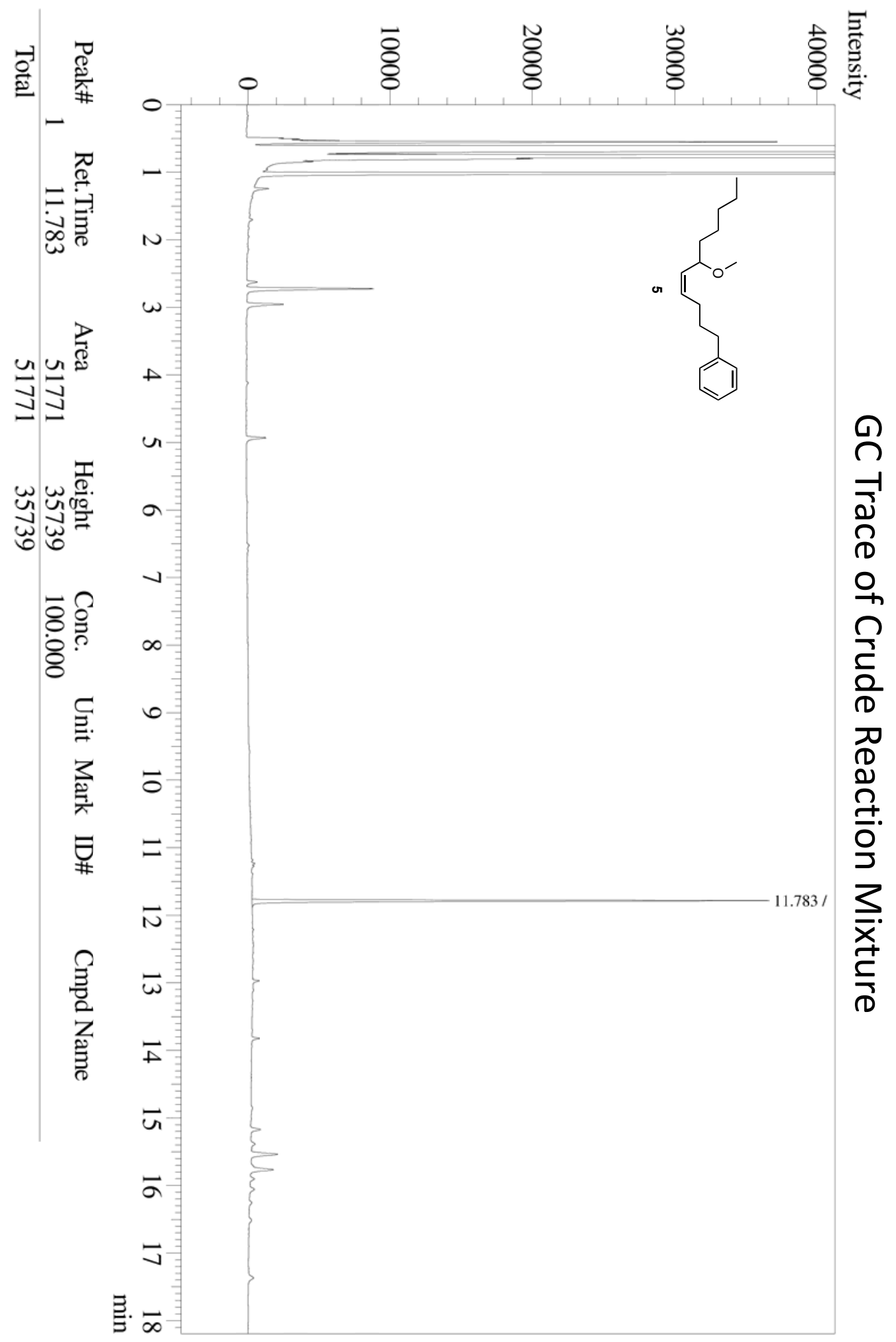




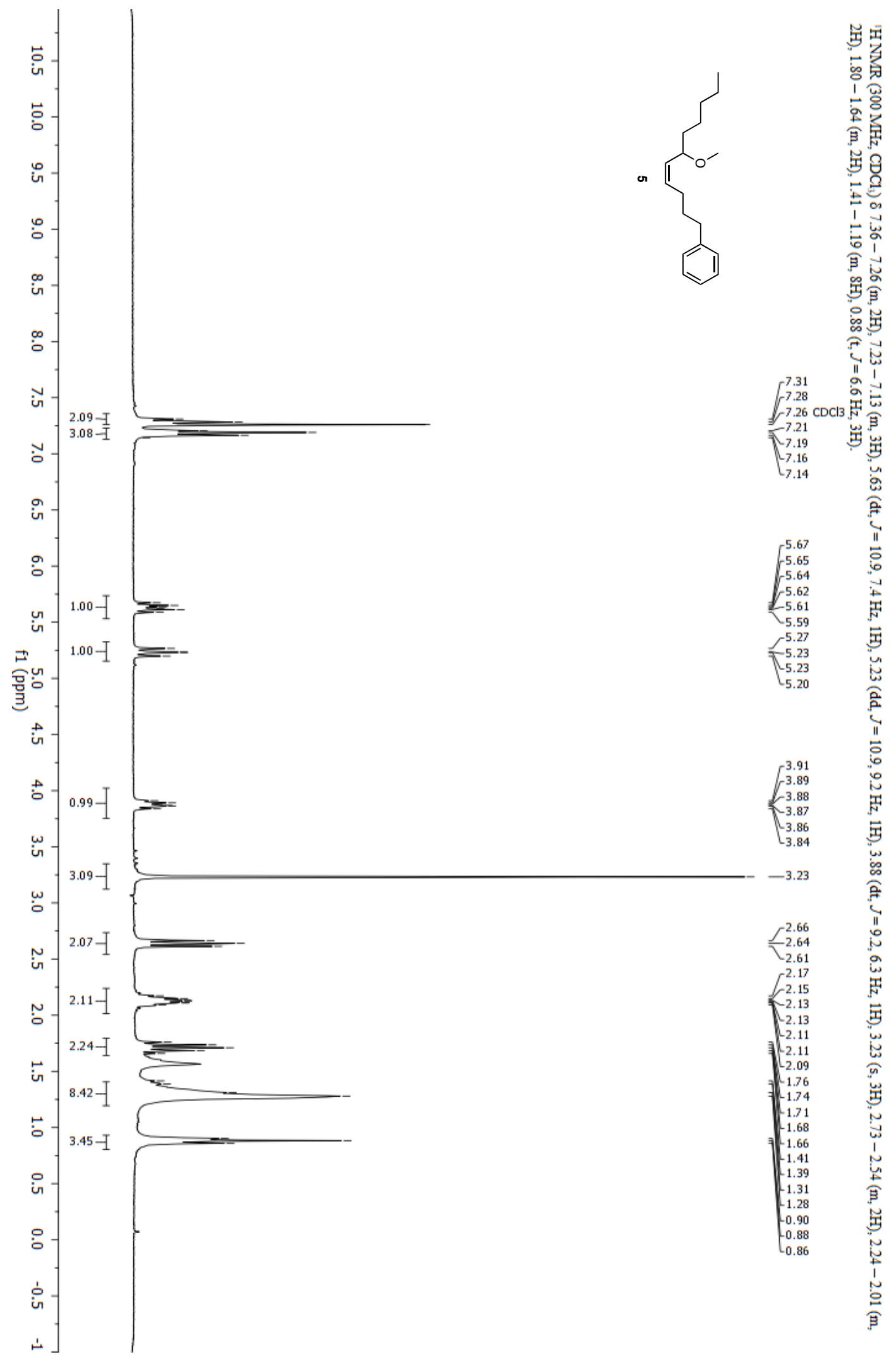



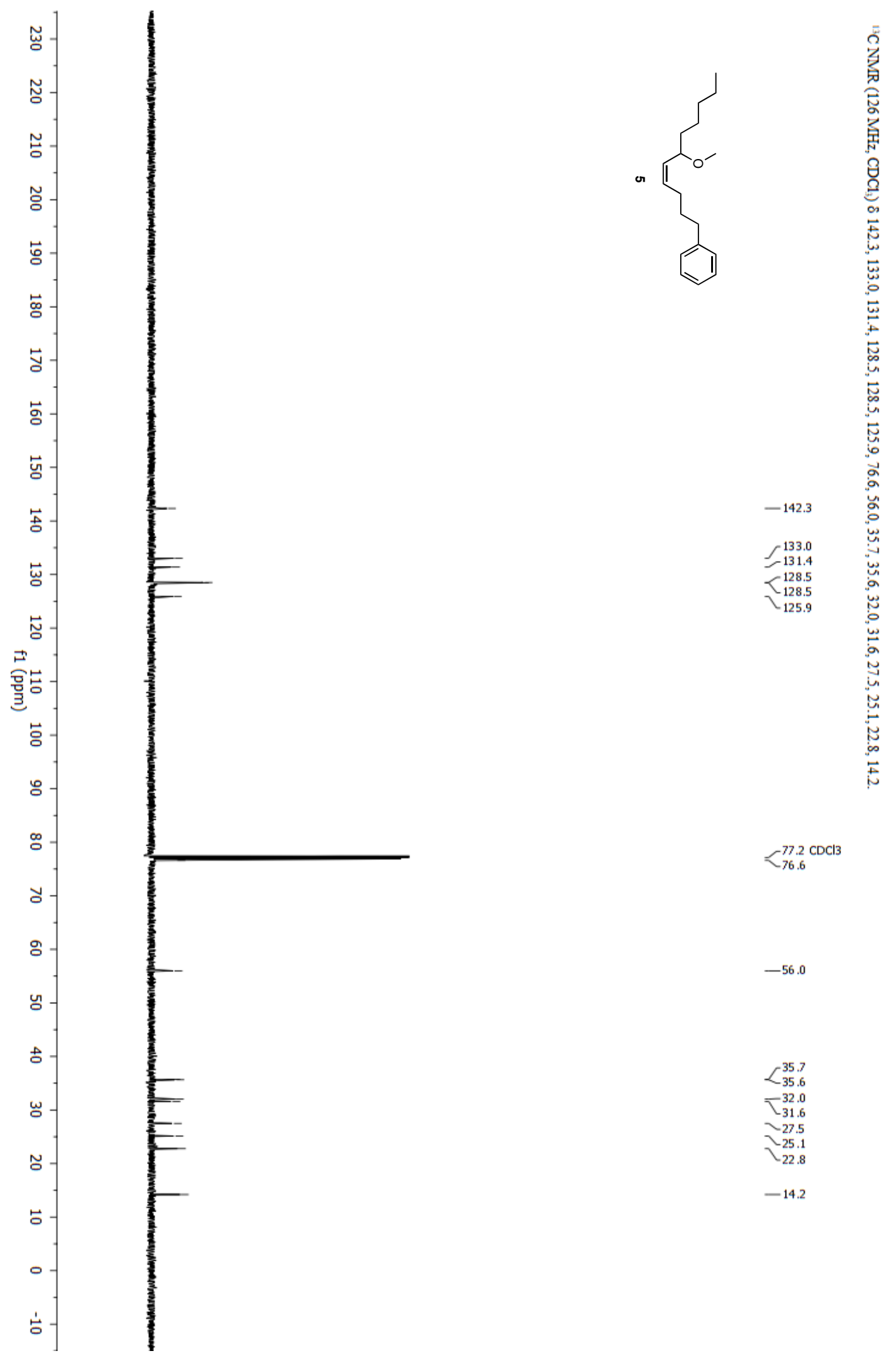

$-56.0$

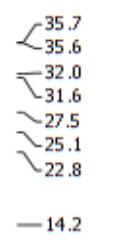




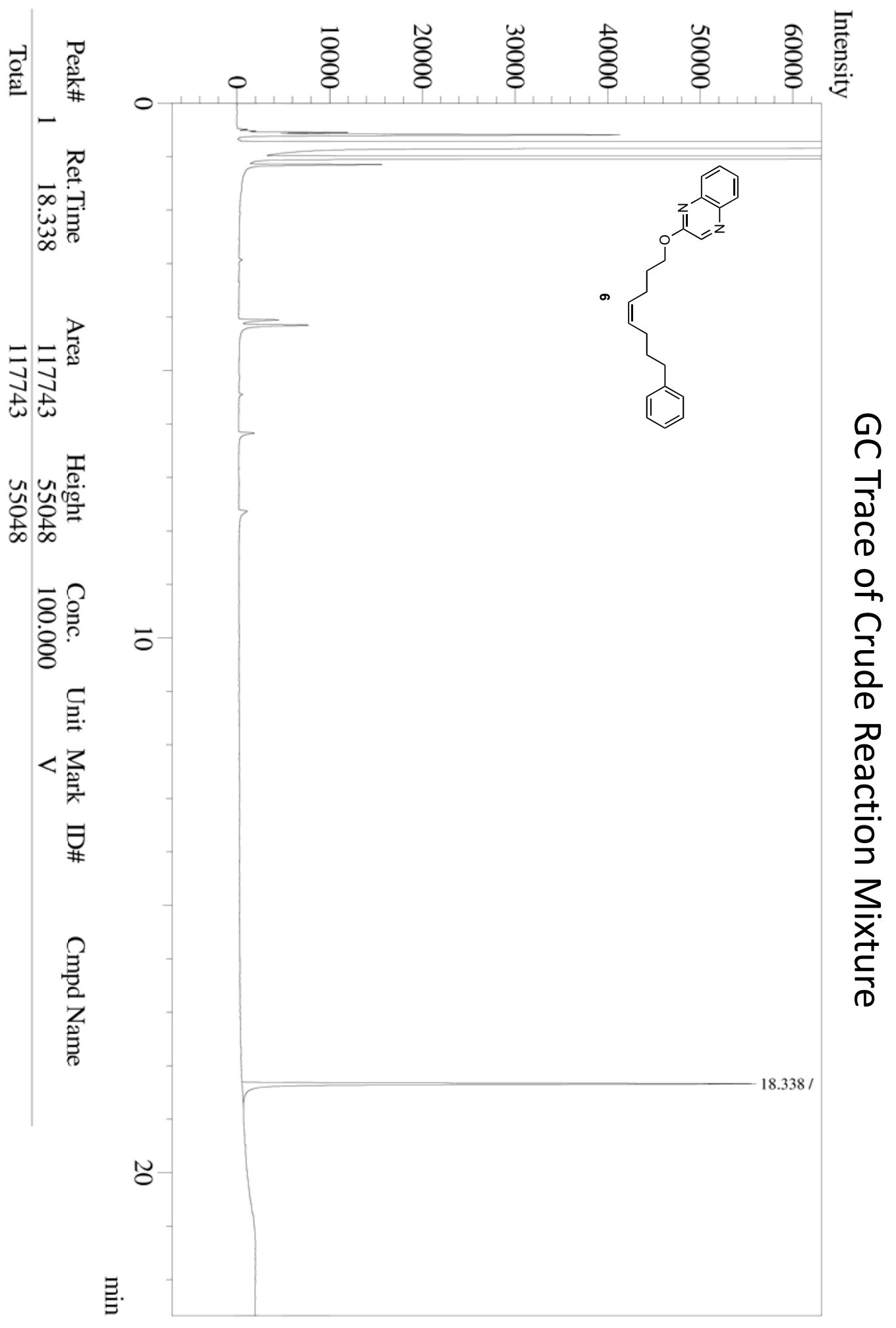




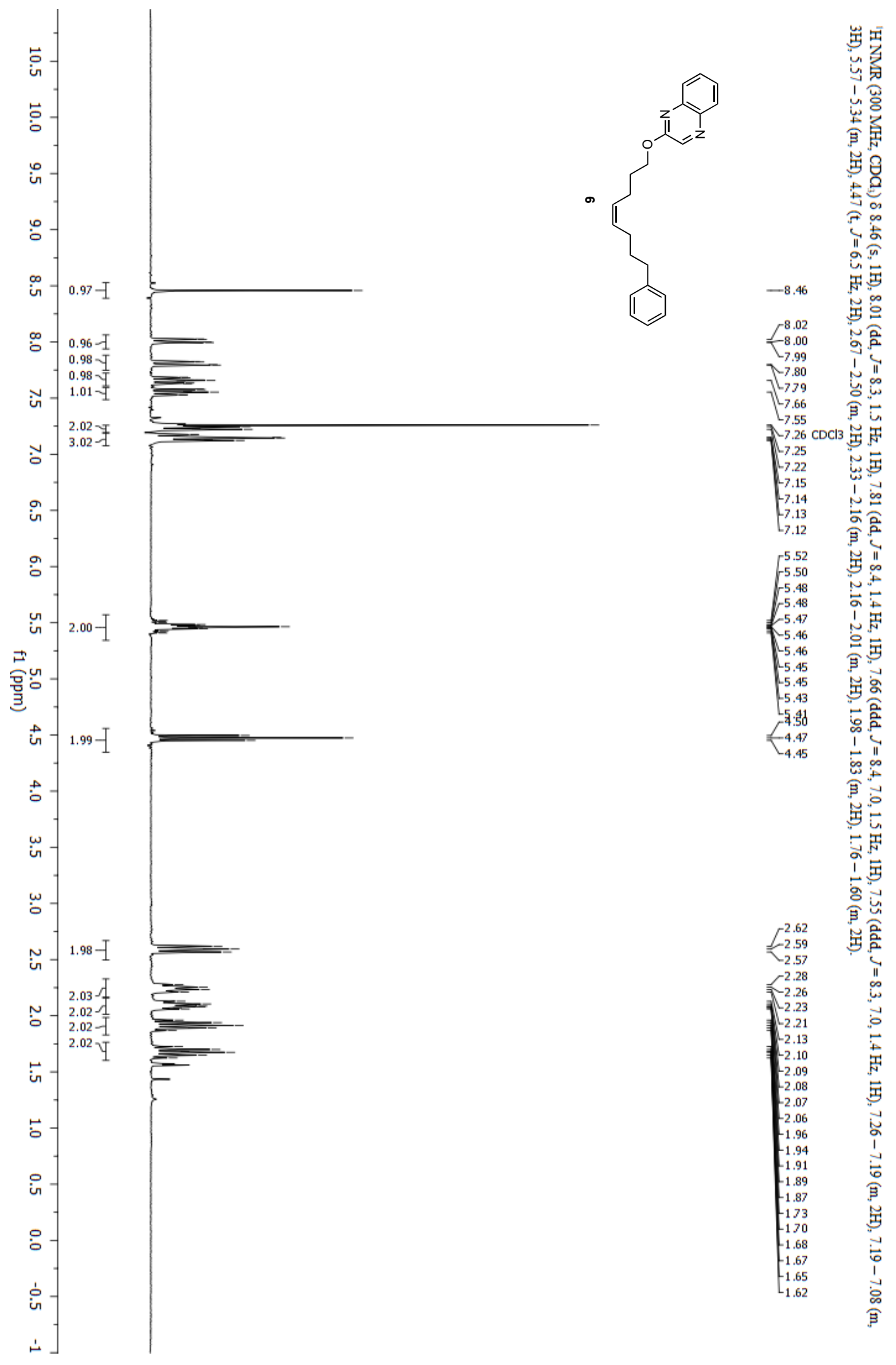



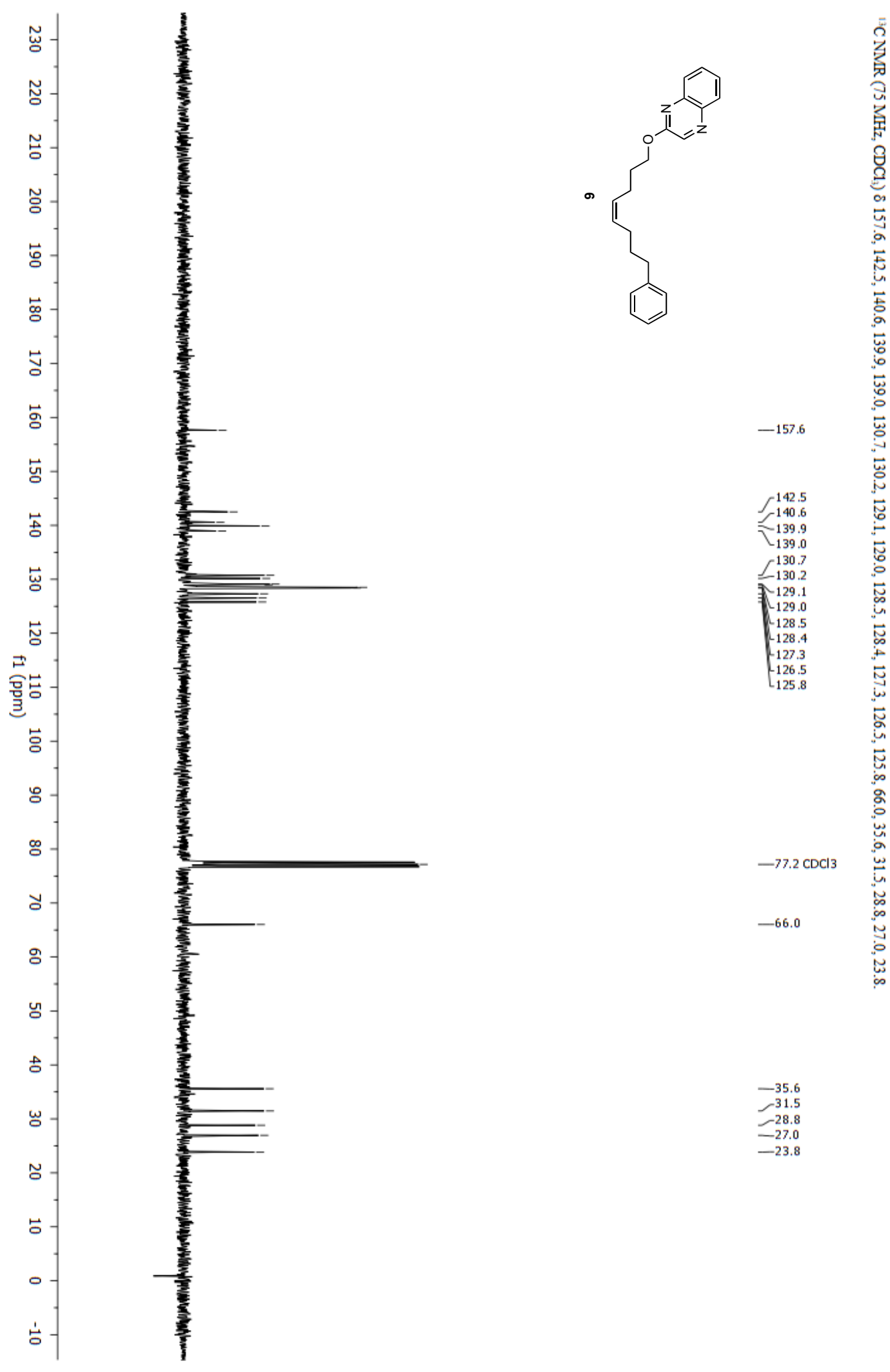


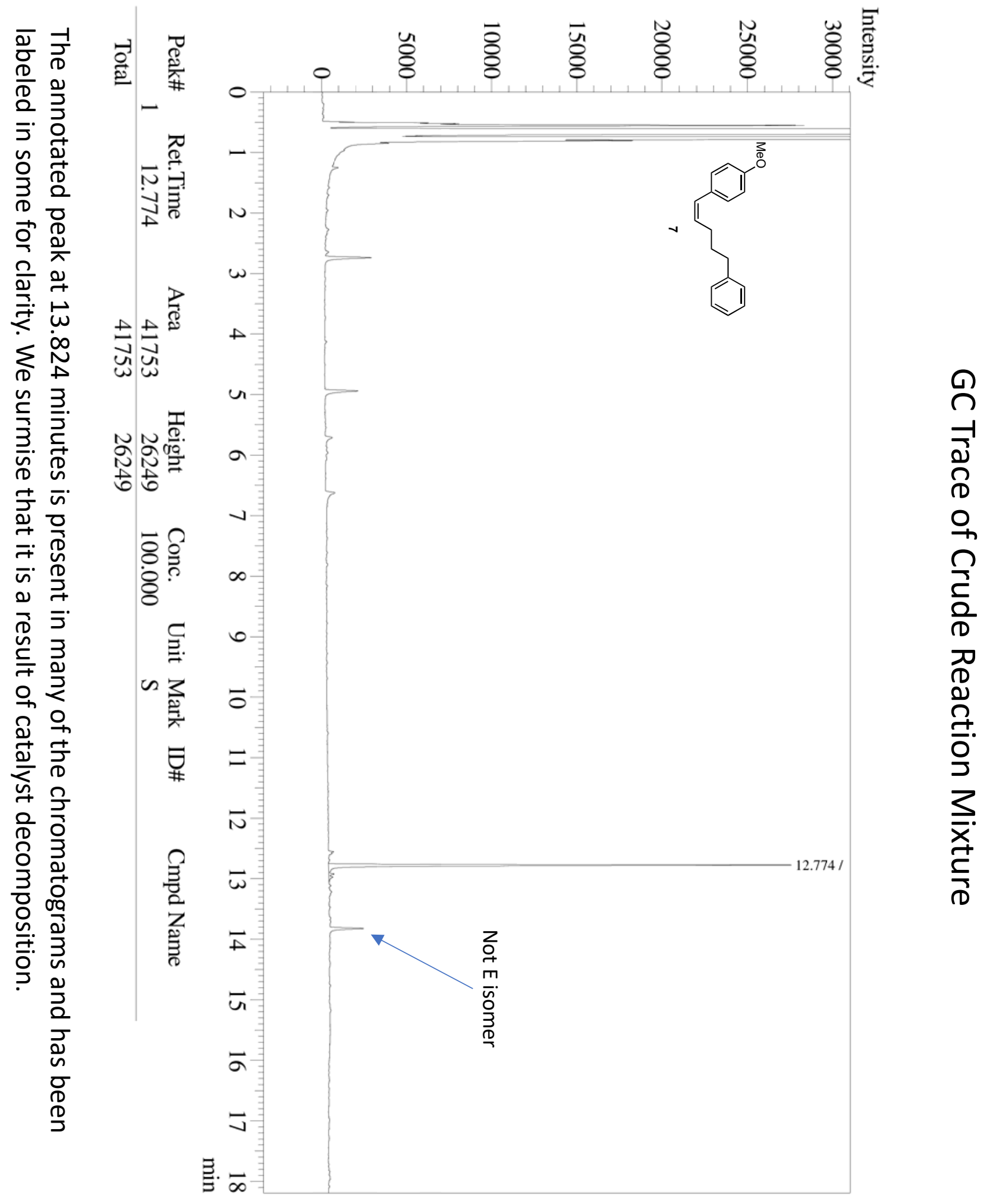



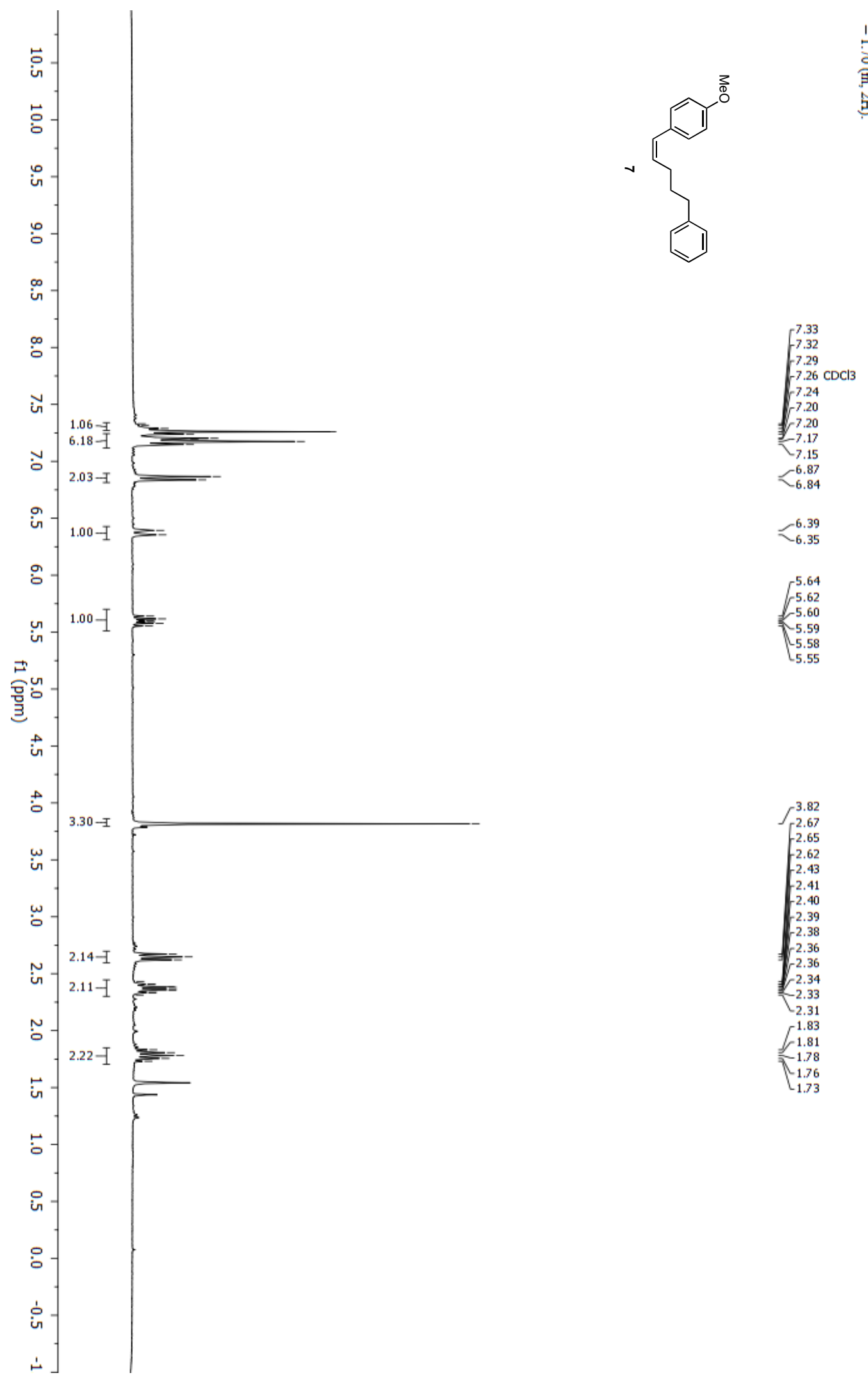

1 实

完

芎客 


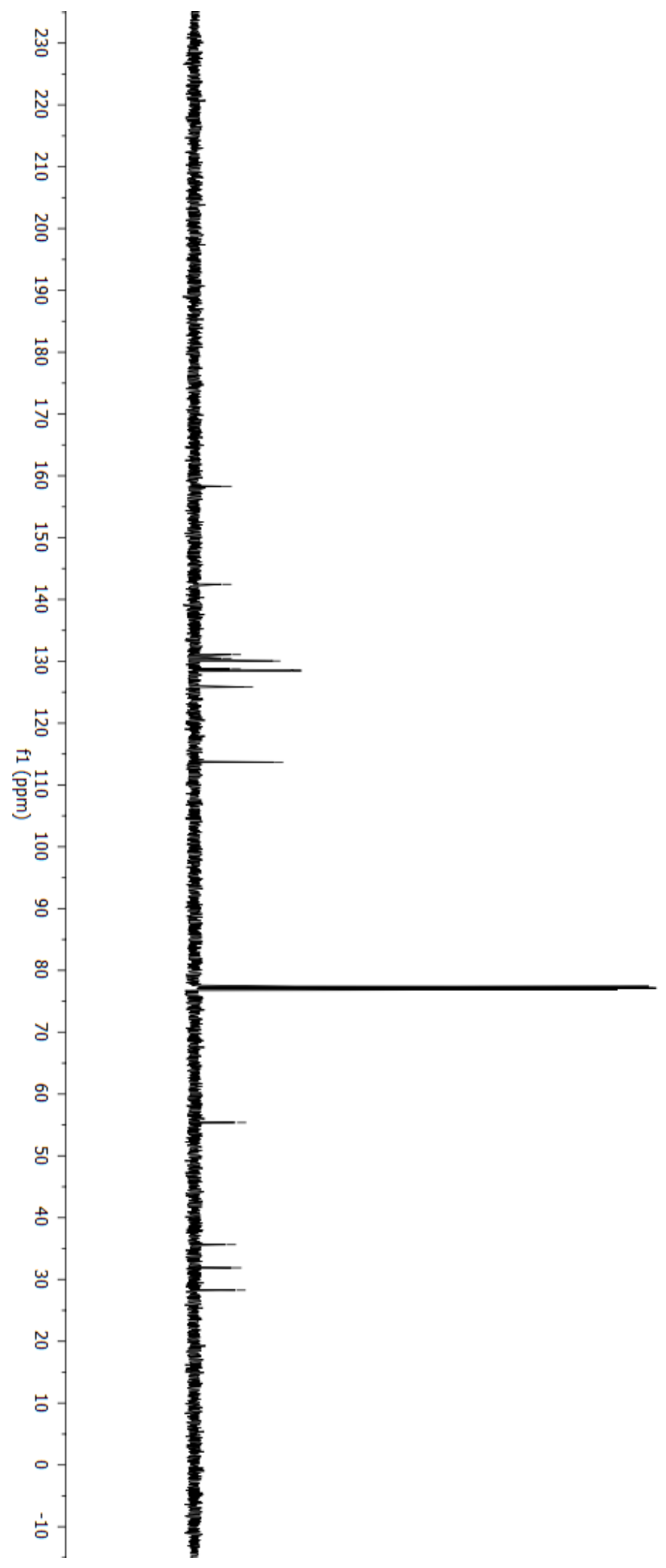

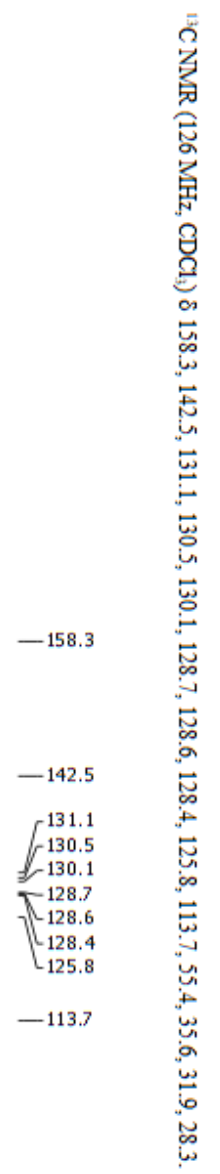

$-77.2 \mathrm{CDCl} 3$

$-55.4$

-35.6
-31.9
-28.3 


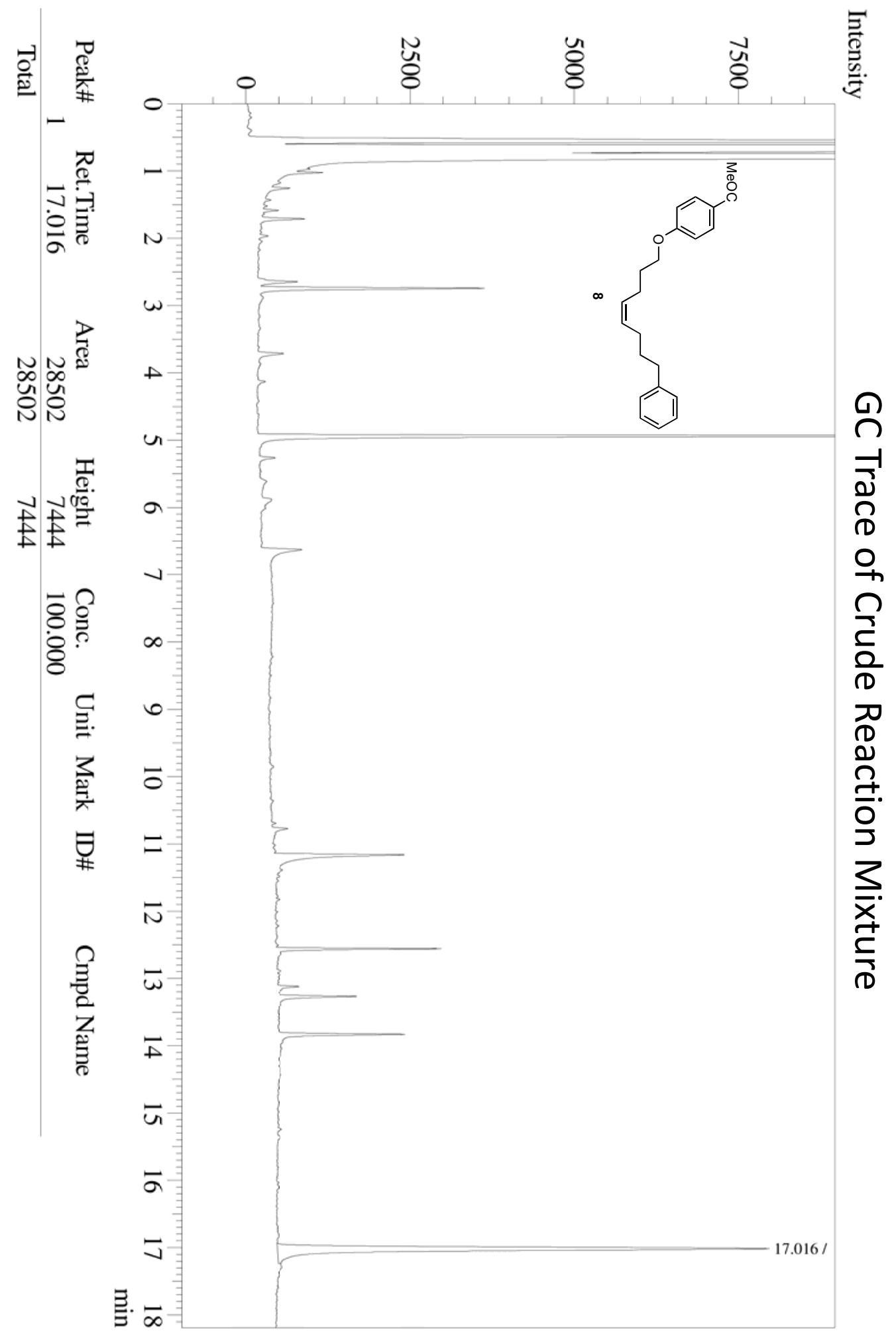



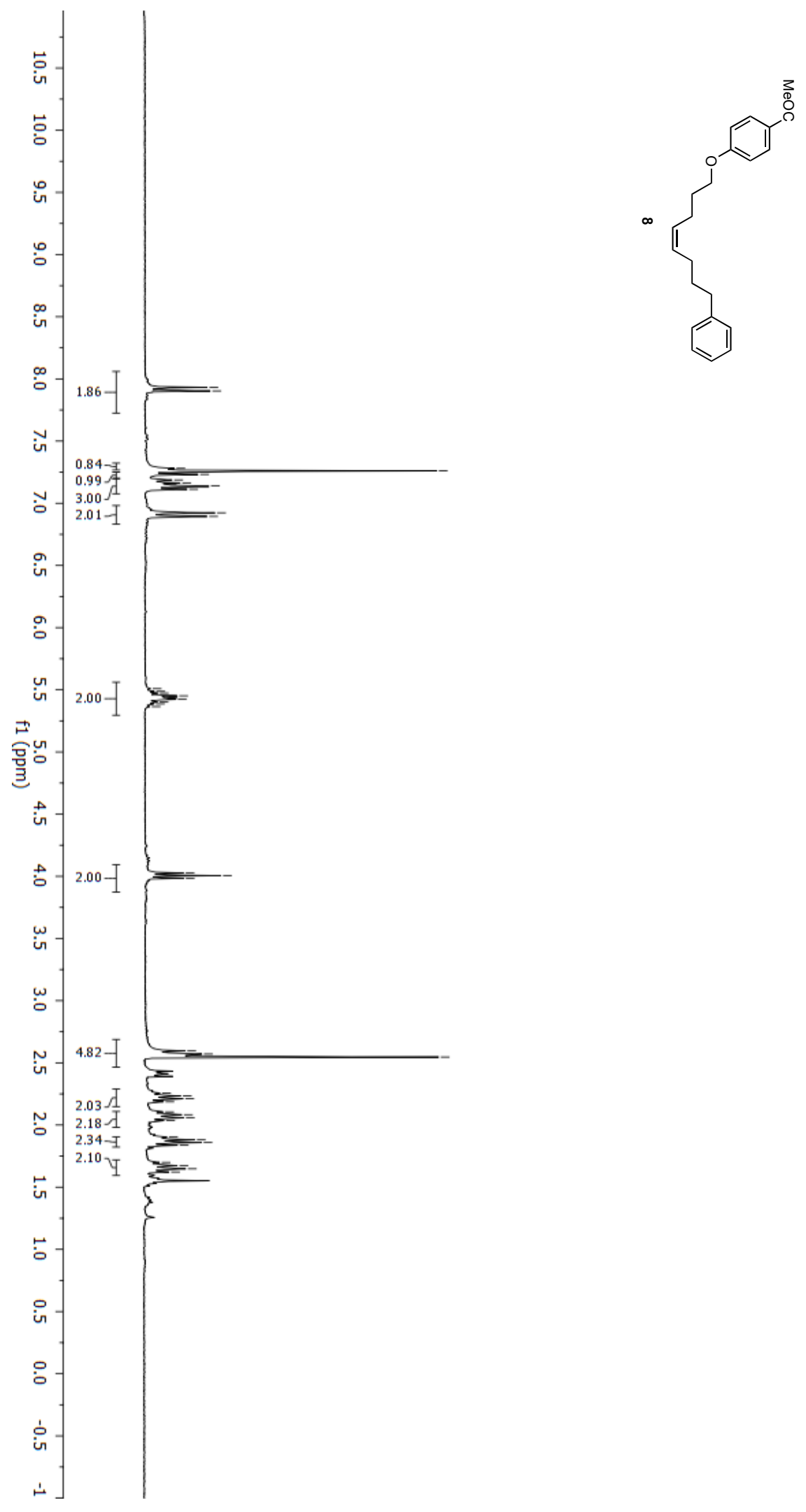

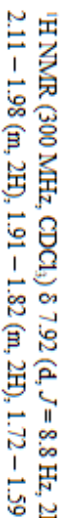

$\succ_{7.90}^{7.93}$ 茴留

$\int_{7.26 \mathrm{CDCl}}^{7.28}$ 舀兽

7.23 क ल

$=7.19$

$\prod_{7.14}^{7.16}$

]$_{7.11}^{7.14}$

$\left[\begin{array}{ll}6.92 \\ 6.89\end{array}\right.$

$\left[\begin{array}{r}5.51 \\ 5.49 \\ 5.47 \\ 5.45 \\ 5.44 \\ 5.44 \\ 5.43 \\ 5.42 \\ 5.40 \\ 5.39 \\ 5.36\end{array}\right.$

$\int_{-4.98}^{4.03}$

(a)

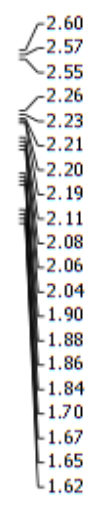

年 

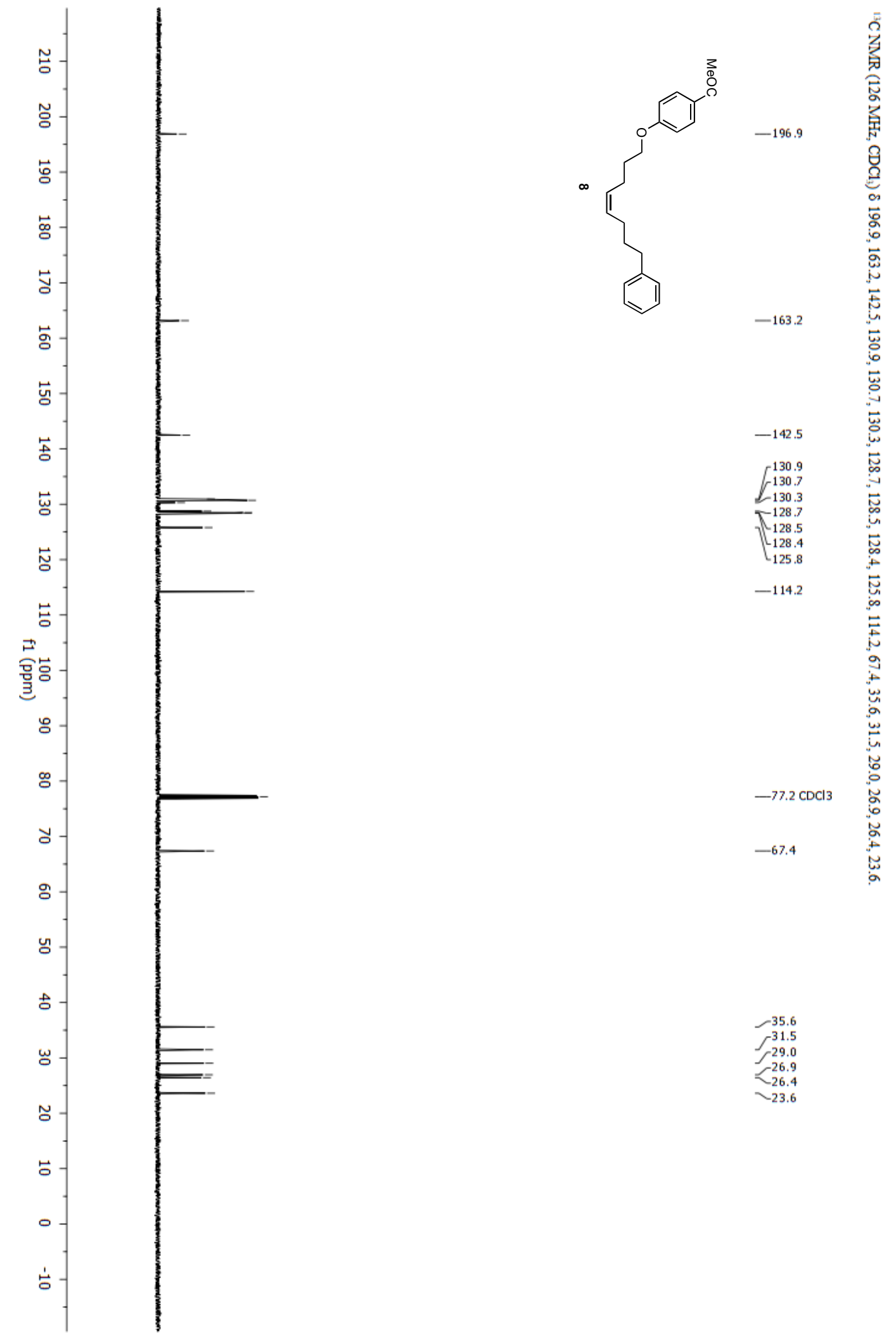


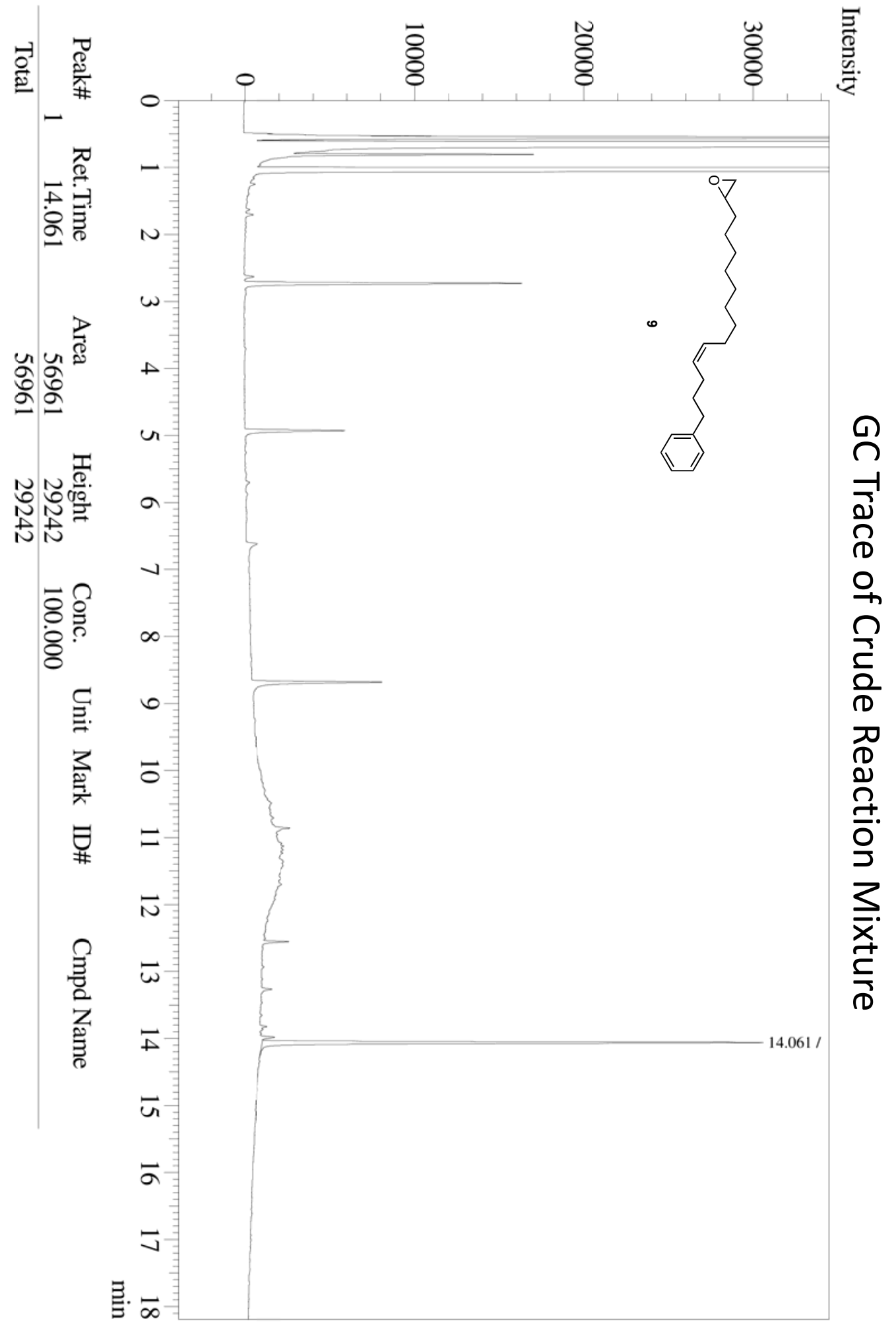



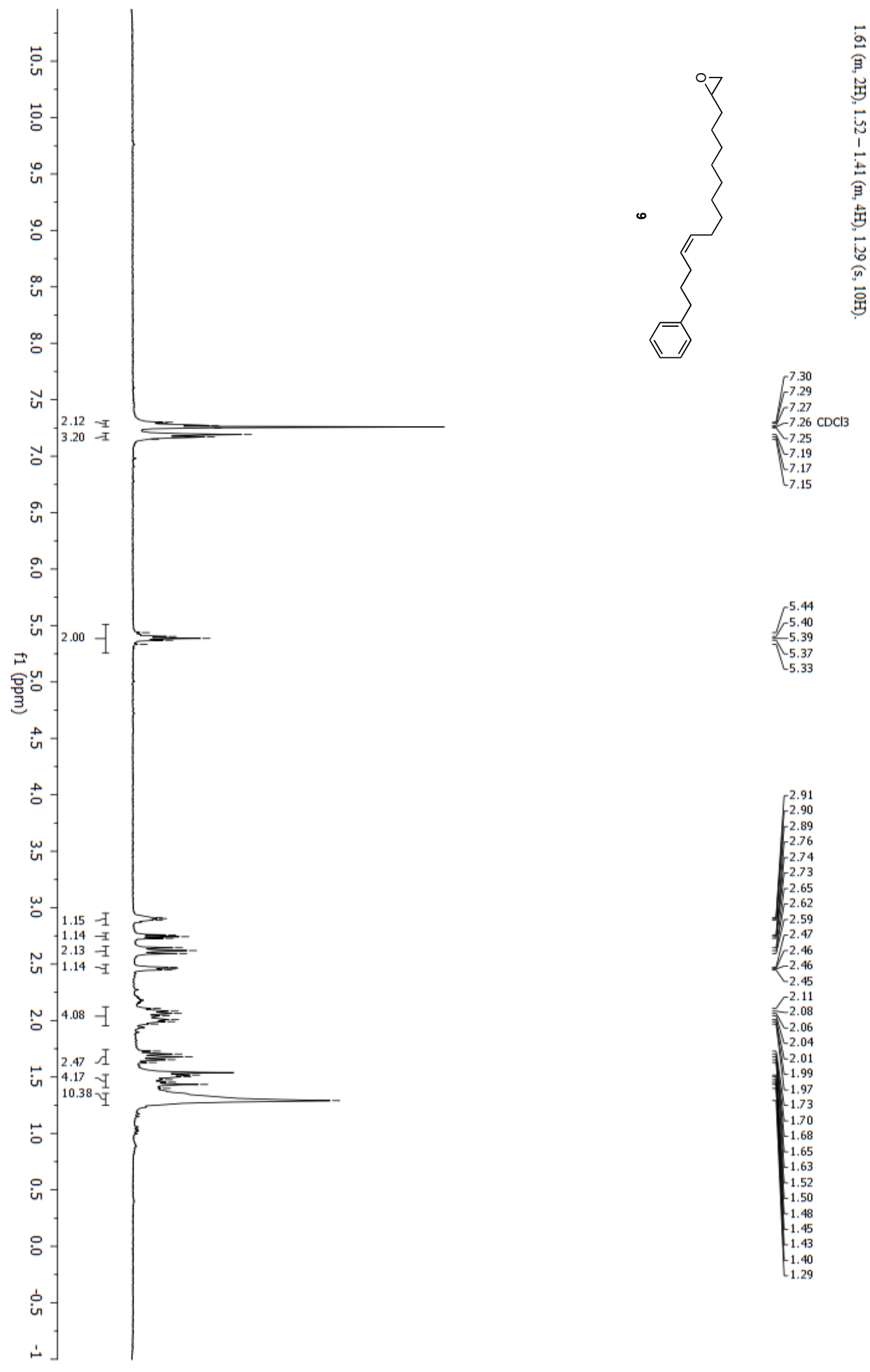

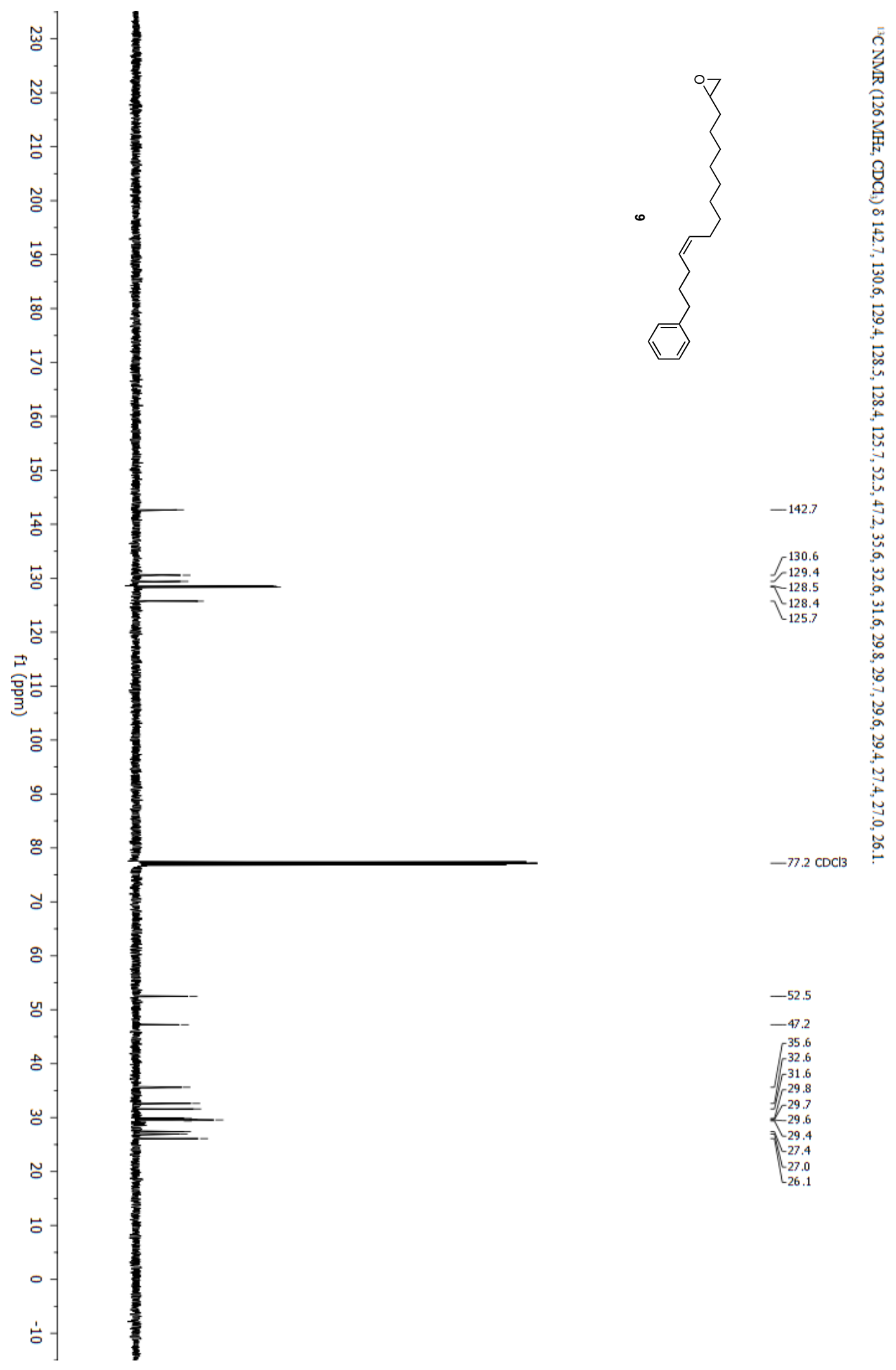


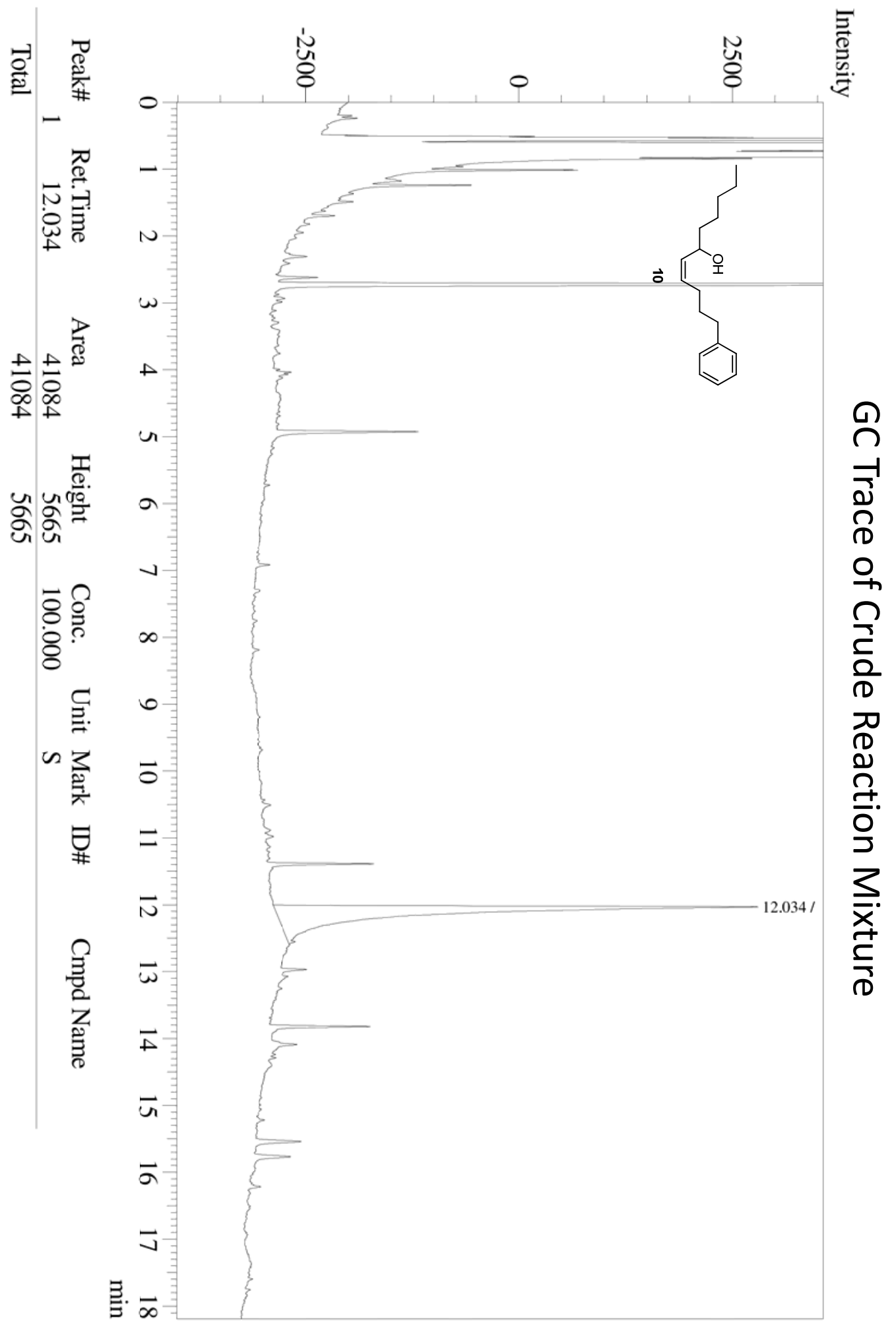



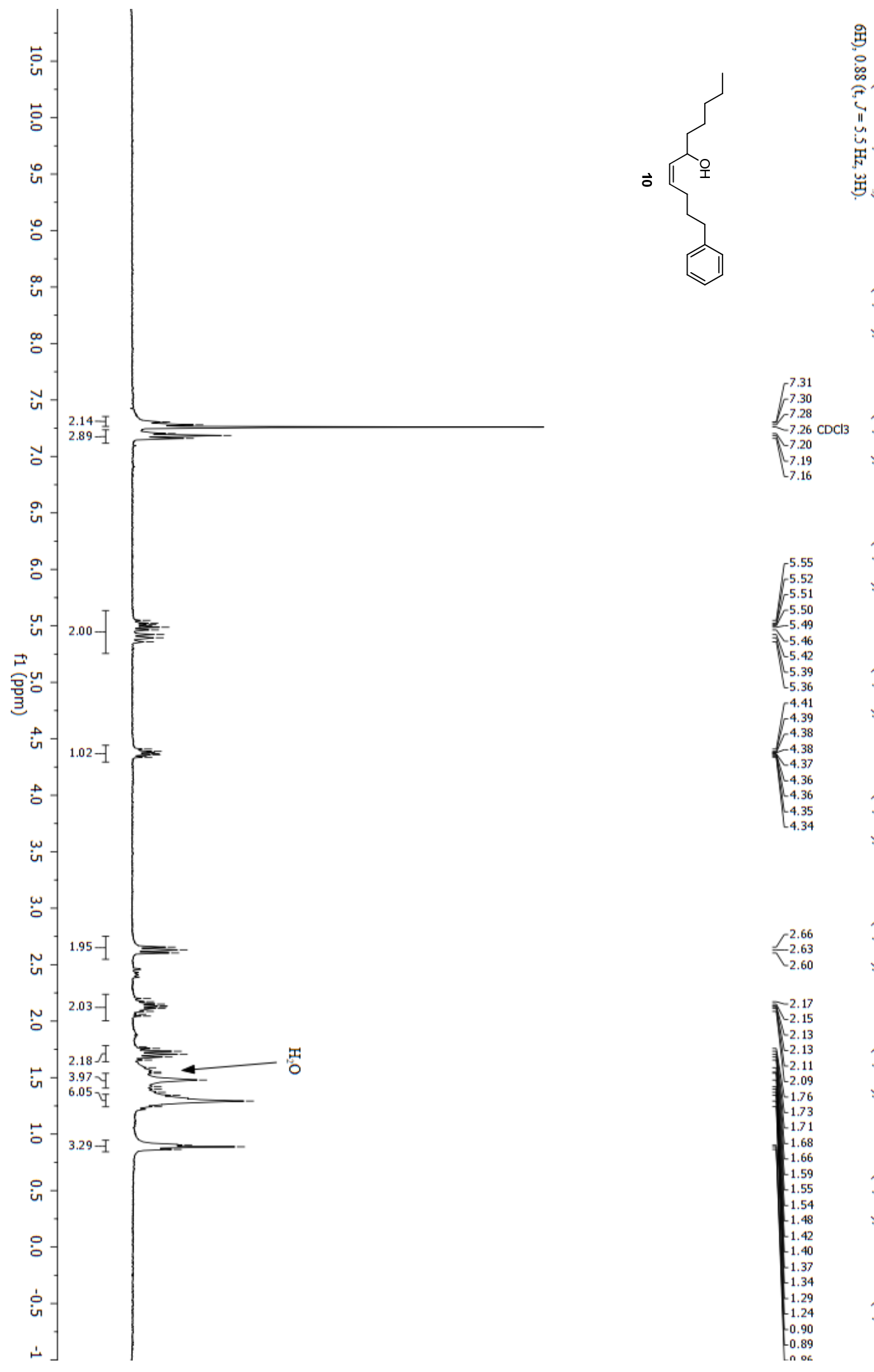

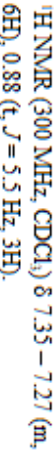

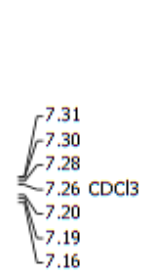

5

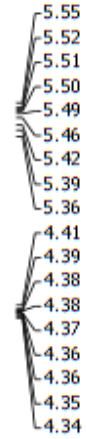

$\tau^{2.17}$

$-2.15$ 

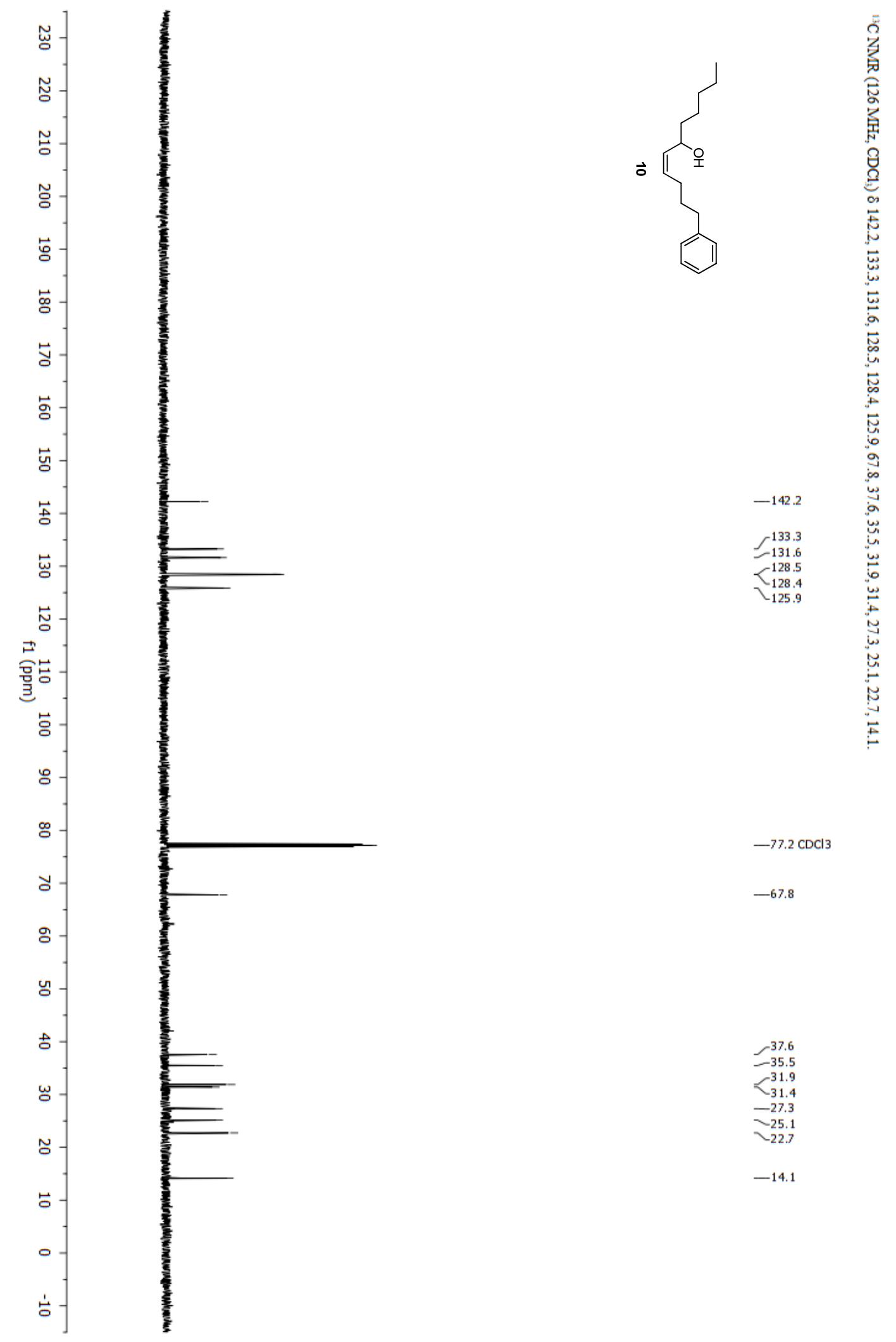


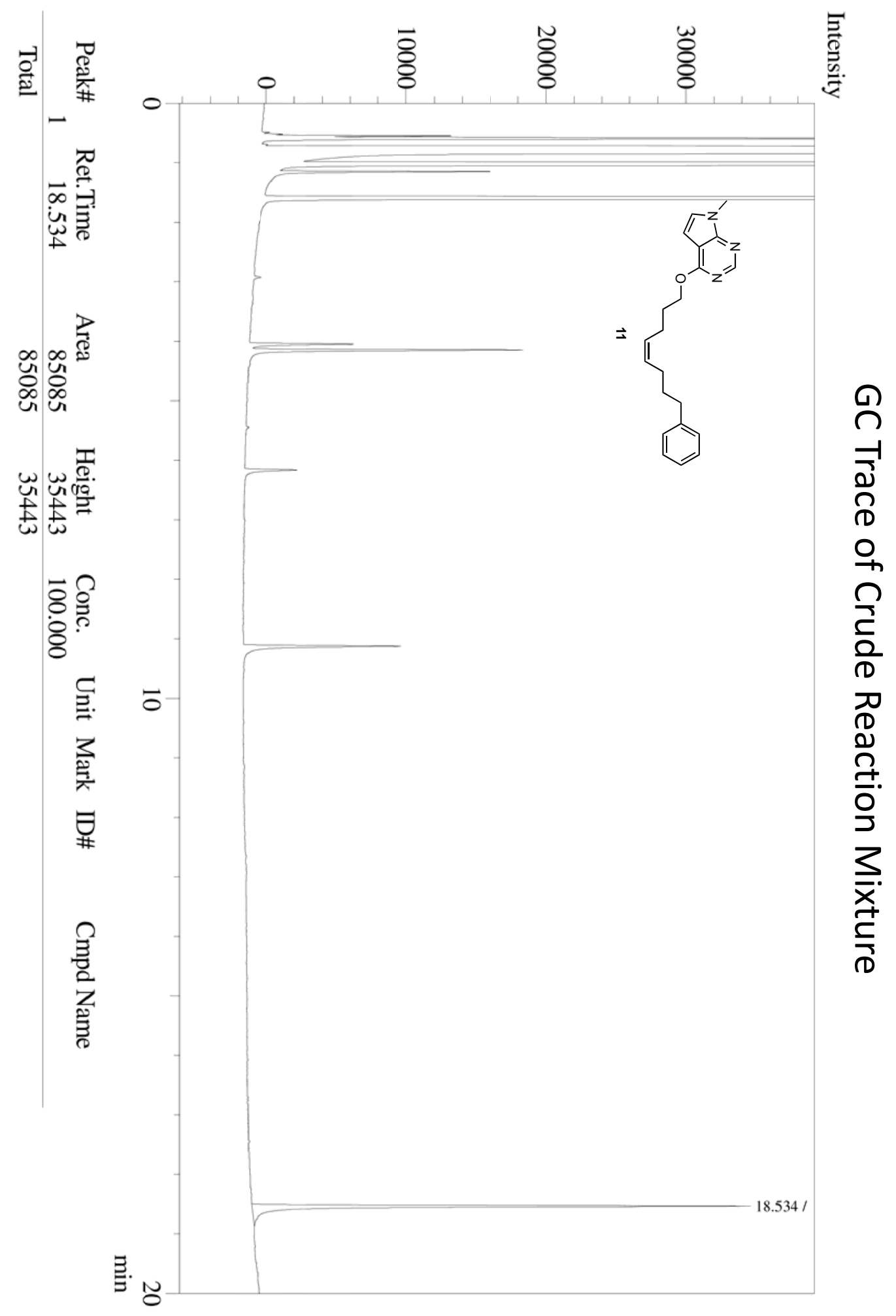



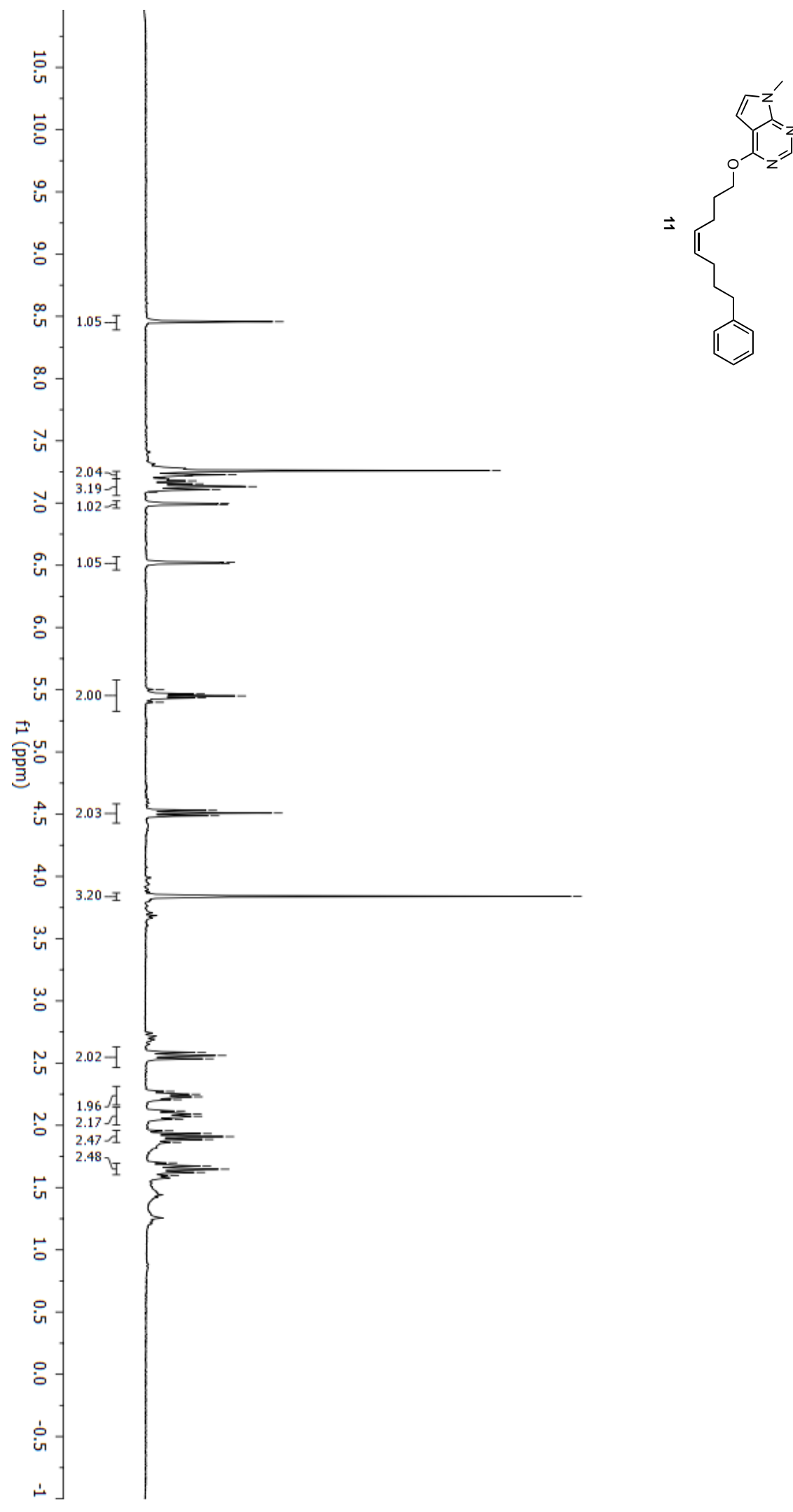

密

1

亩

缶容

点胥

'象

8

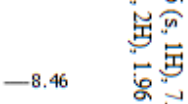

$7.26 \mathrm{CDCl} 3$

7.23 .

-7.22 昰

$7.15-5$

7.13 : 8

$-7.09 \quad \overrightarrow{0}$

7.00
6.99

$<_{6.51}^{6.52} \quad{ }^{3}$

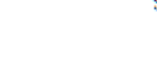

5.50
5.47

$-5.45$

5.43
5.40

(5)

$\int_{-4.53}^{4.51}$

$x_{-3.84}$

$-3.84$

2.59
-2.56
-2.53
-2.27
-2.25
-2.23
-2.21
2.12
2.09
2.09
-2.05
-1.96
1.93
1.91
1.89
1.86
-1.70
1.67
-1.65
1.62
1.60

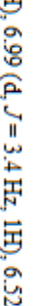



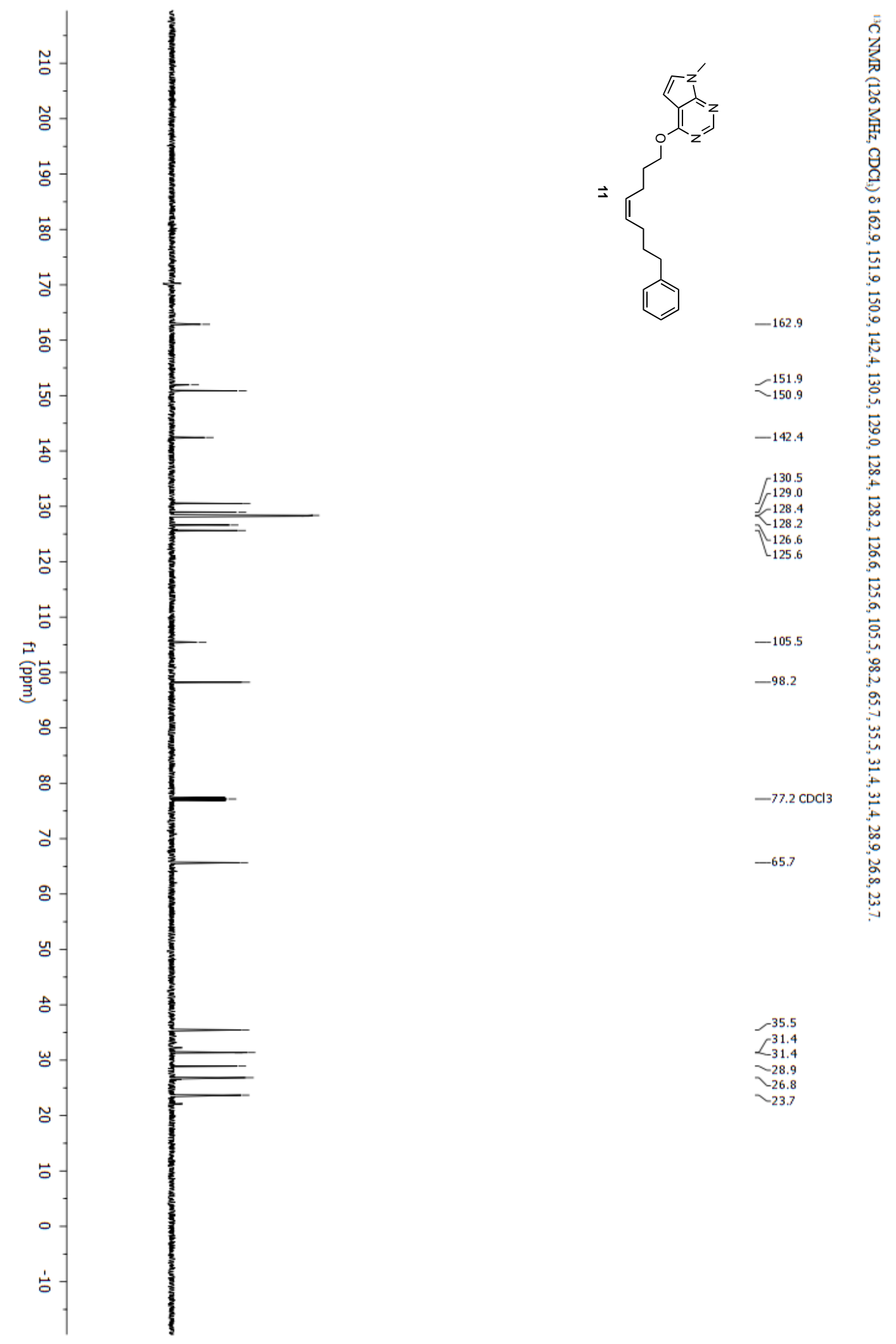


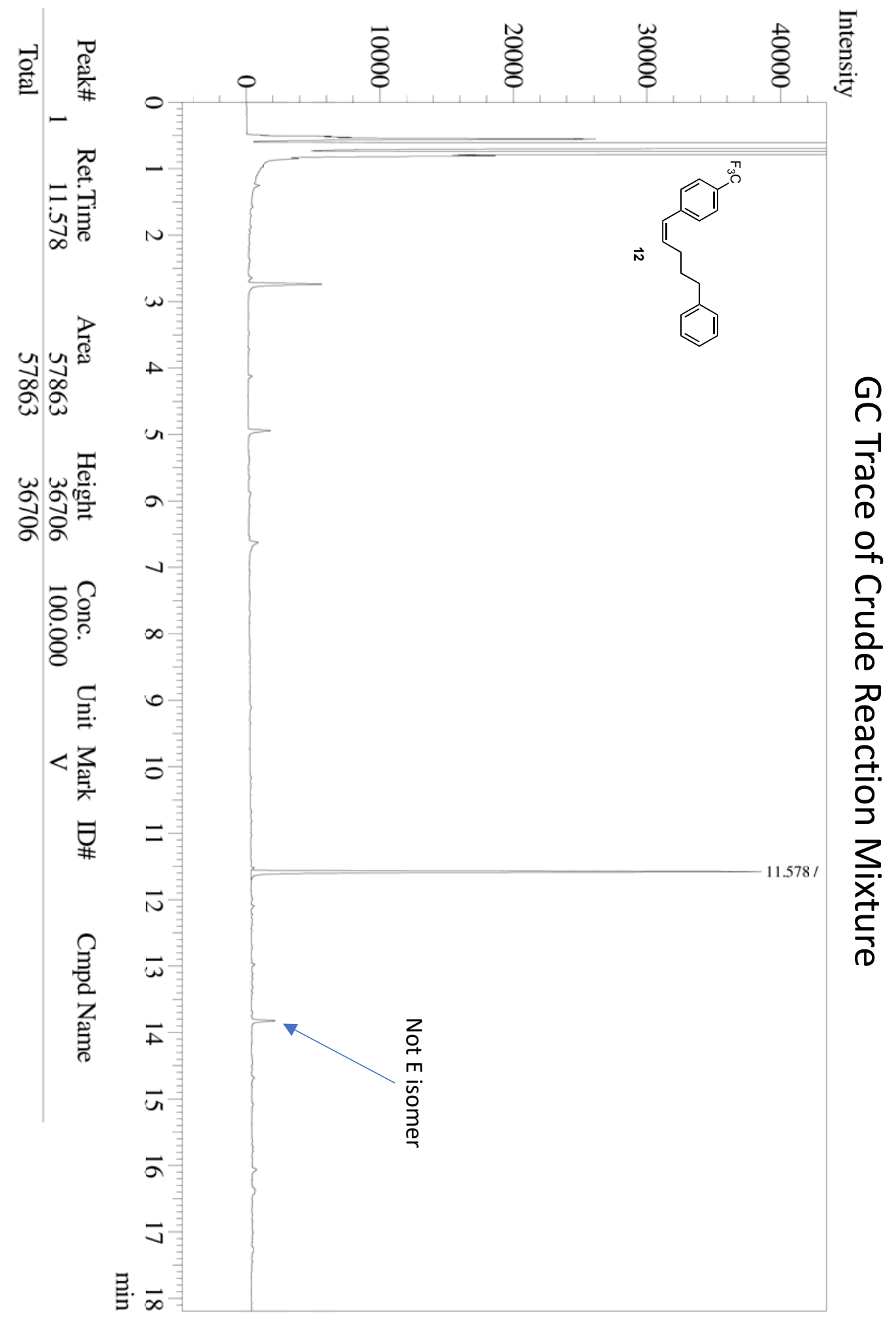



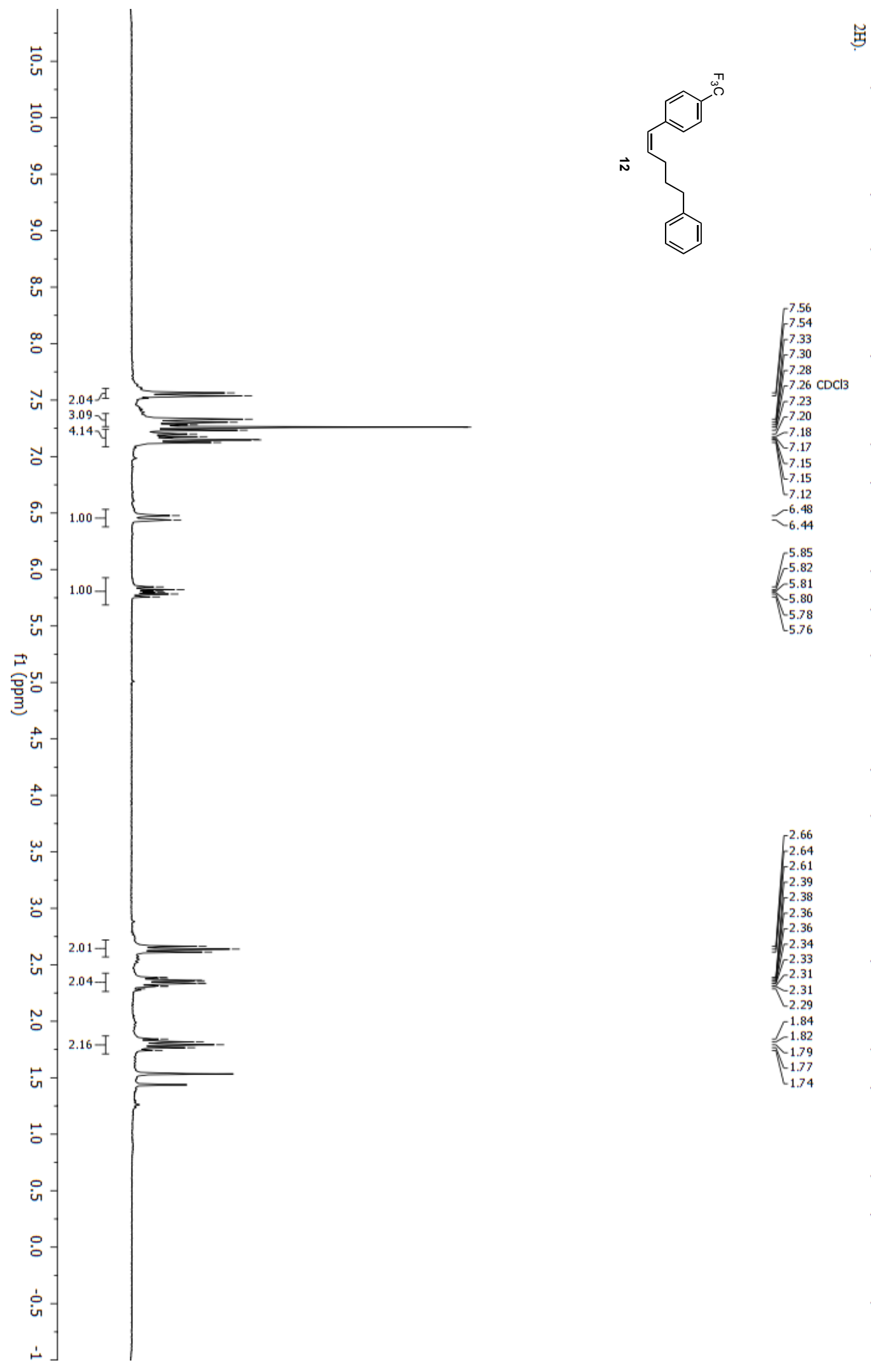

年空

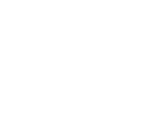

2.66
-2.64
-2.61
-2.39
-2.38
2.36
-2.36
2.34
2.33
2.31
2.31
2.29
$f_{-1.82}^{1.84}$
-1.79
1.77
1.74

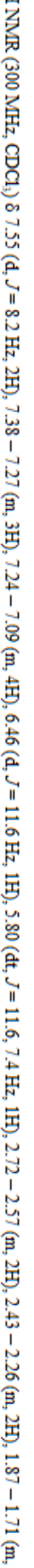




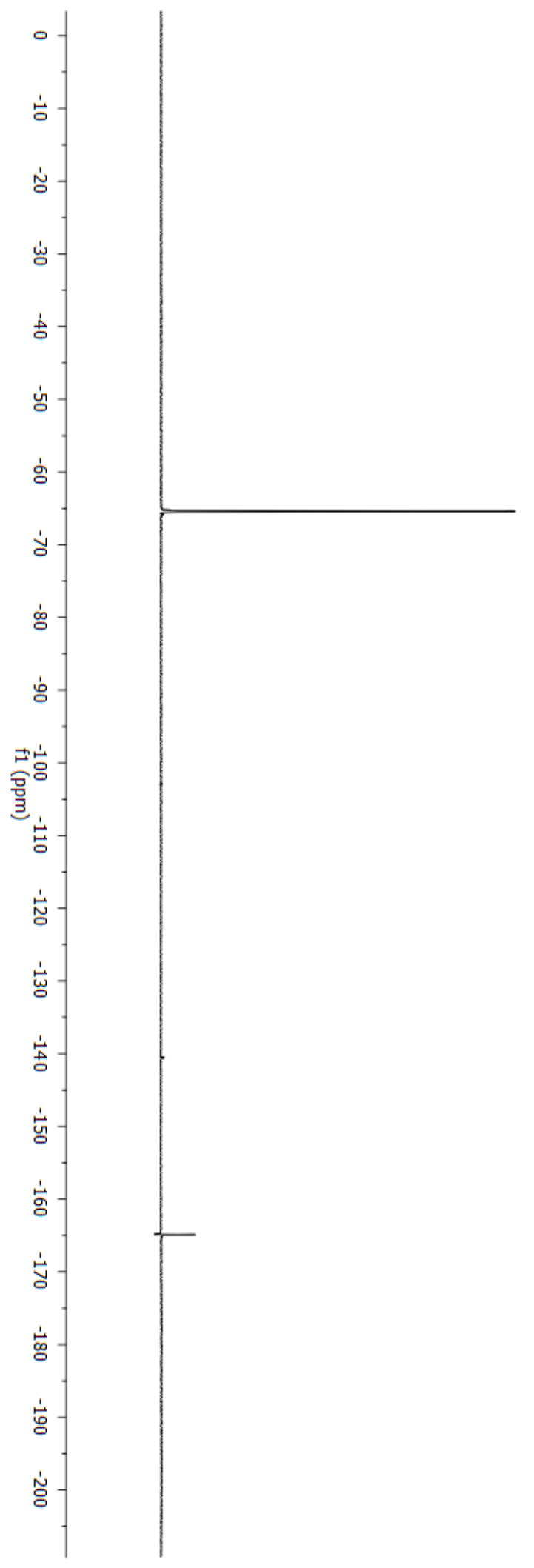

${ }^{-65.4}$ 

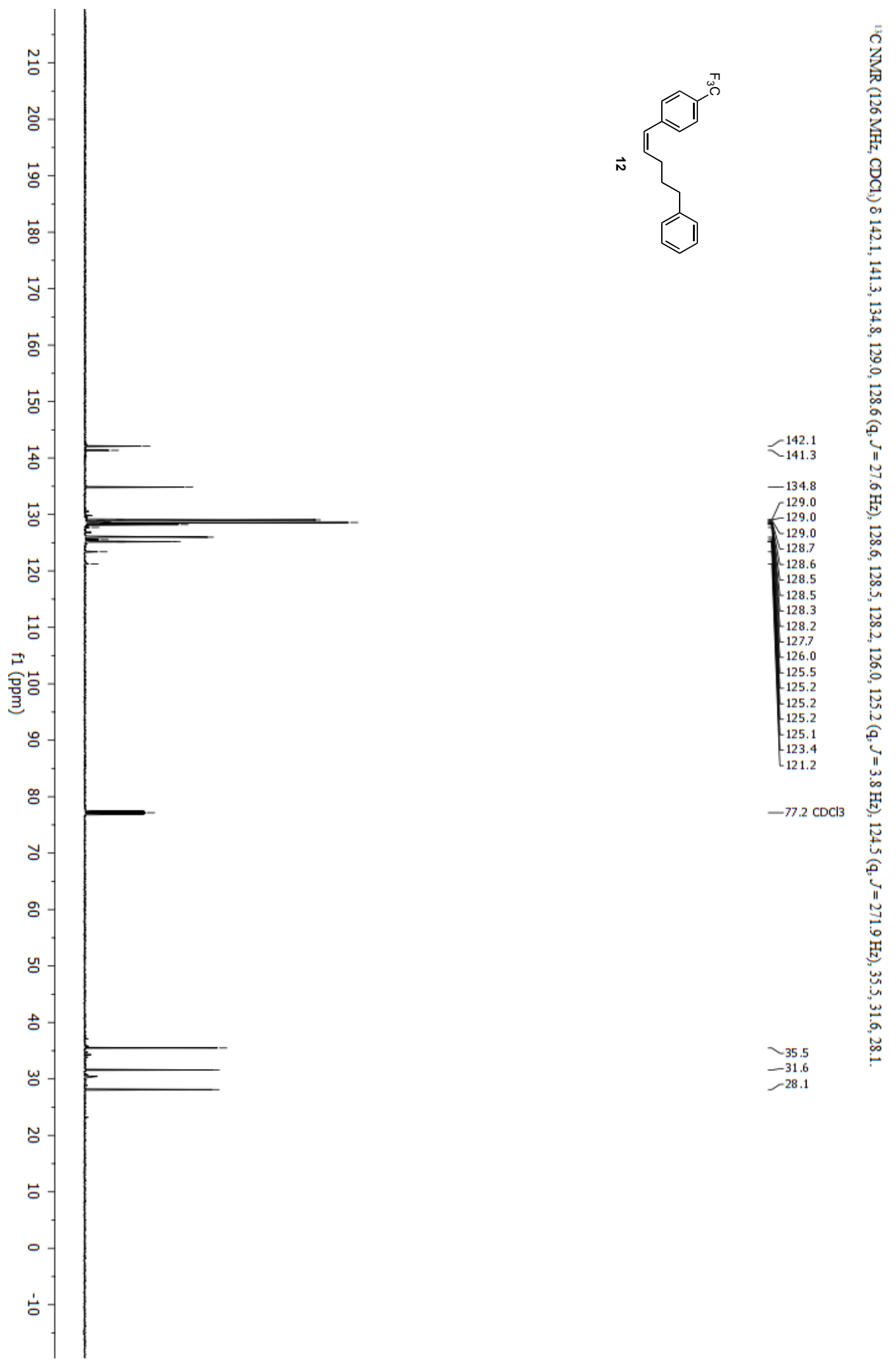


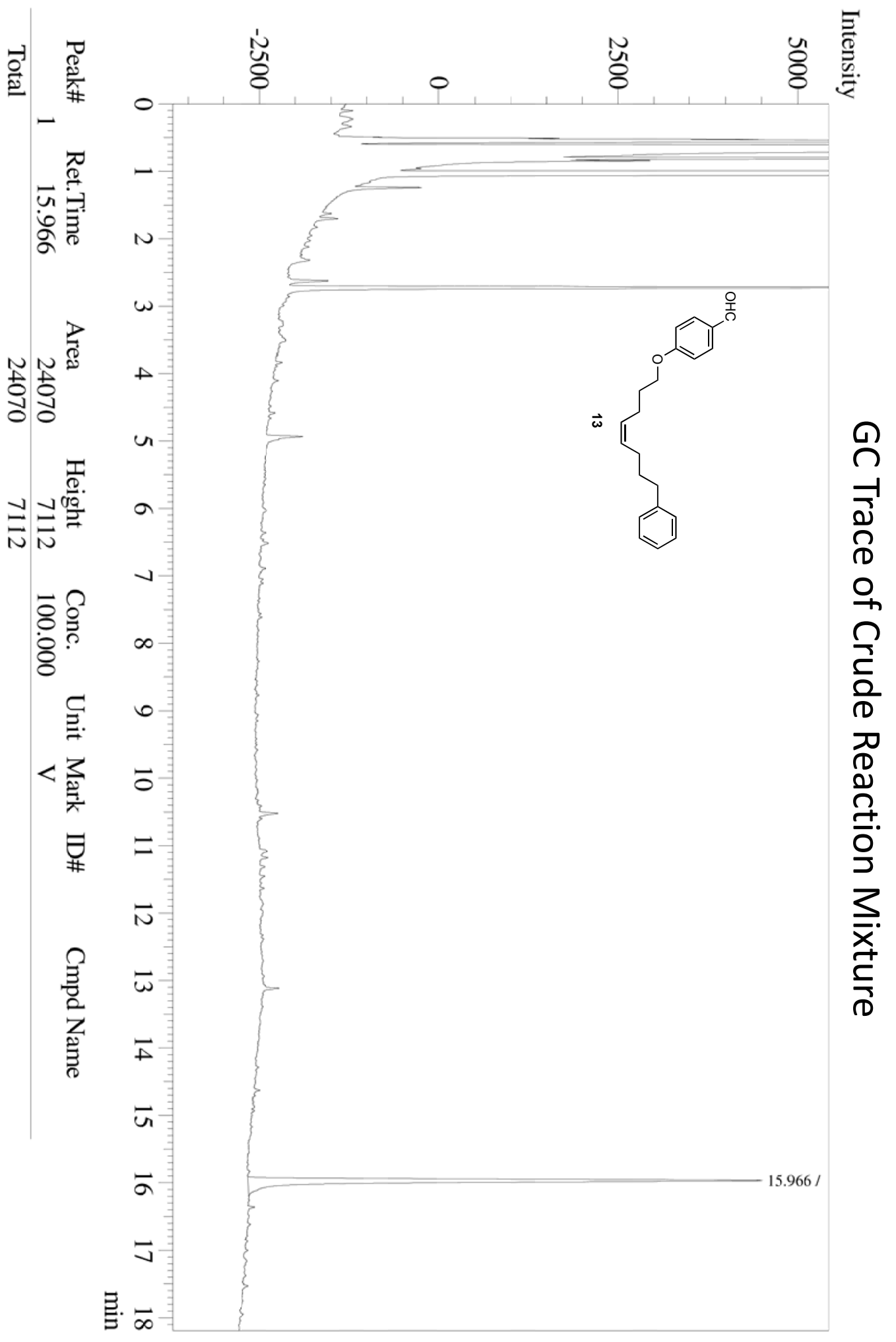



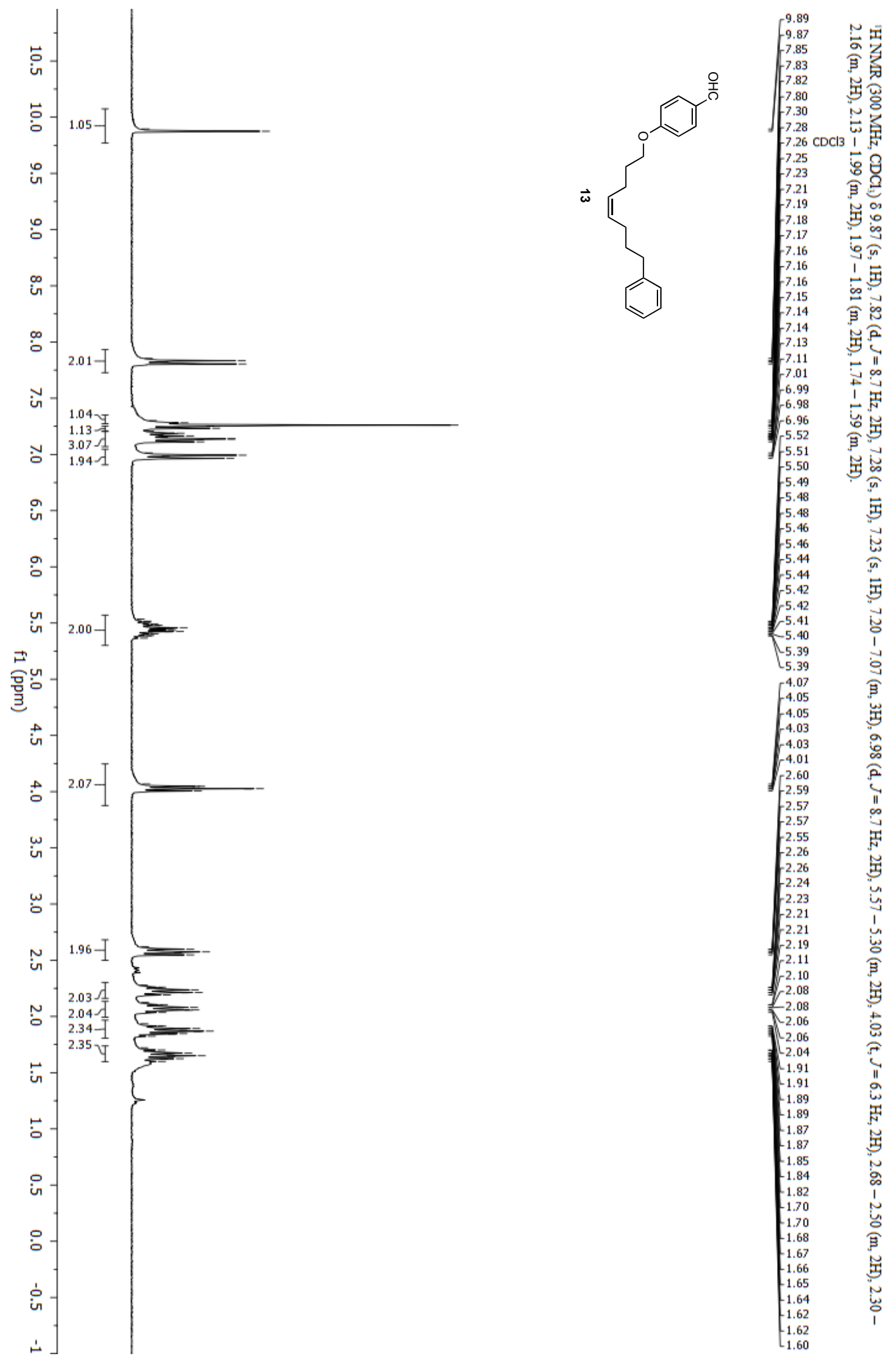

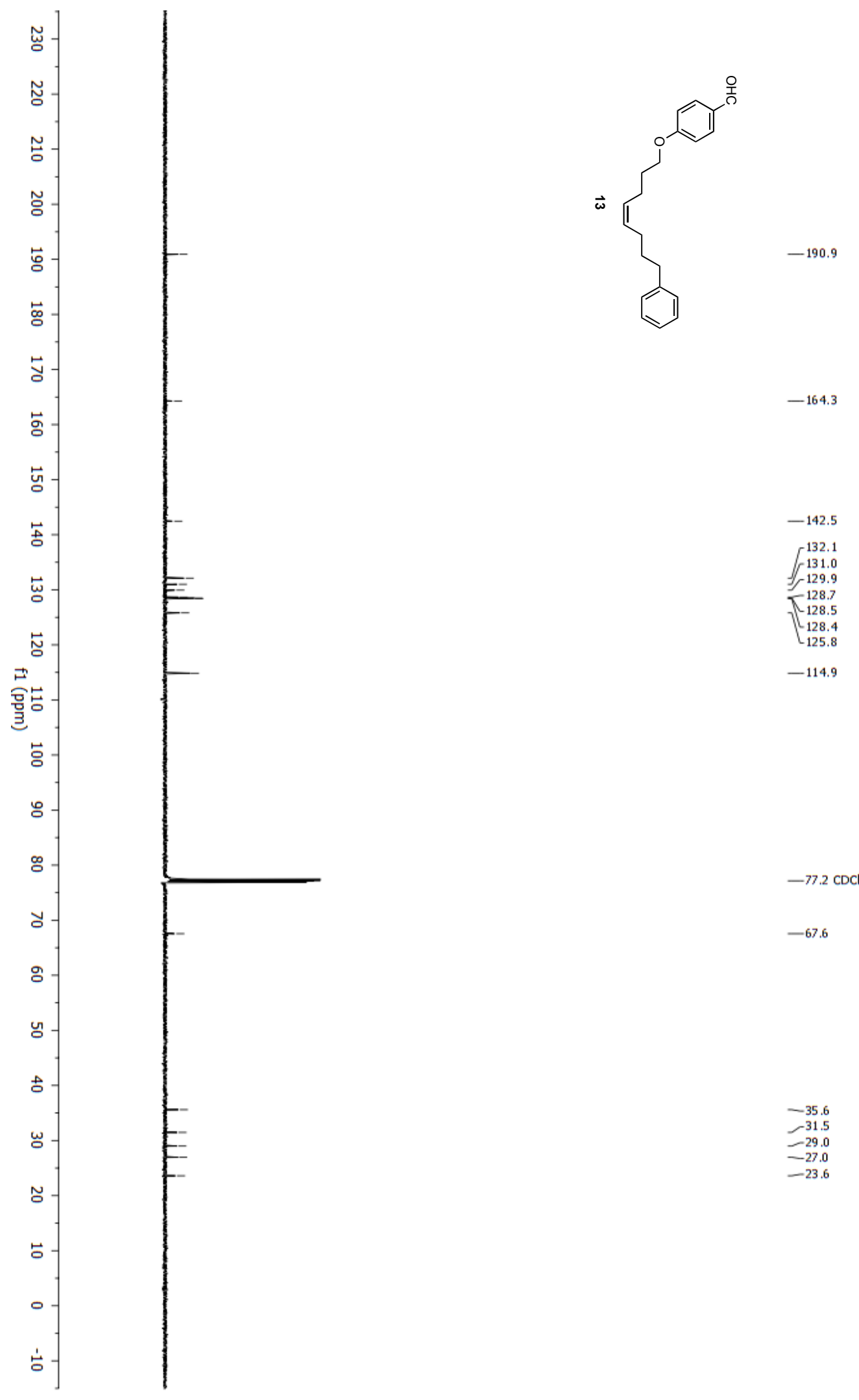

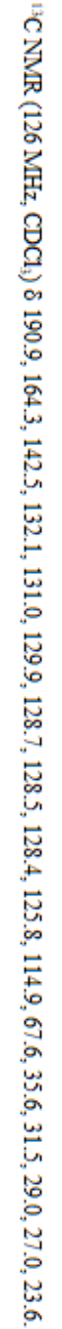

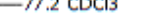

$-67.6$

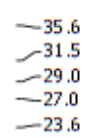




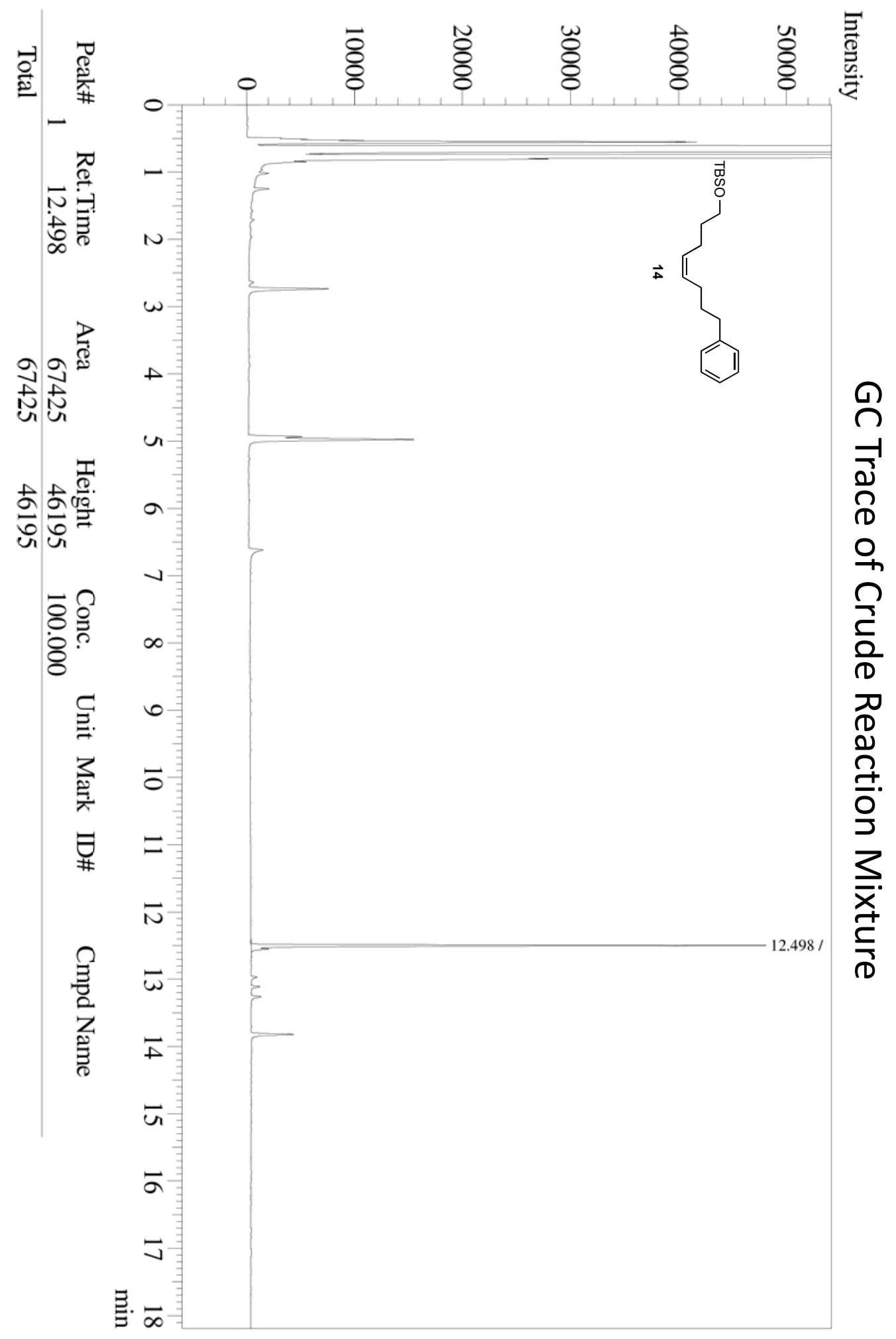




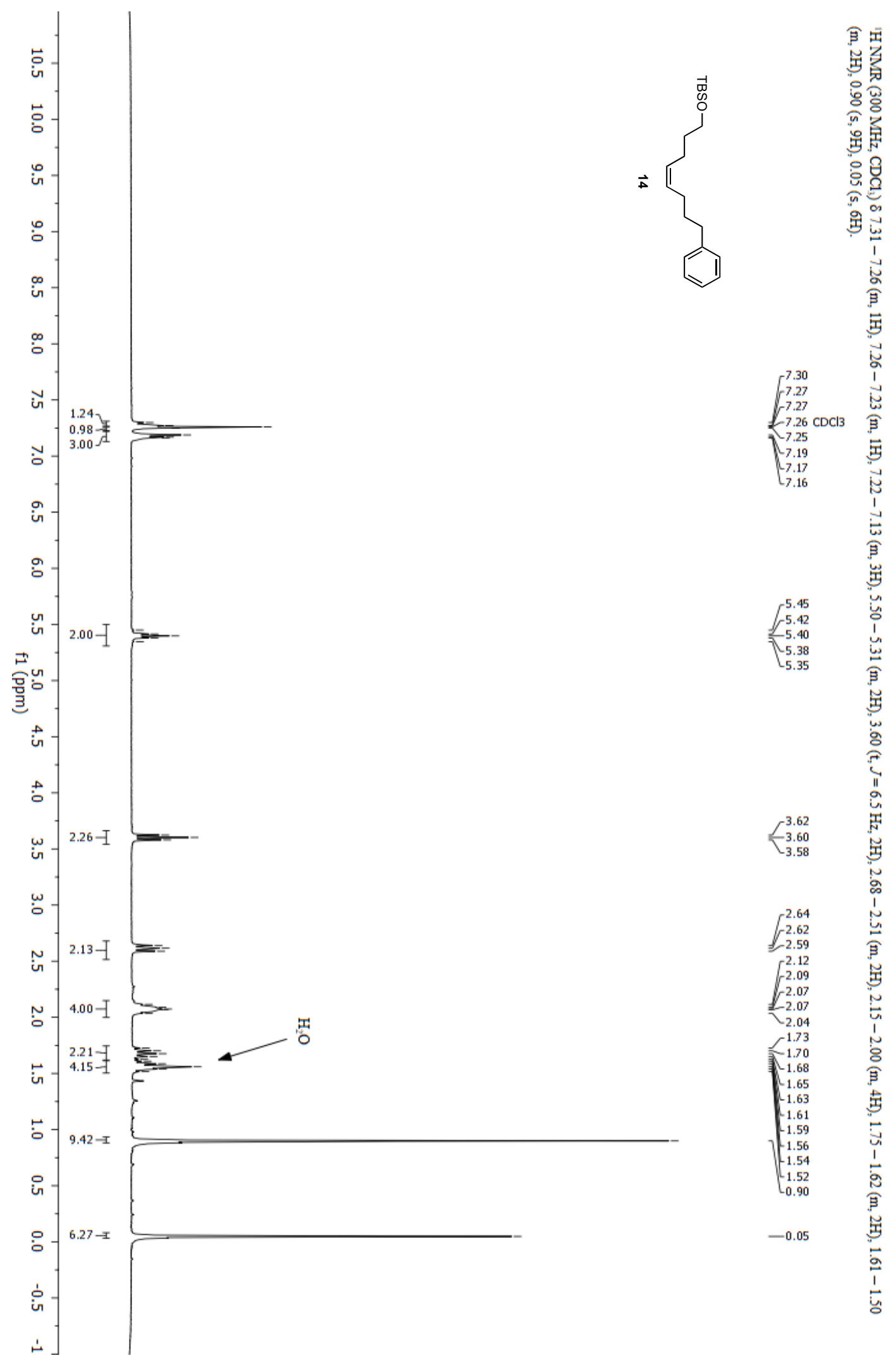



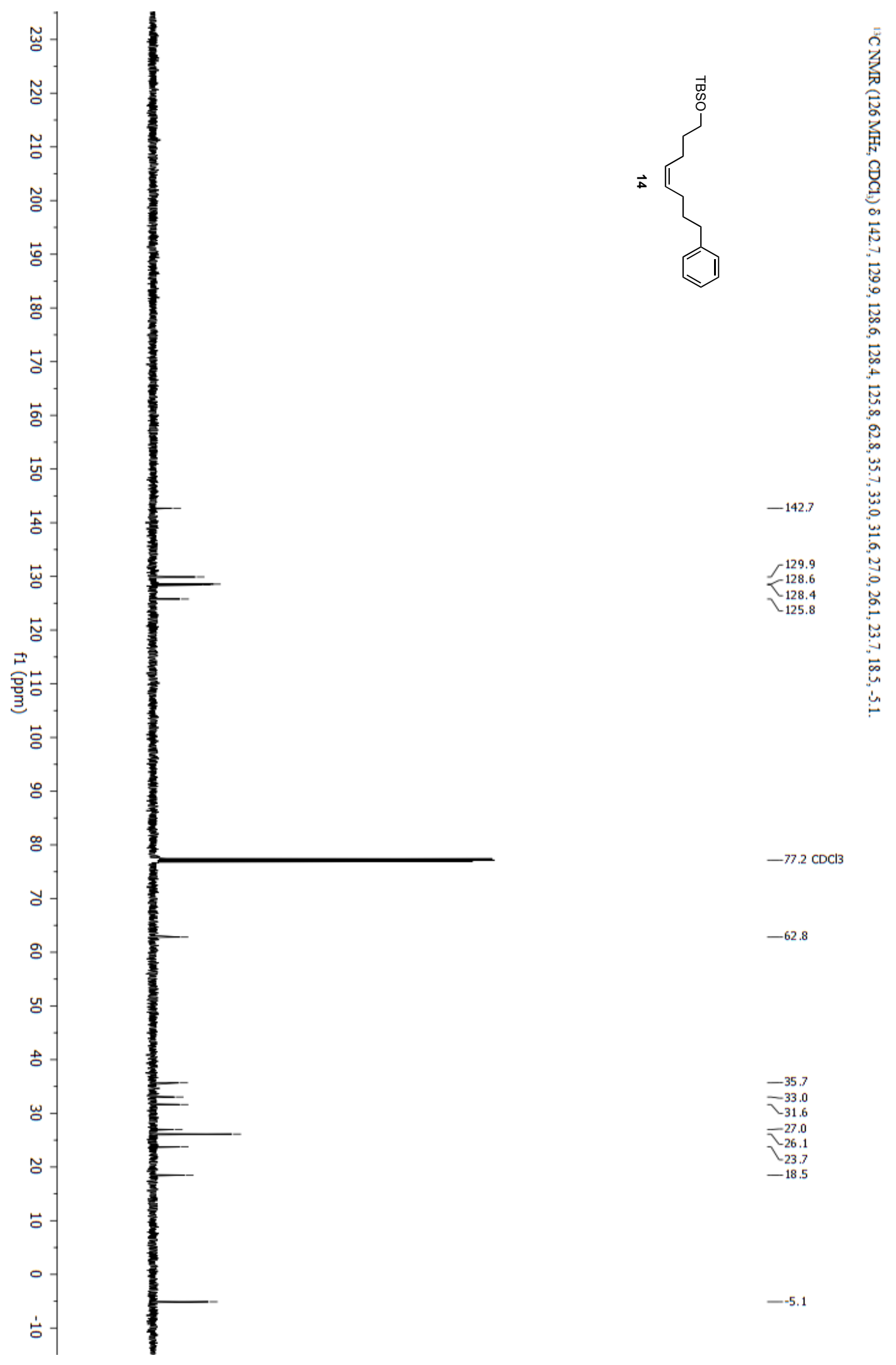

$-77.2 \mathrm{CDCl} 3$

$-62.8$

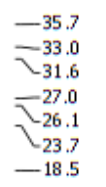

$--5.1$ 


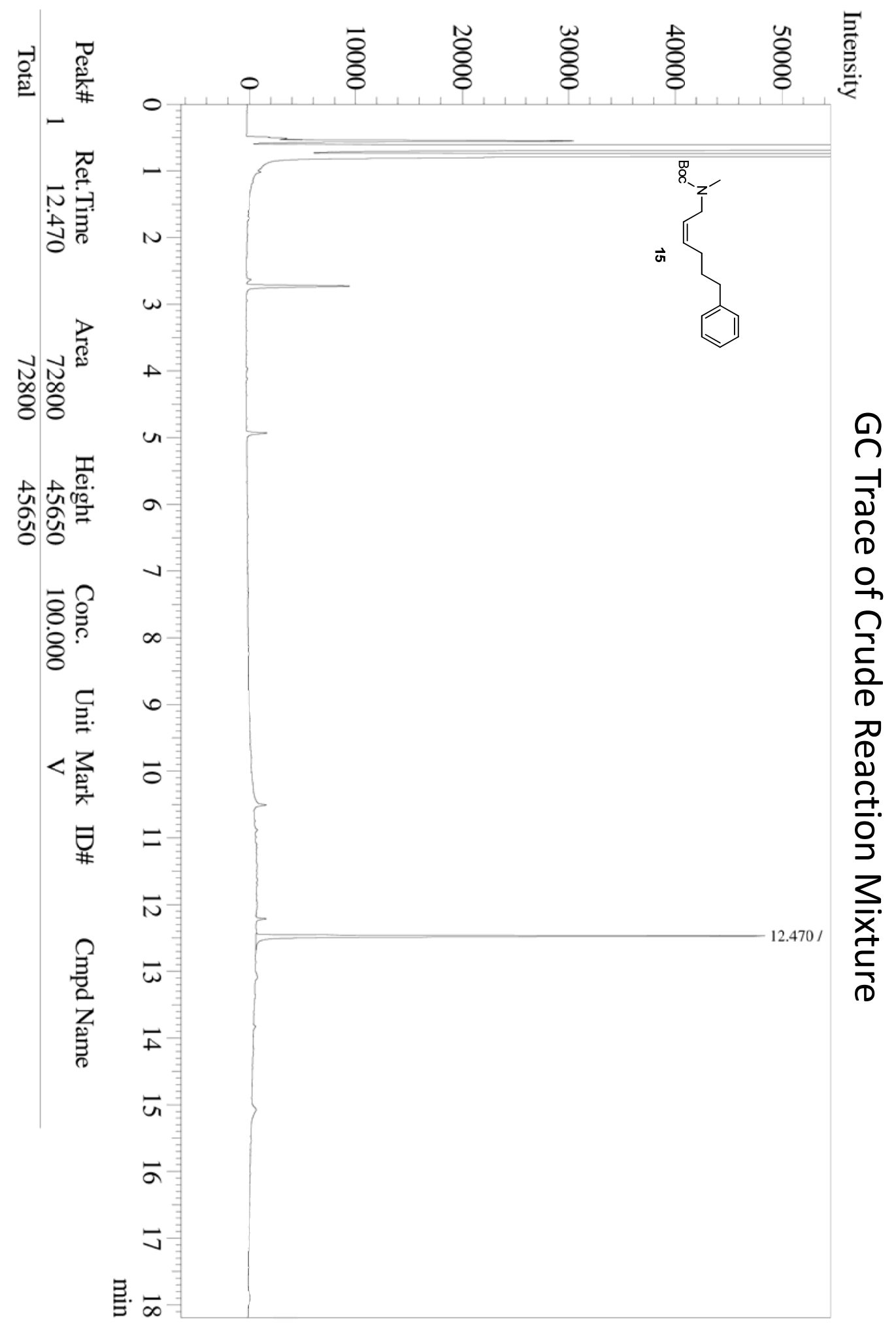




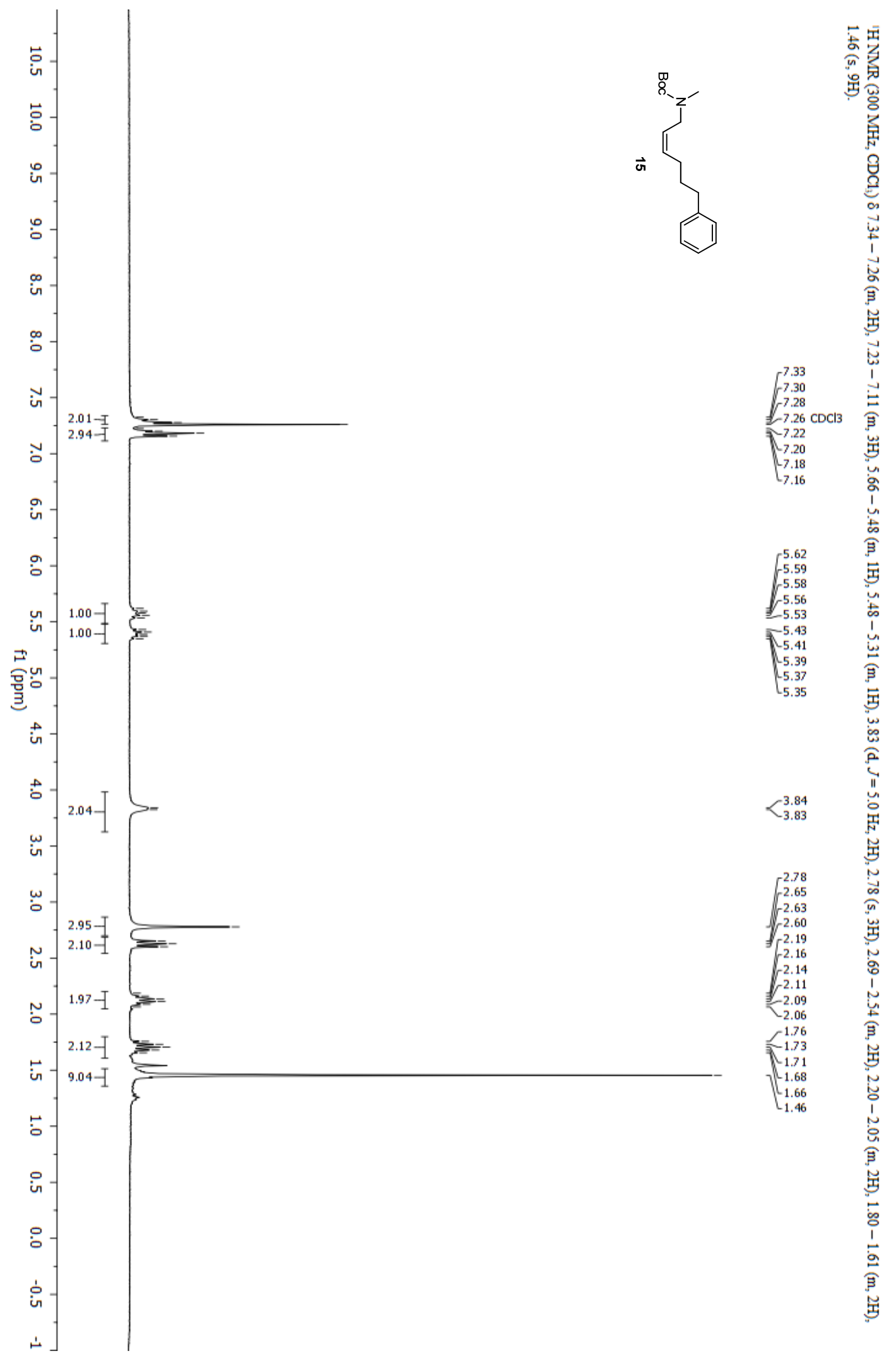




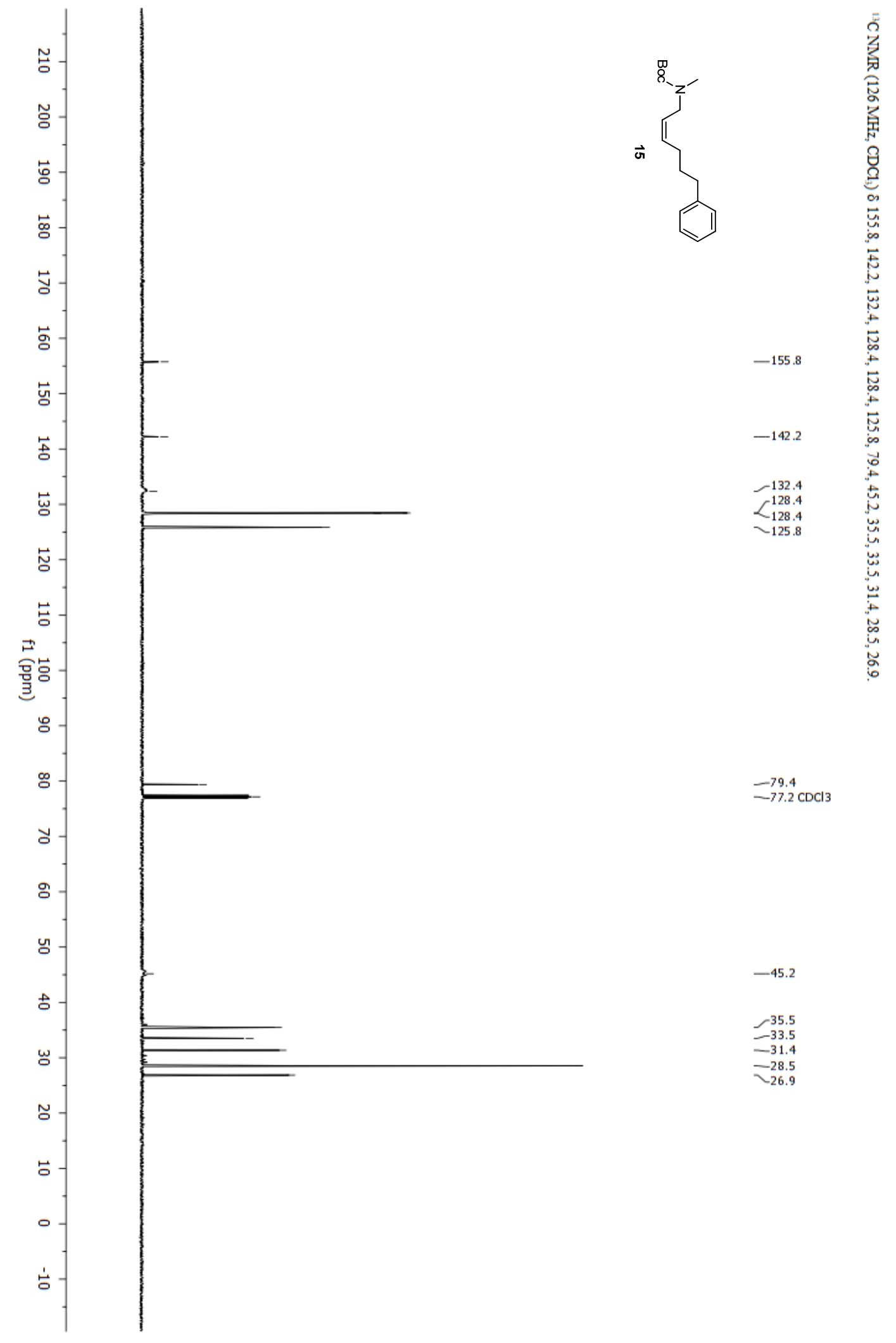




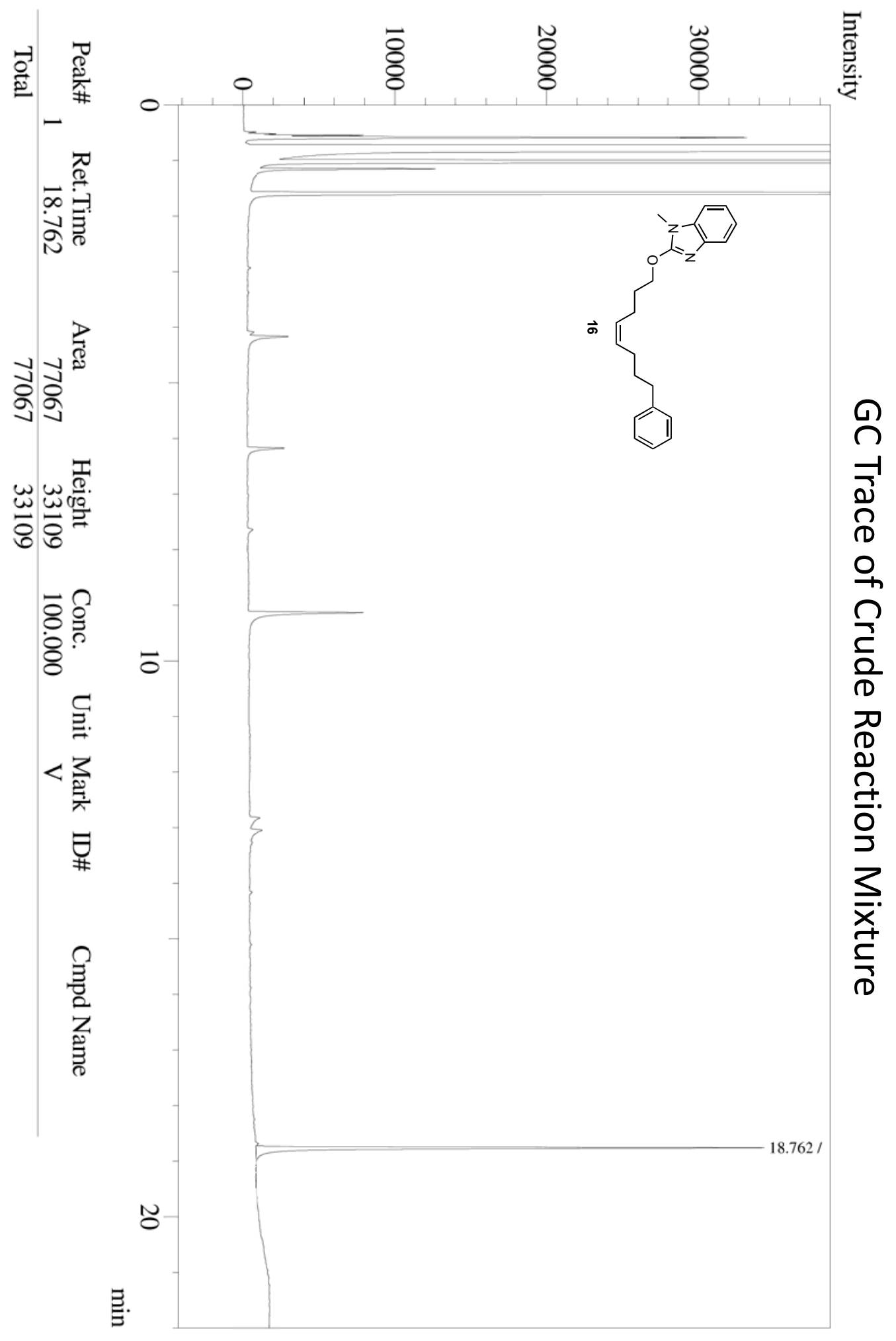



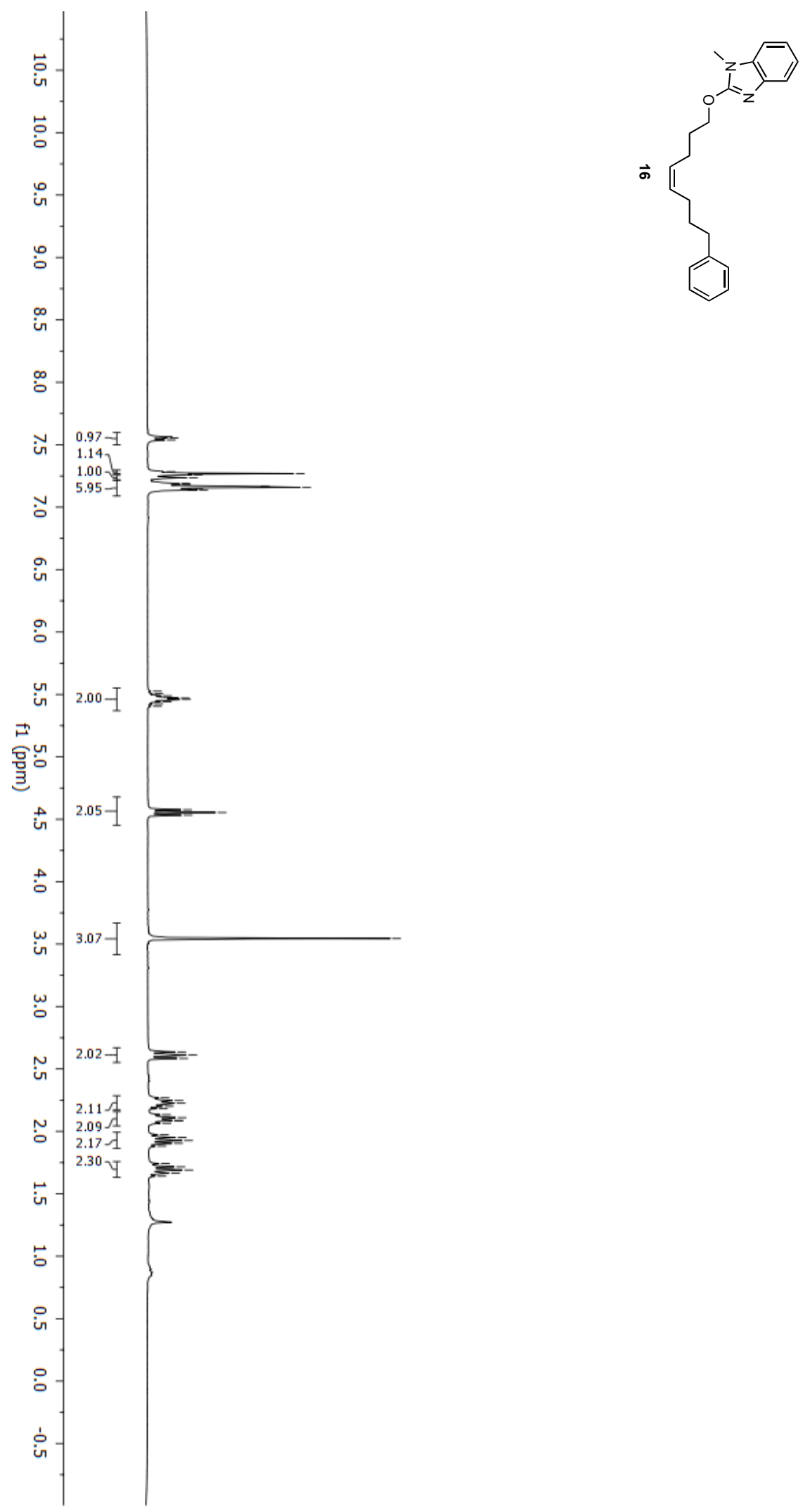

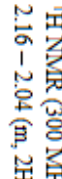

受最

$\div 8$

包

争

前

응

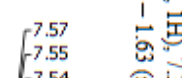

$\int \frac{7.28}{7.27}$

$7.26 \mathrm{CDCl}^{3}$ ?

7.18 苟

7.17

$-7.15$

5.53
5.51
5.49
5.47
5.46
5.44
5.42
5.41

$\left\{_{-4.55}^{4.58}\right.$

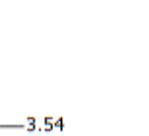

$-3.54$

$\mathcal{f}_{2.61}^{2.64}$

2.58
-2.27

-2.25
-2.22

2.20 in

$-2.13$

$-2.11$

$-2.09$

- 9

$\begin{array}{ll}-2.07 & 1 \\ -1.97 & \quad\end{array}$

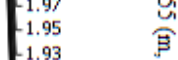

-1.90 年

1.88

-1.74
-1.72

$\begin{array}{rr}-1.72 & 1 \\ -1.69 & \vdots \\ -1.67 & \vdots\end{array}$

1.64

苟 

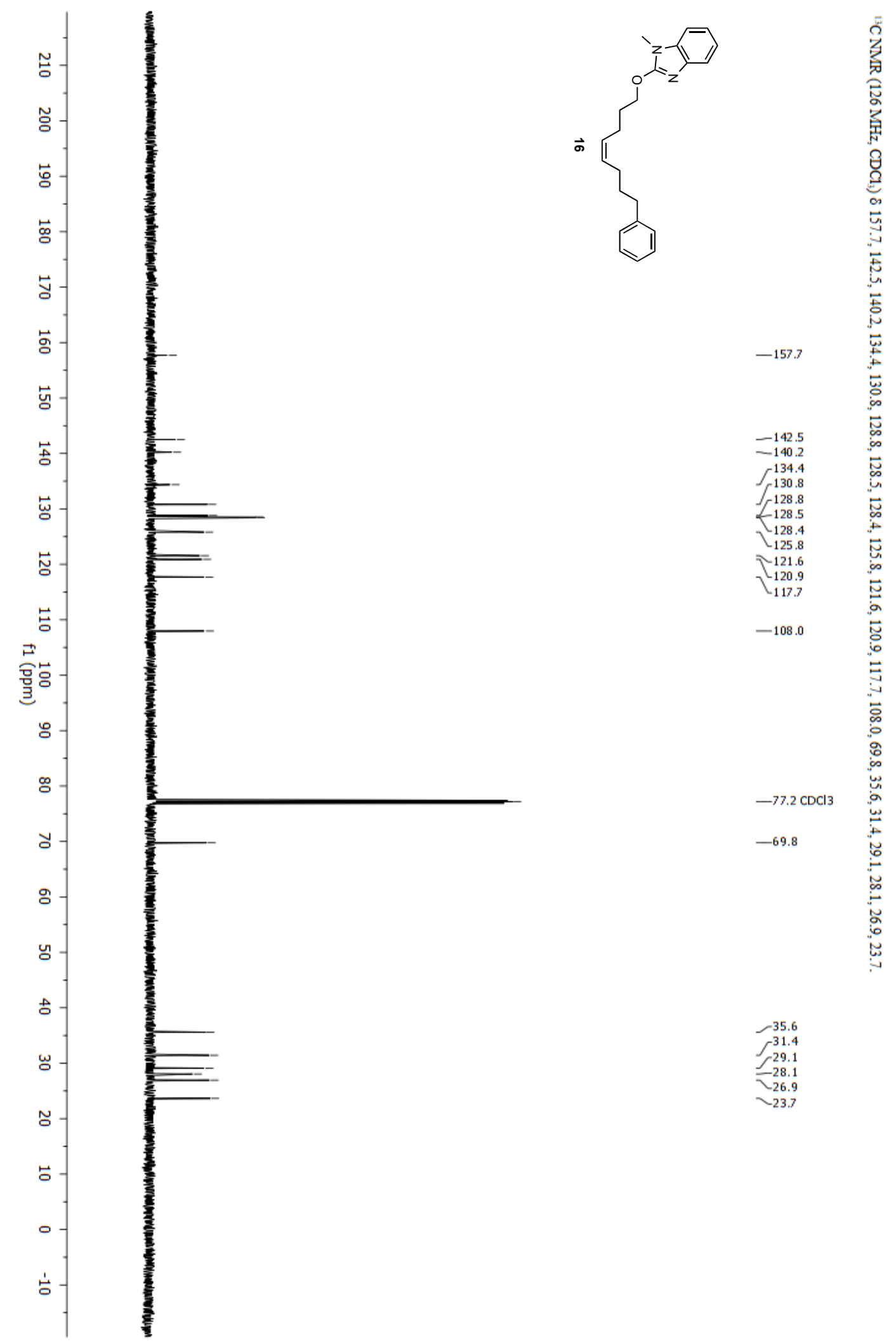


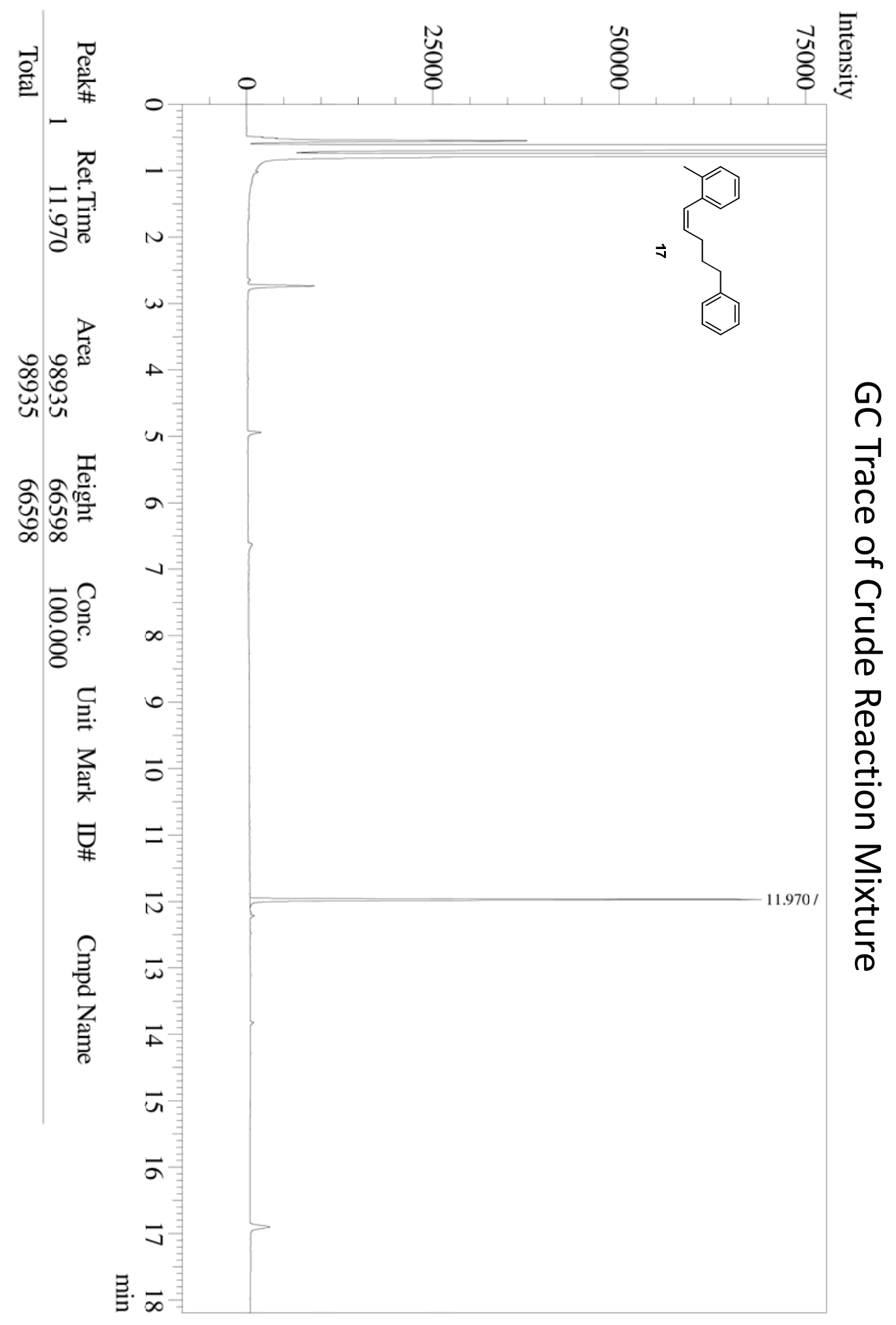



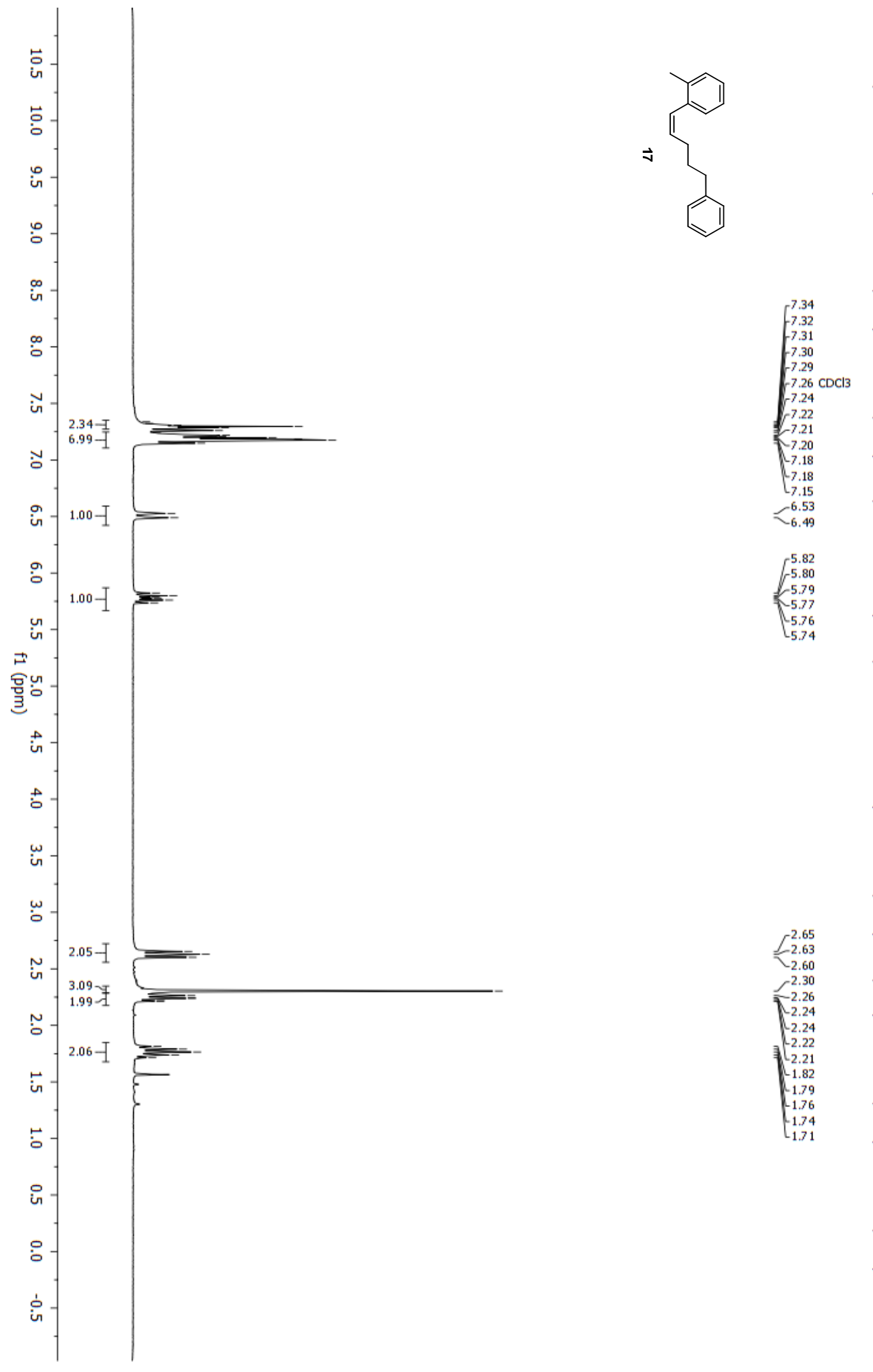


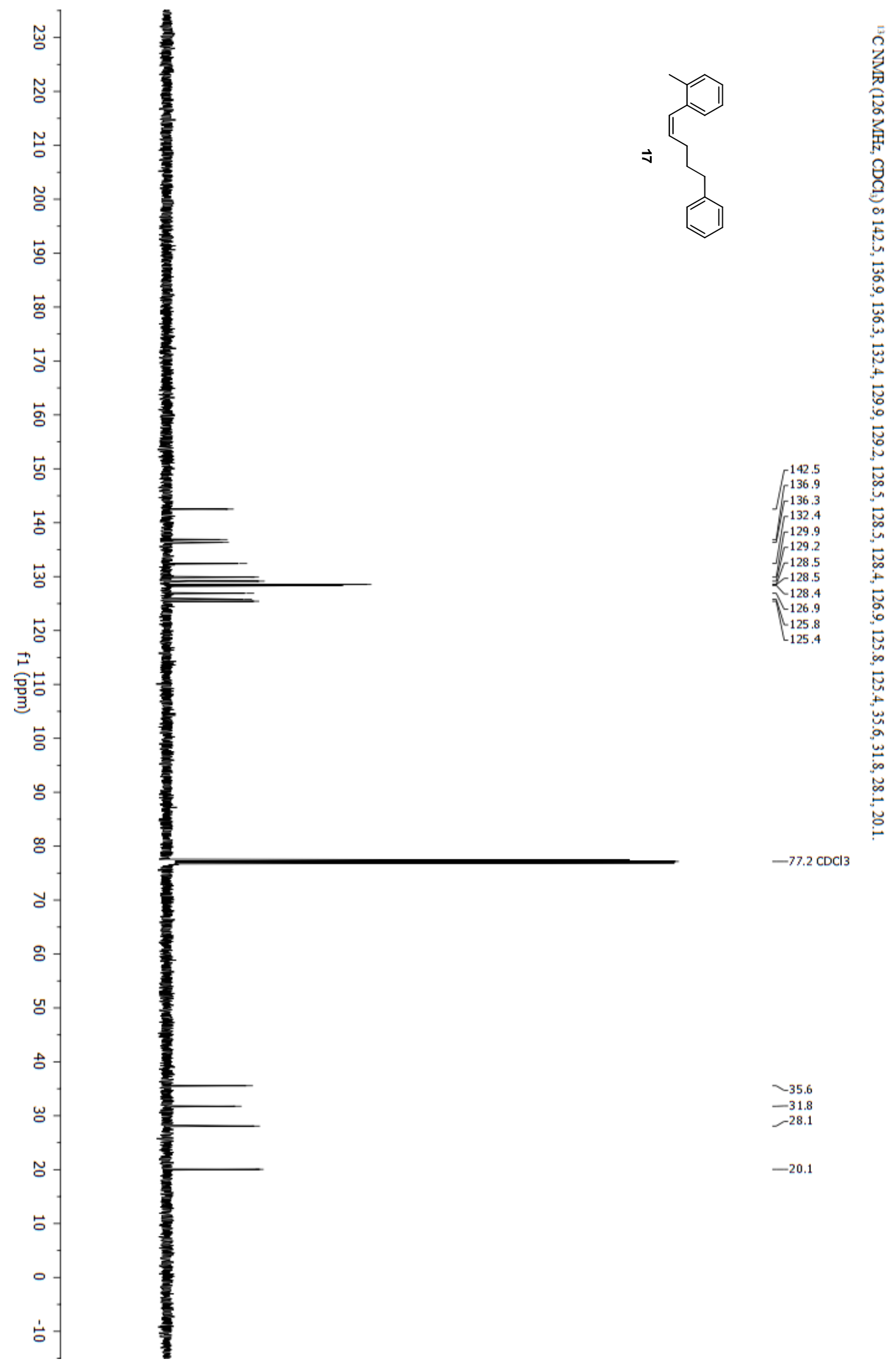




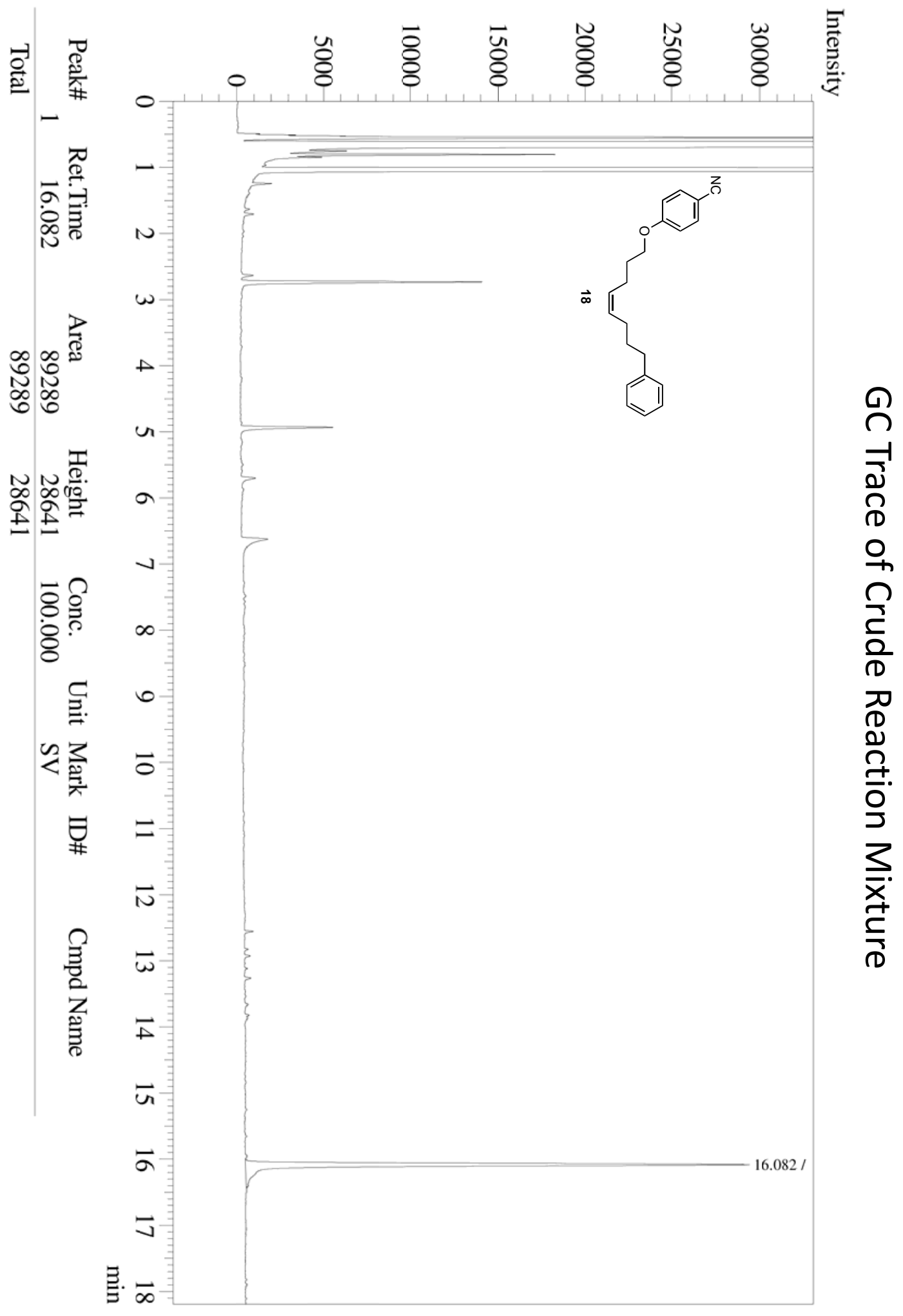



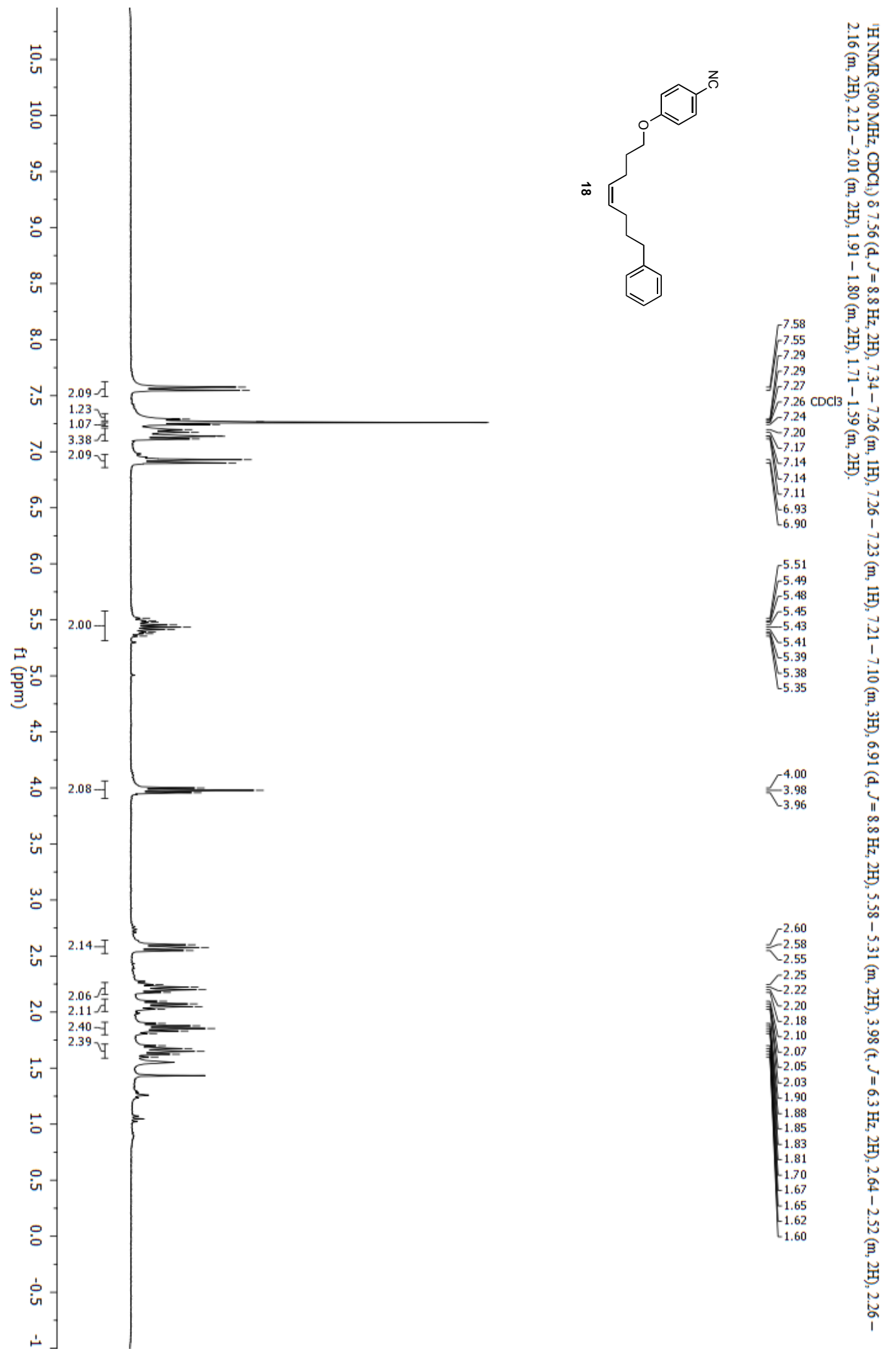

$\begin{cases}7.55 \\ 7.29 \\ 7.29 \\ 7.27 \\ 7.26 \mathrm{cDCl} 13 \\ 7.24 \\ 7.20 \\ 7.17\end{cases}$

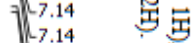

$\left[\begin{array}{ll}-7.11 \\ -6.93\end{array}\right.$

-6.93
6.90

$\left[\begin{array}{r}5.51 \\ 5.49 \\ 5.48 \\ 5 \\ 5.45 \\ 5.43 \\ 5.41 \\ 5.39 \\ 5.35 \\ 5.35\end{array}\right.$

贺

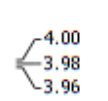

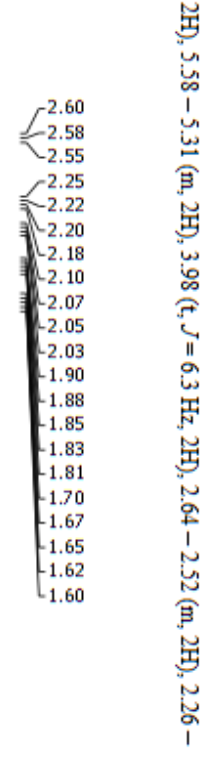

政 

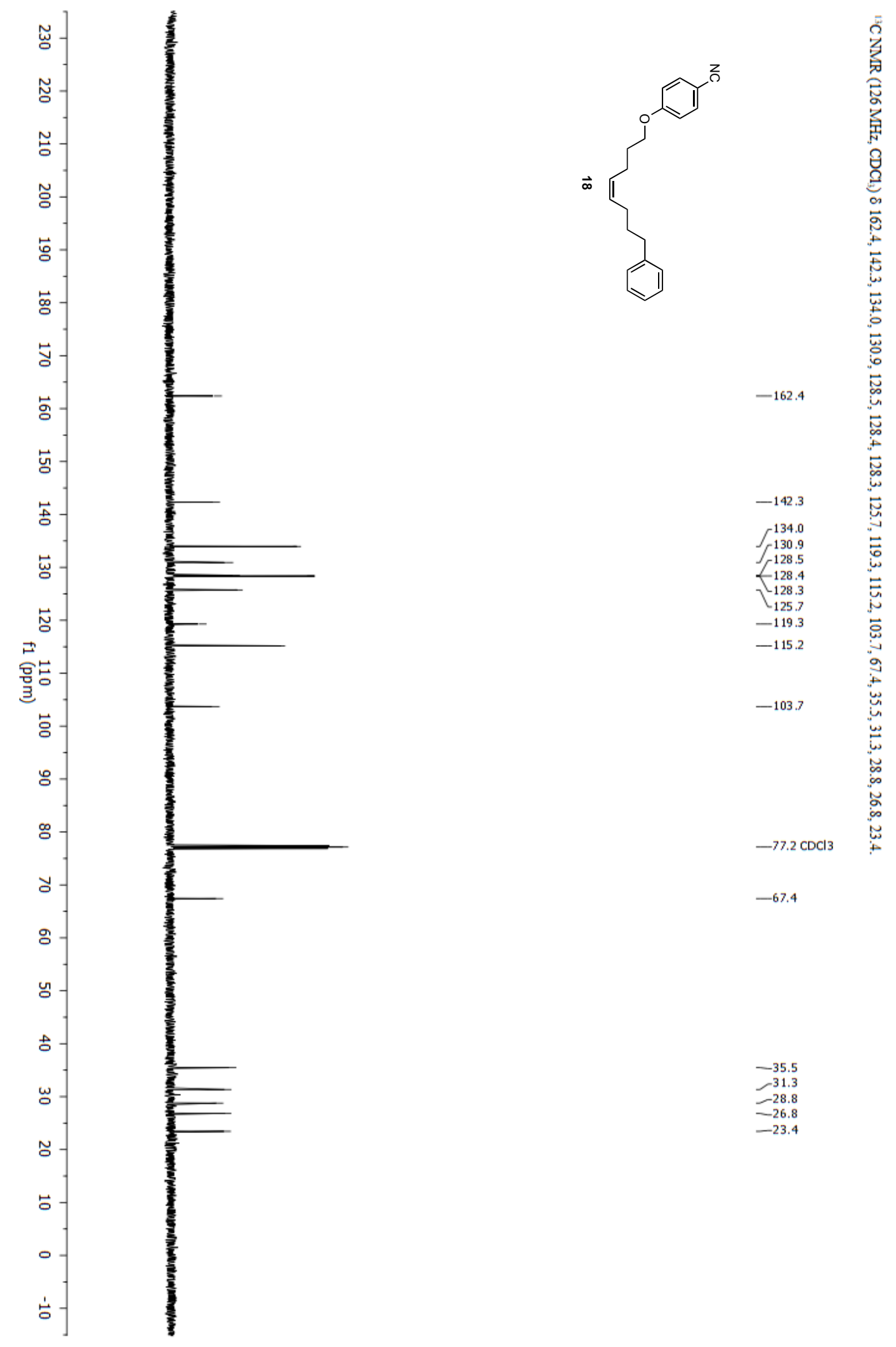


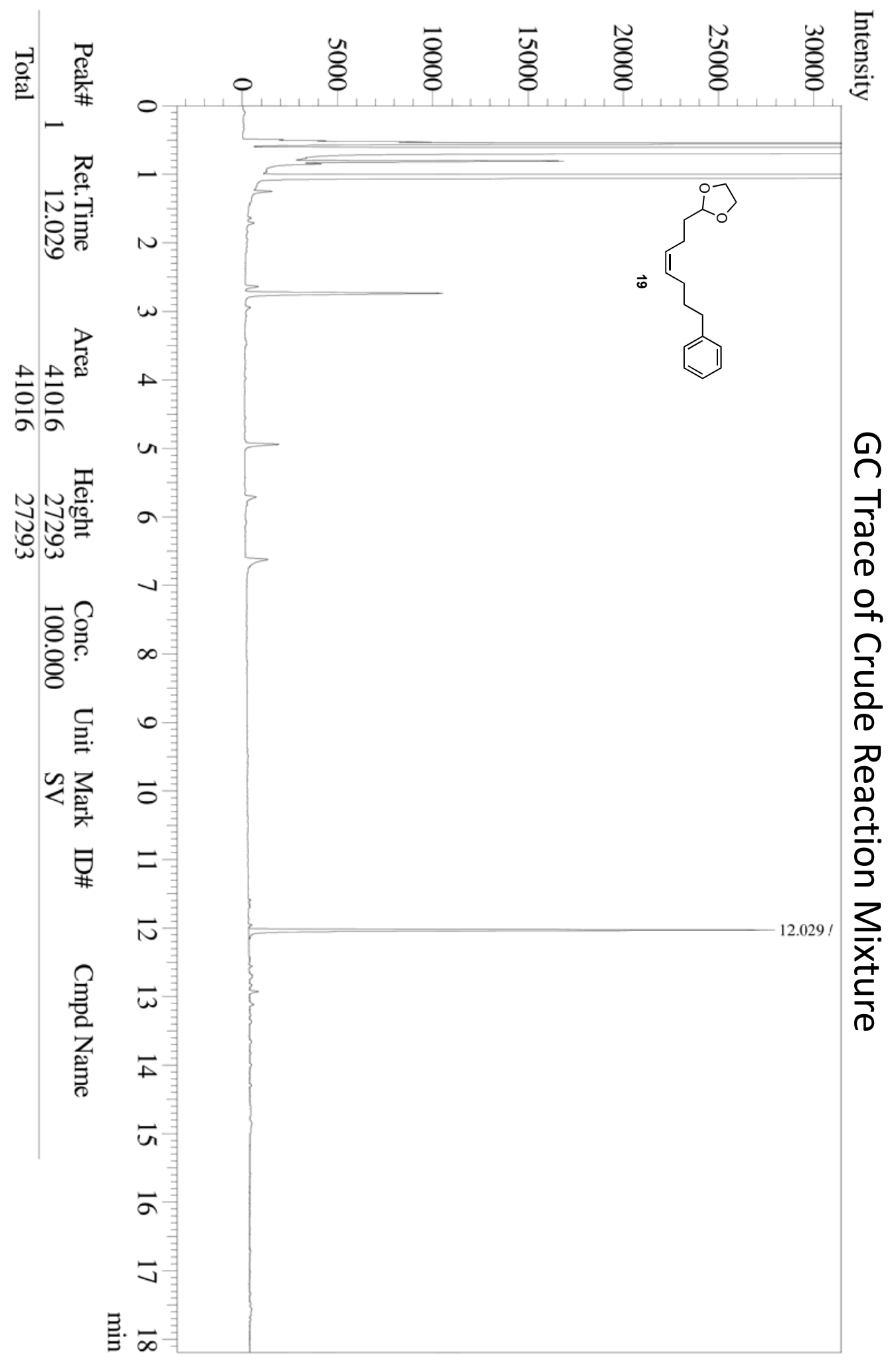



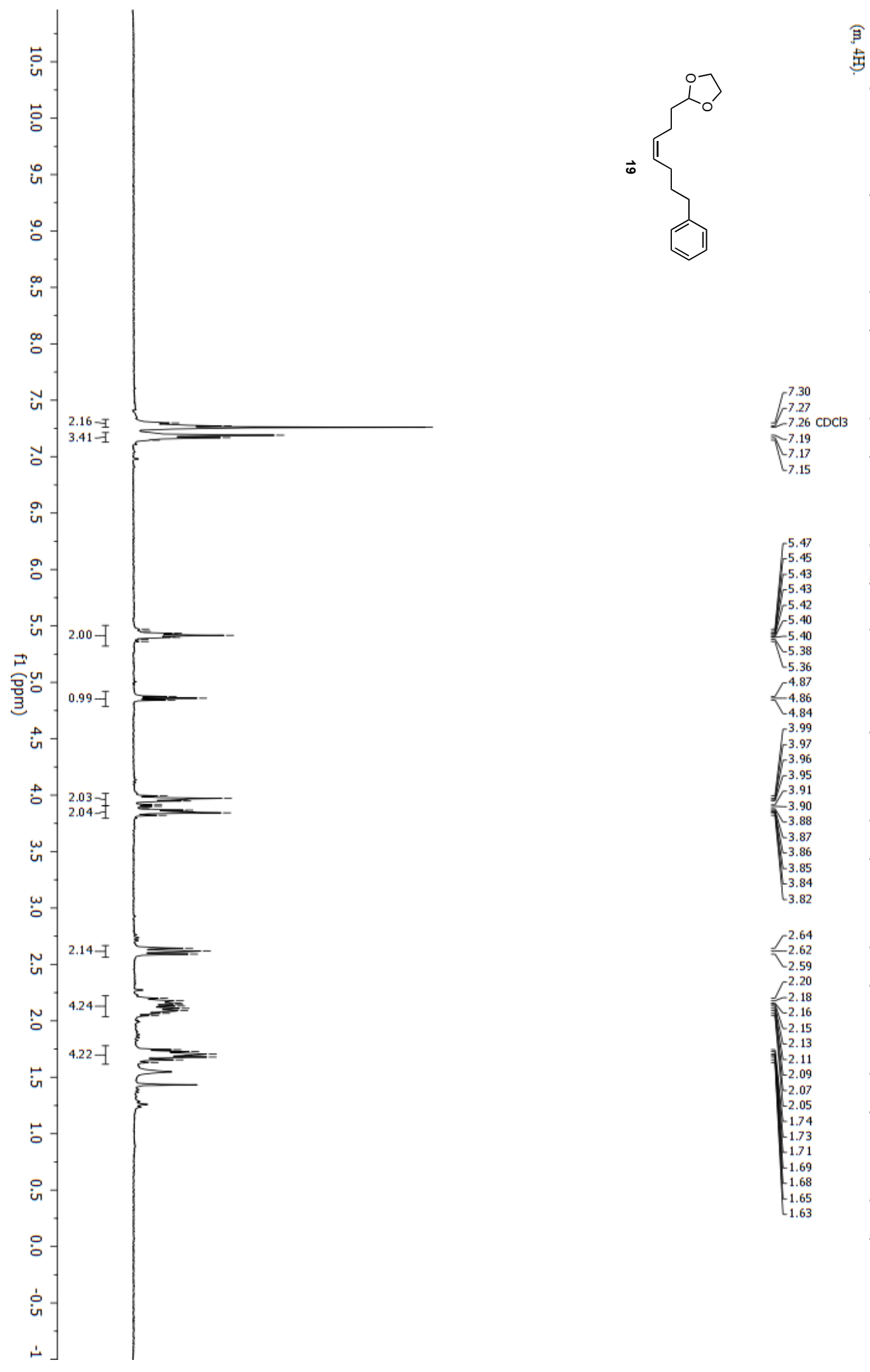

索穿

塄

考 

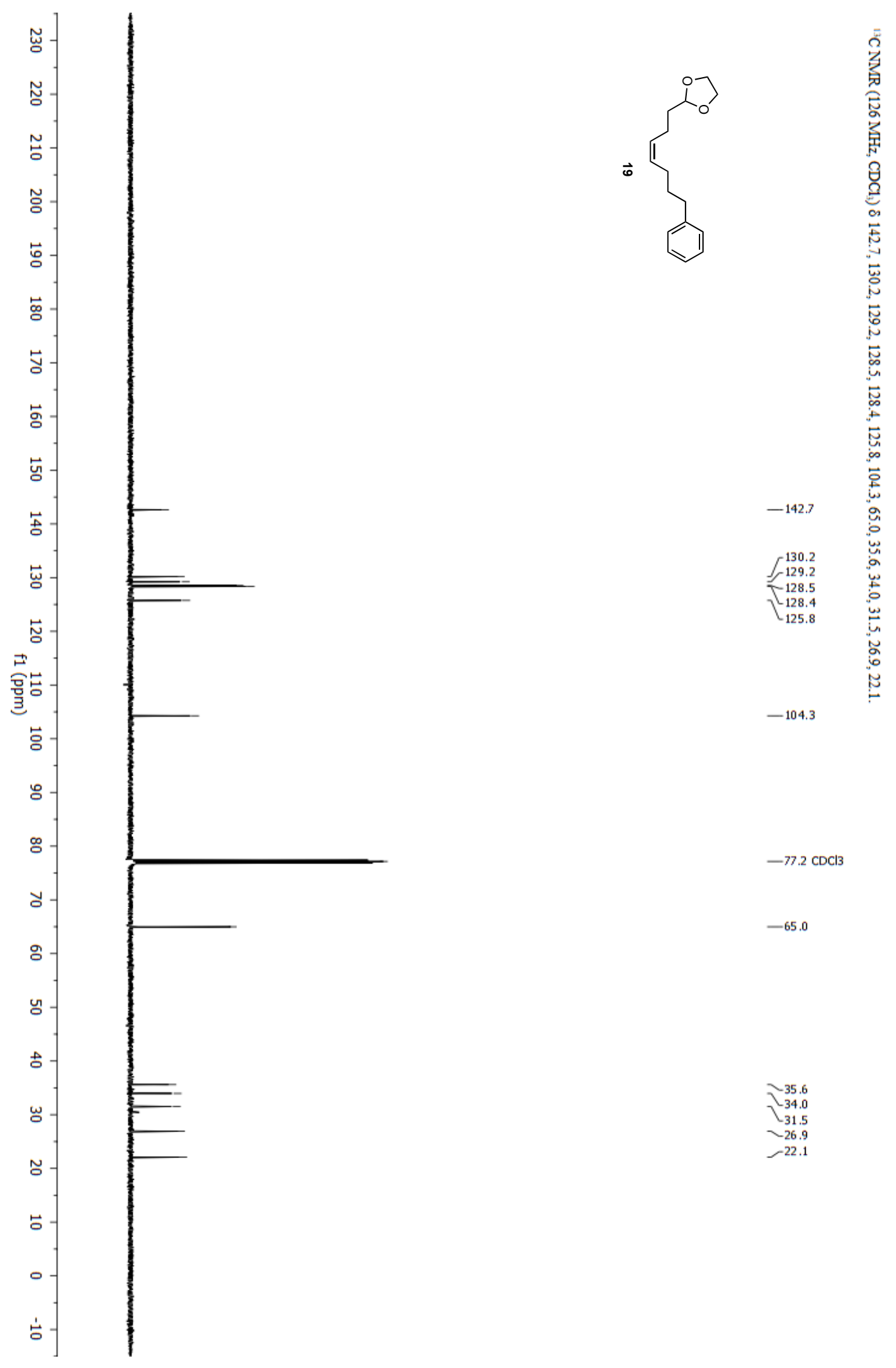

$-77.2 \mathrm{CDCl} 3$

$-65.0$

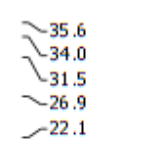




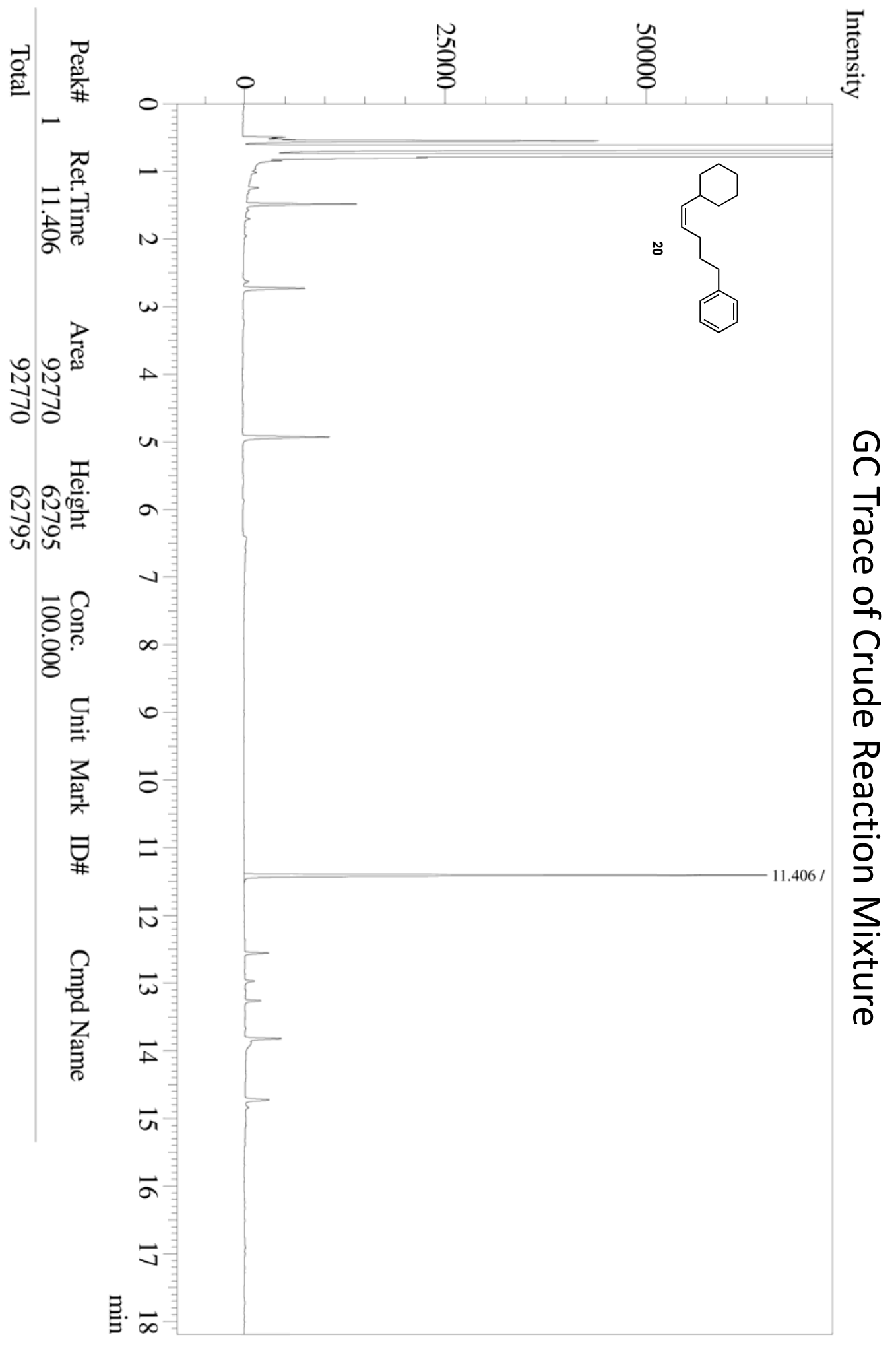



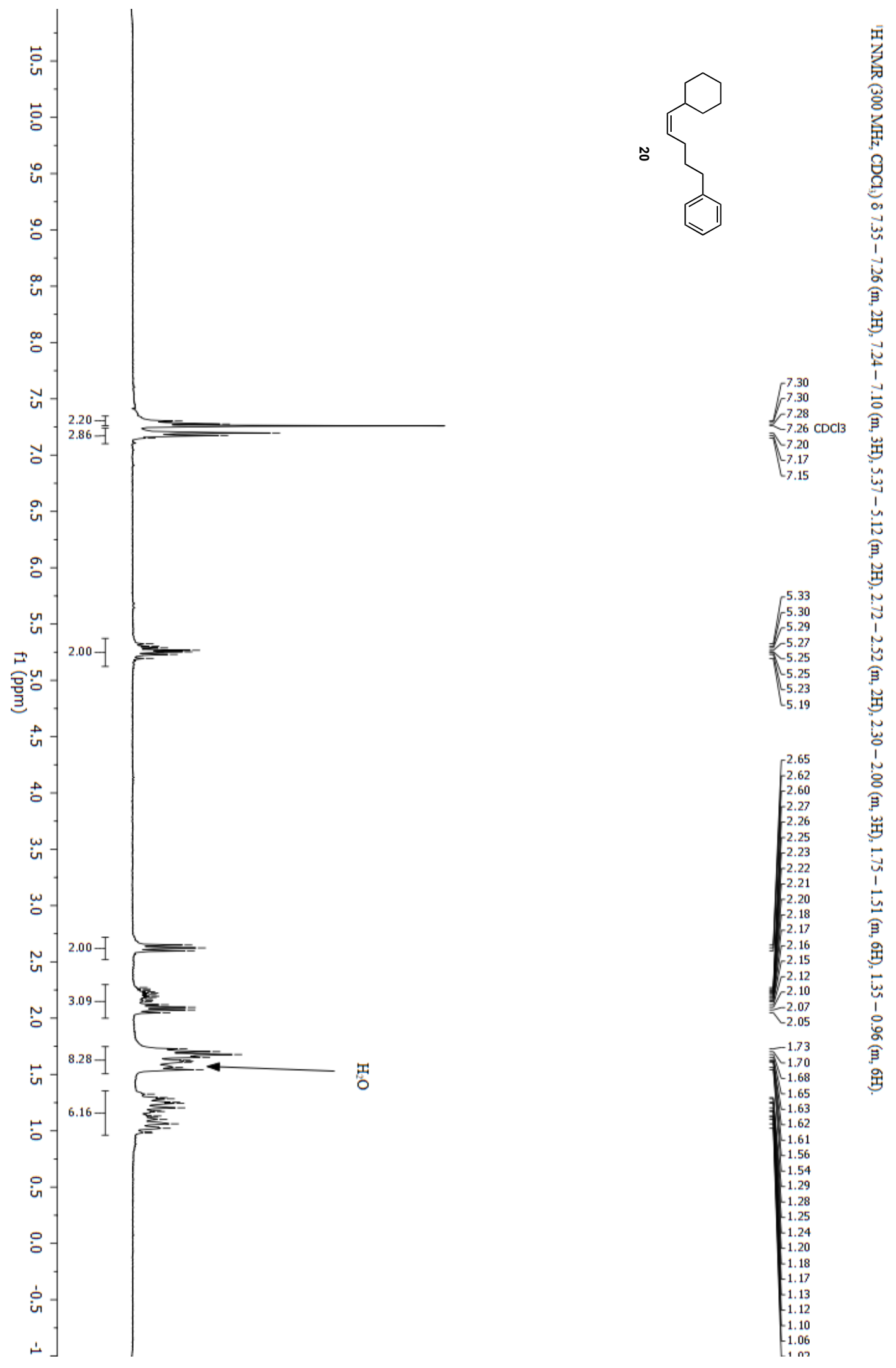

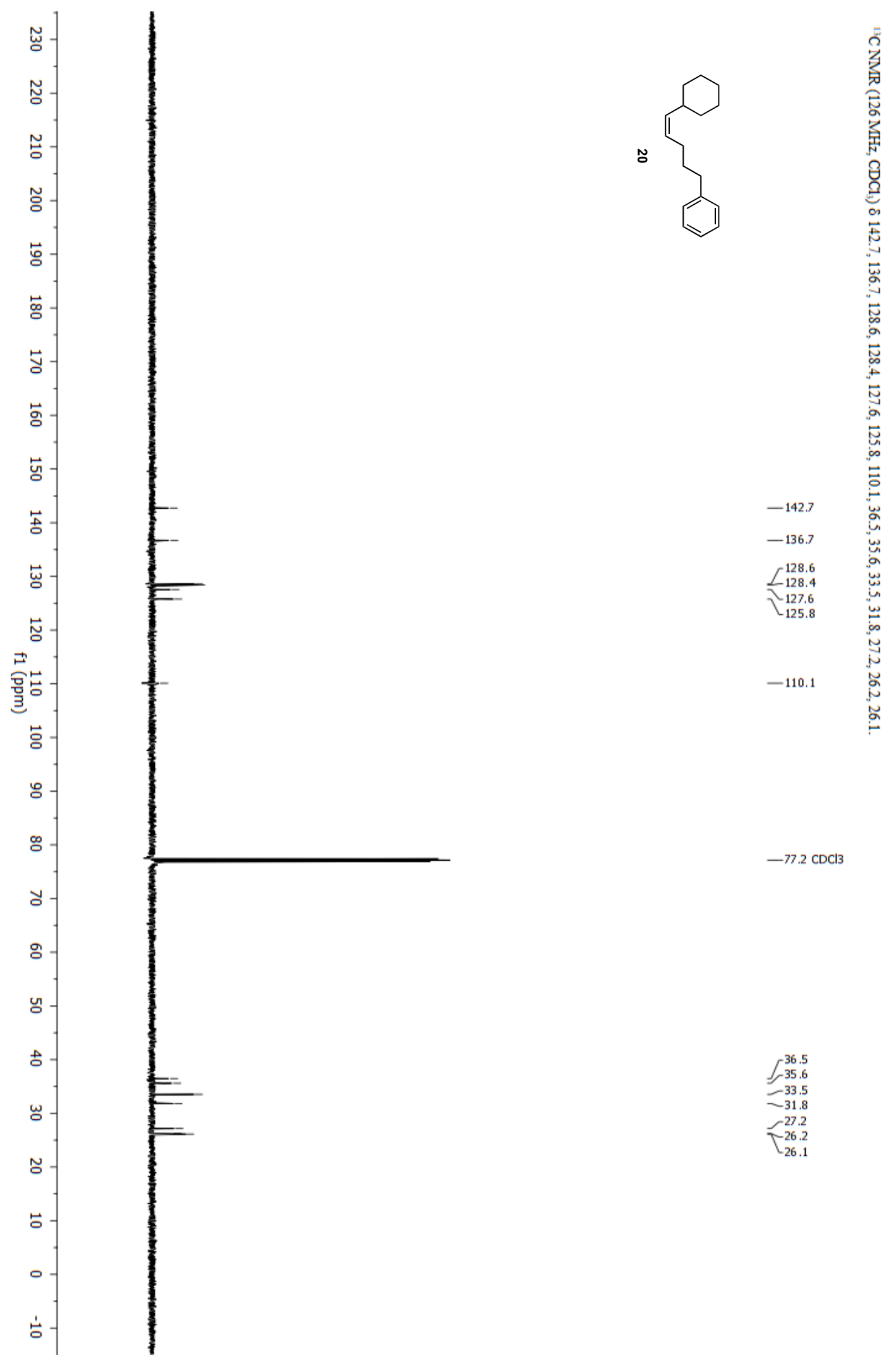

$-77.2 \mathrm{CDCl} 3$

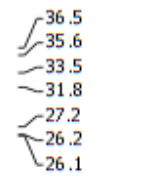




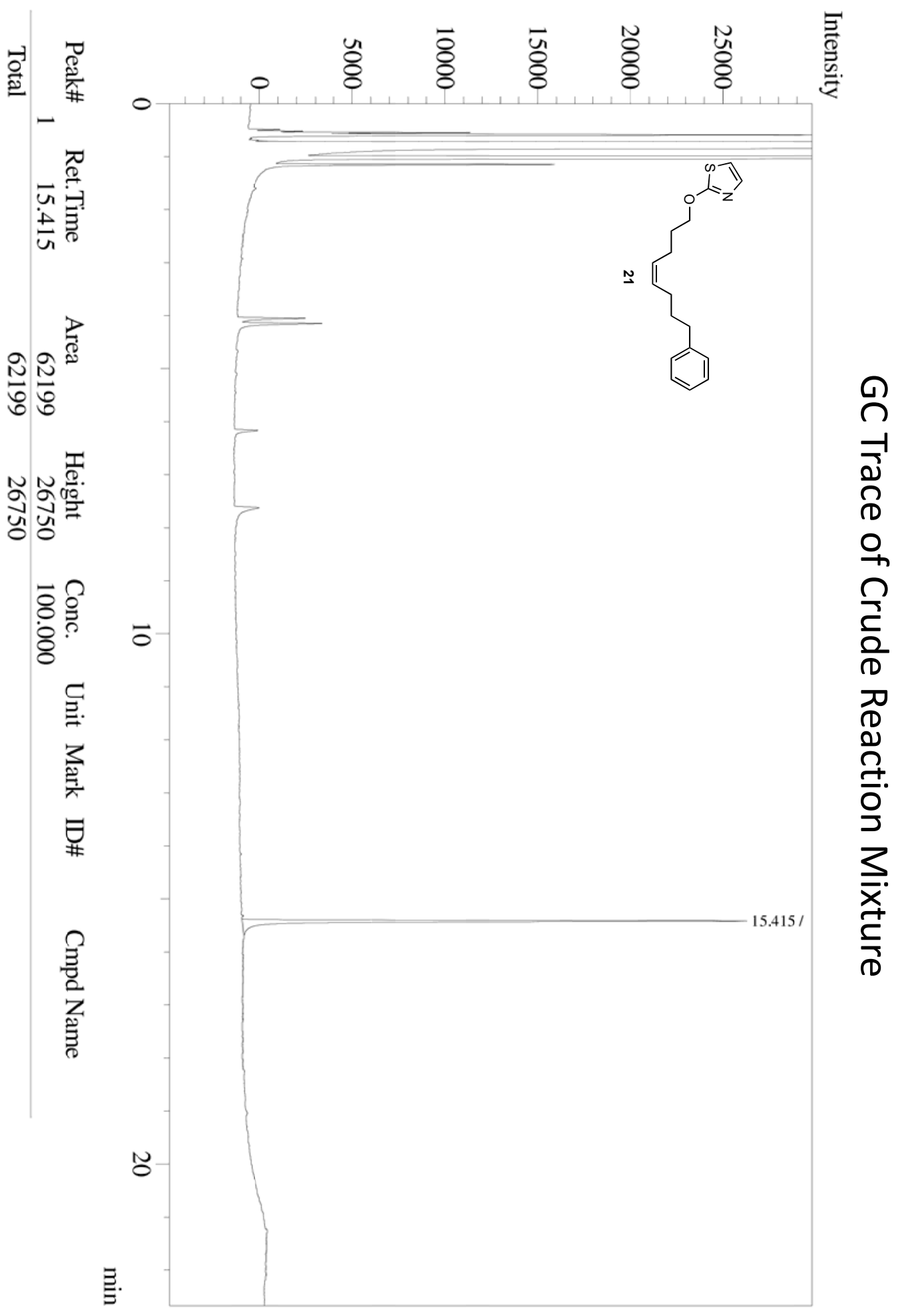



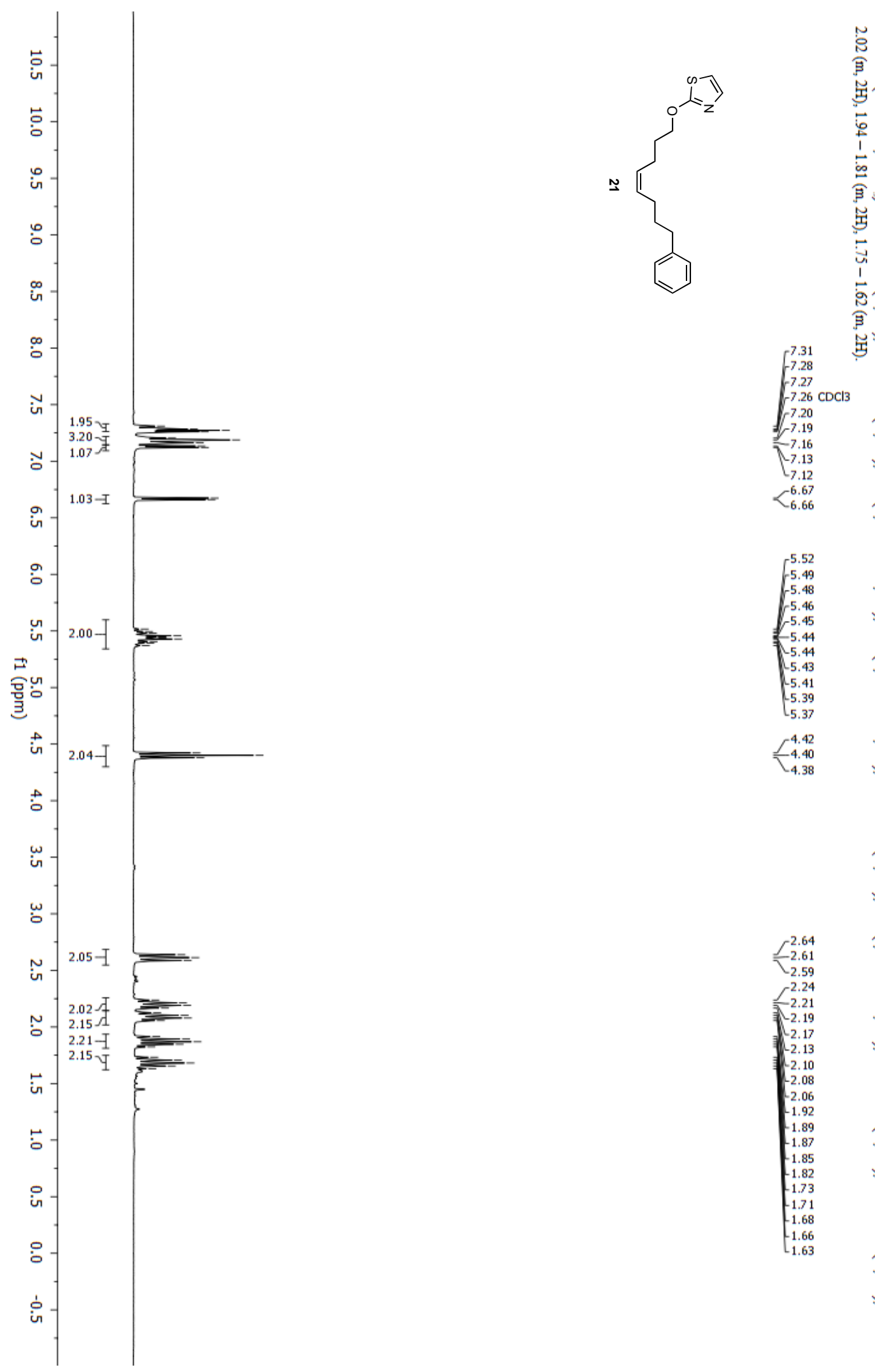

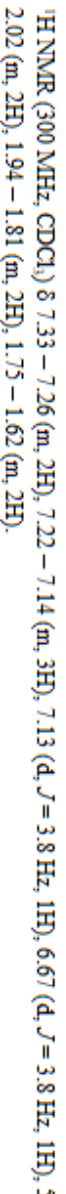

t) 

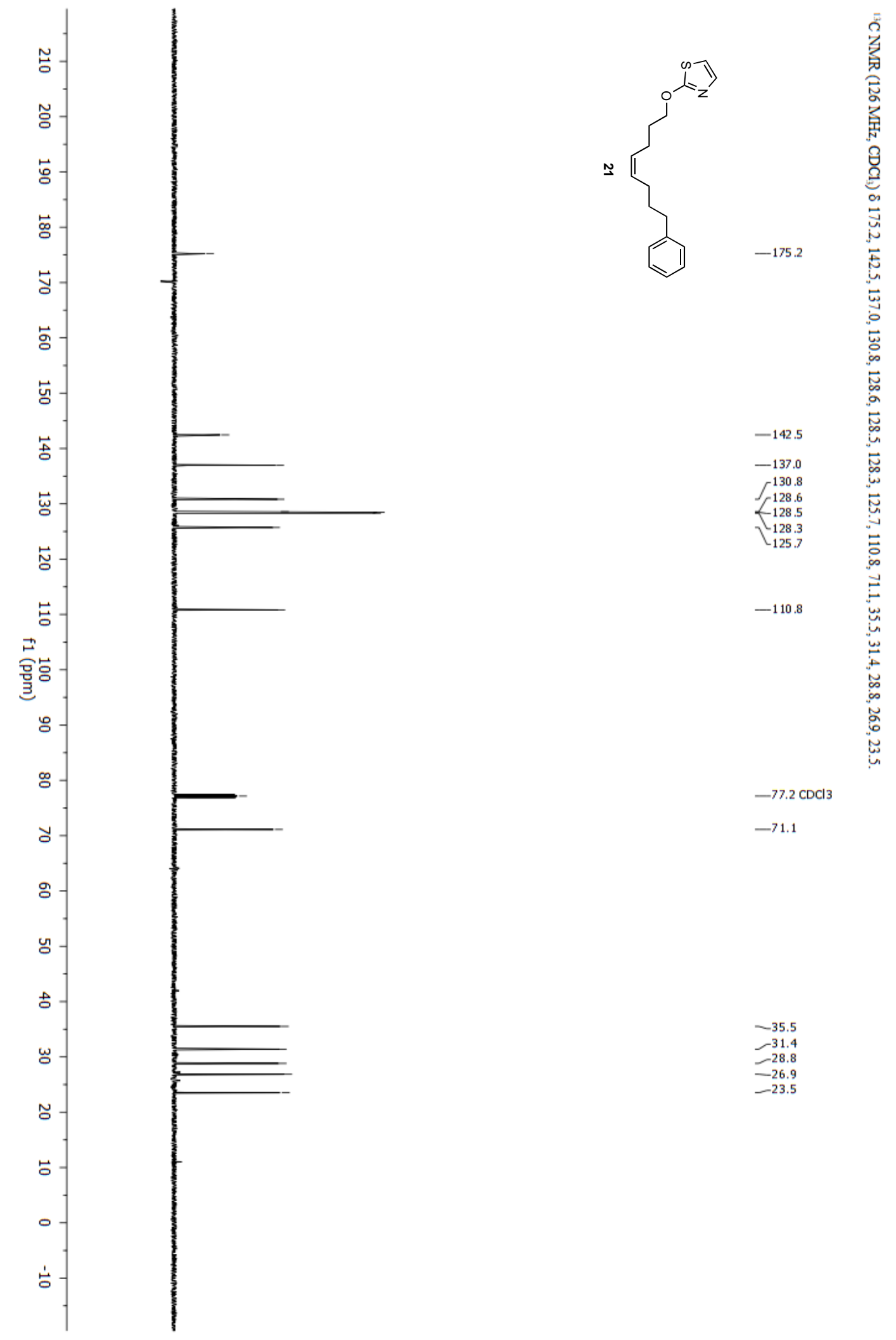


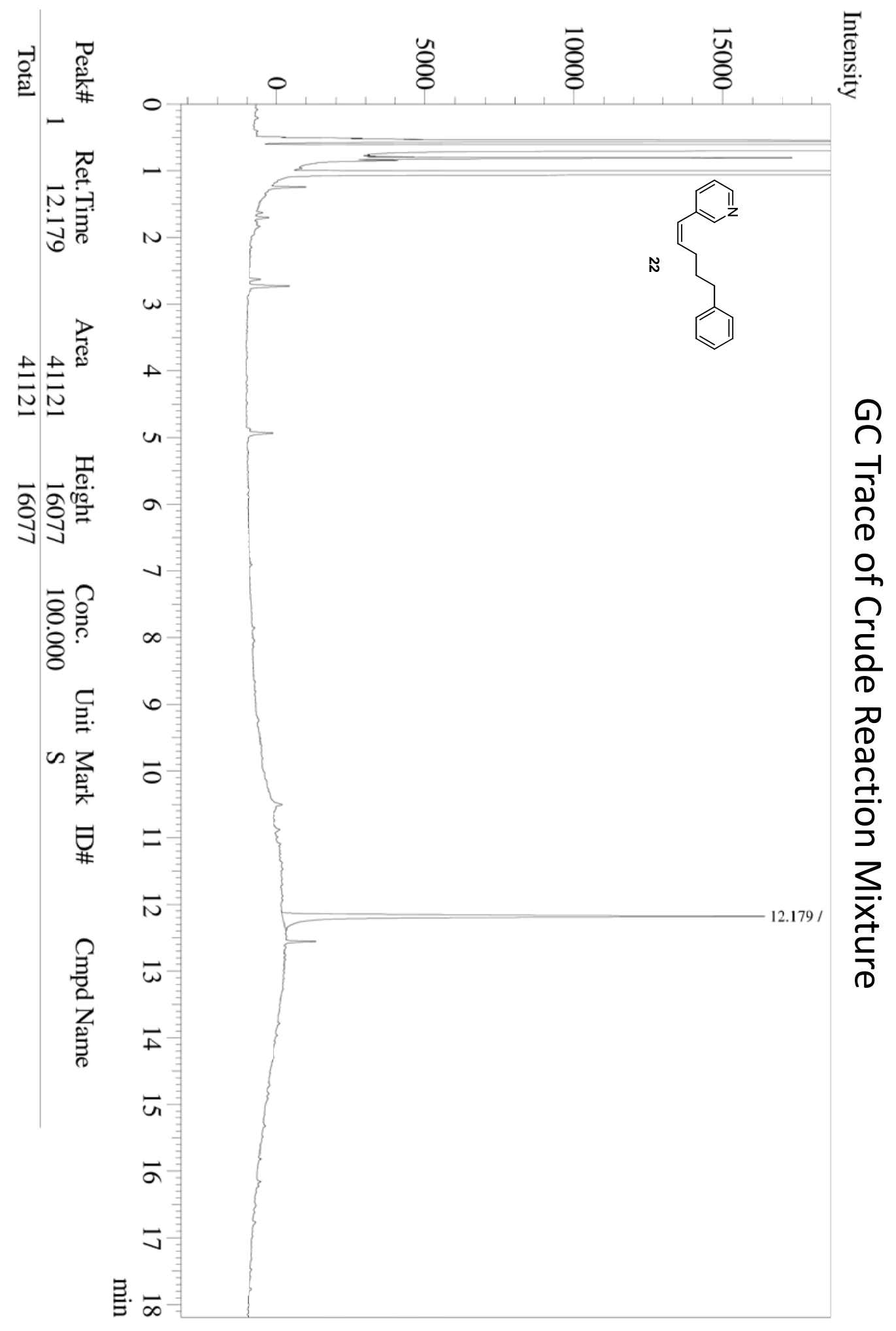



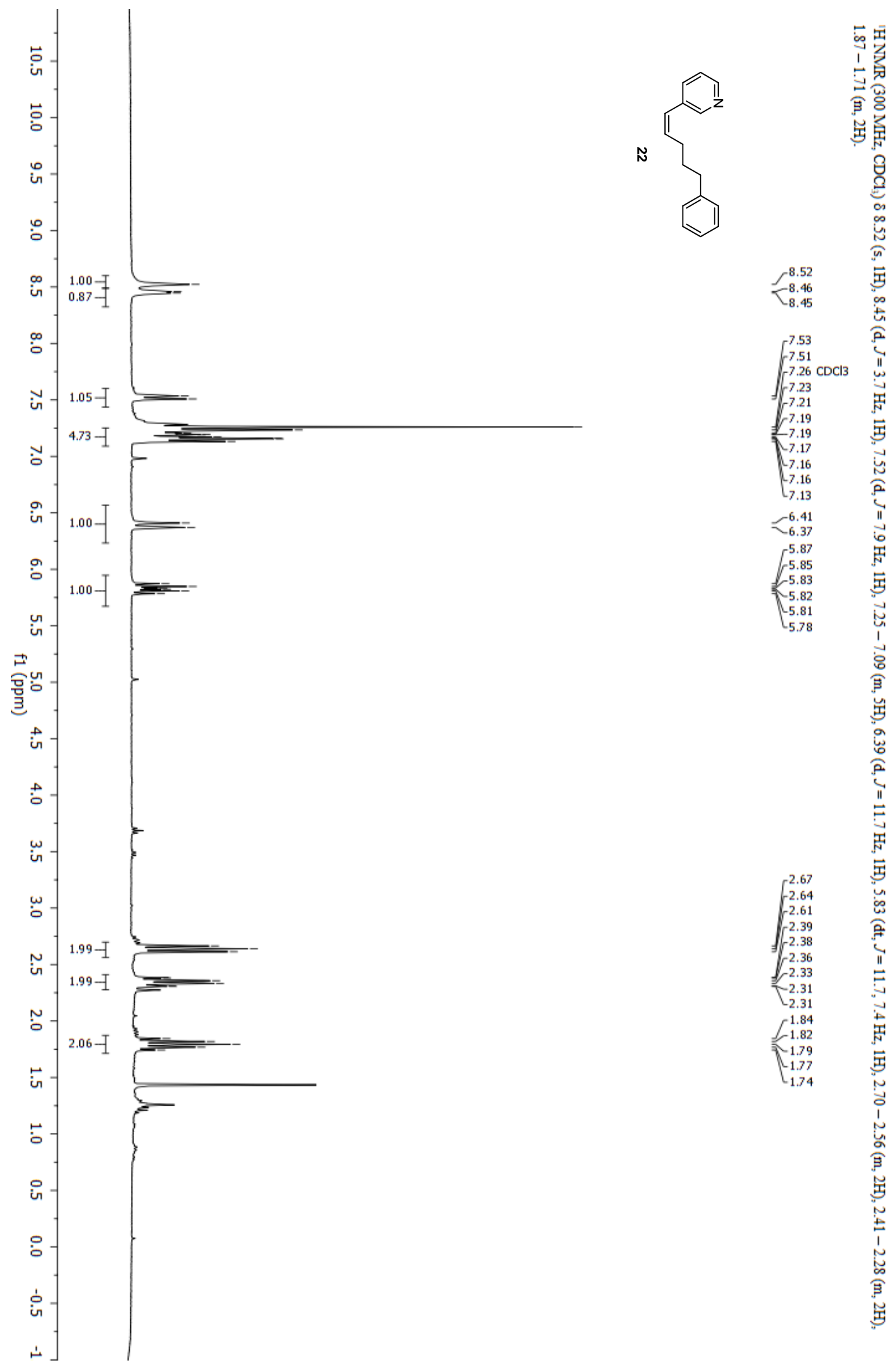

过
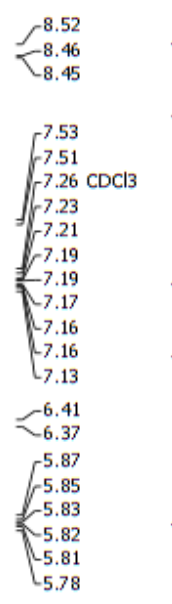

(

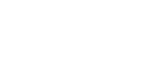

站

2.67 产

$\int^{2.64}$

2.39

$-2.38$

2.36

2.33

2.31
-2.31

1.84

$-1.82$

1.79
1.77

1.74 


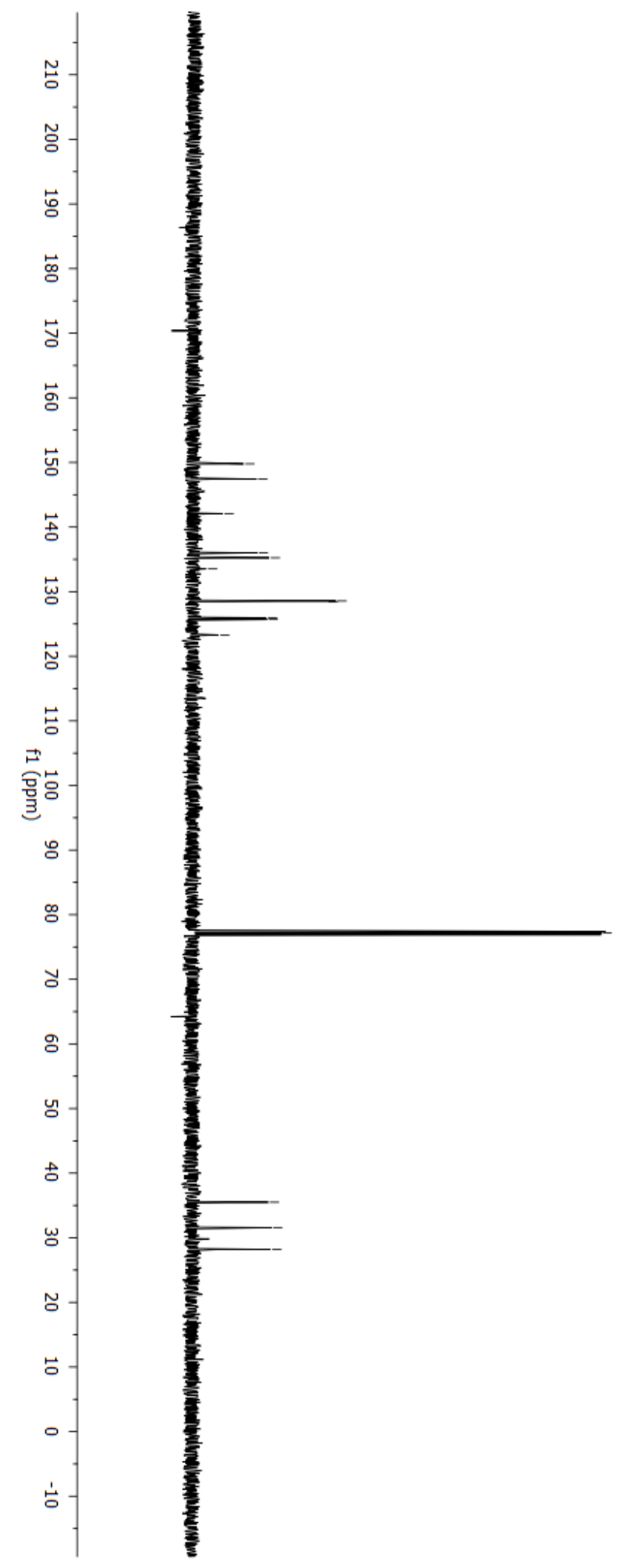

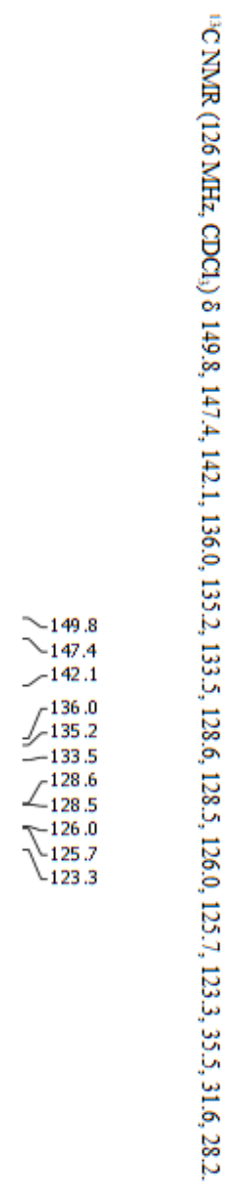

$-77.2 \mathrm{CDCl} 3$
-35.5
-31.6
-28.2 


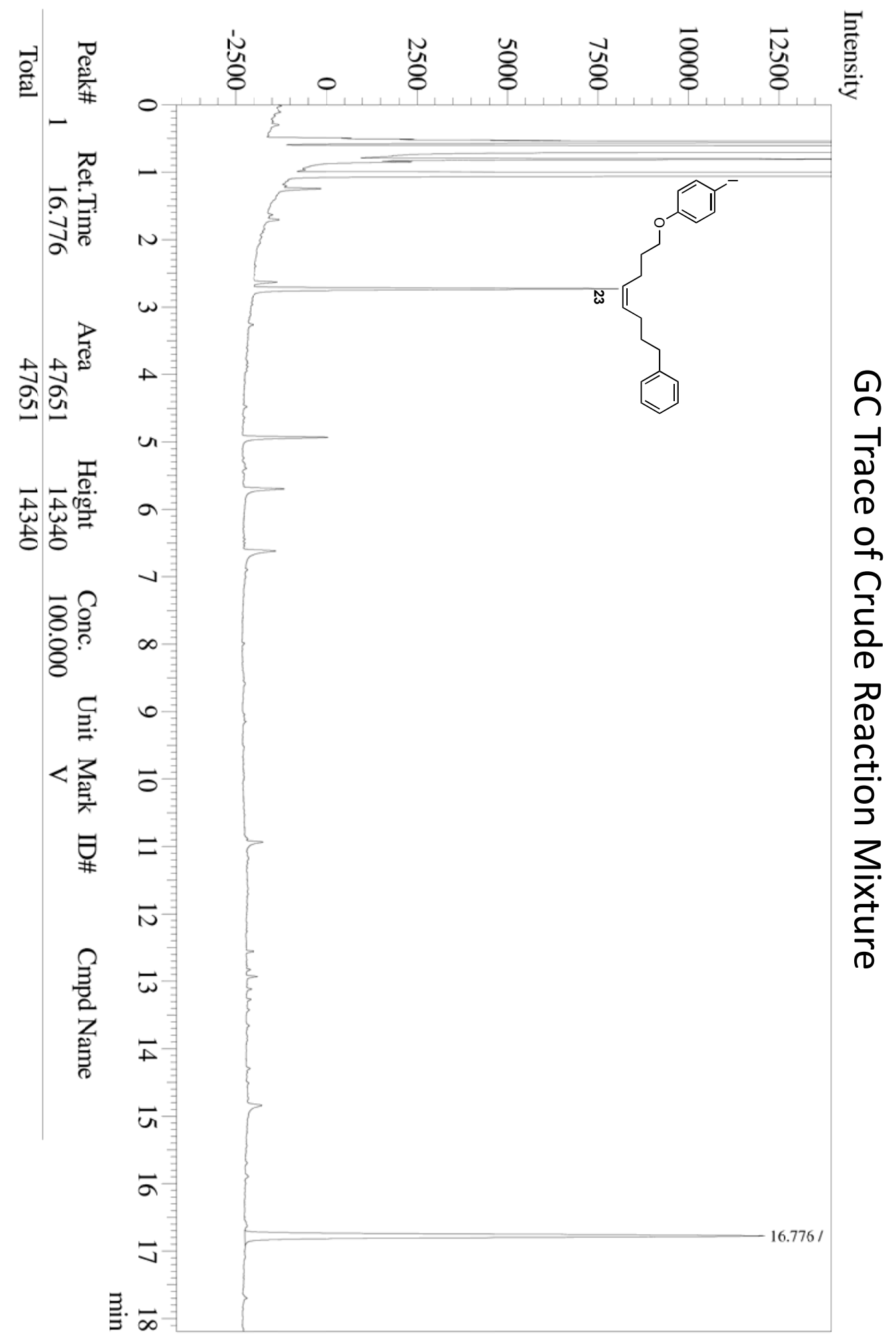



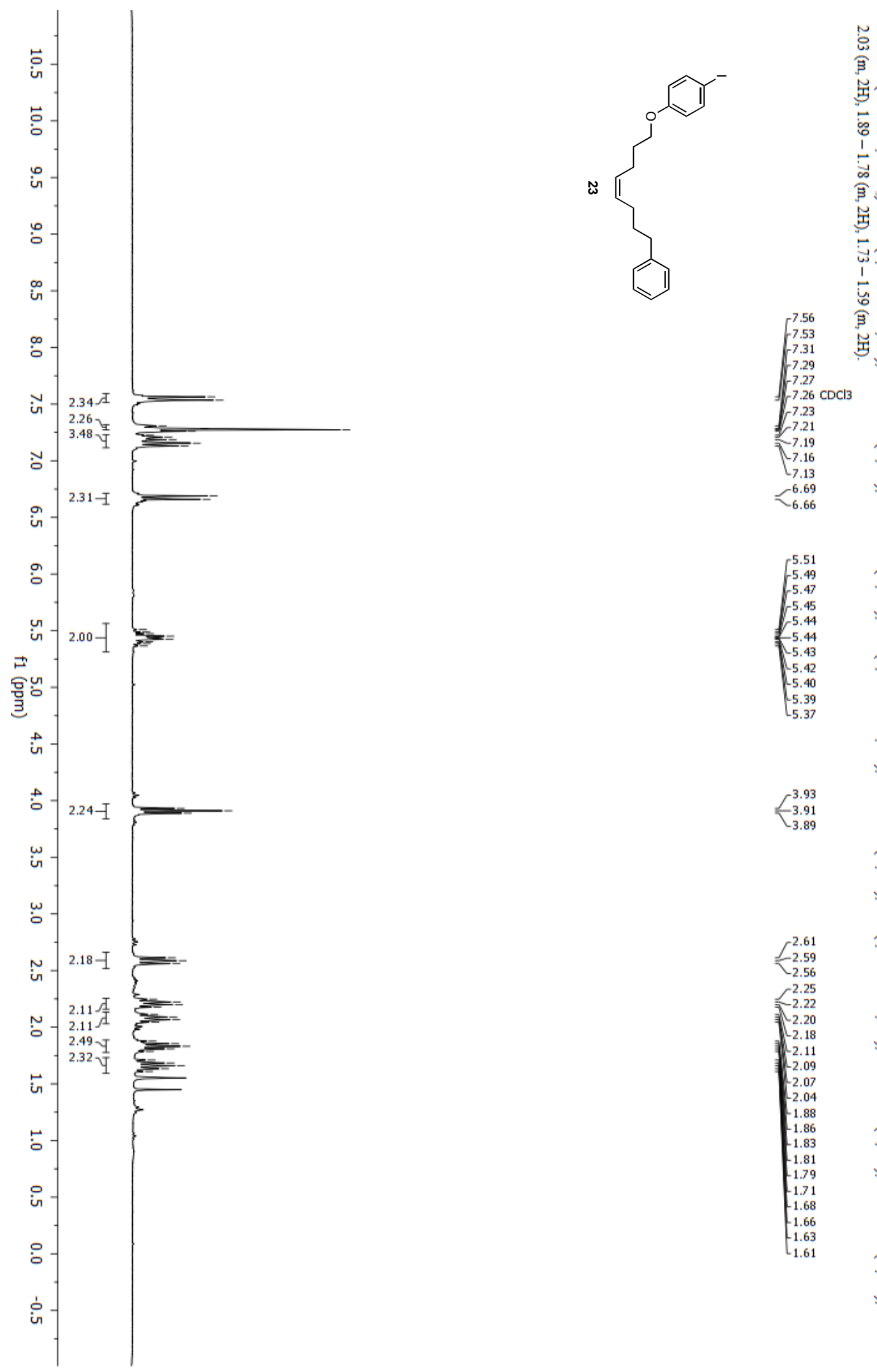

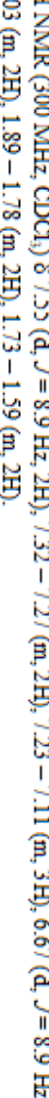

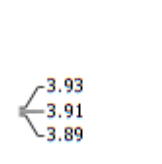

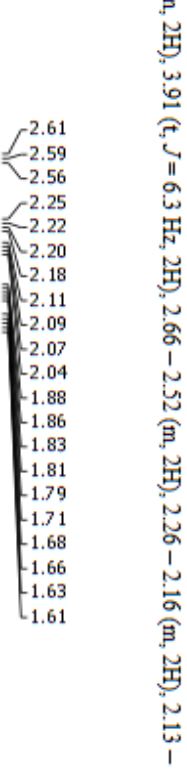



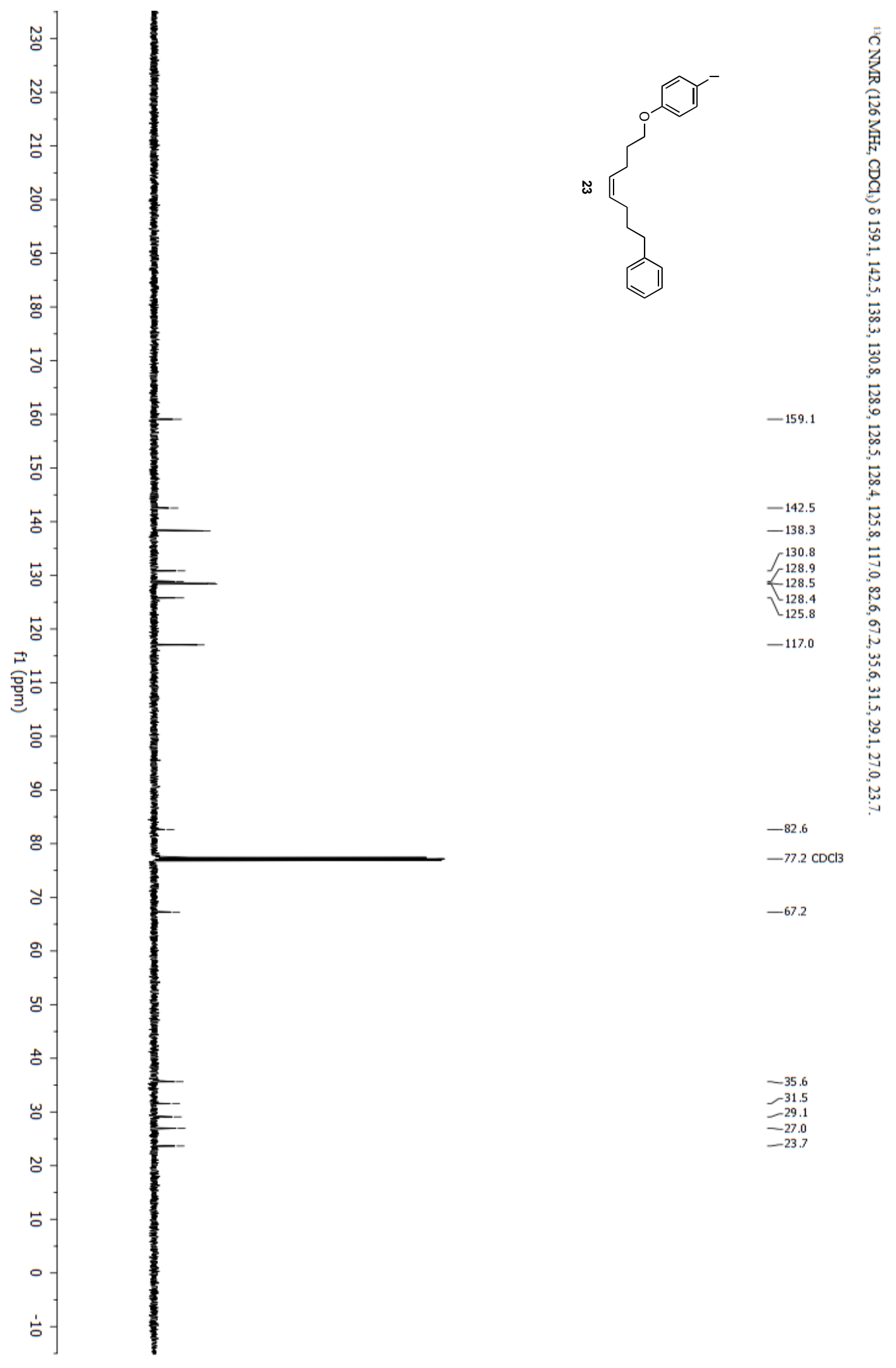

$-67.2$

-35.6
-31.5
-29.1
-27.0
-23.7 


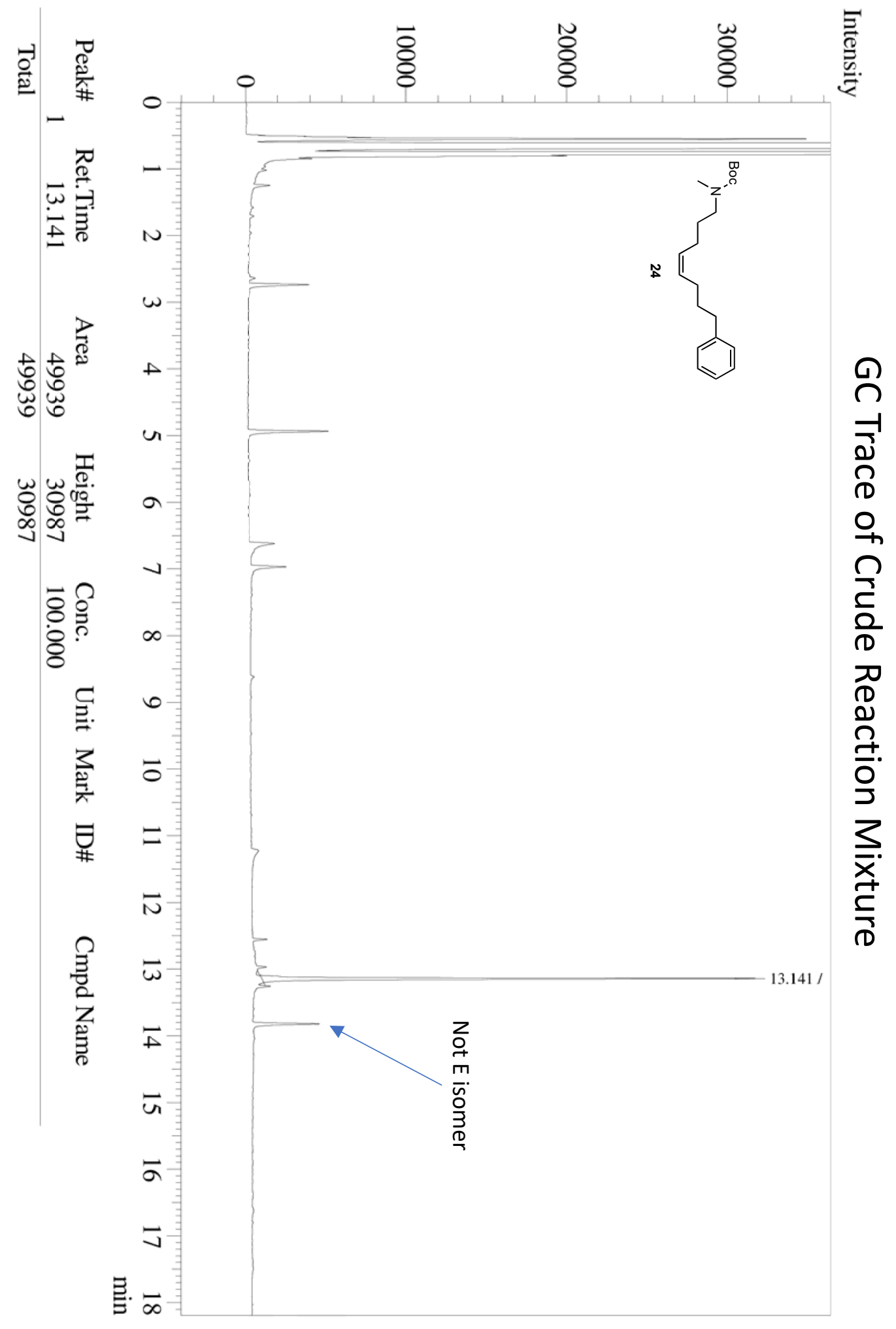




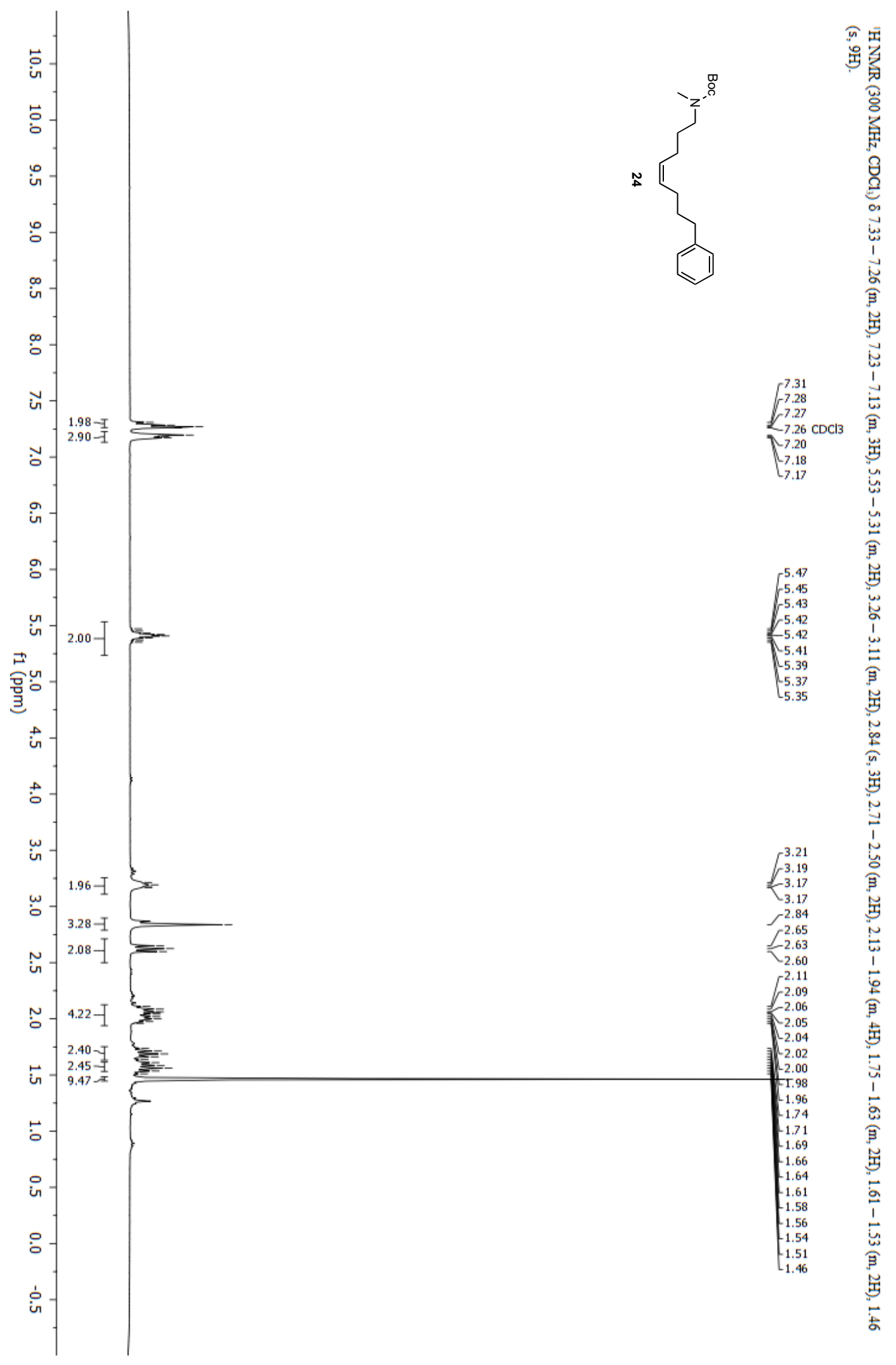



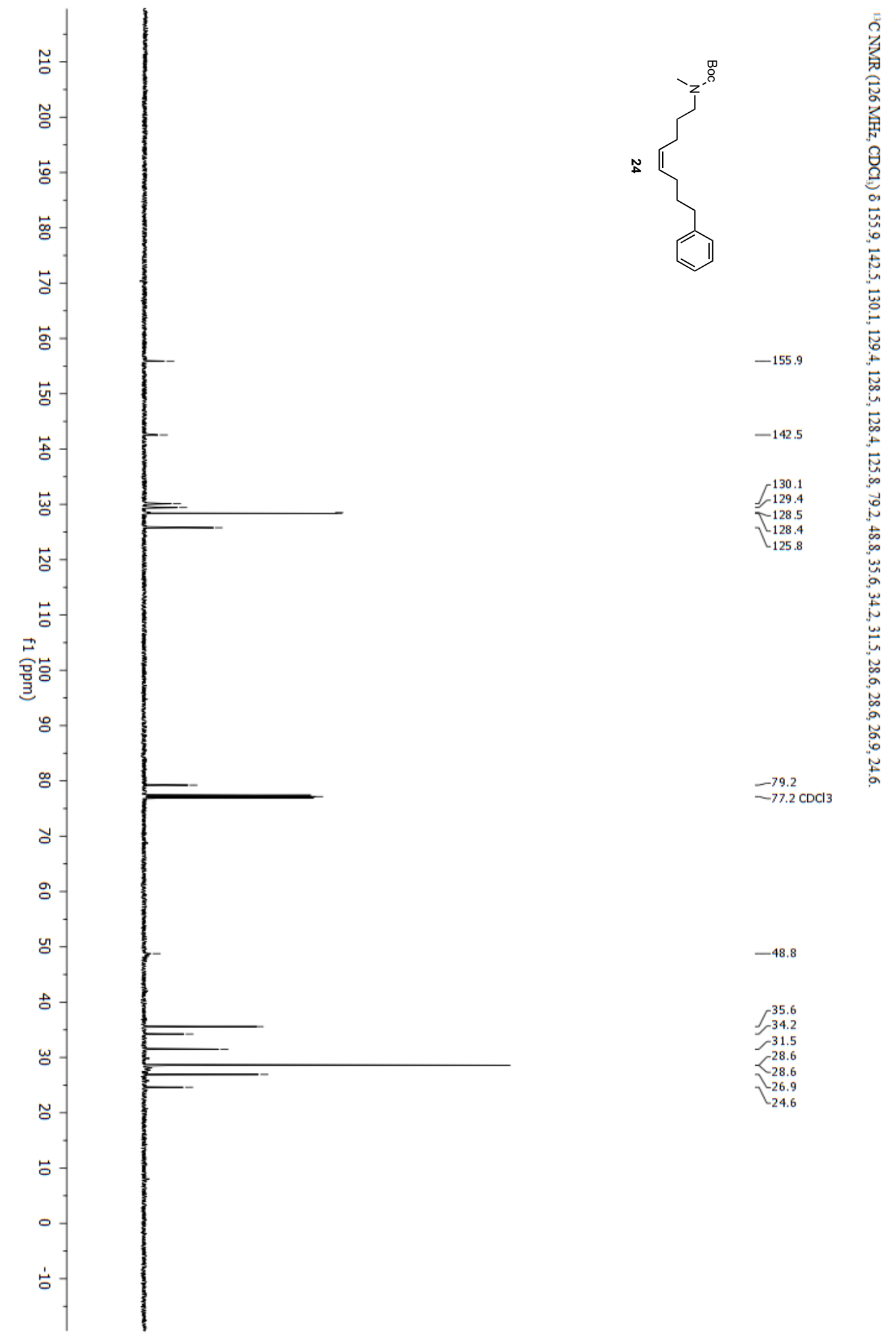

$\sim 77.2 \mathrm{CDCl} 3$

$-48.8$

$\int^{35.6}$

$-31.5$

28.6

$-26.9$ 


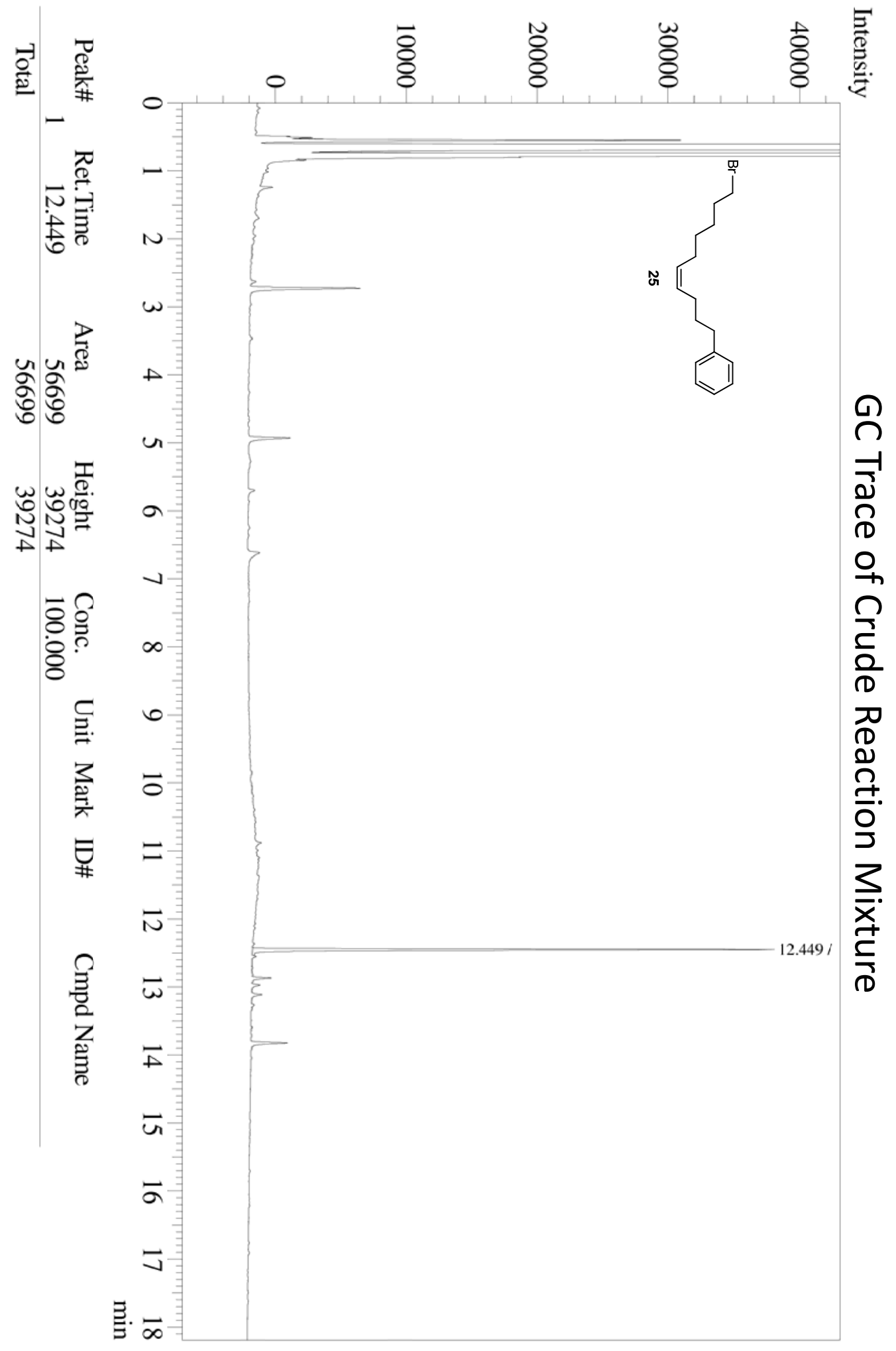



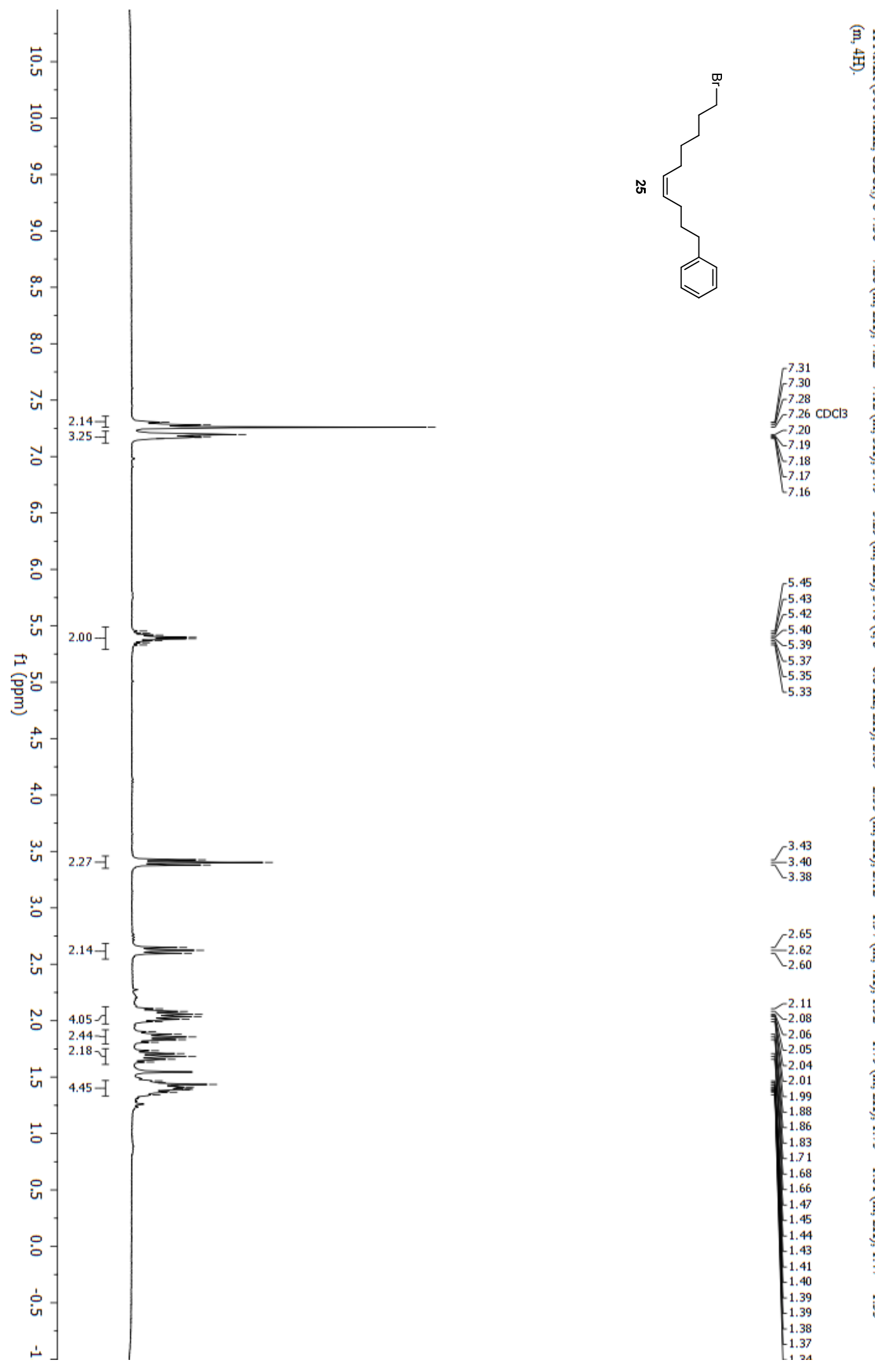

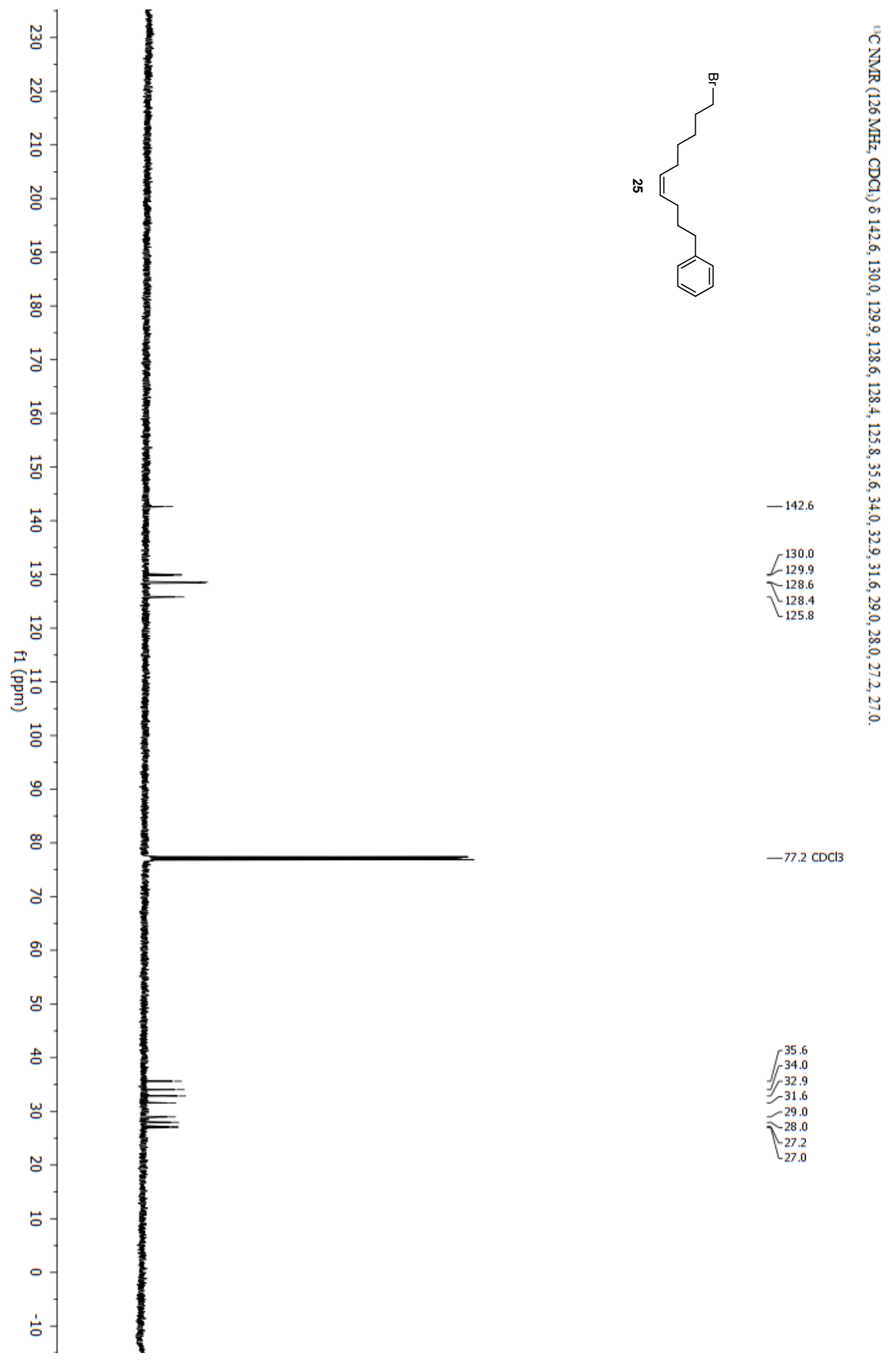

$-77.2 \mathrm{CDCl} 3$

35.6
34.0
32.9
-31.6
-29.0
-28.0
27.2
27.0 


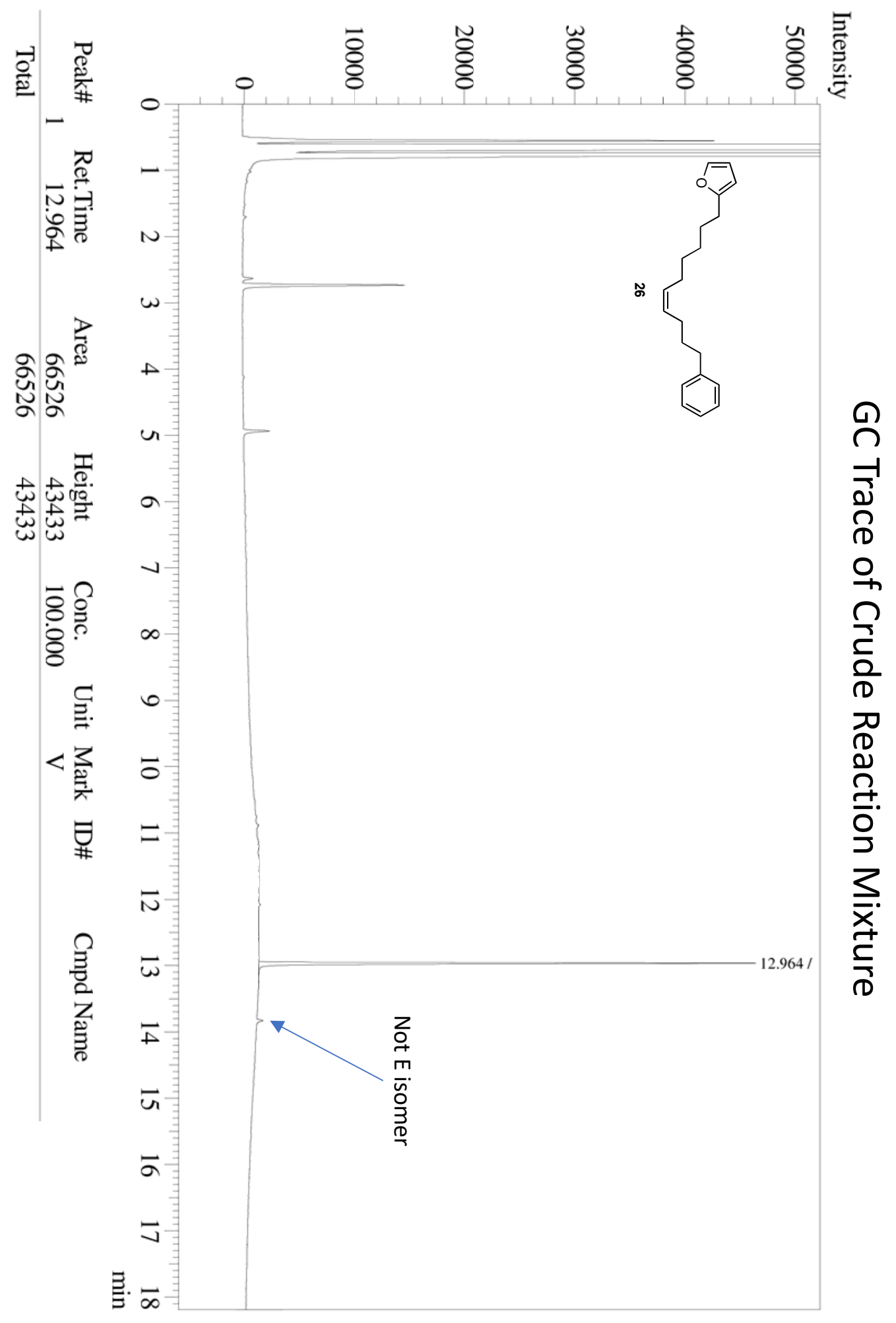



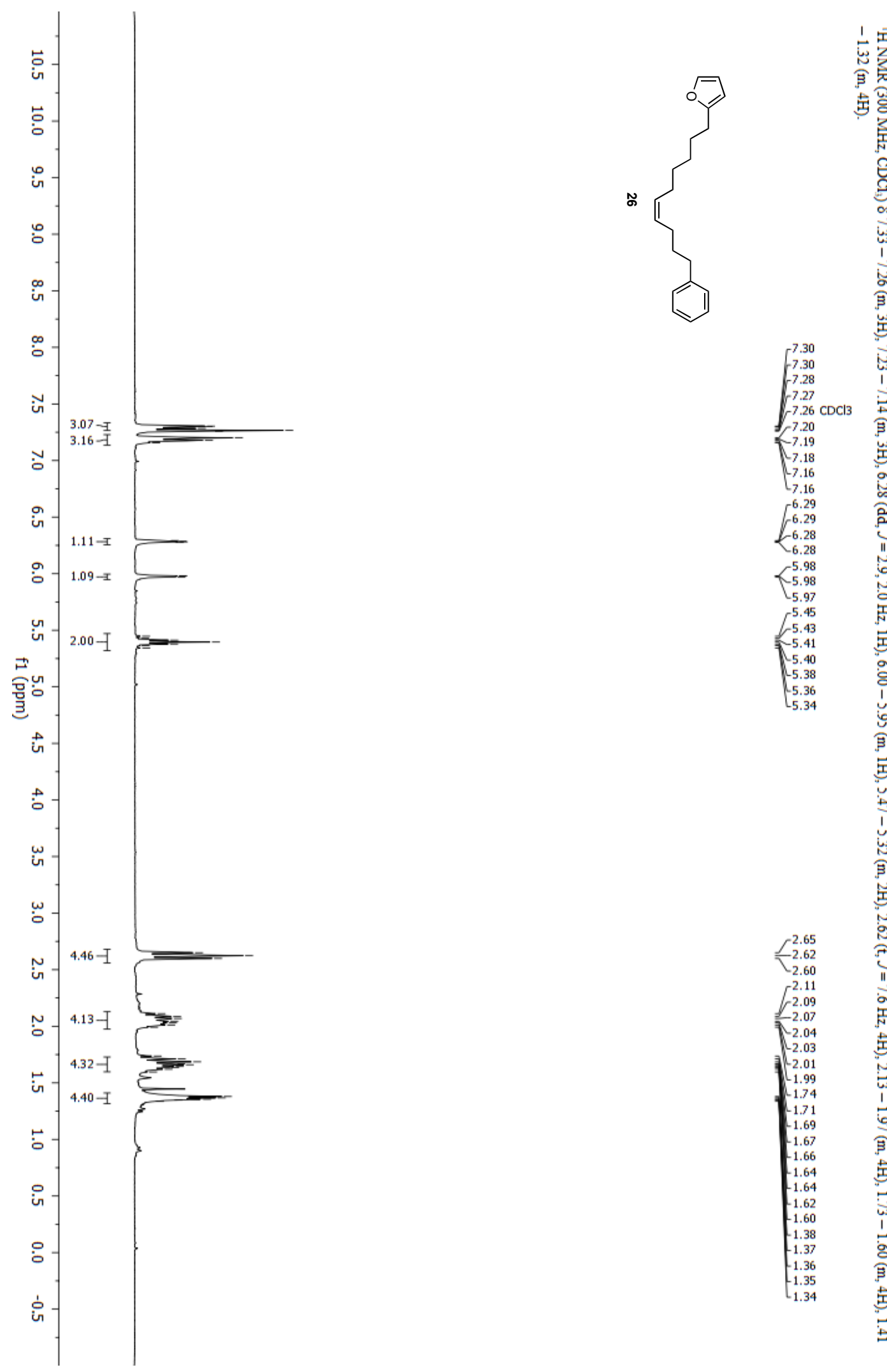

孛

会

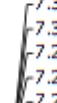

8

7.20

7.19

$\left[\begin{array}{rl}7.16 \\ 7.16\end{array}\right.$

6.29

$\underbrace{6.29}_{6.28}$

$<_{5}^{5.98} \quad$ o

5.97

5.45

5.43
-5.41

5.40

$\left[\begin{array}{rr}5.38 & 8 \\ -5.34 & 1\end{array}\right.$

5.34 i

(2)

实

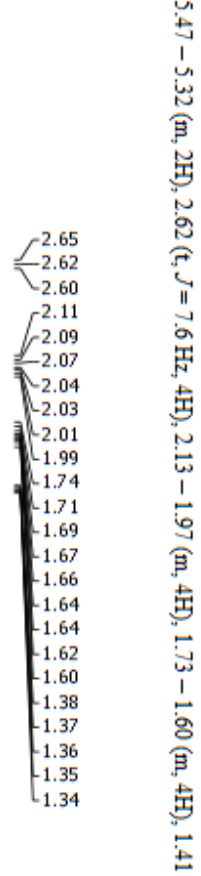



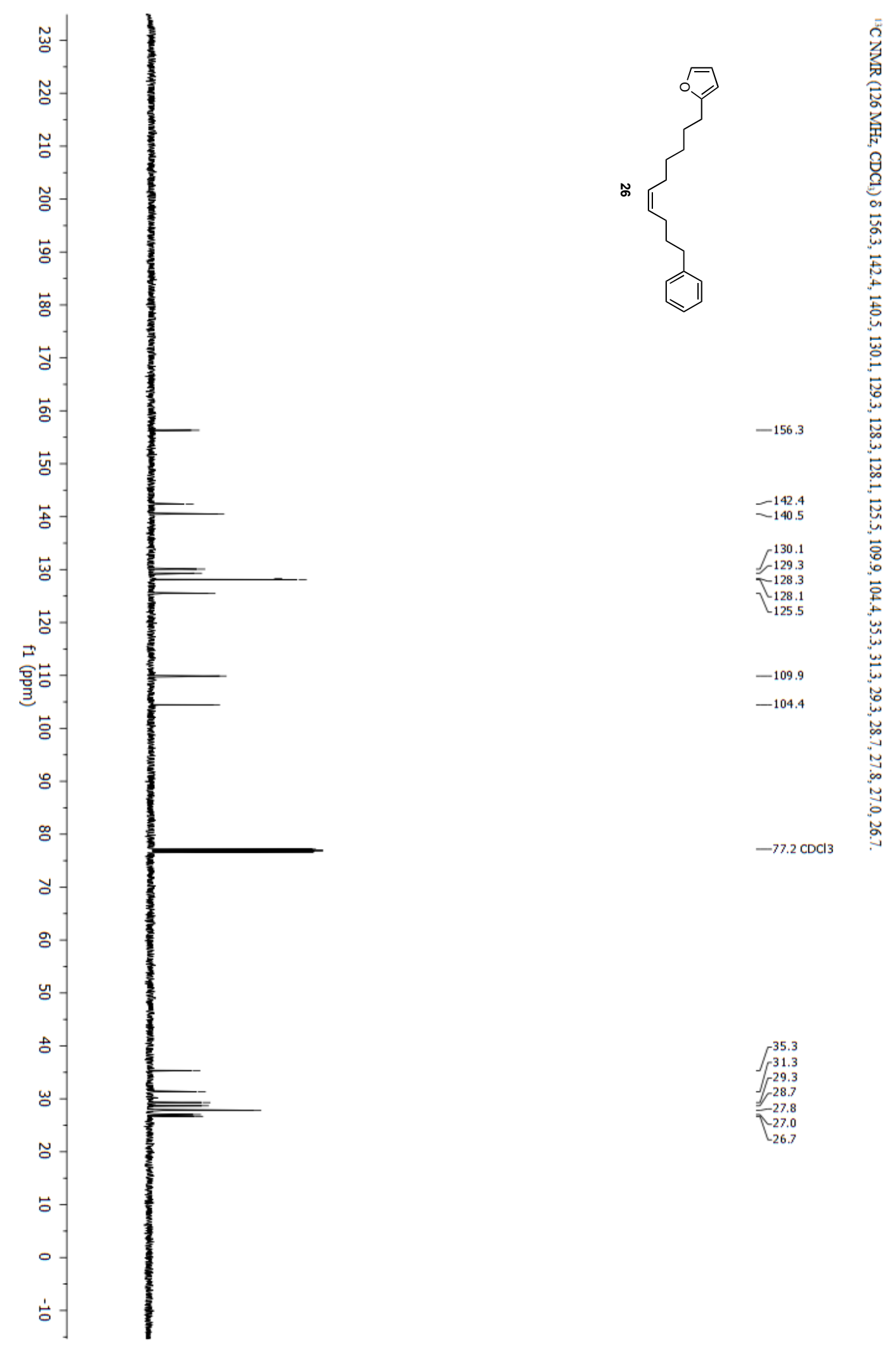


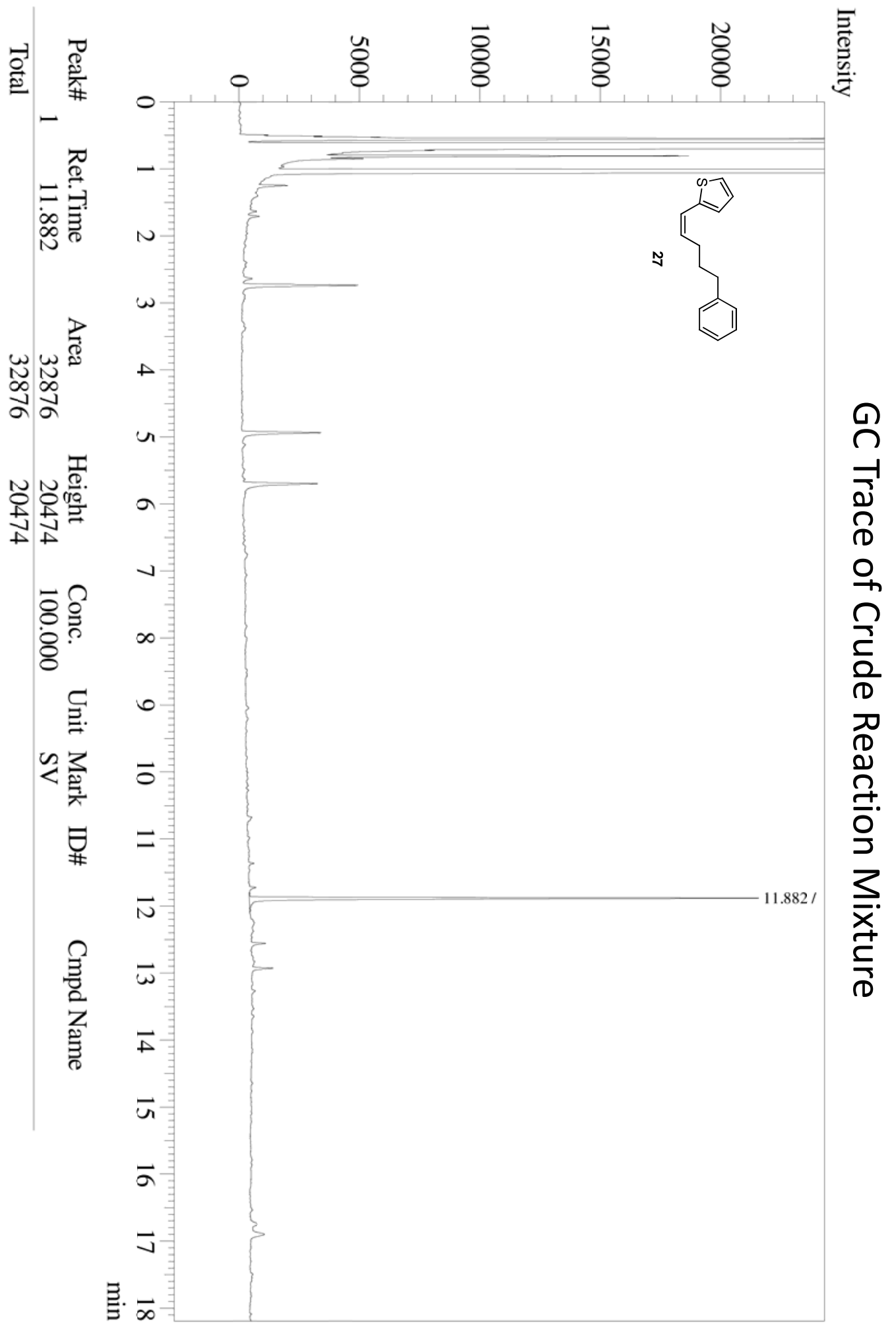



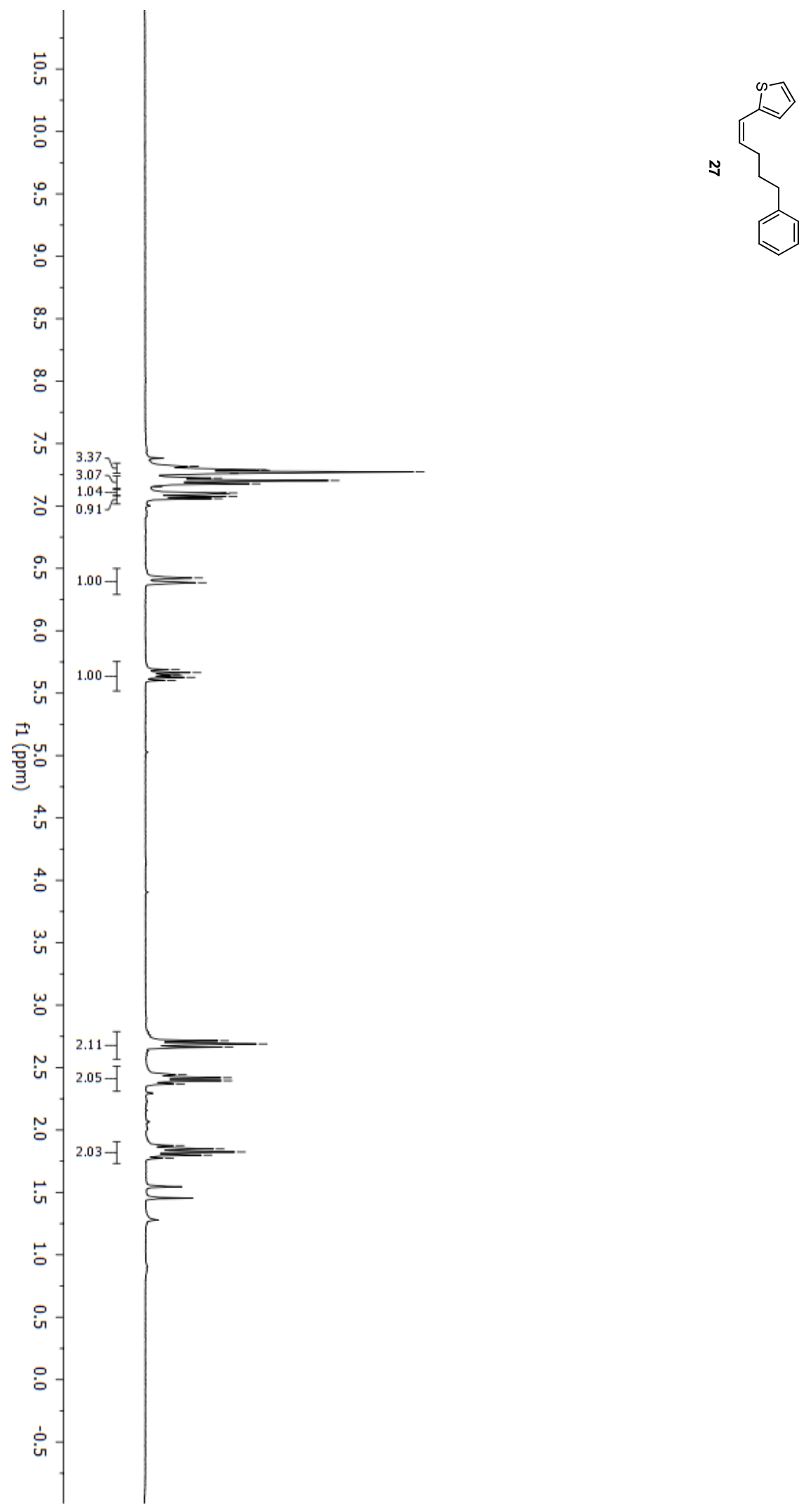

岕窝

要

䍃忿

훙

穴鱼

แิ

荫

苟孚

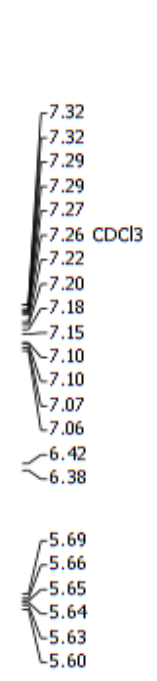

(5)
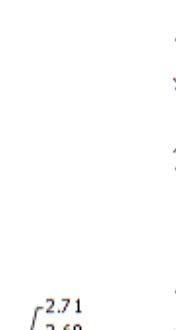

$5^{2.66}$

$-2.42$

2.39
2.37

1.87

$-1.85$

1.82
-1.80

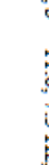

(

11 

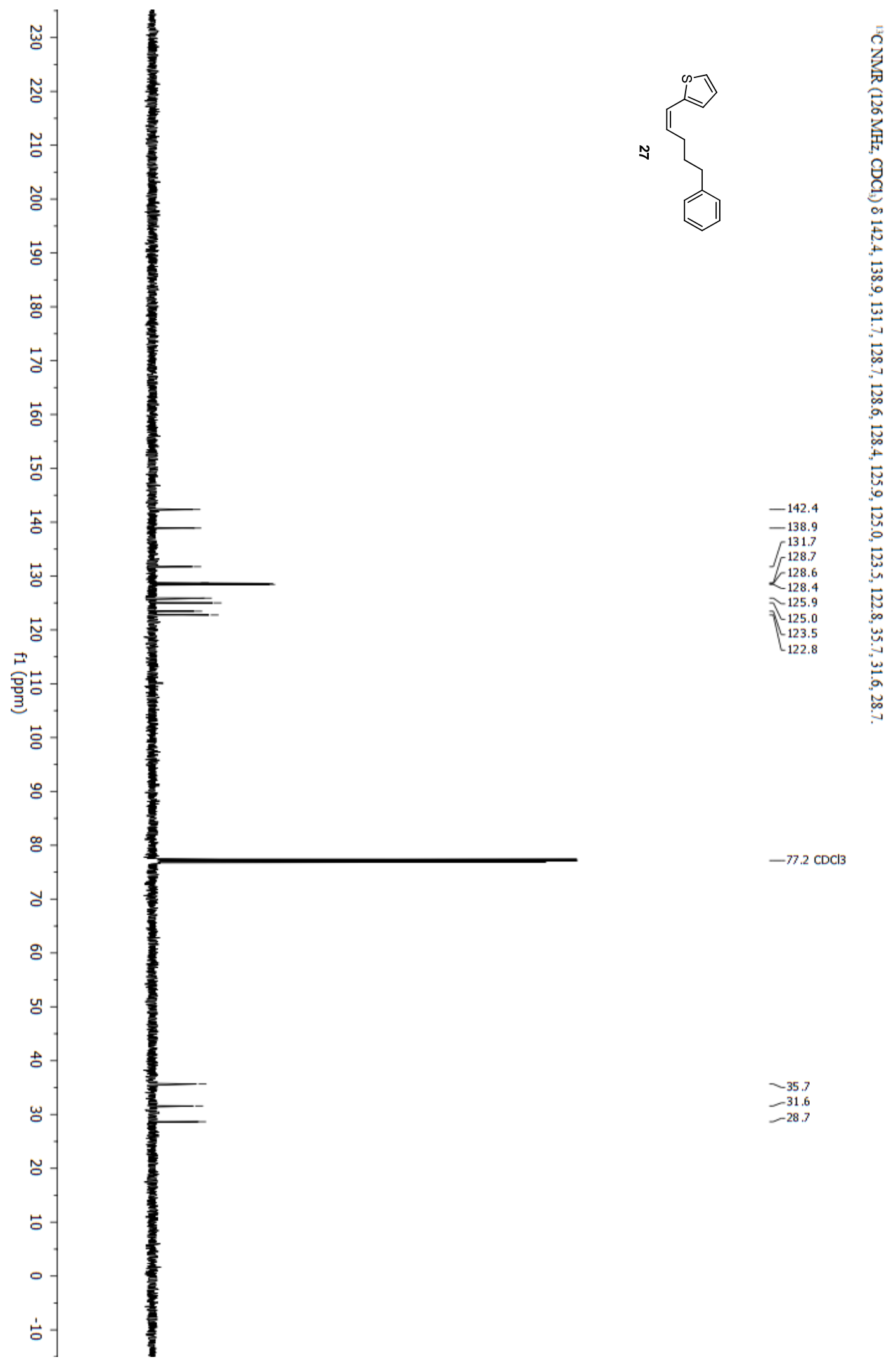

$-77.2 \mathrm{CDCl} 3$

-35.7
$=-31.6$
-28.7 


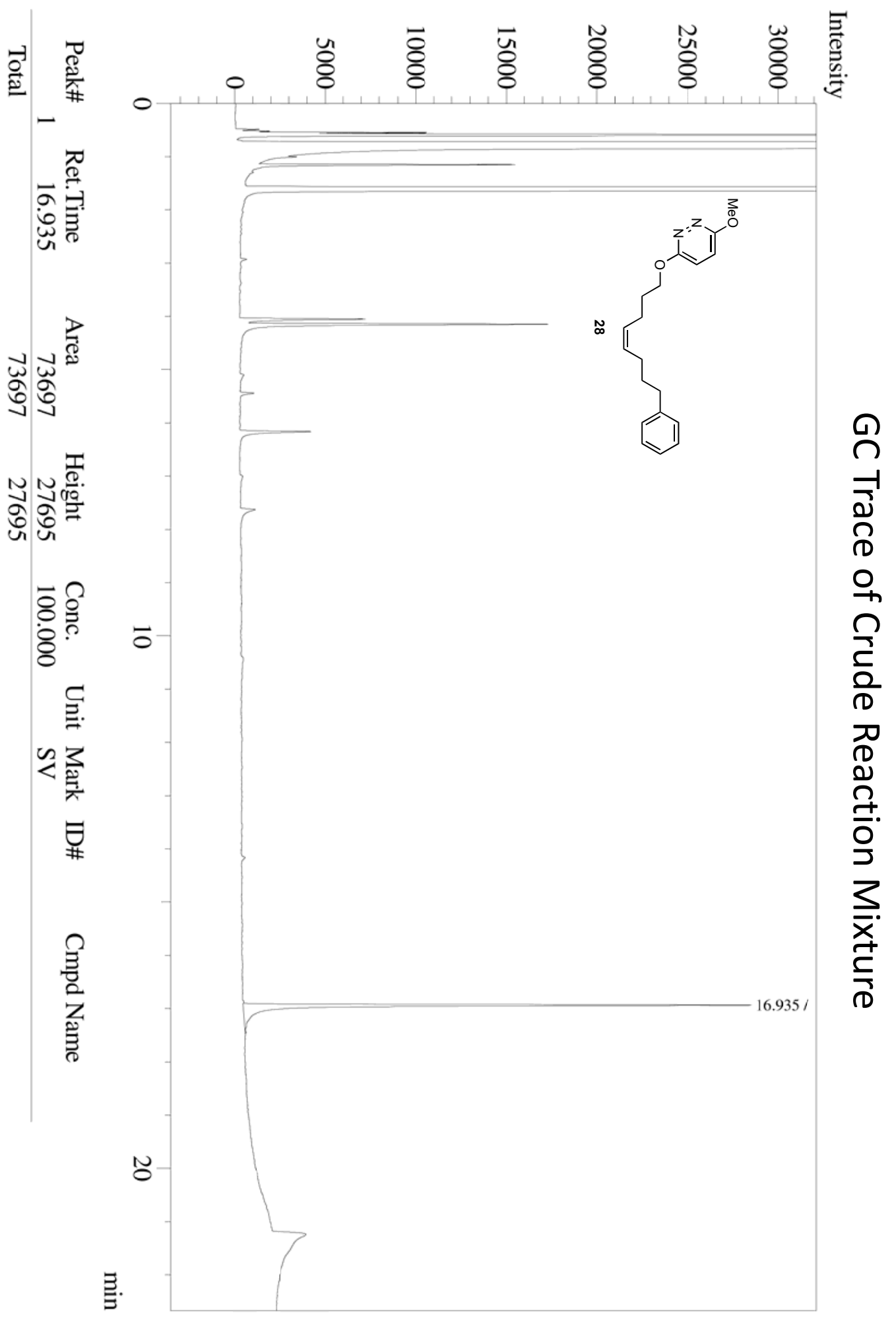



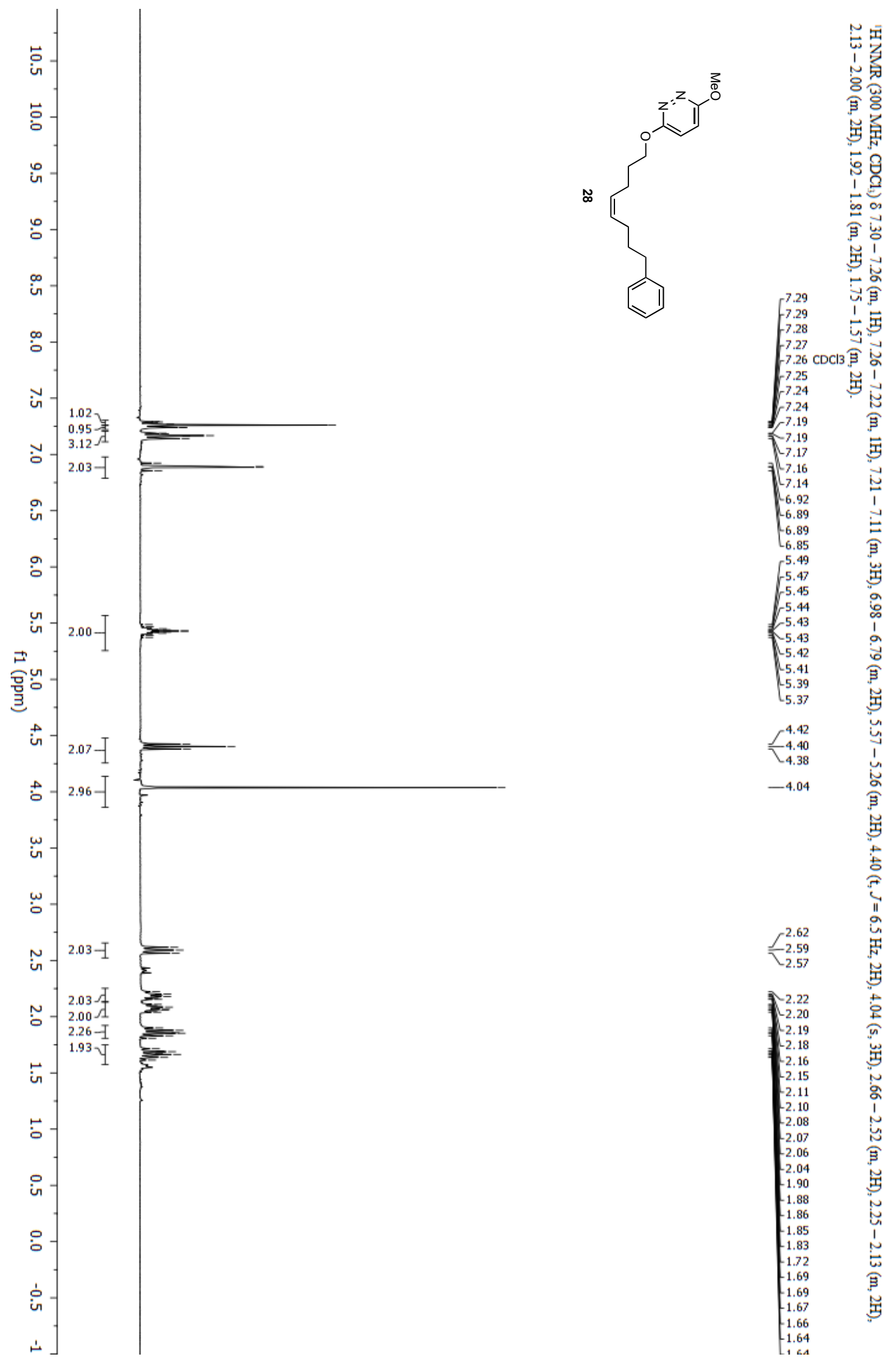

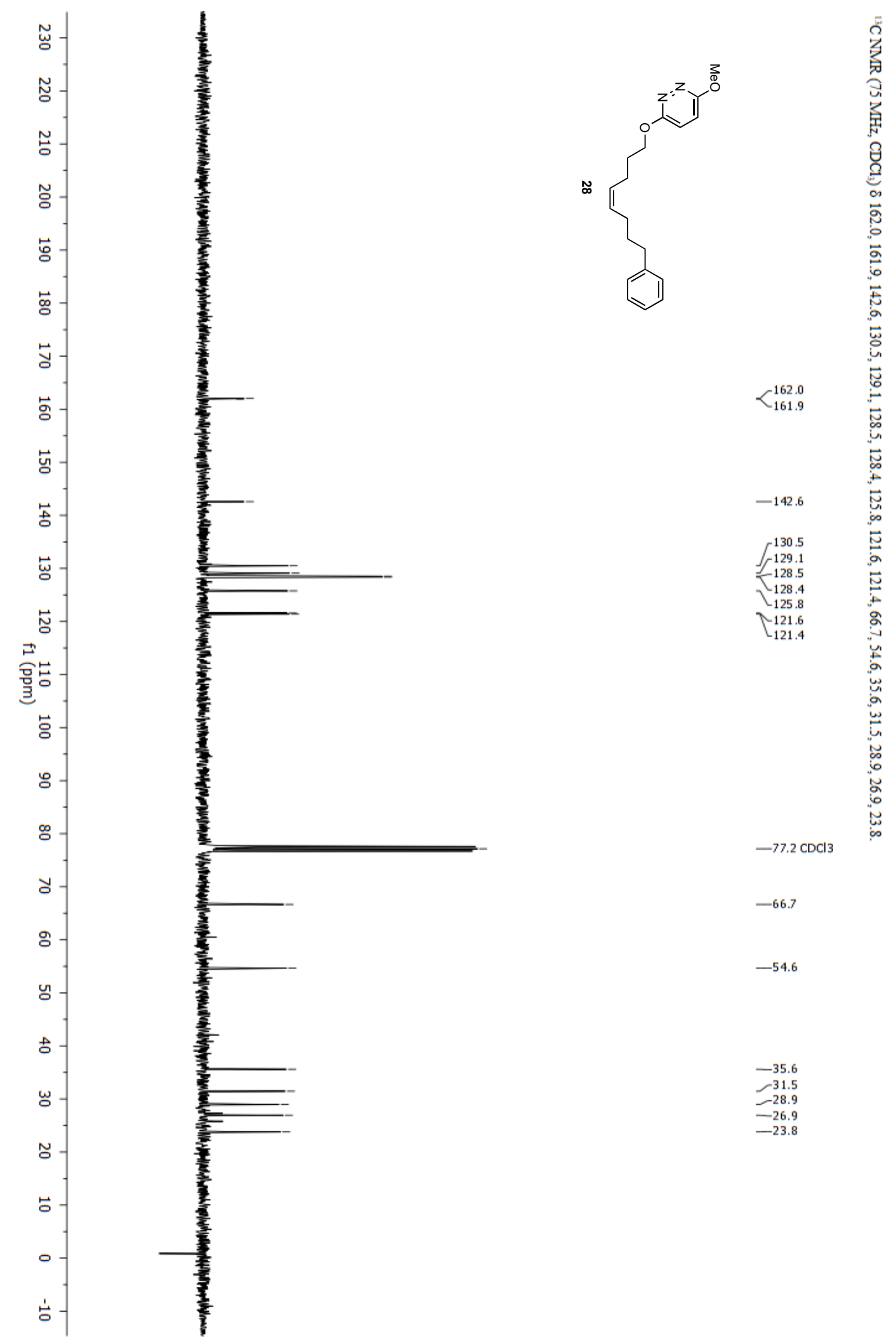

$-77.2 \mathrm{CDCl} 3$

$-66.7$

$-54.6$

-35.6
-31.5

-31.5
-28.9

-26.9
-23.8 


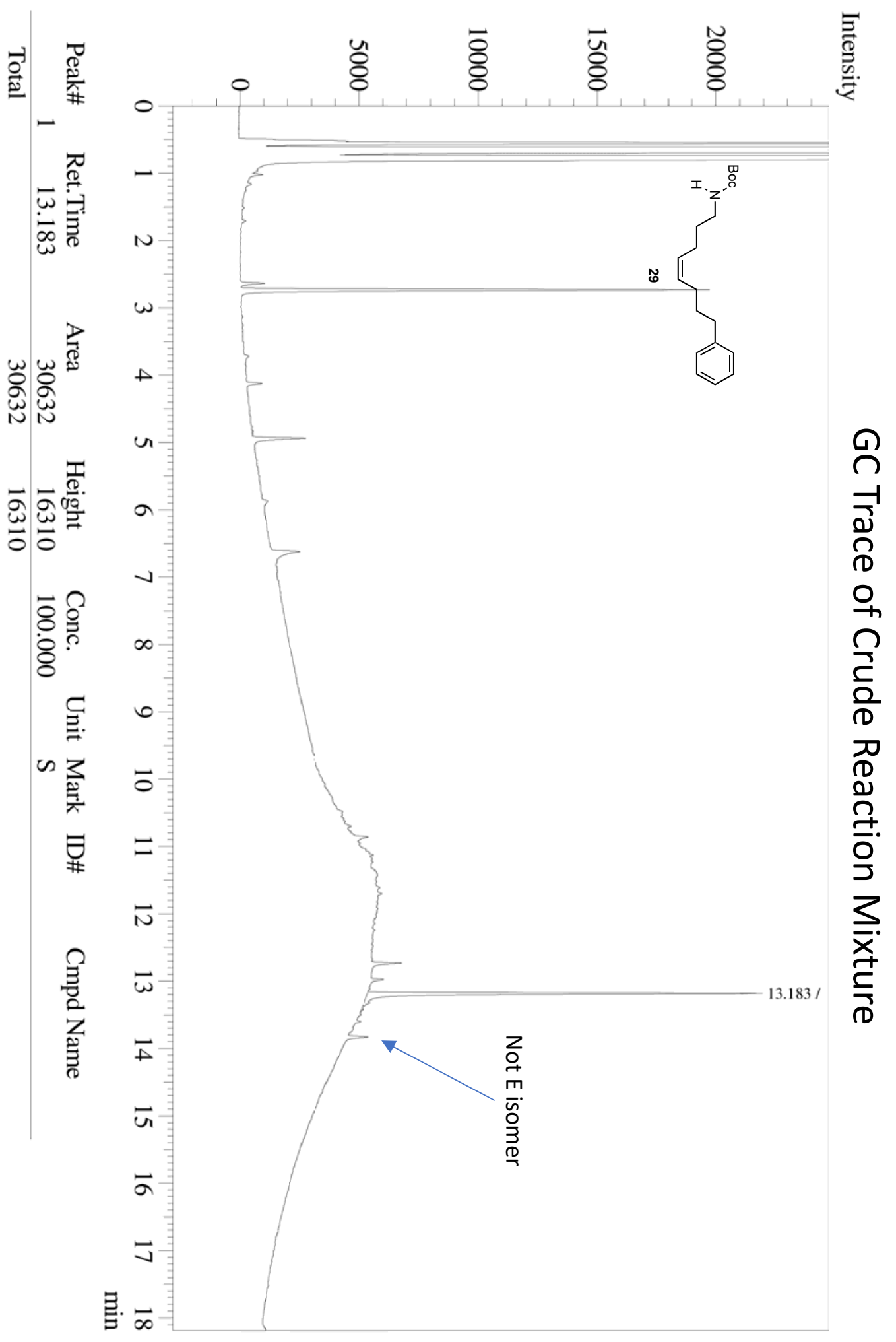



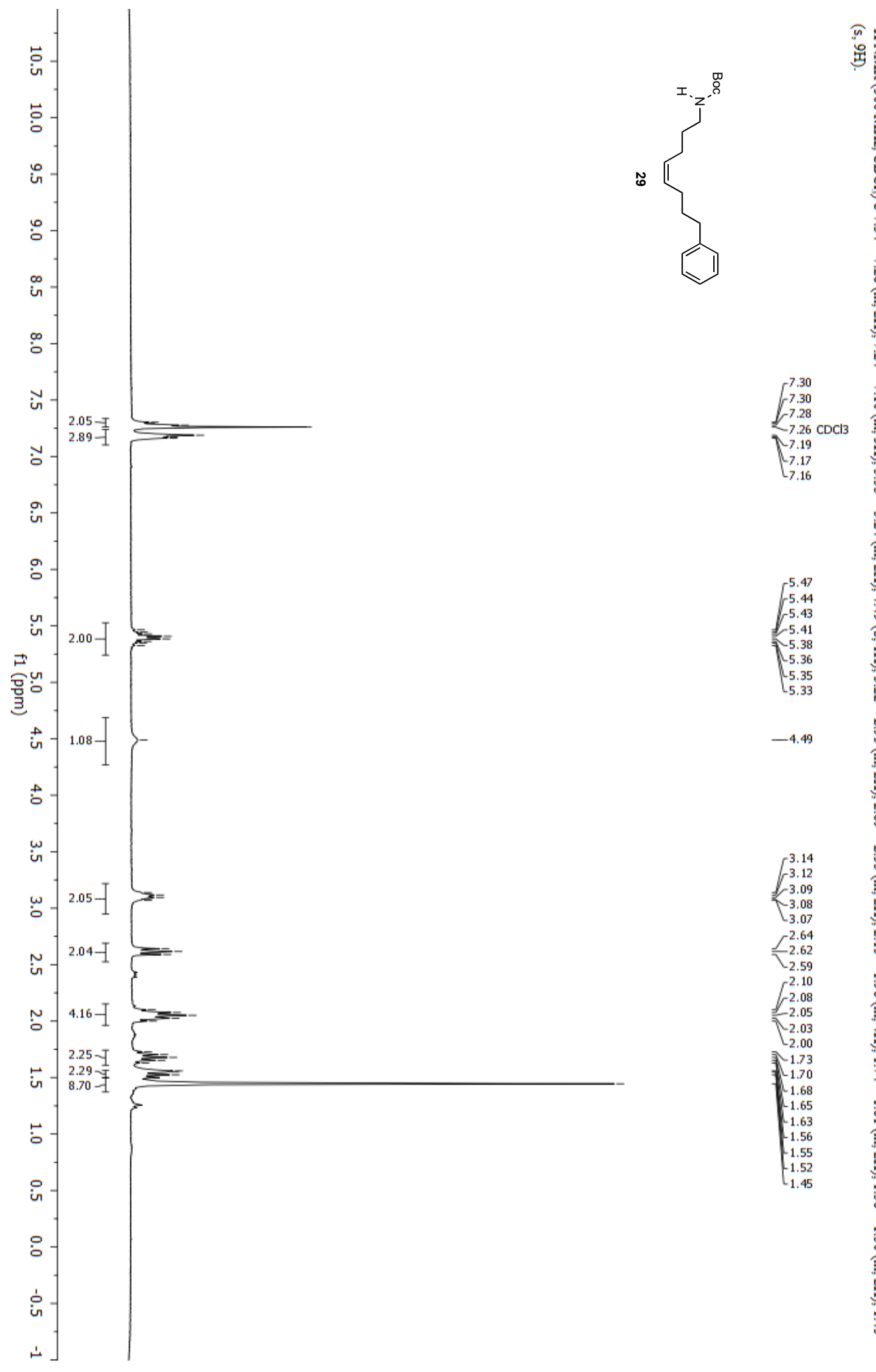

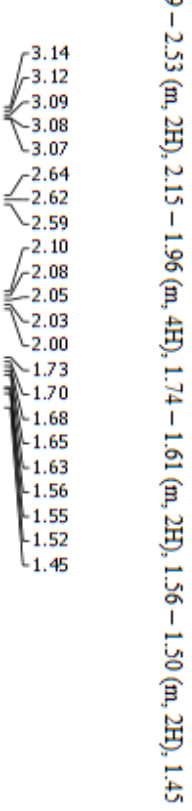



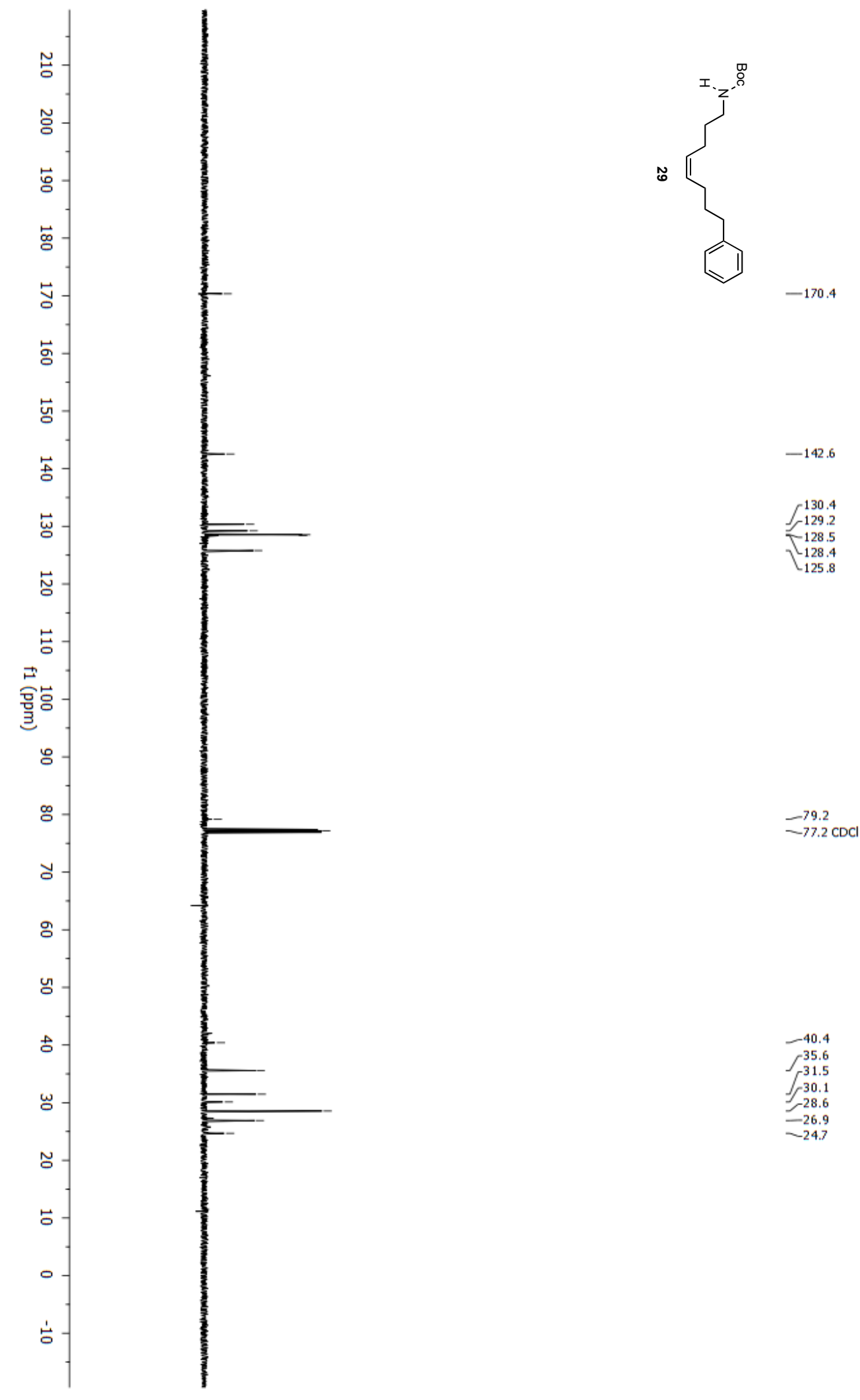

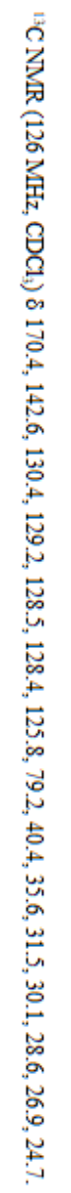

$=79.2$

-40.4
$\int_{-3.6}^{35.6}$

$\int_{-30.1}^{31.5}$

38.6
-26.9
-24.7

-26.9
-24.7 


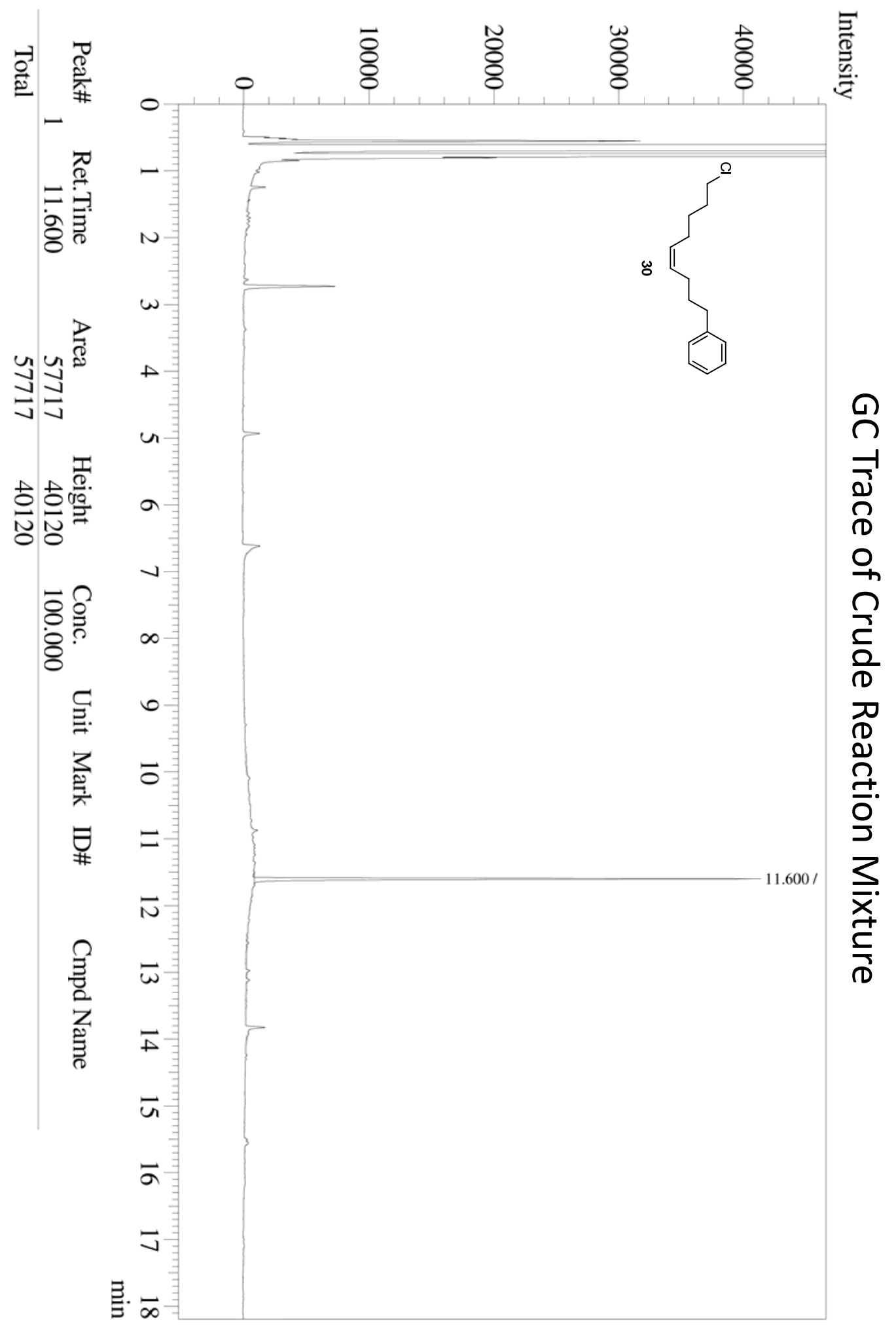



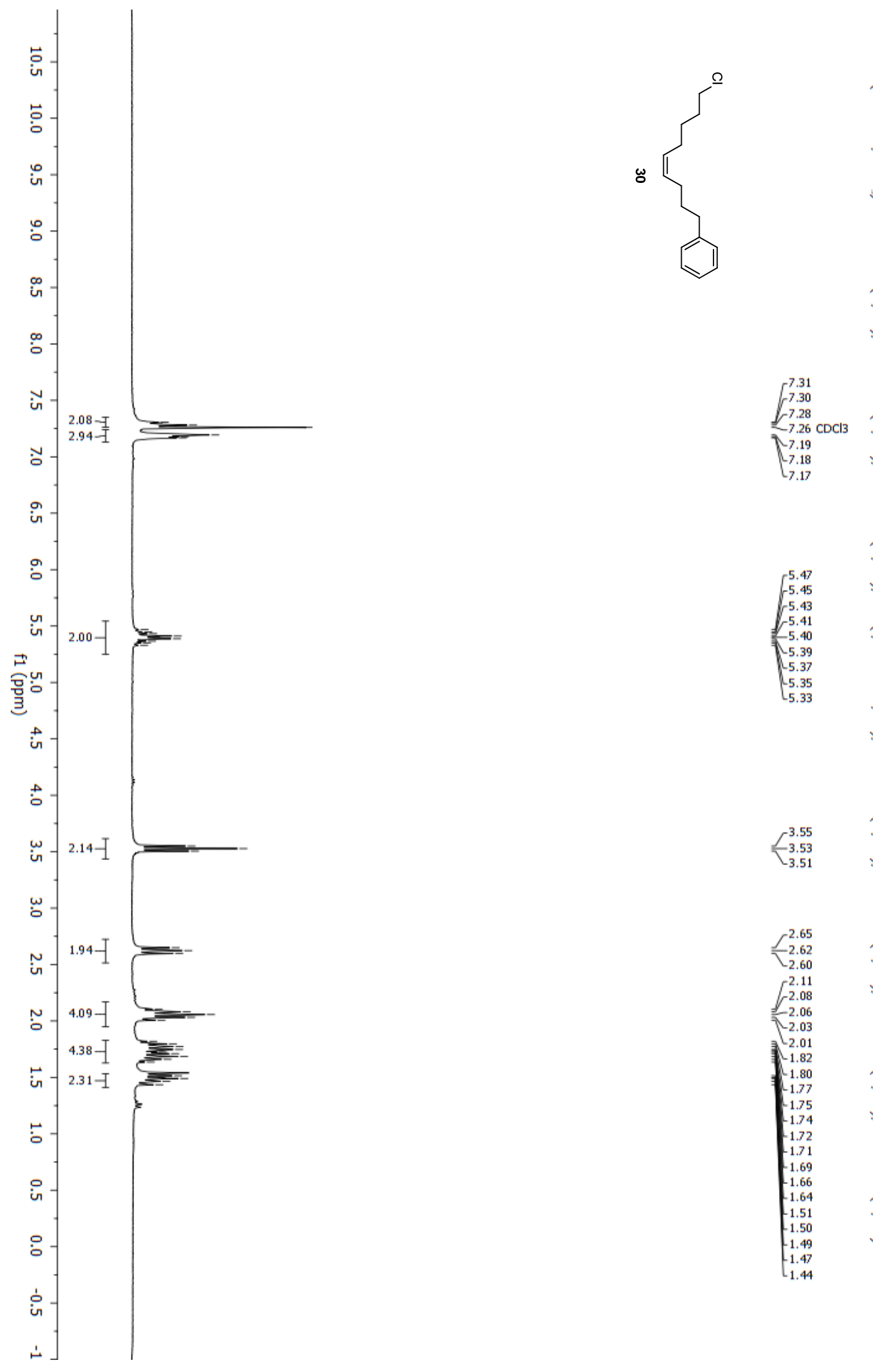

䓵

芩

岸

u.
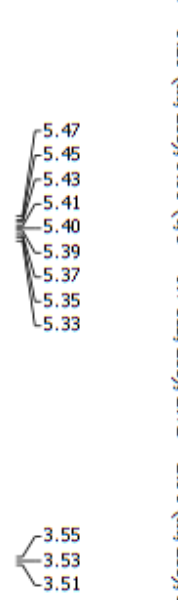

$\int^{2.65}$

击

$y_{-2.06}^{2.08}$

$\underbrace{2.03}_{-2.01}$

$l_{1.80}^{1.82}$

$x_{-1.77}^{1.80} \quad \bar{B}$

-1.75 南

$-1.74$

$-1.71$

1.66

-1.51 每

$\begin{array}{ll}-1.50 & \text { 苟 } \\ -1.49 & \end{array}$ 

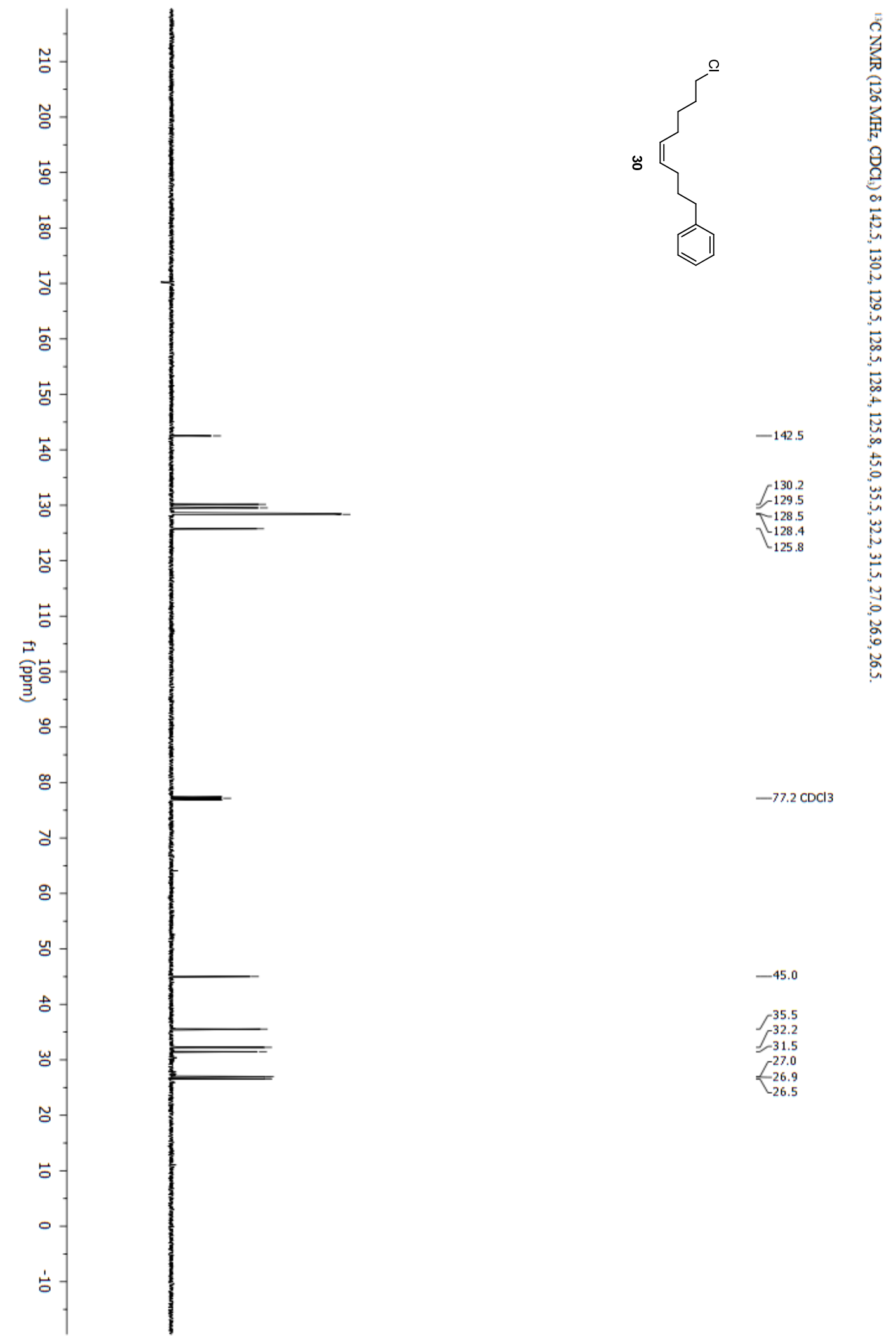

$-77.2 \mathrm{CDCl} 3$

$-45.0$

$\gamma^{35.5}$

$-31.5$

$<_{-26.9}^{27.0}$

26.5 


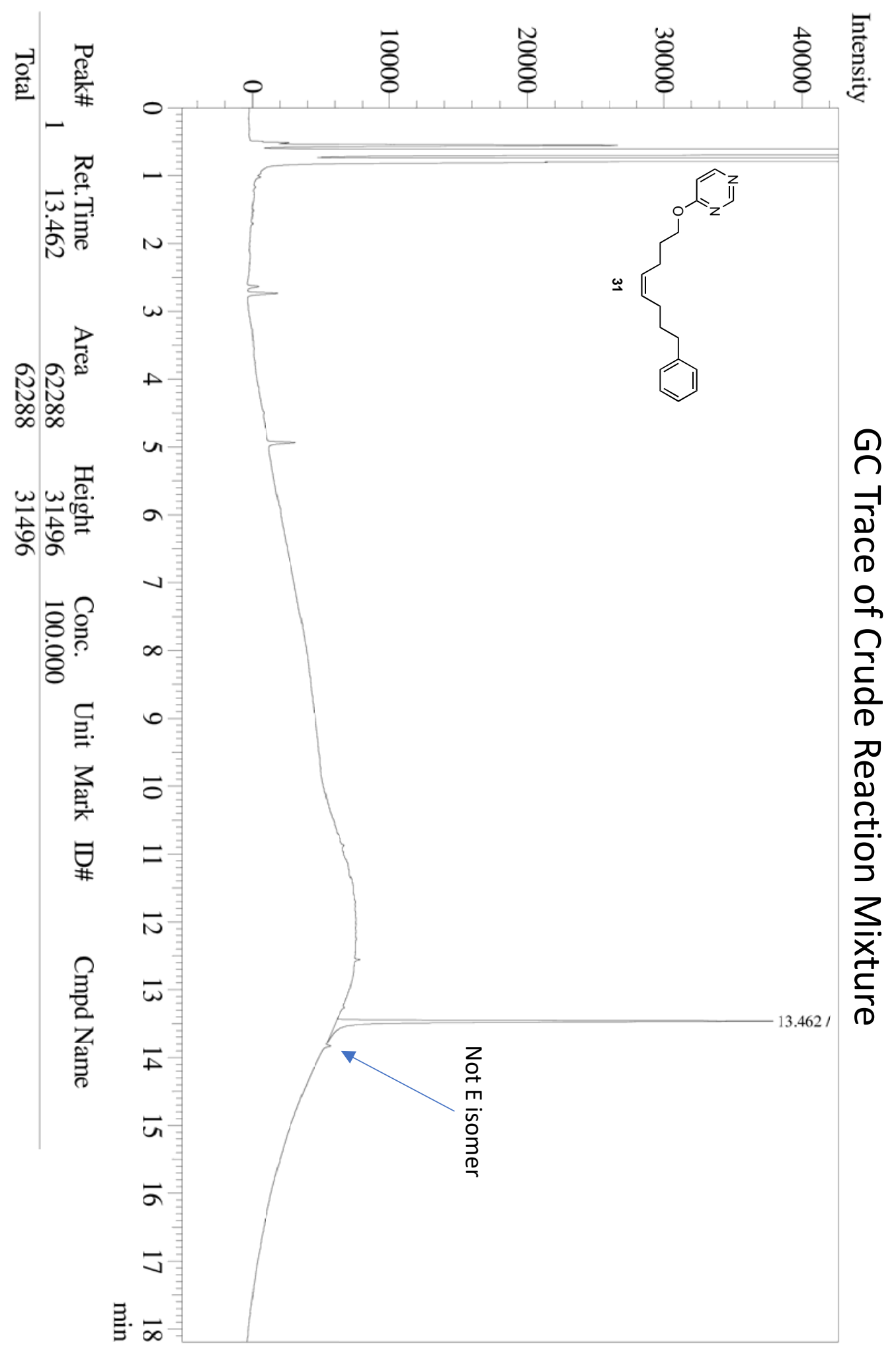



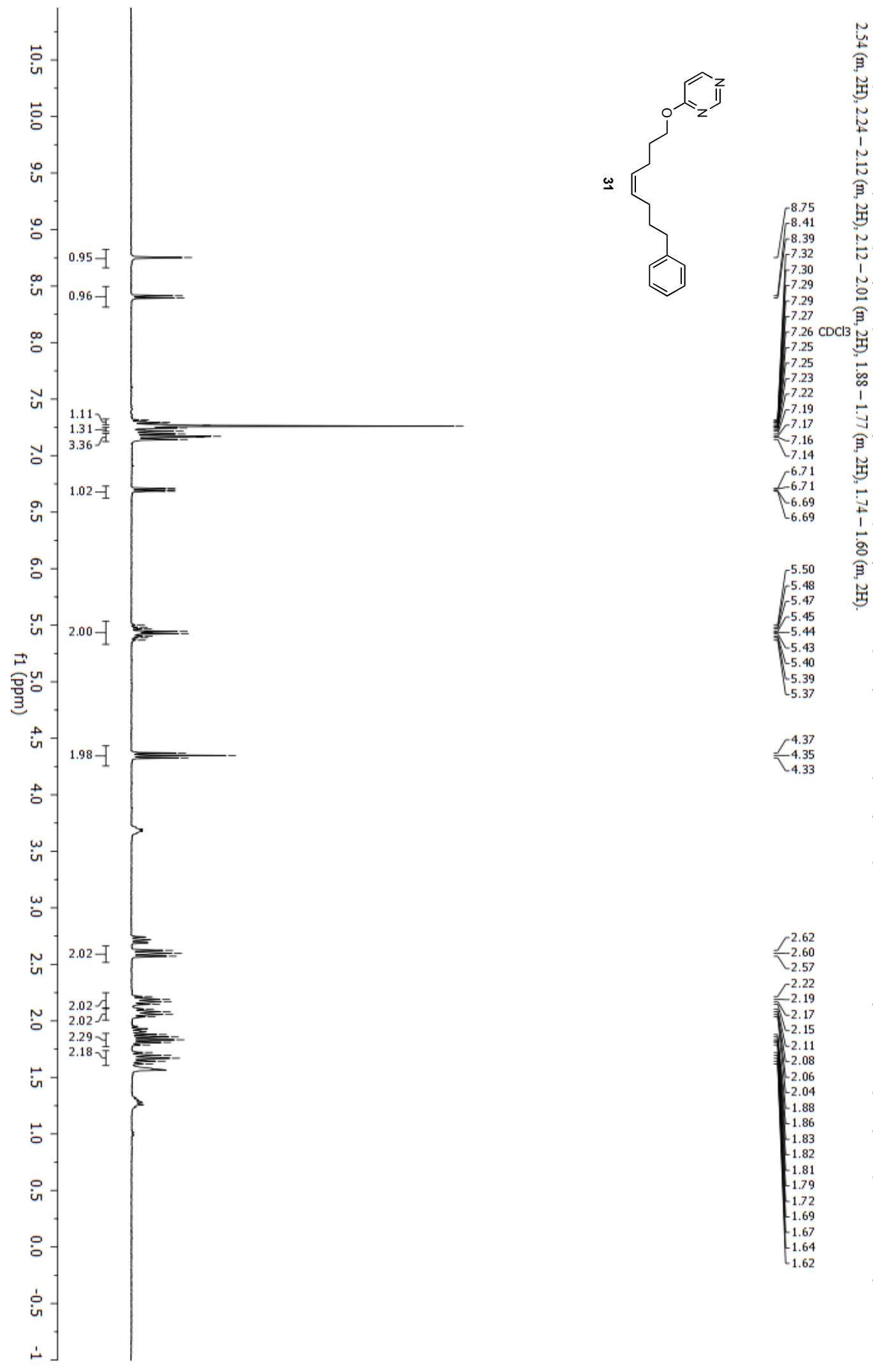

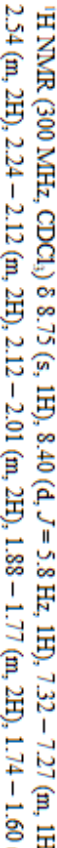

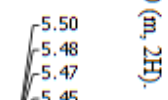

5.47
-5.45

5.44

5.43
5.40

$\begin{array}{rr}5.39 & \text { 年 } \\ 5.37 & \end{array}$

4.37
-4.35
4.33
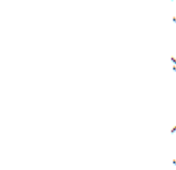

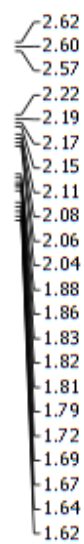

岃

岕 공 

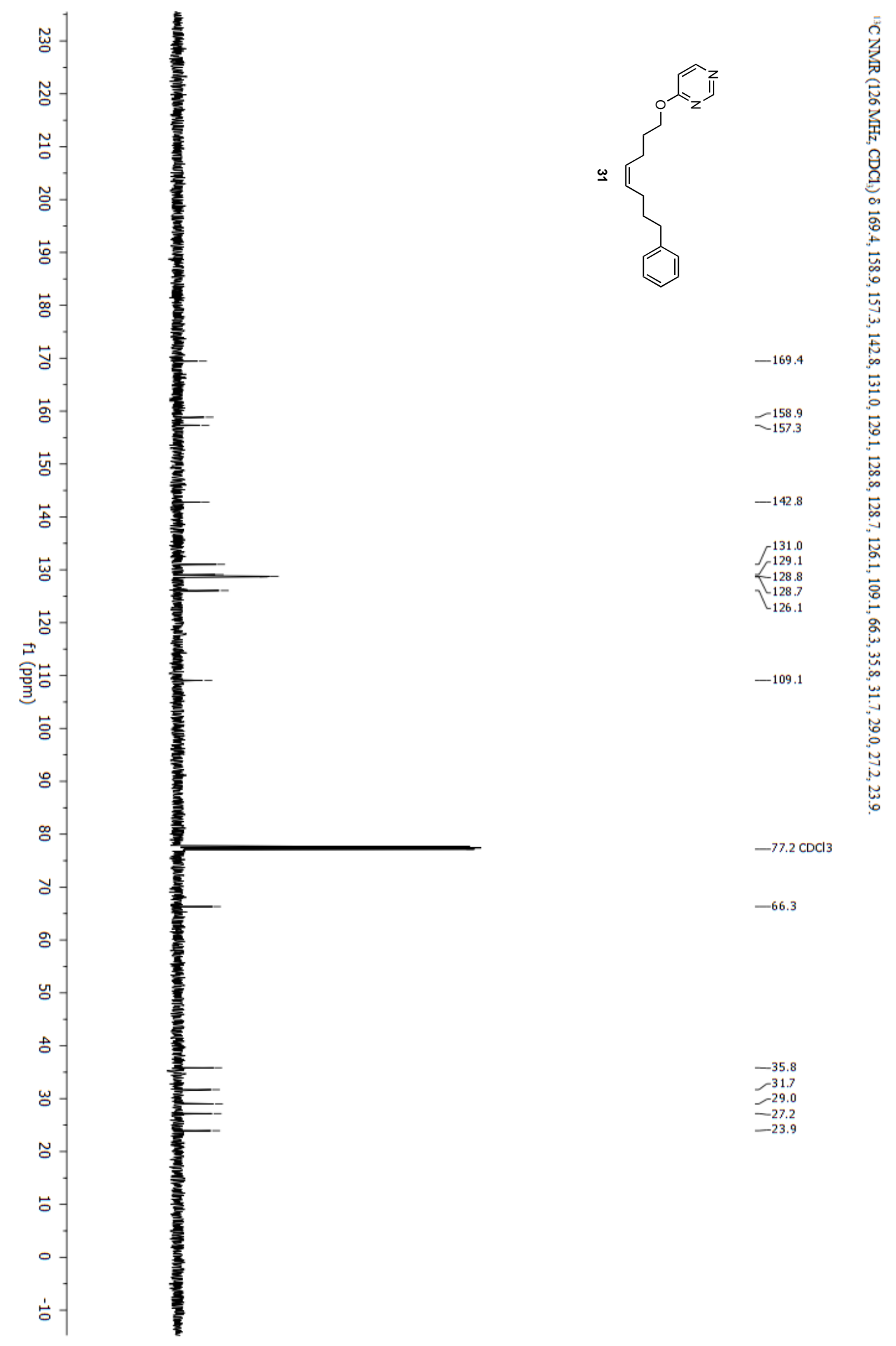

$-77.2 \mathrm{CDCl} 3$

$-66.3$

-35.8
-31.7

$-29.0$

$-27.2$ 


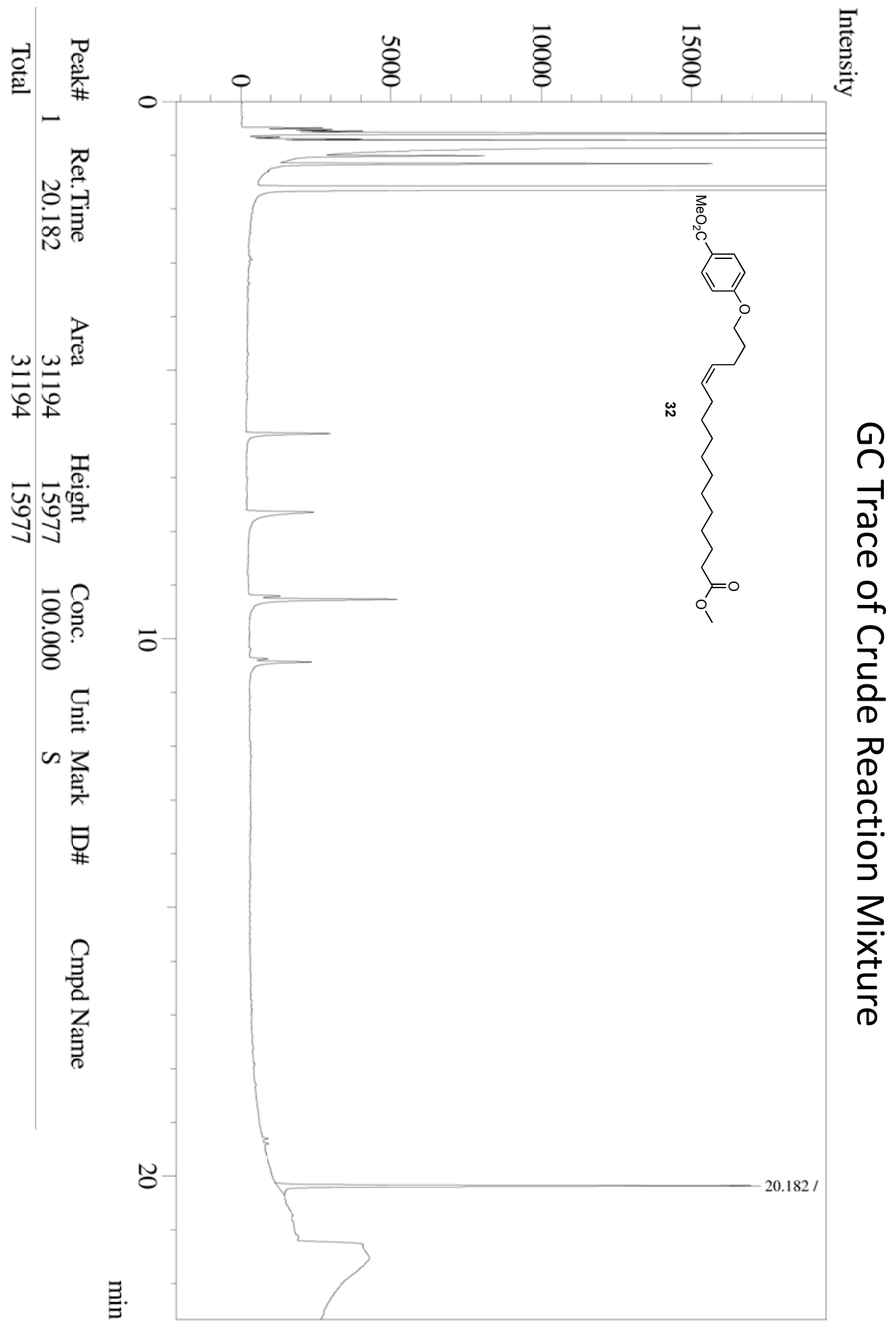



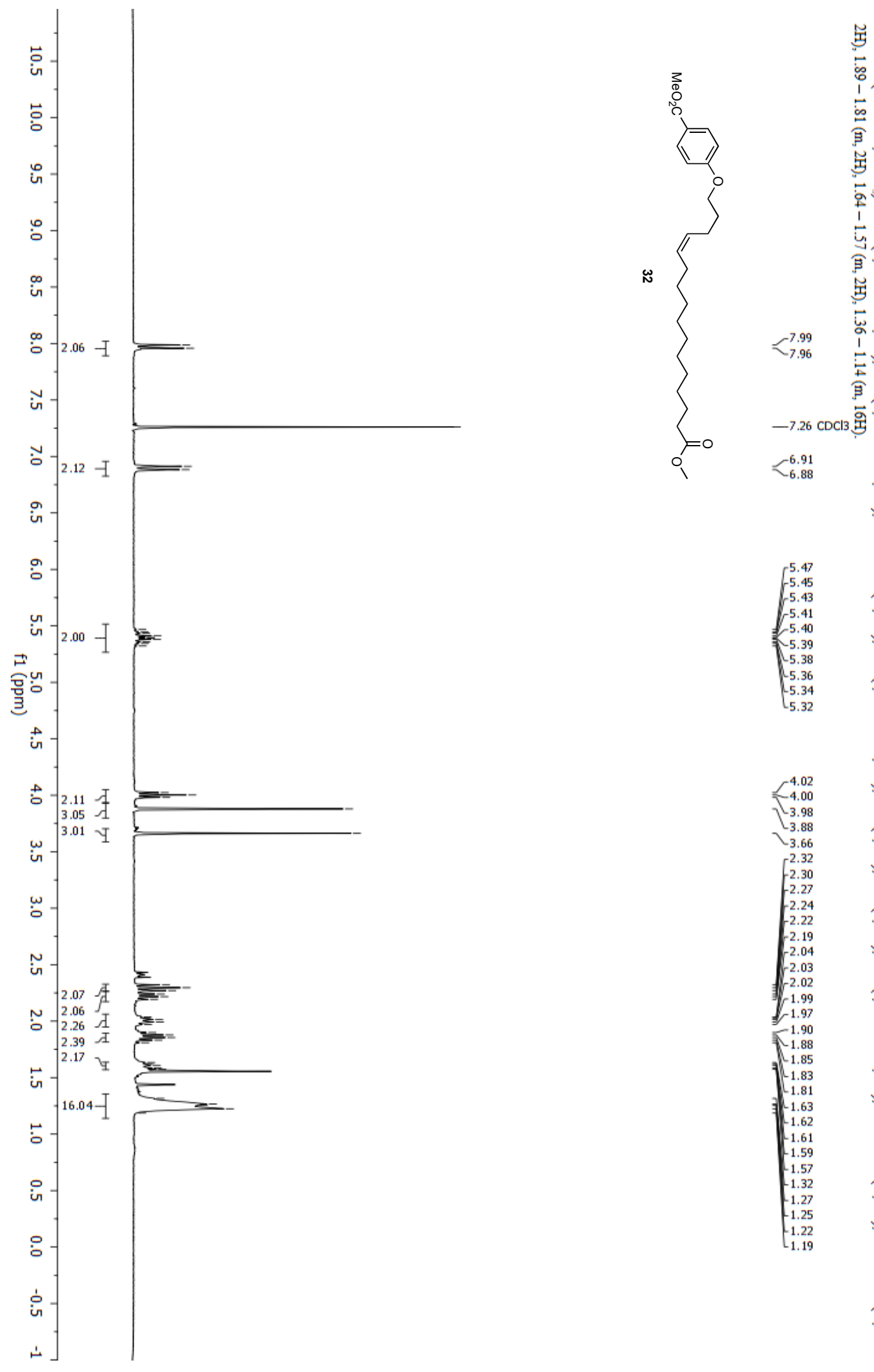

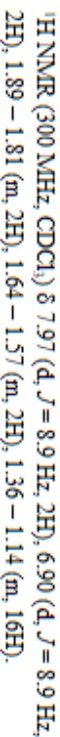

采

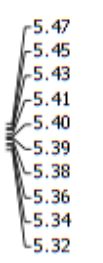

政

$\int^{4.02}$

$\tau_{-3.98}^{-4.00}$

$\sqrt{3.66}$

$\overbrace{}^{2.32}-2.30$

-2.24 กิ

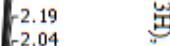

$-2.03 \quad$ 亗

$\left[\begin{array}{r}2.02 \\ 1.99 \\ -1.97 \\ 1.90\end{array}\right.$

$\left\{\begin{array}{l}1.90 \\ 1.88 \\ 1.85\end{array}\right.$

$\begin{cases}1.85 & \text { 苟 } \\ 1.83 & \end{cases}$

年 1.63

-1.61
-1.59
-1.57

$1.57 \quad$

$\begin{array}{ll}-1.32 & \text { B } \\ -1.27 & -1.25\end{array}$

-1.25 采
-1.22

1.19 용

훙

令 

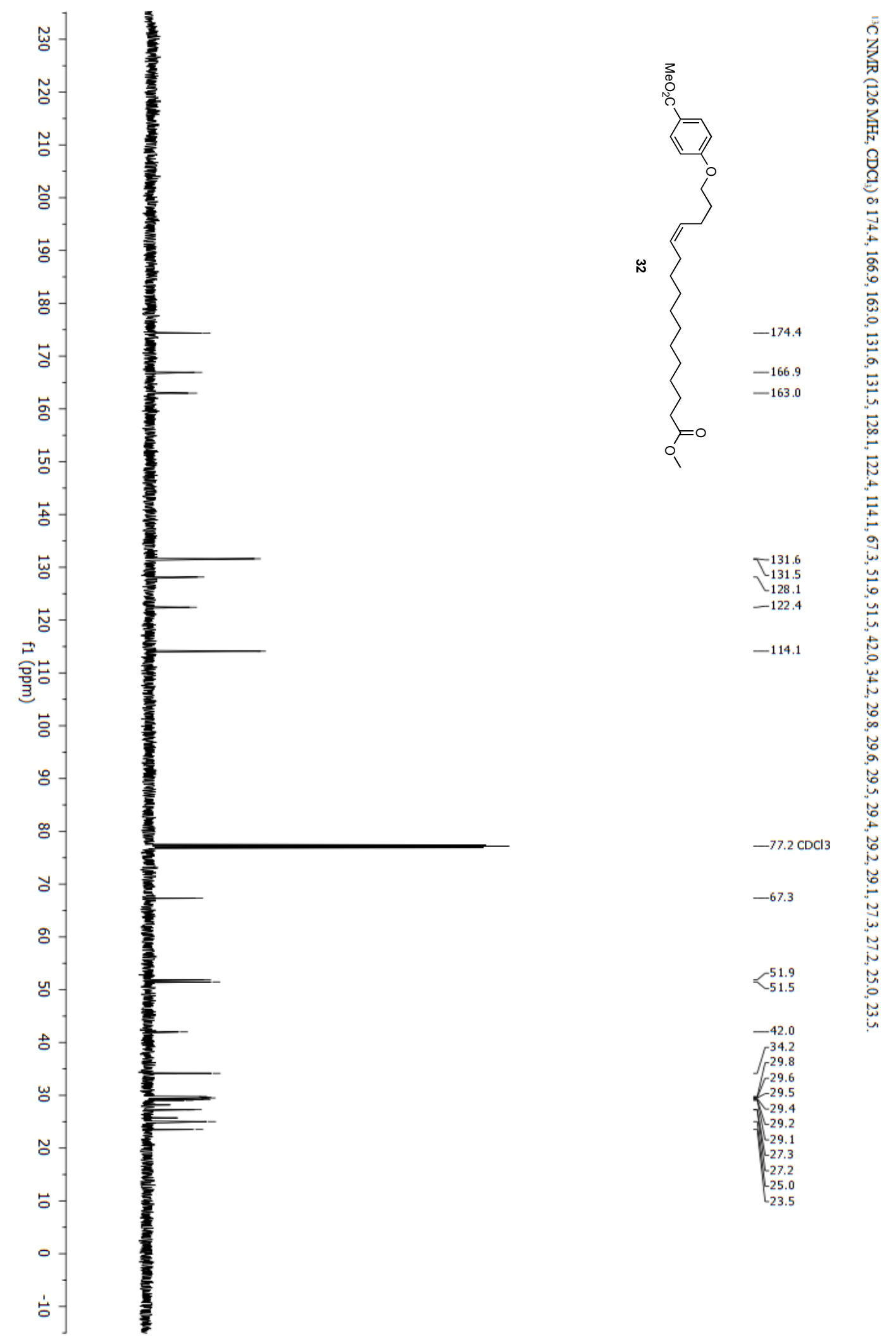


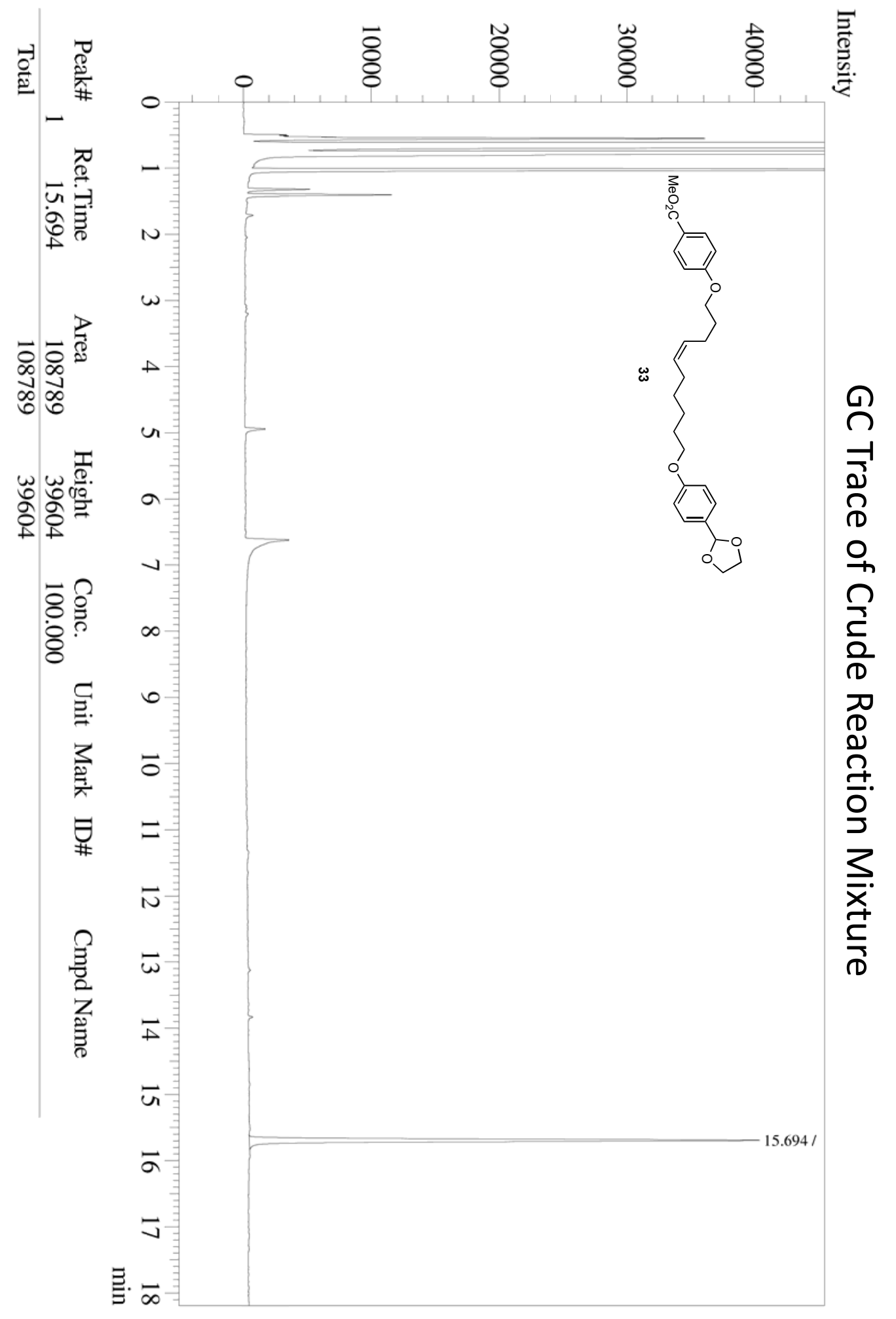



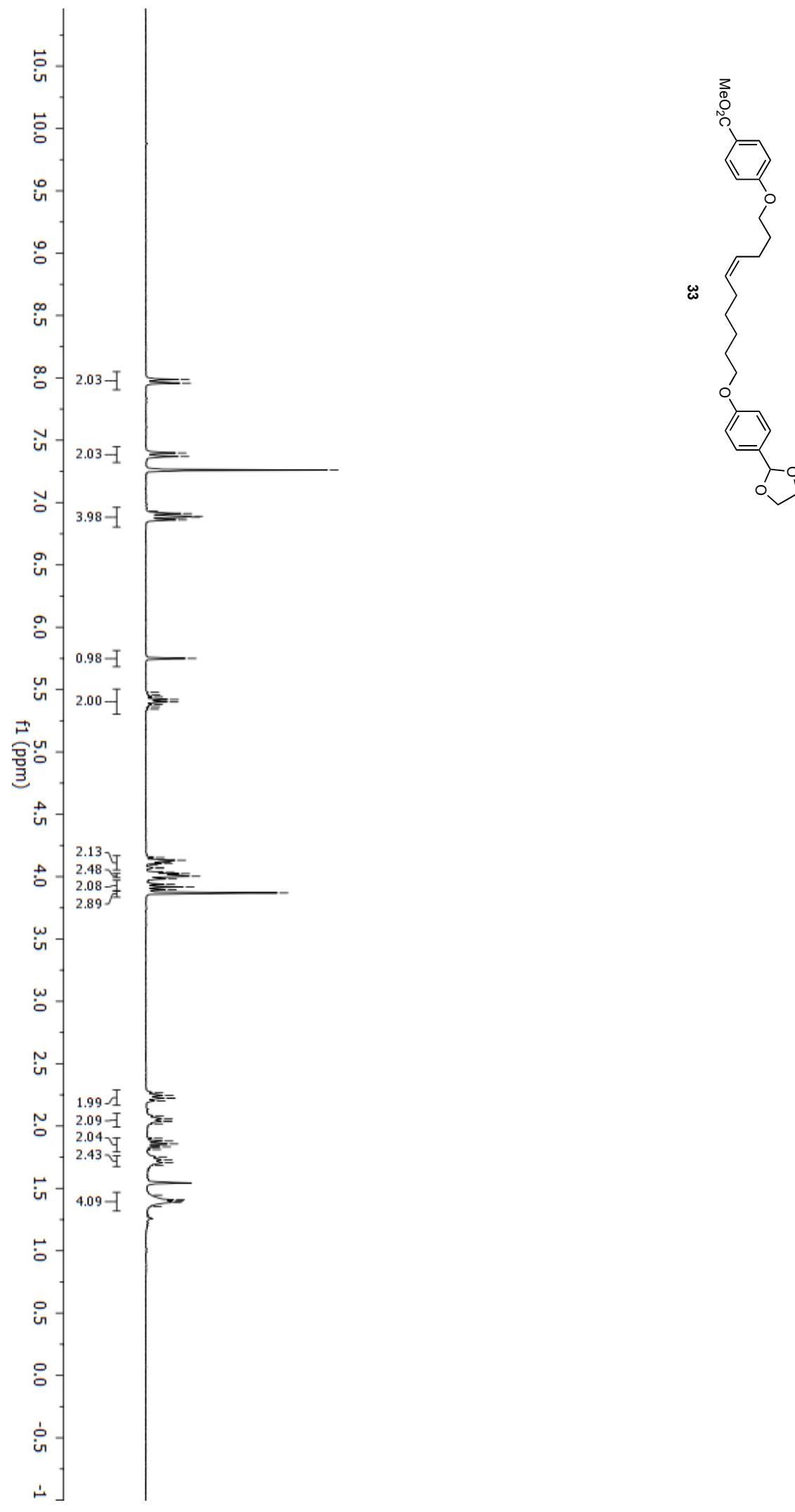

落

悬

光宂

苟負

동

13

8

을

:

我

思

荡

茑崖

点

-j $-7.26 \mathrm{CDCl} 3$ \%

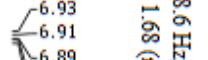

6.88 B

6.86 缹

$\int_{-5.48}^{5.75}-8$

$\begin{array}{rrr}-5.46 & 1 & 1 \\ -5.44 & 1 & 0 \\ 5 & 0 & 0\end{array}$

5.42 .

C5.38 $\mathrm{B}$

- 5.36 齿

5.15
4.13

$-4.13$

$-4.11 \quad$

4.10
-4.07

$4.07 \quad 1$

$-4.02$

$\overbrace{-3.98}^{4.00}$

$-3.94$

-3.92
-3.90

$\left[\begin{array}{ll}-2.27 \\ -2.25 \\ -2.25\end{array}\right.$

-2.22 会

-2.22 苟

2.08

$C_{2.04}^{2.04} 1$

-2.02 is

$1.90 \quad$ B

$\begin{array}{ll}1.88 & \text { 采 } \\ -1.86 & \end{array}$

-1.83 is

-1.81
-1.75

$-1.75$

$-1.73$

1.79

-1.69 \&

$-1.41$

-1.39 要

-1.35

要 

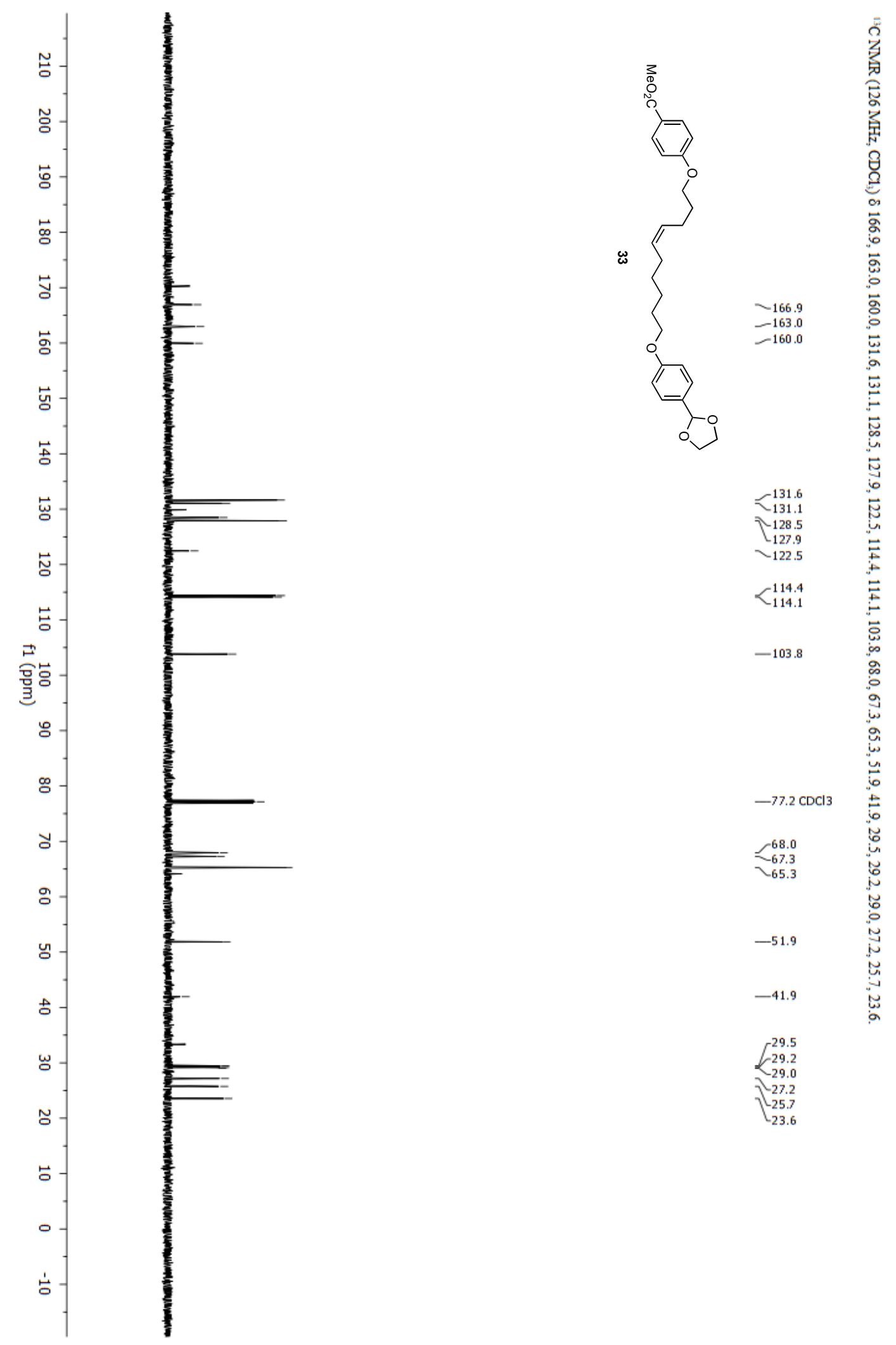


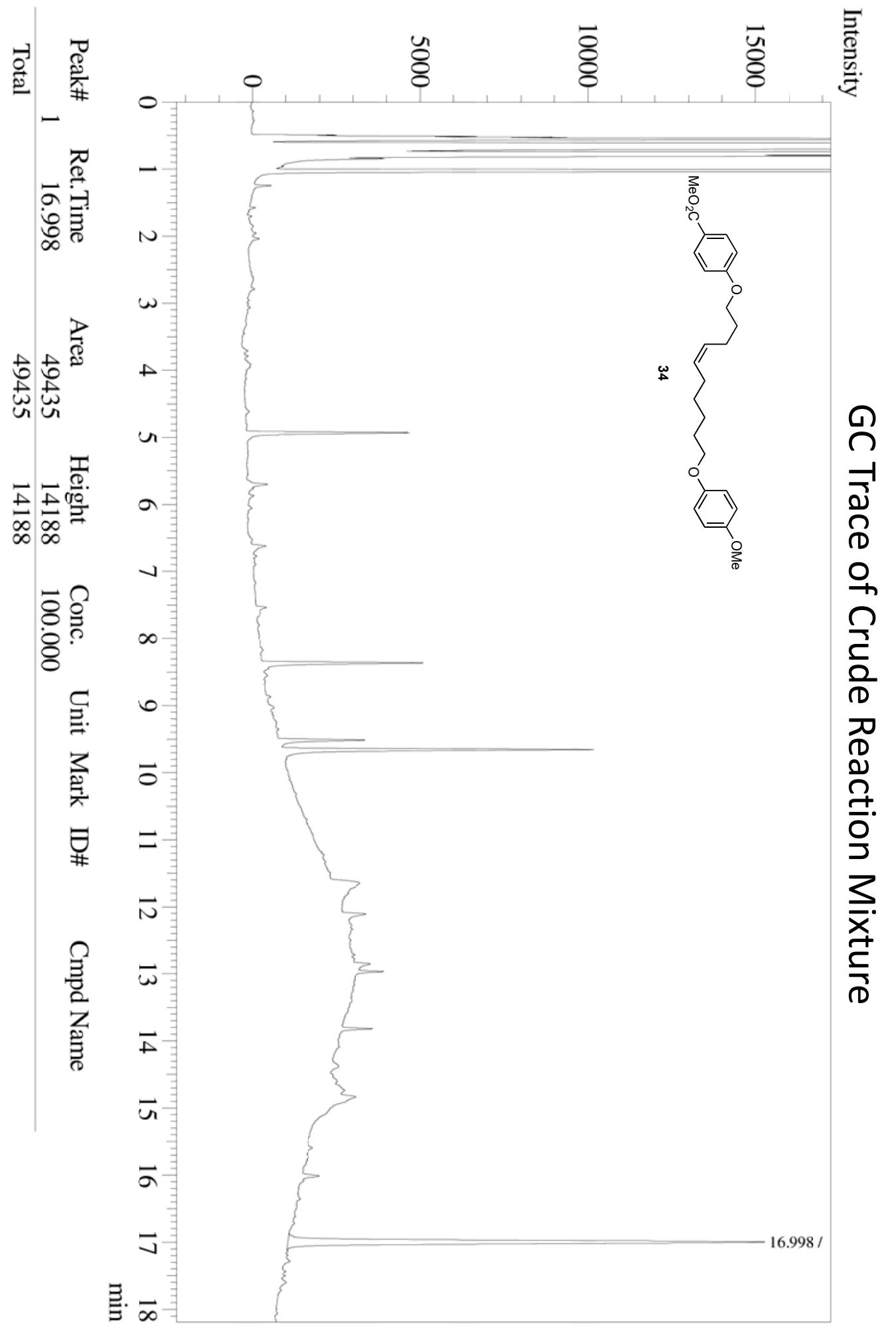




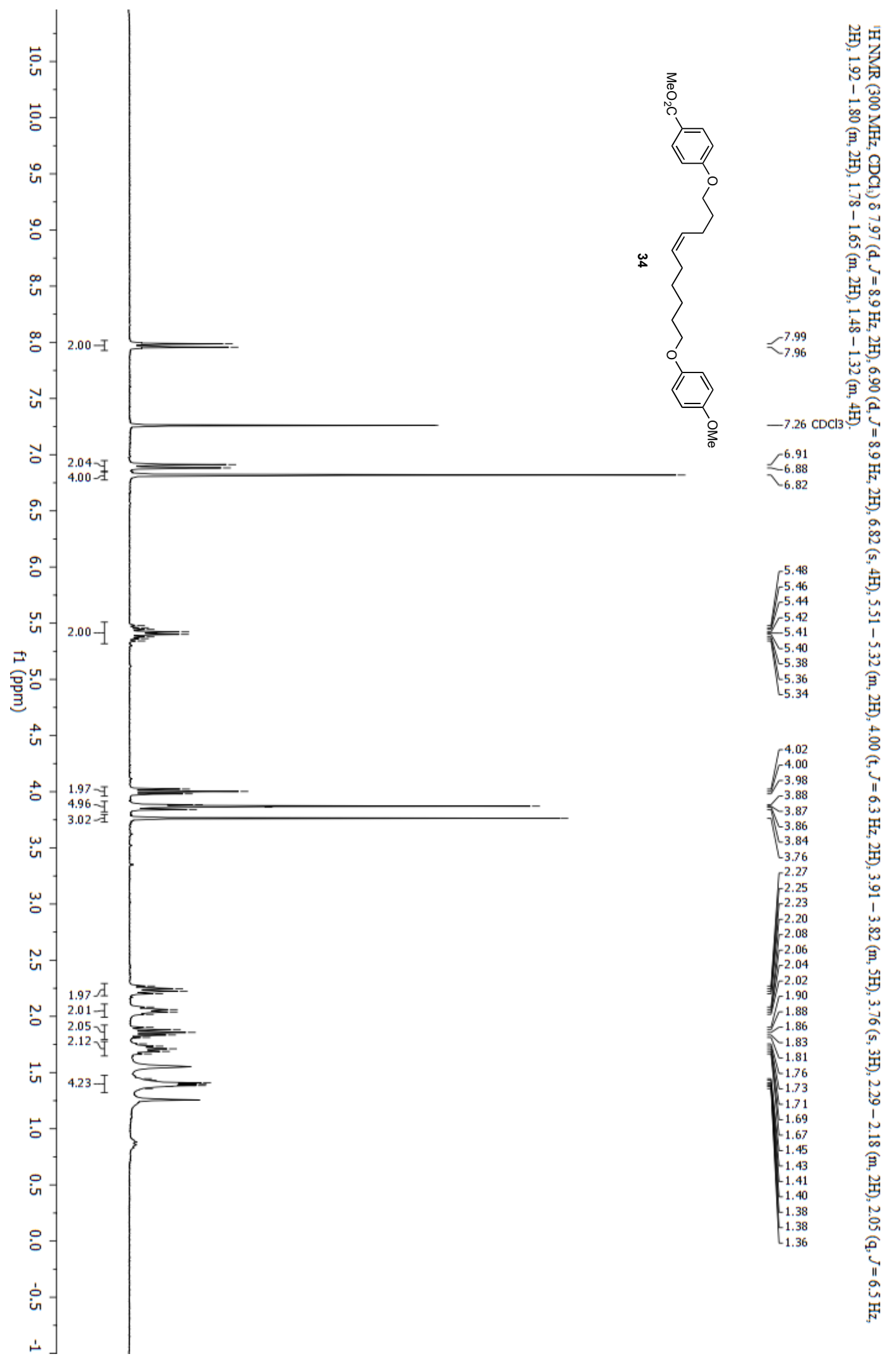



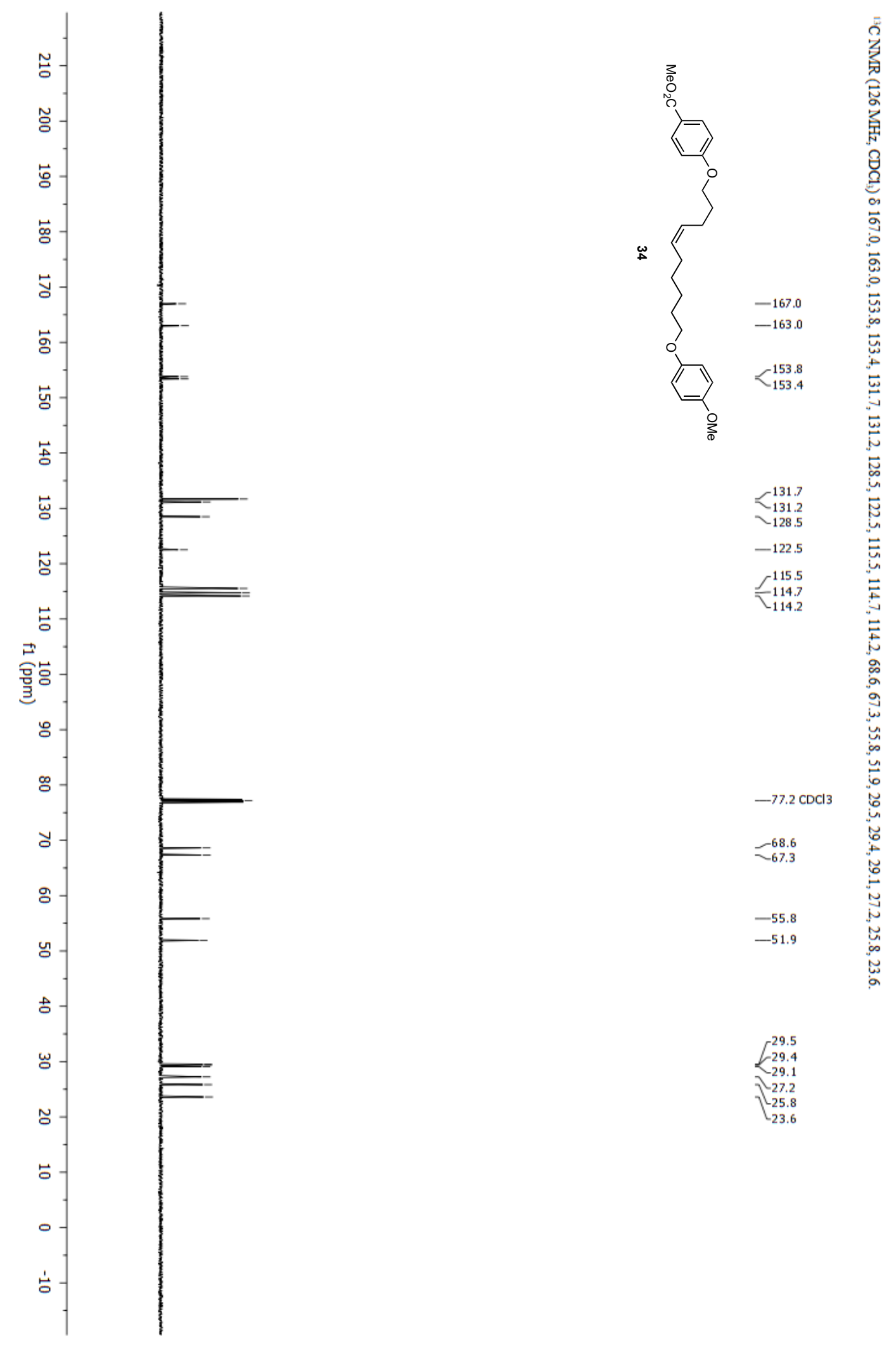


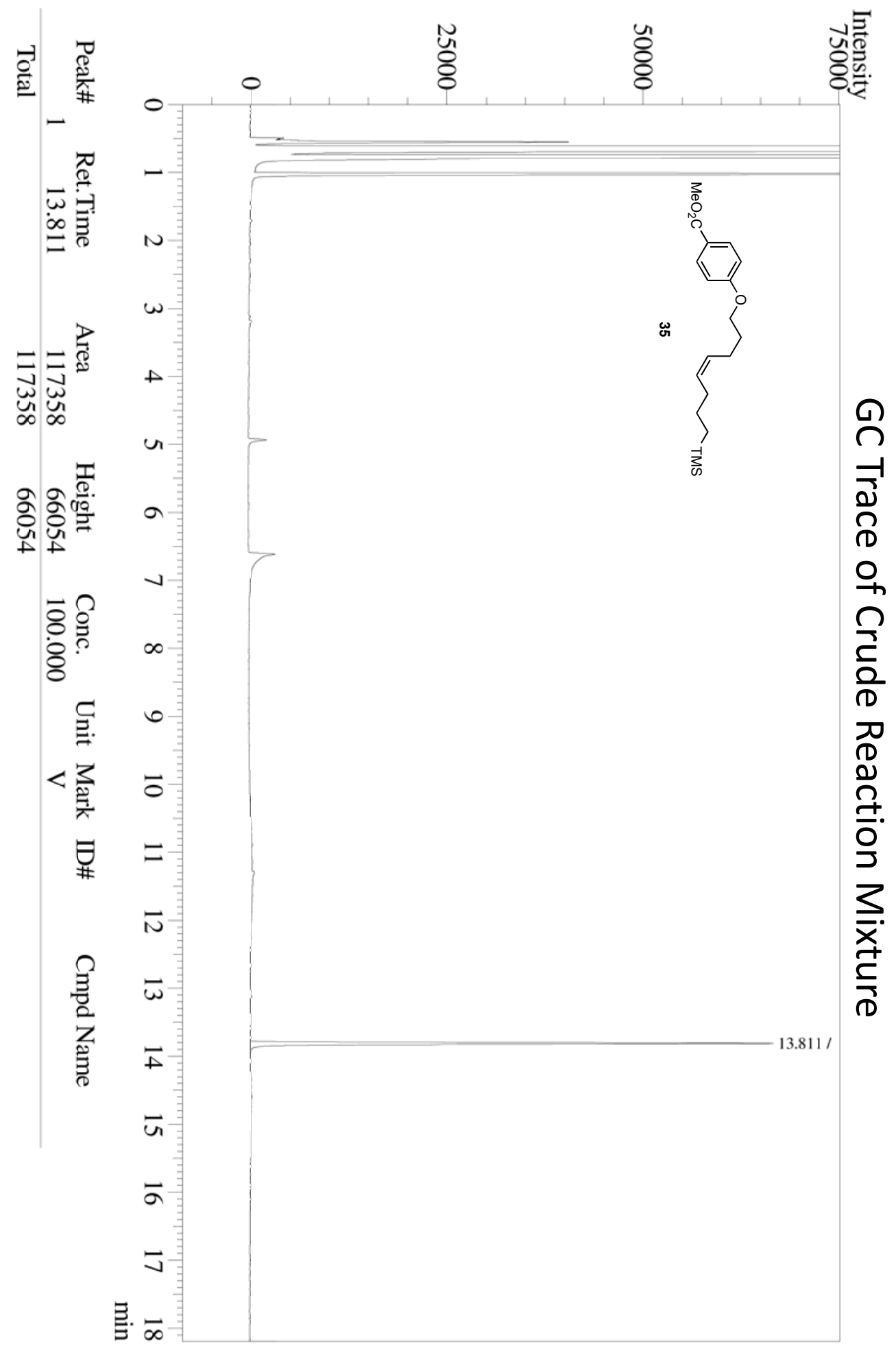




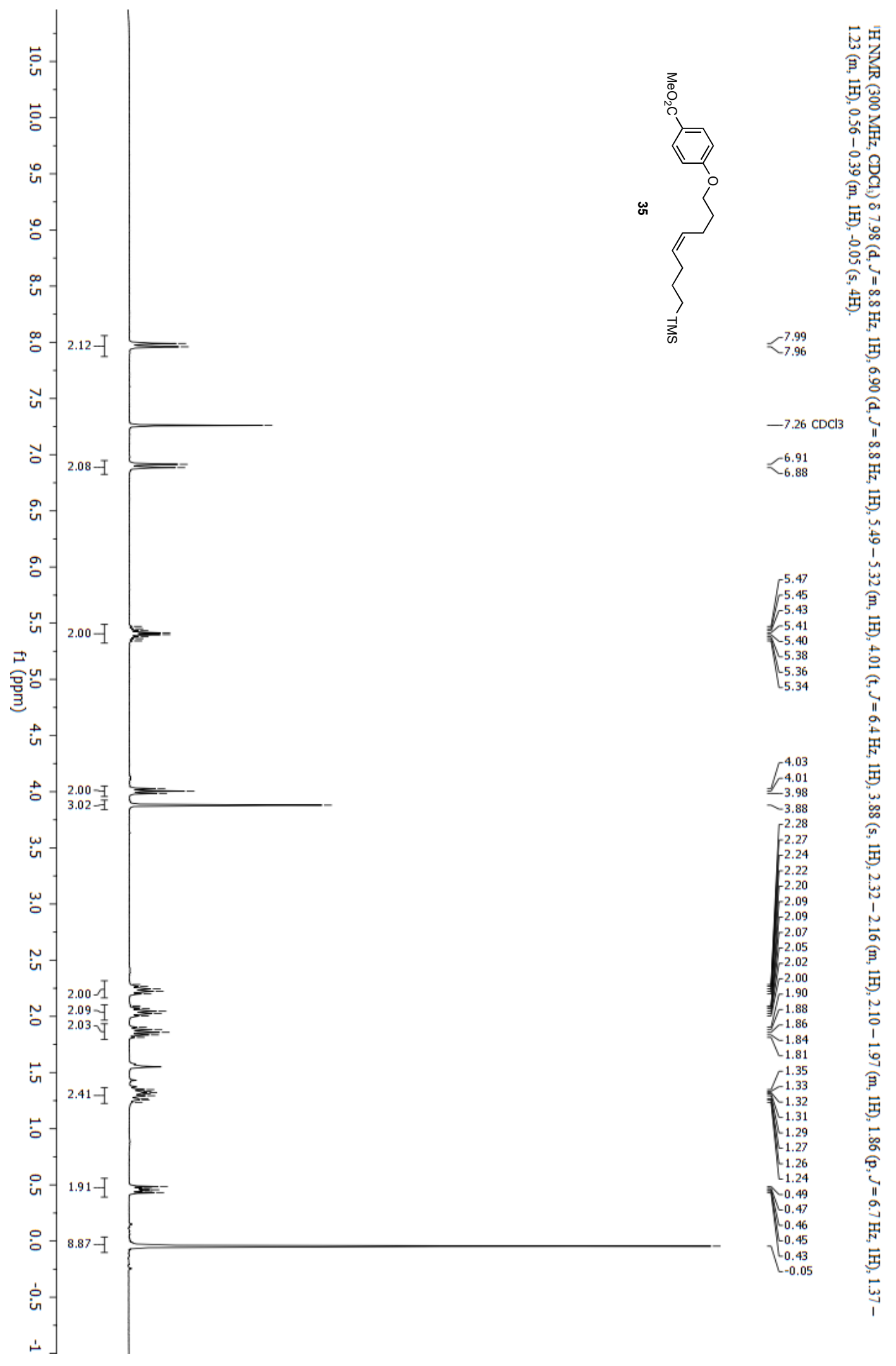



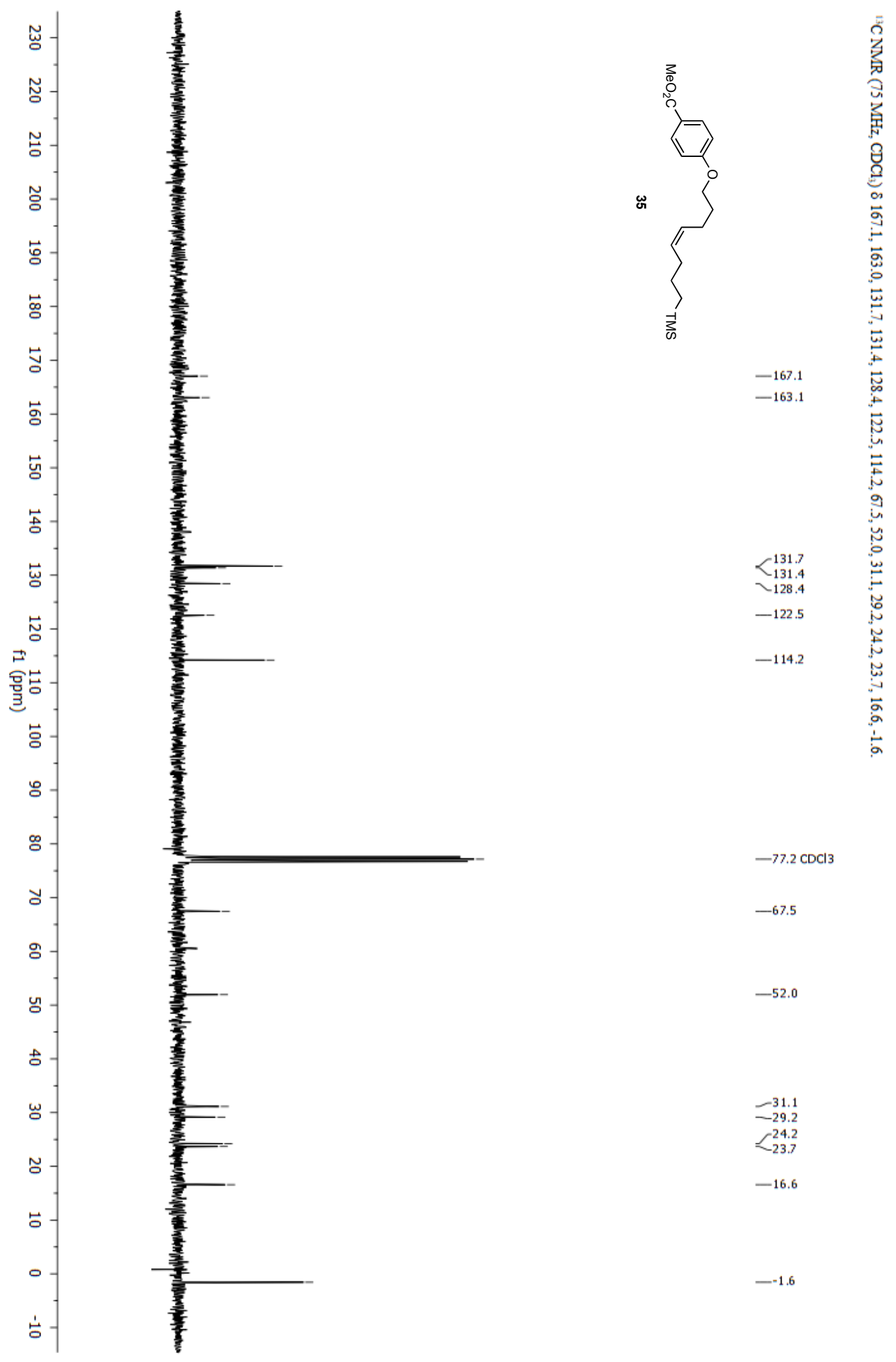


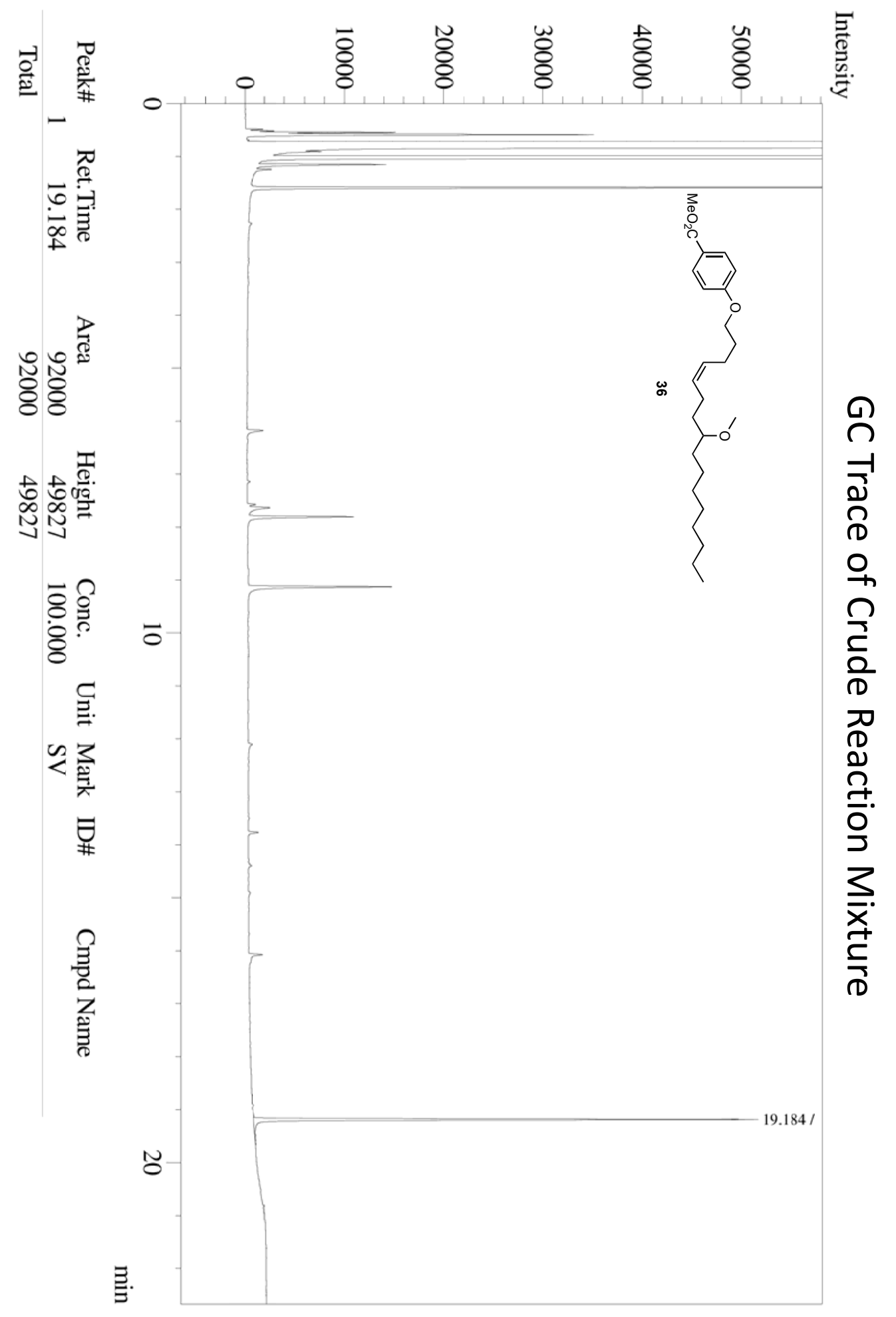



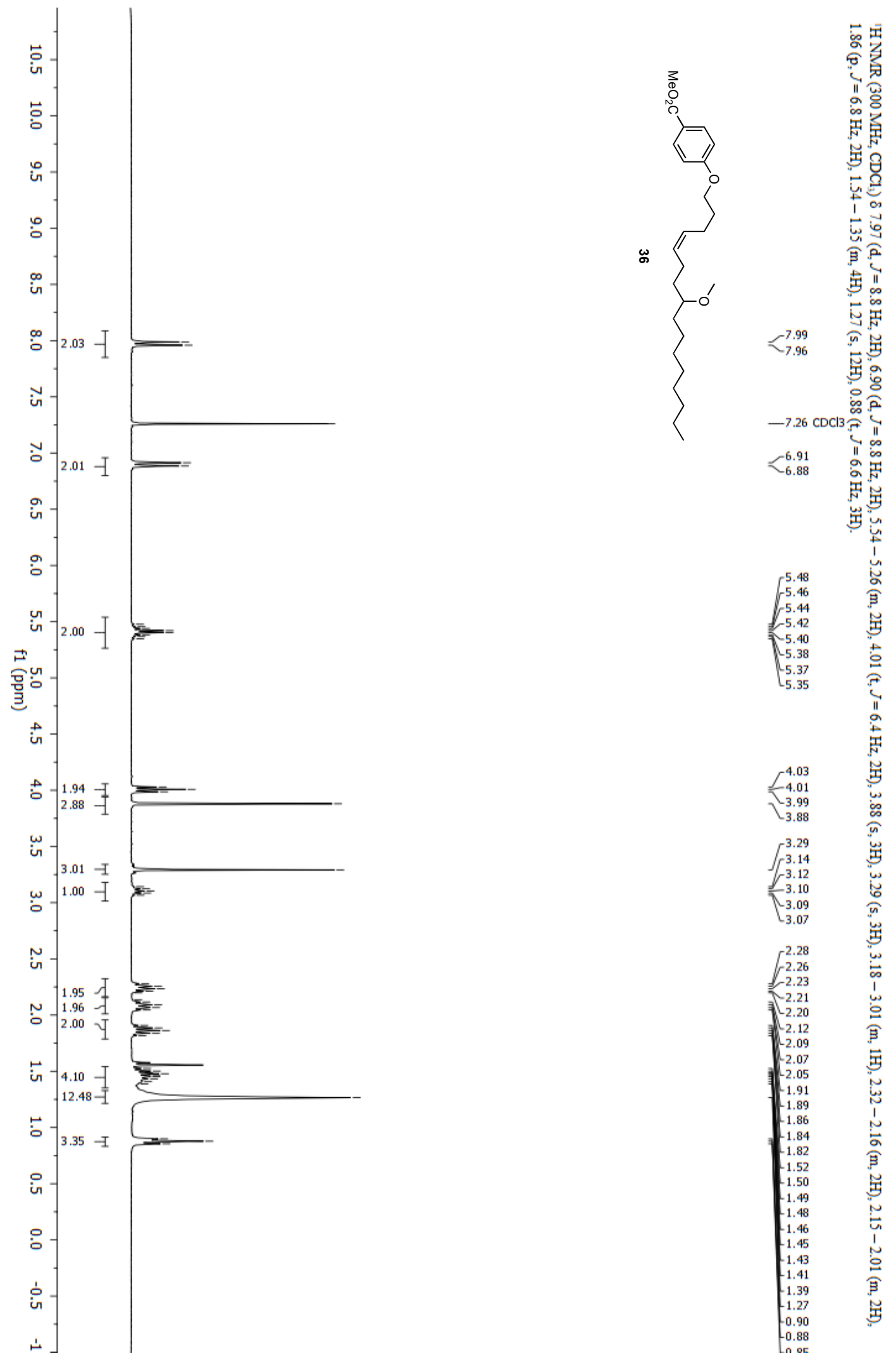

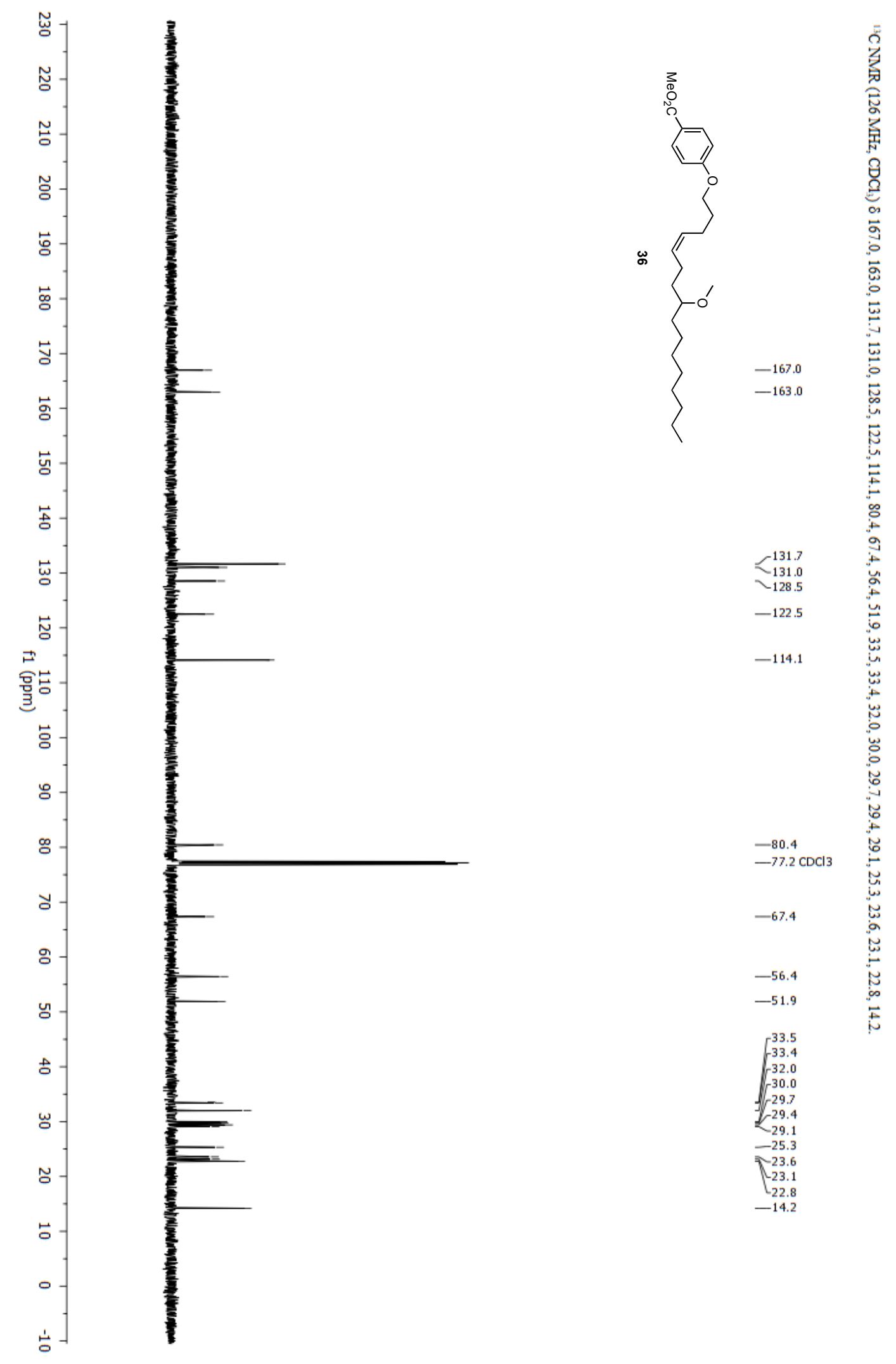


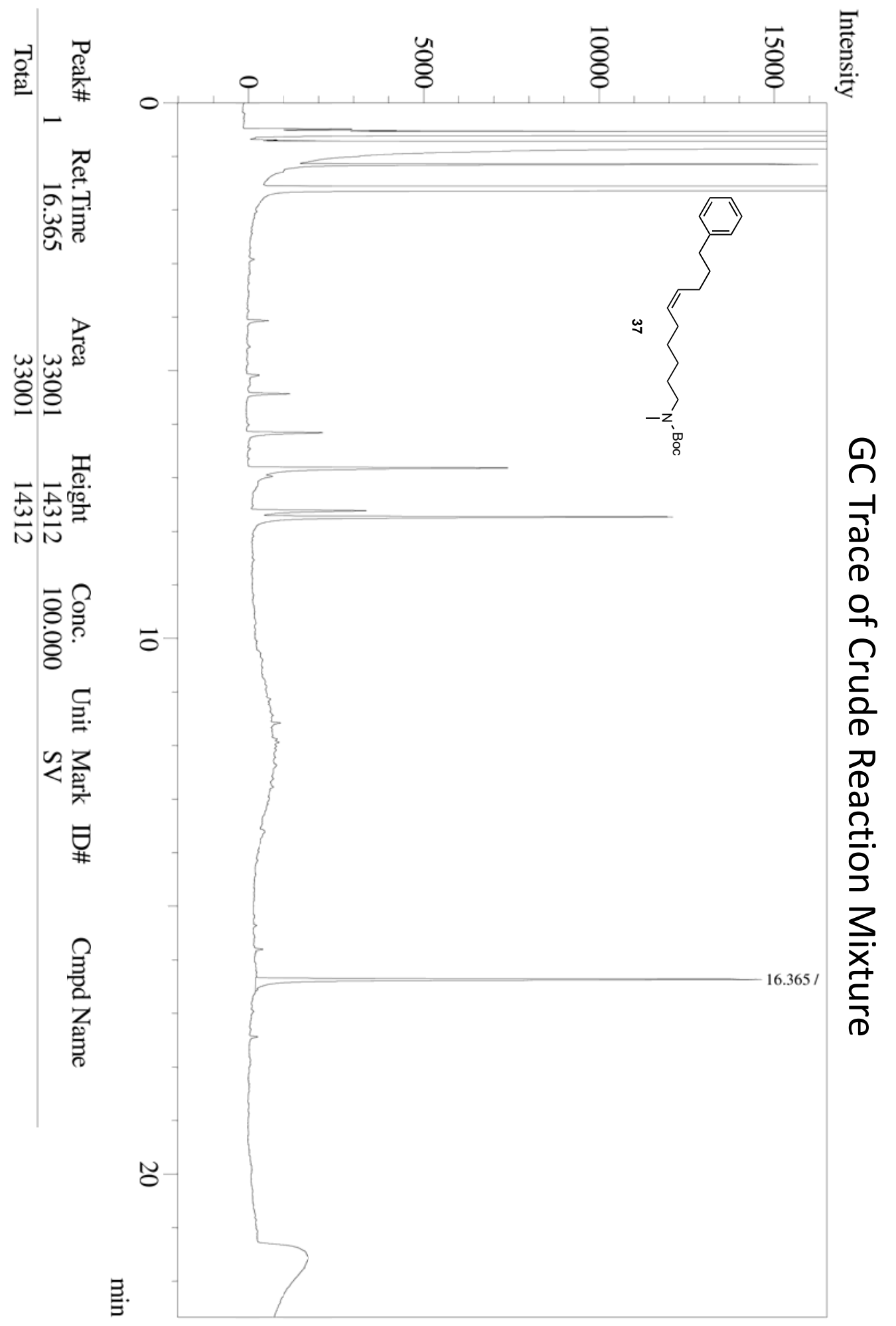



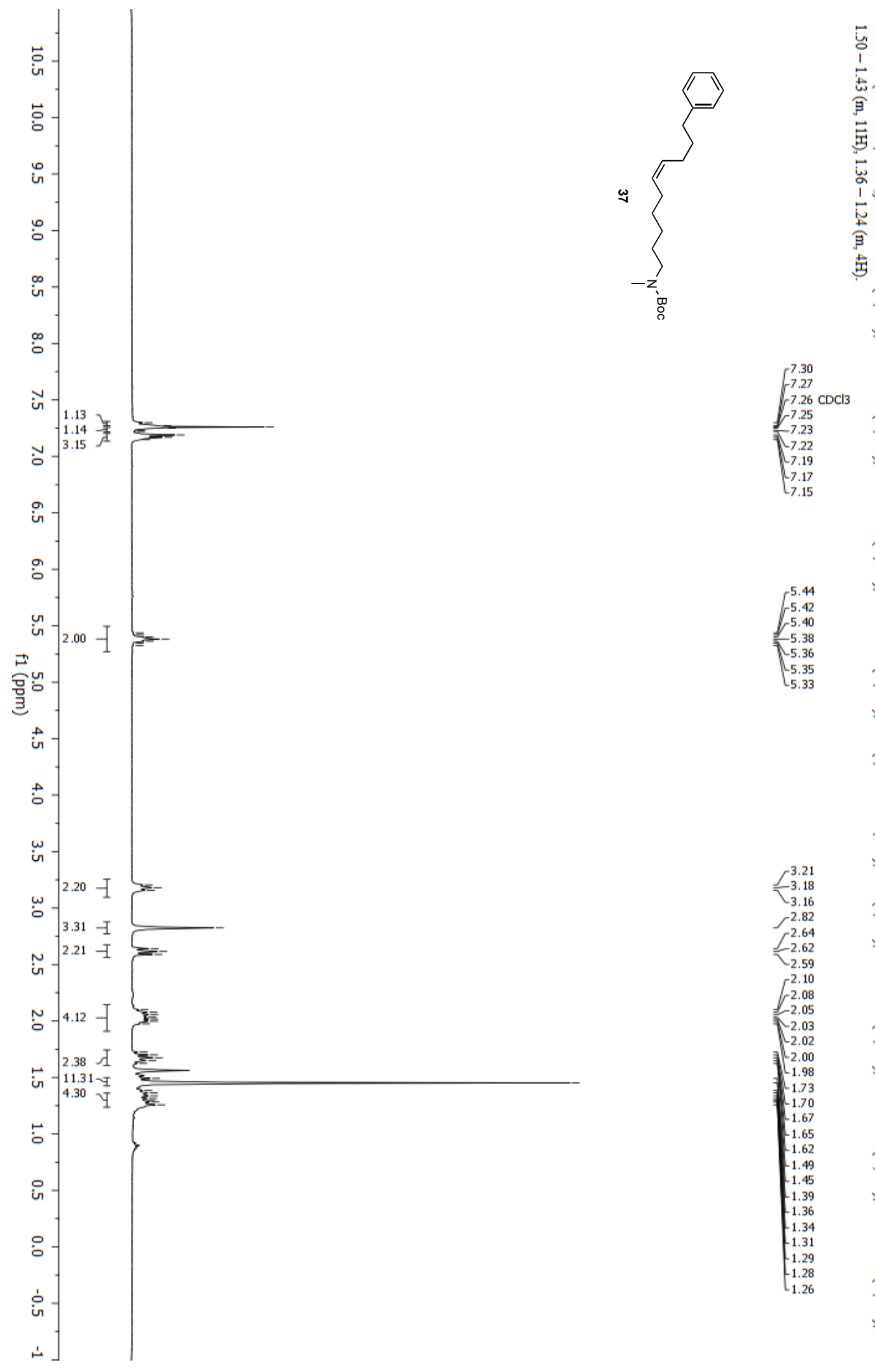

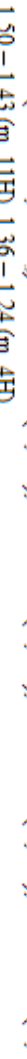




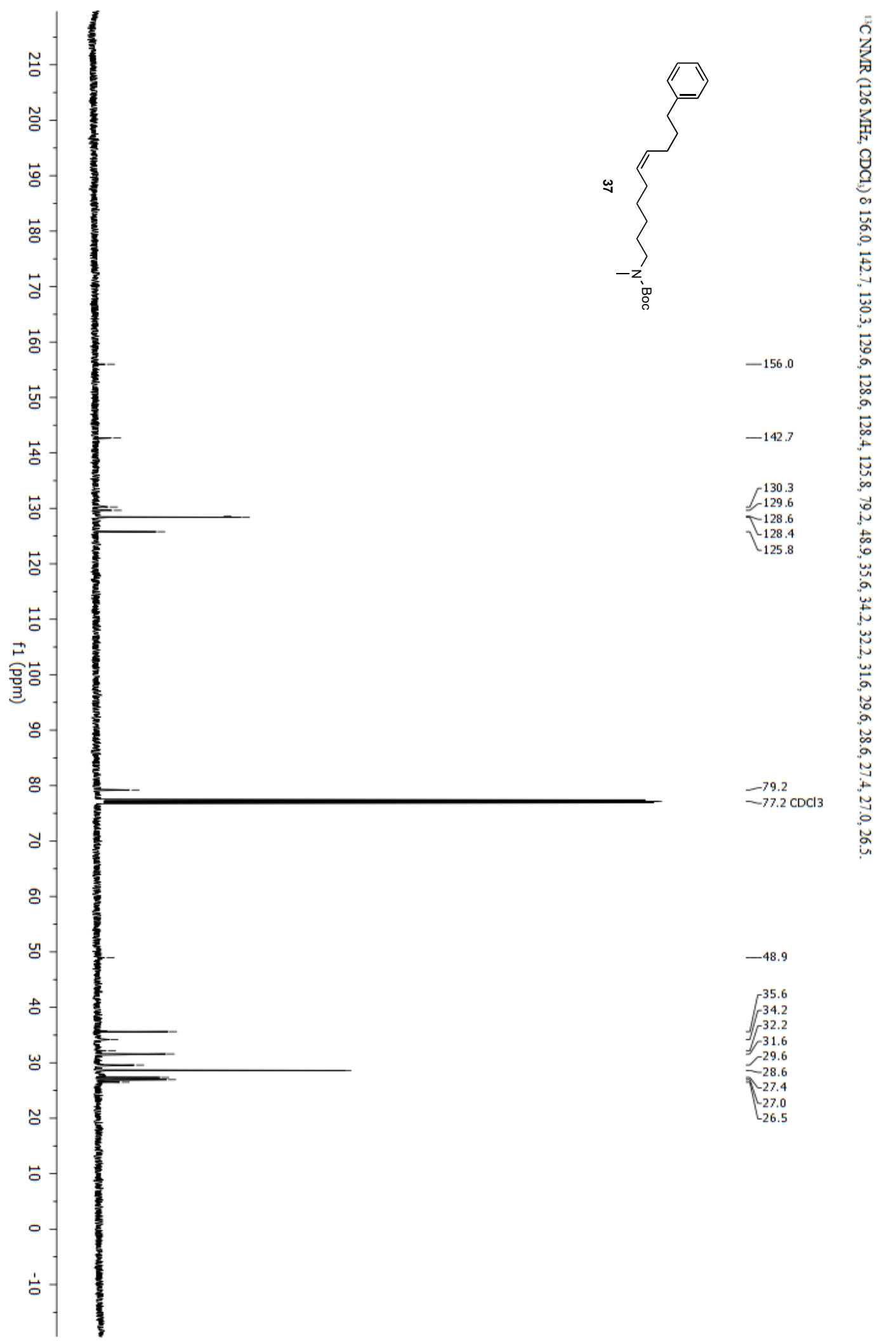




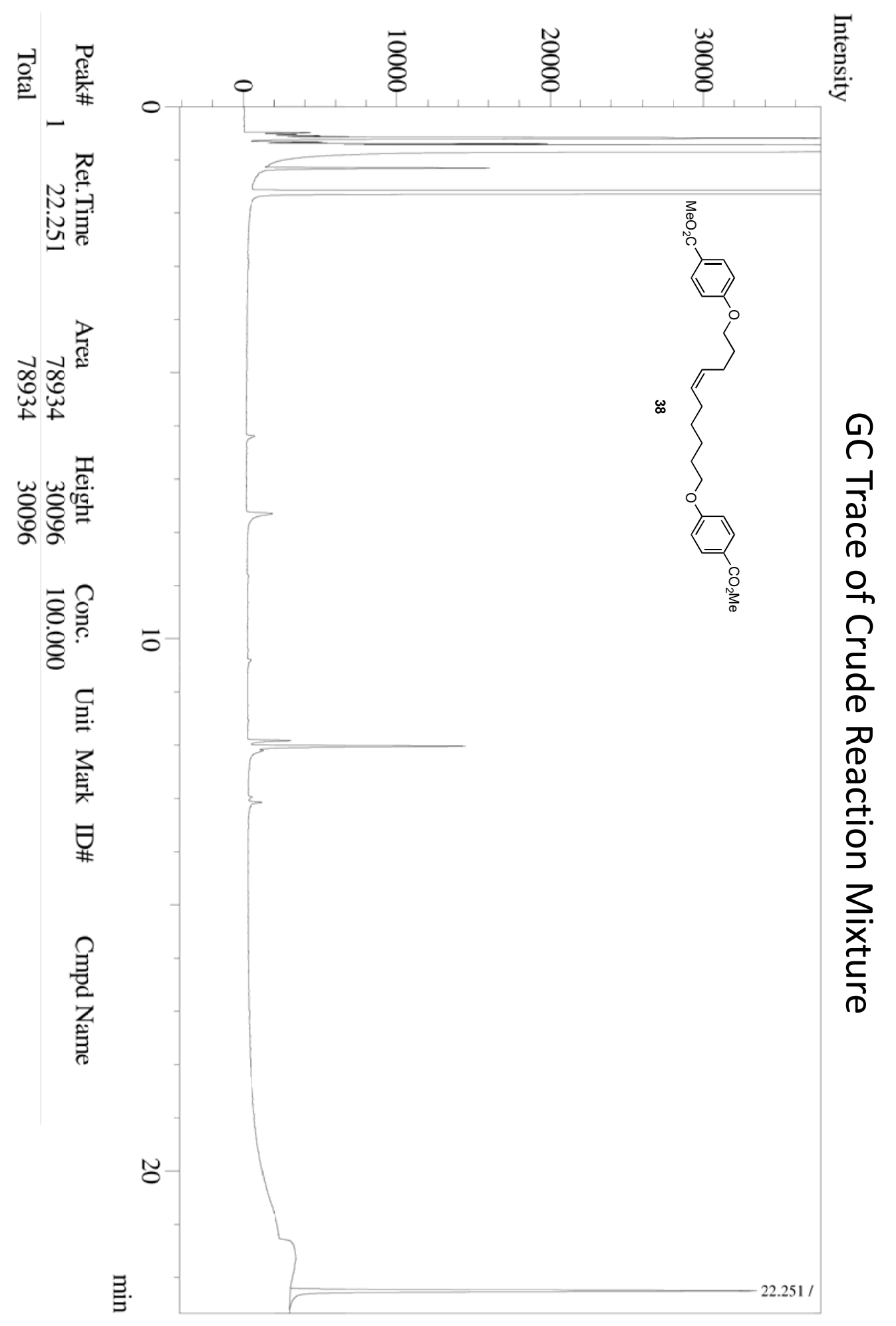



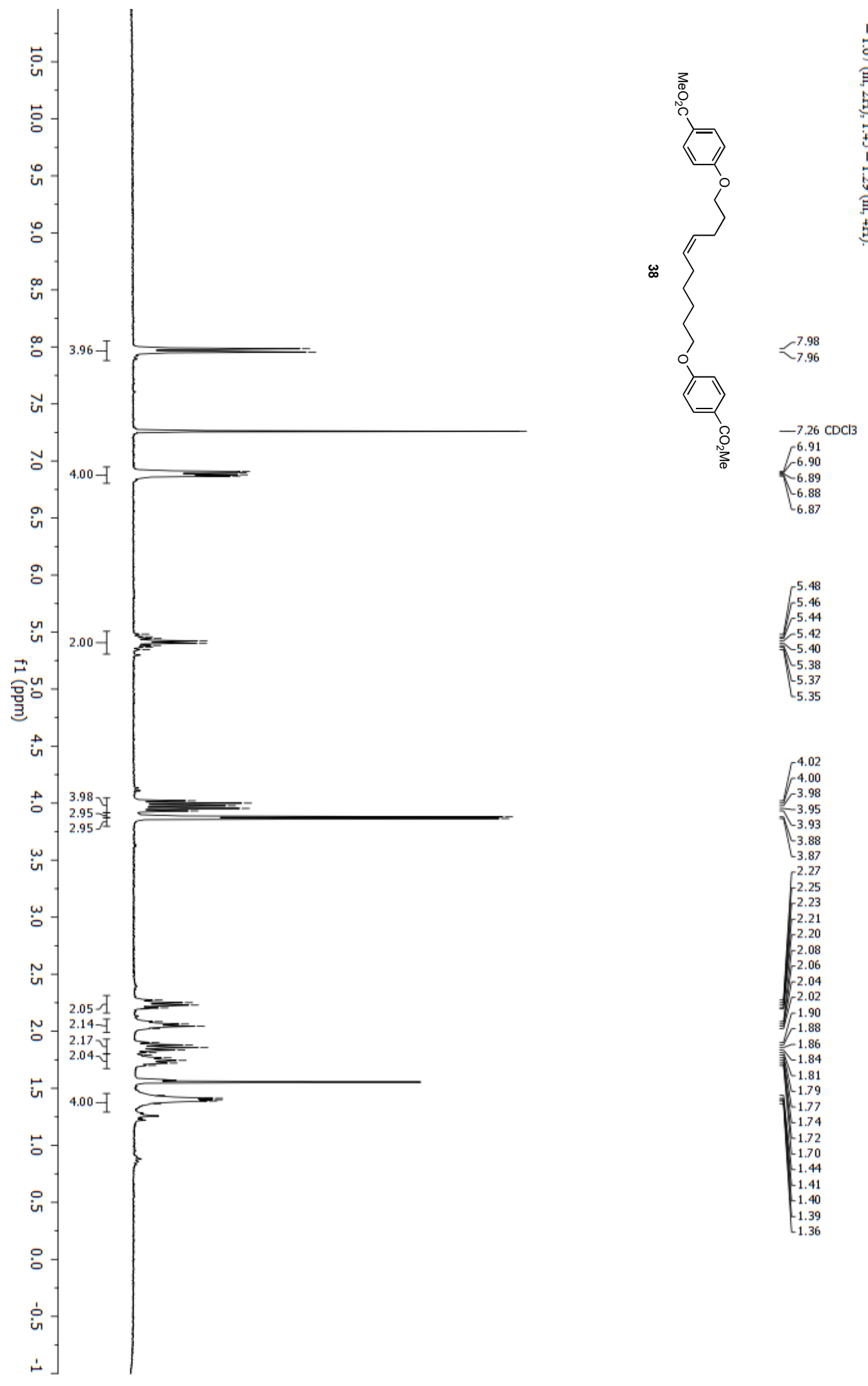

它究

官㫕

画

点界

18

$\div$

专草

e

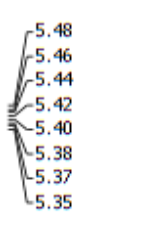

4.02
4.00
-3.98
-3.95

$f_{-3.95}^{-3.98}$

$\mathfrak{\gamma}_{3.88}^{3.93}$

2.87
3.27

$-2.25$

$-2.21$

-2.08 ।

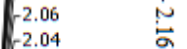

2.02

$=\left[\begin{array}{l}1.90 \\ -1.88\end{array}\right.$

-1.86
-1.84

$n_{1.81}$

$-1.79$

$\begin{array}{ll}-1.77 & 11 \\ -1.74 & \end{array}$

-1.72 i

-1.70
-1.44

-1.41 岁

-1.39
1.36

宛

苛

क 

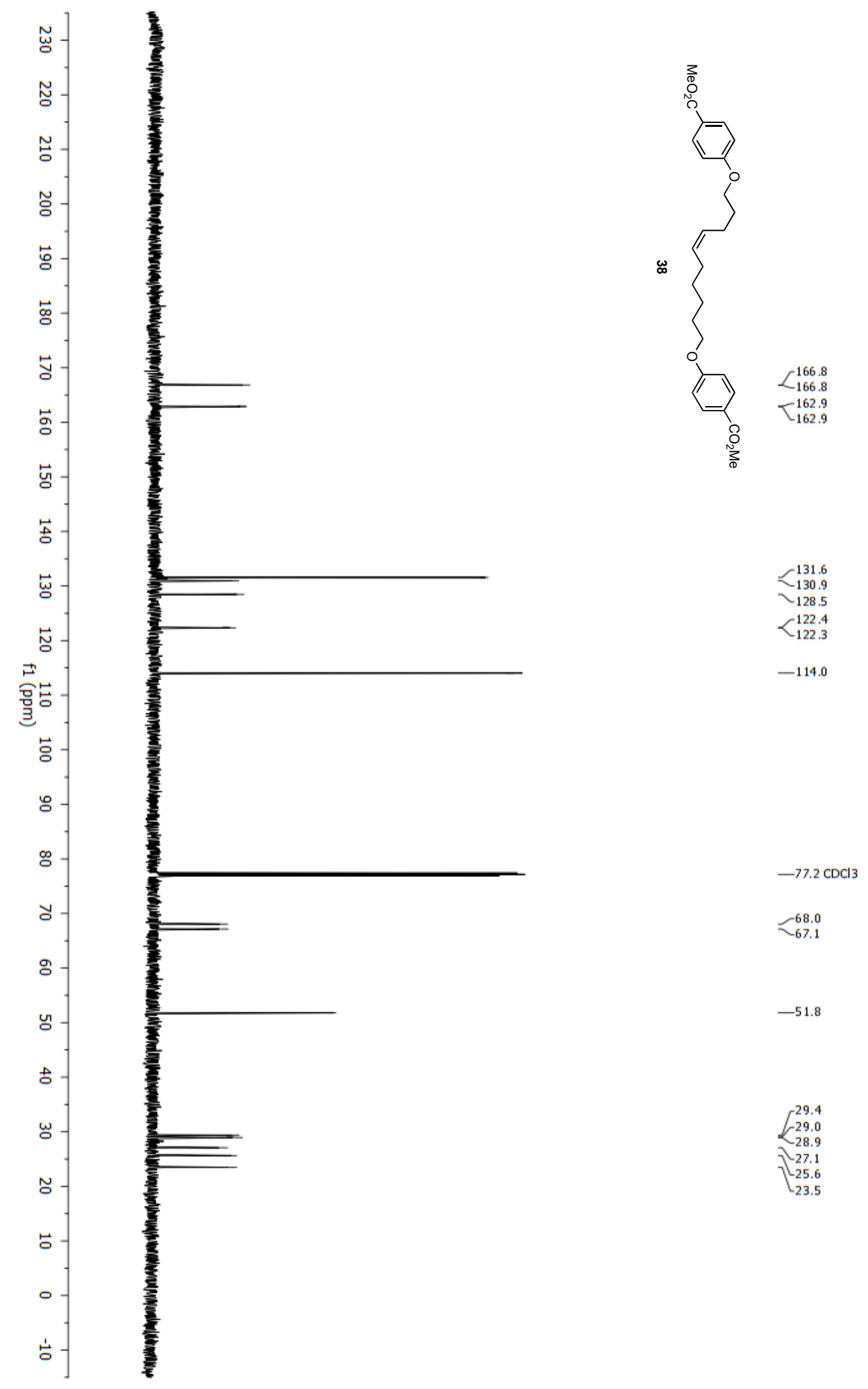

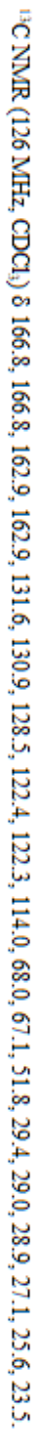

$-51.8$

29.4
$\sum_{28.9}^{29.9}$
\rceil$_{-25.1}^{-25.6}$
-23.5 


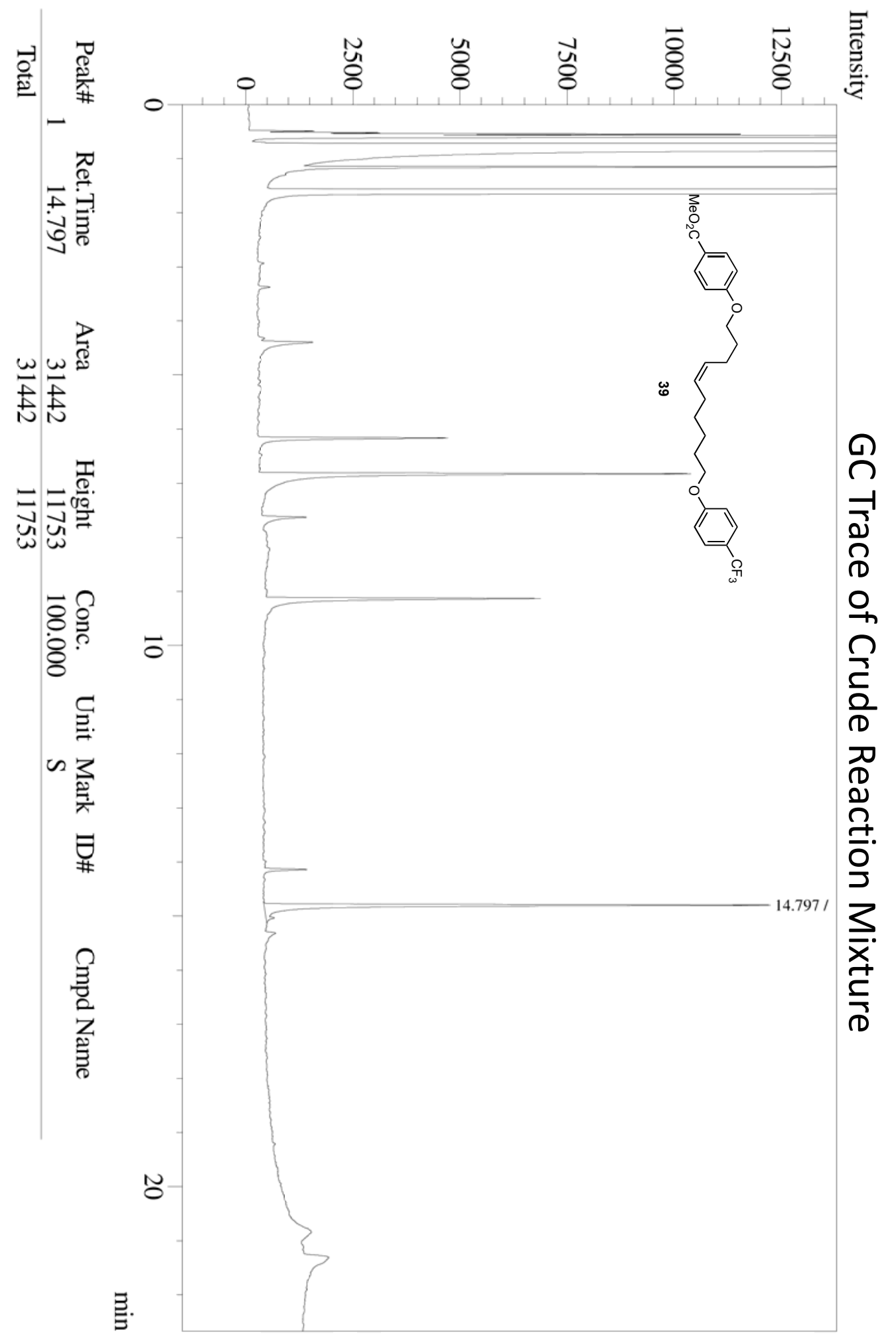



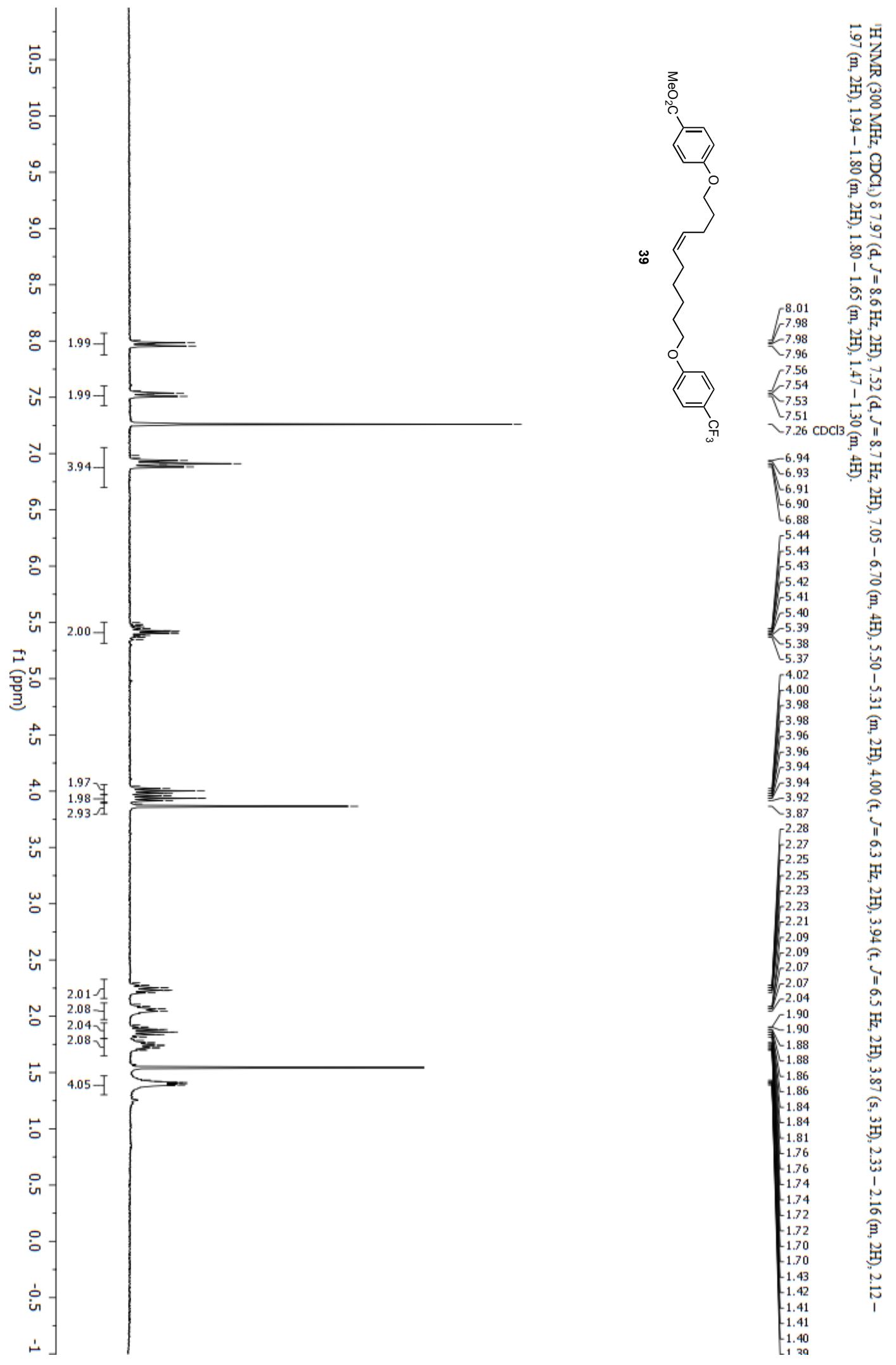

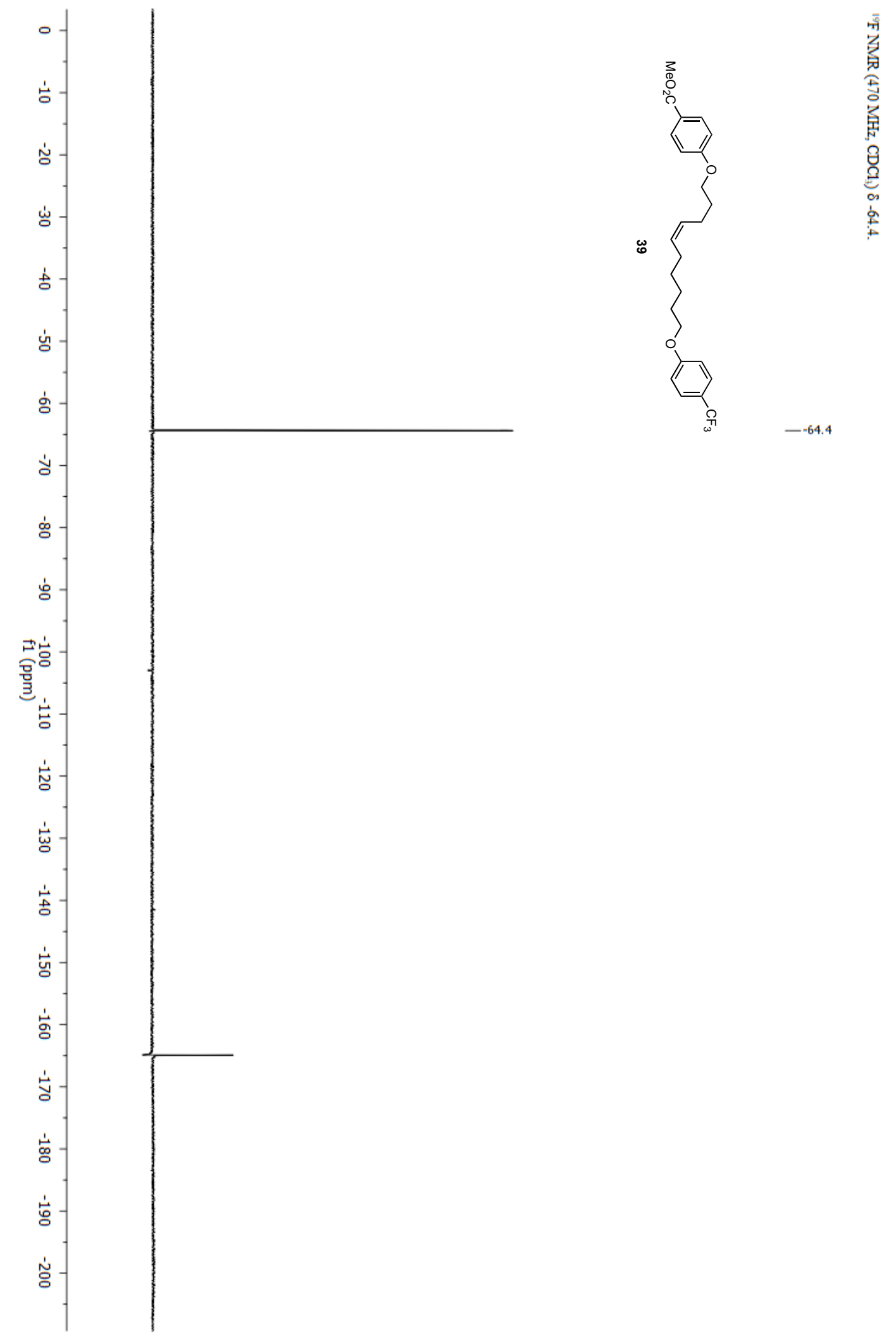

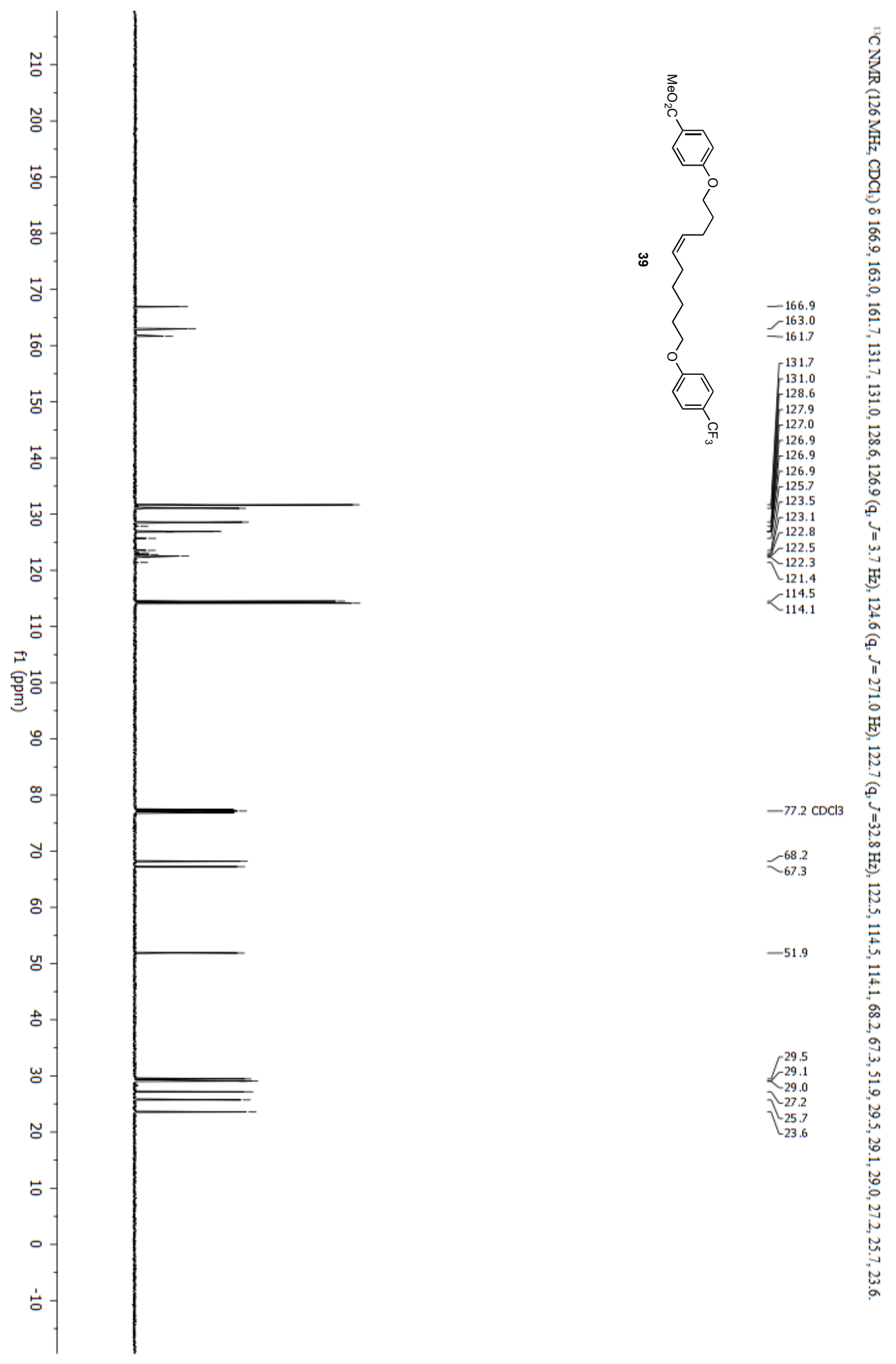


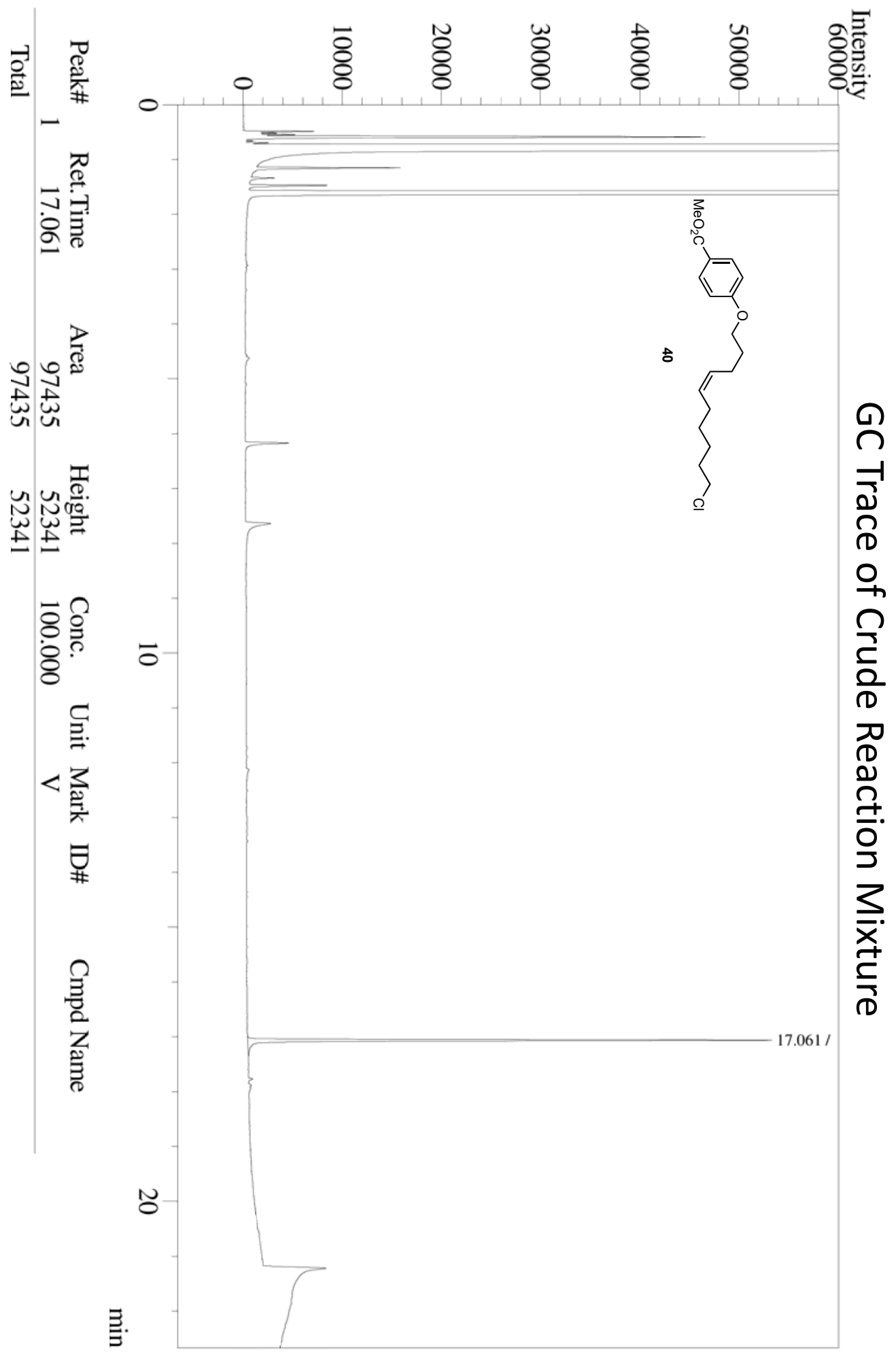



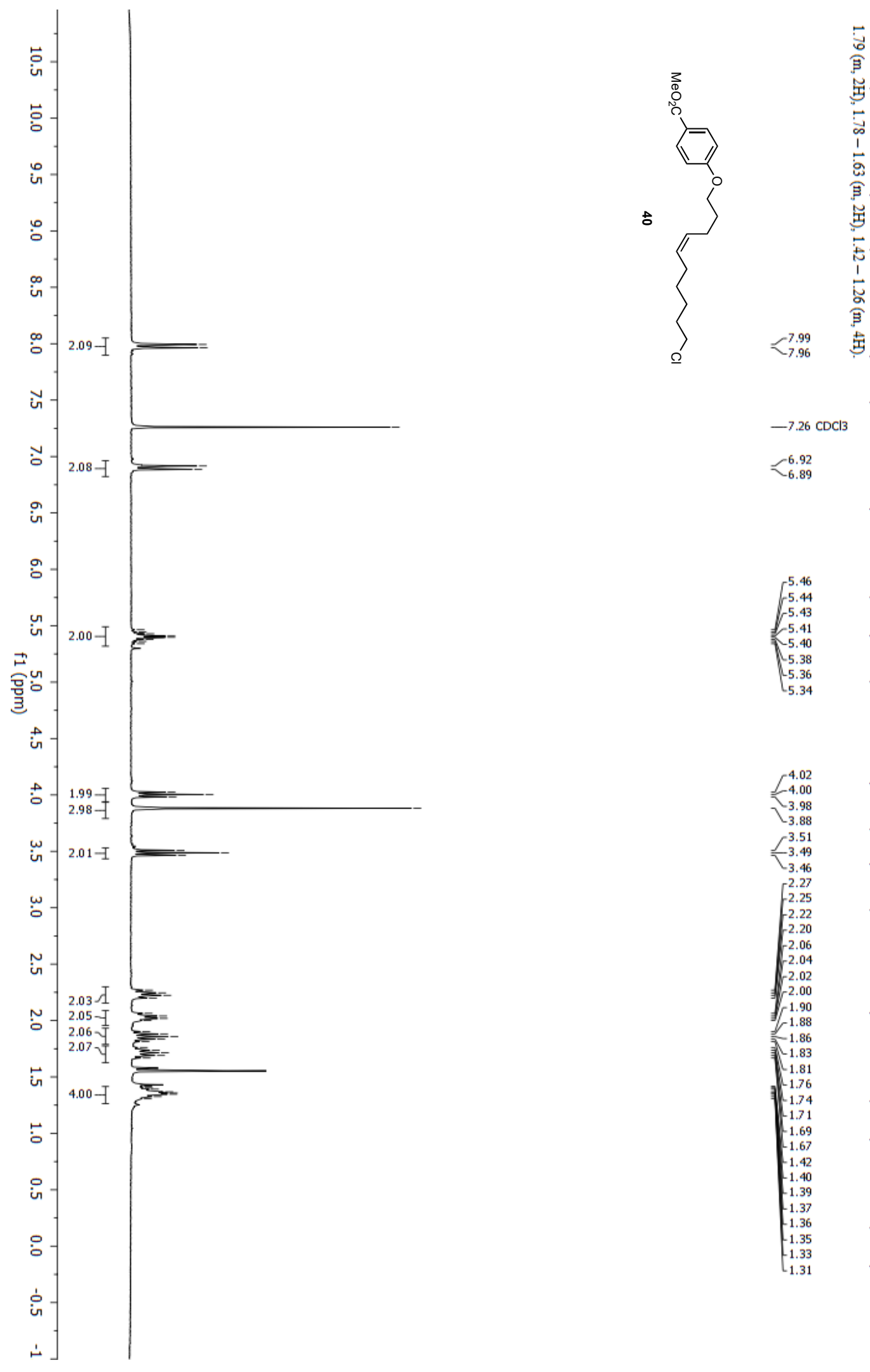

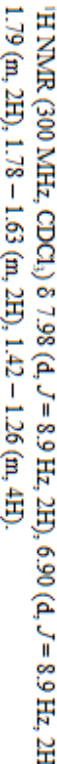

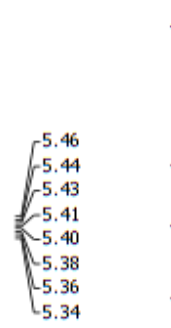

402

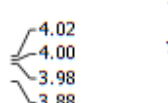

$\int_{3.49}^{3.51}$

$\tau_{3.46}^{3.49}$

$-2.27$

2.25
-2.22
-2.20
-2.06

2.20
-2.06
2.04

$-2.02$

$r_{1.90}^{2.00}$

$-1.88$

1.83

1.81

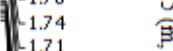

-1.71
-1.69

$-1.67$

$\begin{array}{rr}-1.40 & 1 \\ -1.39 & 8 \\ -1.37 & 8\end{array}$

$-1.36$

$-1.33$

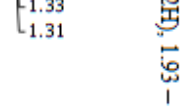



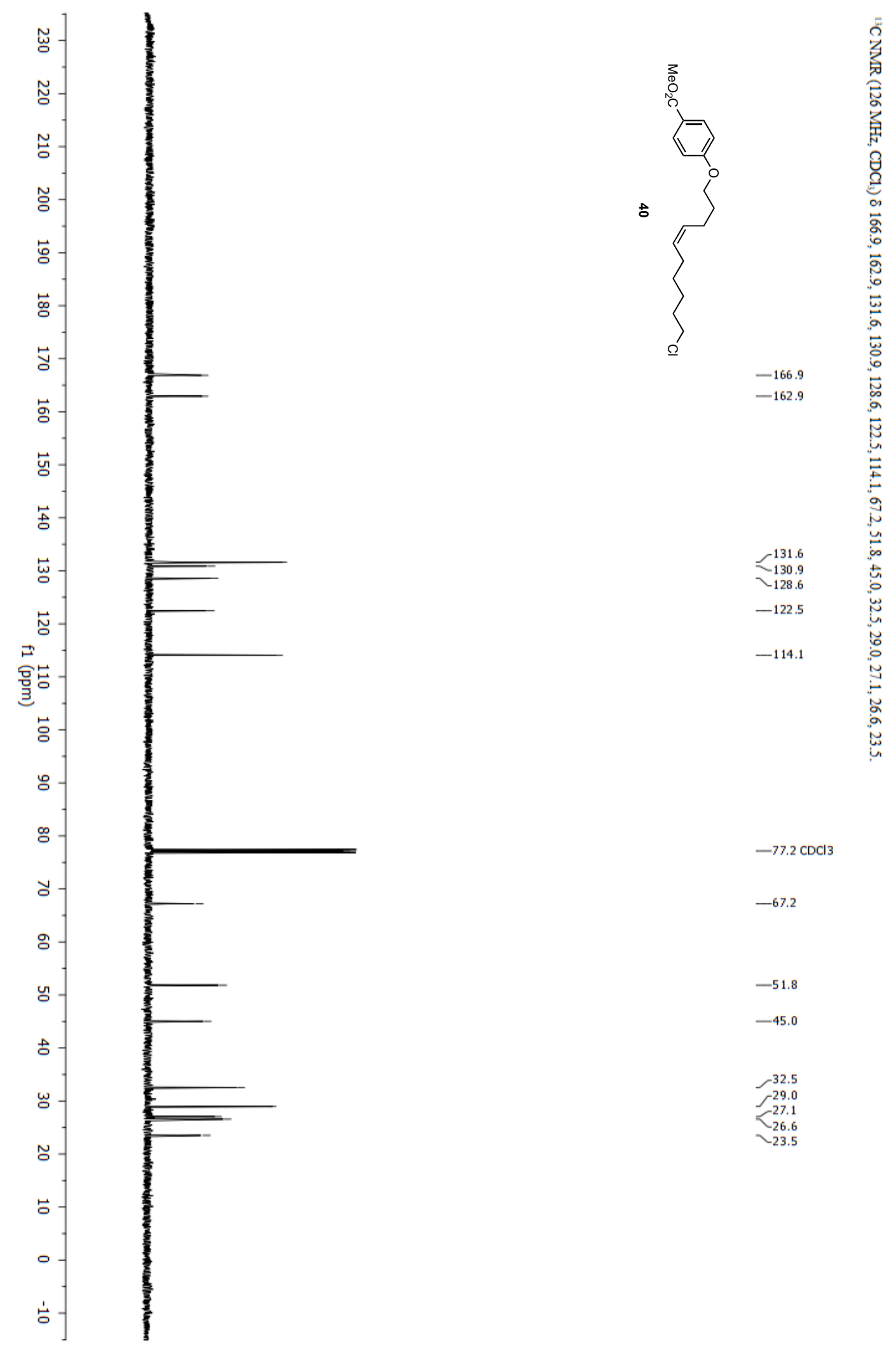


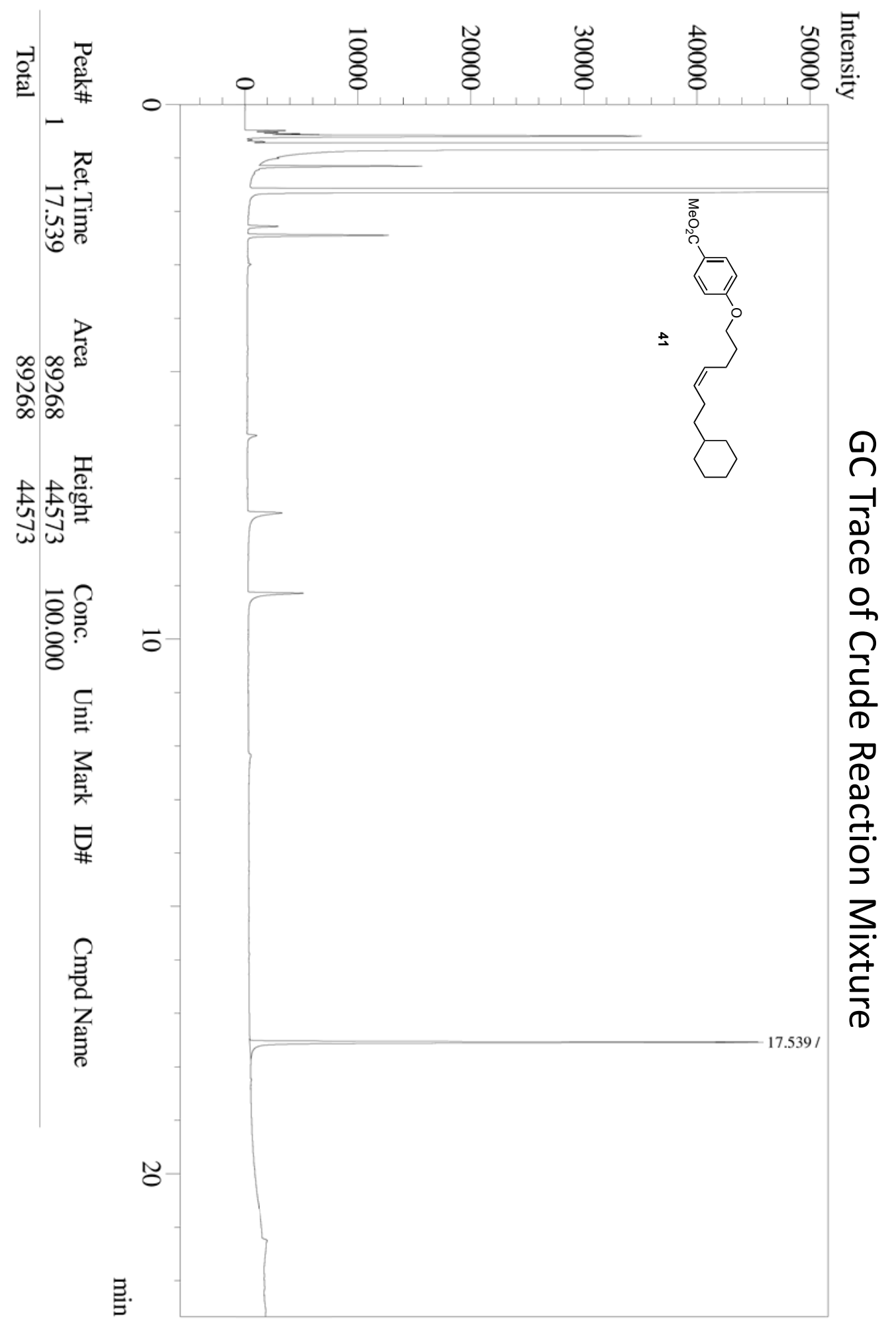



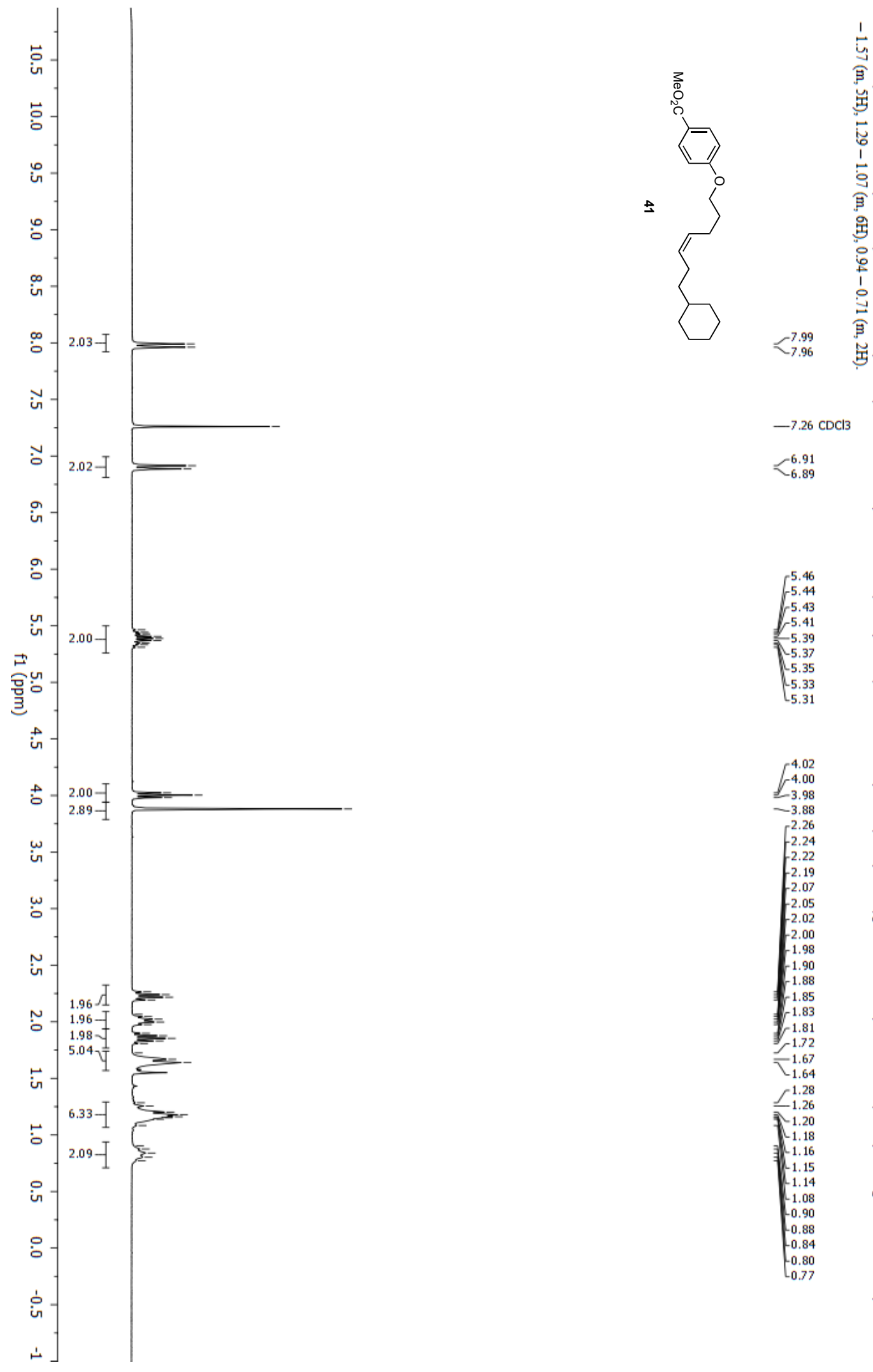

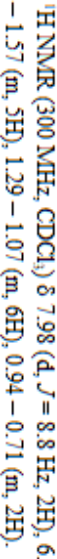

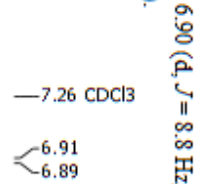

采

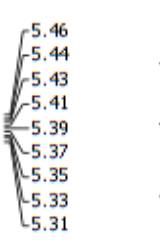

a

$\int_{-4.00}^{4.02}$

-3.88 क

$-2.24$

$-2.22$

$-2.07$

2.05
-2.02
-1.00

-2.00 iा

$-1.88$

1.85
-1.83

$1.81-5$

1.72

$\begin{array}{ll}1.64 & \text { के } \\ -1.28 & \end{array}$

$\int_{1.26}^{1.28}$

$t_{1.18}^{1.20}$

(1.16

-1.14 क

$1.08 \quad 0$

$-0.90 \quad$ II

$\begin{array}{ll}0.88 & \text { o } \\ -0.84 & \text { o } \\ -0.80 & \text { i }\end{array}$

0.77

苟 

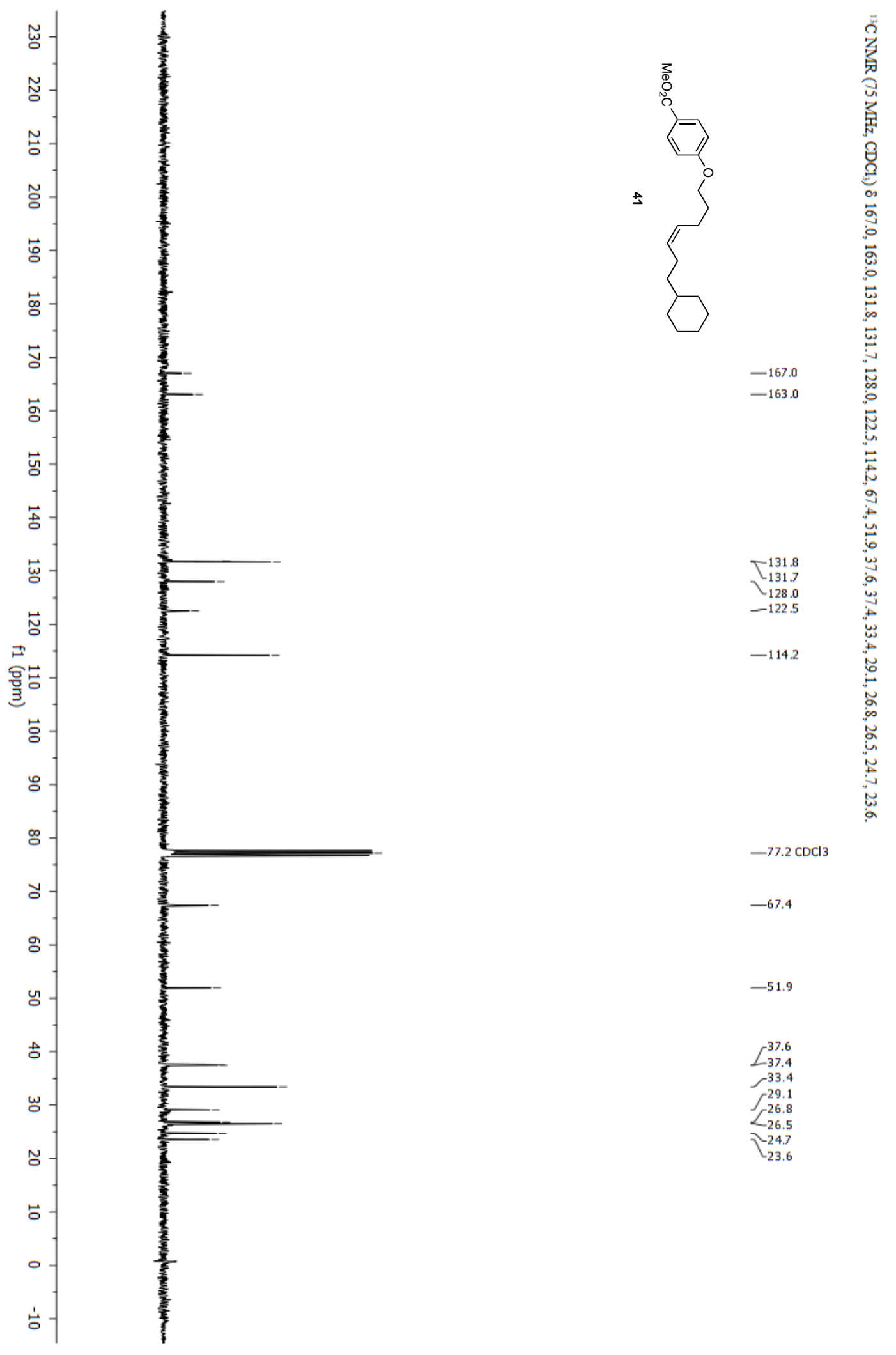

$-77.2 \mathrm{CDCl} 3$

$-67.4$

$-51.9$

$\Sigma_{37.4}^{37.6}$

33.

$\int_{-26.8}^{29.1}$

$\mathcal{L}^{26.8}$

\urcorner$_{-23.6}^{-24.7}$ 


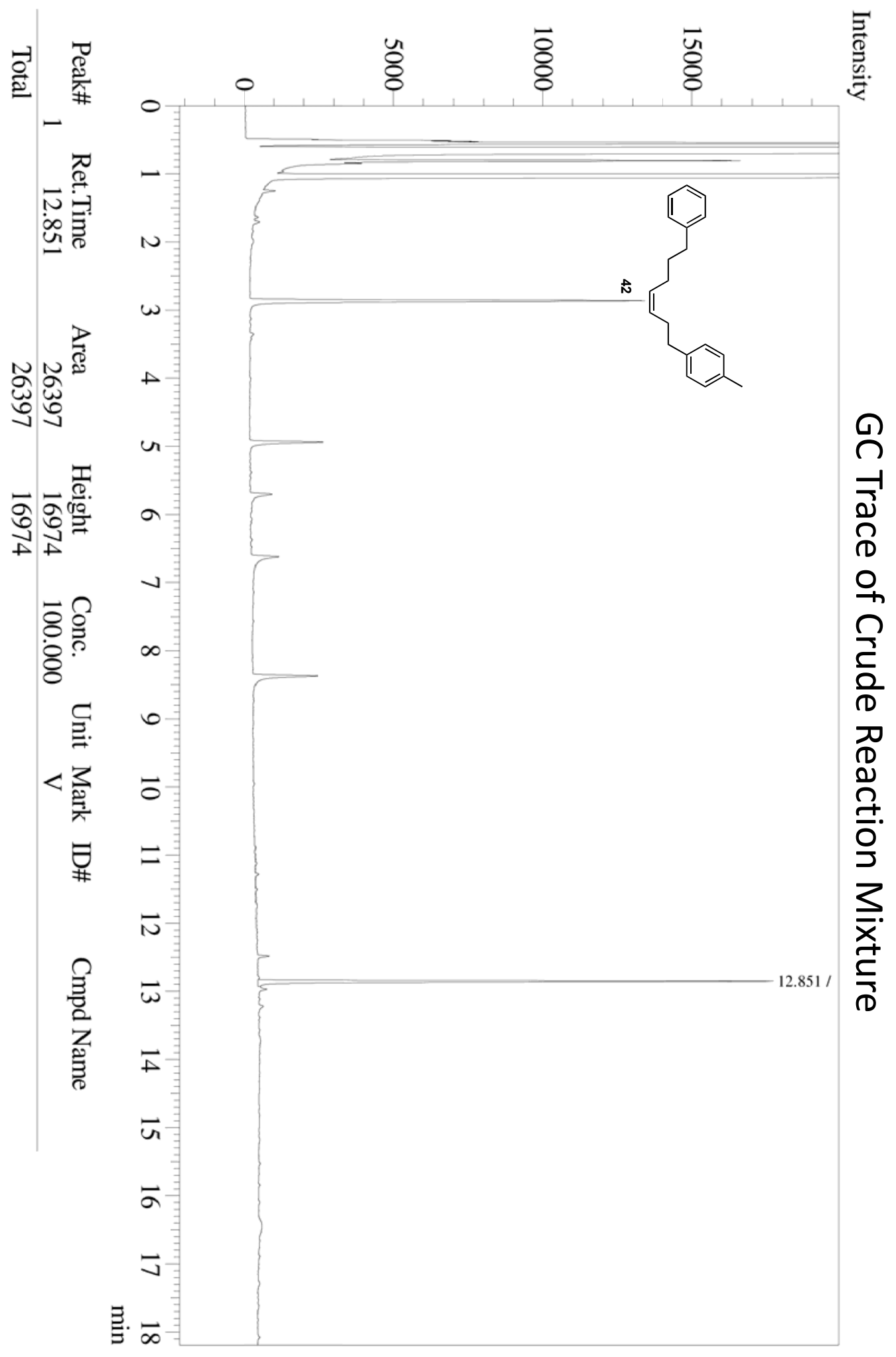



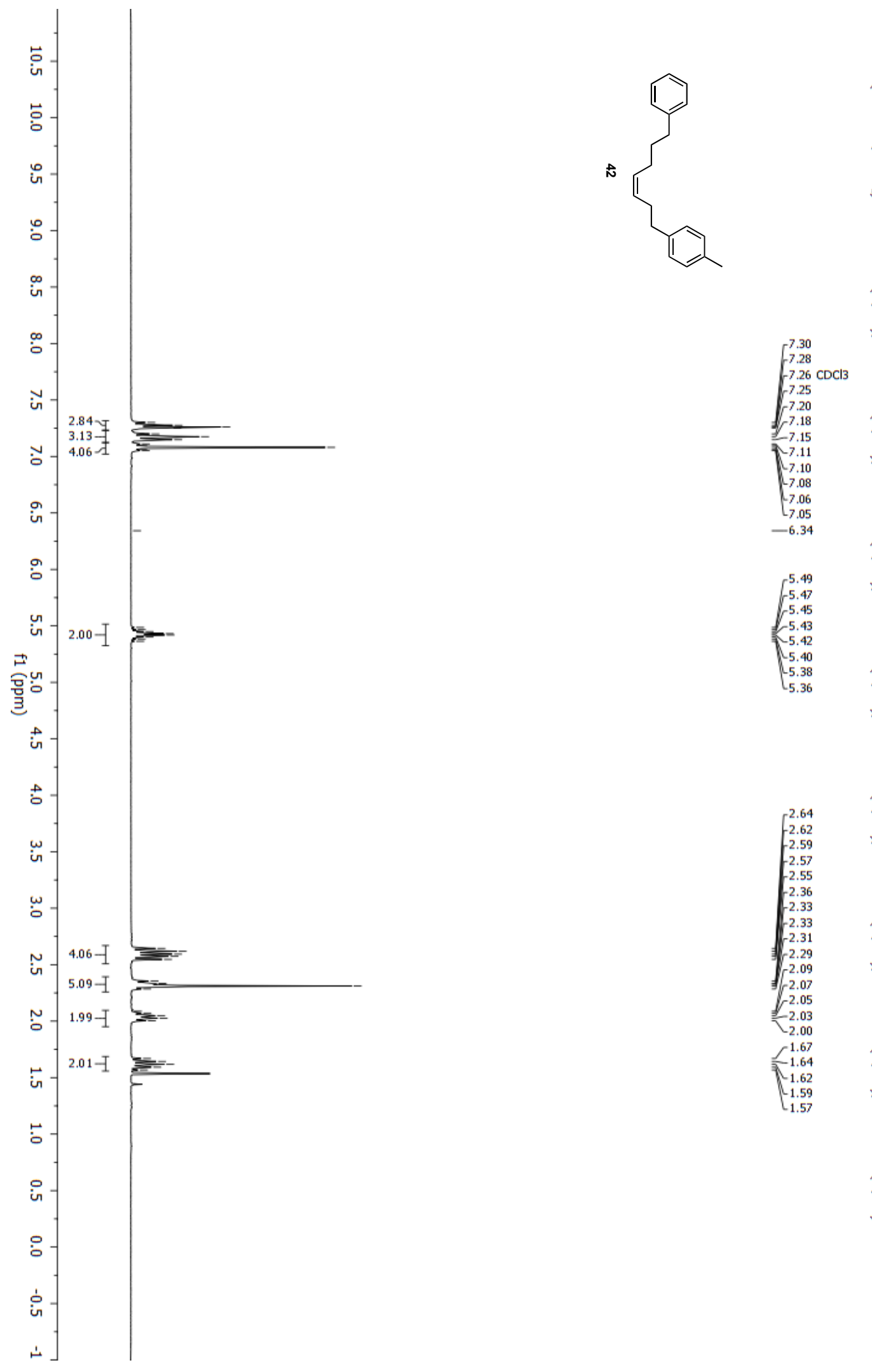

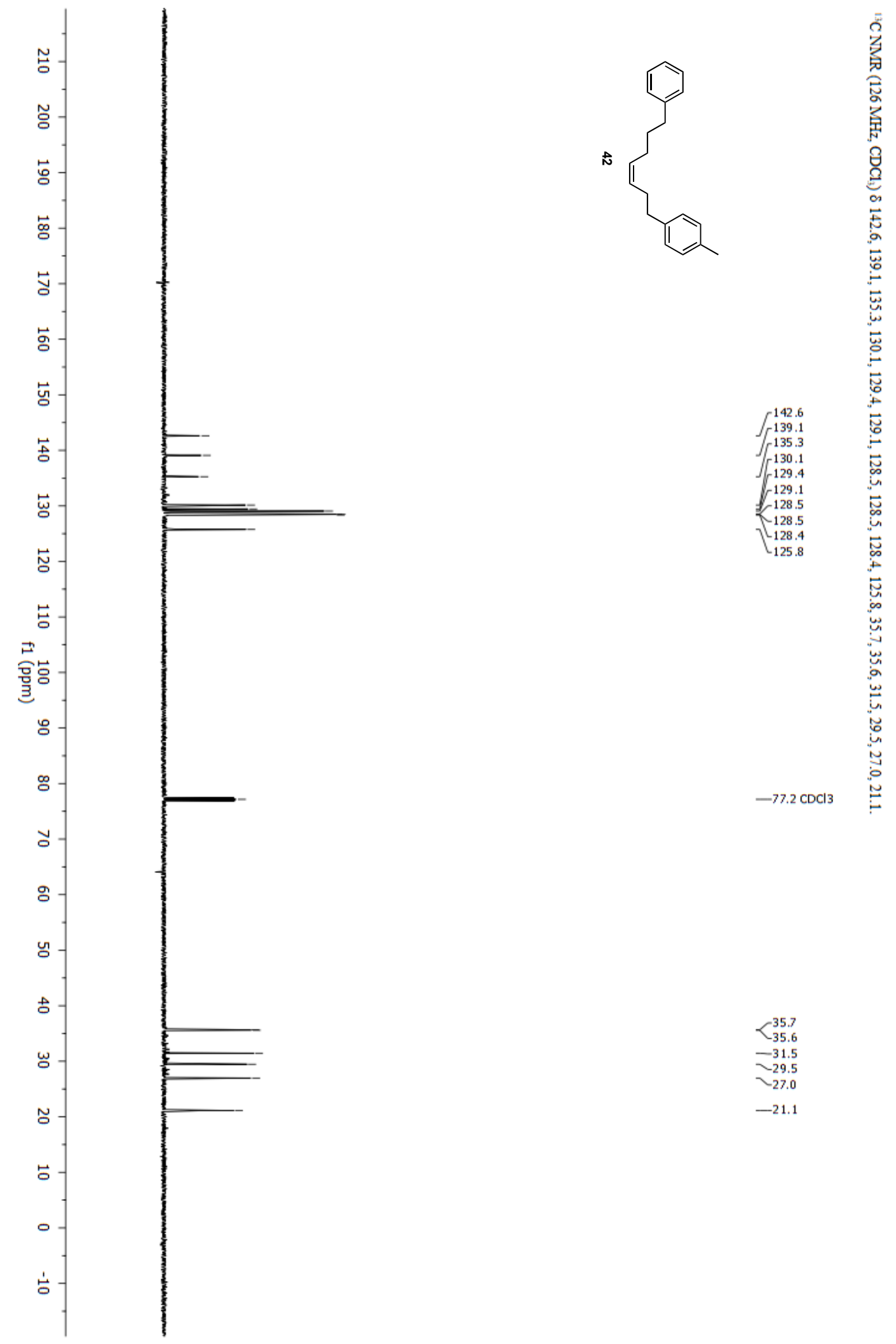

${ }^{35.7}$
-35.6
-31.5
-29.5
-27.0
-21.1 


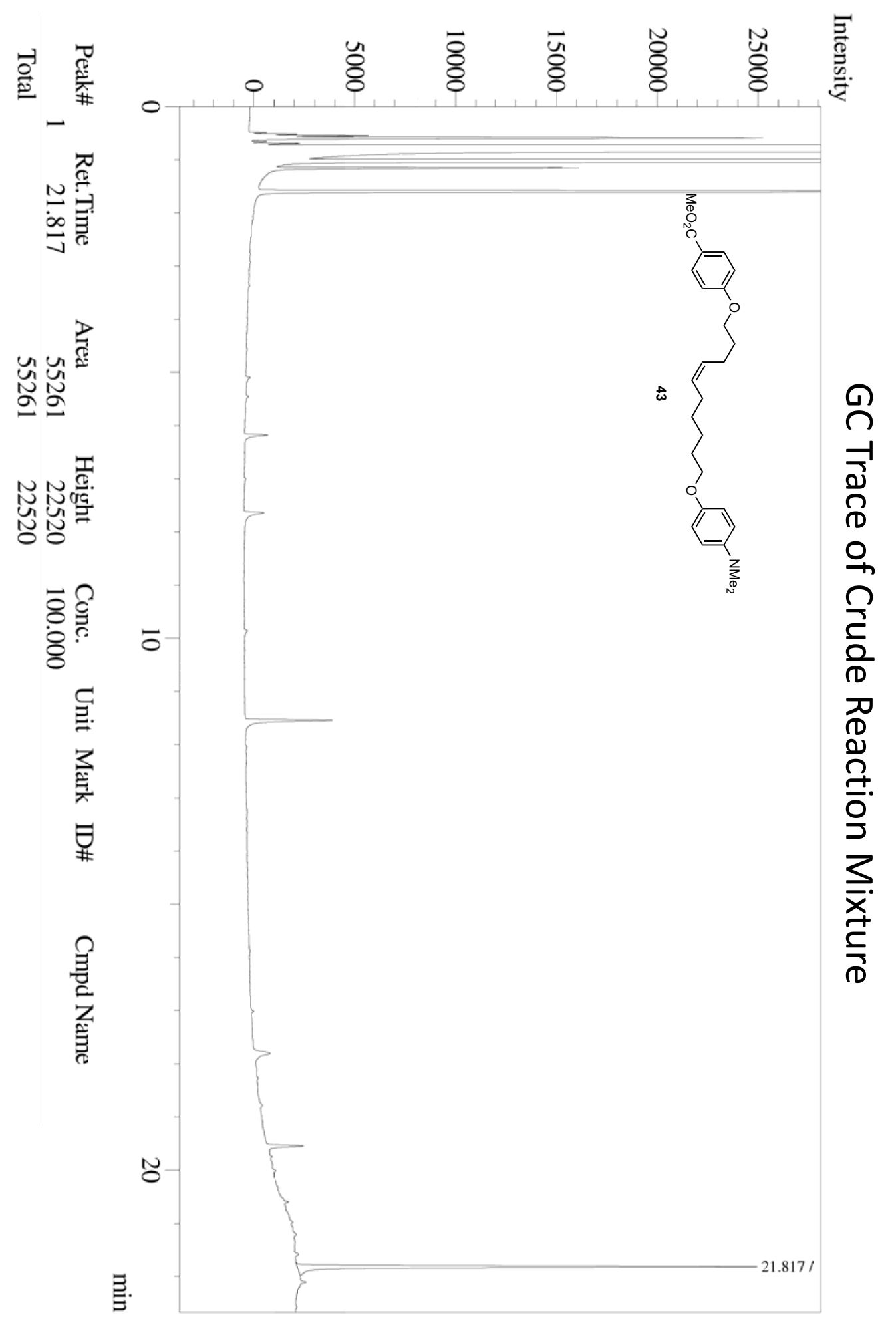




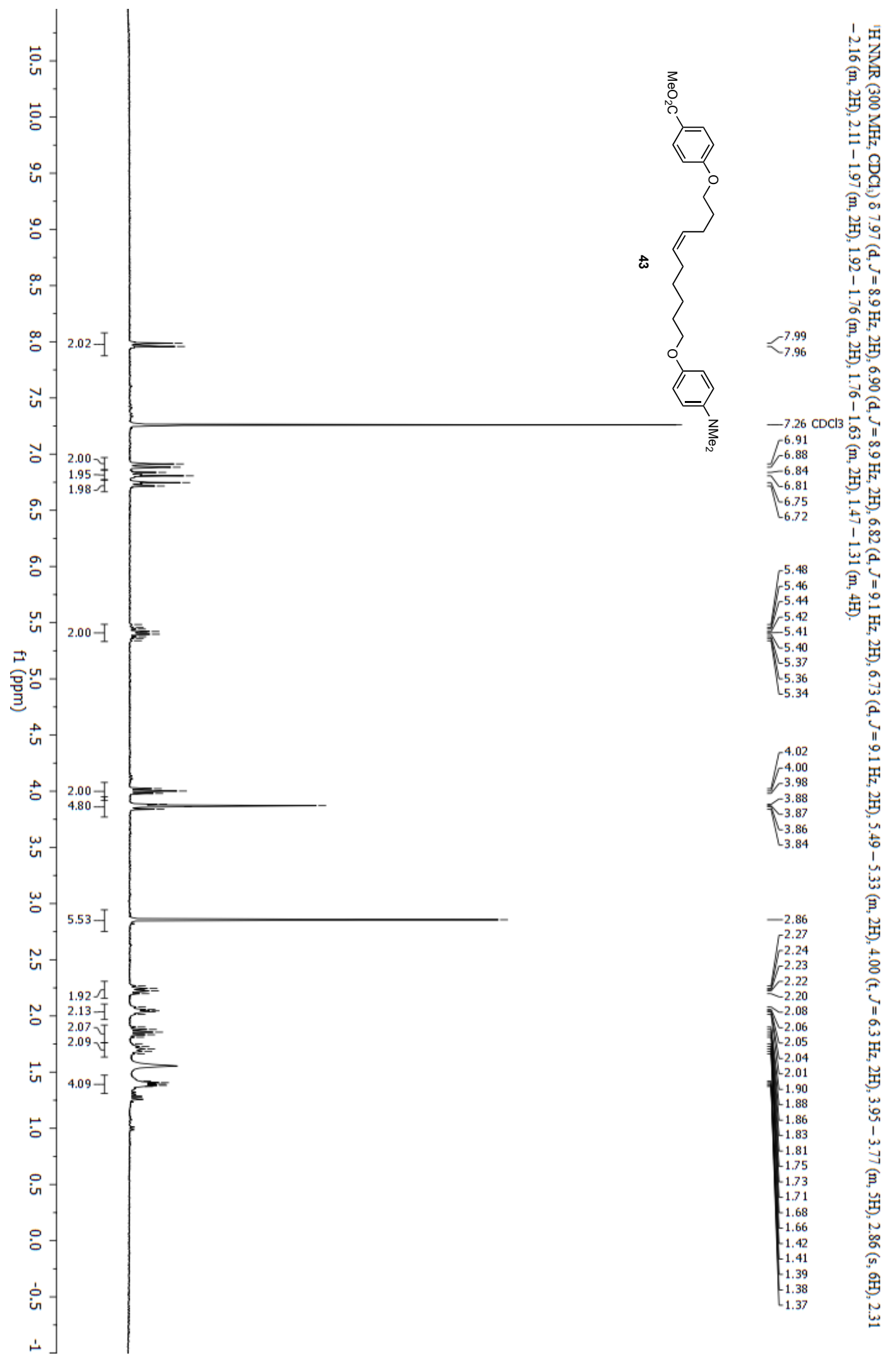



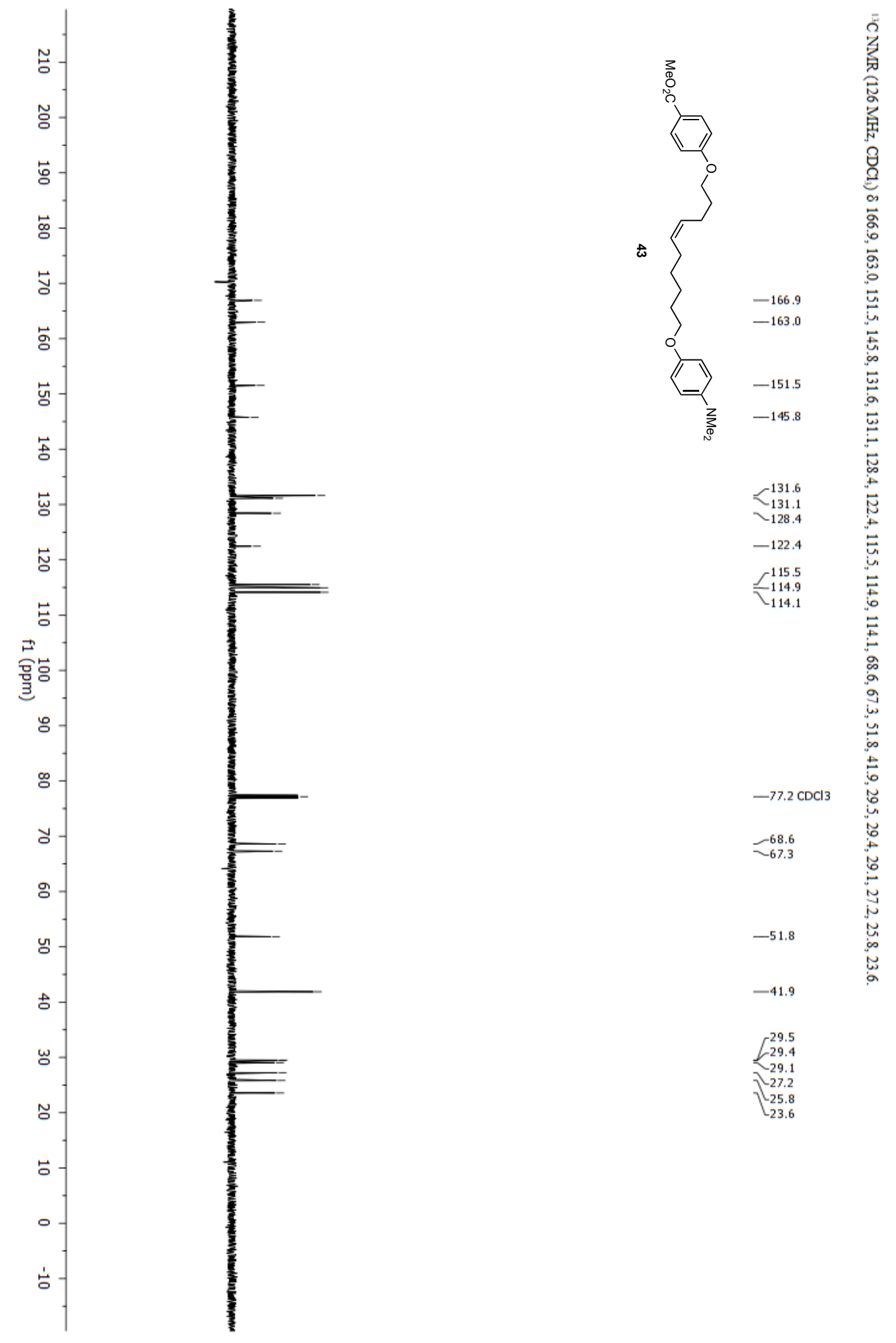


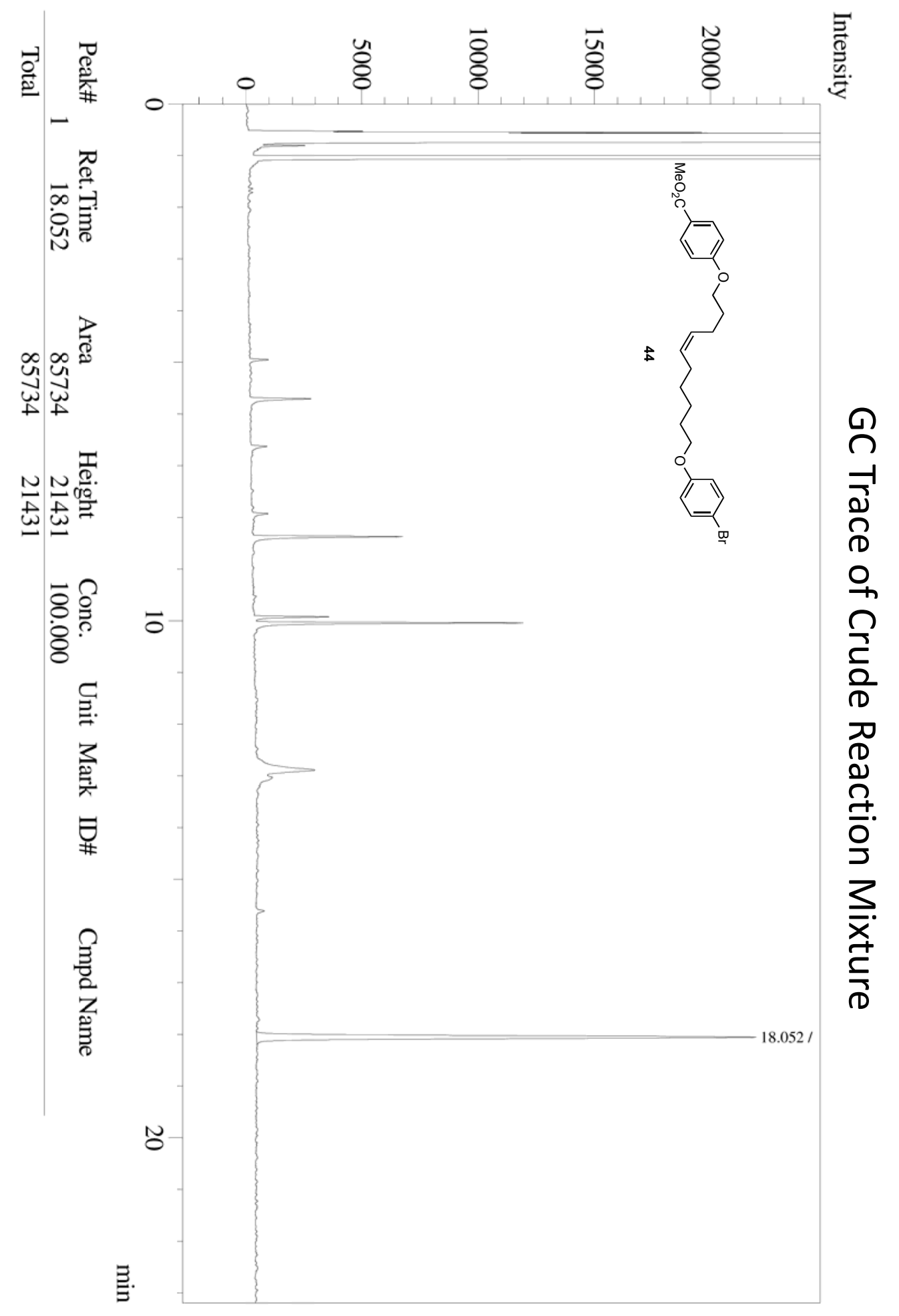




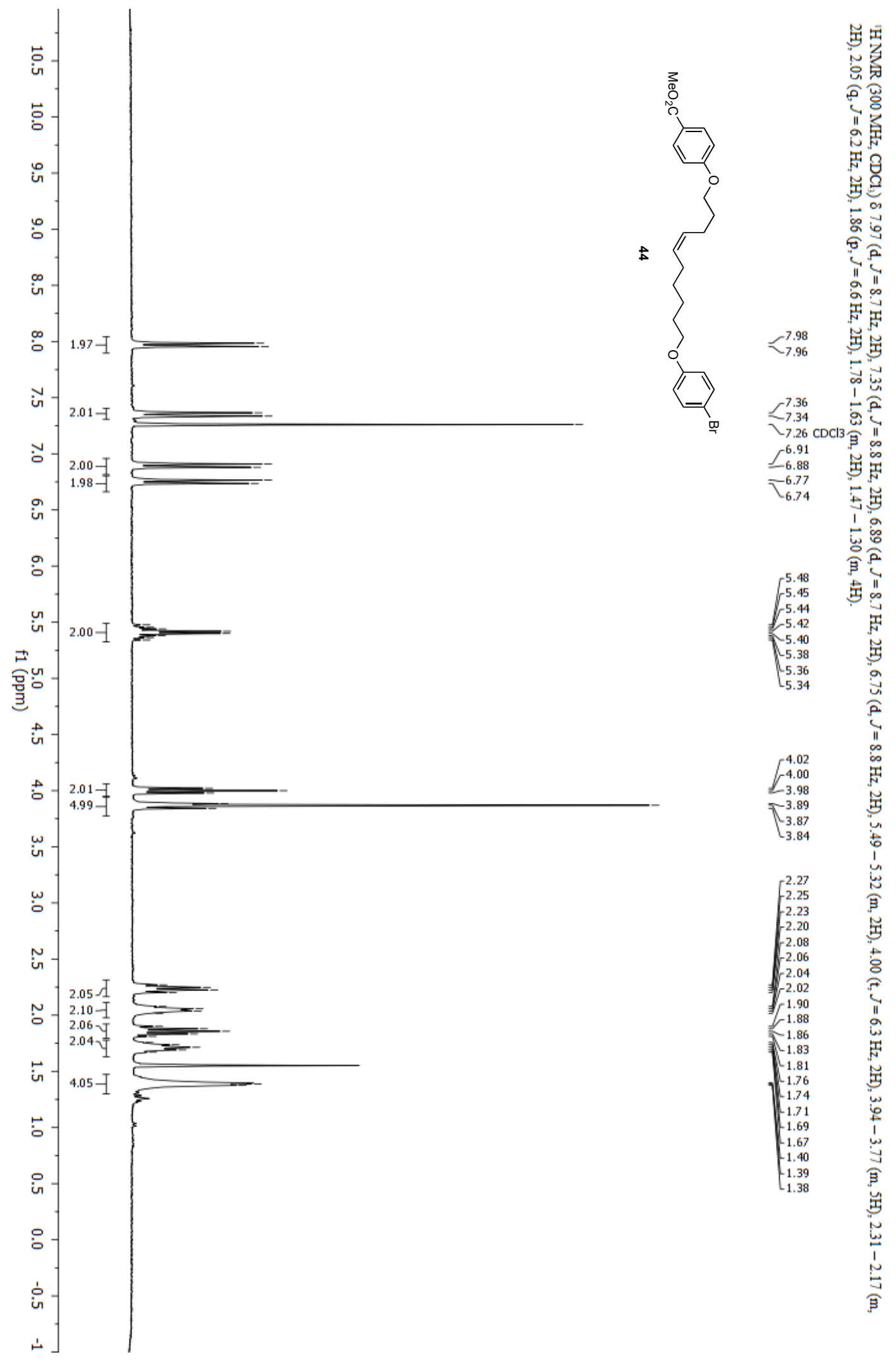



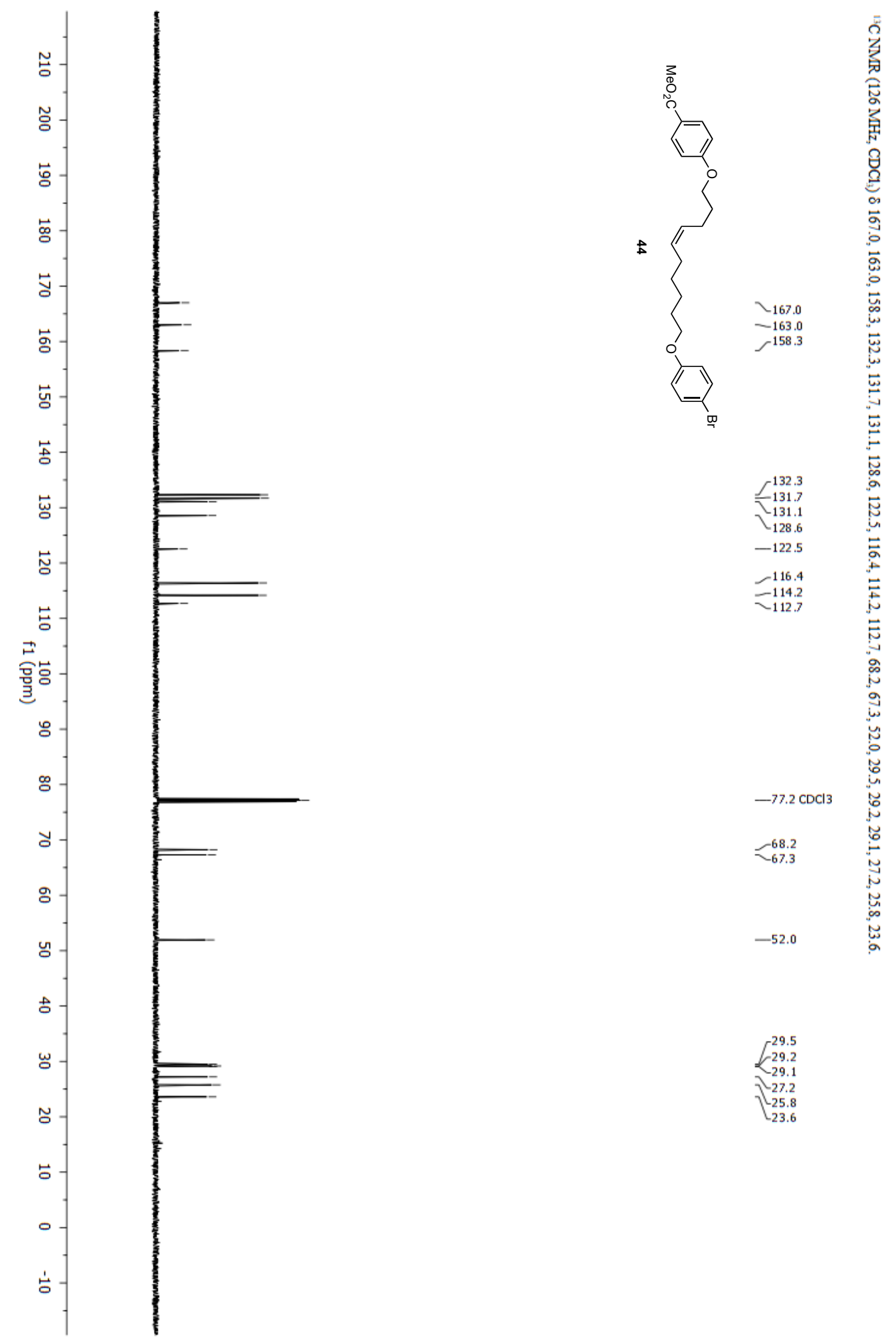


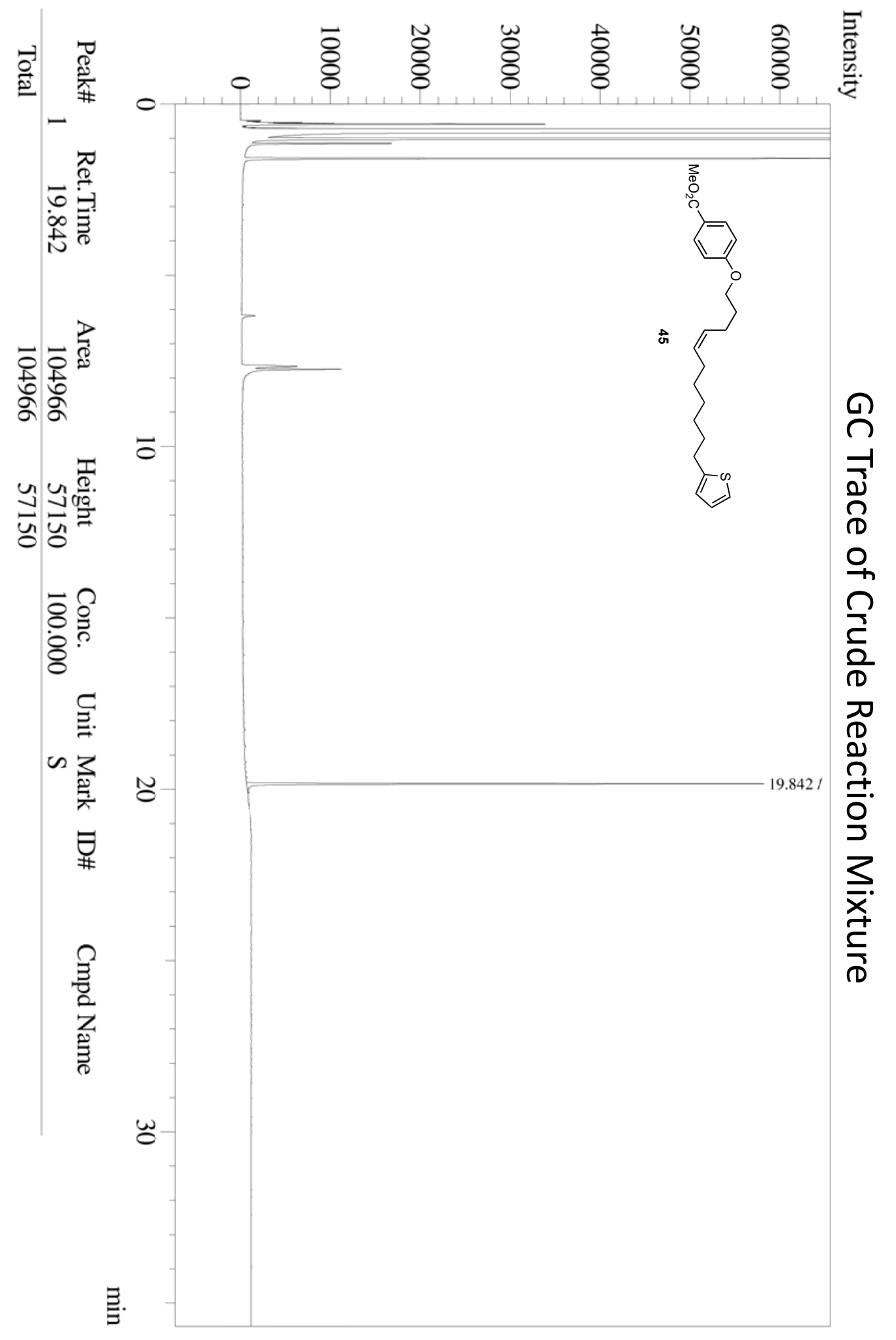



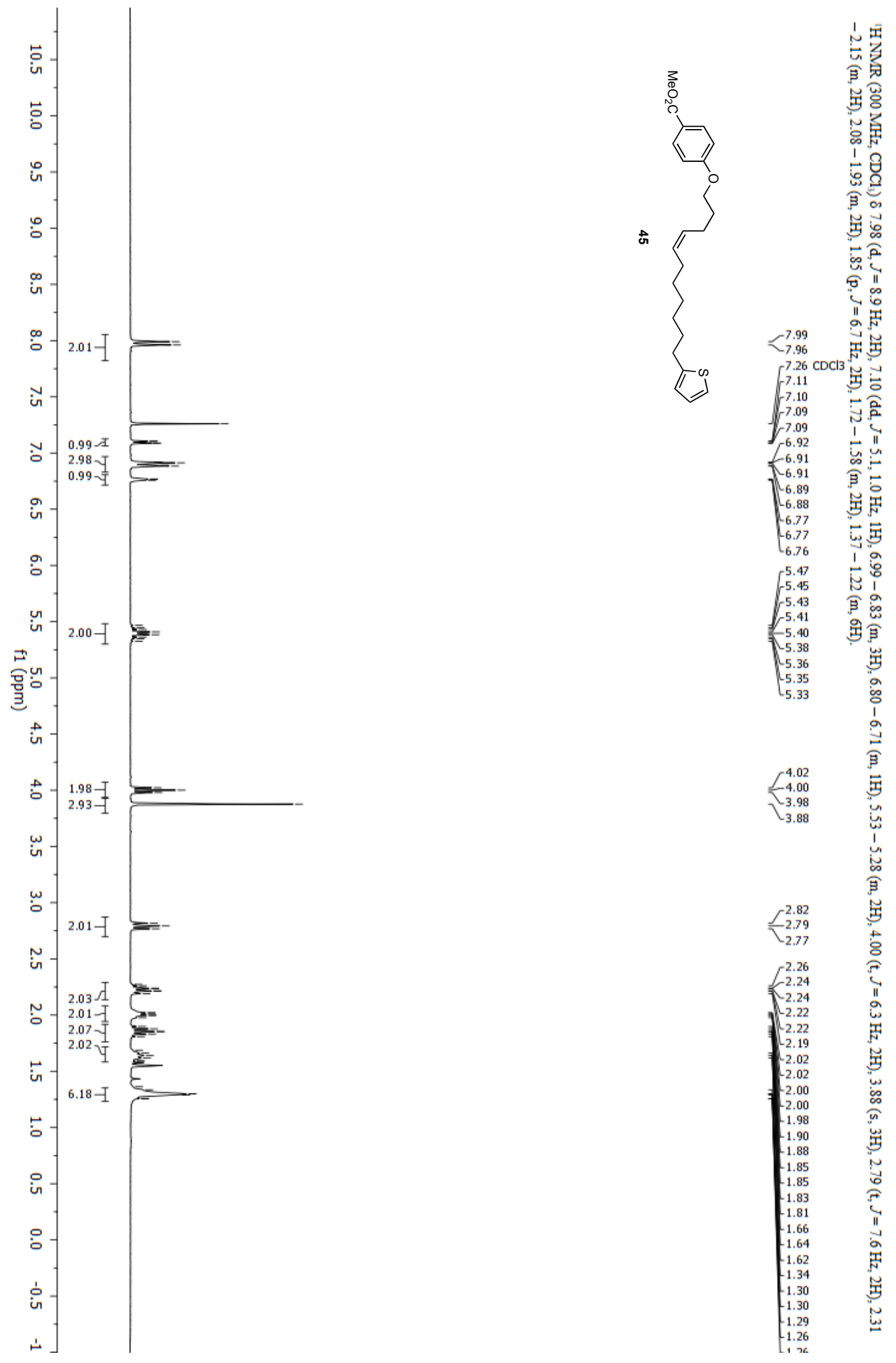

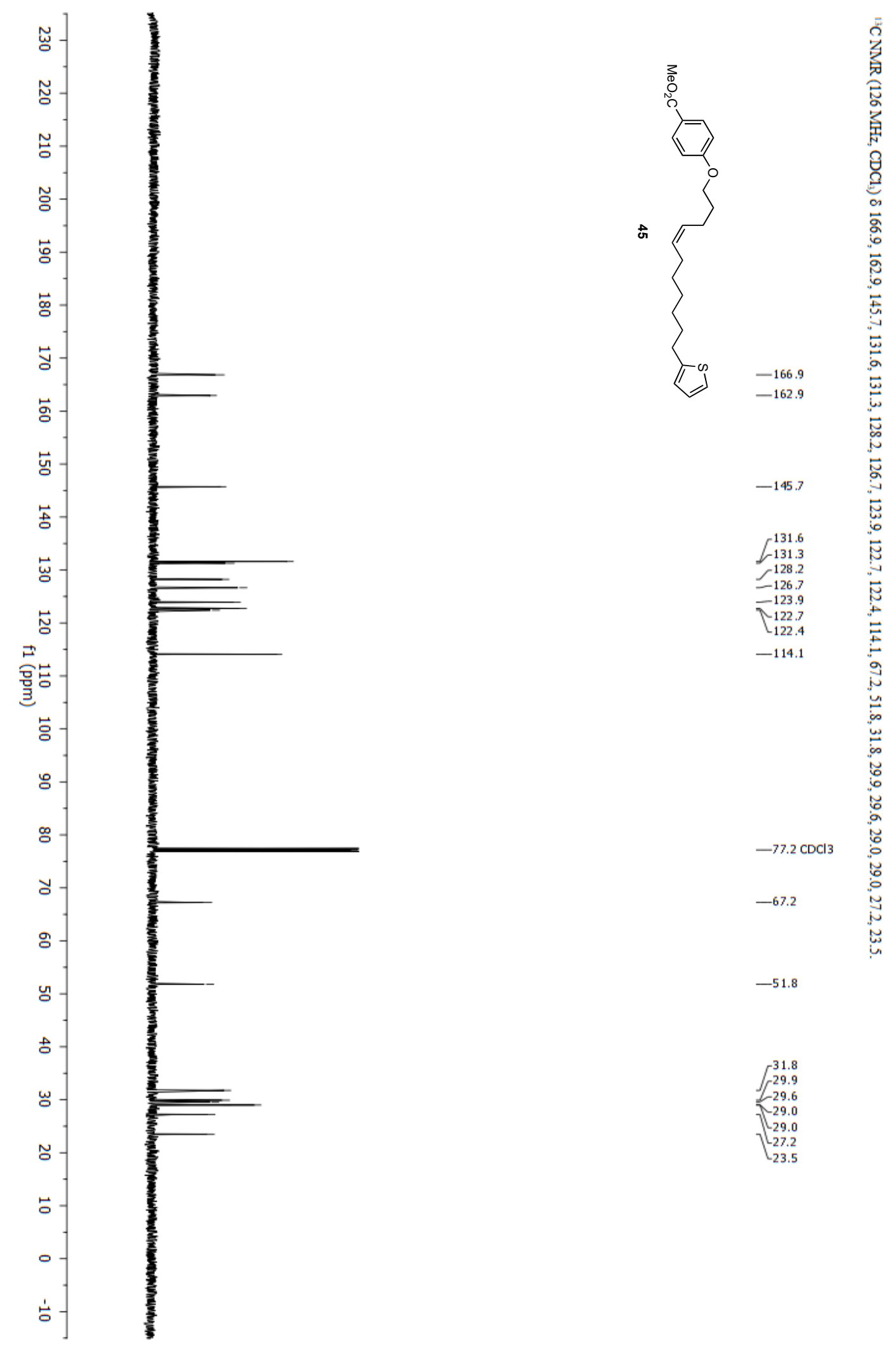


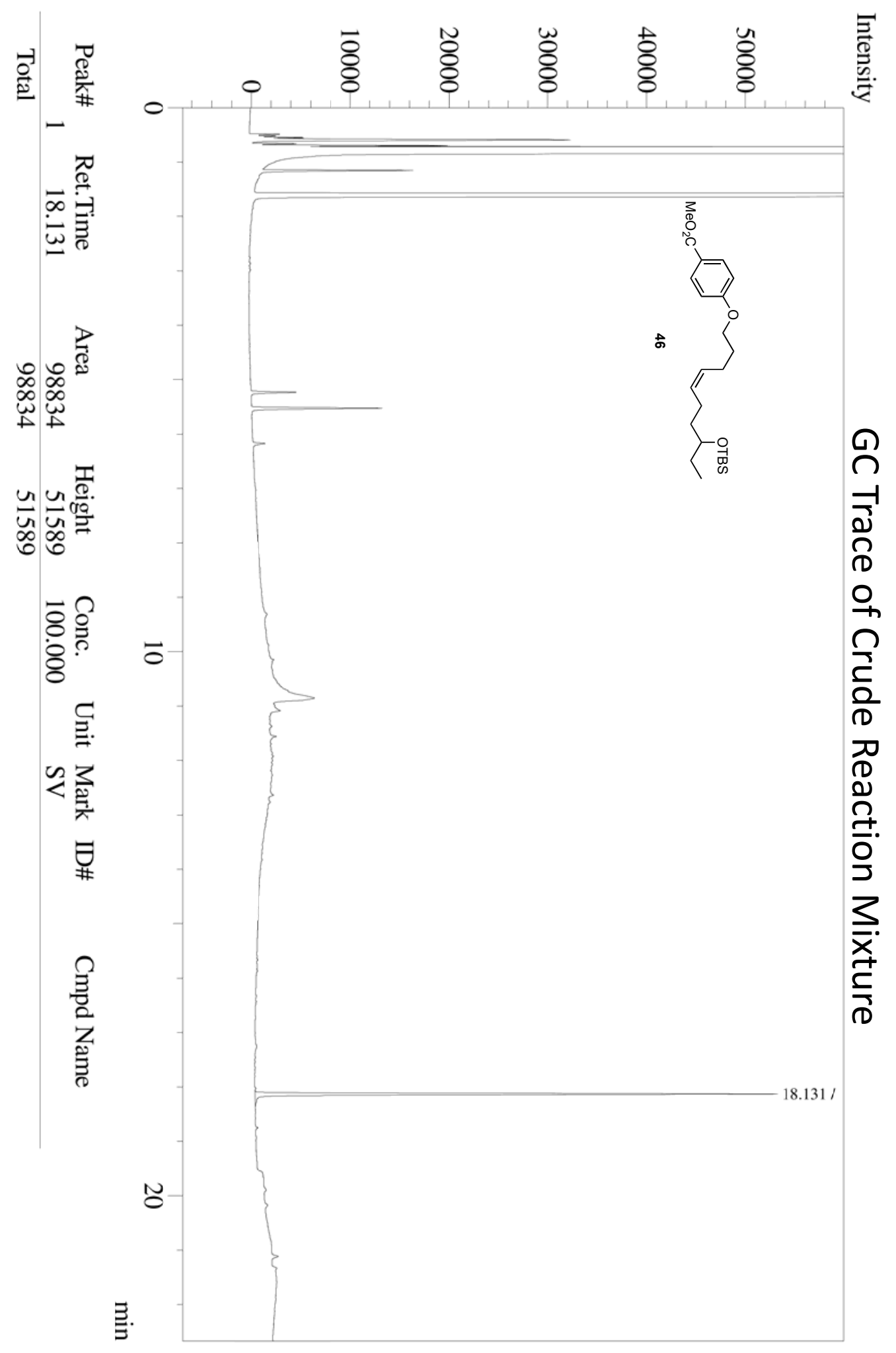




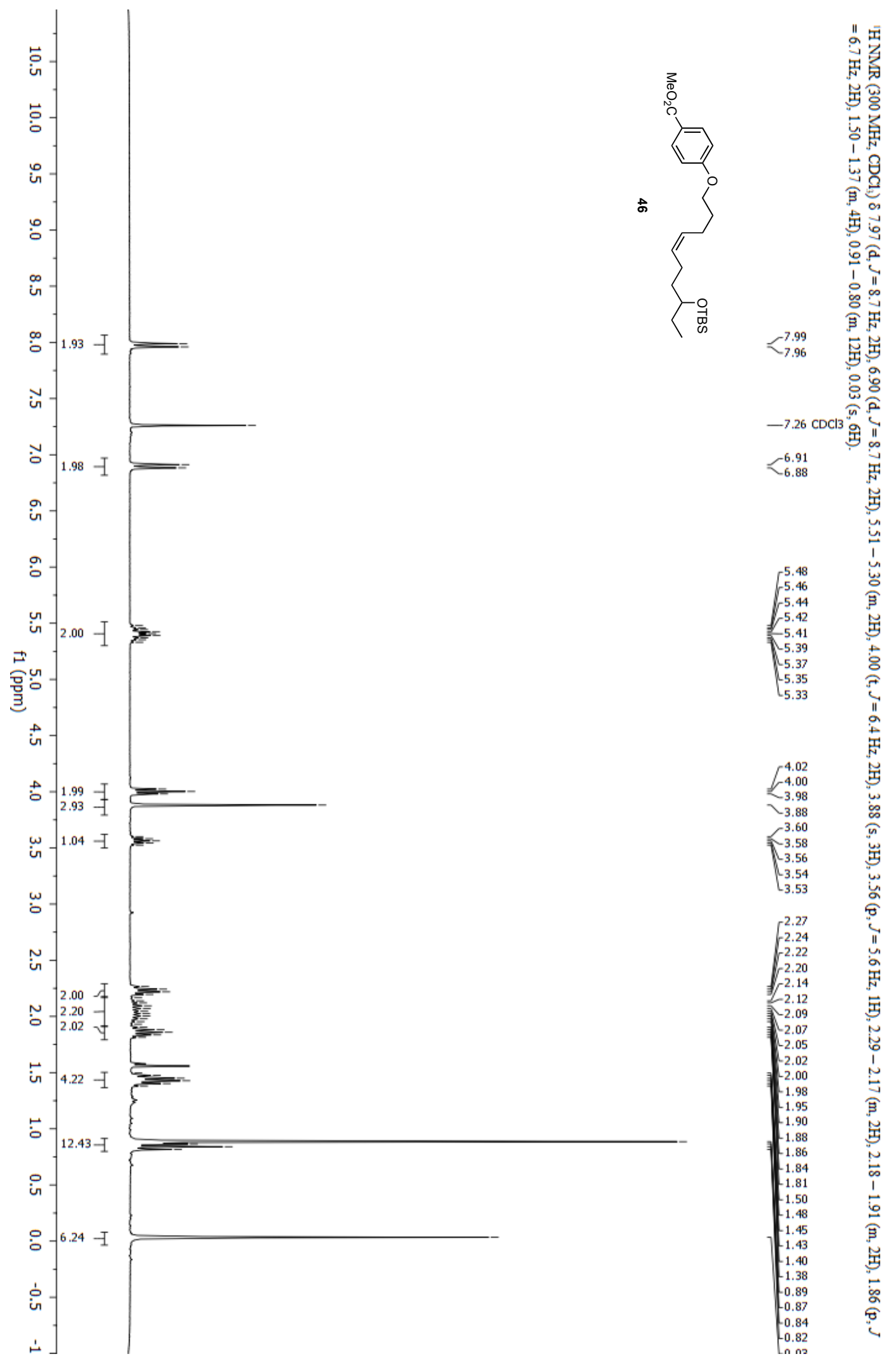



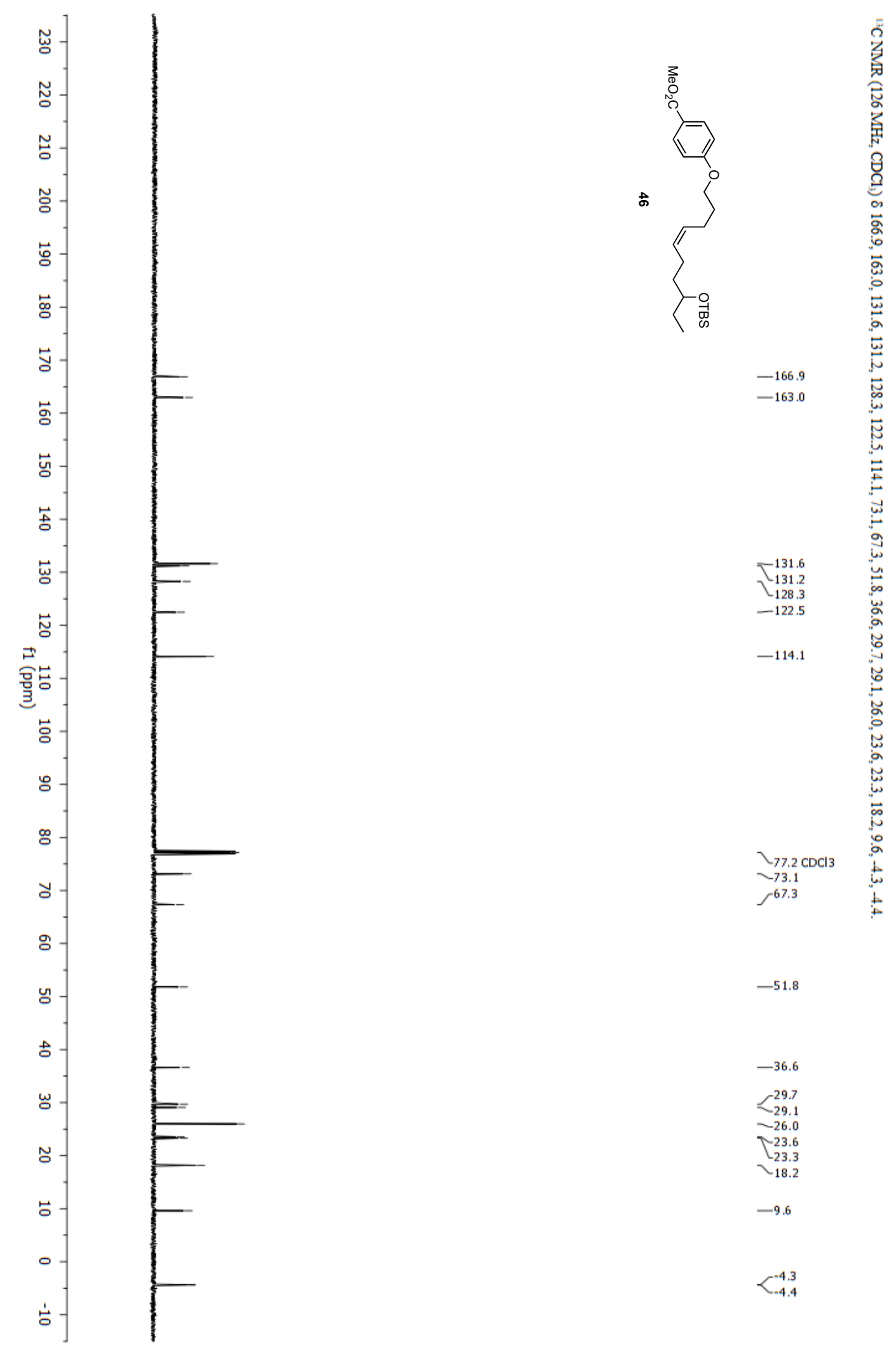

$-51.8$

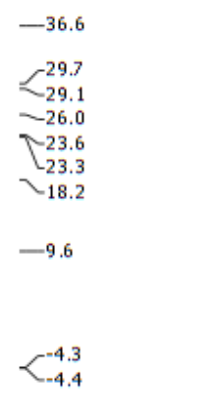




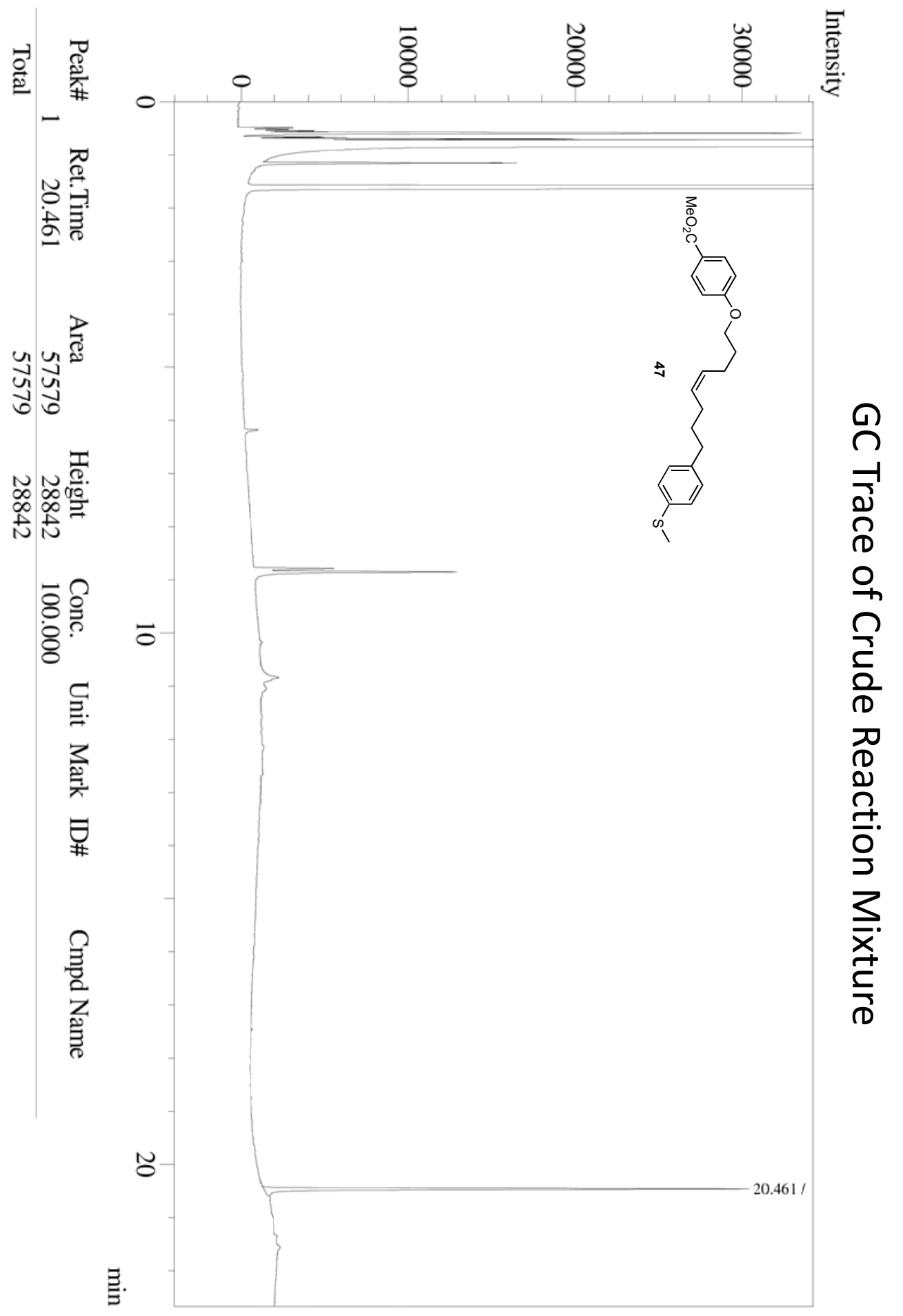



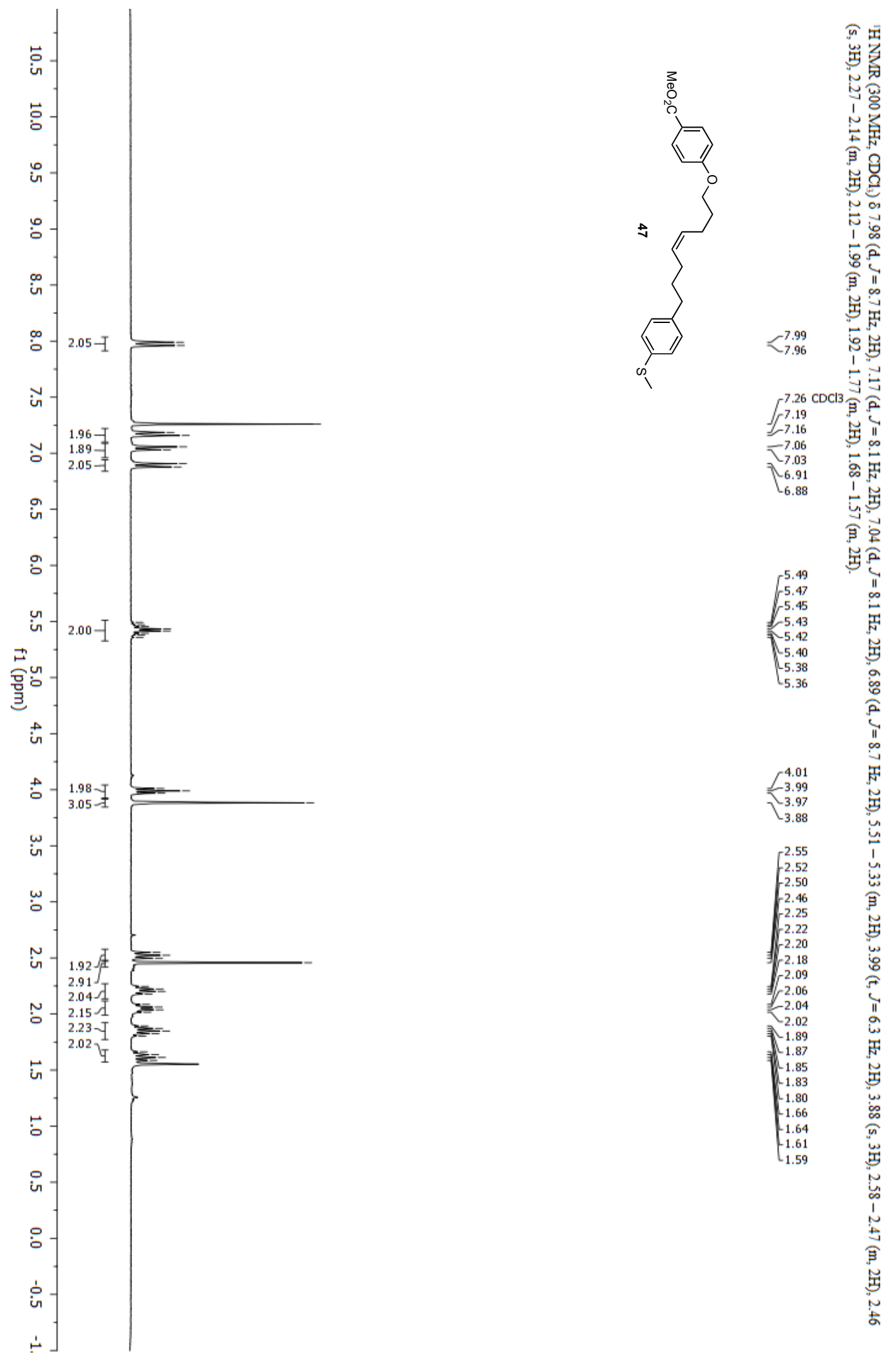

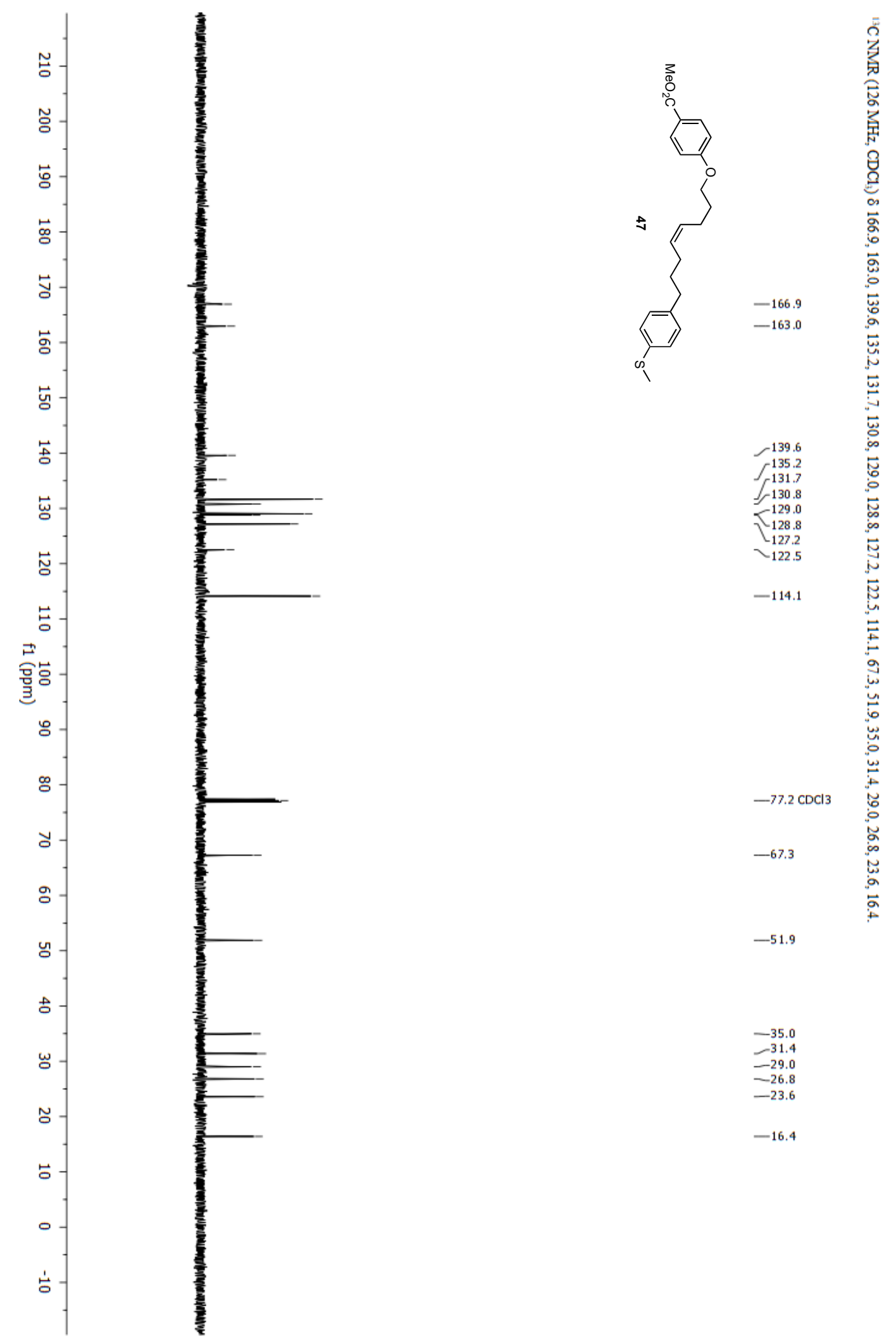


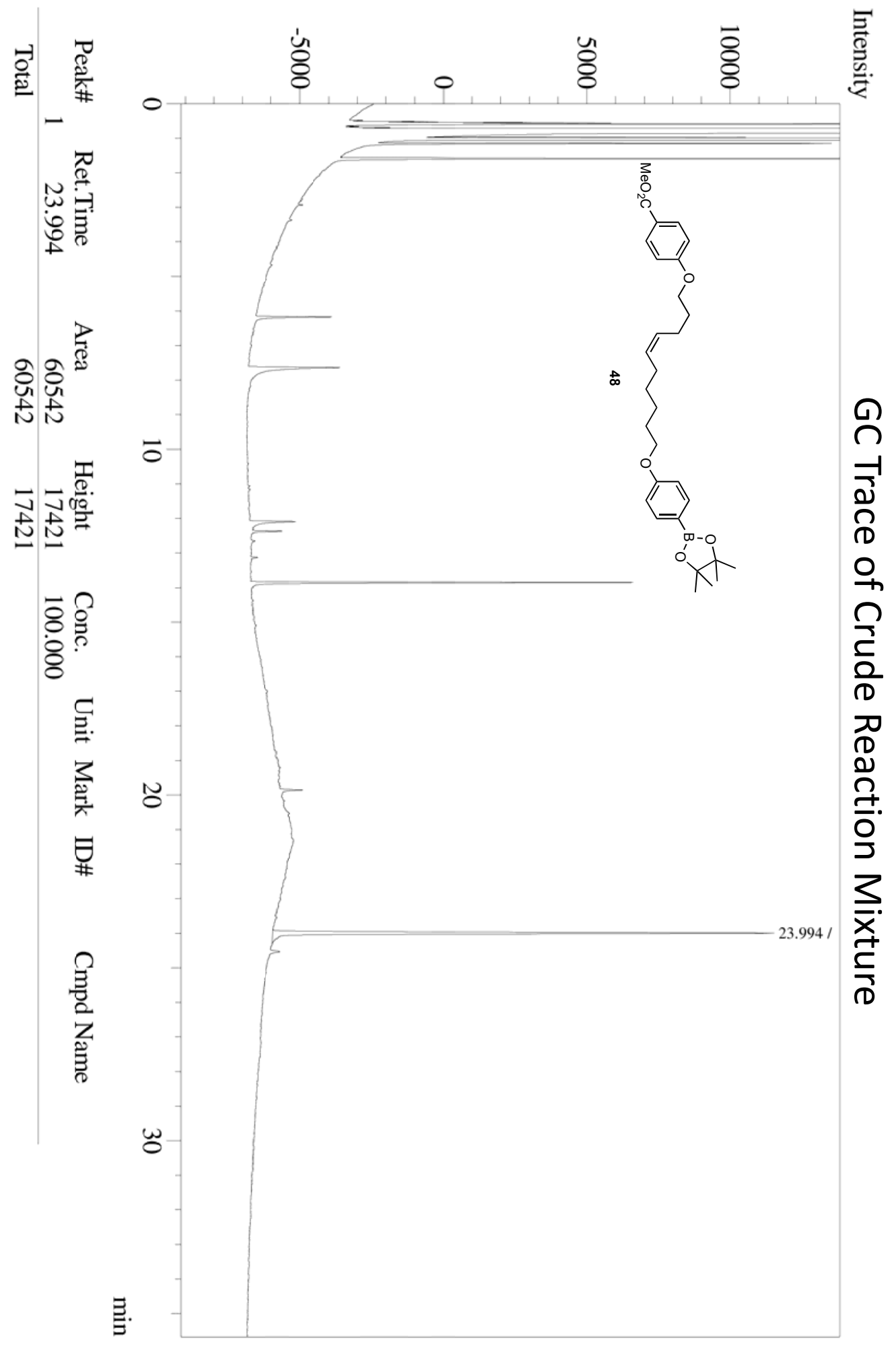



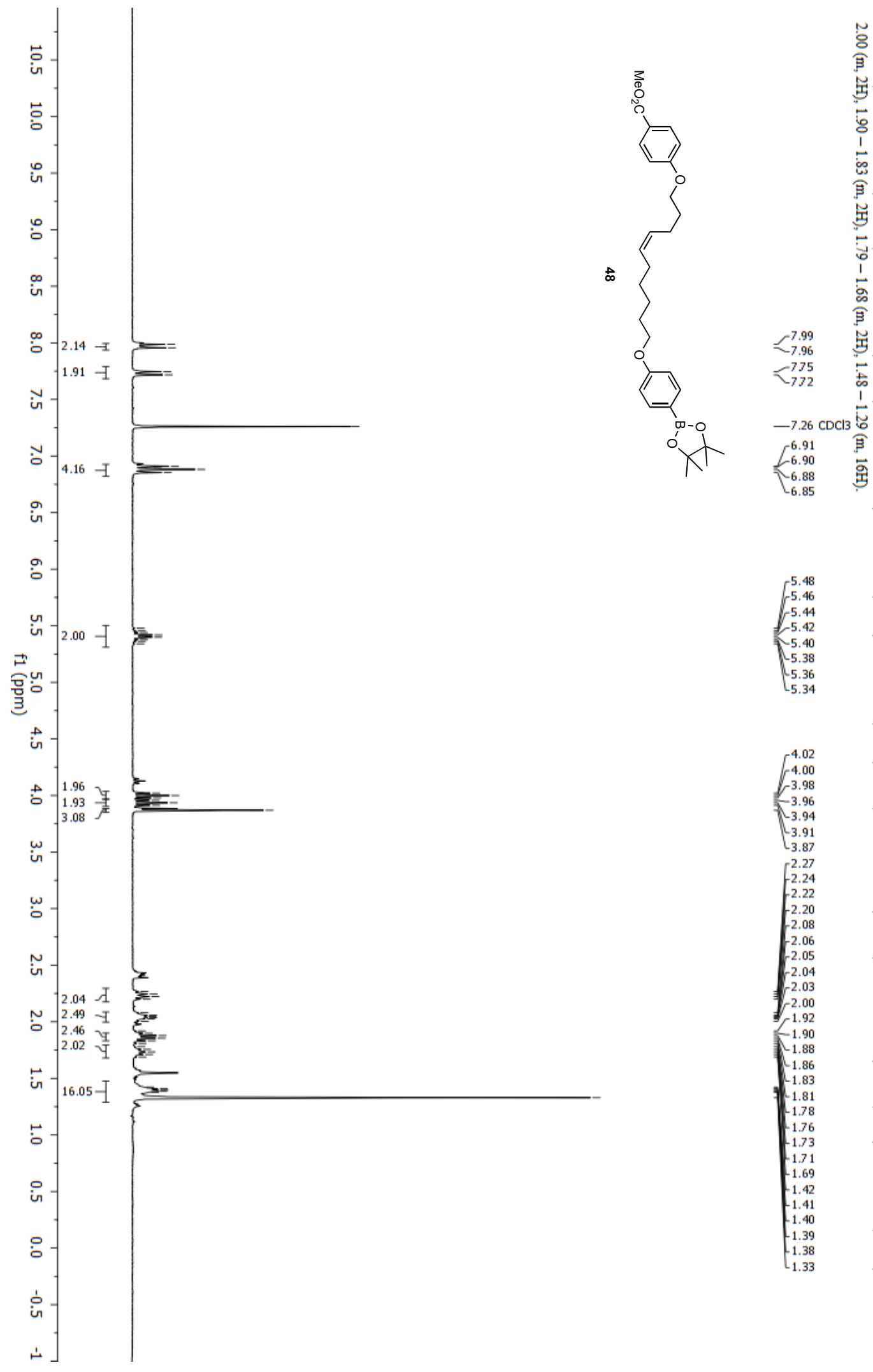

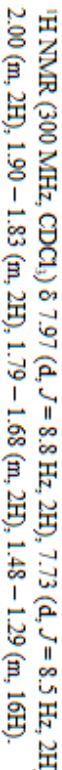

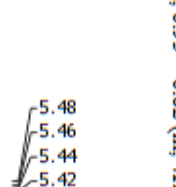

-5.44
-5.42

5.40
5.38

$-5.36$

菑

$r^{4.02}$.

$-3.98$

3.94
3.91

[3.87

2.27
-2.24
-2.22

$-2.20$

-2.08 - 2.06

2.05

$-2.04$

$r_{-1.92}^{2.00} \quad$ o

采

1.88
1.86

1.86

1.83

$-1.81$

$-1.78$

-1.76

-1.73 近

$-1.71$

\begin{tabular}{rr}
1.42 & 1 \\
-1.41 & $\quad$ \\
-1.40 & \multicolumn{1}{c}{}
\end{tabular}

-1.39 今

1.33

苟
0
0
0 

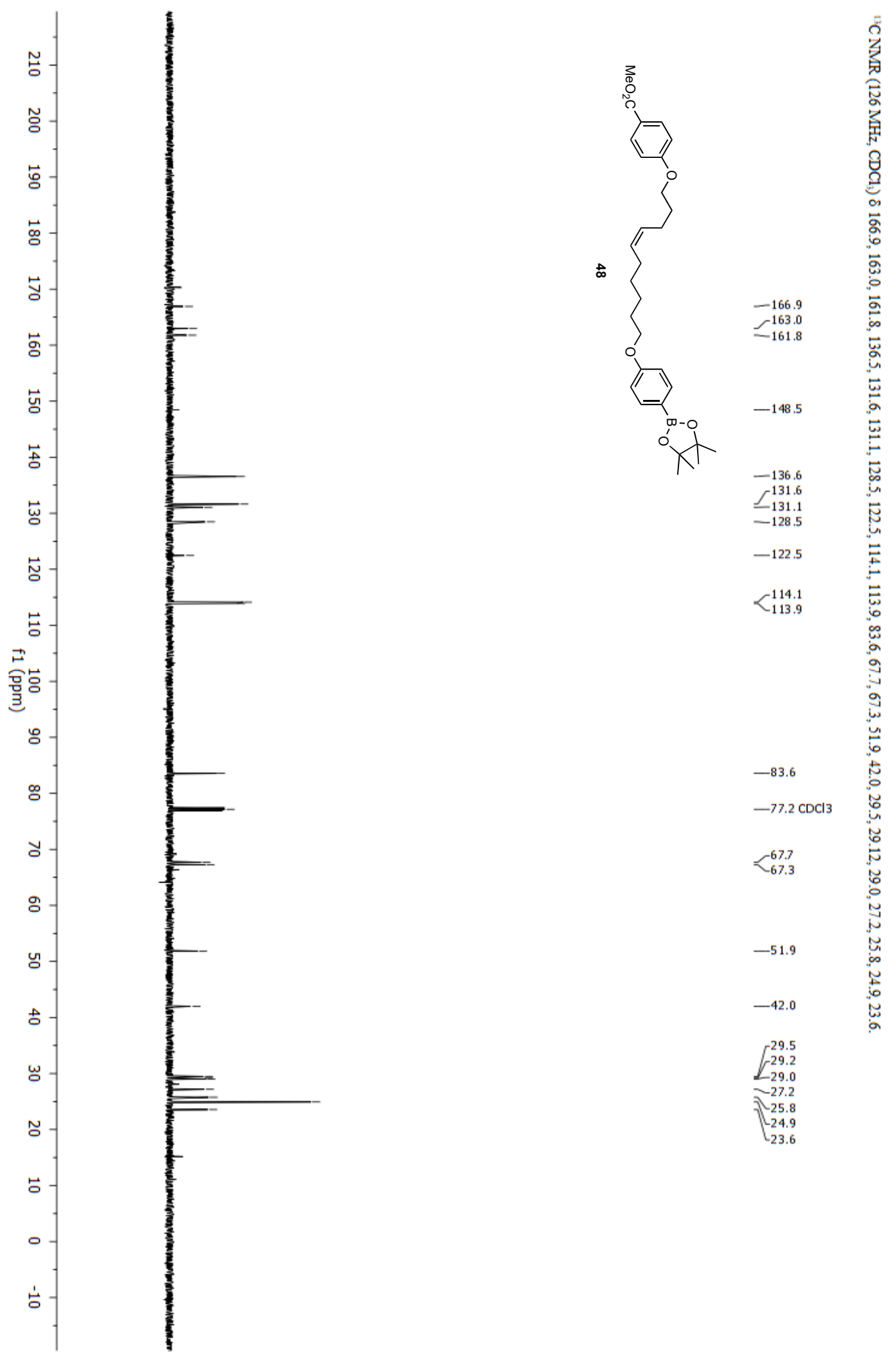

29.5
-29.2

$-29.0$

가. 25.8

-24.9
23.6 


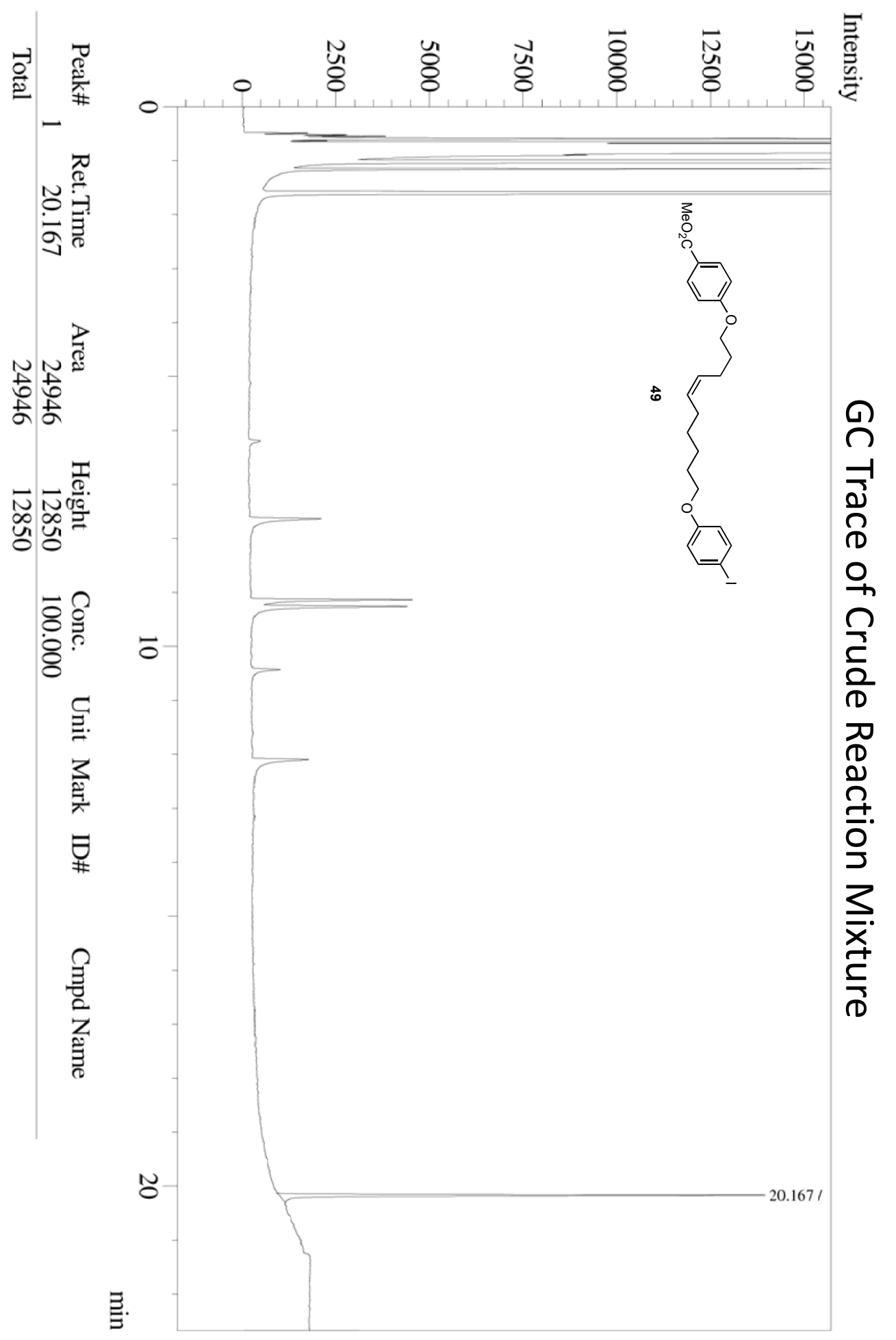



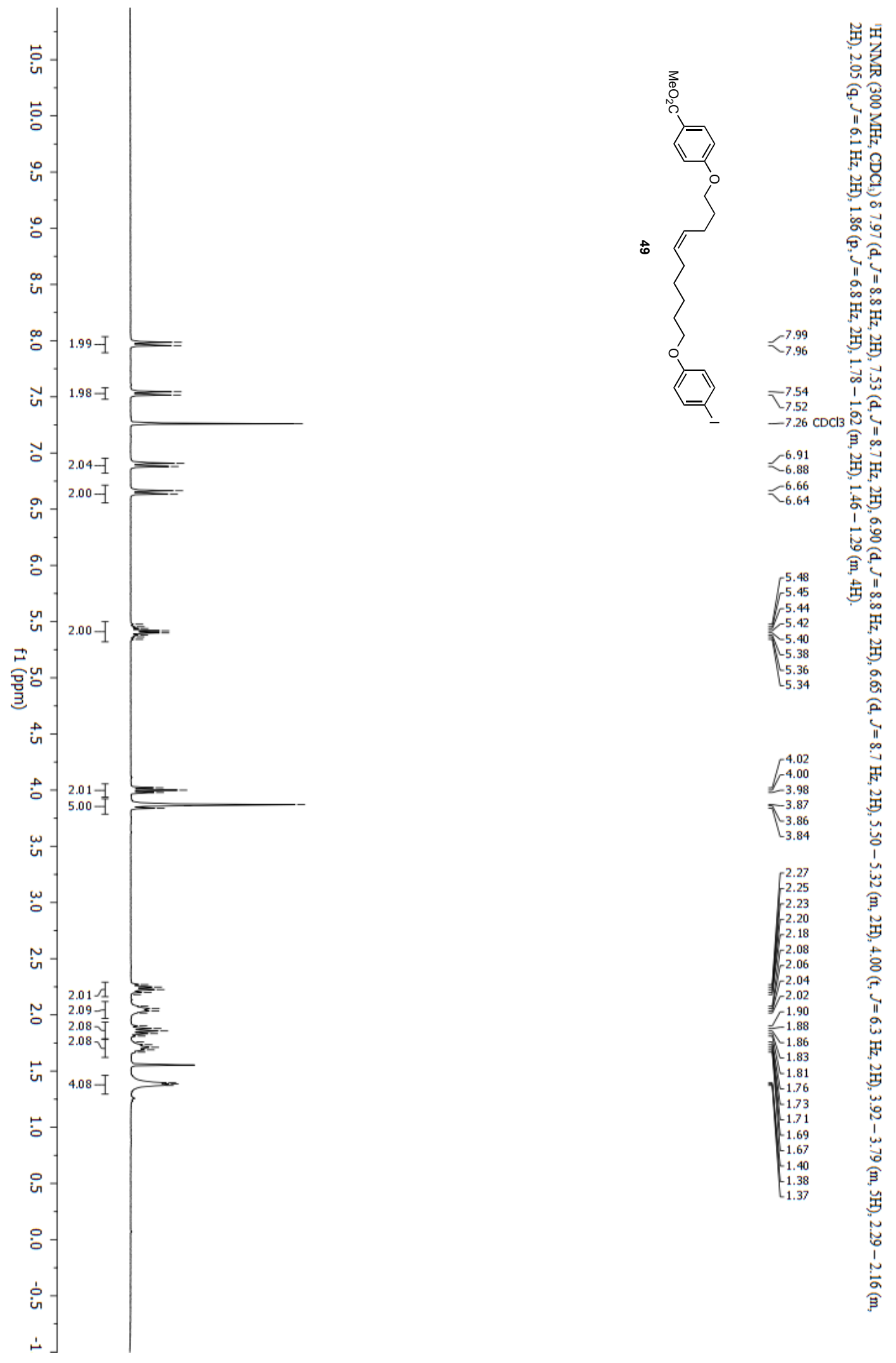

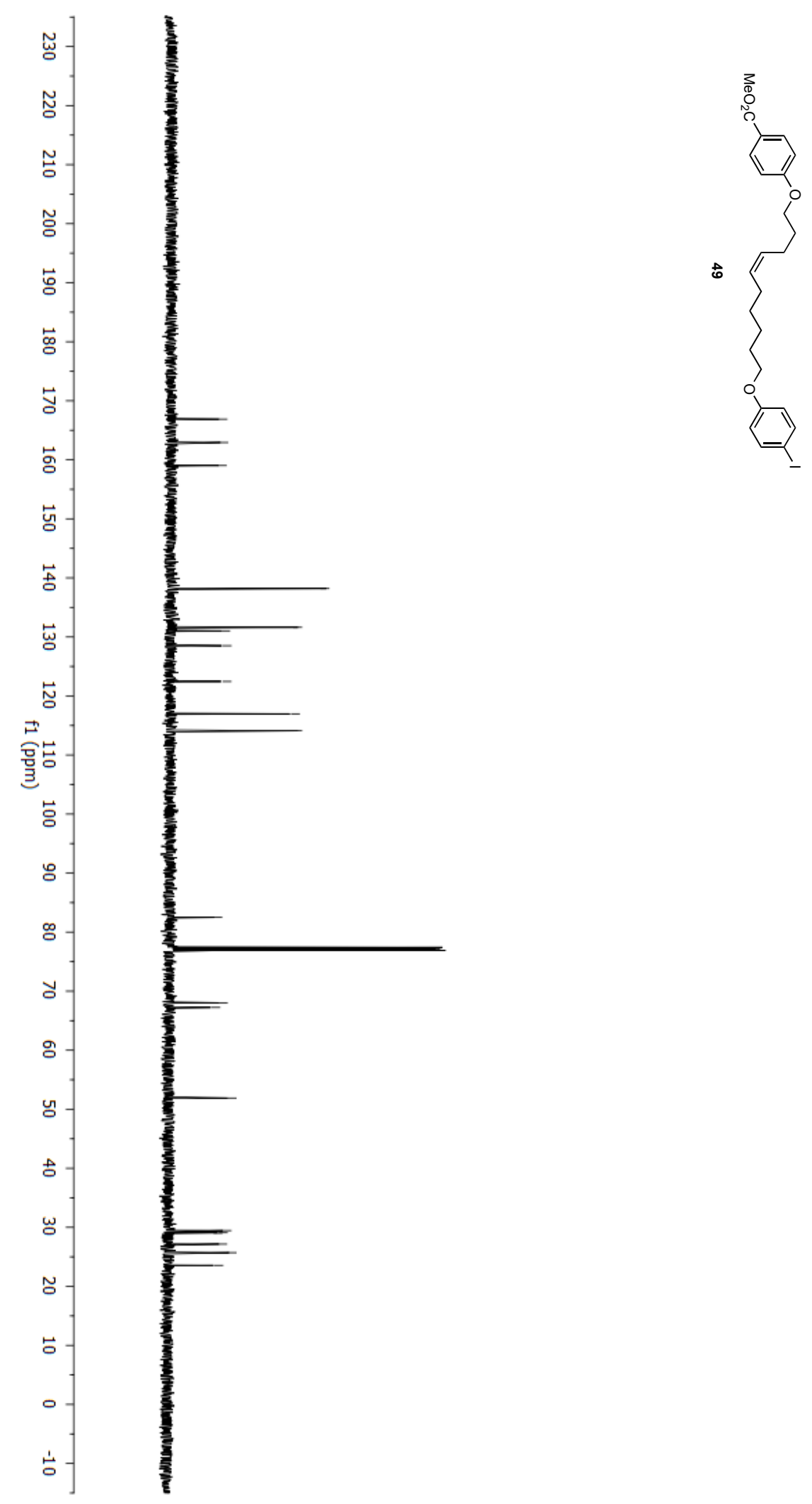

$-51.9$

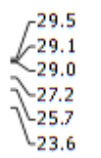




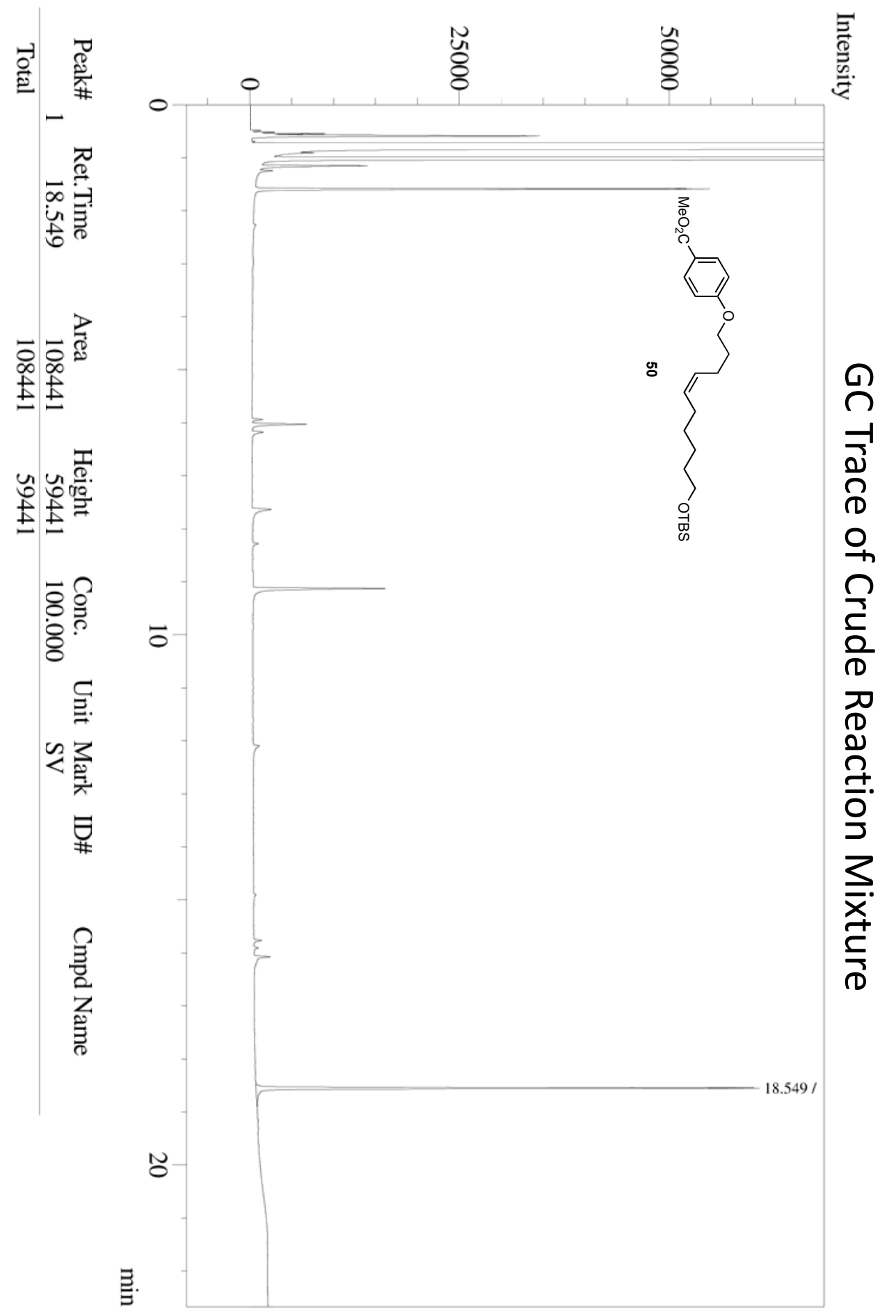




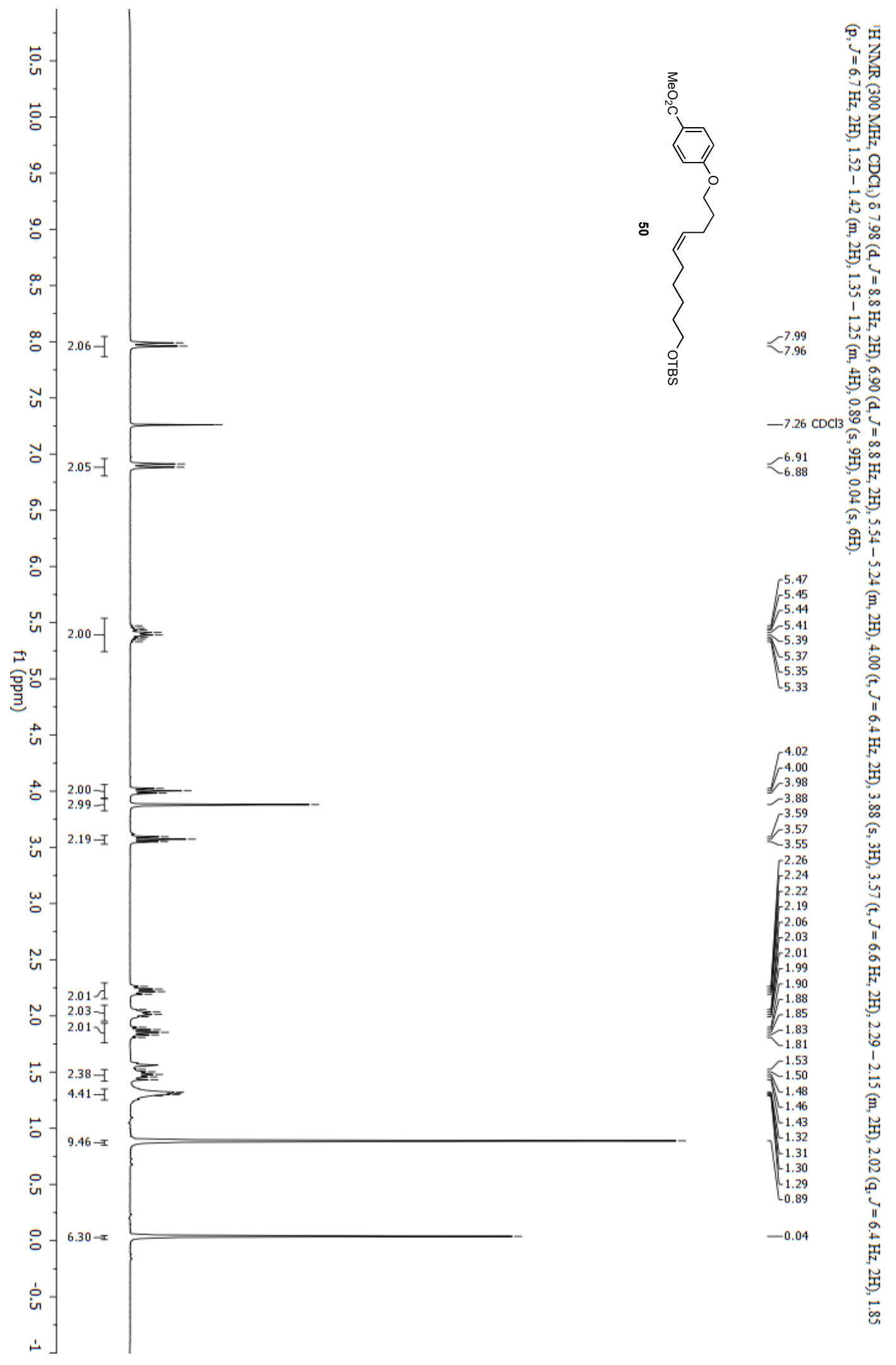



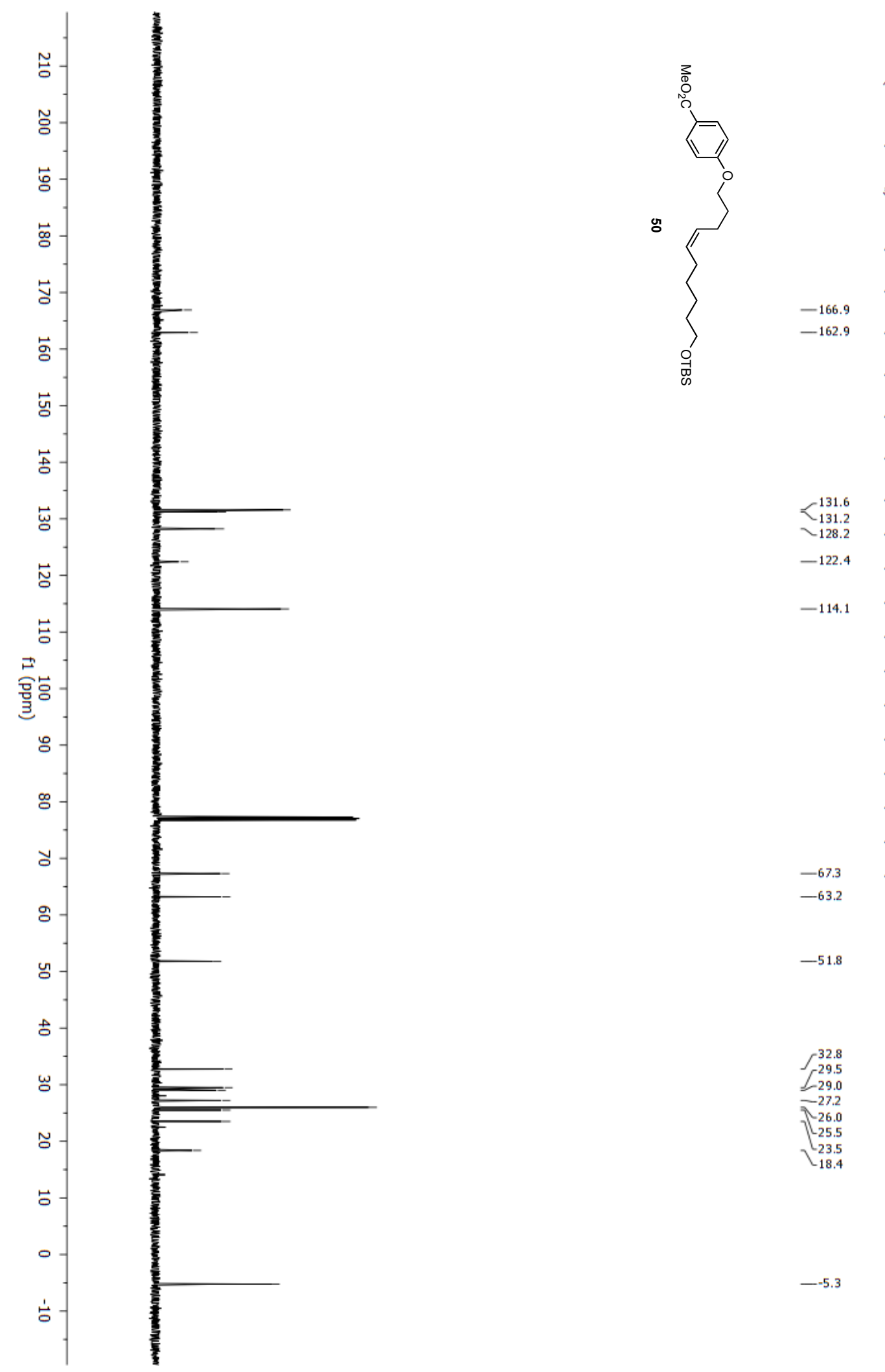

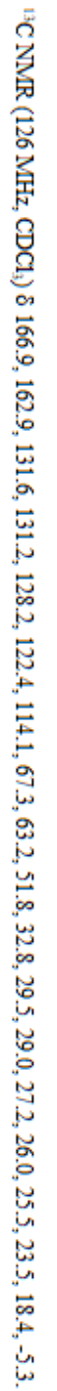

$-51.8$

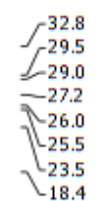

$--5.3$ 


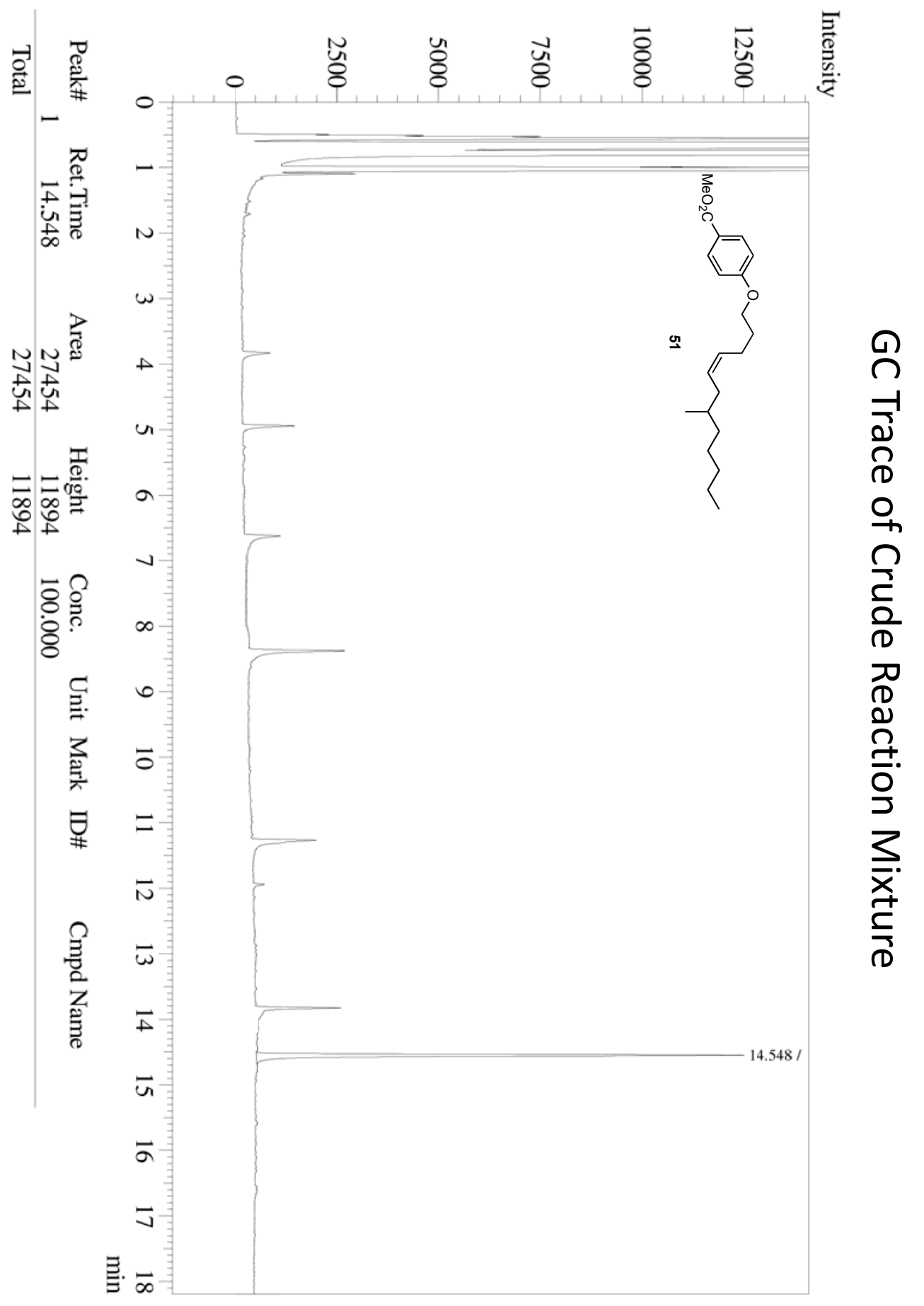



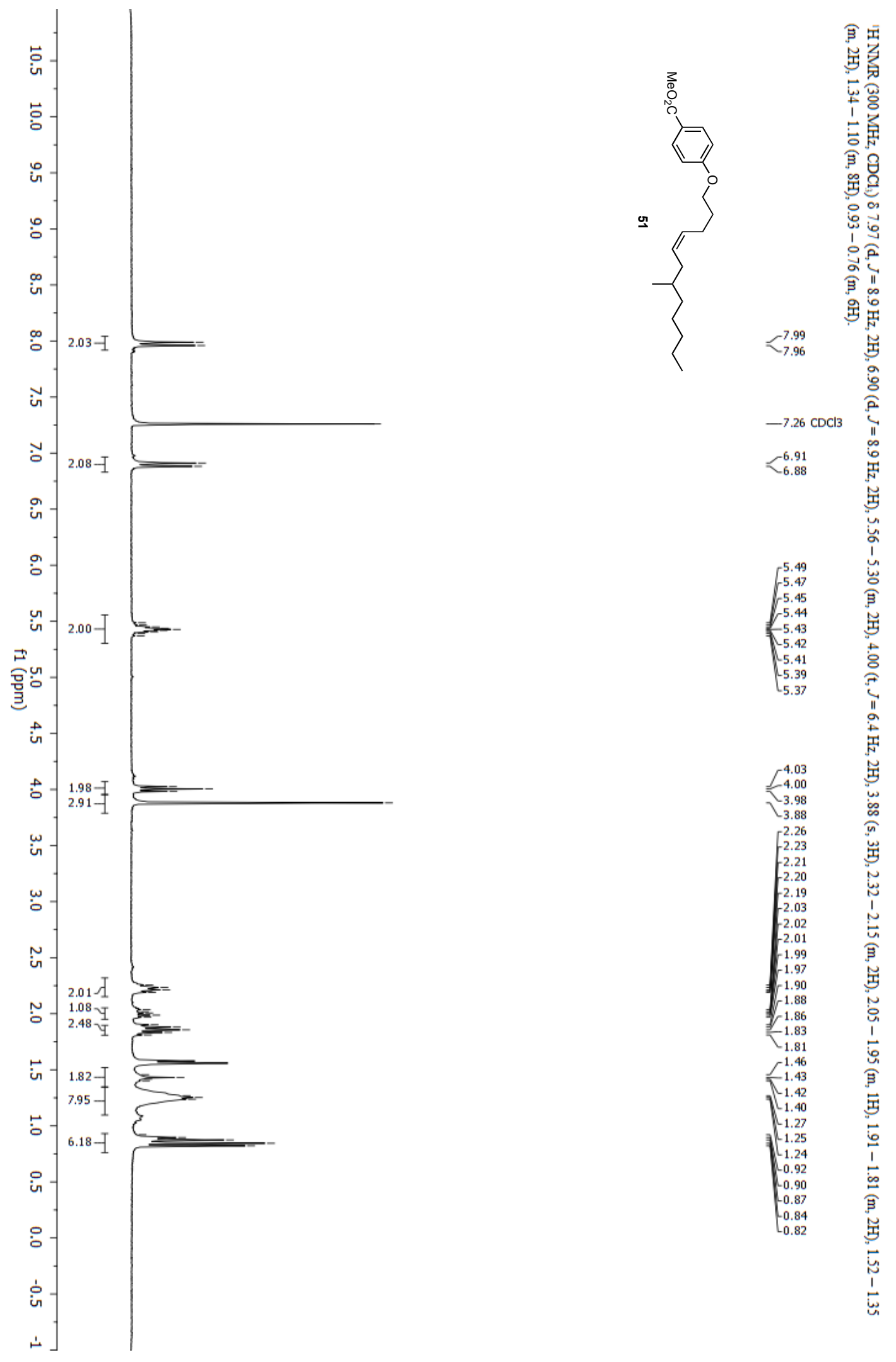

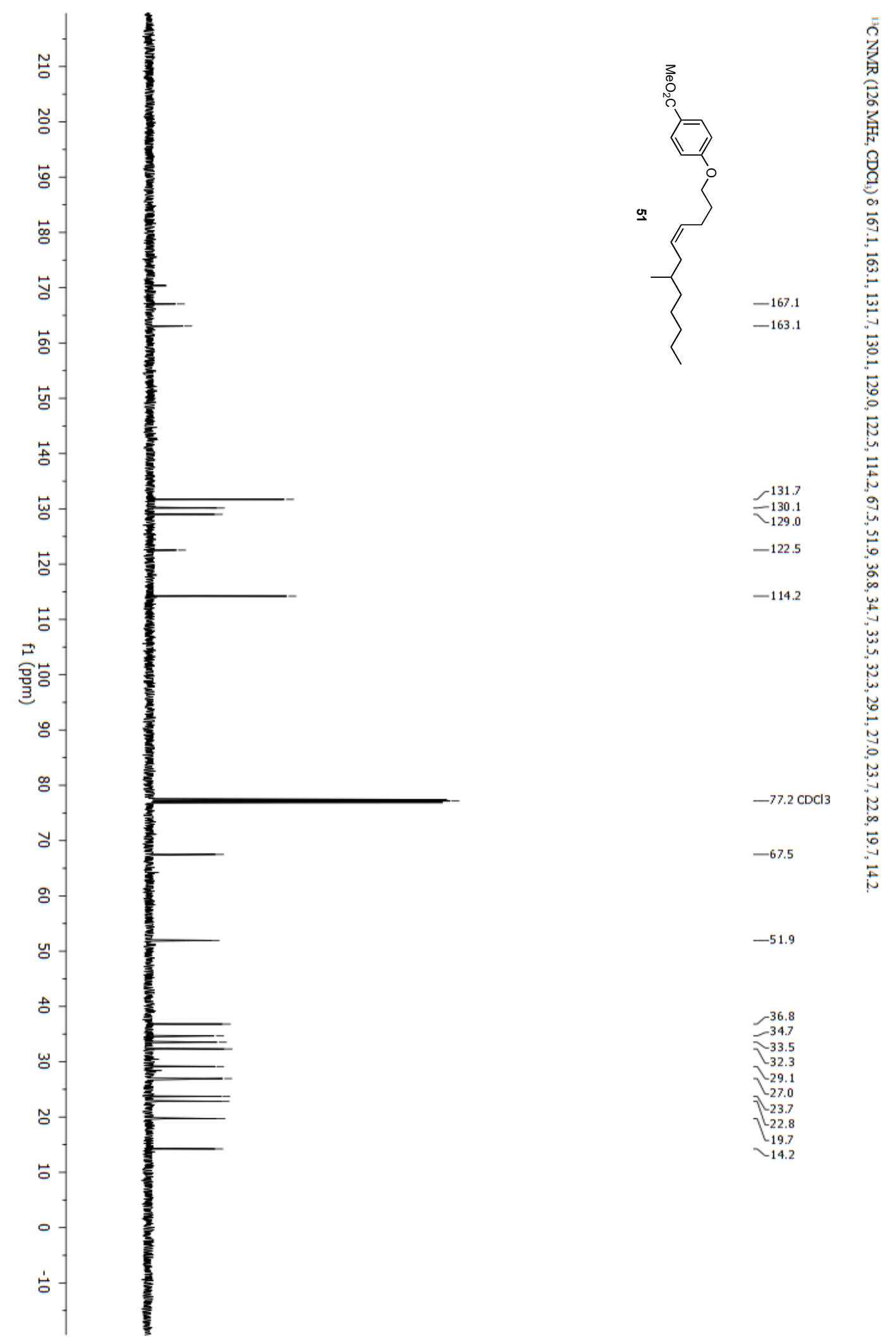


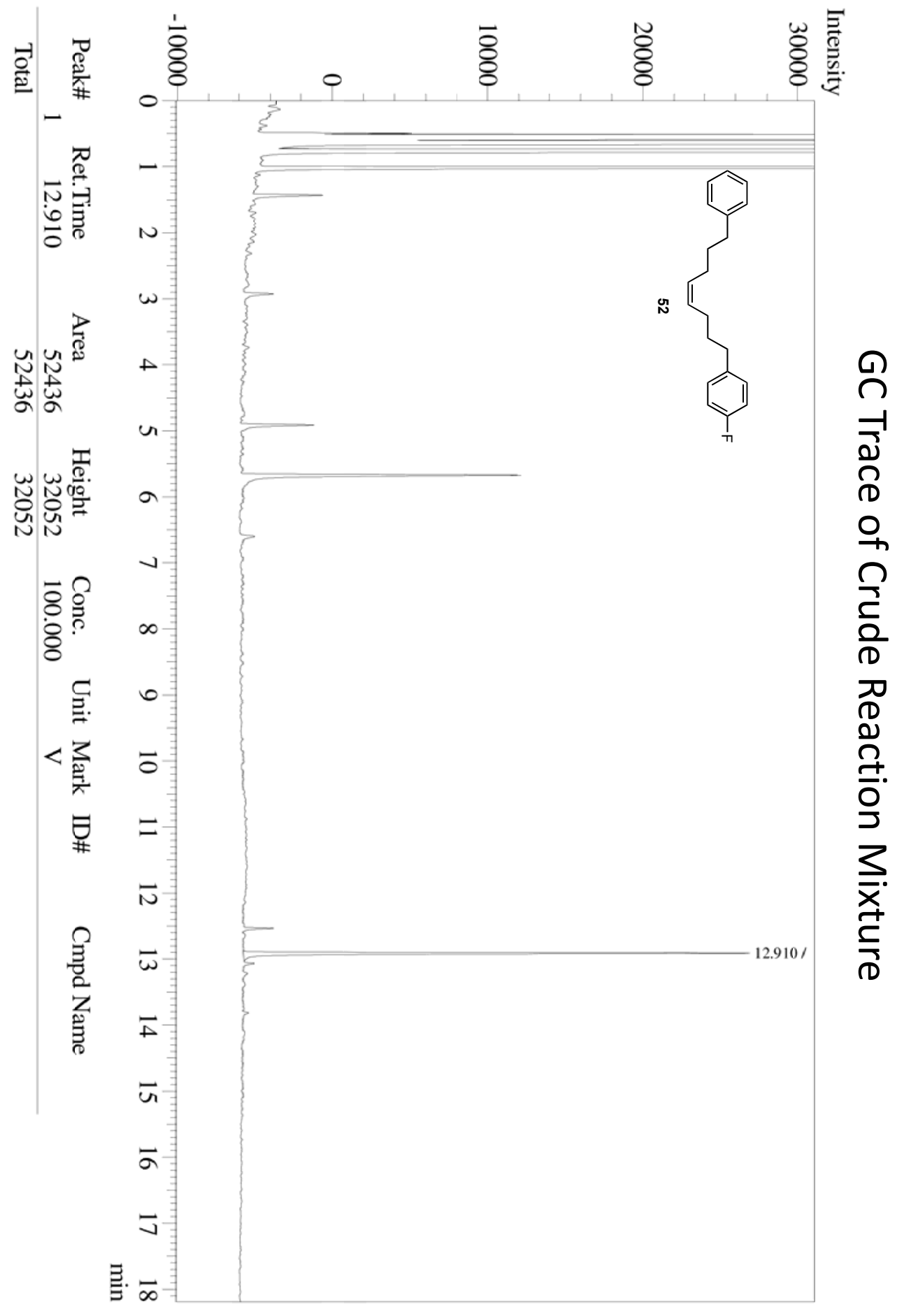



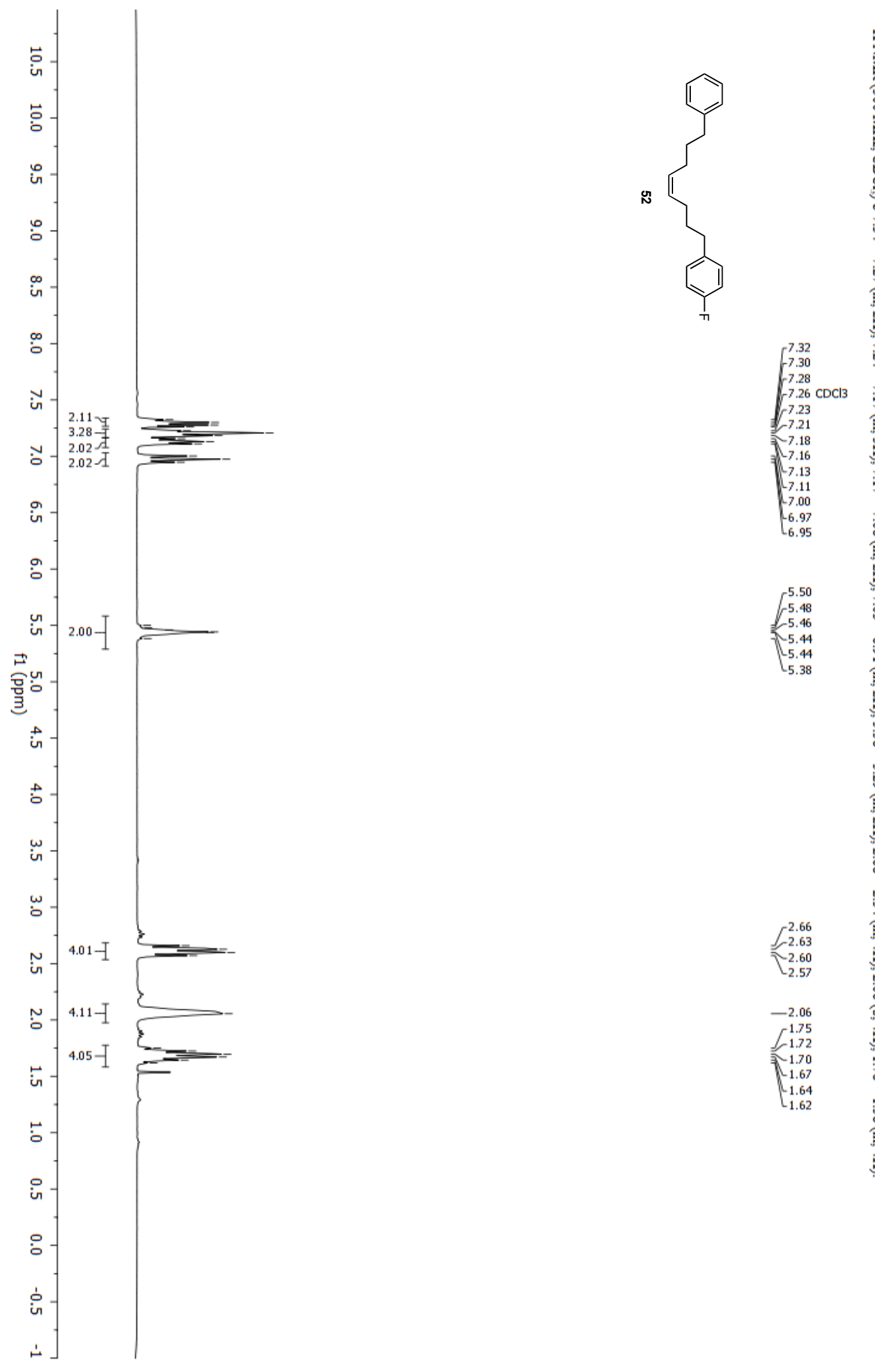

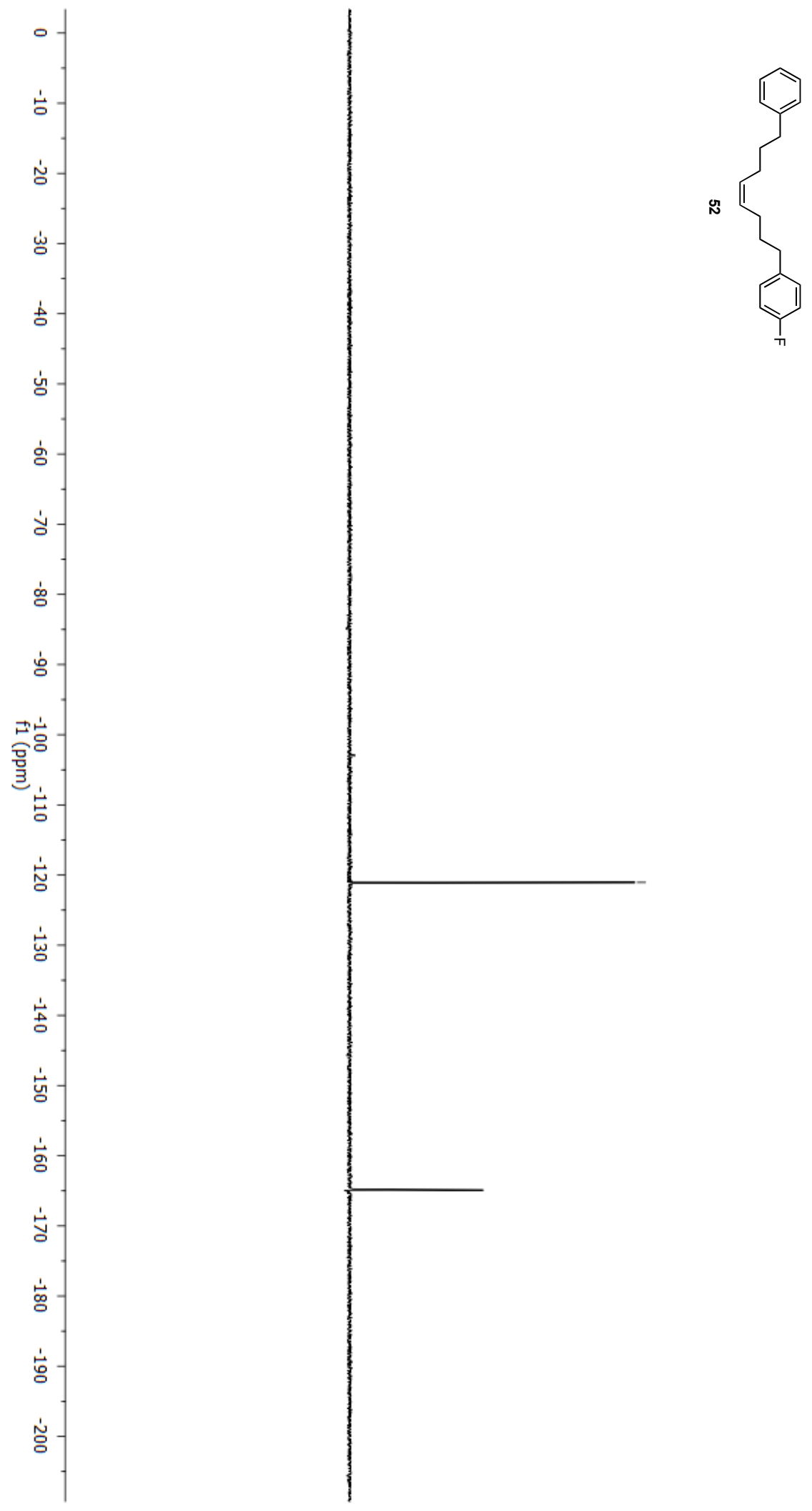

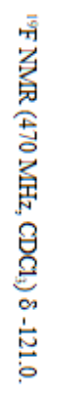

${ }^{-121.0}$

S201 

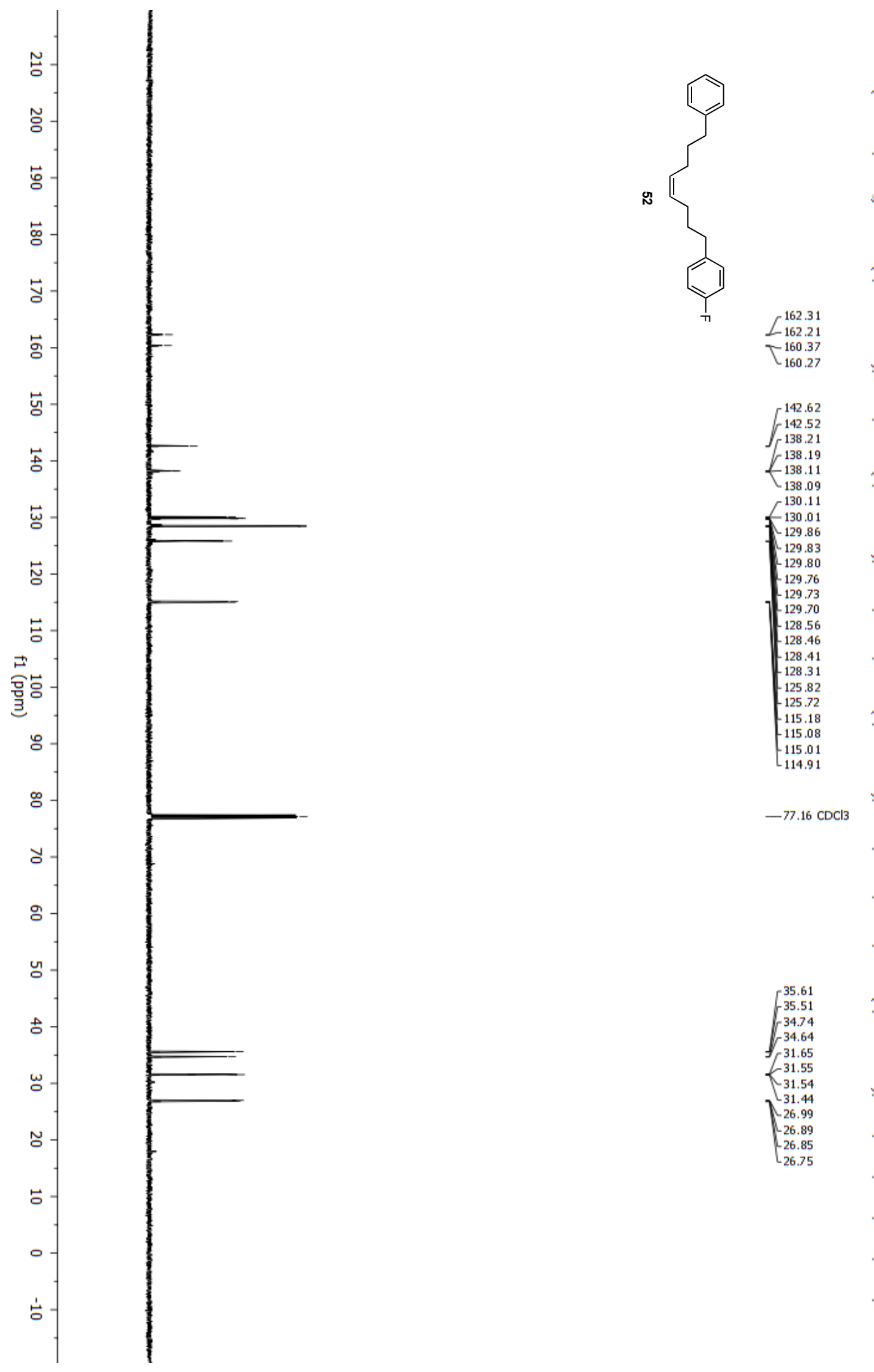

142.62

142.52
-138.21

$K_{138.11}^{138.19}$

138.11

$\Gamma^{130.11}$

$\left\{\begin{array}{l}130.0 \\ 130.0\end{array}\right.$

129.86
-129.83

-129.83
-129.80

$-129.76$

$-129.73$

$-129.70$

-128.56
-128.46

$-128.41$

$-128.31$

125.82

$-125.72$

$-115.18$

-115.08
-115.01

$-114.91$

$-77.16 \mathrm{CDCl}$
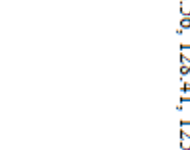

$-35.61$

${ }_{-35.51}^{35.61}$

34.74

$\int^{34.64}$

$K_{31.54}^{31.55}$

31.54
31.44

26.99

26.89

$-26.85$

$-26.75$ 


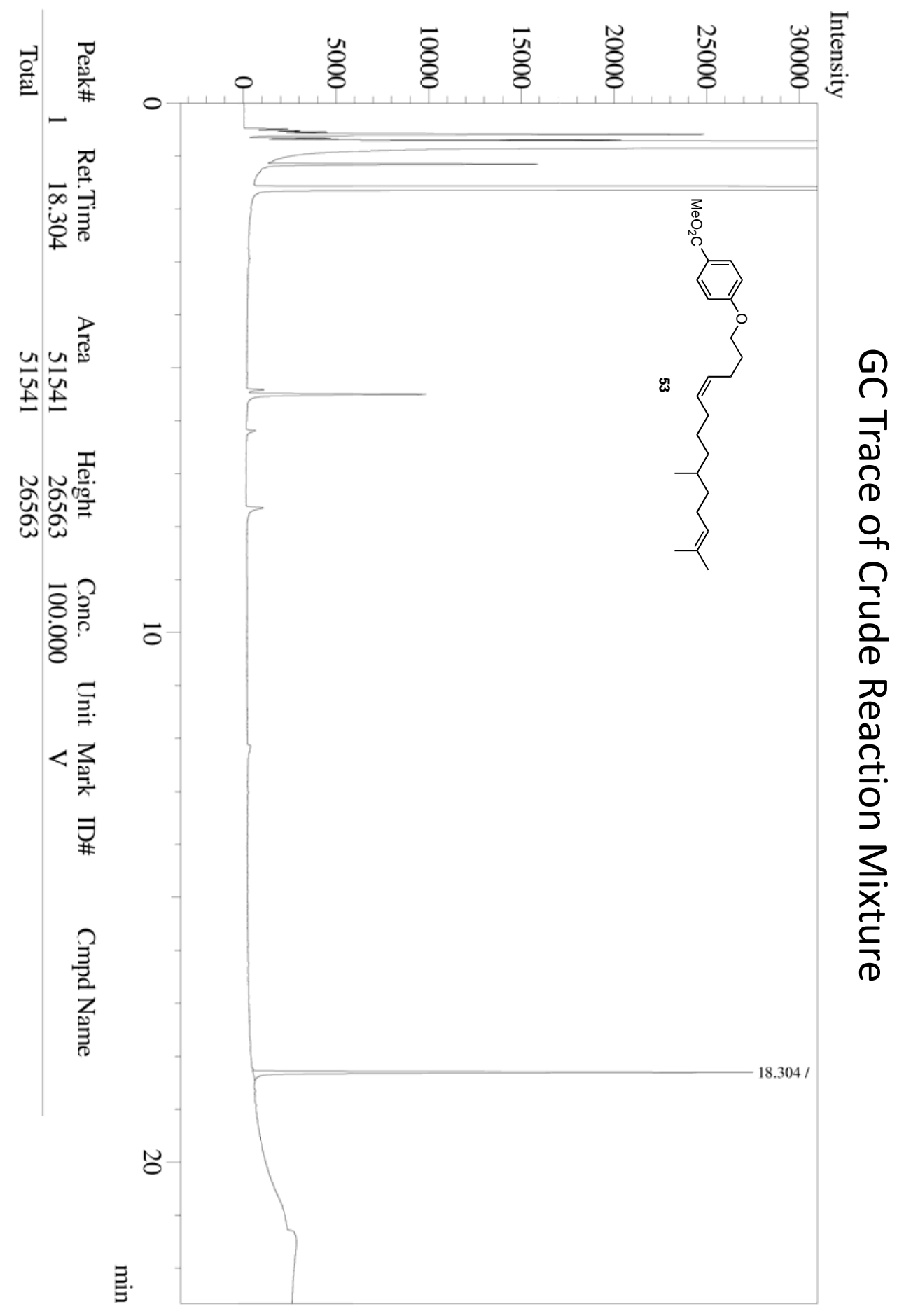



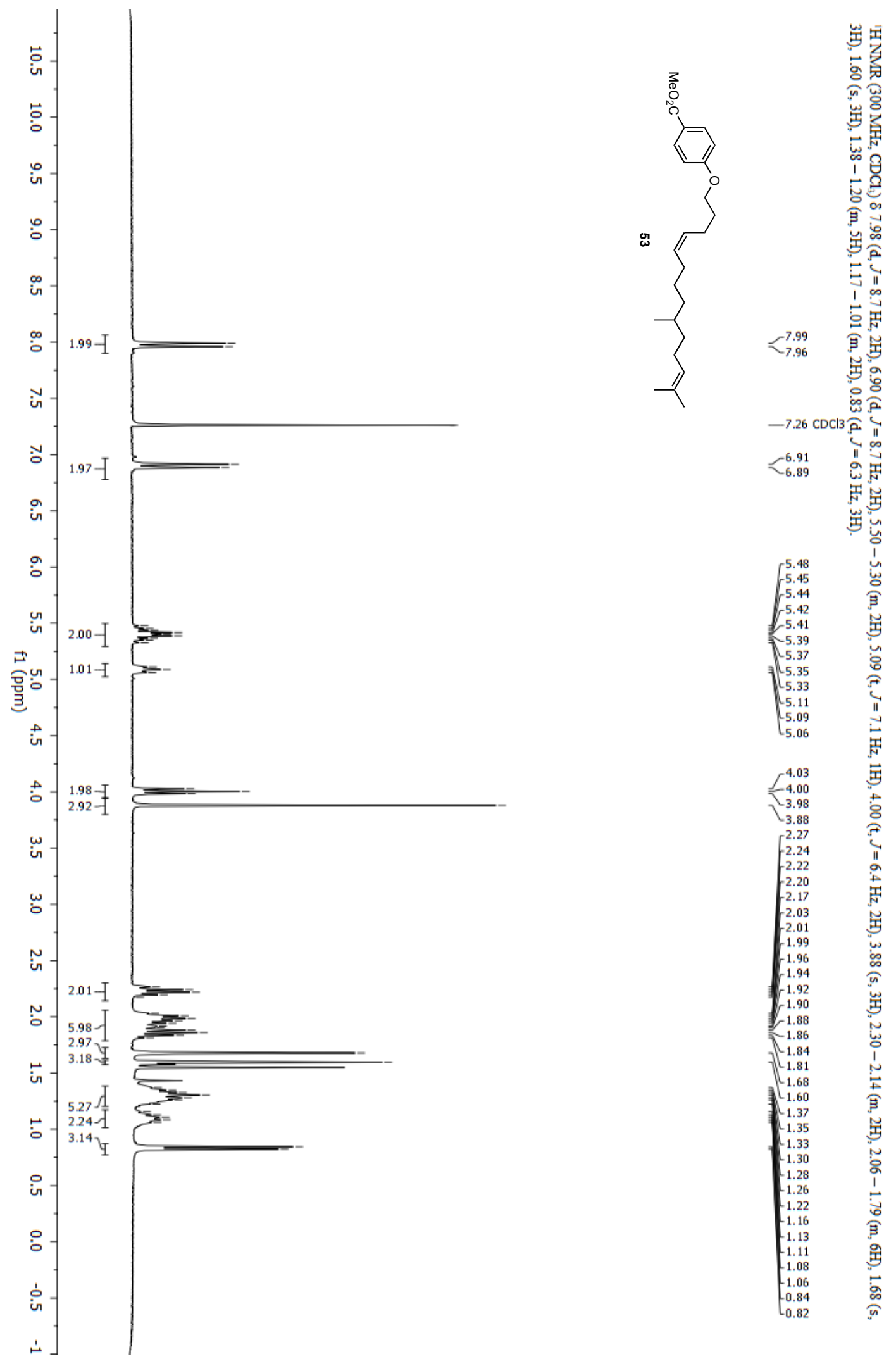

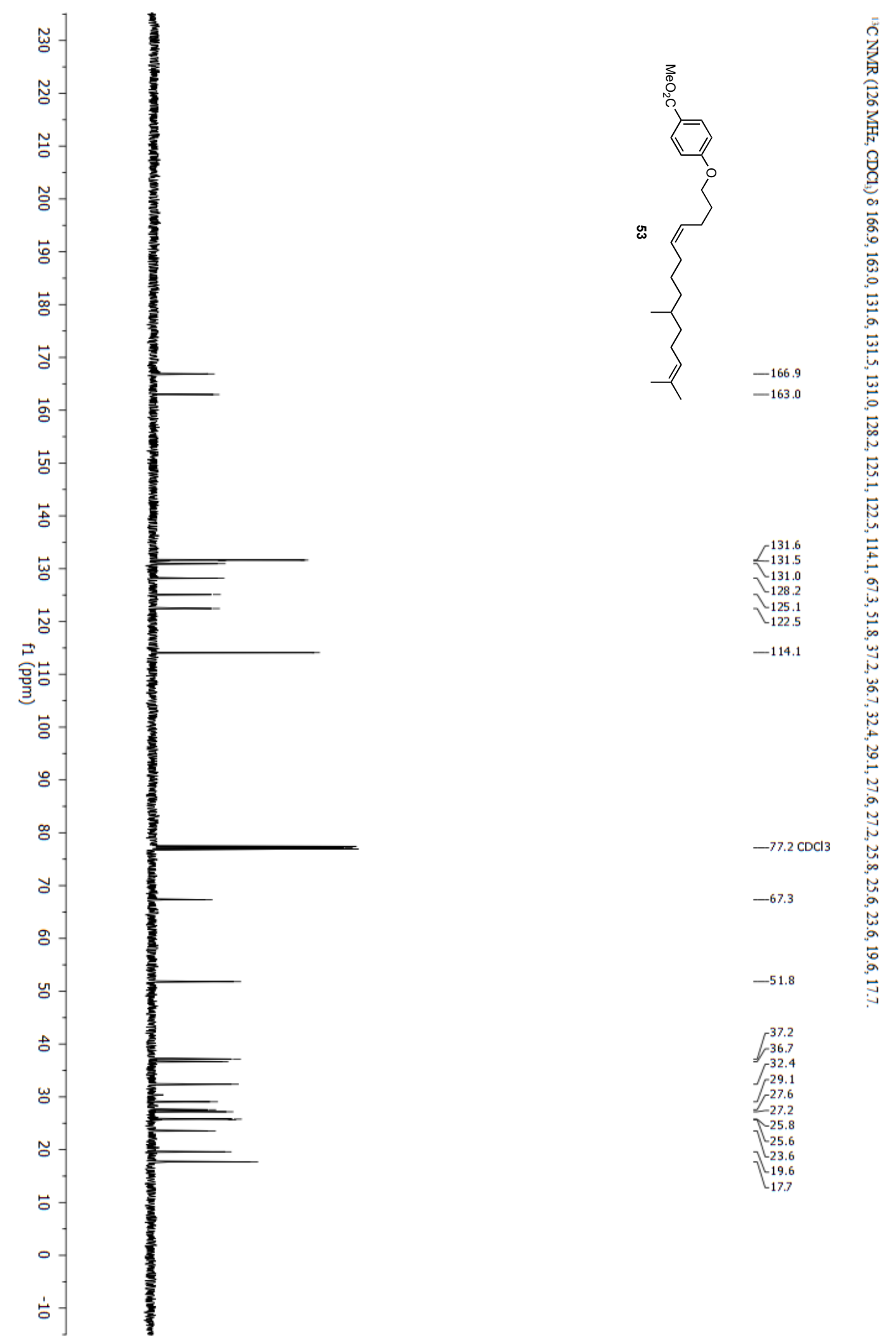


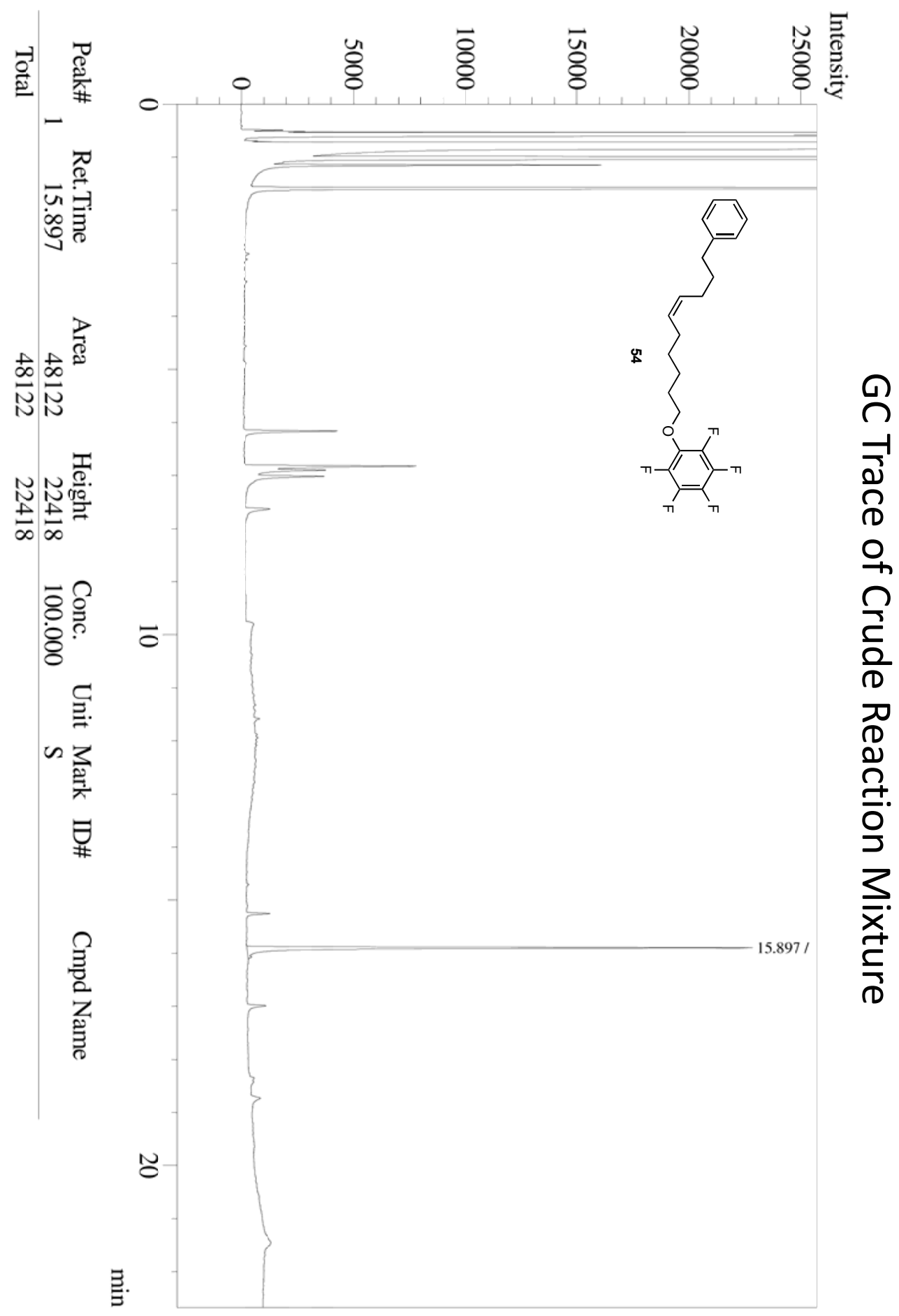



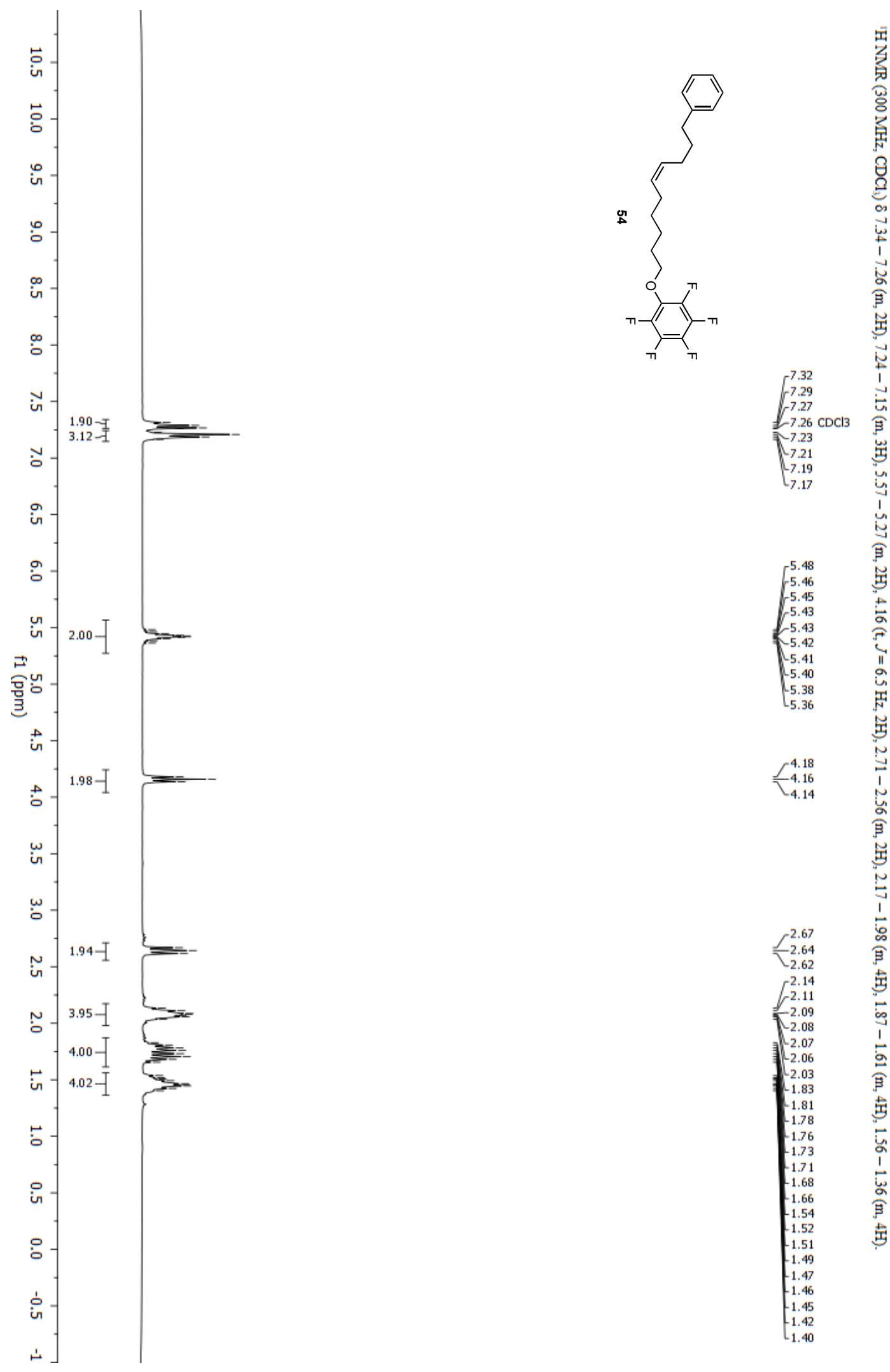

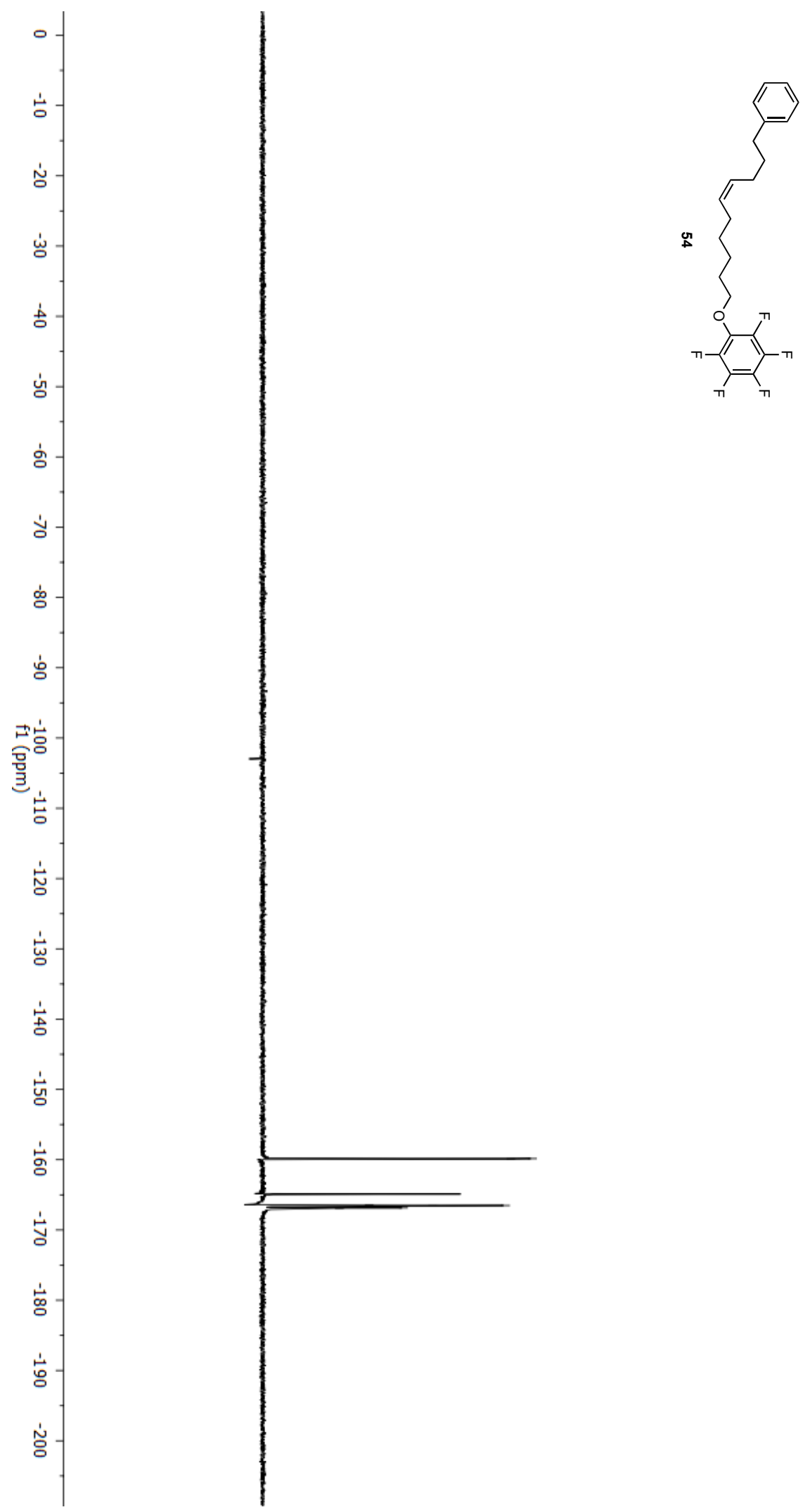

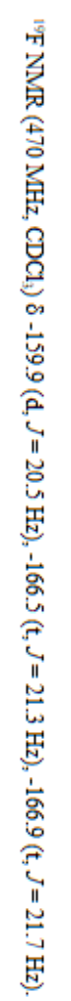

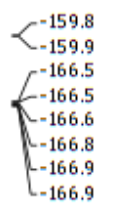



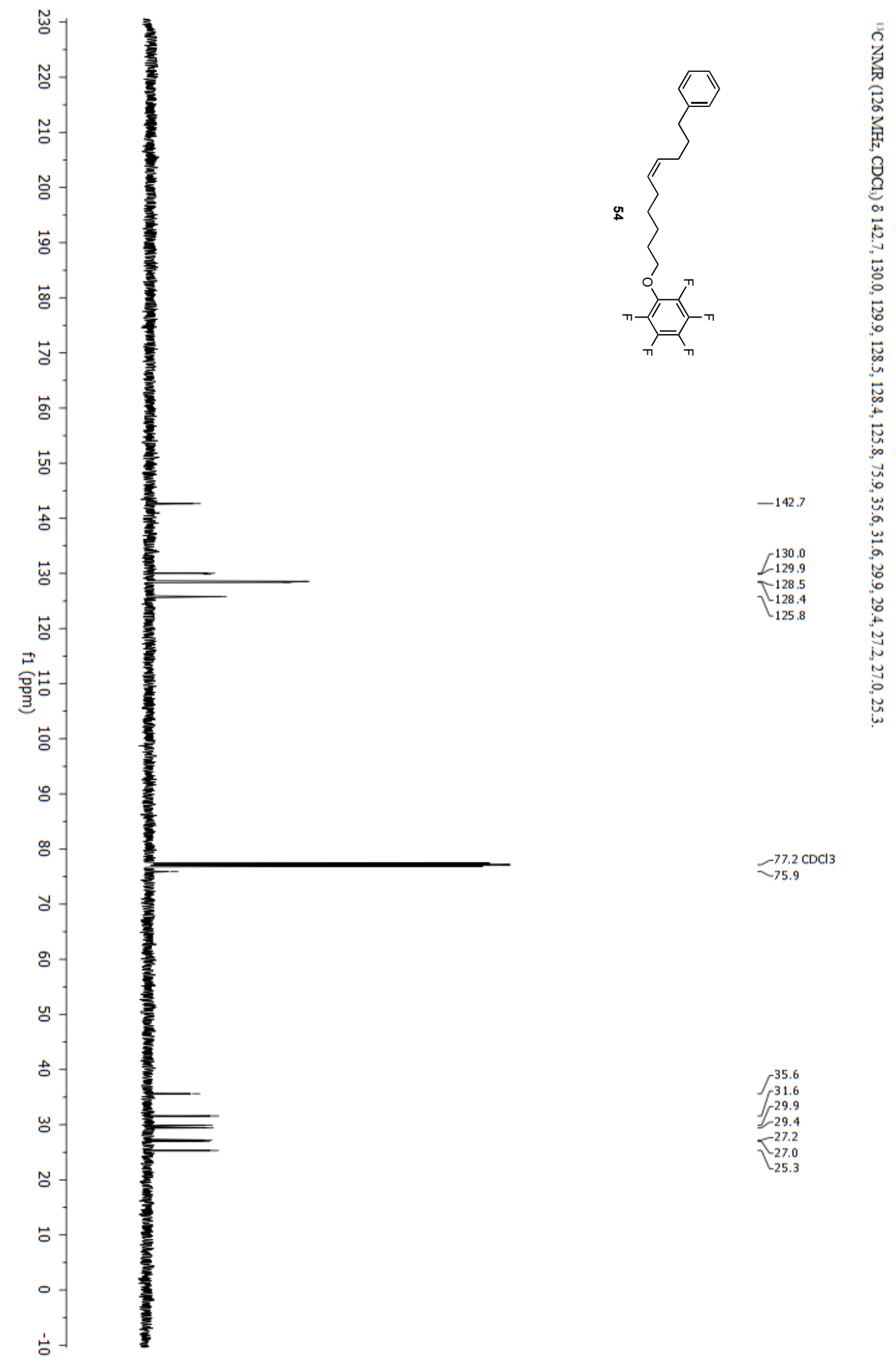

$-77.2 \mathrm{CDCl} 3$

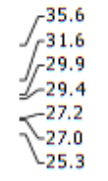



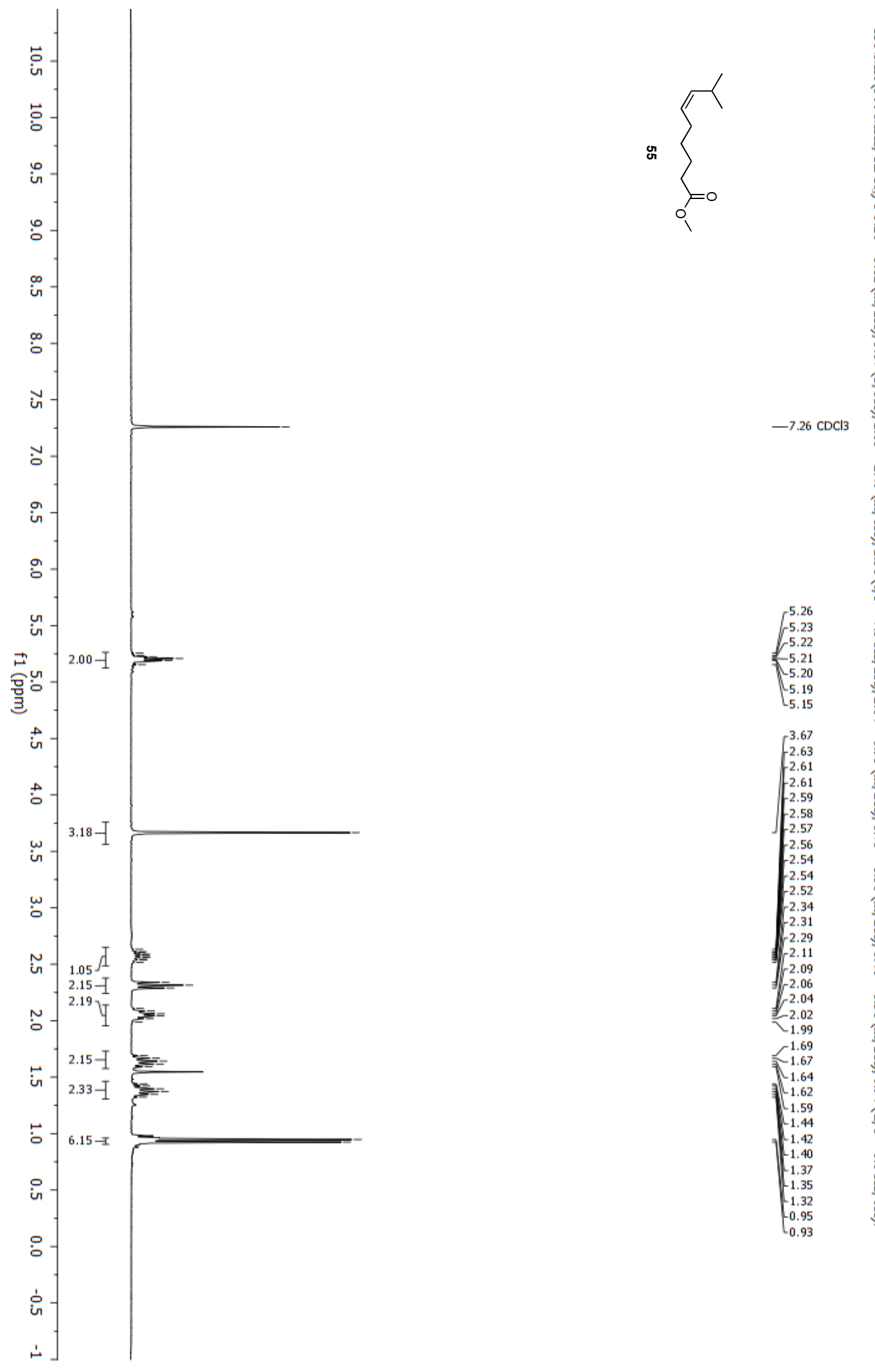

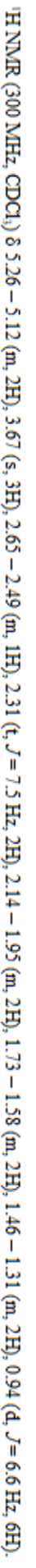




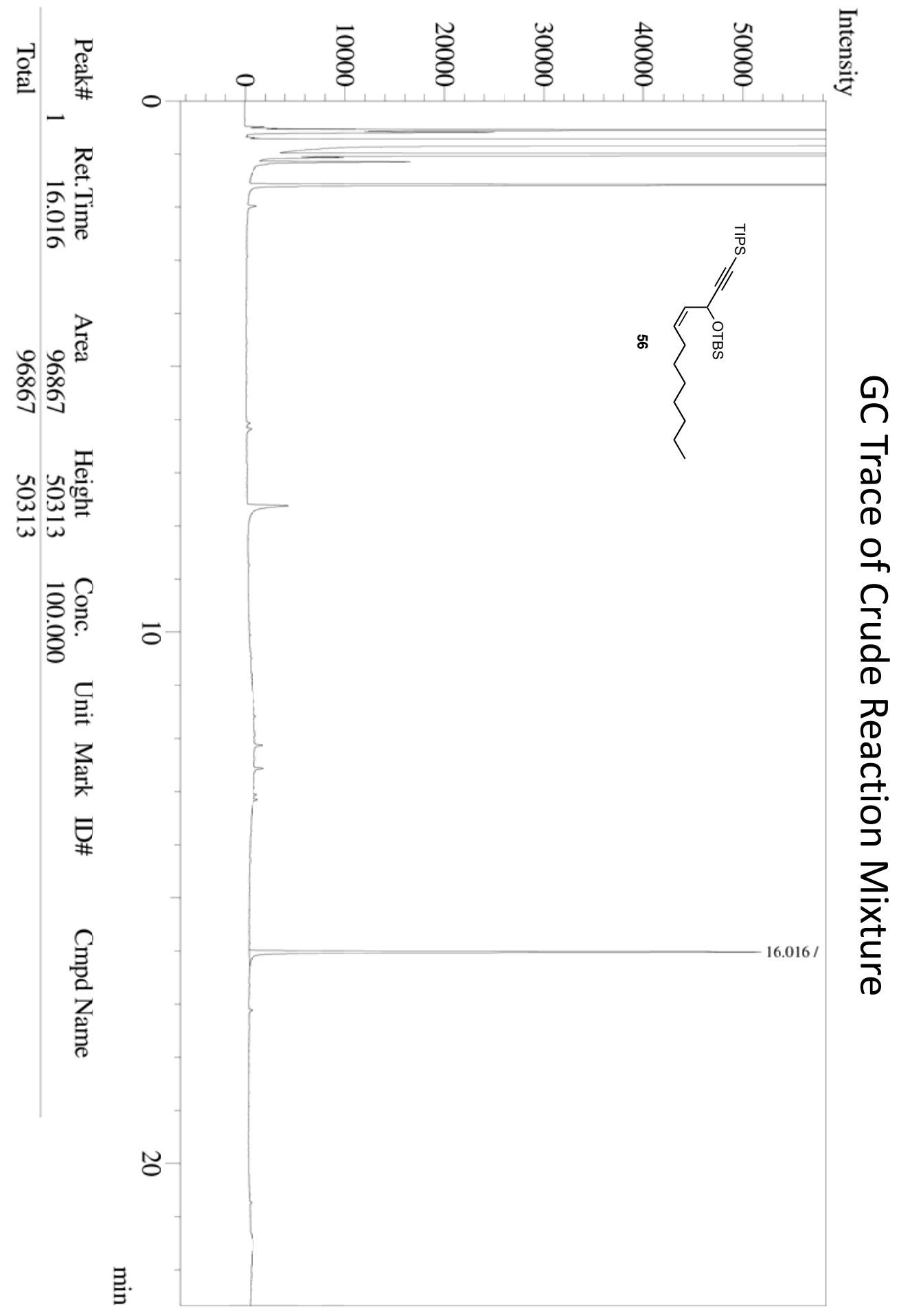




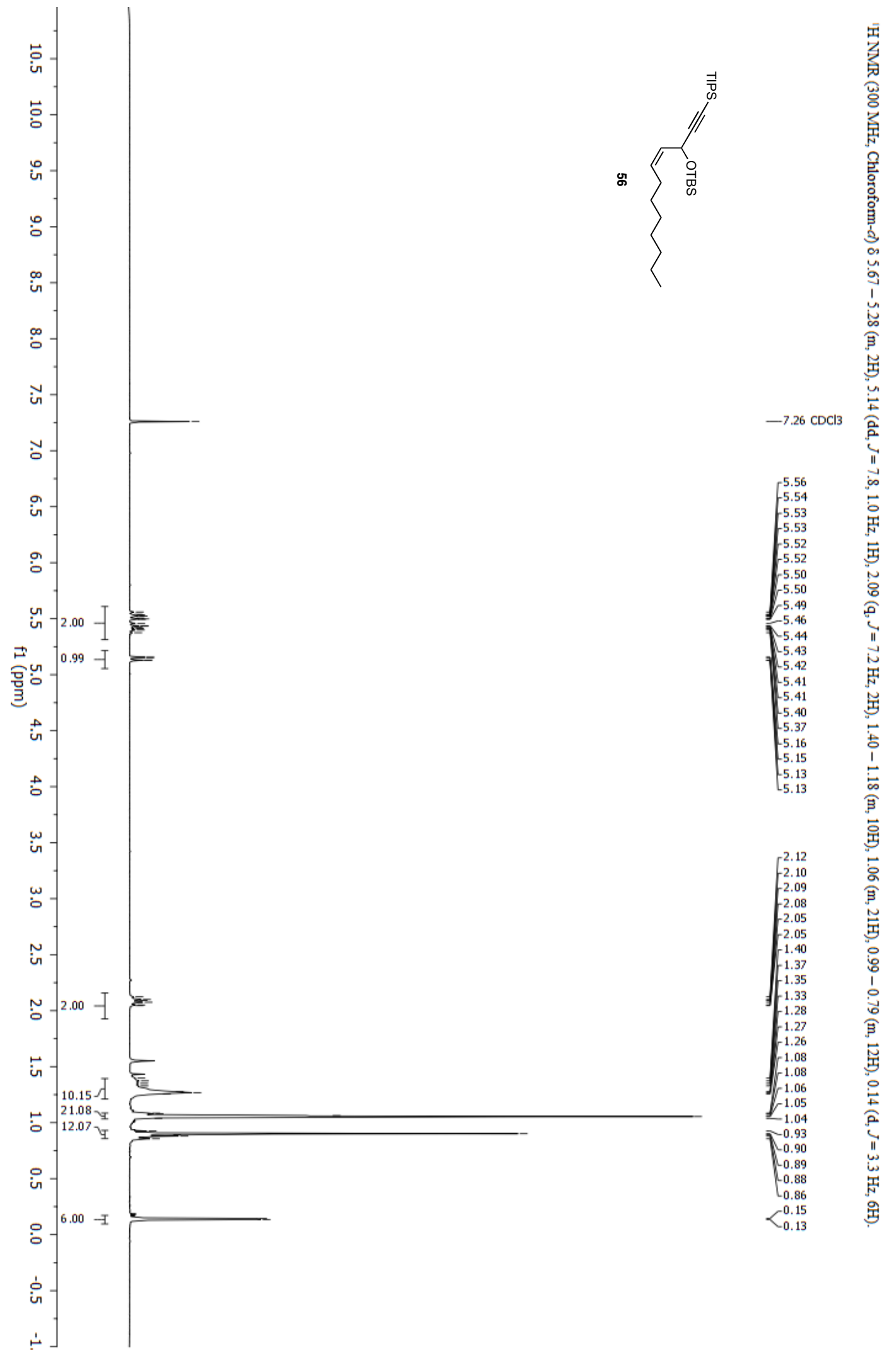



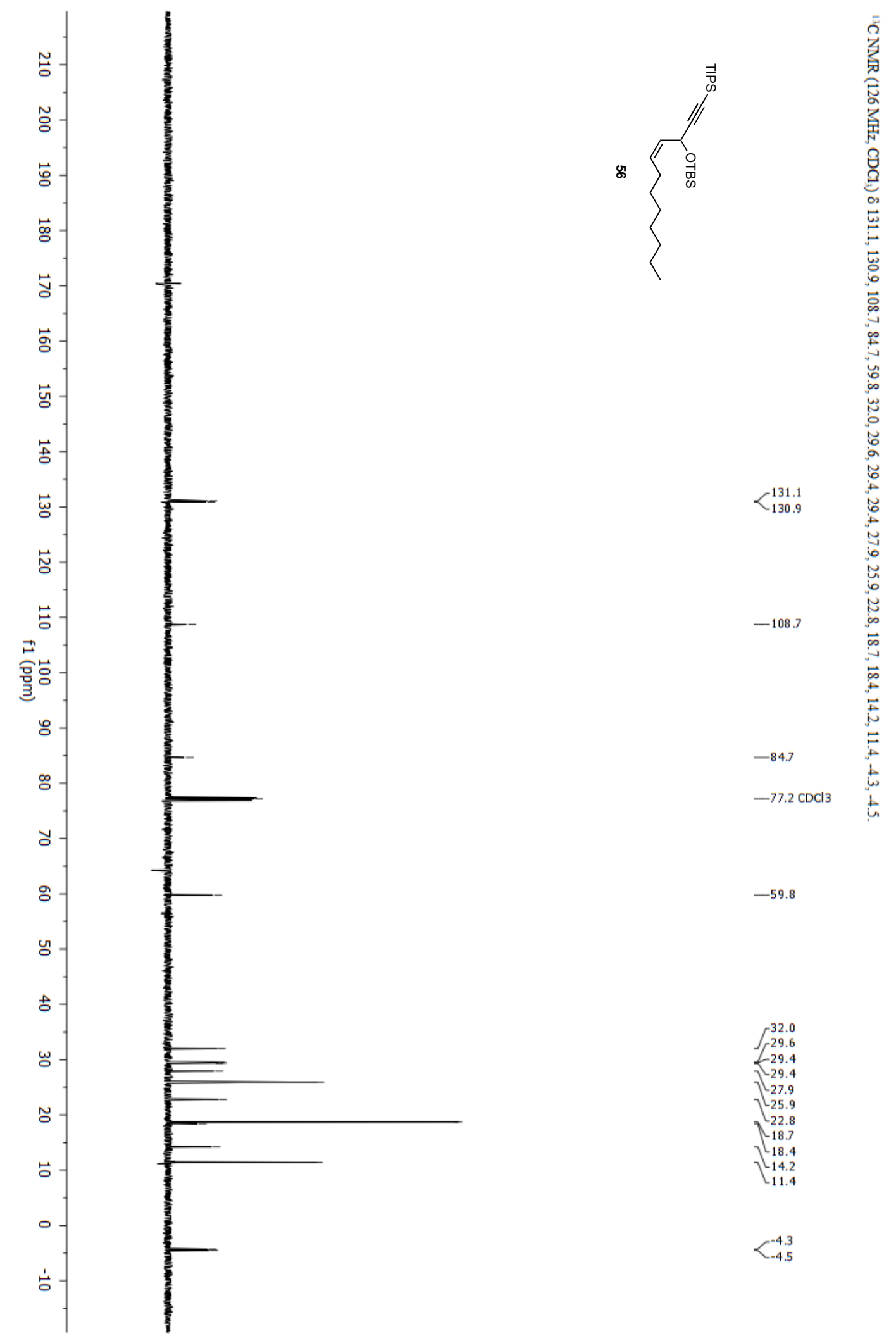

$-59.8$

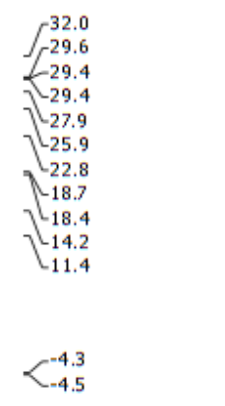




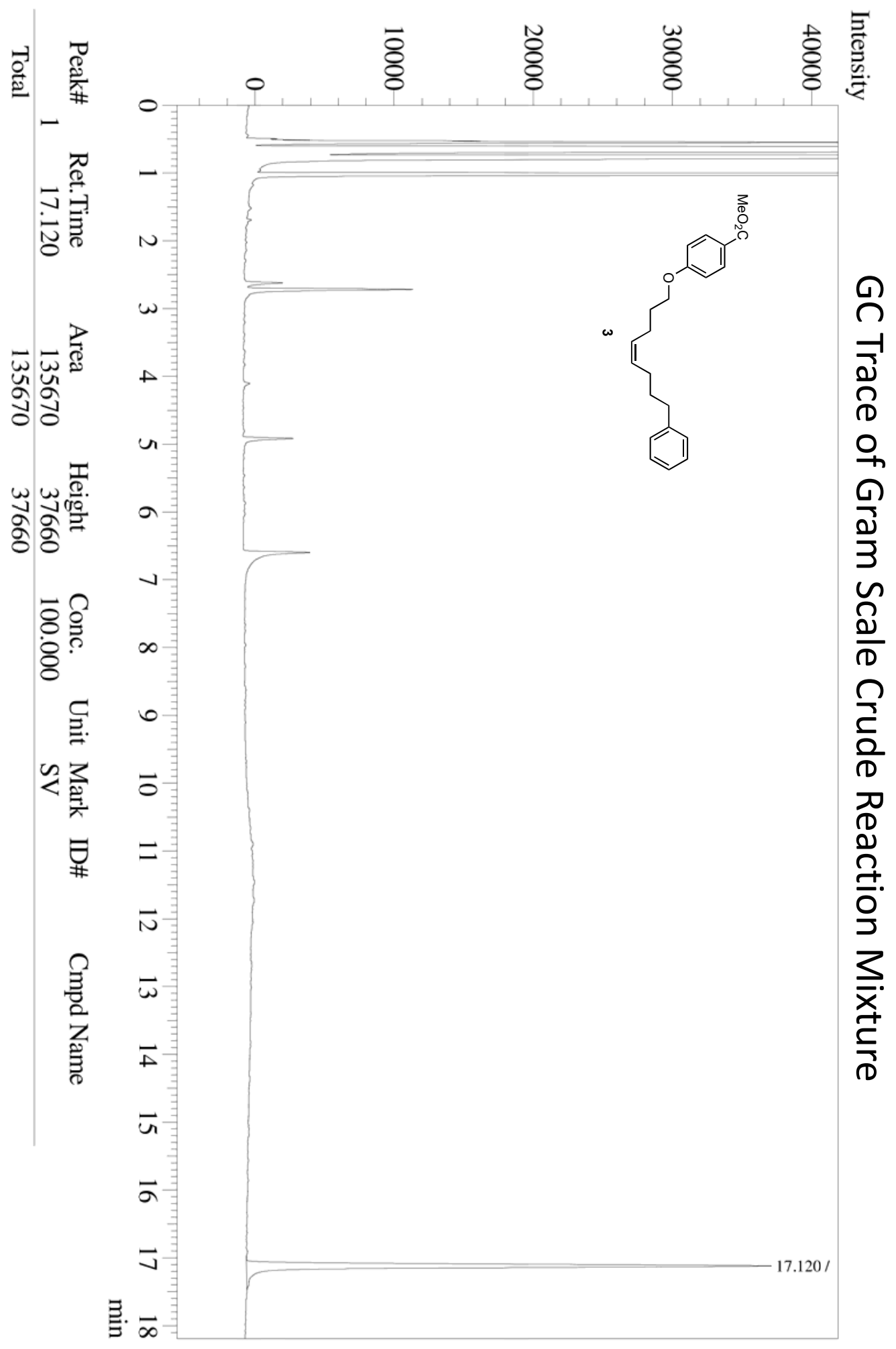




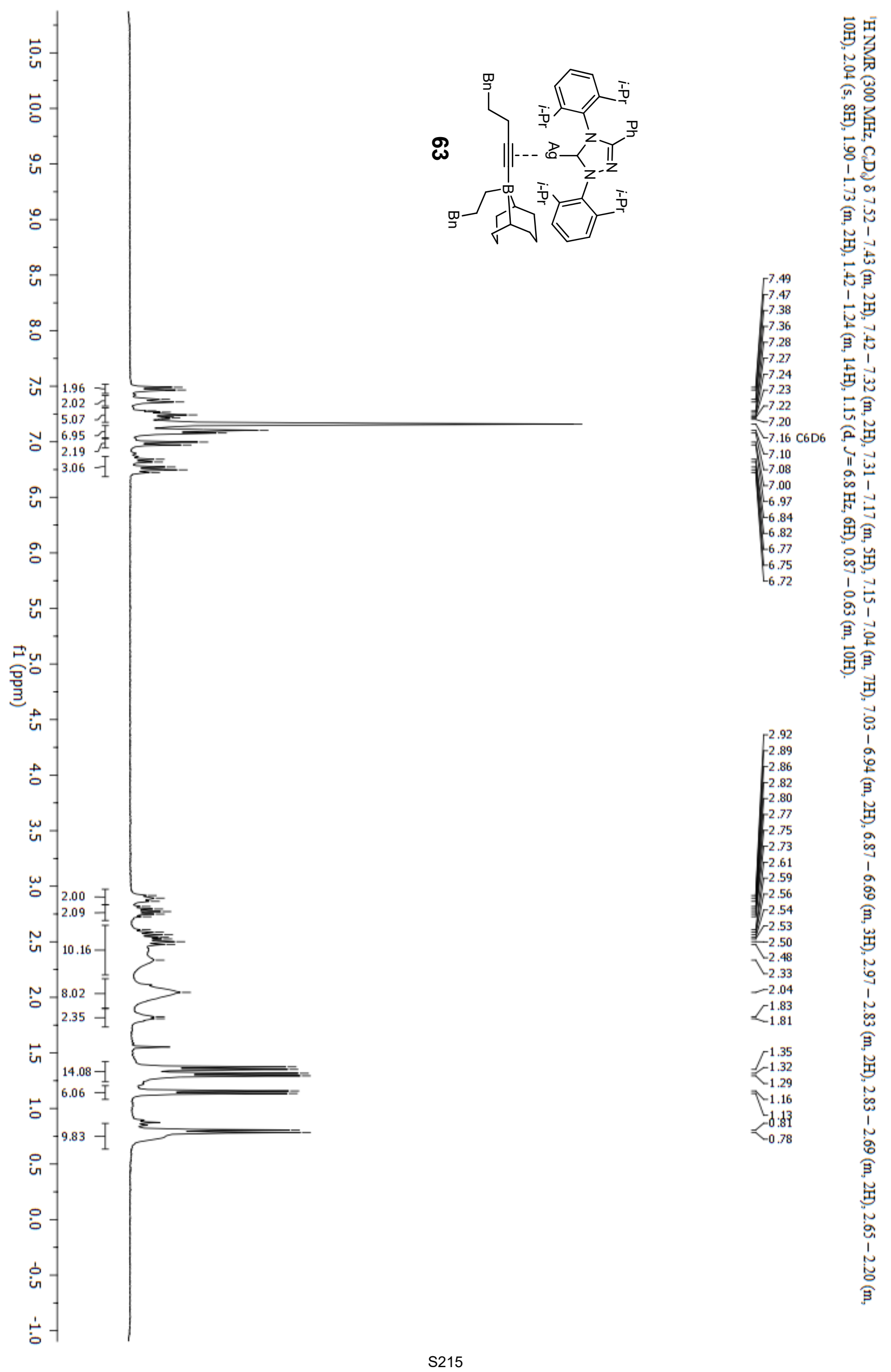

